\title{
Thermal Ignition by Vertical Cylinders
}

\author{
Thesis by \\ Silken Michelle Jones \\ In Partial Fulfillment of the Requirements for the \\ Degree of \\ Ph.D. in Aeronautics
}

\section{Caltech}

\section{CALIFORNIA INSTITUTE OF TECHNOLOGY \\ Pasadena, California}

2021

Defended November 302020 
(C) 2021

Silken Michelle Jones

ORCID: 0000-0003-3496-7191

All rights reserved except where otherwise noted 


\section{ACKNOWLEDGEMENTS}

I am very grateful to my thesis advisor, Professor Shepherd, for his guidance and mentorship over my graduate career. I could not have asked for a better advisor. His encouragement has helped me grow as a scientist and his advice has helped me persevere through research challenges. He trusted me to work independently but was always available for counsel when I needed extra help, despite his busy schedule. I don't think I can overstate how much I appreciate the time and effort he has put in to my education.

I also want to thank my committee members, Professors Guillaume Blanquart, Beverly McKeon, and Joanna Austin. I very much appreciate their time and insights. I also want to thank Professor Dan Meiron for being a committee member on my candidacy exam. I enjoyed participating in Professor Blanquart's group lunches and learning from his students as well as having a chance to present my own work. I would also like to thank Professor McKeon for encouraging me to stay at GALCIT while I was struggling as a master's student. It made a huge difference in my graduate career, and I am so glad that I stayed on for my Ph.D.

Thank you to all of the members of the Explosion Dynamics Laboratory over the years. I have received so much guidance and friendship from the group members

over my time in the lab. In particular, I want to thank Dr. Stephanie Coronel, who showed me the ropes in the laboratory and trusted me to take over (and eventually transform) her experiments. She is a great mentor, and a wonderful friend. I also want to thank Dr. Josue Melguizo-Gavilanes, who was instrumental in providing guidance as I wrote my first academic paper. He helped me figure out how to tell a good scientific story. I want to thank Dr. Jean-Christophe Veilleux; I always looked up to him as an example of a brilliant and dedicated graduate student. I want to thank my current lab members, Conor Martin, Dr. Yunliang Qi, and Donner Schoeffler. I have very much enjoyed my time with them as a fellow lab member, and I look forward to hearing more about their research in the future.

This work was made possible by The Boeing Company through the Strategic Research and Development Relationship Agreement CT-BA-GTA-1. In particular I 
would like to thank Drs. Jason Damazo, Phil Boettcher, and Brad Moravec at Boeing, who were our close collaborators.

I want to thank my friends and classmates, Mallory Neet and Joel Lawson. They were not only my study buddies during the master's year, but also good friends, excellent sources of research advice and encouragment, and always willing to lend a hand in the lab. Thank you also to Mallory for being my roommate for three years; I had a lot of fun with her outside of academic work.

Thank you to my parents, Tiffany and Mike Jones. They always encouraged me to follow my interests, and fostered my love of science from an early age. I remember watching NASA web seminars with my dad and going to science museums with my mom. Thank you for all your love, encouragement, and support. You have guided me towards the person I am today. I couldn't have done it without you.

I also want to thank Anders Knight for all his encouragement, support, and love, and for keeping me sane through the process of writing up my thesis. 


\section{ABSTRACT}

Accidental thermal ignition events present a significant hazard to the aviation industry. There is scarcity of experimental data on ignition by external natural convection flows for surface areas larger than $10 \mathrm{~cm}^{2}$. In this work, thermal ignition of external natural convection flows by vertical cylinders is investigated. The effect of geometry is studied by resistively heating stainless steel cylinders of various sizes in a stoichiometric n-hexane and air mixture at $298 \mathrm{~K}$ and 1 bar. Cylinder lengths range from 12.7 to $25.4 \mathrm{~cm}$, and cylinder surface areas vary from 25 to $200 \mathrm{~cm}^{2}$. Logistic regression is used to provide statistical information about the ignition threshold ( $50 \%$ probability of ignition). The maximum ignition threshold found is $1117 \mathrm{~K}$ for a cylinder $12.7 \mathrm{~cm}$ long and $50 \mathrm{~cm}^{2}$ in surface area. The minimum ignition threshold found is $1019 \mathrm{~K}$ for a cylinder $25.4 \mathrm{~cm}$ long and $200 \mathrm{~cm}^{2}$ in surface area. The maximum uncertainty on these ignition thresholds is $\pm 29 \mathrm{~K}$, which comes from the maximum uncertainty on the pyrometer measurement used to record cylinder surface temperatures. The dependence of ignition threshold on both surface area and length of a cylinder is found to be minor. High speed visualizations of ignition indicated that ignition occurs near the top edge of all cylinders.

The entire experimental setup is heated to allow for ignition tests with multicomponent, heavy-hydrocarbon fuels including Jet A and two surrogate fuels, Aachen and JI. The cylinder used for all testing of heavier fuels is $25.4 \mathrm{~cm}$ long and $200 \mathrm{~cm}^{2}$ in surface area. Hexane is also tested with the heated vessel to investigate the effect of ambient temperature on ignition. At an ambient temperature of $393 \mathrm{~K}$, the ignition threshold of hexane is $933 \mathrm{~K}$. Aachen has an ignition threshold of 947 $\mathrm{K}$ at an ambient temperature of $373 \mathrm{~K}$. JI has an ignition temperature of $984 \mathrm{~K}$ at an ambient temperature of $393 \mathrm{~K}$. Jet $\mathrm{A}$ has an ignition temperature of $971 \mathrm{~K}$ at an ambient temperature of $333 \mathrm{~K}$. The maximum uncertainty on these thresholds is \pm $29 \mathrm{~K} . \mathrm{JI}$ is found to be the most appropriate surrogate for Jet A.

From the experiments, two main conclusions are reached. Ignition threshold temperatures in external natural convection flows are very weakly correlated with surface area. The observed ignition thresholds do not show the drastic transition of ignition temperature with surface area that is observed in internal natural convection situations. Observed ignition thresholds for comparable surface areas (100 to 200 
$\mathrm{cm}^{2}$ ) are 500 to $600 \mathrm{~K}$ higher for external natural convection than internal natural convection. Hexane was found to be a reasonable surrogate for Jet A (38 K difference in ignition threshold) in external natural convection ignition testing. The more complex multi-component JI surrogate, while having an ignition threshold more comparable to Jet A (13 K difference in ignition threshold), requires heating the experimental apparatus and associated difficulties of fuel handling as well as the soot generation by combustion.

Two simplified models of ignition are explored. The first is an investigation of ignition chemistry using a zero-dimensional reactor and a detailed kinetic mechanism for hexane. The temperature history of the reactor is prescribed by an artificial streamline whose rate of temperature increase is parametrically varied. The results from the zero-dimensional reactor computation reveal that a gradually heated streamline exhibits two-stage ignition behavior, while a rapidly heated streamline only experiences one ignition event. The second model of ignition is a one-dimensional simulation of ignition adjacent to a cylinder at a prescribed temperature. The formulation included diffusion of species and thermal energy as well as chemical reaction and employed Lagrangian coordinates. The chemistry is modeled with a reaction mechanism for hydrogen to reduce numerical demand. Heat flux and energy balance are analysed to gain insight into the ignition dynamics. Initially, heat transfer is from the wall into the gas, and a mostly nonreactive thermal boundary layer develops around the cylinder. As reaction in the gas near the surface begins to release energy, the heat transfer decreases, and, near the critical temperature for ignition, the direction of heat flux reverses and is from the gas into the wall. In a case where ignition takes place, there is rapid rise in temperature in the gas within the thermal layer, and a propagating flame is observed to emerge into surrounding cold gas. The heat transfer from the hot combustion products results in a continuous heat flux from the gas into the wall. In a case where ignition does not take place, no flame is observed and the heat flux at the wall is slightly positive. For the critical condition just below the ignition threshold, a balance between energy release and diffusion in the adjacent gas results in a small temperature rise in the thermal layer, but a propagating flame is not created. The Van't Hoff ignition criterion of vanishing heat flux at the ignition threshold is approximately but not exactly satisfied. Contrasting the two modeling ideas, we observe that modeling adiabatic flows along computed nonreactive streamlines is useful in examining the role of detailed chemistry but lacks important diffusion effects. Including mass and thermal transport provides 
more insight into important ignition dynamics but comes at the expense of increased computational complexity. 


\section{PUBLISHED CONTENT AND CONTRIBUTIONS}

Jones, Silken and Joseph Shepherd (2020). "Thermal ignition of n-hexane air mixtures by vertical cylinders". In: International Symposium on Hazards, Prevention, and Mitigation of Industrial Explosion (ISHPMIE). Braunschweig, Germany, pp. 948-959. DOI: $10.7795 / 810.20200724$.

S.M.J. designed, constructed, and conducted the experiments, designed and implemented all software for numerical work, led analysis of results, and was the lead author in writing the manuscript.

Jones, S., J. Melguizo-Gavilanes, and J.E. Shepherd (2019). "Ignition by moving hot spheres in H2-O2-N2 environments". In: Proceedings of the Combustion Institute 37.2, pp. 1597-1604. ISSN: 15407489. DOI: 10.1016/j . proci . 2018 . 07 . 046. URL: https : / / linkinghub . elsevier . com/retrieve / pii / S1540748918304644.

S.M.J. conducted the experiments, analyzed the experimental results, and was the lead author in preparing the manuscript. 


\section{TABLE OF CONTENTS}

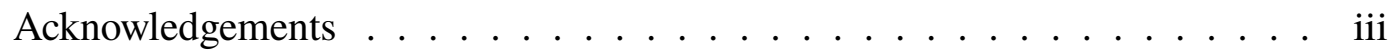

Abstract ......................... $v$

Published Content and Contributions . . . . . . . . . . . . . . . viii

Table of Contents . . . . . . . . . . . . . . . . ix

List of Illustrations . . . . . . . . . . . . . . . . . . . . . . xi

List of Tables . . . . . . . . . . . . . . . . . . . . . . . xvi

Chapter I: Introduction $\ldots \ldots \ldots \ldots$. . . . . . . . . . . . . . . . . . . . . . . . .

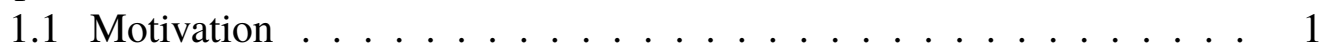

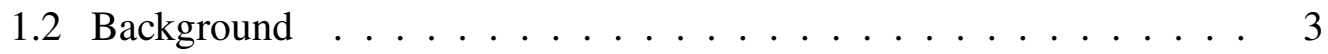

1.3 Goals of Study . . . . . . . . . . . . . . . . . . . . 16

1.4 Thesis Outline . . . . . . . . . . . . . . . . . . 19

Chapter II: Experimental Setup and Diagnostics . . . . . . . . . . . . . . 23

2.1 Combustion Vessel . . . . . . . . . . . . . . . . . . . . . . . . . . . . . . . . . . . 32

2.2 Water Cooling . . . . . . . . . . . . . . . . . . . . . . . . . . . . 32

2.3 Cylinder Characterization . . . . . . . . . . . . . . . . . . . 34

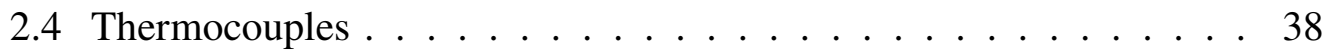

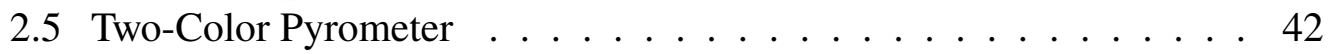

2.6 Interferometer . . . . . . . . . . . . . . . . 51

$2.7 \mathrm{OH}^{*}$ Chemiluminescence . . . . . . . . . . . . . . . . 69

Chapter III: Boundary Layer Modeling . . . . . . . . . . . . . . . . . . . . 74

3.1 Similarity Arguments for Boundary Layer Development . . . . . . 74

3.2 Interferometer Results on Cylinders in Nitrogen $\ldots . . . . . .887$

Chapter IV: Hexane-Air Tests . . . . . . . . . . . . . . . . . . . . . . . . . . . . . 91

4.1 Experimental Conditions . . . . . . . . . . . . . . . . . . 91

4.2 Experiment Procedure . . . . . . . . . . . . . . . . . . . . . 92

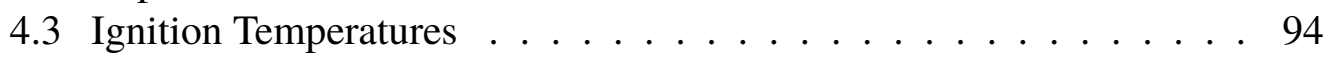

4.4 Effects of Geometry . . . . . . . . . . . . . . . . . . . . . 100

4.5 Comparison with Literature Results . . . . . . . . . . . . . . . . . 104

4.6 Visualization Studies . . . . . . . . . . . . . . . . . . . . . . . . . . 109

Chapter V: Heavier Fuels: Jet A and Surrogates . . . . . . . . . . . . . . . 155

5.1 Setup and Experimental Procedure . . . . . . . . . . . . . 156

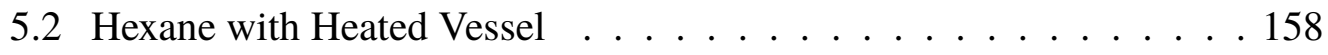

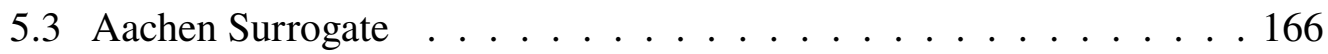

5.4 JI Unified Surrogate . . . . . . . . . . . . . . . . . . . . . . . . . . 169

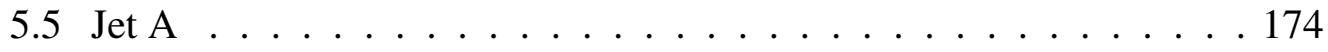

5.6 Discussion and Summary . . . . . . . . . . . . . . . . . . . . . . . . . . . . . . . . . . . . . .

Chapter VI: Modeling . . . . . . . . . . . . . . . . . . . . . . . 190

6.1 Chemistry along Streamlines . . . . . . . . . . . . . 190

6.2 One-Dimensional Simulation in Lagrangian Coordinates . . . . . . . 210 
6.3 One-Dimensional Simulation Results . . . . . . . . . . . . . . . . . 219 Chapter VII: Conclusions and Future Work ～. . . . . . . . . . . . . . . . . 274

7.1 Experimental Development . . . . . . . . . . . . . . . . 276

7.2 Geometry Effects for External Natural Convection . . . . . . . . . . 276

7.3 Jet Fuel and Surrogates . . . . . . . . . . . . . . . . . . 277

7.4 Modeling Efforts . . . . . . . . . . . . . . . . . . . 278

7.5 Future Work . . . . . . . . . . . . . . . . . . . . . 279

Appendix A: Component Lists . . . . . . . . . . . . . . . . . . . 283

A.1 Pyrometer Components . . . . . . . . . . . . . . . . 283

A.2 Interferometer Components . . . . . . . . . . . . . . . . 283

Appendix B: Interferometer Post-Processed Results, Nitrogen . . . . . . . . . 285

Appendix C: Interferometer Post-Processed Results, Hexane . . . . . . . . . 322

Appendix D: Interferometer Post-Processed Results, Hydrogen . . . . . . . . 401

Appendix E: One-Dimensional Simulation Results . . . . . . . . . . . . . . 407

Appendix F: Test Spreadsheet. . . . . . . . . . . . . . . . . . . . . . . . . . 409

F.1 Hexane . . . . . . . . . . . . . . . . . . . . 409

F.2 High-Temperature Hexane . . . . . . . . . . . . . . . . . . . . 421

F.3 Aachen . . . . . . . . . . . . . . . . . . . 424

F.4 JI . . . . . . . . . . . . . . . . . . . . . . 427

F.5 Jet A . . . . . . . . . . . . . . . . . . . . . 430

Appendix G: Engineering Drawings . . . . . . . . . . . . . . . 433

G.1 Support Structure . . . . . . . . . . . . . . . . . . . 433

G.2 Test Cylinders . . . . . . . . . . . . . . . . . . . . . . . . 440

Appendix H: Experimental Checklists . . . . . . . . . . . . . . 478 


\section{LIST OF ILLUSTRATIONS}

Number Page

1.1 Comparison of literature on thermal ignition. . . . . . . . . . . . . 17

1.2 Results of ignition temperature with surface area from Kuchta (1965). 18

2.1 Experimental setup. . . . . . . . . . . . . . . 24

2.2 Trace of typical cylinder heating. . . . . . . . . . . . . 25

2.3 View of connection of test piece to support structure. . . . . . . . . . 26

2.4 Ten test cylinders pictured together. . . . . . . . . . . . . . . 27

2.5 One-dimensional model of heat transfer within stainless steel cylinder. 30

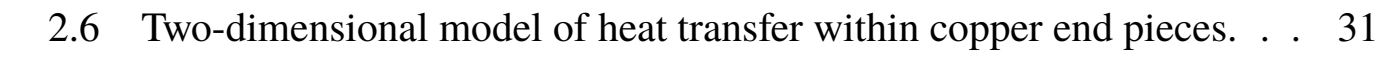

2.7 Benchtop setup for cylinder characterization. . . . . . . . . . . 35

2.8 Results from characterization of cylinders at $800 \mathrm{~K}$. . . . . . . . . . 36

2.9 Schematic of thermocouples . . . . . . . . . . . . . . . 39

2.10 Example of current coupling. . . . . . . . . . . . . . 41

2.11 Top TC calibration. . . . . . . . . . . . . . . . . . 42

2.12 Heating calibration setup. . . . . . . . . . . . . . . . 42

2.13 Blackbody irradiance from Planck's law. . . . . . . . . . . . . . . 44

2.14 Schematic of pyrometer layout. . . . . . . . . . . . . . . 46

2.15 Calibration of pyrometer. . . . . . . . . . . . . 50

2.16 Thin lens ray tracing calculation. . . . . . . . . . . . . . . . 53

2.17 Winlens modeling of the test beam of the interferometer. . . . . . . . 54

2.18 Schematic for fiber Mach-Zehnder interferometer. . . . . . . . . . . 55

2.19 X-shaped fringes, observed at medial focus. . . . . . . . . . . . 56

2.20 Winlens astigmatism prediction. . . . . . . . . . . . 56

2.21 Progression of astigmatic fringe shape. . . . . . . . . . . . . 57

2.22 Winlens model of corrected beam. . . . . . . . . . . . . . . . . 59

2.23 Fringe shape during astigmatism correction. . . . . . . . . . . . 60

2.24 Corrected finite fringe pattern. . . . . . . . . . . . . . . 60

2.25 Raw interferogram of glowplug. . . . . . . . . . . . . . . . . . 66

2.26 Temperature field comparison. . . . . . . . . . . . . . 67

2.27 Thermal boundary layer profile comparison for validation. . . . . . . 67

2.28 Percent error in TBL profiles for validation. . . . . . . . . . . . . . 68

2.29 Schematic of $\mathrm{OH}^{*}$ chemiluminescence setup. . . . . . . . . . . . 70 
3.1 Results of similarity argument. . . . . . . . . . . . . . . . 78

3.2 Comparison of temperature profile along wall-normal direction. . . . 80

3.3 Differences in numerical temperature profile. . . . . . . . . . 81

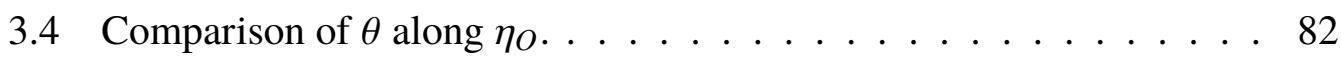

3.5 Differences in $\theta$ along $\eta_{O} . \ldots \ldots \ldots \ldots \ldots$

$3.6 \quad$ Wall-parallel velocity, $s$ along $\eta_{O} . \ldots \ldots \ldots \ldots \ldots$. . . . . 84

3.7 Raw interferograms for nitrogen tests at $700 \mathrm{~K}$ and $1100 \mathrm{~K}$ wall

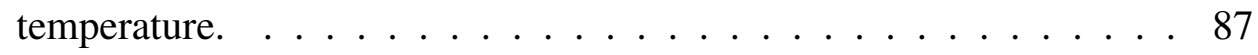

3.8 Interferometry temperature field results at $700 \mathrm{~K} . \ldots \ldots \ldots 8$

3.9 Interferometry temperature field results at $1100 \mathrm{~K} \ldots \ldots . \ldots 88$

3.10 Interferometry results at $700 \mathrm{~K}$ and $1100 \mathrm{~K}$ wall temperature. $\ldots .89$

4.1 Ignition data and probablistic model of ignition for cylinder 50A. . . 95

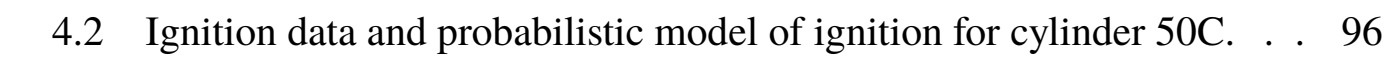

4.3 Ignition data and probabilistic model of ignition for cylinder 75B. . . 96

4.4 Ignition data and probabilistic model of ignition for cylinder 100A. . 96

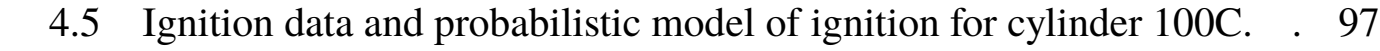

4.6 Ignition data and probabilistic model of ignition for cylinder 200A. . 97

4.7 Ignition data for cylinder 25A. . . . . . . . . . . . . . . . . 97

4.8 Ignition during ramp versus during steady-state beahvior. . . . . . . 99

4.9 Scatter plot of $\tau_{i g n} \mathrm{vs} T_{i g n} . \ldots \ldots \ldots \ldots \ldots$

4.10 Plot of $\mathrm{T}_{i g n}$ vs length for all cylinders. . . . . . . . . . . . 100

4.11 Plot of $\mathrm{T}_{i g n}$ vs diameter for all cylinders. . . . . . . . . . . 101

4.12 Plot of $\mathrm{T}_{i g n} \mathrm{vs} \mathrm{L} / \mathrm{D}$ for all cylinders. $\ldots \ldots \ldots \ldots 10 \ldots \ldots$

$4.13 \mathrm{~T}_{i g n}$ vs surface area at $\mathrm{L}=25.4 \mathrm{~cm} \ldots \ldots \ldots \ldots \ldots \ldots$

4.14 Ignition results compared with Kuchta (1965). . . . . . . . . . . . 105

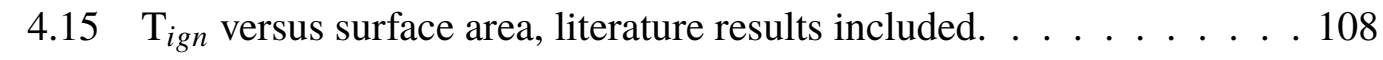

4.16 Shot 66. Temperature fields from interferometry, $\mathrm{N}_{2}$. . . . . . . 112

4.17 Shot 66. Thermal boundary layer profiles. . . . . . . . . . . . . 113

4.18 Shot 66. Percent difference in thermal temperature within the boundary layer profiles. . . . . . . . . . . . . . . . . . . . 114

4.19 Shot 31. Temperature fields from interferometry, hexane. . . . . . . 116

4.20 Shot 31. Thermal boundary layer profiles. . . . . . . . . . . . 117

4.21 Shot 31. Percent difference in thermal boundary layer profiles. . . . . 118

4.22 Shot 164. Temperature fields from interferometry, hexane. . . . . . . 119

4.23 Shot 164. Thermal boundary layer profiles. . . . . . . . . . . 120

4.24 Shot 164. Percent difference in thermal boundary layer profiles. . . . 121 
4.25 Line-of-sight depth through boundary layer. . . . . . . . . . . . . . 123

4.26 Shot 4. Temperature fields from interferometry, $\mathrm{H}_{2}$-air . . . . . . . . 124

4.27 Shot 4. Thermal boundary layer profiles . . . . . . . . . . . . . 125

4.28 Shot 4. Percent difference in thermal boundary layer profiles . . . . . 126

4.29 Mass fraction evolution for hydrogen and hexane mixtures . . . . . . 127

4.30 Dot schlieren visualization of pre-ignition from Shot 175. . . . . . 132

4.31 Dot schlieren visualization of pre-ignition from Shot 176. . . . . . . 133

4.32 Dot schlieren visualization of pre-ignition from Shot 177. . . . . . 134

4.33 Dot schlieren visualization of shots 175,176 , and 177. . . . . . . . 135

4.34 Dot schlieren visualization of ignition from Shot 175. . . . . . . . . . 136

4.35 Dot schlieren visualization of ignition from Shot 176. . . . . . . . . 137

$4.36 \quad$ Scatter plots of ignition location. . . . . . . . . . . . . . . . . 139

4.37 Comparison of Caltech data with Ono correlation. . . . . . . . . . . 141

$4.38 \mathrm{OH}^{*}$ chemiluminescence visualization of ignition from shot 179. . . 142

4.39 Visualization of ignition via interferometry, schlieren, and $\mathrm{OH}^{*}$. . . . 143

$4.40 \quad$ Flame position for Shot 179. . . . . . . . . . . . . . . . . 144

4.41 Schlieren visualization of boundary layer, laminar and disturbed. . . . 150

4.42 Interferometer visualization of boundary layer, laminar and disturbed. 150

4.43 Sequence of images of "vortical-appearing structures". . . . . . . . . 151

5.1 Schematic of heating system for vessel. . . . . . . . . . . . . . . . 158

5.2 Ignition results $200 \mathrm{~A}$, hexane at $393 \mathrm{~K}$. . . . . . . . . . . . 160

5.3 Ignition sequence high-T hexane. . . . . . . . . . . . . 161

5.4 Flame position from shot 239. . . . . . . . . . . . . . . 162

5.5 Ambient and wall temperature effects of BL. . . . . . . . . . . 164

5.6 Ambient and wall temperature effects using ignition threshold values. 165

5.7 Ignition results for Aachen surrogate fuel with cylinder 200A. . . . . 168

5.8 Ignition sequence for Aachen surrogate fuel with cylinder 200A.

Flame front highlighted with dashed orange lines. . . . . . . . . . . . 169

5.9 Flame position from shot 202, for the Aachen mixtures. . . . . . . . 170

$5.10 \quad$ Ignition results for JI Unified surrogate fuel with cylinder 200A. . . . 172

5.11 Ignition sequence for JI unified surrogate fuel with cylinder 200A.

Flame front highlighted with dashed orange lines. . . . . . . . . . . . 173

5.12 Flame position from shot 231, for the JI mixtures. . . . . . . . . . . 174

5.13 Plumbing schematic for combustion vessel. . . . . . . . . . . . . 179

5.14 Ignition results for JI unified surrogate fuel with cylinder 200A. . . . 181 
5.15 Ignition sequence for Jet A testing with cylinder 200A. Flame front highlighted with dashed orange lines. . . . . . . . . . . . . . 181

5.16 Flame position from shot 250, for the Jet A mixtures. . . . . . . . . 182

5.17 Ignition results for all surrogate testing. . . . . . . . . . . . . . 183

5.18 Ignition results for all surrogate testing. . . . . . . . . . . . . . . . 184

5.19 Ignition results for all surrogate testing. . . . . . . . . . . . . . . . 184

5.20 Ignition results for all ignition testing. . . . . . . . . . . . . . 186

5.21 Ignition results for all ignition testing compared with literature values. 187

6.1 Normalized stream function from similarity solutions. . . . . . . . . 192

6.2 Stream function temperature history from similarity solutions. . . . . 193

6.3 Temperature history of streamlines, inert and reactive. . . . . . . . 195

6.4 Temperature history of artificial streamlines. . . . . . . . . . . . . 197

6.5 Reactive temperature history of artificial stream functions. . . . . . . 197

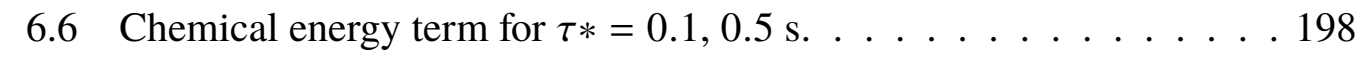

6.7 Major and minor species mole fraction for $\tau *=0.1 \mathrm{~s}$. . . . . . . . 202

6.8 Major and minor species mole fraction for $\tau *=0.5 \mathrm{~s}$. . . . . . . 203

6.9 Reaction pathway diagram for peak of first stage of ignition. . . . . . 206

$6.10 \quad$ Reaction pathway diagram for end of first stage of ignition. . . . . . . 207

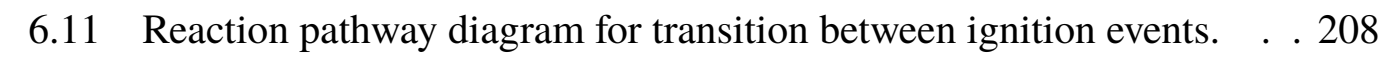

6.12 Reaction pathway diagram for second stage of ignition. . . . . . . . . 209

6.13 Ignition delay based on maximum $\frac{d T}{d t} . \ldots$. . . . . . . . . . . 221

6.14 Ignition delay based on maximum $\frac{d Y_{O H}}{d t}$. . . . . . . . . . . . . 222

6.15 Temperature field vs $\psi$ and $\mathrm{t}, T_{w}=1200 \mathrm{~K}$. . . . . . . . . . . . 224

6.16 Temperature field vs $\Delta \mathrm{r}$ and $\mathrm{t}, T_{w}=1200 \mathrm{~K}$. . . . . . . . . . 225

6.17 Magnified temperature field vs $\Delta \mathrm{r}$ and $\mathrm{t}, T_{w}=1200 \mathrm{~K} . \quad \ldots . . .226$

$6.18 \quad \mathrm{Y}_{H_{2}}$ field vs $\Delta \mathrm{r}$ and $\mathrm{t}, T_{w}=1200 \mathrm{~K} . \ldots \ldots 27$

$6.19 \mathrm{Y}_{\mathrm{HO}_{2}}$ field $\mathrm{vs} \Delta \mathrm{r}$ and $\mathrm{t}, T_{w}=1200 \mathrm{~K}$. . . . . . . . . . . 228

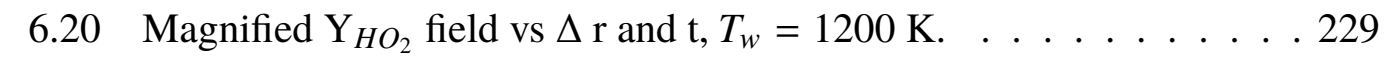

6.21 Graphical representation of flame thickness. . . . . . . . . . . . . 229

6.22 T field vs $\Delta \mathrm{r}$ and $\mathrm{t}, T_{w}=917.1875 \mathrm{~K} . \ldots . \ldots . . \ldots 230$

$6.23 \quad \mathrm{Y}_{H_{2}}$ field vs $\Delta \mathrm{r}$ and $\mathrm{t}, T_{w}=917.1875 \mathrm{~K}$. . . . . . . . . . 231

$6.24 \mathrm{Y}_{\mathrm{HO}_{2}}$ field vs r and $\mathrm{t}, T_{w}=917.1875 \mathrm{~K} . \ldots . \ldots . . \ldots 232$

6.25 Magnified $\mathrm{Y}_{\mathrm{HO}_{2}}$ field vs $\mathrm{r}$ and $\mathrm{t}, T_{w}=917.1875 \mathrm{~K}$. . . . . . . . 233

6.26 T field vs $\Delta \mathrm{r}$ and $\mathrm{t}, T_{w}=915.625 \mathrm{~K} . \ldots . \ldots . . \ldots 234$

$6.27 \quad \mathrm{Y}_{H_{2}}$ field vs $\Delta \mathrm{r}$ and $\mathrm{t}, T_{w}=915.625 \mathrm{~K} . \ldots . \ldots 235$

$6.28 \mathrm{Y}_{O H}$ field vs $\Delta \mathrm{r}$ and $\mathrm{t}, T_{w}=915.625 \mathrm{~K} . \ldots . \ldots . \ldots 235$ 
6.29 Radial profiles of T in time, supercritical case. . . . . . . . . . . 237

6.30 Radial profiles of $\mathrm{Y}_{O H}$ in time, supercritical case. . . . . . . . . . . 237

6.31 Radial profiles of $\mathrm{Y}_{\mathrm{HO}_{2}}$ in time, supercritical case. . . . . . . . . . 238

6.32 Radial profiles of $\mathrm{T}$ in time, critical case . . . . . . . . . . . . . 239

6.33 Radial profiles of $\mathrm{Y}_{O H}$ in time, critical case . . . . . . . . . . . . . 240

6.34 Radial profiles of $\mathrm{Y}_{\mathrm{HO}_{2}}$ in time, critical case . . . . . . . . . . . 241

6.35 Radial profiles of T in time, subcritical case. . . . . . . . . . . . 242

6.36 Radial profiles of $\mathrm{Y}_{O H}$ in time, subcritical case. . . . . . . . . . . . 243

6.37 Radial profiles of $\mathrm{Y}_{\mathrm{HO}_{2}}$ in time, subcritical case. . . . . . . . . . . . 244

6.38 All energy equation terms, supercritical case. . . . . . . . . . . . . 246

6.39 Energy balance during conduction stage, supercritical case. . . . . 247

6.40 Energy balance during early reaction, supercritical case. . . . . . . 247

6.41 Energy balance during strengthening reaction, supercritical case. . . . 248

6.42 Energy balance during flame formation, supercritical case. . . . . . 249

6.43 Energy balance during flame propagation, supercritical case. . . . . . 250

6.44 All energy equation terms, critical case. . . . . . . . . . . . . 251

6.45 Energy balance during conduction stage, critical case. . . . . . . . 252

6.46 Energy balance during early reaction, critical case. . . . . . . . . . 252

6.47 Energy balance during flame formation, critical case. . . . . . . . . 253

6.48 Energy balance during flame propagation, critical case. . . . . . . . 253

6.49 All energy equation terms, subcritical case. . . . . . . . . . . . . 255

6.50 Energy balance during conduction stage, supercritical case. . . . . . 255

6.51 Energy balance during early reaction, subcritical case. . . . . . . . 256

6.52 Energy balance during flame formation, subcritical case. . . . . . . . 256

6.53 Energy balance during flame propagation, subcritical case. . . . . . . 257

6.54 Heat flux next to wall for all $T_{w} \ldots \ldots$. . . . . . . . . . 259

6.55 Heat flux next to wall for critical, subcritical cases. . . . . . . . . . 260

6.56 Temperature profiles of critical case, up to $\mathrm{t}=\tau_{i g n, j_{q}} . \ldots . . . .261$

6.57 Temperature profiles of subcritical case. . . . . . . . . . . . . 261

$6.58 Y_{\mathrm{HO}_{2}}$ profiles of critical case, up to $\mathrm{t}=\tau_{i g n, j_{q}} . \ldots \ldots 262$

$6.59 \quad Y_{\mathrm{HO}_{2}}$ profiles of subcritical case, up to $\mathrm{t}=\tau_{i g n, j_{q}} \cdot \ldots 263$

6.60 Boundary layer profile comparison. . . . . . . . . . . . . . 265

6.61 Wall-parallel velocity profile from similarity solution. . . . . . . . . 266

7.1 Ignition results. . . . . . . . . . . . . . . . . 275 


\section{LIST OF TABLES}

Number Page

2.1 Dimensions of all test cylinders. . . . . . . . . . . . . . . 27

2.2 Processing constants for interferometry. . . . . . . . . . . . . . . 66

4.1 Composition of gas mixture for stoichiometric n-hexane and air tests. 92

4.2 Ignition temperatures and confidence limits for all cylinders. . . . . . 98

4.3 Summary of interferometry results in n-hexane. . . . . . . . . . . . 122

4.4 Effect of varying mixtures on interferometry parameters. . . . . . . . 130

4.5 Average ignition location for all cylinders. . . . . . . . . . . . . . . 137

4.6 Flame speed of hexane-air mixture . . . . . . . . . . . . . . . 145

4.7 Flame speed calculations for hexane at varying temperatures. . . . . . 145

5.1 Comparison of composition of jet fuel blend and surrogate fuels. . . . 156

5.2 Tables of heaters. . . . . . . . . . . . . . . . . . . . . 159

5.3 Composition breakdown of Aachen surrogate. . . . . . . . . . . . 166

5.4 Mixture composition for stoichiometric Aachen-air mixture. . . . . 166

5.5 Composition breakdown of JI surrogate. . . . . . . . . . . . . . 170

5.6 Mixture composition for stoichiometric JI-air mixture. . . . . . . . . 171

5.7 Experimental mixture composition for JI-air mixture. . . . . . . . . . 171

5.8 Mixture composition for stoichiometric Jet A-air mixture. . . . . . 176

5.9 Results of ignition tests with normal and degassed fuel. . . . . . . . 180

5.10 Ignition results for surrogate testing. . . . . . . . . . . . . . 183

6.1 Ignition delay time comparison using $\tau_{i g n, T}$ and $\tau_{i g n, j_{q}} \cdot \ldots$. . . . . . 260

6.2 Timescale comparison between experiments and 1D simulation. . . . 264 
Chapter 1

\section{INTRODUCTION}

\subsection{Motivation}

Understanding combustion hazards is of critical importance to many industries. There are three main types of combustion hazards: fires, deflagrations, and detonations. A fire generally refers to a burning pool of fuel in liquid or solid form. A deflagration is a subsonic combustion wave. These combustion events can propagate at speeds on the order of 0.01 to $100 \mathrm{~m} / \mathrm{s}$ and cause large temperature rises (1000 to $3000 \mathrm{~K}$ ) across the flame front under constant pressure conditions. When contained, a deflagration can increase the pressure by a factor of 5 to 10. Detonations are supersonic propagating shock waves accompanied by exothermic heat release and can reach propagation speeds in excess of $1000 \mathrm{~m} / \mathrm{s}$. Detonations cause very large pressure and temperature increases across the combustion wave; ChapmanJouguet theory predicts pressures and temperatures up to $5 \mathrm{MPa}$ and 2000 to 3000 $\mathrm{K}$, respectively. Deflagrations and detonations are known as explosion hazards and pose a serious threat to structures and human safety. This work focuses on deflagrations in premixed fuel-oxidizer mixtures as a potential source of an explosion hazard.

Deflagrations can ignite by several distinct means. A deflagration can be started by an open flame, for example the pilot flame in an oven or gas fireplace. Ignition can also be started by a spark, such as in the burners of gas stoves. Even when the

presence of open flames and electrical sparks is mitigated, ignition can occur by the presence of a surface at elevated temperature. In such situations, thermal energy is added to the system until ignition spontaneously occurs. Such ignition events are classified as thermal ignition. Thermal ignition is of great interest in industries such as aviation, nuclear power, and chemical processing and is the focus of this investigation.

To mitigate the risk of a thermal ignition event that could lead to an explosion, knowledge surrounding that ignition event must be well understood. This includes factors like mixture composition, initial pressure and temperature, and conditions of the hot surface including size, orientation, and flow configuration. Furthermore, 
an appropriate method of quantifying ignition risk is needed. This compilation of knowledge can inform regulations, rules, and design criteria that will mitigate explosion risks.

The focus for this investigation has been on explosion hazards in the aviation industry. The Federal Aviation Administration (FAA) has regulations on the maximum temperature of a hot surface in an aircraft. These regulations limit the maximum allowable temperature in a flammable leakage zone (any area where flammable liquids or vapors are not intended to be present, but where they might exist due to leakage from flammable fluid carrying components) to $477 \mathrm{~K}$ (Federal Aviation Administration, 2018). This regulation is informed by the results from the ASTM test for autoignition temperatures, which involves injecting fuel into a heated vessel and using visual inspection to determine if ignition occurs within 10 minutes (ASTM International, 2015). The ASTM test considers thermal ignition by natural convection within a heated spherical vessel aproximately $10 \mathrm{~cm}$ in diameter (or 300 $\mathrm{cm}^{2}$ in surface area) (ASTM International, 2015), and the FAA regulations limit the maximum allowable temperature regardless of hazard size or flow configuration (Federal Aviation Administration, 2018).

While these tests and regulations are informative, they also exclude a number of potential hazard situations on an aircraft. For example, the areas on an aircraft that are likely sources of a thermal ignition event are often very different in size and flow configuration than the ASTM test. These include components such as the auxiliary power unit, the engine with its surrounding piping and firewall, and the fuel tank. These components exhibit a wide range of sizes and flow configurations that are not accounted for in the regulations and standardized tests, including characteristic lengths ranging from centimeters to meters and flow configurations from external natural convection to internal forced convection. Section 1.2 details existing literature on a variety of thermal ignition studies, including some with conditions relevant to aircraft thermal ignition hazards. 


\subsection{Background}

\section{Historical Thermal Ignition Work}

Studies of thermal ignition have long been of interest due to their application to safety concerns in many industries. Babrauskas (2003) provides a thorough review of historical efforts on both experiments and modeling of ignition, and is a good source for general information about ignition. Here we will provide a brief review of some relevant historical ignition studies. Early studies primarily approached thermal ignition studies from a mining safety outlook, including work done by Coward and Guest (1927). Coward and Guest investigated the ignition of natural gas by a heated metal strip in conjunction with the U.S. Bureau of Mines by varying strip size, gas composition, and surface material (Coward and Guest, 1927). They found that a larger surface led to a lower ignition temperature by approximately $70 \mathrm{~K}$, and that highly catalytic platinum yielded the highest ignition temperatures when compared to other materials including nickel, gold, copper, tungsten, and stainless steel.

Silver (1937) studied ignition by moving hot particles by injecting spheres ranging from 0.1 to $0.5 \mathrm{~cm}$ in diameter, made from platinum and quartz, into coal-gas, pentane, and hydrogen mixtures. It was found that the material of the sphere had little effect on ignition temperatures and that ignition temperatures were most sensitive to sphere size and temperature (Silver, 1937). The flow velocity ranged from 2 to $5 \mathrm{~m} / \mathrm{s}$ but was not systematically studied. Similarly, Patterson (1940) studied ignition by spherical particles in the same size range for the same fuels but with the flow speed fixed at $1.2 \mathrm{~m} / \mathrm{s}$. The ignition temperatures were found to be lower for these slower moving particles than for Silver's particles otherwise under the same conditions. A variety of particle materials was again investigated, including platinum, quartz, and nickel among others, and Patterson found ignition temperatures were similar for all materials except platinum, which had a relatively high ignition threshold.

Mullen, Fenn, and Irby (1948) studied ignition by a reactive mixture flowing over a heated cylindrical rod arranged horizontally with flow rates from 15 to $150 \mathrm{~m} / \mathrm{s}$. The effect of surface material was studied by testing stainless steel, nichrome, and platinum. Like Patterson (1940), the catalytic effect of platinum was found to cause a significantly higher ignition temperature, while nichrome and stainless steel behaved similarly to one another (Mullen, Fenn, and Irby, 1948). It was noted that flow 
in the vicinity of the stagnation point or recirculation zone of the rod was critical in achieving the lowest possible ignition temperatures.

Babrauskas (2003) provides a more thorough review of historical efforts on both experiments and modeling of ignition, and is generally a useful source on the topic of ignition. The above historical literature hints at some distinctions in thermal ignition that will be explored more fully in the subsequent sections of this chapter, as well as in following chapters of this thesis. This includes literature on thermal ignition by naturally convecting flows, where the flow occur inside a vessel or can flow across the exterior of surfaces such as plates, cylinders, and spheres. Thermal ignition by forced convection will also be explored, including forced convection over plates, cylinders, and spheres. The importance of ignition location and surface material will also be explored throughout these sections.

Non-dimensional numbers like the Grashof and Reynolds number can be used to characterize the flow regime. The Reynolds number, $R e=\frac{\rho U L}{\mu}$, is the ratio of inertial to viscous forces in a fluid flow; $\rho$ is fluid density, $U$ is free stream flow velocity, $L$ is characteristic size, and $\mu$ is dynamic viscosity. The Reynolds number is typically used to characterize the flow regime in forced convection as laminar, turbulent, or transitional. The Grashof number is typically used as an analog to the Reynolds number in free convection flows. $G r=\frac{g \beta\left(T_{s}-T_{\infty}\right) L^{3}}{v^{2}}, g$ is gravitational acceleration, $\beta$ is coefficient of thermal expansion, $T_{s}$ is surface temperature, $T_{\infty}$ is ambient temperture, and $v$ is kinematic viscosity. The Grashof number is the ratio of buoyancy to viscous forces in a free convection flow.

\section{Thermal Ignition by Natural Convection}

We will first review literature on thermal ignition by naturally convecting flows. Natural convection, also known as free convection, is a flow where fluid motion is driven by density differences in the fluid occurring due to temperature gradients. That is, the motion of the flow is driven by buoyancy forces. Natural convection can occur within a heated environment to generate an internal naturally convecting flow, such as within a heated vessel. In such situations, the fluid is generally confined and recirculates within the vessel. External natural convection occurs when the exterior of a heated surface is exposed to a fluid; in contrast to internal flows the fluid is not trapped within the heated surface. The level of confinement and recirculation of the 
flow is determined by the unheated boundary conditions. Studies of both external and internal natural convection flows are documented in the following sections.

\section{Internal Natural Convection: Vessels}

The ASTM E659 test provides a standardized testing method for characterizing the autoignition temperature of fuels (ASTM International, 2015). It consists of injecting up to $5 \mathrm{~mL}$ of fuel into a heated $500-\mathrm{mL}$ flask and monitoring for 10 minutes post-injection to watch for an ignition event. This is an example of a thermal ignition event occuring by internal natural convection. There are a number of shortcomings to this methodology, including unknown mixture compositions as the vessel is open to the atmosphere, and defining ignition events as a flame visible to the naked eye.

Kuchta, Bartkowiak, and Zabetakis (1965) studied ignition by surfaces ranging in area from less than $1 \mathrm{~cm}^{2}$ to $200 \mathrm{~cm}^{2}$ with n-hexane, -octane, -decane, JP-6, and engine oil fuel mixtures. Surfaces with areas greater than approximately $40 \mathrm{~cm}^{2}$ were heated vessels with internal naturally convecting flows. These vessels came in a variety of geometries including spherical, cylindrical, and erlenmeyer flasks. For all fuels except engine oil, they found relatively high ignition temperatures, approximately $900 \mathrm{~K}$, for the smallest vessels and observed a sharp drop in ignition temperature starting around $75 \mathrm{~cm}^{2}$. Ignition temperatures leveled off to approximately $550 \mathrm{~K}$ for vessels larger than approximately $100 \mathrm{~cm}^{2}$.

White (1967) also investigated internal natural convection by studying the effect of vessel size and surface to volume ratio on thermal ignition within heated vessels with keronese fuel mixtures. The vessels ranged in surface area from 250 to over 6600 $\mathrm{cm}^{2}$. At atmospheric pressure, the ignition temperature decreased with increasing vessel size, from $530 \mathrm{~K}$ at $250 \mathrm{~cm}^{2}$ to $481 \mathrm{~K}$ at $6600 \mathrm{~cm}^{2}$. The surface to volume ratio of the largest vessel ( $46 \mathrm{~cm}$ diameter) was also altered by adding inserts with different surfaces areas, and it was found that an increased surface area to volume ratio (in the range of 0.33 to 2.1 ) increased the ignition temperature by approximately $15 \mathrm{~K}$ (White, 1967).

Boettcher (2012) investigated the effects of pressure, mixture composition, and heating rate on thermal ignition by internal natural convection within a vessel $380 \mathrm{~cm}^{2}$ in area. Hexane air mixtures were studied as a surrogate for jet fuel. It was found that a regular ignition event, characterized by a sharp pressure and temperature rise 
and immediate fuel depletion, occurred at approximately $475 \mathrm{~K}$ for a heating rate of $11 \mathrm{~K} / \mathrm{min}$. For a slower heating rate of $4.25 \mathrm{~K} / \mathrm{min}$, no sharp ignition event occurred, and instead, fuel was slowly consumed with minimal pressure rise. Increasing the mixture composition decreased the minimum heating rate at which a standard ignition event occurred.

\section{External Natural Convection: Plates and Cylinders}

Unlike autoignition tests for natural convection within heated vessels (ASTM International, 2015), there is no standardized test methodology for hot surface ignition which generally involves an external natural or forced convection flow. This section will focus on literature documenting studies of thermal ignition by external natural convection flows, and distinctions will be made between planar surfaces and cylindrical ones. The following section will document hot surface ignition by external forced convection flows.

As mentioned previously, Coward and Guest (1927) studied thermal ignition of natural gas mixtures by metal strips. The effect of surface material was investigated by using an inert metal (nickel) and a catalytic one (platinum). Ignition by heated nickel strips was achieved at temperatures over $1273 \mathrm{~K}$. It was found that platinum led to higher ignition temperatures than nickel due to catalytic effects inhibiting ignition. Nickel is assumed to be inert due the the oxide layer that develops during heating. Furthermore, an increase of surface area from 1.6 to $9.7 \mathrm{~cm}^{2}$ led to a decrease in ignition temperature by about $70 \mathrm{~K}$ for nickel strips.

Adomeit (1965) studied thermal ignition by vertical cylinders suddenly heated in a quiescent flow. Hydrogen, propane, and pentane mixtures were studied and the cylinders had a surface area of approximately $3.8 \mathrm{~cm}^{2}$. The cylinders were heated very quickly with a timescale on the order of 0.1 milliseconds, and a Mach-Zehnder interferometer was used to measure the temperature of the gas surrounding the cylinder. Ignition delays were measured to be between 0.002 and 0.04 seconds. Hydrogen, propane, and pentane mixtures achieved ignition at temperatures of 1013, 1163, and $1323 \mathrm{~K}$ respectively. Adomeit (1965) concluded that the temperature gradient near the wall must be small such that heat generation due to reaction could exceed heat losses in order to achieve thermal runaway and start an ignition event. 
Cutler (1974) also studied ignition by heated plates in methane-air mixtures for very short heating durations, on the order of a $1 \mathrm{~ms}$. The surfaces were made of tungsten foil and varied in surface area from 1.5 to $3.8 \mathrm{~cm}^{2}$ by changing the width of the foil. Surface temperature was measured via an optical pyrometer, and the ignition temperature was found to be inversely proportional to surface area. A surface 1.8 $\mathrm{cm}^{2}$ was found to cause ignition at temperatures just over $2000 \mathrm{~K}$. Cutler (1978) furthered this work with propane-air and ethylene-air mixtures for plane surfaces from 2.9 to $7.4 \mathrm{~cm}^{2}$. Propane had a minimum ignition temperature around $1700 \mathrm{~K}$, and ethylene had a minimum ignition temperature just over $1100 \mathrm{~K}$.

Further studies of thermal ignition by external natural convection were carried out by Ono et al. (1976). The surface was a vertical plate made of stainless steel and achieved a heated area from 1.5 to $9 \mathrm{~cm}^{2}$ by varying the height of the plate. Propane-air, methane-air, ethanol-air, and diethyl ether-air mixtures were investigated. Propane ignited around $1290 \mathrm{~K}$ for a $0.5 \mathrm{~cm}$ high $\left(1.5 \mathrm{~cm}^{2}\right)$ plate and about $1230 \mathrm{~K}$ for a $3 \mathrm{~cm}$ high $\left(9 \mathrm{~cm}^{2}\right)$ plate. The surfaces were held just below ignition temperatures, and then the temperature was raised until ignition occurred. As a result, it is possible there are low-temperature chemistry effects that are not considered in the study.

Cairnie, Harrison, and Morgan (1981) studied thermal ignition of diethyl ether-air mixtures with a much larger vertical plate totaling $130 \mathrm{~cm}^{2}$. Diethyl ether was chosen for its high reactivity at low temperatures (Cairnie, Harrison, and Morgan, 1981). They found ignition occurred at the very top of the vertical plates for a surface temperature of $846 \mathrm{~K}$, preceded by the development of a cool flame lower on the plate surface. They noted the large difference in ignition temperature between their vertical plate and that of the standardized ASTM tests (433 K for diethyl ether). Futher, they developed a local non-similarity solution to model the reacting natural convection flow over a vertical plate and used a one-step reaction model, which was found insufficiently complex to capture the cool-flame behavior observed in experiments. This work was extended by Harrison and Cairnie (1988) with experiments using a $270 \mathrm{~cm}^{2}$ vertical plate with acetaldehyde-air mixtures and a more complex 8 -species chemical model. It was found that the more complex chemical model was able to very accurately capture ignition behavior observed in the experiments, and that as simple a model as one with two species did a decent job capturing experi- 
mental results.

Laurendeau and Caron (1982) studied thermal ignition of methane-air mixtures by a vertical strip of foil under free convection conditions. The width of the strip was fixed at $5.0 \mathrm{~cm}$, and its height was varied from 0.2 to $1.0 \mathrm{~cm}$, allowing for surfaces from 1 to $5 \mathrm{~cm}^{2}$ in area. Laurendeau found ignition temperatures between 1283 and $1363 \mathrm{~K}$. They also suggested a the ignition data could be correlated by $L \propto \frac{E}{s R T_{w}}$, where $L$ is the characteristic length, $E$ is the activation energy, $T_{w}$ is the wall temperature at ignition, and $s$ is a scaling parameter; for free convection, they suggested a value of 1/2 (forced convection, $s=1$, and stagnation conditions, $s=2$ ).

There are also a number of studies of thermal ignition in external natural convection flows generated by heated cylinders. Boettcher (2012) studied thermal ignition by a heated glowplug in a quiescent hexane-air mixture and investigated the effects of mixture composition, initial pressure, and surface area. It was found that equivalence ratios from 0.7 to 3.0 had little effect on ignition temperature, which remained nearly constant at $920 \mathrm{~K}$. Increasing the surface area from 0.01 to $1.5 \mathrm{~cm}^{2}$ caused a drop in ignition temperature of $100 \mathrm{~K}$. The temperature of the surface was monitored by a thermocouple in contact with the side of the glowplug, and it is possible that contact resistance between the glowplug surface and thermocouple could have caused temperature readings that were lower than the true surface temperature.

Melguizo-Gavilanes, Nové-Josserand, et al. (2016) revisited this work with a glowplug in hydrogen-air mixtures. Complementary experimental and numerical investigations were performed with a glowplug surface area of $1.6 \mathrm{~cm}^{2}$ and heating rates from $18 \mathrm{~K} / \mathrm{s}$ to $190 \mathrm{~K} / \mathrm{s}$. Experimental results showed ignition temperatures of 1052 $\pm 52 \mathrm{~K}$ and $1028 \pm 53 \mathrm{~K}$ for heating rates of 18 and $190 \mathrm{~K} / \mathrm{s}$, respectively. Experiments also showed ignition occured at the very top of the glowplug. Numerical results also observed this phenomenon and noted that this was a recirculation region with long residence times. An energy balance showed that ignition occured at a distance from the wall where heat release rates were greater than diffusion rates. They noted that separated regions were key in achieving ignition, as convective transport of energy and species was minimal and build up of intermediates helped produce $\mathrm{OH}$ that led to thermal explosion. 
The study of a glowplug as a source of thermal igniton in hexane was revisited in numerical work by Melguizo-Gavilanes, Boeck, et al. (2017). Temperature measurement accuracy in the experiments was improved by use of an optical pyrometer. Numerical results indicated that the thermal layer was thickest in the separated region just above the top of the glowplug. In this region, the flow was essentially stagnant, and convective losses were minimal. It was concluded that these conditions were critical for an ignition event to take place.

Another detailed numerical study by Menon et al. (2016) looks at the effect of fuel decomposition in the presence of a concentrated hot surface; in this case, the glowplug discussed at length above. The numerical model used a n-heptane mechanism due to the similar chemical kinetics of n-hexane and n-heptane. They noted a twostage ignition process: first, a breakdown of the fuel into lighter components and an accompanying small increase in temperature, and second, a main ignition event with fuel depletion. They also noted that the second stage of ignition was primarily caused by a breakdown of hydrogen peroxide at temperatures in excess of $900 \mathrm{~K}$. The decomposition of hydrogen peroxide led to increased production of hydroxyl radicals which in turn caused the thermal explosion typical of a main ignition event. They noted that the breakdown of hydrogen peroxide was facilitated by third body reactions, which accounted for the lack of sensitivity to mixture composition and the sensitivity to initial pressure noted by Boettcher (2012).

The discussion of thermal ignition by external natural convection presented here concludes with the work of Boeck et al. (2017). They studied thermal ignition by a $1 \mathrm{~cm}$ by $1 \mathrm{~cm}$ cylinder (total surface area of $3.14 \mathrm{~cm}^{2}$ in both horizontal and vertical orientations). Mixtures of hydrogen-air, ethylene-air, and n-hexane-air were investigated. For vertical cylinders, hydrogen was found to ignite at $1050 \mathrm{~K}$, ethylene at $1180 \mathrm{~K}$, and $\mathrm{n}$-hexane at $1270 \mathrm{~K}$. For all fuels, mixture composition had little effect on the ignition temperature for a total of less than $100 \mathrm{~K}$ difference between flammability limits. The ignition temperature was minimally effected by cylinder orientation for hydrogen, but it was noted that ignition temperatures in ethylene and n-hexane were significantly lower for a horizontal cylinder than a vertical one. For both cylinder orientations, ignition occurred at the topmost location of the cylinder where the boundary layer was thickest. 


\section{External Natural Convection: Particles}

Roth, Sharma, et al. (2014) studied thermal ignition by stationary spherical submillimeter particles in a hydrogen-air mixture. The effects of particle material, particle size, and mixture stoichiometry were studied. The spherical particles were laser heated, and their surface temperature was measured via two-color pyrometer. The particle materials investigated were silicon nitride, tungsten carbide, steel types 1.3505, 1.3541, and 1.4034; all of these were spherical balls. Casting steel and aluminum were also investigated, but these materials were available as shot-blasting particles and were not strictly spherical in shape. Silicon nitride had the lowest ignition temperature out of all the materials investigated and was considered chemically inert. The steel particles were found to ignite at temperatures approximately $150 \mathrm{~K}$ higher than the silicon nitride particles and were considered to have catalytic effects. The diameter of the particles varied from 0.3 to $0.8 \mathrm{~mm}$ in diameter, and it was found that the larger particles ignited at lower temperatures. Finally, the mixture stoichiometry was found to only have a minor effect on ignition temperature. The lowest ignition temperature achieved in this study was approximately $1050 \mathrm{~K}$ for a $0.8 \mathrm{~mm}$ diameter particle in a 15/85 volume percent hydrogen/air mixture.

Roth, Haber, and Bockhorn (2017) extended their previous work with stationary submilimeter particles (Roth, Sharma, et al., 2014) by studying ignition with a wide variety of fuels and mixture stoichiometries. The particle material and diameter was fixed to a silicon nitride sphere $0.8 \mathrm{~mm}$ in diameter. Laser heating once again raised the surface temperature of the particle until ignition was achieved. The fuels investigated were methane, ethane, propane, n-butane, ethylene, acetylene, hydrogen, and diethyl ether. The fuel used had a significant effect on ignition temperatures; methane ignited at temperatures above $1600 \mathrm{~K}$, while hydrogen mixtures ignited as low as $1070 \mathrm{~K}$. Most fuels showed limited effect of mixture composition on ignition temperature in a range of equivalence ratios surrounding their stoichiometric compositions. Generally, the effect of mixture composition on ignition temperature was found to be strongest near the flammability limits. A simple correlation of the ignition temperature with laminar burning speed, $S_{L}$, was proposed, $T_{i g n}=3300 S_{L}^{-0.2}$. 


\section{Thermal Ignition by Forced Convection}

There are a number of studies of thermal ignition by forced convection flows. They include hot surface geometries such as millimeter-scale moving spheres, flow past cylindrical rods, and flow past a heated plate.

Early studies of thermal ignition by moving spheres includes the work of Silver (1937) and Patterson (1940) mentioned previously. Silver (1937) studied ignition of coal-gas, pentane, and hydrogen mixtures by spheres with diameters on the order of millimeters. These spheres were injected into the reactive environment at speeds from 2 to $5 \mathrm{~m} / \mathrm{s}$. Patterson (1940) revisited this work with similarly sized spheres in the same mixtures but with a well-controlled injection speed of $1.2 \mathrm{~m} / \mathrm{s}$ for the spheres. An increase in the size of the sphere was found to decrease the minimum ignition temperature, and a decrease in injection speed of the sphere was found to decrease the minimum ignition temperature.

Coronel (2016) studied the effect of sphere size and mixture composition on ignition thresholds in n-hexane mixtures. Increasing the sphere diameter decreased the ignition threshold by a total of approximately $80 \mathrm{~K}$ for diameters from 0.18 to 0.6 $\mathrm{cm}$. For equivalence ratios from 0.75 to 2.2 , the mixture composition of an $\mathrm{n}$-hexane and air mixture was found to have no significant effect on ignition temperature. It was observed that ignition occured near the seperation point of the flow over the sphere. Further work from Coronel et al. (2018) looked at thermal ignition by a 0.6 $\mathrm{cm}$ diameter sphere moving at $2.4 \mathrm{~m} / \mathrm{s}$ in n-hexane-air mixtures and found a $50 \%$ probability of ignition at $1176 \mathrm{~K}$. They also observed that ignition occured near the region of flow separation. Detained flow simulations were performed. At the minimum temperature at which ignition occurs, simulations indicated ignition was localized at the separation region. The gas mixture in this ignition location was composed primarily of ethylene, and thus it was mostly ethylene which first ignited. Very little hexane was found at the ignition location immediately prior to the main ignition event, which indicates the importance of fuel decomposition in the ignition process.

In addition to the study of ignition by natural convection flows within a vessel, Kuchta, Bartkowiak, and Zabetakis (1965) studied ignition by horizontal cylinders with surfaces areas from less than $1 \mathrm{~cm}^{2}$ to $40 \mathrm{~cm}^{2}$ in n-hexane, -octane, -decane, 
and JP- 6 mixtures. All cylinders were $5 \mathrm{~cm}$ long and their surface area was varied by changing the radius of the heating element (typically a metal wire or rod). The flow rate was estimated at $0.35 \mathrm{~cm} / \mathrm{s}$. The ignition temperature was found to decrease in a relatively linear fashion as the surface area of the cylinder increased, from $1273 \mathrm{~K}$ at $0.65 \mathrm{~cm}^{2}$ to $833 \mathrm{~K}$ at $30 \mathrm{~cm}^{2}$.

The work of Mullen, Fenn, and Irby (1948) mentioned previously investigated ignition by horizontal cylinders with total surface areas of $1.26 \mathrm{~cm}^{2}$. The flow rate was varied from 15 to $150 \mathrm{~m} / \mathrm{s}$, and the diameter of the cylinders was also varied. Mullen, Fenn, and Irby (1948) found that a higher flow rate led to higher ignition temperatures, while a larger diameter led to lower ignition temperatures. Furthermore, the effect of increased diameter was found to be more pronounced as the flow velocity increased. Experiments were conducted wherein either the upstream or downstream half of the cylinder was heated, and it was found that heating only in the downstream region had little effect on ignition temperature, while heating only in the upstream half of the cylinder would increase the ignition temperature by as much as $220 \mathrm{~K}$. It was concluded that ignition behavior was dominated by conditions in the recirculation zone at the downstream side of the cylinder. Effects of surface material were also investigated; little difference in ignition temperature was observed between stainless steel and nichrome, while a platinum surface required a significantly higher ignition temperature under the same conditions. Nominal ignition temperatures in pentane were found to be $1366 \mathrm{~K}$.

Smyth and Bryner (1997) studied ignition by a flat plate $25 \mathrm{~cm}^{2}$ in area at an angle of $45^{\circ}$ from horizontal. The flow rate was set at 15 cubic centimeters per second. Mixtures of methane, ethylene, and n-hexane among many other fuels were studied at equivalence ratios of $0.7,1.0$, and 1.3. The effect of surface material was also investigated. For the same conditions, stainless steel led to the lowest ignition temperatures and nickel the highest for a total difference in ignition temperature of about $130 \mathrm{~K}$ for $\mathrm{n}$-hexane. The average ignition temperatures for methane, ethylene, and n-hexane were found to be $1233 \mathrm{~K}, 1052 \mathrm{~K}$, and $1105 \mathrm{~K}$ respectively. 


\section{Modeling Efforts}

We also provide a brief overview of some efforts made to model ignition under various flow configurations. A simple way of conceptualizing ignition is that there is a critical balance between chemical reaction, energy release, and diffusion of thermal energy and species. When this balance is tipped, ignition can occur. One common way of providing a quantitative criteria for ignition is to use the so-called Van't Hoff ignition criteria, which states that ignition occurs when the thermal gradient as the wall is zero: $\left.\frac{d T}{d x}\right|_{\text {wall }}=0$. Interestingly, few literature sources cite the original source for the Van't Hoff ignition criterion. Ono et al. (1976) cites the source for the above equation of the ignition criterion as Semenov (1942). The orignal work of Hoff (1896) describes the criteria for ignition to occur as "the ignition temperature is the temperature at which the initial loss of heat, due to conduction etc., is equal to the heat evolved in the same time by the chemical reaction". There is no equation for the ignition criterion presented in that work. Regardless, we will proceed with common

nomenclature of $\left.\frac{d T}{d x}\right|_{\text {wall }}=0$ as the Van't Hoff ignition criterion when discussing previous modeling efforts.

Law and Law (1979) used matched asymptotic analysis to investigate the ignition location of two-dimensional steady flow over a flat plate. The flow was broken into two separate regions: a weakly reactive region near the surface that is locally similar and a chemically frozen outer region that is nonsimilar. Using similarity arguments, Law solved for the weakly reactive flow to identify the ignition location. The criterion for ignition was a unity Damkohler number. This is similar to the Van't Hoff ignition criterion which states that ignition occurs when the thermal gradient at the wall is equal to zero. Using this analysis, Law and Law (1979) developed an analytical expression for the ignition location in a chemically reacting boundary layer over a flat plate. Note that analytical analysis of stagnation point flows by Alkidas and Durbetaki (1973) found that the Van't Hoff criterion was reliable in finding the ignition temperature, but the critical Damkohler number (ratio of chemical time to convection time) was found to be in error by 25 to $50 \%$.

A similar approach was taken by Law (1979) when investigating transient ignition in one-dimension. Law first treated transient ignition by a flat plate and broke the boundary layers into two separate regions much in the manner of Law and Law (1979). The region near the surface was treated as weakly reactive and locally 
similar; a similarity argument was used to solve the flow in this region. An analytical expression was developed for the ignition delay time. Both analyses by Law assumed a one-step Arrhenius-type reaction for the chemistry.

Laurendeau (1982) performed a steady-state analysis of ignition using the energy conservation equation. Chemistry was assumed to occur via a one-step Arrheniustype reaction, and ignition was described by the Van't Hoff criterion and was assumed to occur in a stagnant film immediately next to the surface. An analytical relationship between characteristic length, pressure, and surface temperature was developed for a stagnant, free convection, and forced convection flow. For a free convection flow, this relation was given as $\ln \left(P L^{1 / 2}\right) \propto-E / R T_{w}$. Laurendeau also performed a transient analysis of ignition in a similar manner using an estimate of the transient temperature profile of gas near a hot slab as the starting point. Again, a one step Arrhenius-type reaction was used to model chemistry, and the Van't Hoff ignition criterion described ignition. The analytical ignition delay relation was given as $\ln \left(\frac{T_{w} \tau}{\left(T_{w}-T_{\infty}\right)}\right) \propto-E / R T_{w}$.

Maas and Warnatz (1988) modeled the ignition process of a hydrogen-oxygen mixture in one dimensional geometries (cartesian, cylindrical, and spherical) using a Lagrangian formulation of the conservation equations. The Lagrangian transformation reduced the number of governing equations to integrate, particularly if uniform pressure was assumed. A multi-species kinetic mechanism and detailed transport properties were used to model the chemistry. The one dimensional simulation was capable of capturing the three explosion limits present in a hydrogen-oxygen system when an appropriate set of surface reactions were included.

Peters et al. (2002) developed a reduced kinetic mechanism to describe the twostage ignition behavior of $n$-heptane. This mechanism was used in a homogenous reactor to simulate ignition of $n$-heptane air mixtures. They also introduced steady state assumptions about many of the intermediate species in order to develop a global mechanism for the low-temperature ignition regime and a separate global mechanism for the intermediate- to high-temperature ignition regime. Peters found that the dissociation of ketohydroperoxide dominated the low-temperature ignition regime and the first stage of ignition, while the dissociation of hydrogen peroxide dominated the intermediate- to high-temperature regime and the second stage of 
ignition. Analytical expressions were presented for the delay times of the first and second stages of ignition. The results from the analytical expressions for delay time were found to be good approximations for the results from performing a reactor calculation with a detailed kinetic mechanism.

Menon et al. (2016) performed detailed two-dimensional axisymmetric and transient simulations of ignition of a n-heptane air mixture by a glowplug. The variable density low Mach number Navier-Stokes equations were solved along with transport equations for energy and species. The simulations were designed to match corresponding experimental conditions, and so the temperature of the glowplug surface was continuously ramped. n-heptane was used in the simulations as opposed to the n-hexane mixture used in the corresponding experiments due to the availability of validated and detailed kinetic mechanisms for $\mathrm{n}$-heptane. The simulations were able to reproduce trends observed in the corresponding experiments like the location of the ignition kernel, the relative insensitivity of ignition temperature to equivalence ratio, and the strong dependence of ignition temperature on pressure. Furthermore, a two-stage ignition process much like the one described by Peters et al. (2002) was observed for $\mathrm{n}$-heptane. However, differences in the second stage of ignition were observed due to the modeling of diffusion and convection and continuous increase in surface temperature. Specifically, it was noted that an acceleration in the decomposition of hydrogen peroxide drove the second stage ignition event, and diffusion prevented local depletion of the n-heptane fuel, which was cited as the cause for second-stage ignition by Peters et al. (2002).

Haber et al. (2017) simulated the ignition of stationary submillimeter particles. They examined the effects of stoichiometry, heating rate, fuel type, and particle size on ignition. Simulations were performed using one-dimensional and two-dimensional formulations. The one-dimensional simulation used the unsteady conservation equations in radial coordinates, and simulated both the particle and the gas surrounding it. The resulting system of partial differential equation was solved using the method of lines. The two-dimensional simulation solved the governing equations (mass, momentum, species mass, energy) in cylindrical coordinates using a finite volume method. It was found that the one-dimensional simulation was sufficient to capture the details of ignition for a spherical particle. The results agreed well with experimental work. The ignition temperature was found to depend strongly on fuel type, 
while mixture stoichiometry and heating rate only had minor effects on ignition temperature. Additionally, an increase in particle size was found to decrease the ignition temperature.

Melguizo-Gavilanes, Boeck, et al. (2017) performed two-dimensional transient simulations of ignition of hydrogen-air mixutes by a glowplug. The governing equations are the axisymmetric variable-density reactive Navier-Stokes equations with temperature-dependent transport properties. A detailed kinetic mechanism is used to model the chemistry. The simulation is designed to mimick the conditions of corresponding experiments. The simulations are able to recreate the results seen with experiments and provide insight on some experimental phenomena. In particular, the simulations reproduce the ignition threshold temperature and the trend of ignition to occur at the top of the glowplug. Further analysis indicates the important role a separation region plays in setting up conditions favorable for ignition: minimal convective losses and long residence times allow for buildup of radical species.

Coronel et al. (2018) presents a joint experimental and numerical study of thermal ignition by moving spheres in a n-hexane air mixture. The numerical simulations solved the two-dimensional axisymmetric formulation of the variable-density reactive Navier-Stokes equations with temperature-dependent transport properties. Four different detailed kinetic mechanisms for n-hexane were used to model the chemistry. The numerical simulations reproduced the experimental threshold on ignition within $6-12 \%$, depending on the mechanism used. Like in the experiments, the location of ignition was observed at the separation angle of the sphere. The simulations also revealed that a significant amount of hexane decomposed into lighter species like ethylene prior to ignition.

\subsection{Goals of Study}

A review of the literature on natural convection flows for thermal ignition indicates that ignition by external flows at surface areas greater than $10 \mathrm{~cm}^{2}$ has not been thoroughly studied, as shown in Fig. 1.1. The only study of external natural convection flows over surfaces larger than $10 \mathrm{~cm}^{2}$ is the work of Cairnie, Harrison, and Morgan (1981) with vertical plates in diethyl ether mixtures, which is not a suitable surrogate fuel for aviation kerosene and therefore not particularly useful 


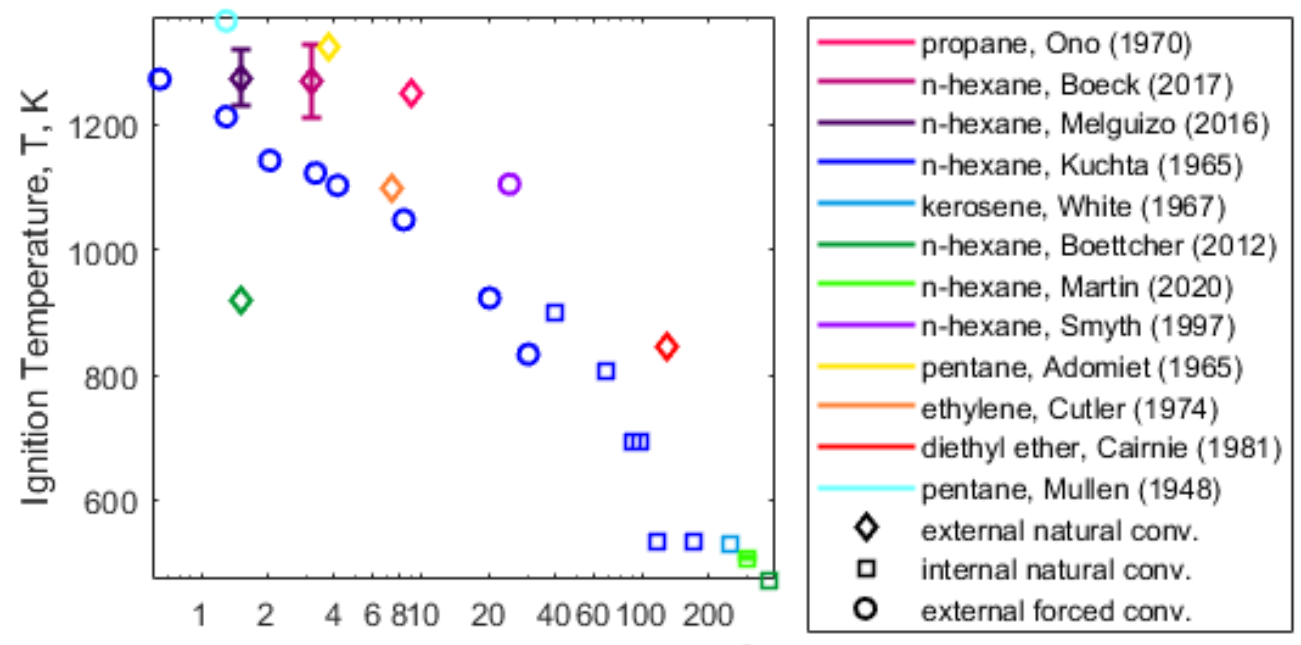

Surface Area of Heat Source, A, $\mathrm{cm}^{2}$

Figure 1.1: Comparison of literature on thermal ignition of various flow types.

in the context of aviation safety concerns. Additionally, the potential for a sudden change in ignition temperature around surface areas over $70 \mathrm{~cm}^{2}$ (as indicated by the results of Kuchta, Bartkowiak, and Zabetakis (1965) for internal flows) has not been investigated systematically. We propose a study of thermal ignition with the purpose of investigating these underexplored areas of thermal ignition by external natural convection flows.

The particular goals of this investigation are:

1. Experimentally investigate the ignition behavior of large $\left(>10 \mathrm{~cm}^{2}\right)$ surfaces subject to external natural convection flows:

a) Develop and characterize a well-defined large hot surface using vertical cylinders.

b) Obtain statistically based ignition thresholds.

c) Observe ignition dynamics with several visualization techniques.

d) Study the effect of geometrical parameters on ignition behavior:

i. Investigate surface areas ranging from 25 to $200 \mathrm{~cm}^{2}$.

ii. Investigate lengths ranging from 12.7 to $25.4 \mathrm{~cm}$.

e) Study the effect of fuel on ignition behavior:

i. Effect of elevated temperature on simple surrogate (n-hexane). 


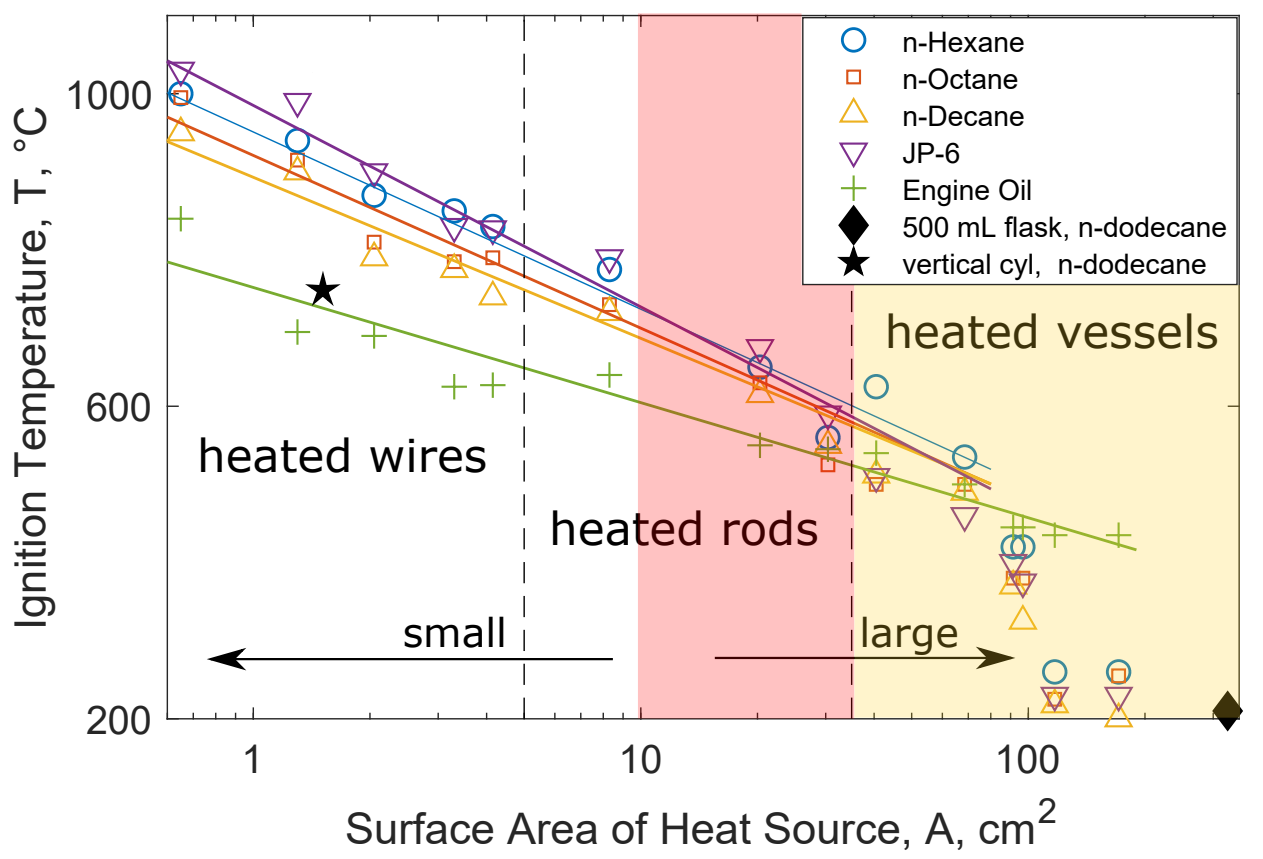

Figure 1.2: Results of ignition temperature with surface area from Kuchta, Bartkowiak, and Zabetakis (1965). Large surfaces highlighted in color, external flows highlighted in red, internal flows highlighted in yellow. Black diamond from Martin and Shepherd (2020), black star from Sund (2019).

ii. Jet fuel and multicomponent surrogate fuels designed to replicate jet fuel.

2. Use simple modeling techniques with detailed chemical kinetics to gain insight into the physics, chemistry, and energy transport phenomena of ignition:

a) Model the chemistry along streamlines of a natural convection flow.

b) Perform a one-dimensional transient simulation of ignition.

We hypothesize that surface area is not a key parameter governing ignition, and it is not correct to think of it as impacting the ignition behavior for either scientific or engineering purposes. However, we will investigate the effects of surface area and occasionally report ignition thresholds as a function of surface area in order to make comparisons with historical data on thermal ignition, which is often reported in terms of surface area of the ignition source. 


\subsection{Thesis Outline}

Chapter 1 provides an introduction to thermal ignition. It discusses the motivation for studying thermal ignition under varying flow conditions and provides a review of previous experimental investigations of thermal ignition by free and forced convection flows, as well as a brief review of some thermal ignition modeling efforts. Chapter 2describes the experimental setup that produces a larger vertical cylinder by which to study thermal ignition by external free convection. The diagnostics used in the experiments are described as well, and these include pyrometery, interferometry, schlieren imaging, and $\mathrm{OH}^{*}$ chemiluminescence. Chapter 3 describes modeling the boundary layer of a natural convection flow via similarity solutions and uses these solutions to validate interferometry resutls. Chapter 4 discusses the experimental results obtained for thermal ignition using cylinders of varying geometries to ignite hexane-air mixtures. Chapter 5 documents the results of studying the effect of fuel type on ignition behavior. This includes the effect of ambient temperature on a single-component jet fuel surrogate (n-hexane) as well as studying ignition of jet fuel and multicomponent surrogates. Chapter 6 documents the efforts to use some simple modeling techniques to gain insights into important ignition chemistry and behaviors. The chemistry along flow streamines is computed for a rudimentary model of ignition that still provides useful insights into important chemical phenomena. One-dimensional simulations of ignition are also performed that capture the physics of diffusion and conduction transverse to the heated surface.

\section{References}

Adomeit, G. (1965). "Ignition of gases at hot surfaces under nonsteady-state conditions". In: Symposium (International) on Combustion. Tenth Symposium (International) on Combustion 10.1, pp. 237-243.

Alkidas, A. and P. Durbetaki (1973). “A Simplified Model for Stagnation Heat Transfer and Ignition of a Gaseous Mixture". In: Combustion Science and Technology 7, pp. 135-140.

ASTM International (2015). ASTM E659-15, Standard Test Method for Autoignition Temperature of Chemicals.

Babrauskas, V. (2003). Ignition Handbook: Principles and Applications to Fire Safety Engineering, Fire Investigation, Risk Management and Forensic Science. Fire Science Publishers. ISBN: 978-0-9728111-3-2.

Boeck, Lorenz R. et al. (2017). "Ignition of fuel-air mixtures from a hot circular cylinder". In: Combustion and Flame 185, pp. 265-277. 
Boettcher, Philipp (2012). "Thermal Ignition". PhD thesis. California Institute of Technology.

Cairnie, L.R., A.J. Harrison, and P.A. Morgan (1981). "Autoignition in a free convection boundary layer". In: Symposium (International) on Combustion 18.1.

Coronel, Stephanie A. (2016). “Thermal Ignition Using Moving Hot Particles". $\mathrm{PhD}$ thesis. California Institute of Technology.

Coronel, Stephanie A. et al. (2018). "Experimental and numerical study on moving hot particle ignition". In: Combustion and Flame 192, pp. 495-506.

Coward, H. F. and P. G. Guest (1927). "Ignition of Natural Gas-Air Mixtures by Heated Metal Bars". In: Journal of the American Chemical Society 49.10, pp. 2479-2486.

Cutler, D.P. (1974). "The ignition of gases by rapidly heated surfaces". In: Combustion and Flame 22.1, pp. 105-109.

- (1978). "Further studies of the ignition of gases by transiently heated surfaces". In: Combustion and Flame 33, pp. 85-91.

Federal Aviation Administration (2018). Advisory Circular 25.981-1D.

Haber, Thomas et al. (2017). "Numerical Simulation of the Ignition of Fuel/Air Gas Mixtures Around Small Hot Particles". In: Zeitschrift fur Physikalische Chemie 231.10, pp. 1625-1654.

Harrison, A.J. and L.R. Cairnie (1988). "The development and experimental validation of a mathematical model for predicting hot-surface autoignition hazards using complex chemistry”. In: Combustion and Flame 71, pp. 1-21.

Hoff, J. H. Van't (1896). Studies in Chemical Dynamics. F. Muller.

Kuchta, J., A. Bartkowiak, and M. Zabetakis (1965). "Hot Surface Ignition Temperatures of Hydrocarbon Fuel Vapor-Air Mixtures". In: Journal of Chemical Engineering Data 10.3, pp. 282-288.

Laurendeau, Normand M. (1982). "Thermal ignition of methane-air mixtures by hot surfaces: A critical examination”. In: Combustion and Flame 46, pp. 29-49.

Laurendeau, Normand M. and Richard N. Caron (1982). "Influence of hot surface size on methane-air ignition temperature". In: Combustion and Flame 46, pp. 213218.

Law, C. K. and H. K. Law (1979). "Thermal-ignition analysis in boundary-layer flows". In: Journal of Fluid Mechanics 92.1, pp. 97-108. (Visited on 04/01/2020).

Law, C.K. (1979). "Transient ignition of a combustible by stationary isothermal bodies”. In: Combustion Science and Technology 19, pp. 237-242.

Maas, U and J Warnatz (1988). "Ignition processes in hydrogen-oxygen mixtures". In: Combustion and Flame 74, pp. 53-69. 
Martin, C. and J.E. Shepherd (2020). "Low Temperature Autoignition of Jet A and Surrogate Jet Fuels". In: 13th International Symposium on Hazards, Prevention, and Mitigation of Industrial Explosions.

Melguizo-Gavilanes, J., L.R. Boeck, et al. (2017). "Hot surface ignition of stoichiometric hydrogen-air mixtures". In: International Journal of Hydrogen Energy 42.11, pp. 7393-7403.

Melguizo-Gavilanes, Josué, Augustin Nové-Josserand, et al. (2016). "Hot Surface Ignition of $n$-Hexane Mixtures Using Simplified Kinetics". In: Combustion Science and Technology 188.11-12, pp. 2060-2076.

Menon, Shyam K. et al. (2016). "Hot surface ignition of n-hexane in air". In: Combustion and Flame 163, pp. 42-53.

Mullen, James W., John B. Fenn, and Moreland R. Irby (1948). "The ignition of high velocity streams of combustible gases by heated cylindrical rods". In: Symposium on Combustion and Flame, and Explosion Phenomena 3.1, pp. 317-329.

Ono, S. et al. (1976). "Ignition in a Free Convection from Vertical Hot Plate". In: Bulletin of JSME 19.132, pp. 676-683.

Patterson, S (1940). "The ignition of inflammable gases by hot moving particles II". In: The London, Edinburgh, and Dublin Philosophical Magazine and Journal of Science, 30.203, pp. 437-457.

Peters, N. et al. (2002). "Temperature cross-over and non-thermal runaway at twostage ignition of n-heptane". In: Combustion and Flame 128.1-2, pp. 38-59.

Roth, David, Thomas Haber, and Henning Bockhorn (2017). "Experimental and numerical study on the ignition of fuel/air mixtures at laser heated silicon nitride particles". In: Proceedings of the Combustion Institute 36, pp. 1475-1484.

Roth, David, Pratyush Sharma, et al. (2014). "Ignition by Mechanical Sparks: Ignition of Hydrogen/Air Mixtures by Submillimeter-Sized Hot Particles". In: Combustion Science and Technology 186.10-11, pp. 1606-1617. DoI: 10.1080/ 00102202.2014 .935606 .

Semenov, N. N. (1942). THERMAL THEORY OF COMBUSTION AND EXPLOSION. Tech. rep. NACA-TM-1024.

Silver, R.S. (1937). "The ignition of gaseous mixtures by hot particles". In: The London, Edinburgh, and Dublin Philosophical Magazine and Journal of Science, 23.156, pp. 633-657.

Smyth, K.C. and N.P. Bryner (1997). "Short-duration autoignition temperature measurements for hydrocarbon fuels near heated metal surfaces". In: Combustion Science and Technology 126.16, pp. 225-253.

Sund, Sveinung M (2019). "Thermal Ignition of Surrogate Jet Fuels". PhD thesis. University of South-Eastern Norway. 
White, R.G. (1967). Spontaneous ignition of kerosene/AVTUR/vapour - the effect of the ratio, vessel surface area to volume (effect of vessel surface area on minimum spontaneous ignition temperature of kerosene). Tech. rep. 67107. Royal Aircraft Establishment. 
Chapter 2

\section{EXPERIMENTAL SETUP AND DIAGNOSTICS}

\subsection{Combustion Vessel}

The experiments are performed in a $40 \mathrm{~L}$ combustion vessel, made of a $22 \mathrm{~L}$ stainless steel vessel with aluminum extensions to provide additional space for the experimental test surfaces. The vessel has a height of $66.0 \mathrm{~cm}$ and an inner diameter of $30.2 \mathrm{~cm}$. Two parallel flanges are used to mount windows for interferometer visualization, and the windows have a diameter of approximately $12 \mathrm{~cm}$. A flange perpendicular to the interferometer windows is used to mount a window approximately $5 \mathrm{~cm}$ in diameter for pyrometer optical access. A fourth flange that is parallel to the flange for pyrometer access is the mounting and access point for the test structure. Figure 2.1 shows a schematic of the experimental setup.

The surfaces tested in this investigation are vertical cylinders. The cylinders are tested one at a time, and there are ten total cylinders with a range of lengths, diameters, and surface areas. The axis of the cylinder tested is aligned with the axis of the cylindrical combustion vessel and the top edge of the cylinder is lined up with the top edge of the interferometer access windows, providing optical access to the top $12 \mathrm{~cm}$ of the cylinder. The cylinder is held in place within the vessel by a modular copper support structure. This support structure can be adjusted to accommodate the length of any of the test cylinders. The support structure also provides a path for current to flow and resistively heat the cylinders.

A remotely controlled plumbing system is used to evacuate the combustion vessel to less than 100 mTorr and accurately fill it with the reactive mixture using the method of partial pressures. A Heise manometer with a precise digital readout measures the static pressure so the gases can be filled to within $0.01 \mathrm{kPa}$ of the desired gas pressure, providing control over the mixture composition. The reactive mixture of the experiments is premixed. A Kulite piezoresistive pressure sensor with a thermal protection system measures the dynamic pressure of the mixture during the experiments. 


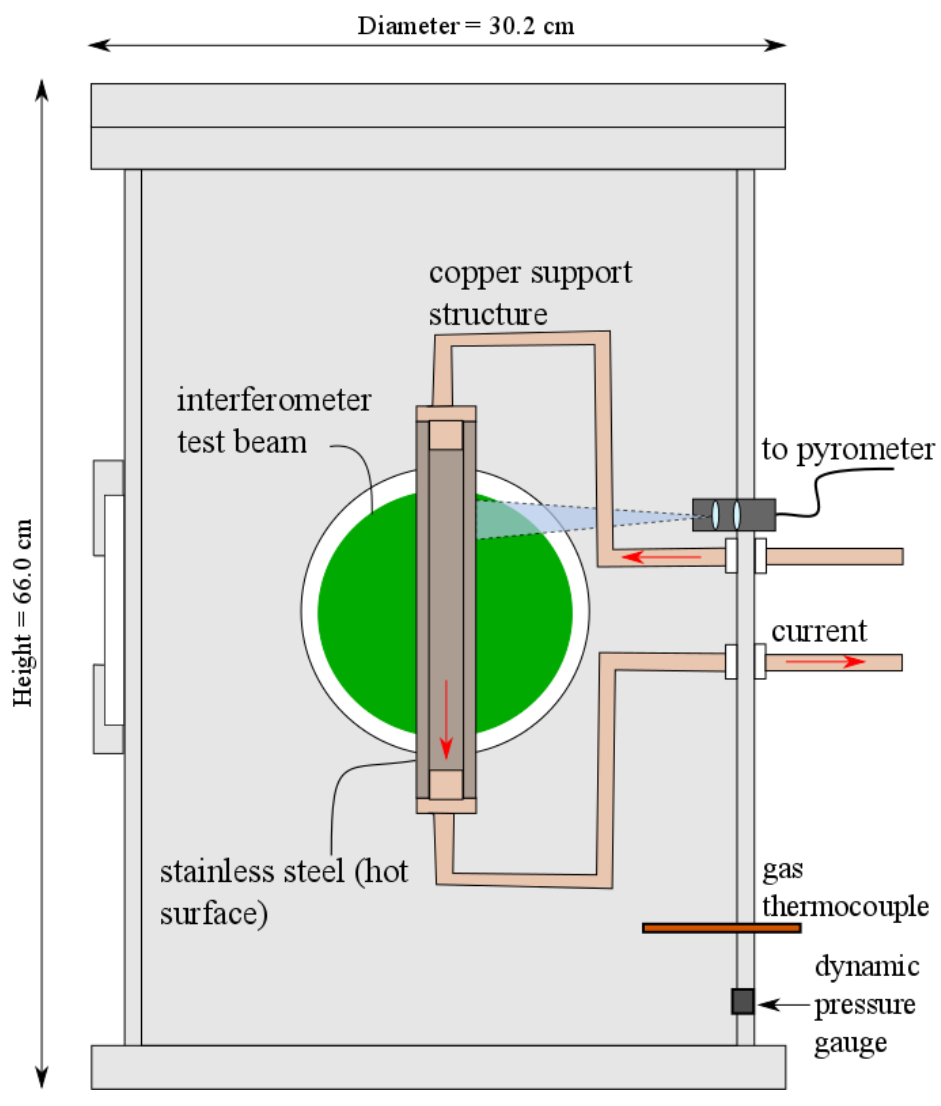

Figure 2.1: Experimental setup.

Once the vessel is filled with the desired reactive mixture, the heating power supply provides a constant current to the resistive heating circuit. The surface temperature of the cylinder is monitored by a thermocouple (placed above the point where ignition occurs so that the presence of the thermocouple does not disturb or alter the thermal boundary layer in that region) and by a two-color pyrometer. The surface temperature of the cylinder is fed back to a LabView script that provides closed-loop control on the surface temperature. When the temperature of the cylinder is within five percent of the set steady-state temperature in LabView, the control loop kicks in and drives the temperature to the set value. A temperature trace of this process is shown in Fig.2.2. Note that the pyrometer reading is noisy at the start of the heating process due to a small signal-to-noise ratio from minimal light emission at the two pyrometer-measured wavelengths when the surface temperature is low.

Various methods are used for ignition detection. The Kulite pressure sensor records 


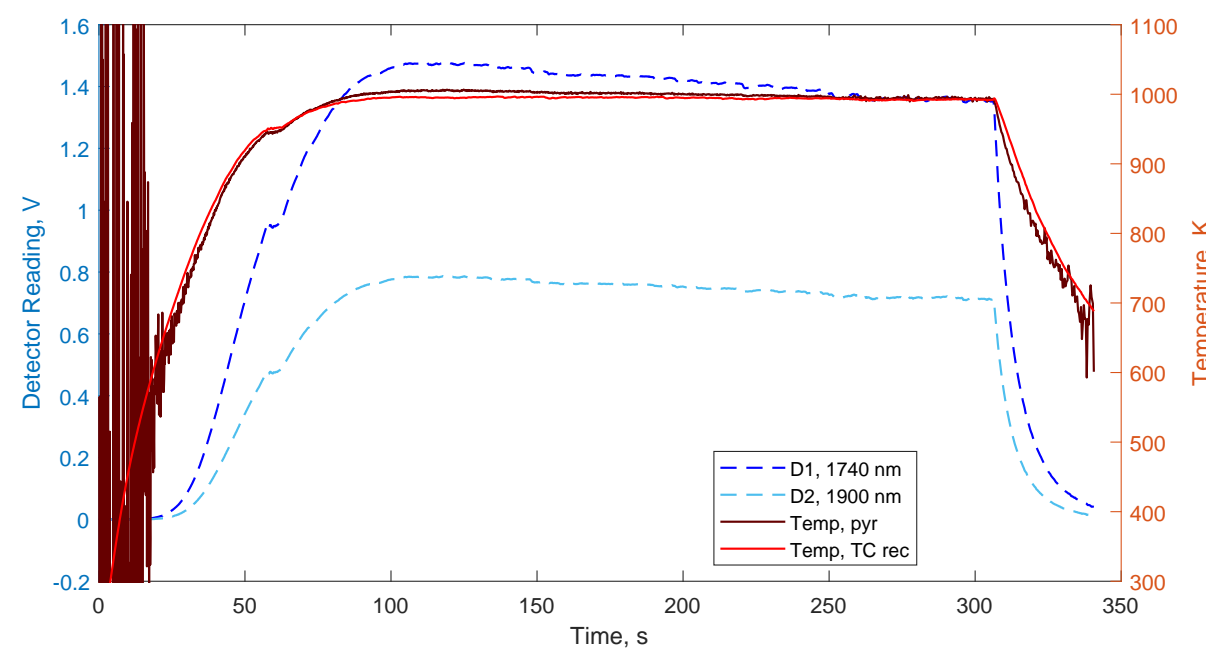

Figure 2.2: Trace of typical cylinder heating.

the dynamic pressure within the vessel, and a sharp rise in pressure is noted as an ignition event. This pressure data is also used to determine the peak pressure during an ignition event. A K-type thermocouple monitors the temperature within the vessel, placed about $3 \mathrm{~cm}$ normal to the inner wall of the vessel. A sharp rise in temperature also indicates an ignition event. Third, the flame emission in the infrared is observed using the two-color pyrometer photodetectors. The fourth method is a Mach-Zehnder interferometer that uses a $532 \mathrm{~nm}$ single-mode laser. This method is used to visualize the ignition and flame propagation using a high-speed camera at up to 10,000 frames per second and a field of view of approximately $12 \mathrm{~cm}$ in diameter. Further details on the interferometer are found in Section 2.6 .

The test procedure is described in Chapters 4 and 5. The procedure varies slightly depending on the fuel used in the ignition testing. For example, hexane fully vaporizes at room temperature whereas heavier multicomponent fuels require heating of the experimental setup to fully vaporize. The particulars of the test procedure for each fuel are given in the chapters corresponding to those fuels.

\section{Test Cylinders}

Ten distinct cylinder geometries were used for the experiments. Each cylinder was made out of a thin-walled 304 stainless steel tube that acted both as test surface and as resistive heating element in the heating circuit. A copper plug was pressed 
into each end of the stainless steel cylinder to provide an attachment point to the rest of the circuit. Good electrical connection between the copper plug and the stainless steel cylinder was ensured by either brazing or soldering the joint between the stainless steel cylinder and the copper plug. The outer end of the plug was threaded with female threads. A copper cooling piece with male threads at one end threaded into the copper plug. The other end of the cooling piece had a NPT fitting, and a hollow inner diameter of $0.6 \mathrm{~cm}$ to connect to plumbing for water cooling. The middle section of the cooling piece had a diameter of $1.9 \mathrm{~cm}$ that was $1 \mathrm{~cm}$ long for all cylinders, and this section is where the connection to the modular support structure was made. A detailed view of this connection scheme is show in Fig. 2.3.

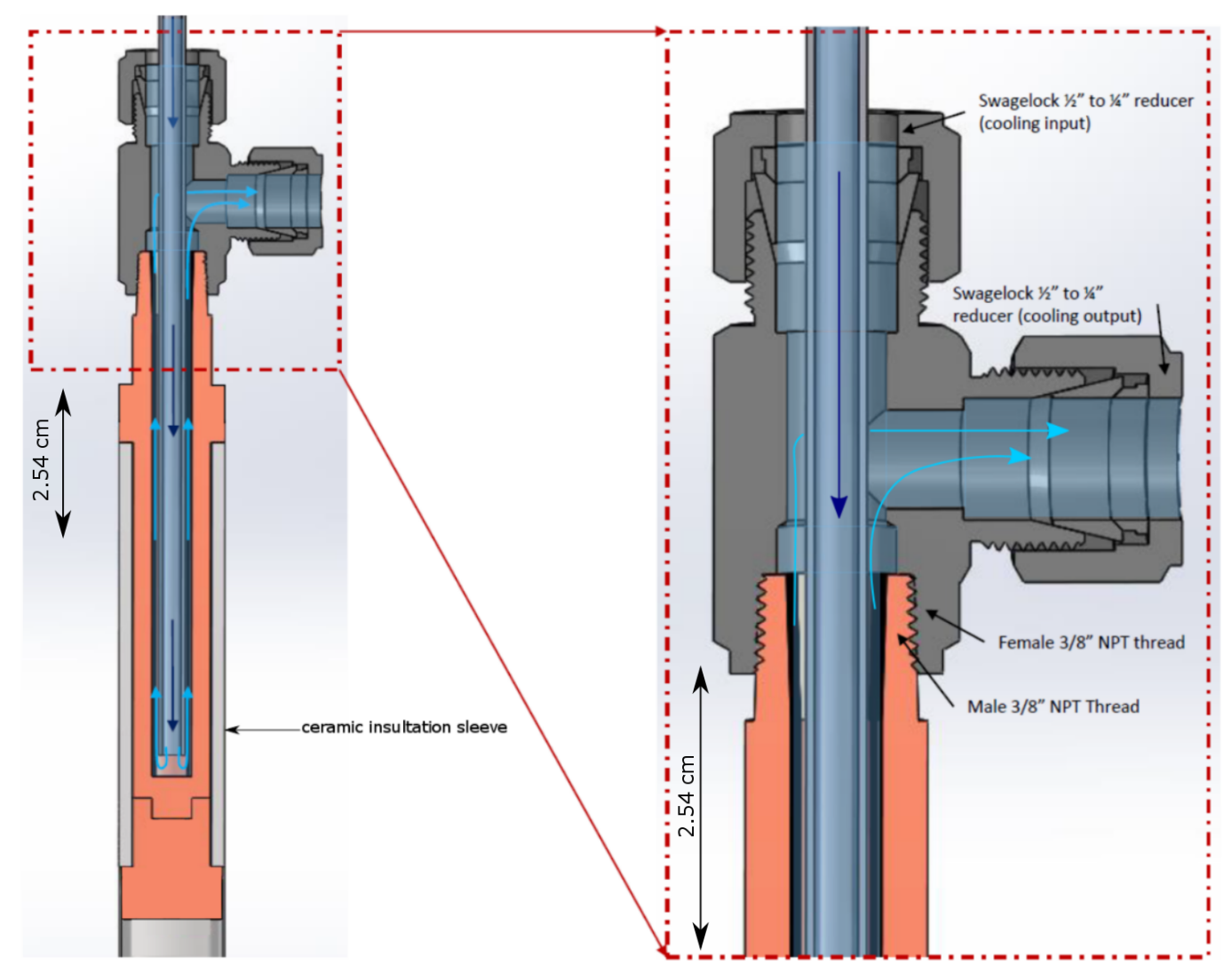

Figure 2.3: View of connection of test piece to support structure.

The surface area, length, and diameter of the test cylinders were varied to investigate the effect of different parameters on ignition. Fig. 2.4 shows the ten different cylinders, and Table 2.1 lists their important dimensions. 


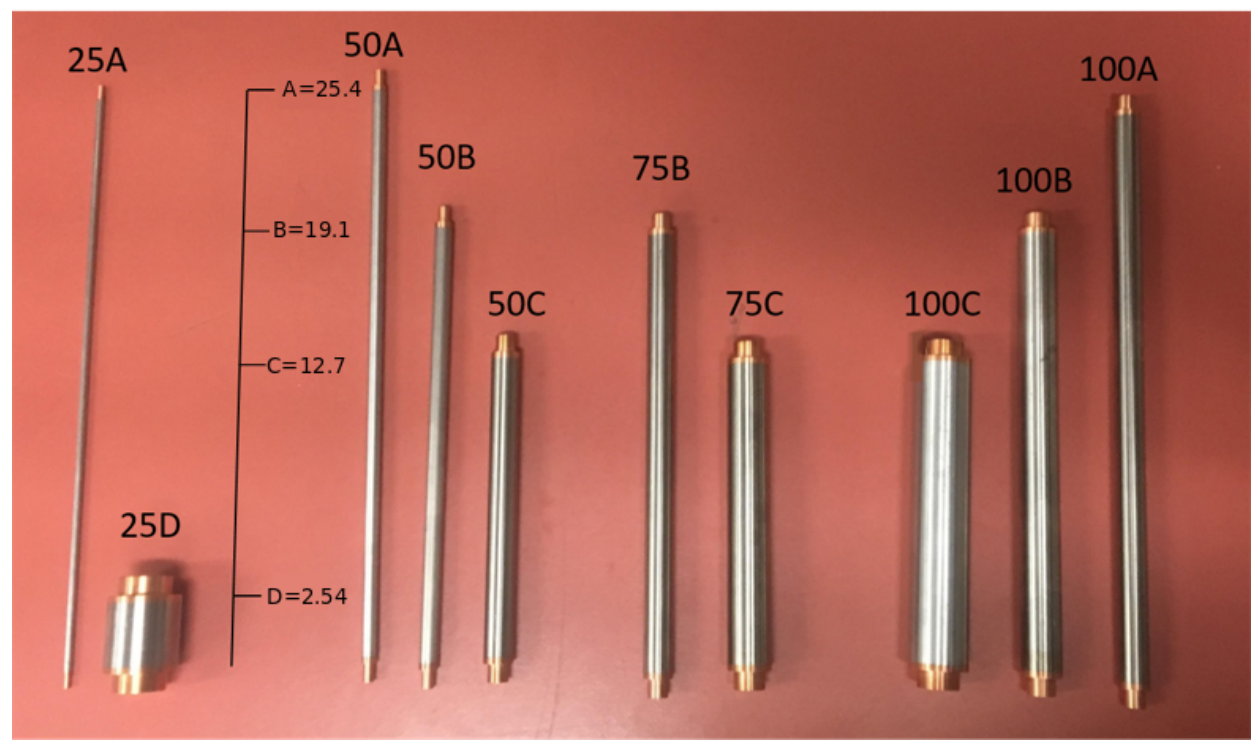

Figure 2.4: Ten test cylinders pictured together. The number above each cylinder denotes its surface area in $\mathrm{cm}^{2}$, and the letter indicates the length of the cylinder, ranging from $\mathrm{A}=25.4 \mathrm{~cm}$ to $\mathrm{D}=2.54 \mathrm{~cm}$.

\begin{tabular}{llllll}
\hline Label & $\begin{array}{l}\text { Surface } \\
\text { Area }\left(\mathrm{cm}^{2}\right)\end{array}$ & $\begin{array}{l}\text { Length } \\
(\mathrm{cm})\end{array}$ & $\begin{array}{l}\text { Diameter } \\
(\mathrm{cm})\end{array}$ & L/D & $\begin{array}{l}\text { Wall Thickness } \\
(\mathrm{cm})\end{array}$ \\
\hline 25A & 25 & 25.4 & 0.31 & 84.6 & 0.05 \\
25D & 25 & 2.5 & 2.90 & 0.9 & 0.05 \\
$50 \mathrm{~A}$ & 50 & 25.4 & 0.64 & 39.7 & 0.05 \\
$50 \mathrm{~B}$ & 50 & 19.1 & 0.80 & 23.9 & 0.05 \\
$50 \mathrm{C}$ & 50 & 12.7 & 1.27 & 10.0 & 0.05 \\
$75 \mathrm{~B}$ & 75 & 19.1 & 1.27 & 15.0 & 0.05 \\
$75 \mathrm{C}$ & 75 & 12.7 & 1.91 & 6.6 & 0.09 \\
$100 \mathrm{~A}$ & 100 & 25.4 & 1.27 & 20.0 & 0.05 \\
$100 \mathrm{~B}$ & 100 & 19.1 & 1.75 & 10.9 & 0.17 \\
$100 \mathrm{C}$ & 100 & 12.7 & 2.54 & 5.0 & 0.05 \\
200A & 100 & 25.4 & 2.54 & 10.0 & 0.05 \\
\hline
\end{tabular}

Table 2.1: Dimensions of all test cylinders.

\section{Power Supply Requirements}

The surfaces under investigation in this work are larger than $10 \mathrm{~cm}^{2}$. In order to ensure that ignition could be achieved, the test surfaces were designed to be capable of reaching temperatures of $1000 \mathrm{~K}$ or more when heated. Lumped mass heat transfer calculations were performed to determine the power requirements to reach predicted ignition temperatures. The calculations for power requirements are listed below. 


$$
\begin{gathered}
P_{\text {heating }}=P_{\text {convection }}+P_{\text {radiation }} \\
I^{2} R=h A_{s}\left(T_{s}-T_{\infty}\right)+\sigma \epsilon A_{s}\left(T_{s}^{4}-T_{\infty}^{4}\right)
\end{gathered}
$$

$I$ is the heating current, $R$ is the resistance of the cylinder, $h$ is the convective heat transfer coefficient, $A_{s}$ is the surface area of the cylinder, $T_{S}$ and $T_{\infty}$ are the surface and ambient temperature respectively, $\sigma$ is the Stephan-Boltzmann constant, and $\epsilon$ is the emissivity of the surface. Resistance of the cylinder, $R$, can be calculated as:

$$
R=\rho \frac{L}{A_{c}},
$$

where $\rho$ is resistivity of stainless steel, $L$ is the length of the cylinder, and $A_{c}$ is the cross-sectional area of the cylinder.

The convective heat transfer coefficient, $h$, is calculated using Churchill et al.'s approximation for a vertical flat plate and laminar flow (Churchill:etal:1975):

$$
h=\frac{k}{L}\left(0.68+\frac{0.67 R a_{L}^{1 / 4}}{\left(1+(0.492 / P r)^{9 / 16}\right)^{4 / 9}}\right), 10^{-1}<\operatorname{Ra}_{\mathrm{L}}<10^{9}
$$

$k$ is the thermal conductivity of the fluid, $L$ is the length, $R a_{L}$ is the Rayleigh number, and $P r$ is the Prandtl number of the fluid. All fluid properties are evaluated at the film temperature, $T_{f}=\frac{T_{s}+T_{\infty}}{2}$.

Equation 2.2 is solved for $I$ to find the current required to generate enough heat for the cylinder to reach the desired surface temperature, $T_{s}$. Voltage drop across the cylinder can then be calculated as $V=I R$, and the power requirements for the resistive heating determined accordingly. A Magna Power XR5-600 computercontrolled power supply was selected, capable of providing up to 600 Amps and 5 Volts. 
The validity of using the lumped mass approximation can be checked by calculating the Biot number of the cylinder, which determines if temperatures within a body are reasonably uniform.

$$
B i=\frac{h L_{c}}{k},
$$

$h$ is the convective heat transfer coefficient, $k$ is the thermal conductivity of the

cylinder, and $L_{c}=\frac{V}{A_{s}}$ is the characteristic length. The Biot number for a typical cylinder comes out to approximately 0.3 compared to the limit of 0.1 for the use of the lumped mass approximation to produce less than 5\% error. While the Biot number of the cylinder is larger than the typically accepted limit for the lumped mass approximation, the Biot number of 0.3 is close enough to the limit that using this approximation is acceptable in the context of first-order calculations to estimate the power supply requirements. More appropriate models that include the distribution of heat within the cylinder are discussed in the following section.

\section{Cylinder Heat Transfer Models}

It was also necessary to understand how the temperature would be distributed along the length of the test cylinders and how much heat would be transferred to the copper pieces. Heat transfer models were made for both the stainless steel cylinder and the copper end pieces. These models predicted the temperature distribution along the cylinder's length and temperature rise in the copper end pieces.

\section{Model for Test Cylinder}

First, the heat transfer in the stainless steel cylinder is modeled to predict the temperature distribution along the length of the cylinder. The stainless steel cylinder is treated as a one-dimensional solid conductor in the shape of a cylindrical shell and neglects radial conduction for simplicity. One half of the cylindrical shell is modeled, using a symmetry boundary condition at the center and a lumped mass boundary condition at the end of the cylinder, where the cylinder is in contact with the copper end pieces. Fig. 2.5 shows a schematic of the problem.

Conduction, convection, and radiation were all included in this model, as well as the heat generated via resistive heating. The heat transfer equation is as follows: 


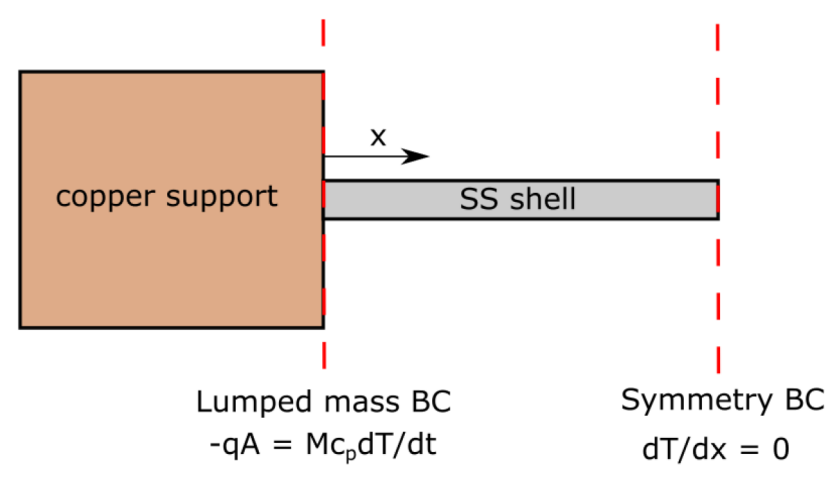

Figure 2.5: One-dimensional model of heat transfer within stainless steel cylinder.

$$
\frac{\partial T}{\partial t}=\alpha \frac{\partial^{2} T}{\partial x^{2}}+\frac{I^{2}}{\rho c_{p} A_{c}^{2} \sigma_{S S}}-\frac{h\left(T-T_{\infty}\right)}{\rho c_{p} w_{t}}-\frac{\sigma \epsilon\left(T^{4}-T_{\infty}^{4}\right)}{\rho c_{p} w_{t}}
$$

where $T$ is temperature, $t$ is time, and $x$ is position along the cylinder. $\alpha, \rho, c_{p}$, $\epsilon$, and $\sigma_{S S}$ are thermal diffusivity, density, heat capacity, emissivity, and electrical conductivity of stainless steel respectively. $A_{c}$ and $w_{t}$ are cross-sectional area and wall thickness of the shell, respectively. $I$ is current, $h$ is convection constant, $T_{\infty}$ is ambient temperature, and $\sigma$ is the Boltzmann constant. The equation was modeled as using the method of lines to transform this into a system of ODEs in MATLAB and solved with a stiff ODE solver, ode15i.

\section{Model for copper end pieces}

The copper end pieces were also modeled in order to carry out heat transfer calculations. These calculations were necessary to predict the temperature rise in the supports during heating, and if it was large enough to require water cooling in order to keep the supports from a significant temperature rise that could disturb the axisymmetry of the setup. The copper end pieces are modeled as one solid, axisymmetric piece of copper for simplicity. Heat generation within the copper pieces due to current is neglected, as these components were designed specifically with low electrical resistance to avoid heat generation. The boundary conditions on this model are more complex than the cylindrical shell and are divided into five separate regions:

1. axis of symmetry;

2. bottom, horizontal circular face with convection and radiation removing heat; 
3. side, vertical cylindrical face with convection and radiation removing heat;

4. top, horizontal circular face $0<r<r_{i}$, with convection and radiation removing heat;

5. top, horizontal annular face $r_{i}<r<r_{o}$, with a $200 \mathrm{~W}$ influx (approximately half of the heat generated within the cylinder).

This model of the copper end pieces is depicted in Fig.2.6. The heat transfer equation in cylindrical coordinates is shown in Equation 2.7. The boundary conditions are shown in Equations 2.8 through 2.10

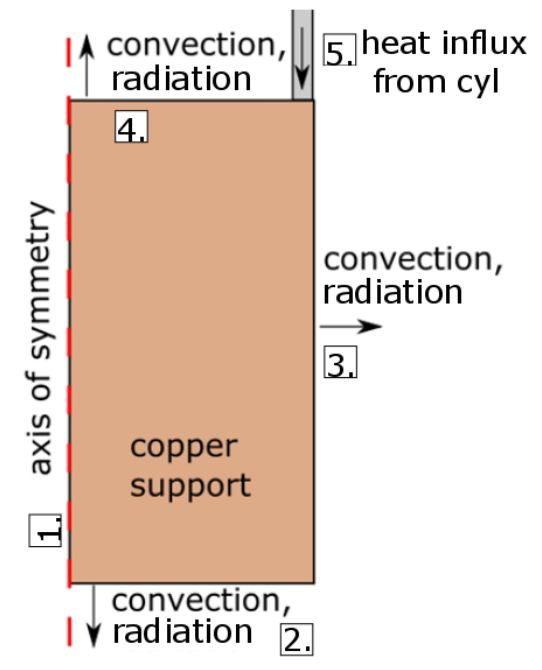

Figure 2.6: Two-dimensional model of heat transfer within copper end pieces.

$$
\frac{\partial T}{\partial t}=\alpha\left(\frac{1}{r} \frac{\partial T}{\partial r}+\frac{\partial^{2} T}{\partial r^{2}}+\frac{\partial^{2} T}{\partial z^{2}}\right)+q
$$

$T$ is temperature, $t$ is time, $r$ is radial position, $z$ is vertical position, $\alpha$ is thermal diffusivity, and $q$ is heat generation.

$$
\text { Boundary Condition, Region 1: } \frac{\partial T}{\partial r}=0, q=0
$$

Boundary Condition, Region 2-4: $q=-\frac{h A\left(T-T_{\infty}\right)+\sigma \epsilon\left(T^{4}-T_{\infty}^{4}\right)}{\rho c_{p}}$ 
Boundary Condition, Region 5: $q=\frac{P / A_{\text {shell }}}{\rho c_{p}}$

$\alpha, \rho, c_{p}, \epsilon$, and $\sigma_{S S}$ are thermal diffusivity, density, heat capacity, emissivity, and electrical conductivity of stainless steel respectively. $A_{c}$ and $w_{t}$ are cross-sectional area and wall thickness of the shell, respectively. $I$ is current, $h$ is convective heat transfer constant, $T_{\infty}$ is ambient temperature, and $\sigma$ is the Boltzmann constant. $P$ is the power input from the cylindrical shell, and $A_{\text {shell }}$ is the cross-sectional area of the cylindrical shell.

It is assumed that the heat generated from passing current through the copper supports is negligible. Heat can be convected and radiated away at boundary regions $2-4$, and heat is conducted into the supports at region 5 where the copper is in contact with the stainless steel shell. The power conducted into the support is assumed to be $200 \mathrm{~W}$ (400 W total, with half going into each copper piece at the end of the shell). The results from these calculations showed a temperature rise of at least $60 \mathrm{~K}$ in the copper end pieces over a $60 \mathrm{~s}$ test time. This temperature rise is enough to cause significant buoyancy effects in fluid exposed to the support structure and as a result would disturb the axisymmetry of the setup if that temperature rise bled into the horizontal bars of the support structure.

\subsection{Water Cooling}

In order to prevent the temperature change seen in the heat transfer calculations for the copper supports, water cooling was implemented on the copper end pieces to effectively force a constant temperature condition at the outermost edge of the end pieces. This would effectively prevent excess heat from transferring to the horizontal part of the support structure. Simple calculations were performed to select the flow rate appropriate for cooling.

$$
\frac{d V}{d t}=\frac{P}{c_{p_{w}} T_{w} \rho_{w}}=\frac{500 \mathrm{~W}}{\left(4162 \frac{\mathrm{J}}{\mathrm{kgK}}\right)(298 \mathrm{~K})\left(960 \frac{\mathrm{kg}}{\mathrm{m}^{3}}\right)}=4.2 \times 10^{-7} \frac{\mathrm{m}^{3}}{\mathrm{~s}}
$$

Where $\frac{d V}{d t}$ is the flow rate in $\mathrm{m}^{3} / \mathrm{s}, P$ is half of the total power input of the resistive heating system (using a worst-case estimate that half of the total power input must be removed from the supports at each end), $\rho_{w}$ is density of water, $c_{p_{w}}$ is the heat 
capacity of water, and $T_{w}$ is the water temperature.

Additionally, a "plume plate" was installed on the underside of the vessel lid to cool the hot, buoyant plume of gas coming off the test cylinders. Without removing heat from the buoyant plume, the ambient gas temperature in the vessel would rise at a rate of roughly $20 \mathrm{~K} / \mathrm{s}$, as shown in 2.12

$$
\frac{d T}{d t}=\frac{P}{c_{P_{g}} V_{g} \rho_{g}}=\frac{1000 \mathrm{~W}}{\left(1000 \frac{\mathrm{J}}{\mathrm{kgK}}\right)\left(0.04 \mathrm{~m}^{3}\right)\left(1.28 \frac{\mathrm{kg}}{\mathrm{m}^{3}}\right)}=19.5 \frac{\mathrm{K}}{\mathrm{s}}
$$

Where $\frac{d T}{d t}$ is the temperature rise rate of the gas in the vessel in $\mathrm{K} / \mathrm{s}, P$ is the total power input of the resistive heating system (using a worst-case estimate that all of the power input is transferred to the buoyant plume around the cylinder), $\rho_{g}$ is density of the gas, $c_{p_{g}}$ is the heat capacity of the gas, and $V_{g}$ is the volume of gas inside the vessel (40L). This estimated temperature rise required adding a water-cooled "plume plate" inside the vessel just beneath the top lid to remove the excess heat from the gas and maintain minimal temperature increase in the ambient gas inside the vessel. Flow rate calculations very similar to Equation 2.11 were performed, this time assuming the total power input was transferred to the buoyant plume, and thus had to be removed by the cooled "plume plate". These calculations yielded a minimum flow rate of $8.4 \times 10^{-7} \mathrm{~m}^{3} / \mathrm{s}$ for the plume plate. The plume plate was constructed from $0.6 \mathrm{~cm}$-thick copper plate. Copper tubing was coiled in a zig-zag pattern across the back side of the plate, and soldered in place to ensure heat conduction between the point of impingement from the buoyant plume to the cooling water.

The water cooling system implemented used a Thermo-Fisher Scientific NESLAB System III water-to-water heat exchanger to maintain a cooled water temperature of 298K. Plumbing was installed around and inside the vessel to supply the supports and the plume plate with cooled water. The overall flow rate of the installed cooling system was $3.8 \times 10^{-5} \mathrm{~m}^{3} / \mathrm{s}\left(2280 \mathrm{~cm}^{3} / \mathrm{min}\right)$. This is a far greater flow rate than the calculations above required (minimum flow rate of $1.68 \times 10^{-6} \mathrm{~m}^{3} / \mathrm{s}\left(101 \mathrm{~cm}^{3} / \mathrm{min}\right)$, and thus supports and plume plate are assumed to be held at $298 \mathrm{~K}$. The maximum temperature rise in the ambient gas in the vessel after a 5 minute test period was observed to be $20 \mathrm{~K}$, for a temperature rise in the ambient gas of $0.067 \mathrm{~K} / \mathrm{s}$ instead 
of the predicted rise of $20 \mathrm{~K} / \mathrm{s}$ without water cooling. The water cooling system is key to proper functioning of the experiment, preserving ambient gas temperature as well as the axisymmetry of the setup.

\subsection{Cylinder Characterization}

It was important to characterize the temperature distribution along cylinders of different lengths. Four cylinders were selected for characterization, each with a diameter of $1.27 \mathrm{~cm}$ and a wall thickness of $0.05 \mathrm{~cm}$. The lengths of the cylinders for characterization were $2.54,12.7,19.1$, and $25.4 \mathrm{~cm}$ long to match the four different lengths of the test cylinders. Variations in diameter and wall thickness were not considered for characterization, as the results of the heat transfer models from Section 2.1 did not show significant variation in temperature distribution when diameter and wall thickness values were varied within the range of values that would be tested as specified by Table 2.1 .

The entire support structure was mounted on the lab bench, including vessel flange, power feedthroughs, and modular copper support structure. Each cylinder for characterization was then mounted into the support structure, and the water cooling plumbing was connected to the copper end pieces to ensure the same cooling setup would be used for final tests. Five K-type thermocouples (see Section 2.4 for details) were spot-welded on to the surface of the cylinder using a capacitance welder (HotSpot I from DCC Corporation) with a weld energy of $20 \mathrm{~J}$. Welding the thermocouples to the metal surface is critical to minimize contact resistance and thus get accurate temperature readings. Due to the direct connection to the high current circuit via thermocouple leads, isolation modules are used to take measurements from the thermocouples. The module used was Analog Devices 5 B37 Isolated Thermocouple Input, which uses transformer coupling to isolate the signal. These modules prevent any stray current from reaching and potentially damaging the computer system. Figure 2.9p shows the wiring schematic for using the thermocouples with the isolation module.

The two outermost thermocouples are placed about $0.6 \mathrm{~cm}$ in from the edge of the stainless steel cylinder to sample temperature in the "cool layer" of the cylinder, and the rest were spaced evenly between the outermost thermocouples. A pyrometer, described in detail in Section 2.5, takes non-contact measurements of surface tem- 
perature. The pyrometer was put on a remotely controlled translation stage with a travel length of $25.4 \mathrm{~cm}$ to scan the full length of all characterization cylinders. This allows the pyrometer to measure the temperature distribution along each cylinder. The characterization setup is shown in Figure 2.7

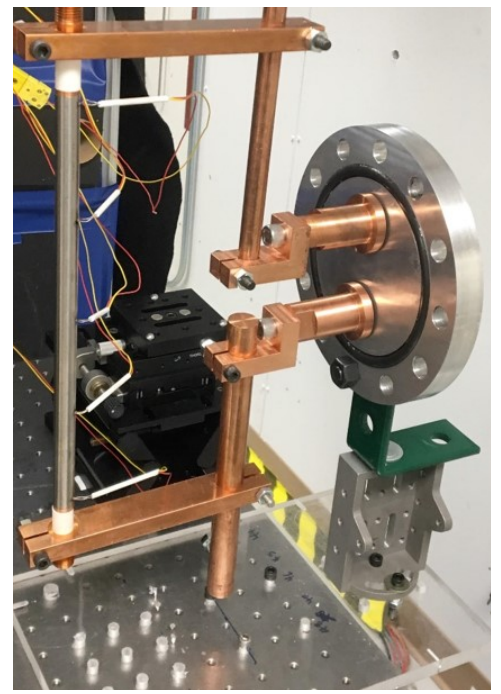

no heat

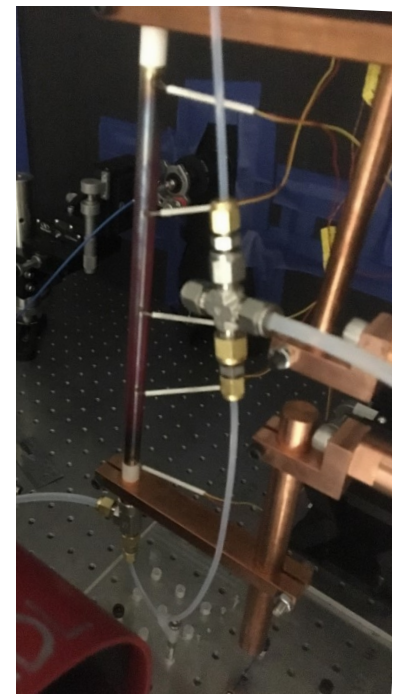

$773 \mathrm{~K}$

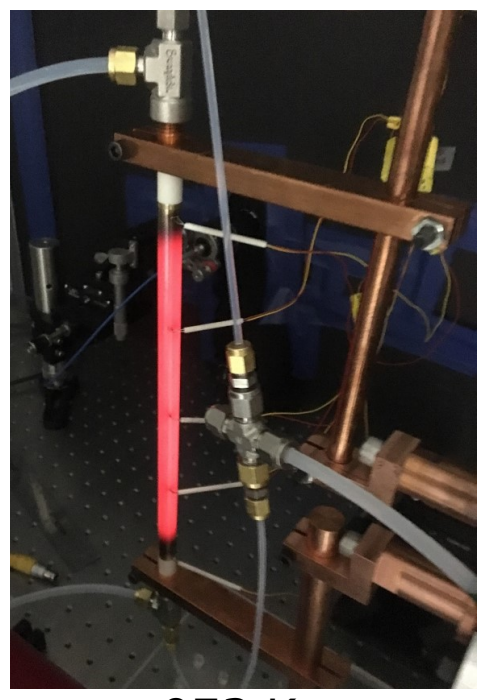

$973 \mathrm{~K}$

Figure 2.7: Benchtop setup for cylinder characterization. Left to right: $25.4-\mathrm{cm}-$ long cylinder with no heating, heated to $773 \mathrm{~K}$, and heated to $973 \mathrm{~K}$.

The procedure for cylinder characterization was as follows: power was applied to the cylinder, and temperature was controlled off of the thermocouple placed at the center of the cylinder. The temperature was driven to a preset steady-state value by LabView. Once temperature at the center of the cylinder reached steady-state, the translation stage was turned on and allowed the pyrometer to scan the full length of the cylinder. At least two full cycles of the translation stage movement were completed while the cylinder temperature was held constant. The tests were repeated for steady state temperatures ranging from 700 to $1000 \mathrm{~K}$. The translation staged moved at a rate of $1.27 \mathrm{~cm} / \mathrm{s}$, and the pyrometer sampled at a rate of $1000 \mathrm{~Hz}$, with an average taken every 100 samples for a final rate of $10 \mathrm{~Hz}$. This meant the pyrometer was taking a surface temperature average over a $0.12 \mathrm{~cm}$ length. The spot size of the pyrometer was measured to $0.32 \mathrm{~cm}$, so that the effect of the pyrometer movement during measurement is to create an effective measurement area that is at most 0.44 $\mathrm{cm}$ in diameter.

Figure 2.8 shows some results from this characterization work. All cylinders are brought to steady-state temperature at $800 \mathrm{~K}$, at which point the translation stage 

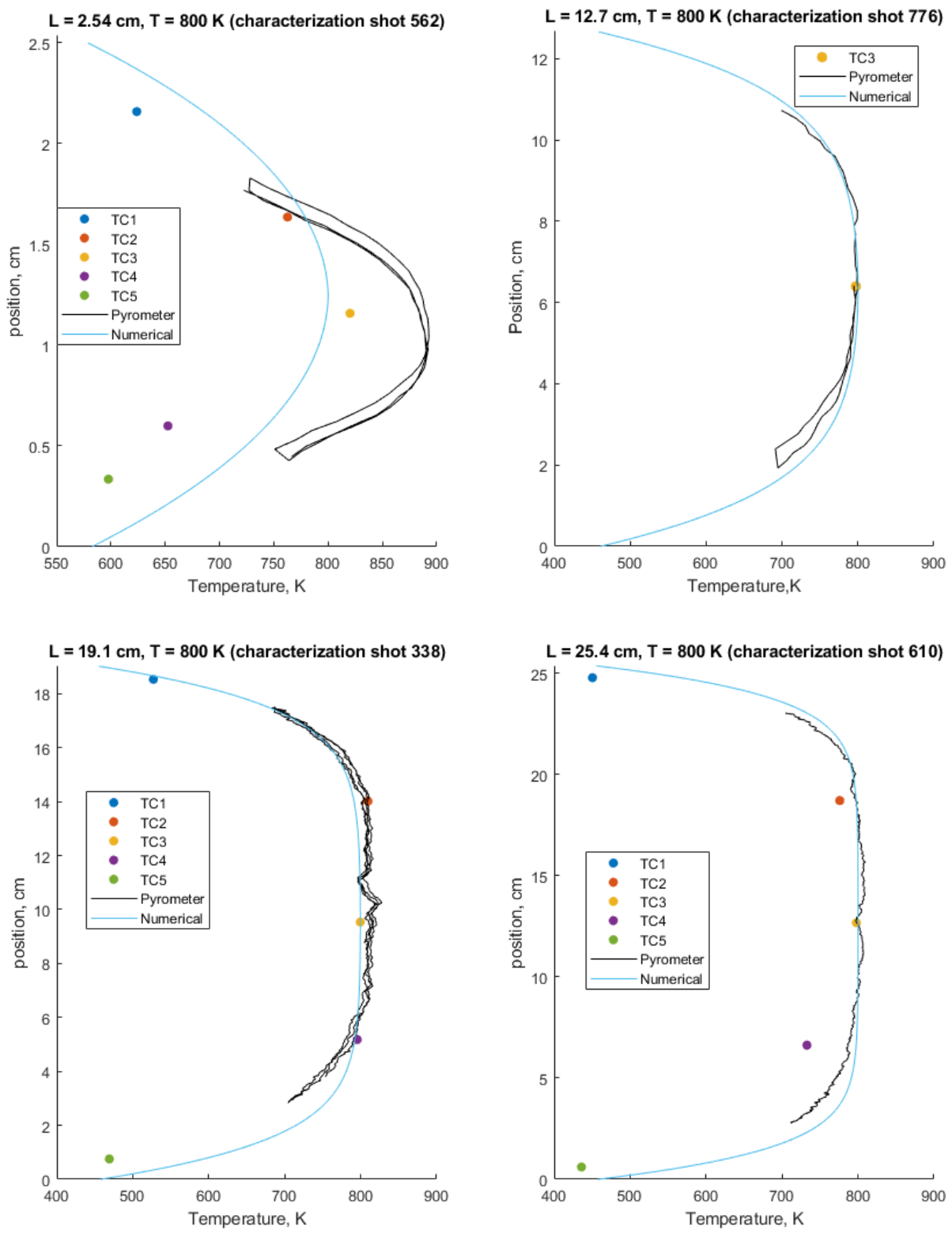

Figure 2.8: Results from characterization of cylinders at $800 \mathrm{~K}$.

began moving to scan the length of the cylinder under characterization testing. The pyrometer temperature is shown as a black line, and cuts out around $700 \mathrm{~K}$. While the pyrometer scans the full length of the cylinders, pyrometer measurements are reported only when signal-to-noise ratio is greater than 10 , which typically occurs at a temperature of about $700 \mathrm{~K}$. Temperature measured by thermocouples is also reported as the colorful filled dots. Note the error in location for all thermocouples is approximately $\pm 0.2 \mathrm{~cm}$. The numerical prediction of temperature distribution along the cylinder from the one-dimensional heat transfer calculations in Section 
2.1 is also included as the light blue line.

Pyrometer measurements of cylinders from 12.7 to $25.4 \mathrm{~cm}$ in length show a large central portion of the cylinder reaching a high and relatively uniform temperature. The pyrometer measurements are in very good agreement with the thermocouple measurements with the exception of TC4 for the cylinder with a length of 25.4 $\mathrm{cm}$. This anomaly could be be due to a poor quality weld between the thermocouple and the cylinder causing an artificially low temperature reading by TC4. This thermocouple is at least $1 \mathrm{~cm}$ into the uniform high temperature region and reads a significantly lower temperature than the other two thermocouples in the uniform high temperature region. We concluded that the temperature reported by TC4 for the 25.4-cm-long cylinder is incorrect. The numerical results as shown in Fig. 2.8 are calculated by adjusting the power input in the heat transfer calculations until the maximum temperature in the numerical calculations matched the set temperature. The experimentally observed temperature distribution is very similar to the numerical predictions with the same maximum temperature. All together this indicates that the design of these longer test cylinders is successful and that the observed temperature distributions are similar to those predicted from previous heat transfer calculations.

There are a number of difficulties with the characterization of the $2.54 \mathrm{~cm}$ long cylinder. The thermocouple readings, pyrometer readings, and numerical results all give different answers for the temperature at any given place on the cylinder. In particular, the pyrometer measurements are far hotter than either the thermocouple or numerical results show. The thermocouples indicate a narrower peak in temperature distribution than predicted by the numerical calculations, but both reach a peak temperature of about $800 \mathrm{~K}$. When comparing the pyrometer and thermocouple measurements, both indicate similar widths in temperature distribution, but the pyrometer reading is approximately $100 \mathrm{~K}$ higher than the thermocouples. There are likely two things happening simultaneously to yield such results: first, heat losses are more pronounced in the physical setup than predicted by the numerical model which leads to steeper temperature gradients and thus a narrower peak in temperature as seen by the thermocouples and the pyrometer. A reason for the discrepancies between the experimental and numerical temperature profiles is that for the very short cylinder, the one dimensional model for the shell and simplified 
treatment of contact with the support structure are not accurate representations of the true situation. Second, the pyrometer is measuring artificially high temperatures. This is most likely also due to the steep temperature gradients, as the spot size of the pyrometer is $0.32 \mathrm{~cm}$ and the temperature can vary by more than $100 \mathrm{~K}$ over that distance. As described in Section 2.5, the spot size used for calibration has a significant effect on the temperature read by the pyrometer. A source smaller than the size used for calibration gives an artificially high temperature reading. A similar effect could be occurring with the $2.54 \mathrm{~cm}$ long cylinder. Gradients are so steep that the spot size of 0.32 is far too large to look at an area small enough to be relatively uniform. In effect, the collection optics image a small area of high temperature (and strong radiation) and a larger area of low temperature that radiates less strongly; ultimately this is similar in effect to looking at a source that is smaller than the size used for calibration and would produce the same effect of an artificially high temperature reading. This indicates caution should be used with pyrometer measurements taken from the shortest cylinder, 25D, used for ignition experiments.

\subsection{Thermocouples}

Thermocouples are used in both cylinder characterization and igniton testing. The thermocouples were made in the laboratory for this experiment out of 24 AWG chromel and alumel wire to create a K-type thermocouple. The thermocouple wire is stripped of its plastic insulation for the last $2.5 \mathrm{~cm}$, and each wire is threaded through a 2-cm-long piece of double-bore alumina ceramic rod. The ends of the wires are welded together using a capacitance welder (HotSpot I from the DCC Corporation). The ceramic rod serves as insulation to separate the wires from each other and protect the rest of the plastic wire insulation from the high temperatures of the heated surface. Figure 2.9 a shows a schematic of the thermocouple construction.

As mentioned previously, the thermocouples are welded to the cylinder surface to ensure low contact resistance. Poor contact between the surface and the thermocouple may cause a temperature reading that is lower than the actual temperature (Michalski, Strąk, and Piasecka, 2017). There are thermally conductive epoxies designed to help ensure good thermal contact between thermocouple and surface, but the operational temperature range of such epoxies is limited and thus not useful here. Welding the thermocouples to the surface is possible in this case since both thermocouple and surface are metallic, and this method of attachment is chosen as 
it creates excellent contact and works over a very large range of surface temperatures.

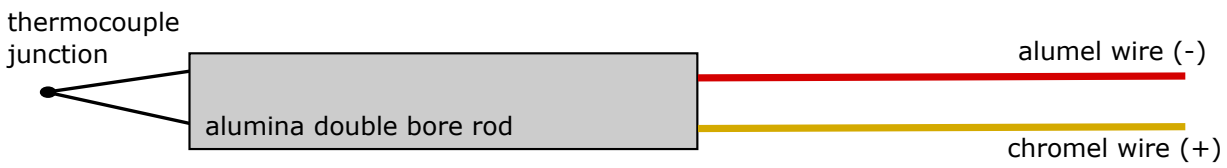

(a)

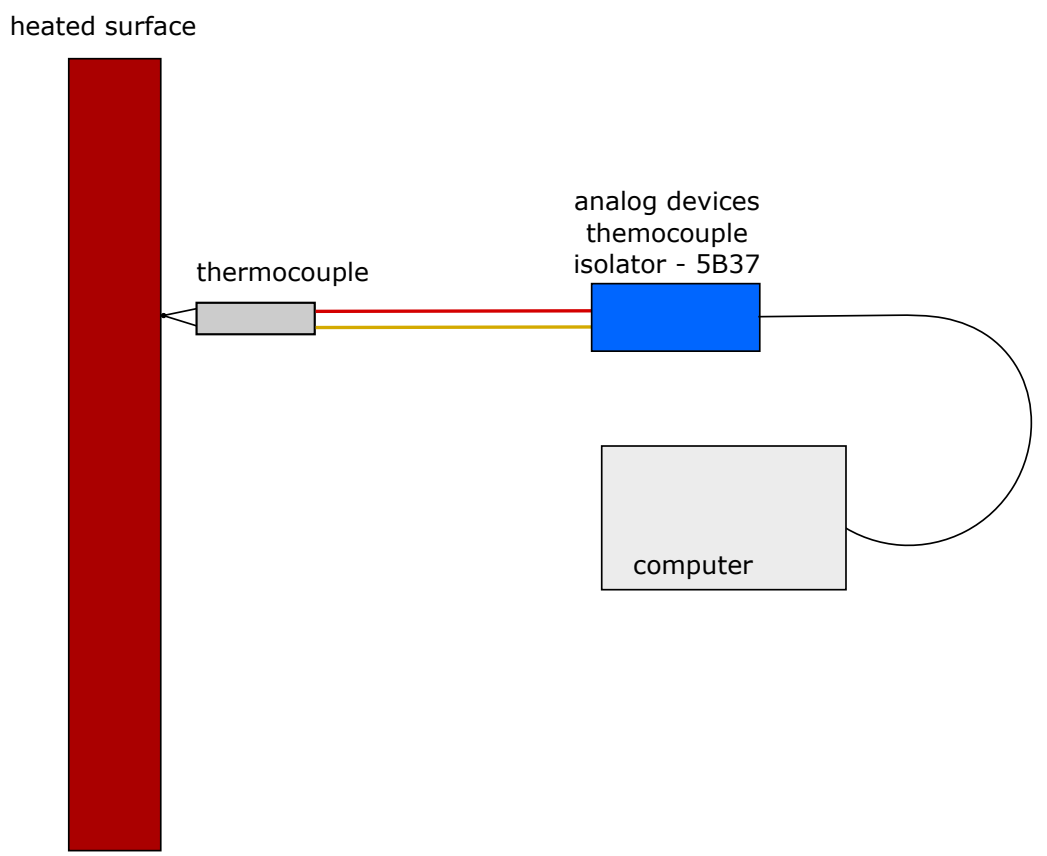

(b)

Figure 2.9: (a) Thermocouple construction schematic. (b) Themocouple wiring schematic.

The first method for taking surface temperature measurements of a test cylinder during ignition experiments uses a thermocouple. However, the presence of a thermocouple disturbs the boundary layer over a vertical cylinder by introducing stagnation points at the top and bottom of the thermocouple leads. In order to avoid disturbing the portion of the thermal boundary layer that leads to ignition, the location of ignition along the length of the cylinder needed to be identified. Preliminary visualization experiments showed that ignition occurred about $3 \mathrm{~cm}$ from the top edge of the cylinder, and consequently the testing thermocouple was placed $1.5 \mathrm{~cm}$ from the top edge of the cylinder so that no stagnation points were introduced in regions along the cylinder where ignition would occur. 
The thermocouple also has to be calibrated for its placement in the cool region of the cylinder (see Section 2.3), as the temperature $1.5 \mathrm{~cm}$ from the end of the cylinder is significantly cooler than the temperature near the center line. To calibrate the thermocouple for measuring center line temperature, two thermocouples are welded to the surface; one along the center line, and the other at the final testing placement $1.5 \mathrm{~cm}$ below the top edge of the cylinder. Welding the thermocouples to the metal surface is critical to minimize contact resistance and thus get accurate temperature readings. Because direct exposure of the thermocouple leads to high current levels from resistive heating, thermocouple isolation modules (5B37) from Analog Devices are used to take measurements from the thermocouples. These modules prevent any stray current from reaching and potentially damaging the computer system.

Calibration tests are run, heating up the cylinder to steady-state temperatures from 500 to $1100 \mathrm{~K}$. For each test, the cylinder is allowed to reach steady state for at least $60 \mathrm{~s}$ so that the calibration between center line temperature and temperature at $1.5 \mathrm{~cm}$ from the top edge can be taken for the ramp up period and for steady-state behavior.

The thermocouple readings can contain artifacts due to coupling with with the current flowing through the circuit. This can be seen as a sharp rise or fall in temperature reading at the moment when current is applied or tuned off. This current coupling can be corrected easily. The change in temperature during current shut off is described as a linear function of the current applied before shut off. The thermocouple reading is corrected by adding the linear function for change in temperature with current to raw temperature measurement, as shown in Eq. 2.13.

$$
T_{\text {corrected }}=T_{\text {raw }}+\Delta T, \text { and } \Delta T=c_{1} I+c_{2},
$$

where $c_{1}$ and $c_{2}$ are found by fitting a linear function to data points ( $I_{\text {pre-shutoff }}$, $\Delta T_{\text {shutoff }}$ ) found from shutting off current at at least six discrete values. Figure 2.10 shows an example of the uncorrected temperature reading (dark blue dashed line) compared with the corrected temperature reading (light blue solid line). At the moment when current (red) shuts off, the uncorrected temperature spikes up by approximately $40 \mathrm{~K}$ and stays at this level to slowly cool. This is a sign of current coupling, as the temperature should instead decrease once current and thus 
heat generation is stopped. The corrected temperature also shows a spike, but it is brief and quickly returns to the temperature before current was shutoff, and then slowly cools. Uncorrected and corrected temperatures produce the same reading once current is off.
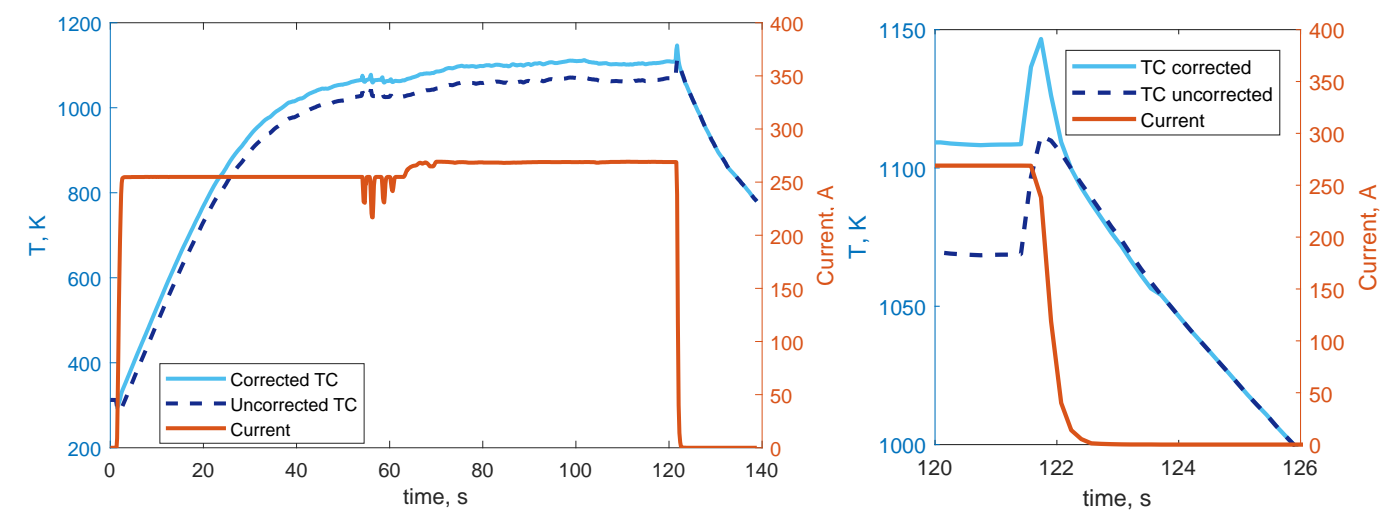

Figure 2.10: Example of current coupling of thermocouple signal and the correction implemented. Left: Full time trace of current, uncorrected, and corrected temperature. Right: Zoom to shutoff period showing spikes in temperature reading.

Once the thermocouple measurement is corrected for current coupling, the thermocouple measurement can be fully calibrated by taking the center line temperature ( $\left.T_{\text {central }}\right)$ as a function of the temperature measured by the testing thermocouple $\left(T_{t o p}\right)$. The function used to describe this relationship is a polynomial whose order is raised until the residuals fluctuate around a mean of zero. That is, with a polynomial of sufficient order, there are no trends in the residual. The raw data of $\left(T_{t o p}\right.$, $\left.T_{\text {central }}\right)$, polynomial fit for $T_{\text {central }}=f\left(T_{\text {top }}\right)$, and residuals of the polynomial fit are shown in Fig. 2.11.

The correlation between the temperatures measured by the testing and central thermocouple is then used during testing to determine the central location temperature. The thermocouple on the center line is removed so that only the testing thermocouple remains for ignition experiments. Figure 2.12 shows a schematic of the setup for both heating and ignition tests. 

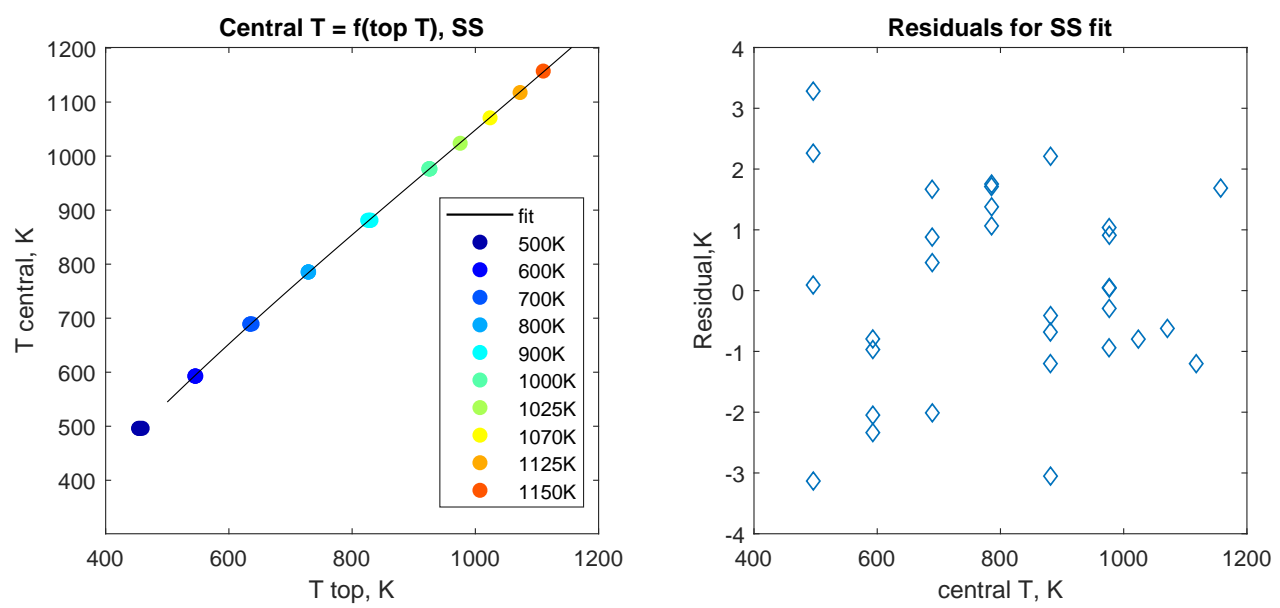

Figure 2.11: Fit for calibration of top TC to reconstruct central TC, left. Right: Residuals of fit.

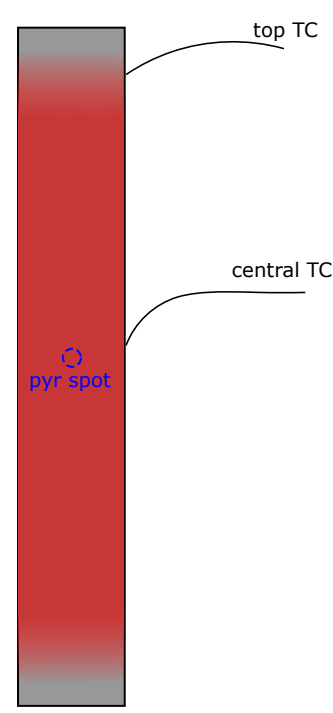

a. heating tests

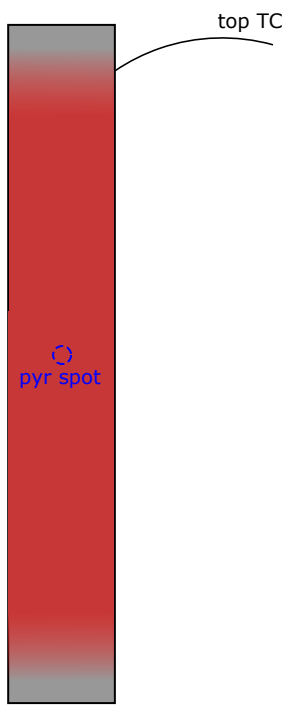

b. ignition tests

Figure 2.12: Schematic of (a) heating calibration setup and (b) ignition testing setup.

\subsection{Two-Color Pyrometer}

In addition to the testing thermocouple, a two-color pyrometer takes high-speed measurements of surface temperature (approximately $100 \mathrm{kHz}$ ). The benefit of the pyrometer is that measurements are taken without disturbing the boundary layer development over the test surface. The design of the pyrometer is a variation of the pyrometer described by Coronel (2016). 


\section{Theory}

Here we describe the theory behind the operation of the pyrometer, the fundamentals of which are described in Michalski, Eckersdorf, et al. (2001). In ignition studies alone, a pyrometer is frequently used to measure the surface temperature of a hot element (see Silver (1937), Patterson (1940), Kuchta, Bartkowiak, and Zabetakis (1965), Ono et al. (1976), and Roth, Haber, and Bockhorn (2017), among many others).

As laid out in Michalski, Eckersdorf, et al. (2001), the fundamental phenomenon that enables pyrometers to make temperatures measurements is Planck's law, which describes the relationship between a blackbody's spectral irradiance, wavelength, and temperature. Planck's law is shown in Equation 2.14:

$$
\begin{gathered}
L_{\lambda, b}(T)=\frac{C_{1}}{\lambda^{5}\left[\exp \left(C_{2} / \lambda T\right)-1\right]}, \\
C_{1}=8 \pi h c^{2} \text { and } C_{2}=\frac{h c}{k_{b}} .
\end{gathered}
$$

$L_{\lambda, b}$ is irradiance, $T$ is temperature, $\lambda$ is wavelength, $h$ is Planck's contant, $c$ is the speed of light in a vacuum, and $k_{b}$ is Boltzmann's constant. The Planck constants are calculated as shown by Equation 2.15 and have values of $C_{1}=1.496 \times 10^{-15} \mathrm{~W} \cdot \mathrm{m}^{2}$ and $C_{2}=0.0144 \mathrm{~m} \cdot \mathrm{K}$. Figure 2.13 shows the spectral irradiance of a blackbody as a function of wavelength and temperature.

Equation 2.14 is only valid for true blackbodies (perfect emission of radiation). For non-ideal bodies, a correction factor for spectral emissivity, $\epsilon_{\lambda}$, is introduced as shown in Equation 2.16 .

$$
L_{\lambda}(T)=\epsilon_{\lambda} \frac{C_{1}}{\lambda^{5}\left[\exp \left(C_{2} / \lambda T\right)-1\right]} .
$$

This can be further simplified in the infrared region by using the Wein approximation that $\lambda T \ll C_{2}$ :

$$
L_{\lambda}(T) \approx \epsilon_{\lambda} \frac{C_{1}}{\lambda^{5}} \exp \left(-C_{2} / \lambda T\right)
$$




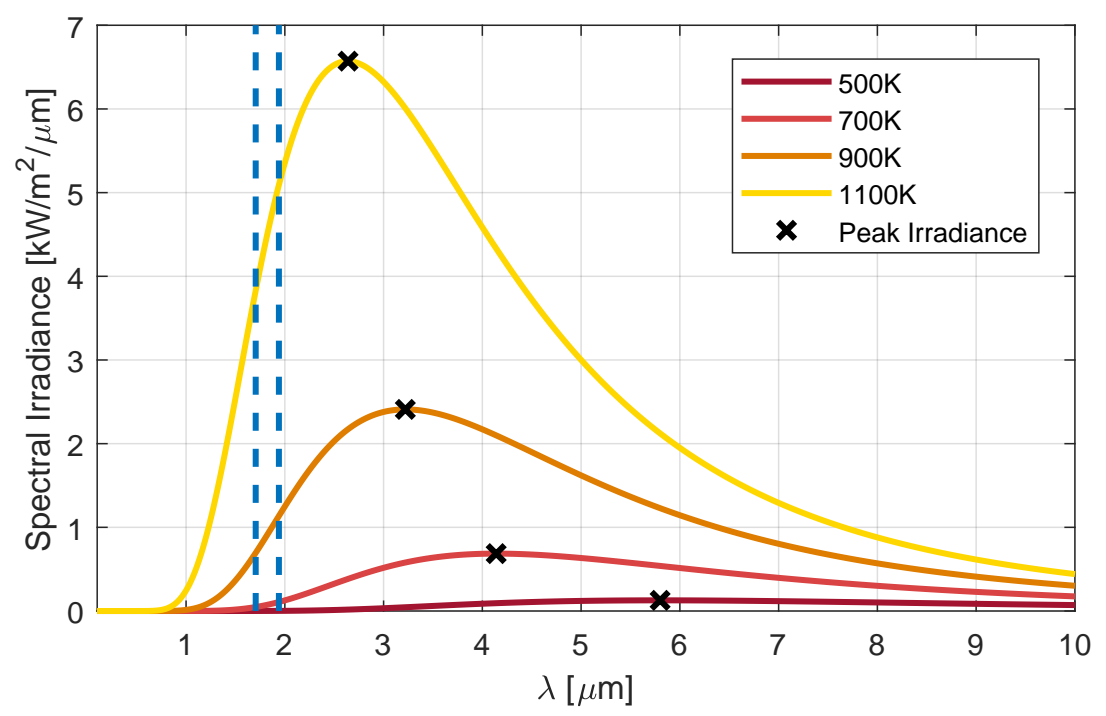

Figure 2.13: Blackbody irradiance from Planck's law. X's mark wavelength that produces peak irradiance for each temperature. Blue dashed lines represent wavelengths of the filters used in the two-color pyrometer.

Note that $\lambda T$ is approximately one order of magnitude less than $C_{2}$. Equation 2.17 is integrated over a wavelength region, $\Delta \lambda$, to find total intensity $I$ :

$$
I(T)=\int_{\lambda-\Delta \lambda / 2}^{\lambda+\Delta \lambda / 2} \epsilon_{\lambda} \frac{C_{1}}{\lambda^{5}} \exp \left(-C_{2} / \lambda T\right) d \lambda .
$$

Assuming that that the wavelength region used for integration is small (achieved in the pyrometer by using narrow bandpass filters), Eq. 2.18 is approximated as shown:

$$
I(T)=\epsilon_{\lambda} \frac{C_{1}}{\lambda^{5}} \exp \left(-C_{2} / \lambda T\right) \Delta \lambda
$$

Equation 2.19 is applied for two wavelength regions to yield a ratio of total intensities given by,

$$
\frac{I_{1}}{I_{2}}=\frac{\epsilon_{\lambda_{1}}}{\epsilon_{\lambda_{2}}} \frac{\lambda_{2}^{5}}{\lambda_{1}^{5}} \frac{\Delta \lambda_{1}}{\Delta \lambda_{2}} \exp \left(\frac{-C_{2}}{T\left(\frac{1}{\lambda_{1}}-\frac{1}{\lambda_{2}}\right)}\right) .
$$

If the radiation source is assumed to be a graybody, that is the emissivity is constant across all wavelengths, $\epsilon_{\lambda_{1}}=\epsilon_{\lambda_{2}}$ and the intensity ratio is reduced to 


$$
\frac{I_{1}}{I_{2}}=\frac{\lambda_{2}^{5}}{\lambda_{1}^{5}} \frac{\Delta \lambda_{1}}{\Delta \lambda_{2}} \exp \left(\frac{-C_{2}}{T\left(\frac{1}{\lambda_{1}}-\frac{1}{\lambda_{2}}\right)}\right) .
$$

The wavelengths $\lambda_{1}$ and $\lambda_{2}$ are known, as are the wavelength bandwidths $\Delta \lambda_{1}$ and $\Delta \lambda_{2}$ due to the bandpass filters chosen in the pyrometer. This means the intensity ratio given by Eq. 2.21 is reduced to a function of temperature only. Further manipulation yields:

$$
\ln \left(\frac{I_{1}}{I_{2}}\right)=\frac{A}{T}+B
$$

where,

$$
A=\frac{C_{2}}{\left(\frac{1}{\lambda_{1}}-\frac{1}{\lambda_{2}}\right)} \text { and } B=\ln \left(\frac{\lambda_{2}^{5}}{\lambda_{1}^{5}} \frac{\Delta \lambda_{1}}{\Delta \lambda_{2}}\right)
$$

Equation 2.22 is the fundamental equation that the pyrometer shown in Figure 2.14 operates on. The pyrometer measures a portion the intensity of two wavelengths of light emitted by a radiating surface. Eq. 2.22 calculated the temperature of the surface given the measured intensities.

Note that if the Wein approximation was not used in Eq. 2.17, the intensity ratio of the two wavelengths used in the two-color pyrometer (1705 and $1940 \mathrm{~nm}$ ) would only be $0.015 \%$ different than the intensity ratio found using the Wein approximation. This is a very small difference in results and justifies the use of the Wein approximation in the pyrometer measurement formulation.

\section{Description of the Two-Color Pyrometer}

The pyrometer used consists of three main parts: a collection head, a rigid pyrometer body, and a fiber optic cable used to pass light from the collection head to the pyrometer body. This is shown in Fig. 2.14. The optical components of the pyrometer are listed in Table A.1. This pyrometer is an adaptation of the one documented by Coronel (2016). The major modifications include using a single fiber input, different body optical components, and a small rigid lens tube system for 
the body of the pyrometer that makes the entire pyrometer portable and simplifies alignment of the optical components.

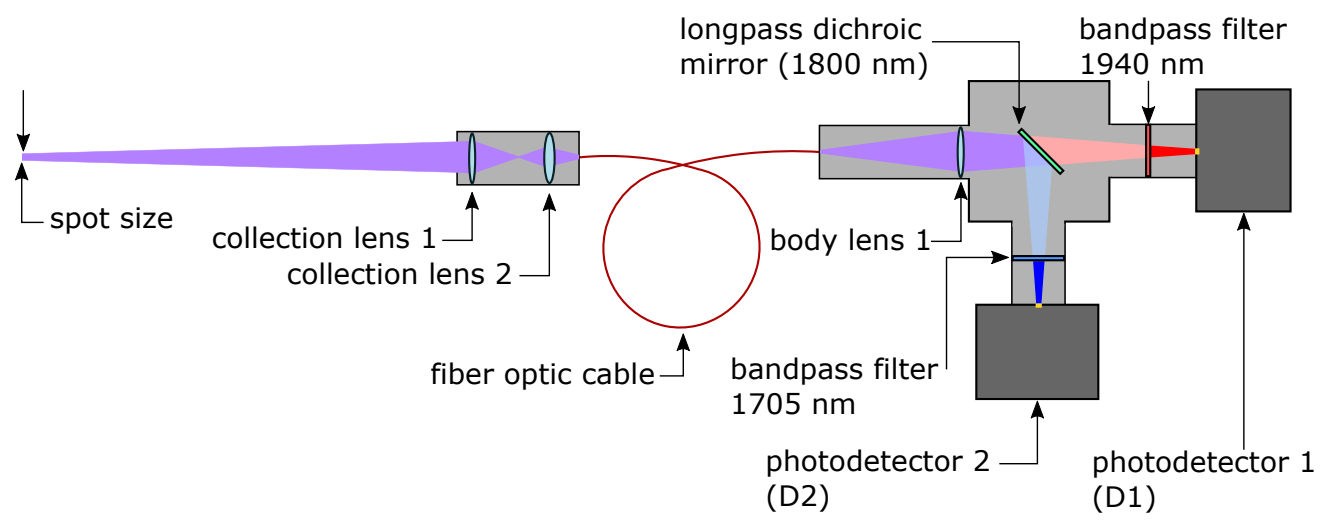

Figure 2.14: Schematic of pyrometer layout. Purple represents unsplit wavelengths, red represents long wavelengths $(>1800 \mathrm{~nm})$, blue represents short wavelengths $(<1800 \mathrm{~nm})$, and saturated red and blue represent the narrow bandpass wavelengths (1940 and $1705 \mathrm{~nm}$ ) that reach the active detector face (yellow).

The collection head is designed to collect light from a surface at a distance of 38.1 $\mathrm{cm}$ and has a collection spot diameter of $0.32 \mathrm{~cm}$. The first focusing lens collects light from the surface being measured, and the second lens focuses that light onto the fiber optic cable. For maximum light intensity (and thus high signal-to-noise ratios), all the light collected must be focused on to the 1-mm-diameter core of the fiber optic cable.

The fiber optic cable is a multi-mode silica fiber with a $1 \mathrm{~mm}$ core diameter that transmits light from 400-2200 $\mathrm{nm}$. The core diameter is large to maximize the amount of light collected by the fiber and thus transmitted to the photodetectors. Light from the collection head is focused into the fiber and projected out the back end of the fiber into the pyrometer body.

The lens in the body of the pyrometer is used to focus the light coming out of the fiber optic onto the 1-mm-diameter active area of the photodetectors. The light passes through a longpass dichroic mirror with a splitting wavelength of $1800 \mathrm{~nm}$. Wavelengths longer than that pass through the mirror, while shorter wavelengths are reflected. The light that passes through the dichroic is further filtered by a narrow 
bandpass filter centered at $1940 \mathrm{~nm}$ with a FWHM of $105 \mathrm{~nm}$ such that the light collected by the photodetector is of a narrow bandwidth. Light reflected from the mirror is further filtered by a bandpass filter of $1705 \mathrm{~nm}$ with a FWHM of $97 \mathrm{~nm}$. The photodetectors measure light intensity via a voltage reading and the ratio of intensity is used to calculate surface temperature, $T_{S}$, by Eq. 2.24 adapted from Eq. 2.22

$$
T_{S}=\frac{A}{\ln \left(\frac{I_{1}}{I_{2}}\right)-B}
$$

$I_{1}$ and $I_{2}$ are voltage readings from photodetectors D1 and D2 respectively. Constants $A$ and $B$ are determined by calibration with a blackbody radiation source (Process Sensors BBS1200). The parts list for the pyrometer is documented in Table A.1.

\section{Glass Pyrometer Variation}

A variation on the previously described pyrometer was constructed and tested. The principle goal was to allow reliable pyrometer readings at temperatures as low as $500 \mathrm{~K}$ (vs the lower bound of $700 \mathrm{~K}$ with the fiber based pyrometer). This low temperature would be achieved by two main differences in the pyrometer design: first, the wavelengths monitored would be shifted to longer wavelengths (2100 and 2300 $\mathrm{nm}$ ) to collect more of the radiation emitted by low-temperature sources. Second, the pyrometer would increase the amount of light collected across all wavelengths by eliminating the fiber optic cable shown in Fig. 2.14 and integrating the collection optics directly into the body of the pyrometer.

The reasoning behind shifting wavelengths longer is twofold. For a blackbody at $500 \mathrm{~K}$, there is about 10 times the spectral irradiance at 2100 verus $1705 \mathrm{~nm}$ and about 4 times the spectral irradiance at 2300 versus $1940 \mathrm{~nm}$. There is just more irradiance to observe at longer wavelength. Additionally, the photodetectors used in the pyrometer are nearly twice as sensitive at these longer wavelengths than the original pair. These factors together indicate that using longer wavelength would increase the signal produced by the pyrometer at low temperature when compared with the original wavelengths. It is important to note that selection of these wavelengths alone necessitated eliminating the fiber optic cable, as no cable was avaliable that would transmit light at wavelengths longer than $2200 \mathrm{~nm}$. 
This necessary elimination of the fiber optic cable aligned well with the design of the new pyrometer since it was planned to eliminate the fiber anyway by incorporating the collection optics directly into the pyrometer body. The goal of this design was to increase the amount of light collected, since there is always some light lost when using the fiber due to imperfect focusing of the collected light into the fiber core. This can occur in two ways: by the numerical aperture of the light focused by the collection optics being greater than the accepted numerical aperture of the fiber (i.e. the fiber views a narrower "cone" of light than the optics of the collection heat create), and by the diameter of the focal point being larger than the diameter of the fiber core. Eliminating the fiber means the amount of light collected is limited only by the solid angle of the collection optics. While it was difficult to quantitatively predict how much this change would increase the signal of the pyrometer, it was expected that this would significantly increase the signal at low temperatures when compared with a fiber-based pyrometer with similar collection optics.

Custom bandpass filters centered at $2100 \mathrm{~nm}$ and $2300 \mathrm{~nm}$ were ordered, as was a custom longpass dichroic mirror with a split wavelength of $2200 \mathrm{~nm}$. The glassbased pyrometer was set up very similarly to the body of the fiber-based pyrometer shown in Fig. 2.14, with slightly different lenses used before the dichroic mirror in order to properly incorporate the collection optics. It was observed during early tests that the glass pyrometer has signals approximately 10 times larger than the fiber-based pyrometer at temperatures around $500 \mathrm{~K}$. However, alignment of the glass pyrometer for calibration and measurement was extremely difficult.

The fiber acts like a fixed point of light emission in the pyrometer, such that if the collection head is misaligned (at an angle or displaced from center), the only difference in light emission is an overall dip in intensity; the point of light emission is fixed in place and thus there is no misalignment within the pyrometer body. This means slight misalignment results only in a small overall signal strength decrease and not in a change of temperature reading (the intensity of both wavelengths decreases proportionally). If the collection head is severely misaligned, then no light will enter the fiber in the first place. This misalignment will be immediately obvious and easy to correct.

However, for the glass pyrometer, even a small misalignment of the collection op- 
tics with the source will cause misaligment from intended positioning downstream in the optical system that ultimately leads the light to strike detectors off center in complex ways that are difficult to compensate for. Several dozen calibrations were attempted, and each time tiny differences in the positioning of the pyrometer between calibration and measurement setups caused large ( $>100 \mathrm{~K})$ discrepancies in the measured temperature. Ultimately, the glass pyrometer was abandoned due to these difficulties. Fortunately, the test cylinders ignite at temperatures well above the lower limit of the fiber-based pyrometer and are easily monitored with this more reliable and easily aligned version.

\section{Pyrometer Calibration}

The calibration process consists of aligning the pyrometer collection head with the aperture on the blackbody source that most closely matches the spot size of the pyrometer. It is important to note that calibrating off an aperture that is similar to the spot size of the pyrometer is key to getting accurate temperature readings. For example, calibrating with a $0.3 \mathrm{~cm}$ aperture and then trying to measure a source 0.1 $\mathrm{cm}$ in characteristic length will lead to inaccurately high temperature readings. It is also important that the pyrometer spot is centered on the aperture and is viewing it head-on and not at an angle. A proper alignment will give the best possible calibration. The window used to view inside the vessel is placed in the line of sight of the pyrometer to replicate experimental operation as closely as possible during calibration. The temperature of the blackbody source is varied from 723 to $998 \mathrm{~K}$ at $25 \mathrm{~K}$ increments. This temperature range is chosen in order to calibrate over the full dynamic range of the detectors without changing the gain, as gain cannot be changed during a full ignition experiment. At each temperature, detector readings are collected for at least 10 seconds. The data from each temperature calibration point is collected as $\left(1000 / T, \ln \left(V_{1} / V_{2}\right)\right)$. The calibration plot is shown in Fig. 2.15 , and constants $A$ and $B$ are found by fitting a linear model following Eq. 2.22 to the collected data. The following values are reported:

$$
A=950.3 \text { and } B=-0.3147 \text {. }
$$

The left side of Fig. 2.15 shows the linear calibration curve in red, which is determined applying the calibration data to the standard "fit" function in MATLAB. Constants $A$ and $B$ are found from this fit. Also $95 \%$ confidence limits on each constant are returned and can be used to contruct the $95 \%$ confidence limits on 

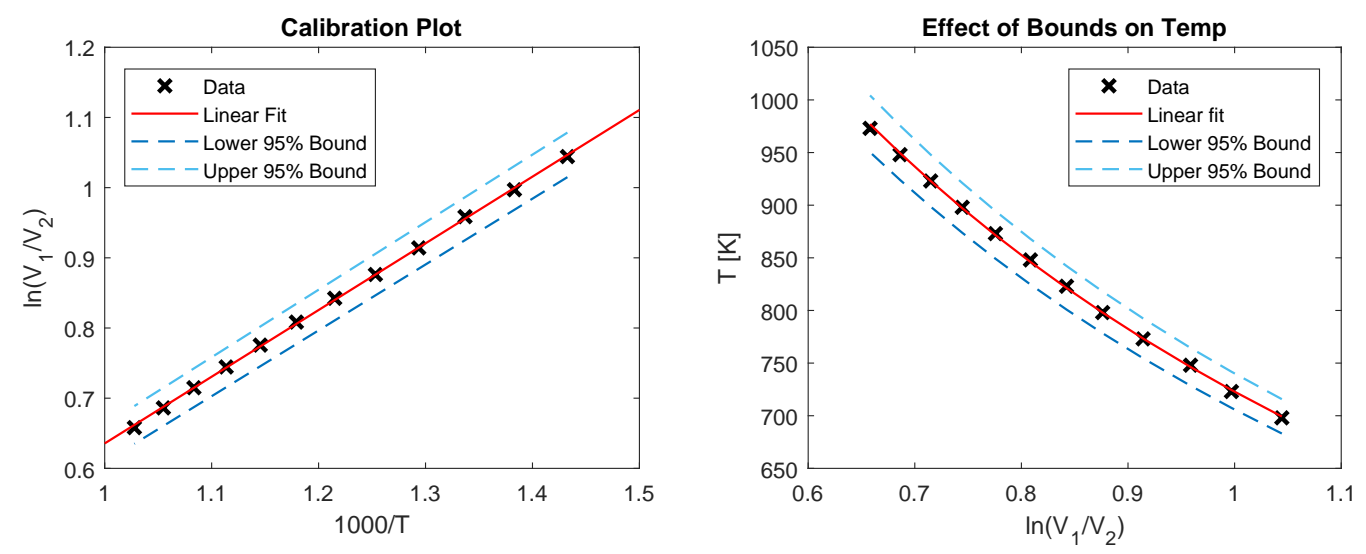

Figure 2.15: Calibration of pyrometer. Left: linear calibration model (red) fit to calibration data (black) using Eq. 2.22, including 95\% confidence bounds. Right: Temperature reconstruction (red) using constants $A, B$ applied to Eq. 2.24 compared with calibration data(black), including $95 \%$ confidence bounds.

the calibration as a whole. The right side of Fig. 2.15 shows the reconstruction of temperature using values of $A$ and $B$ found from calibration and compares that with the temperature data from calibration. The reconstruction of temperature using the calibration constants $A$ and $B$ is in good agreement with the raw temperature data. Furthermore, uncertainty on the pyrometer measurement can be quanitified by using the $95 \%$ confidence limits on $A$ and $B$ to construct upper and lower bounds on temperature measurements, shown as blue dashed lines in the right side of Fig. 2.15, These 95\% confidence limits are returned form MATLAB's standard "fit" function. This results in an uncertainty of $\pm 28 \mathrm{~K}$ for temperature measurements taken by the pyrometer. This is likely an upper bound that overestimates the actual uncertainty; comparison of the pyrometer reading with thermocouple measurements indicated that the pyrometer uncertainty is approximately $\pm 10 \mathrm{~K}$.

Additional uncertainty arises from pyrometer temperature measurements made during the heating ramp. The pyrometer samples at a rate of $1000 \mathrm{~Hz}$, but the measurements are taken by averaging over 100 samples at a time, for a final measurement rate of $10 \mathrm{~Hz}$. The heating rate of the cylinder is modest by the time ignition temperatures are approached; leading up to ignition, a conservative estimate of the maximum heating rate would be $10 \mathrm{~K} / \mathrm{s}$. This would be a maximum uncertainty on the pyrometer of $\pm 1 \mathrm{~K}$ due to heating transients. This would add on to the measurement uncertainty on the pyrometer itself for a total uncertainty of $\pm 11 \mathrm{~K} / \mathrm{s}$, and a conservative upper bound of $\pm 29 \mathrm{~K}$ for the pyrometer measurement uncertainty. 
The pyrometer calibration was not found to drift significantly. The calibration was checked every few weeks for the first two months. Additionally, the pyrometer calibration was indirectly checked by regular comparison with thermocouple measurements taken from the same surface during the setup of each cylinder. Over months of running experiments, the pyrometer temperature reading was consistently found to be within approximately $10 \mathrm{~K}$ of the thermocouple measurement.

\subsection{Interferometer}

Interferometers are instruments that can measure changes in the index of refraction of a medium by interfering beams of electromagnetic radiation. Interferometers can be used for a variety of purposes; for this work, the interferometer is used to make quantiative temperature field measurements. Merzkirch (1987) describes in detail the fundamental principles behind interferometry, and lays out much of the theory that allows for an interference pattern from an interferometer to be translated back into measurements of index of refraction (and ultimately temperature, in this work). Other researchers have used interferometry to study combustion; notably, Adomeit (1965) used a Mach-Zehnder interferometer to measure the development of temperature fields surrounding suddenly heated vertical cylinders over time. The cylinders Adomeit (1965) studied are smaller than the ones investigated here (0.3$0.4 \mathrm{~cm}$ diameter and $3.5 \mathrm{~cm}$ long, surface area of $3.8 \mathrm{~cm}^{2}$ ) so the design of the interferometer for our large cylinders is substantially different, but the application of interferometry is remarkably similar.

Due to the size of the surfaces studied, the interferometer designed for this investigation needed to have a test beam with a diameter of $101.6 \mathrm{~mm}$ (to match the window aperture). This allows the interferometer to collect the maximum amount of information possible about the gas surrounding the test cylinders, as limited by the size of the windows on the vessel. The interferometer presented here is a variation on the classic Mach-Zehnder interferometer. In this version, the test beam alone is expanded to the test section diameter of $101.6 \mathrm{~mm}$, and then condensed before recombining with the reference beam. The reference beam is routed through a single-mode polarization preserving fiber. The fiber protects the reference beam from disturbances from the air currents in the laboratory without building a protective structure over the entire bench. The test beam of the interferometer is a free-space beam and is contained within such a protective structure, but space con- 
straints make a similar structure for a free-space reference beam infeasible. It also preserves the flatness of the wavefront of the reference beam.

\section{Design of Interferometer}

This design of the interferometer presented two main constraints: the diameter of the interferometer output beam must just match the size of the camera detector to maximize resolution without cutting out part of the beam, and the focal plane must coincide with the center of the vessel. It was not possible to achieve this with a single focusing element on the camera side of the interferometer, so an arrangement with three focusing elements was chosen. The three focusing elements were selected first through use of the thin lens equations to perform a simple ray tracing analysis and by using focal lengths of optical elements available for purchase.

$$
\frac{1}{F L}=\frac{1}{p}+\frac{1}{q}, M=\frac{-q}{p}=\frac{h_{i}}{h_{o}},
$$

where $F L$ is the focal length of a lens, $p$ is distance from the lens to the object, $q$ is distance from the lens to the image, $M$ is the magnification, and $h_{o}$ and $h_{i}$ are the height of the object and image respectively. Calculations with a parabolic mirror as the first focusing element with $F L_{1}=863.6 \mathrm{~mm}$, a silica lens with $F L_{2}=100 \mathrm{~mm}$ as the second focusing element, and a silica lens with $F L_{3}=150 \mathrm{~mm}$ as the third and final focusing element yielded the following arrangement of optical elements as shown in Fig. 2.16. This calculation treated all focusing elements as simple thin lenses. The image of one lens (for example FM2) is treated as the object of the next lens in order to estimate the optical elements needed to produce a image of the desired magnification on the camera chip.

Once the optical elements were chosen, the system was modeled in WinLens to include realistic effects such as folding of the beam on the benchtop, thick lenses, and optical aberrations.

The spacing and folding pattern shown in Fig. 2.17 was replicated on the benchtop. A resolution target (2"x2" USAF 1951 negative) was placed in the center of the vessel, and the placement of the camera (i.e. image plane) was altered until maximum resolution was achieved. In this case, the optical resolution of the system was higher than the camera resolution, meaning the limit at which the USAF target markings could not be distinguished was due to pixel spacing on the camera and not 


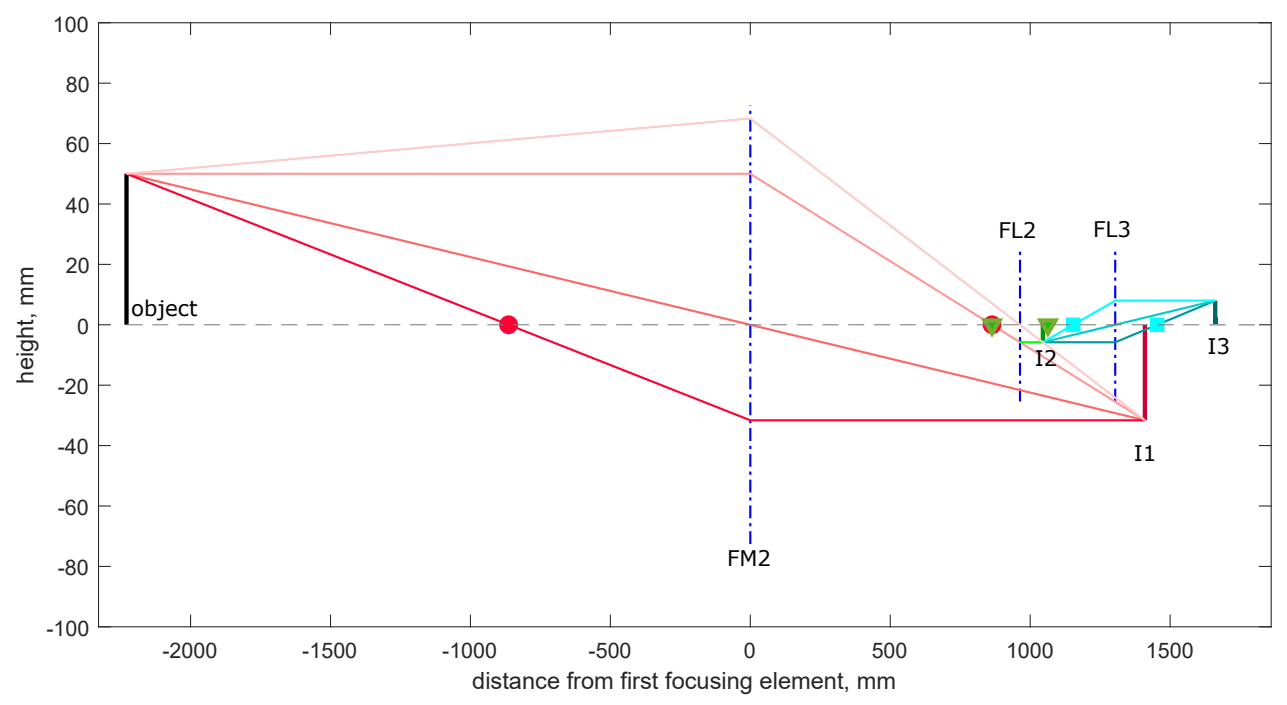

Figure 2.16: Thin lens ray tracing calculation. All distances are measured from the placement of the first focusing element. The object is placed at the center of the vessel, and the image from each of the three focusing elements is labeled I1, I2, or I3. I3 is the final image recorded by high speed camera and matches the chip size. The focal points for each focusing element are shown as red (FM2), green (FL2), or blue (FL3) shapes on the axis.

due to poor optical resolution. This resolution came out to about $8 \mathrm{px} / \mathrm{mm}$ at the focal plane.

\section{Interferometer Description}

$532 \mathrm{~nm}$ light from a Coherent Sapphire laser emerges at the laser head with a $0.7 \mathrm{~mm}$ diameter. It is expanded once by a $5 \mathrm{X}$ beam expander to $3.5 \mathrm{~mm}$ diameter. The light is then split by a 50:50 unpolarized cubic beamsplitter into a test beam and a reference beam. The test beam is expanded again by a factor of $5 \mathrm{X}$ to $17.5 \mathrm{~mm}$ diameter, and a focusing lens and a concave mirror are used to collimate the light at a diameter of $151.3 \mathrm{~mm}$. Some of this light is lost due to the beam diameter being larger than some of the folding mirrors used to direct it. The actual beam diameter comes out to approximately $101.6 \mathrm{~mm}$. This matches the aperture of the windows on the combustion vessel and maximizes the amount of information that the interferometer can collect at the focal plane. After the test beam passes through the combustion vessel, it is condensed by an identical concave mirror and focusing lens pair to a diameter slightly less that $17.5 \mathrm{~mm}$ due to the light lost before passing through the vessel. It then passes through the second beamsplitter to recombine with the reference beam. 


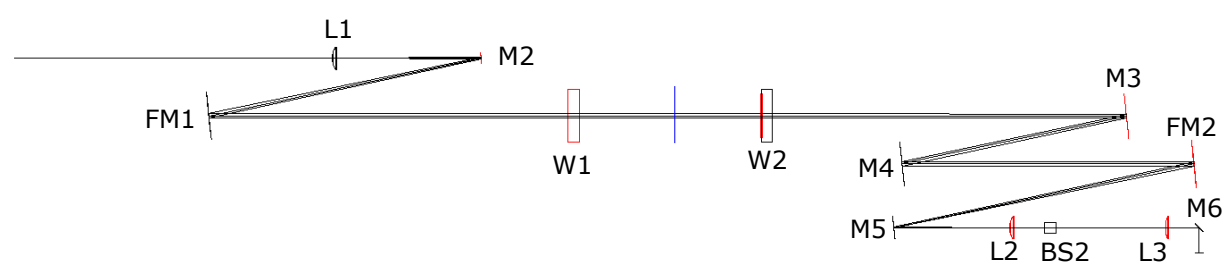

\begin{tabular}{|c|c|c|c|c|}
\hline$\#$ & Stop & Dirn 0 & Component & Sepn \\
\hline 1 & & Nom & lens & 328.610 \\
\hline 2 & & & mirror & -635.000 \\
\hline 3 & & & mirror & 819.600 \\
\hline 4 & & Nom & lens & 415.000 \\
\hline 5 & Stop & Nom & lens & 807.000 \\
\hline 6 & & & mirror & -523.000 \\
\hline 7 & & & mirror & 666.000 \\
\hline 8 & & & mirror & .700 .000 \\
\hline 9 & & & mirror & 263.610 \\
\hline 10 & & Nom & lens & 70.000 \\
\hline 11 & & Nom & lens & 249.400 \\
\hline 12 & & Nom & lens & 69.000 \\
\hline 13 & & & mirror & \\
\hline ThLeng & Lens & $\underset{\text { Mirror }}{\mathbb{D} \text { Block }}$ & ck $\underset{\text { Priem }}{\mathbb{B}} \underset{\text { Brosk }}{\mathbb{H i}}$ & 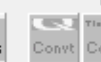 \\
\hline
\end{tabular}

Figure 2.17: Winlens modeling of the test beam of the interferometer. Treatments starts after the beam passes through BE2 and calculates the placement of focusing optics on the camera side of the interferometer (FM2, L2, L3) to create a focused image of the center of the vessel on the camera.

After splitting from the test beam, the reference beam couples into a single-mode polarization preserving fiber with the use of an $18 \mathrm{~mm}$ focal length collimation package. The collimation package is compatible with the $3.5 \mathrm{~mm}$ diameter of the test beam. The fiber is routed to the other side of the interferometer. An identical collimation package is used at the fiber output to return the reference beam to a $3.5 \mathrm{~mm}$ diameter. A third $5 \mathrm{X}$ beam expander is used to match the reference beam diameter to the test beam. The two beams recombine at the second beam splitter. The combined beams pass through a final focusing lens to ensure the the focal plane coincides with the center of the test cylinder. A Phantom V711 camera is used to record the interferometer at frame rates in excess of 10,000 fps. 800 by 800 pixels of the sensor were used, for an effective resolution of approximately $8 \mathrm{px} / \mathrm{mm}$ in the object plane. Typically, each fringe pair was 15 pixels wide. The interferometer layout is shown in Fig. 2.18 . 


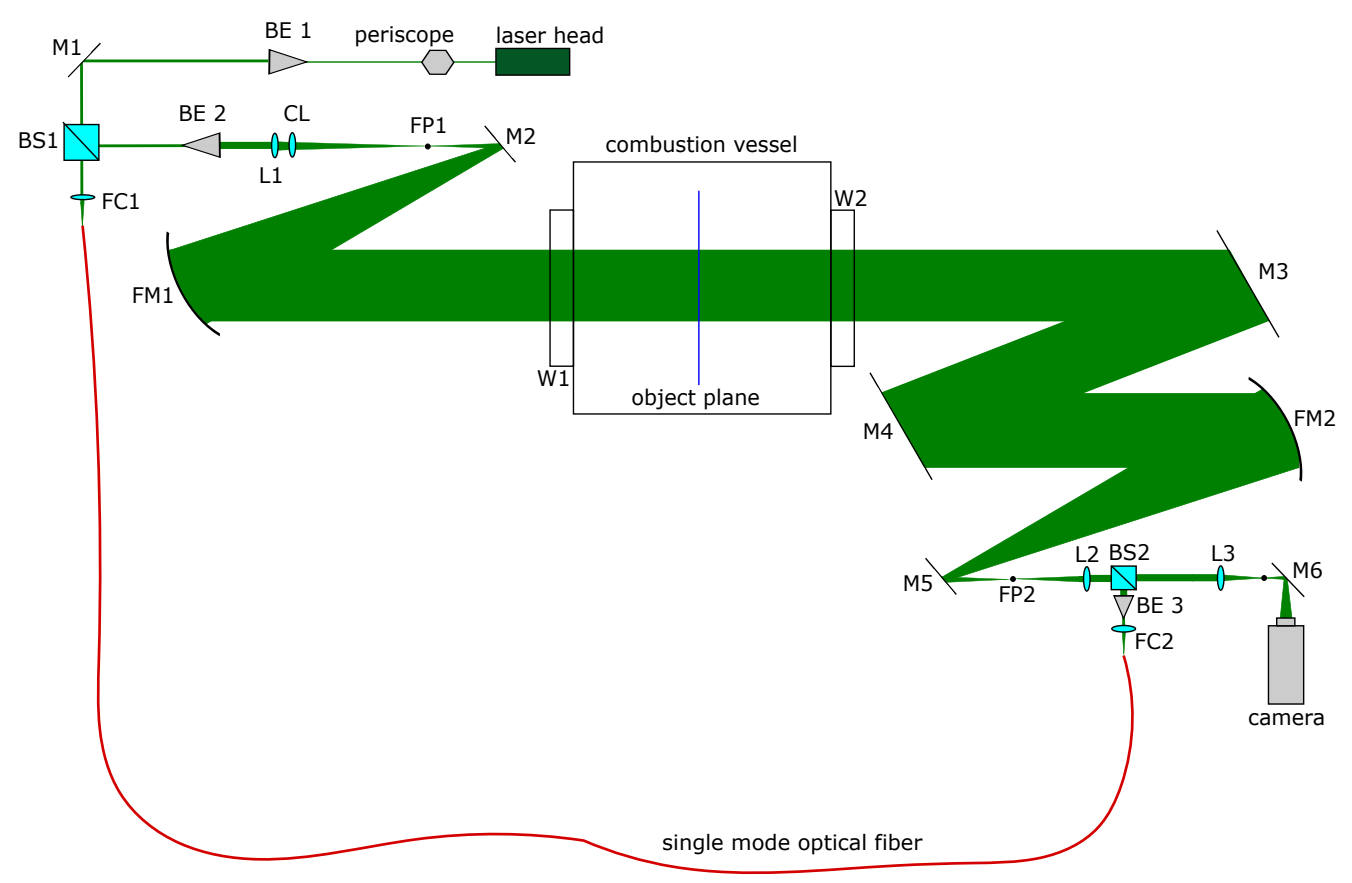

Figure 2.18: Schematic for fiber Mach-Zehnder interferometer.

\section{Correcting Astigmatism}

When this setup was first tested, an X-shaped fringe pattern emerged as shown in Fig. 2.19. Investigation of possible sources for this shape revealed that the $\mathrm{X}$ shape of the fringes was due to large astigmatism (Wyant, 2016). This astigmatism comes from the off-axis focusing that occurs with the $100 \mathrm{~mm}$ lens and $864.6 \mathrm{~mm}$ parabolic mirror pair on either side of the vessel. Astigmatism can be reduced by minimizing the folding angle, but due to relatively large beam diameters and small focal lengths, it is still enough to severely distort the fringe shape.

Astigmatism occurs when the saggital and tangential planes are not focused at the same location (Guenther and Steel, 2018). At the medial focus (i.e. "best compromise" in focus) astigmatism distorts the wavefront of the test beam into a saddle shape (Telescope astigmatism 2019). The reference beam remains very flat from transmission through a single mode fiber. Attempting to interfere these two wavefronts produces the shape of the fringes seen in Fig. 2.19. Furthermore, the Winlens model of the interferometer predicts both the X-shaped fringes and the saddle shape of the wavefront as seen in Fig. 2.20 .

The progression of fringe shape as the focus shifts from saggital to tangential focus 


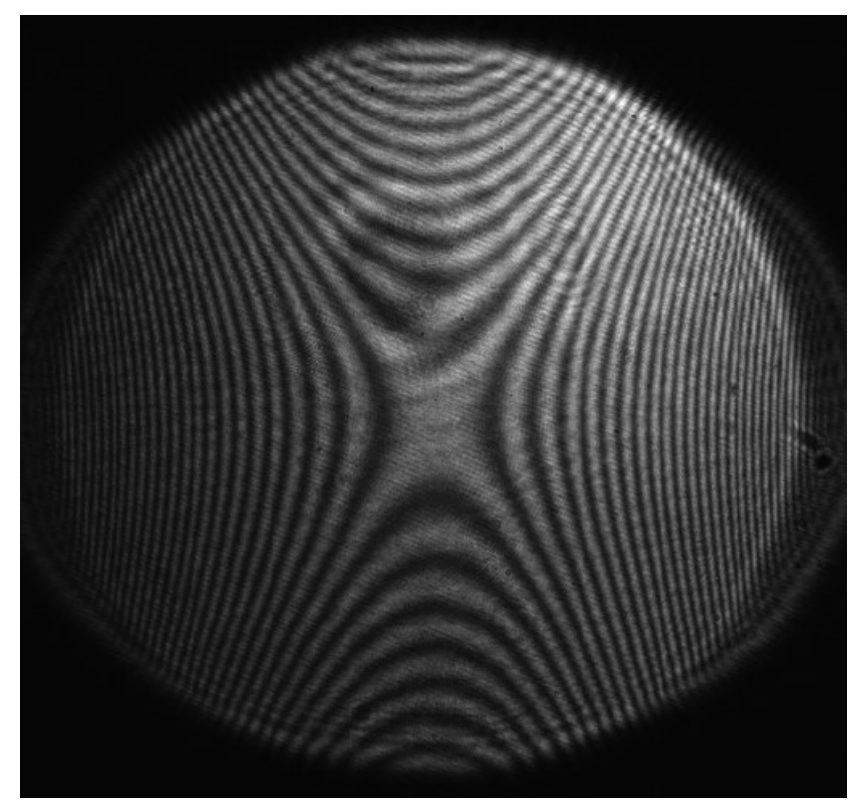

Figure 2.19: X-shaped fringes, observed at medial focus.

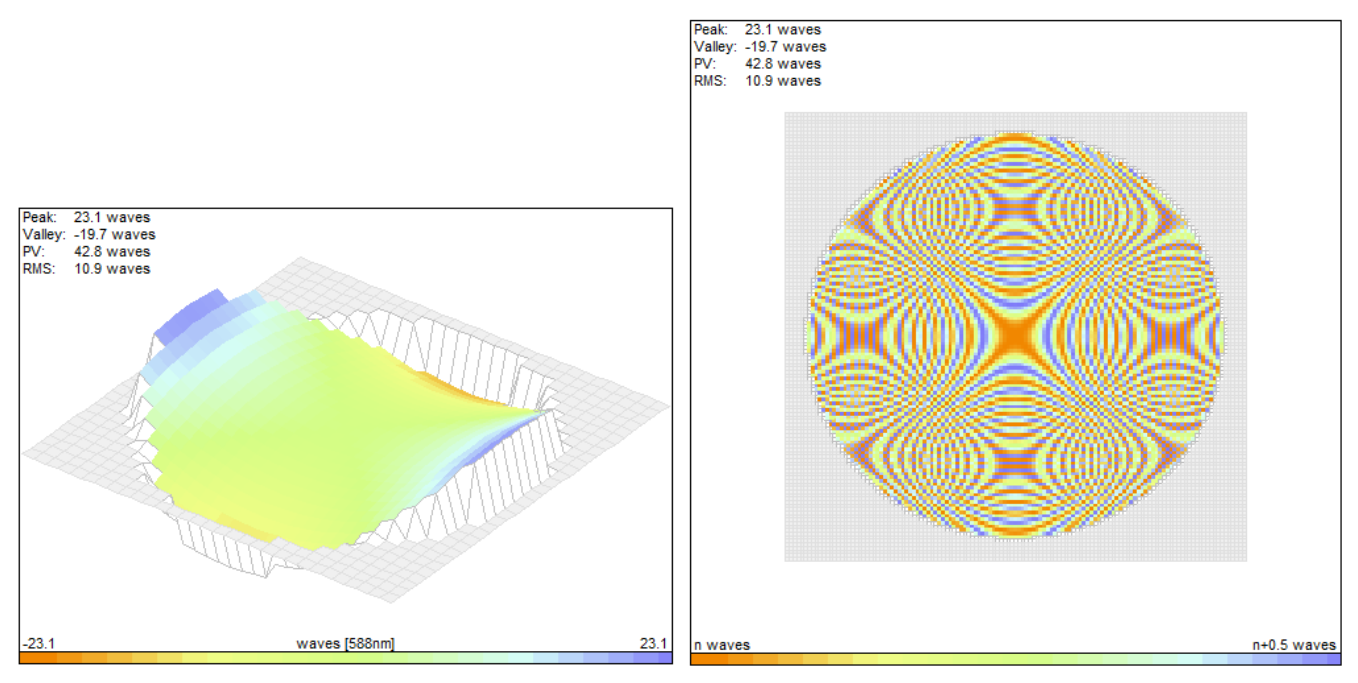

Figure 2.20: Winlens model showing saddle shaped wavefront (left) and predicted interference pattern (right) due to astigmatism at medial focus.

is shown in Fig. 2.21. Saggital focus means the saggital rays are focused and the wavefront is flat along the saggital plane and curved only in the tangential plane, producing horizontal fringes. Tangential focus means the tangential rays are focused and the wavefront is flat along the tangential plane and curved in the saggital plane, producing vertical fringes. The transition between saggital, medial, and tangential focus is marked by a stretch in the $\mathrm{X}$ shape of the fringes as seen in Fig. 2.21. This progression matches the fringe patterns described by Wyant (2016) for large astigmatism. 


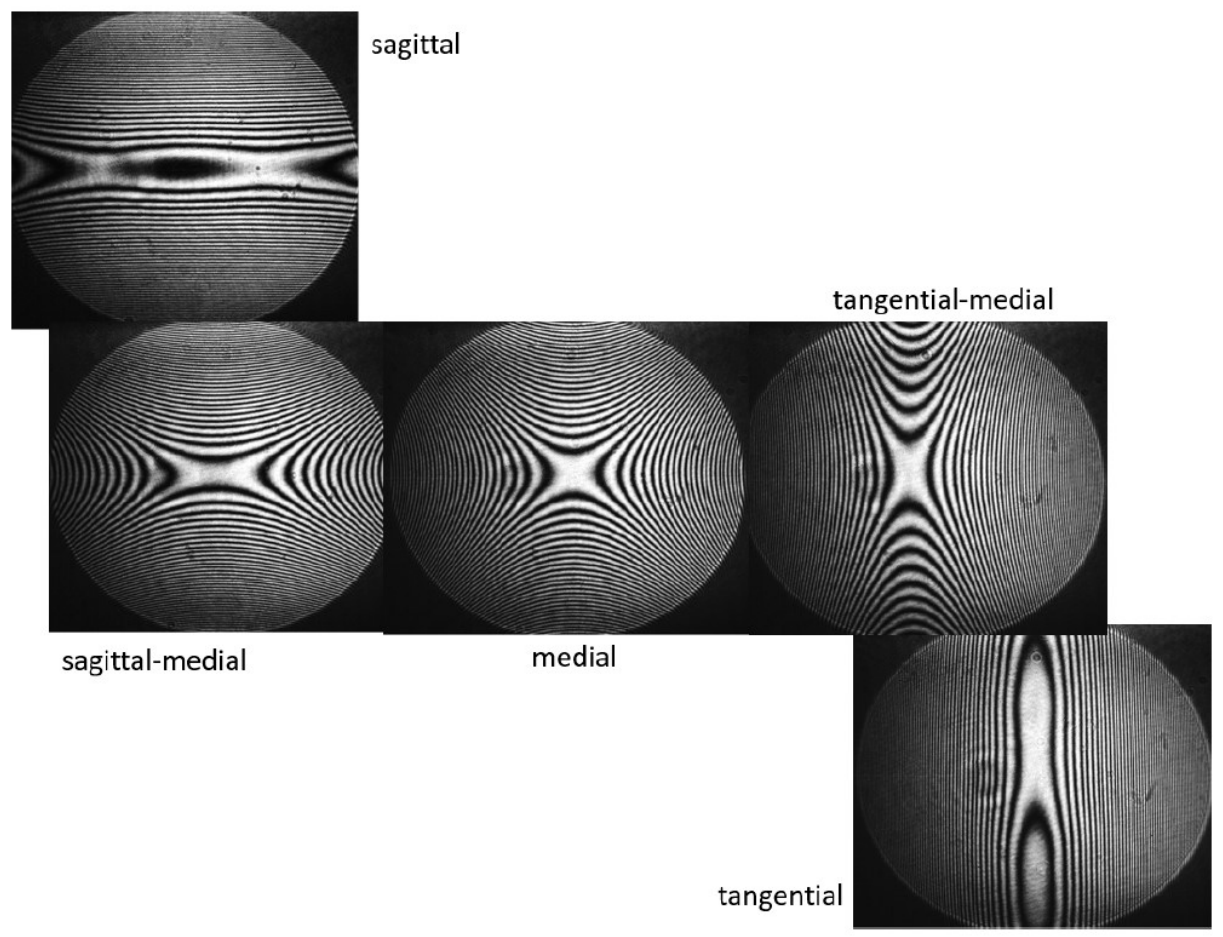

Figure 2.21: Progression of fringe shape from point of saggital focus (top left) through medial focus (center) all the way to tangential focus (bottom right).

Astigmatism can be corrected by use of a cylindrical lens (Howes, 1984). A cylindrical lens focuses light in one plane and, when used properly, can adjust the focus of one plane so that the saggital and tangential planes are focused at the same location. Howes (1984) corrects the astigmatism of an large-aperture local reference beam interferometer with off-axis focusing by introducing a cylindrical lens near the light source. The astigmatism in the interferometer described here was corrected with a cylindrical lens through the process of making estimates in Winlens and practical trial and error.

The goal of introducing the cylindrical lens into the interferometer was to correct the astigmatism present in the setup. This correction is most easily observed by monitoring the fringe pattern, which indicates the shape of the wavefront. An Xshaped fringe pattern indicates a saddle-shaped wavefront as demonstrated in Fig. 2.20. A horizontal fringe pattern indicates curvature along the vertical axis (e.g. the tangential plane), and a vertical fringe pattern indicates curvature along the horizontal axis (e.g. saggital plane). No fringe pattern, also known as the infinite fringe 
mode, indicates a flat wavefront (saggital and tangential planes in focus at the same location) and thus fully corrected astigmatism. Progress on astigmatism correction was monitored by examining the infinite fringe pattern.

The steps to correct astigmatism on the interferometer are as follows:

1. Place interferometer in saggital focus, such that:

- Horizontal fringes are observed,

- The wavefront is curved in tangential plane only.

2. Introduce cylindrical lens with curvature oriented along tangential plane, such that:

- All focal alterations occur in tangential plane,

- Wavefront curvature is only altered in tangential plane,

- Lens axis of curvature is parallel to saggital plane.

3. Adjust placement of cylindrical lens such that:

- No fringes are observed (infinite fringe),

- The wavefront curvature in the tangential plane is flattened,

- The tangential focus is altered to coincide with the saggital focus,

- Astigmatism is fully corrected.

The required focal length of cylindrical lens was roughly estimated using Winlens. It is important to note that Winlens only shows wavefront shape at the medial focus, so what we are looking for in the Winlens model is for the wavefront to be flattened in one direction. This means that the cylindrical lens has corrected curvature in one plane and a flat fringe pattern appears perpendicular to that plane. Since Winlens only reports at the medial focus, the specifications on the cylindrical lens, including placement location and focal length, will be rough approximations and loosely inform the focal length and placement of the actual lens used to correct the interferometer. The modeled cylindrical lens that best flattened the wavefront along the tangential plane had a focal length of approximately $300 \mathrm{~mm}$. 

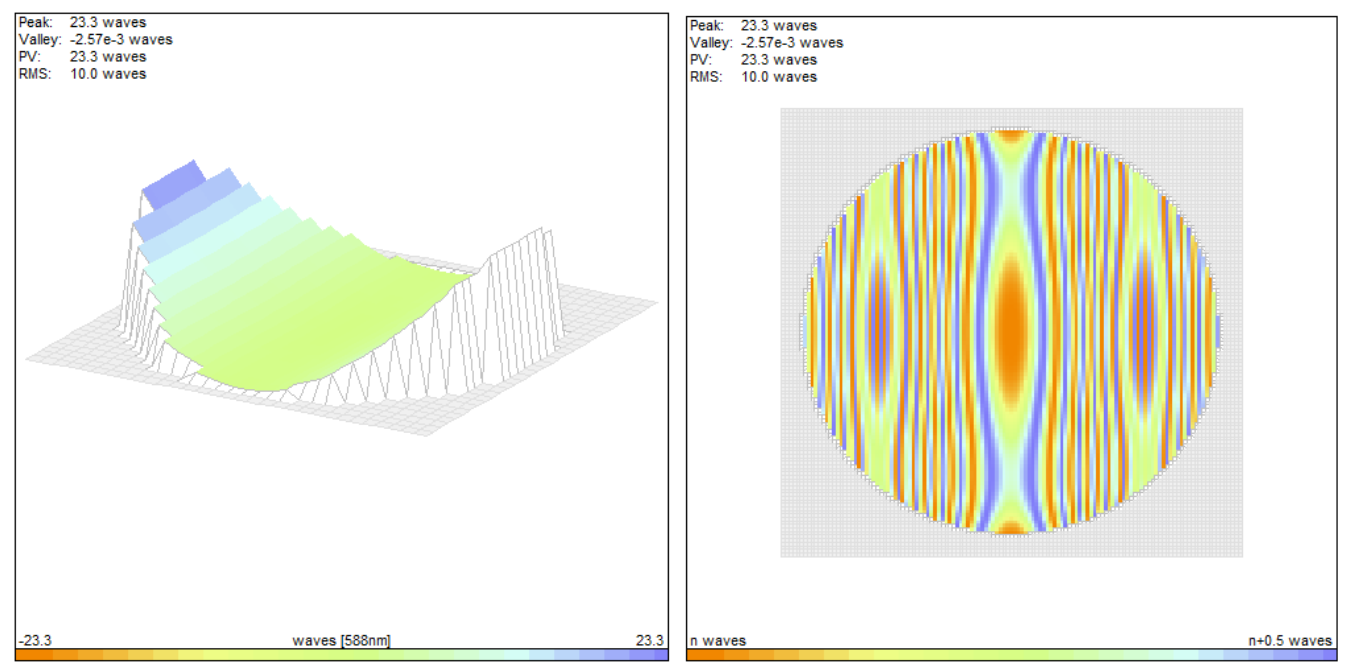

Figure 2.22: Winlens model showing corrected wavefront (left) and predicted interference pattern (right) at medial focus.

The actual interferometer is then corrected. It is put into saggital focus to ensure that the fringes are horizontal, and thus, curvature of the wavefront is only along the tangential plane. A cylindrical lens is added between BE1 and L1, and the curvature of the lens is aligned with the tangential plane such that the axis of curvature is aligned with the saggital plane. This adjusts the focus of rays in the tangential plane only. Cylindrical lenses with focal lengths of 200, 250, and $300 \mathrm{~mm}$ were tested, and the $250 \mathrm{~mm}$ focal length lens was found to make the best correction at a distance of $10 \mathrm{~mm}$ from BE2. That is, the fringe pattern was as close to infinite fringe mode as possible and the wavefront was thus flattened as much as possible. This corrected fringe pattern is shown in Fig. 2.23.

This correction does not perfectly correct astigmatism, but it is close enough to produce usable fringes for interferometry. The finite fringe pattern of the corrected interferometer is shown in Fig. 2.24. The fringes are mostly horizontal but show a bit of curvature due to the imperfect correction of astigmatism. Despite imperfections, this corrected fringe pattern is easily post-processed to extract temperature fields, as is discussed in the following section.

\section{Interferometer Post-Processing}

The fringe pattern presented in Fig. 2.24 represents the difference in path length between light traveling through a field of view with refractivity $n(z)$, where $z$ is 


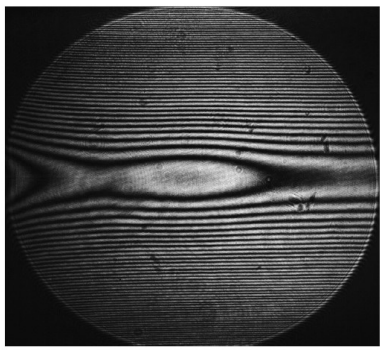

sagittal, no cyl. lens

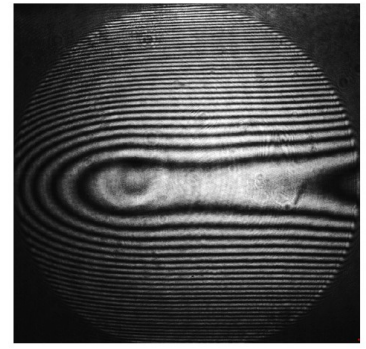

partially corrected

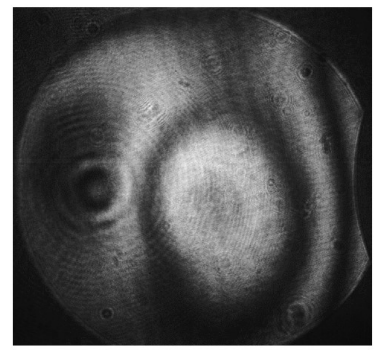

fully corrected with cyl. lens

Figure 2.23: Fringe shape while correcting astigmatism in the interferometer. Left: no cylindrical lens correction, sagittal focus. Fringes become very tightly spaced away from the center. Middle: partial cylindrical lens correction, sagittal focus. Fringes are less densely spaced. Right: best correction, sagittal focus. Few to no fringes in either direction.

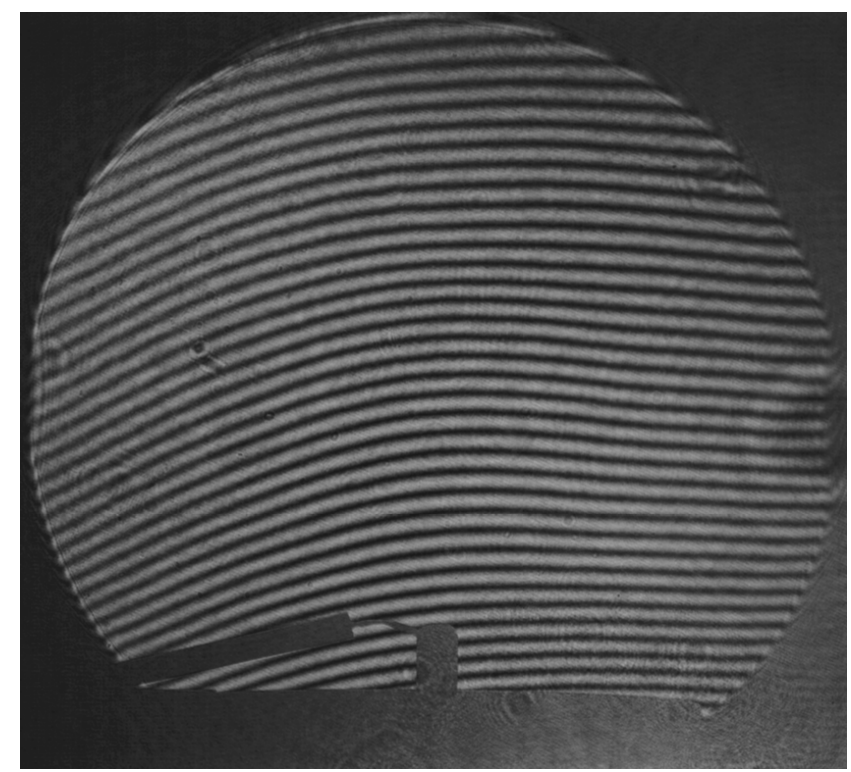

Figure 2.24: Corrected finite fringe pattern. Object in field of view is the glowplug used for verification of the pyrometer. The glowplug is not at an elevated temperature, and the fringes are undisturbed in this image.

along the optical axis of the test beam, and light traveling through a reference field with constant refractivity, $n_{0}$. The difference in phase, $\Delta \varphi$, between the two beams of light can be related to the index of refraction by:

$$
\Delta \varphi=\varphi-\varphi_{0}=\frac{2 \pi}{\lambda} \int_{\zeta_{1}}^{\zeta_{2}}\left(n(z)-n_{0}\right) d z
$$

where $\zeta_{1}$ and $\zeta_{2}$ are the points along the z-axis where the ray enters and leaves the 
test section, respectively, and $\lambda$ is the wavelength of light in a vacuum $(\lambda=532 \mathrm{~nm}$ in this work).

The intensity, $I$, of a two-dimensional fringe pattern is represented by the following equation amplitude and frequency modulated function:

$$
I(x, y)=a(x, y)+b(x, y) \cos (\Delta \varphi(x, y)),
$$

where $a$ represents the background illumination and noise, $b$ the amplitude, and $\varphi$ the optical phase.

Before the phase is extracted from the raw interferogram, a Gaussian filter is applied to the image with the goal of filtering out high-frequency noise. The phase demodulation of the interferograms, the process by which $\Delta \varphi$ is obtained, is then performed using the 2D Windowed Fourier Filtering method (WFF2) presented in detail by Kemao (2013). Here, an abbreviated overview of the method used for phase extraction is presented. The phase demodulation begins with the following windowed Fourier basis,

$$
g_{\xi_{x}, \xi_{y}}(x, y)=g(x, y) \exp \left[i\left(\xi_{x} x+\xi_{y} y\right)\right]
$$

where $g(x, y)$ is a Gaussian window,

$$
g(x, y)=\frac{1}{2 \pi \sqrt{\sigma_{x} \sigma_{y}}} \exp \left[\frac{-x^{2}}{2 \sigma_{x}^{2}}-\frac{y^{2}}{2 \sigma_{y}^{2}}\right] .
$$

The windowed Fourier transform (WFT), $F_{W}$, and inverse WFT, $f$, are related to another by use of a convolution operator, $\otimes$, as shown in Eq. 2.31 and 2.33.

$$
\begin{gathered}
F_{W}\left(u, v ; \xi_{x}, \xi_{y}\right)=f(u, v) \otimes g_{\xi_{x}, \xi_{y}}(x, y), \\
f(x, y)=\frac{1}{4 \pi^{2}} \int_{-\infty}^{\infty} \int_{-\infty}^{\infty} F_{W}\left(u, v ; \xi_{x}, \xi_{y}\right) \otimes g_{\xi_{x}, \xi_{y}}(x, y) d \xi_{x} d \xi_{y} .
\end{gathered}
$$

Equation 2.33 can be written in summation form as: 


$$
f(x, y)=\frac{\xi_{x}^{(i)} \xi_{y}^{(i)}}{4 \pi^{2}} \sum_{\xi_{y}=-\pi}^{\pi} \sum_{\xi_{x}=-\pi}^{\pi} F_{W}\left(u, v ; \xi_{x}, \xi_{y}\right) \otimes g_{\xi_{x}, \xi_{y}}(x, y),
$$

where $\xi_{x}^{(i)}$ and $\xi_{y}^{(i)}$ are sampling intervals of $\xi_{x}$ and $\xi_{y}$. To accurately reconstruct the inverse WFT, signal noise must be eliminated by setting a predetermined threshold, $t h r$, such that all coefficients lower than $t h r$ are discarded. The thresholded inverse WFT is then,

$$
\bar{f}(x, y)=\frac{\xi_{x}^{(i)} \xi_{y}^{(i)}}{4 \pi^{2}} \sum_{\xi_{y}=-\pi}^{\pi} \sum_{\xi_{x}=-\pi}^{\pi} \bar{F}_{W}\left(u, v ; \xi_{x}, \xi_{y}\right) \otimes g_{\xi_{x}, \xi_{y}}(x, y),
$$

where thresholded WFT, $\bar{F}_{W}$ is

$$
\bar{F}_{W}\left(u, v ; \xi_{x}, \xi_{y}\right)= \begin{cases}F_{W}\left(u, v ; \xi_{x}, \xi_{y}\right) & \text { if }\left|F_{W}\left(u, v ; \xi_{x}, \xi_{y}\right)\right| \geq t h r \\ 0 & \text { if }\left|F_{W}\left(u, v ; \xi_{x}, \xi_{y}\right)\right|<t h r .\end{cases}
$$

The thresholded phase, $\overline{\Delta \varphi_{W}}(x, y)$ is then found,

$$
\overline{\Delta \varphi_{W}}(x, y)=\angle \bar{f}(x, y),
$$

where $\angle \bar{f}(x, y)$ refers to the angle of the thresholded signal $\bar{f}$. The subscript $W$ refers to a wrapped signal, that is the phase difference is bounded between $-\pi$ and $\pi$. A quality-guided phase map using a flood-filling algorithm is used to unwrap the phase to construct a continuous optical phase difference. A path-following method is selected with a quality-guided algorithm. The quality-guided algorithm uses a quality map, $Q(x, y)$, to determine the path along which the phase is unwrapped,

$$
Q(x, y)=1-\frac{\Delta \phi_{r}(x, y)}{2 \pi},
$$

where $\Delta \phi_{r}$ is the phase variance,

$$
\begin{aligned}
\Delta \phi_{r}(x, y)= & \frac{1}{4}\left(\left|\phi_{r}(x, y)-\phi_{r}(x, y-\Delta y)\right|+\left|\phi_{r}(x, y)-\phi_{r}(x, y+\Delta y)\right|\right. \\
& \left.+\left|\phi_{r}(x, y)-\phi_{r}(x-\Delta x, y)\right|+\left|\phi_{r}(x, y)-\phi_{r}(x+\Delta x, y)\right|\right),
\end{aligned}
$$


where $\phi_{r}$ is the wrapped thresholded optical phase, $\overline{\Delta \varphi_{W}}$. The quality-guided algorithm begins with a pixel with the highest quality $Q$ where the phase is unwrapped. Unwrapped phase is denoted by $\phi$. The quality of the 4 pixels immediately surrounding the unwrapped pixel is checked, and the next highest quality pixel is chosen to unwrap using Eq. 2.39;

$$
\phi(n)= \begin{cases}\phi(n-1)+\Delta \phi_{r}(n)+2 \pi & \text { if } \Delta \phi_{r}(n) \leq-\pi, \\ \phi(n-1)+\Delta \phi_{r}(n) & \text { if }-\pi<\Delta \phi_{r}(n)<\pi, \\ \phi(n-1)+\Delta \phi_{r}(n)-2 \pi & \text { if } \Delta \phi_{r}(n) \geq \pi,\end{cases}
$$

where $n$ is the chosen pixel location and $\phi(n)$ is the unwrapped phase at that location. Subsequent discussion will refer to unwrapped optical phase by $\Delta \varphi$.

The next step is to remove the bias from the image. That is, the continuous change in unwrapped optical phase from the bottom to the top of image must be removed. This is accomplished by subtracting the unwrapped optical phase of an undisturbed image from all subsequent images.

If we assume an axisymmetric setup, a radial distribition, $f(r)$, can be transformed into a line of sight quantity, $F(x)$ by use of the Abel transform:

$$
F(x)=2 \int_{x}^{\infty} \frac{f(r) r}{\left(r^{2}-x^{2}\right)^{1 / 2}} d r
$$

and a line of sight quantity can be transformed into a radial distribution by use of the inverse Abel transform:

$$
f(r)=-\frac{1}{\pi} \int_{r}^{\infty} \frac{d F}{d x} \frac{d x}{\left(x^{2}-r^{2}\right)^{1 / 2}} .
$$

In the case of optical phase differences and refractive indices,

$$
f(r)=\frac{2 \pi}{\lambda}\left(n(r)-n_{0}\right), \text { and } F(x)=\Delta \varphi .
$$


The inverse Abel transformation can be calculated numerically by using the NestorOlsen algorithm Nestor and Olsen (1960). This method approximates Eq. 2.41 by the following:

$$
f(r)=\frac{-2}{\pi \Delta x} \sum_{i=j}^{N-1} F\left(x_{i}\right) B_{j, i},
$$

where

$$
B_{j, i}= \begin{cases}A_{j, i-1}-A j, i & \text { for } i \geq j+1, \\ -A_{j, i} & \text { for } i=j,\end{cases}
$$

and

$$
A_{j, i}=\frac{\left[i^{2}-(j-1)^{2}\right]^{1 / 2}-\left[(i-1)^{2}-(j-1)^{2}\right]^{1 / 2}}{2 i-1} .
$$

The refractive index, $n(r)$, is found after obtaining $f(r)$ from Eq. 2.43 and 2.45 , where $F\left(x_{i}\right)=\Delta \varphi$. Manipulation of Eq. 2.42 produces an expression for $n(r)$ the radial distribution of the index of refraction,

$$
n(r)=n_{0}+\frac{\lambda}{2 \pi} f(r)
$$

The index of refraction depends of the composition, pressure, temperature of the medium, and the wavelength of the light passing through the medium. The density can then be calculated through use of the Gladstone-Dale relation,

$$
n-1=K \rho,
$$

where $K=2.274 \times 10^{-4} \mathrm{~m}^{3} / \mathrm{kg}$ for air at $300 \mathrm{~K}$ and $100 \mathrm{kPa}$ (Merzkirch, 1987). This yields a density relation,

$$
\rho(r)=\frac{n(r)-1}{K}
$$

Assuming a medium of non-reacting ideal gas at constant pressure, a radial temperature distribution can be found from density by the following, 


$$
T(r)=\frac{P}{\rho(r) R_{s p}},
$$

where $P$ is the pressure of the gas and $R_{s p}$ is the specific gas constant.

Gas composition must be known accurately in order to select the proper index of refraction, Gladstone-Dale constant, and specific gas constant that are critical to accurate post-processing of interferometry results. These parameters can be calculated following the procedure laid out by Gardiner, Hidaka, and Tanzawa (1981), who tabulated the molar refractivity, $R_{L_{i}}$, of common combustion gases. The molar refractivity for the entire mixture is calculated by a molar weighted average:

$$
R_{L}=\sum_{i=1}^{n} R_{L_{i}} X_{i}
$$

Subsequently, the index of refraction, $\mathrm{n}_{0}$, and Gladstone-Dale constant can be calculated from the mixture molar (note $\rho_{m}$ is the molar density of the mixture):

$$
n_{0}=\left(\frac{1+2 \rho_{m} R_{L}}{1-\rho_{m}}\right)^{\frac{1}{2}}
$$

The Gladstone-Dale constant is calculated from the index of refraction:

$$
K=\frac{n_{0}-1}{\rho} \text {. }
$$

Finally, the specific gas constant of the mixture is calculated from the universal gas constant and the mixture-averaged molar weight:

$$
R_{s}=\frac{\tilde{R}}{M_{m i x}} .
$$

When this methodology is followed in the image-processing routine, this produces a two-dimensional radial temperature field. This is how quantitative, radial temperature fields are obtained from a raw interferogram. Table 2.2 contains processing constants for a pure nitrogen mixture as well as the stoichiometric hexane and diluted hydrogen mixtures that will be discussed in Chapter 4 


\begin{tabular}{l|ccc}
\hline Mixture & $\mathrm{n}_{0}$ & $\mathrm{~K}$ & $\mathrm{R}_{s}$ \\
\hline $100 \% \mathrm{~N}_{2}$ & 1.00027423353 & $2.40109673708 \mathrm{e}-4$ & 296.802103844 \\
stoich hexane & 1.00029769777 & $2.46787492823 \mathrm{e}-4$ & 276.328826446 \\
diluted $\mathrm{H}_{2}$ & 1.00024352237 & $2.52971821446 \mathrm{e}-4$ & 346.267636518 \\
\hline
\end{tabular}

Table 2.2: Processing constants for interferometry.

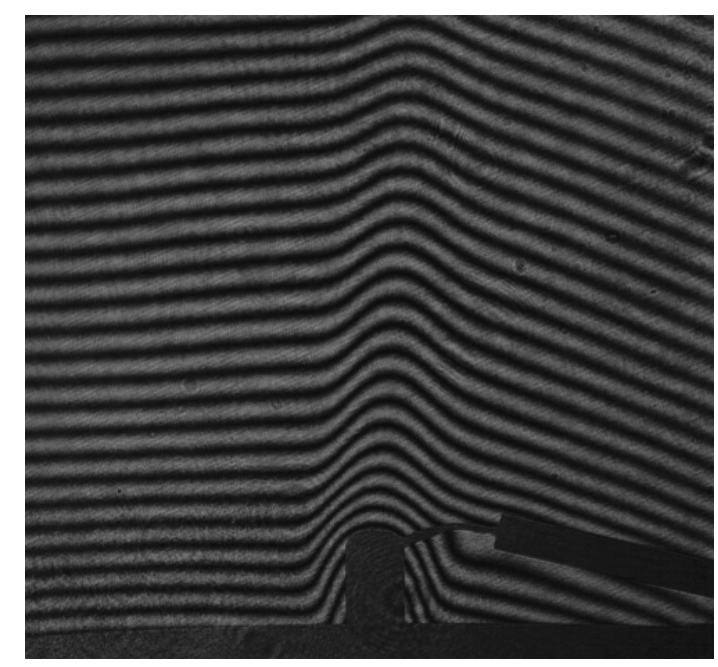

Figure 2.25: Raw interferogram of glowplug.

\section{Interferometer Validation}

The interferometer is validated by investigating the temperature fields it captures from heating a well-characterized surface (Autolite glowplug from Boettcher (2012), Melguizo-Gavilanes et al. (2016)). The glowplug is placed in the vessel in the interferometer field of view (Fig. 2.25). The vessel is filled with nitrogen and the glowplug is heated while its temperature is monitored by a k-type thermocouple and by pyrometry. Interferometer images are captured and post processed. The temperature fields from interferometry are compared to a simulation from MelguizoGavilanes et al. (2016), shown in Fig. 2.26, The interferometer temperature fields compare well with previous numerical results, with less than $10 \%$ error everywhere except right along the centerline above the glowplug as shown in Figures 2.27 and 2.28. A narrow region of higher error occurs along the centerline due to the nature of Nestor-Olsen algorithm, which converts line of sight integrated quantities into radial quantities by working from the outside of the image in towards the centerline. This causes a buildup of errors along the centerline of the processed image. 


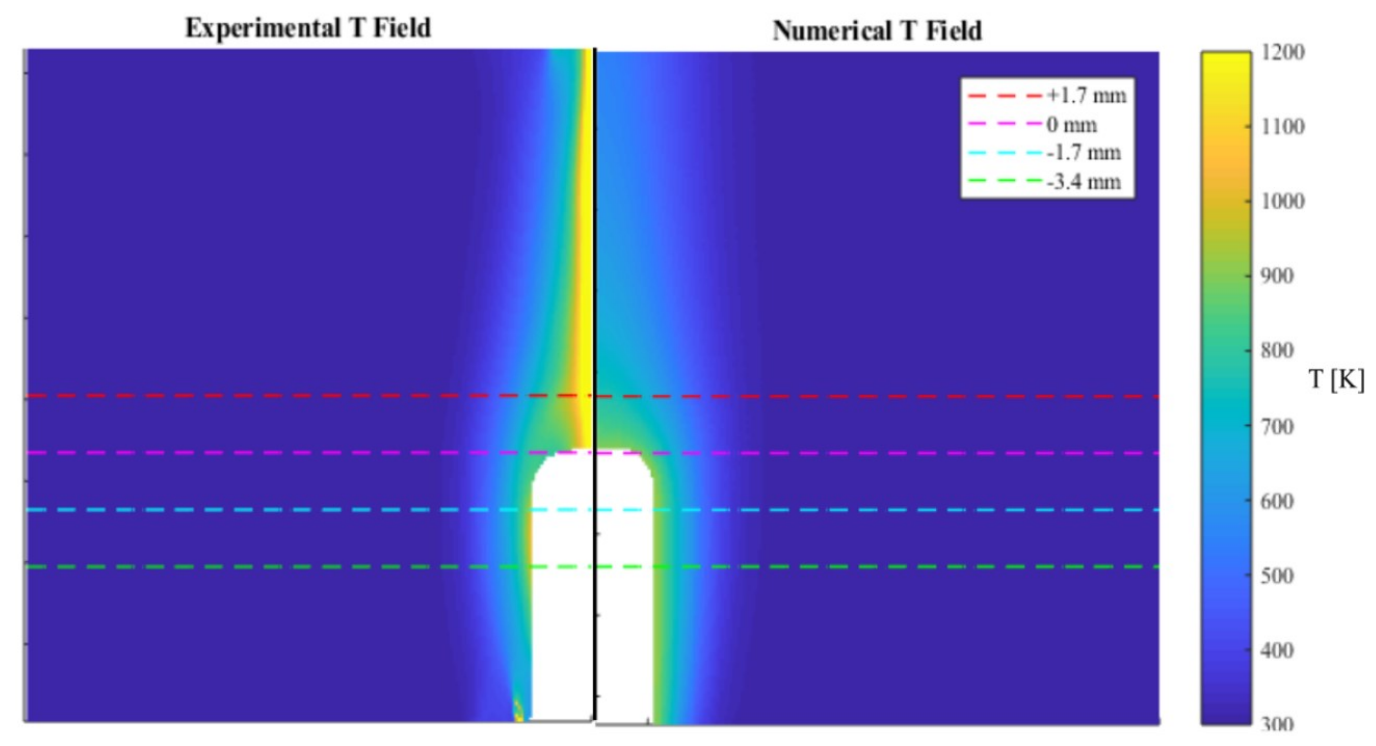

Figure 2.26: Temperature fields from interferometry (left) compared with simulations from Melguizo-Gavilanes et al. (2016) (right).
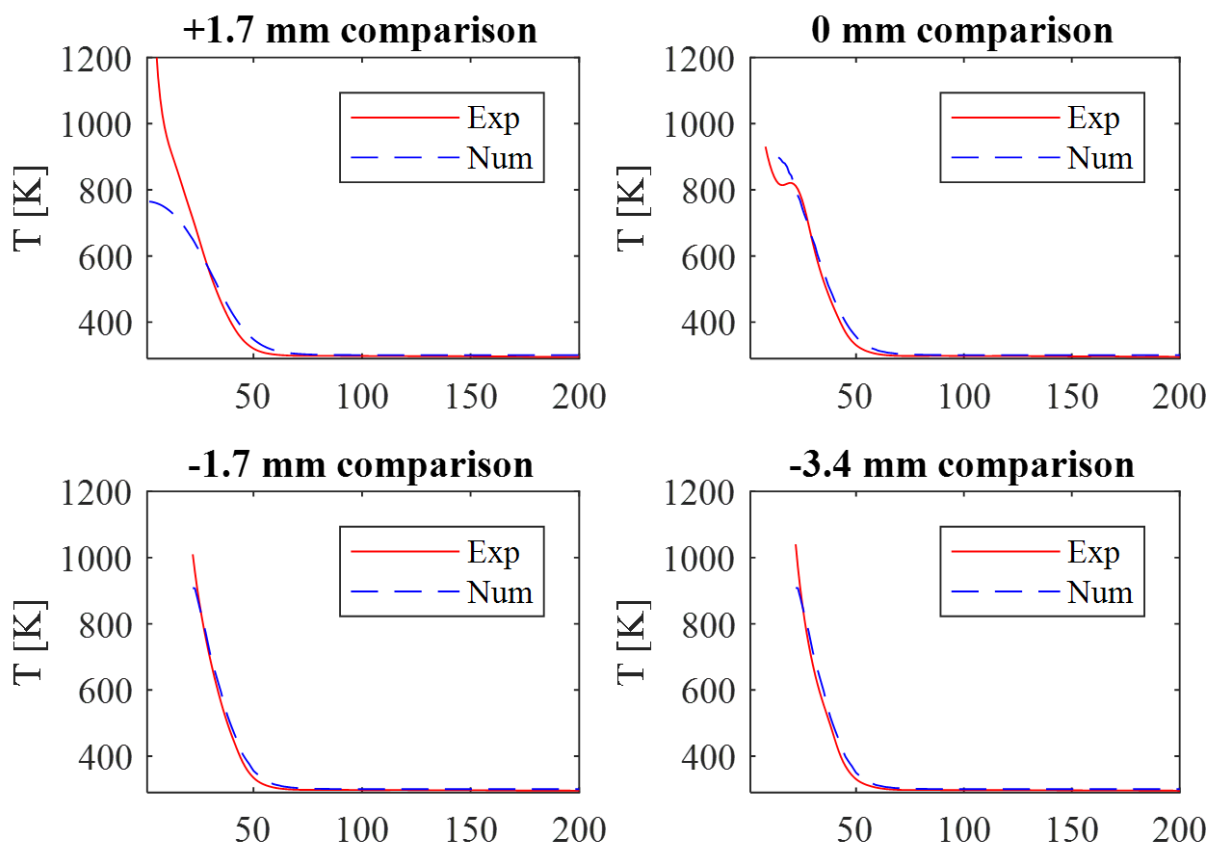

Figure 2.27: Thermal boundary layer profiles of experimental and numerical results compared for each of the four slices as indicated in Figure 2.26. 

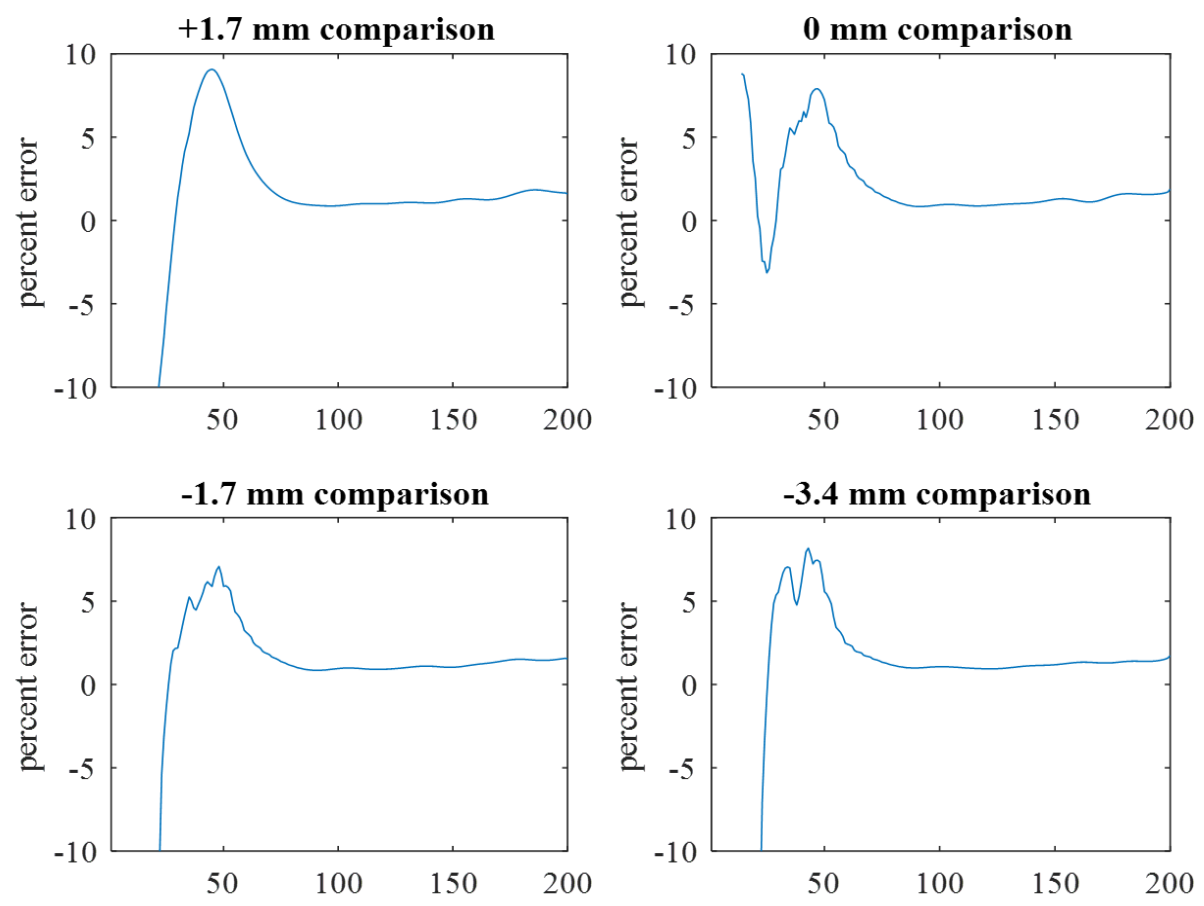

Figure 2.28: Percent error in thermal boundary layer between experimental and numerical results for each of the four slices as indicated in Figure 2.26 .

\section{Note on Conversion to Schlieren Imaging System}

It is sometimes desirable to take schlieren images instead of interferograms in order to take more qualitative and intuitive images of the cylinders during heating and ignition processes. This interferometer is easily converted to and from a schlieren imaging system without altering any of the precisely aligned interferometer components. The setup described here is for a dot schlieren system but is compatible with any type of schlieren stop.

An opaque insert is added between BE3 and BS2 in order to block transmission of the reference beam such that only light from the test beam is collected. A stop, in this case an opaque $0.5 \mathrm{~mm}$ diameter dot on a transparent glass pane, is placed in FP2. This transforms the optical system into a dot schlieren system. The dot stop acts as a high-pass filter in Fourier space, removing the "DC" component of the signal. This means the schlieren images show changes from the basic or unheated configuration. 


\subsection{OH* Chemiluminescence}

$\mathrm{OH}^{*}$ Chemiluminescence is the natural phenomenon by which naturally excited $\mathrm{OH}$ molecules return to the ground state by emitting UV radiation at a wavelength of approximately $306 \mu \mathrm{m}$ (Tinaut et al., 2011). Liu et al. (2020) note the formation and emission process of excited radicals. The formation of an excited radical, $\mathrm{R}^{*}$, can be generated by the reaction of two ground state radicals, $A$ and $B$ :

$$
A+B \longrightarrow R *+\text { others }
$$

and the emission of chemiluminescence occurs as a radiative transition:

$$
R * \longrightarrow R+h v
$$

It is important to note that not all $\mathrm{OH}$ radicals formed during combustion will enter an excited state, and of the excited $\mathrm{OH}$ that do form, not all of them will participate in chemiluminescence. Some will enounter third-body particles and return to the ground state without emitting radiation. Still, even though only a very small fraction of the $\mathrm{OH}$ are naturally excited and emit chemiluminescence, it is still enough radiation to capture with an intensifier and track the progress of the reaction zone during the ignition transient.

In the literature, this phenomenon has been utilized to quantify the heat release rate during combustion (Sardeshmukh, Bedard, and Anderson, 2017), (Tinaut et al., 2011). Due to the fact that $\mathrm{OH}^{*}$ has been shown to be generated in the reaction zone (Deleo et al., 2007), here we will use the presence of $\mathrm{OH}^{*}$ chemiluminescence as a marker of the flame front. This is a technique by which the ignition kernel and flame front can be observed directly.

A high-speed intensifier (LaVision HS-IRO) is coupled to a high-speed Phantom V7.3 camera, and a UV Nikon lens (UV-Nikkor $105 \mathrm{~mm} \mathrm{f/4.5s)} \mathrm{is} \mathrm{used} \mathrm{at} \mathrm{the} \mathrm{collec-}$ tion end of the intensifier to permit the UV radiation to reach the intensifier while setting the focal plane at the center of the combustion vessel where the cylinder is placed. A narrow bandpass filter centered at $310 \mathrm{~nm}$ is mounted to the front of the UV lens; this ensures only wavelengths appropriate for $\mathrm{OH}^{*}$ chemiluminescence are observed by the intensifier. Figure 2.29 shows a schematic of the setup used to collect the $\mathrm{OH}^{*}$ chemiluminescence images. A enhanced aluminum mirror is 


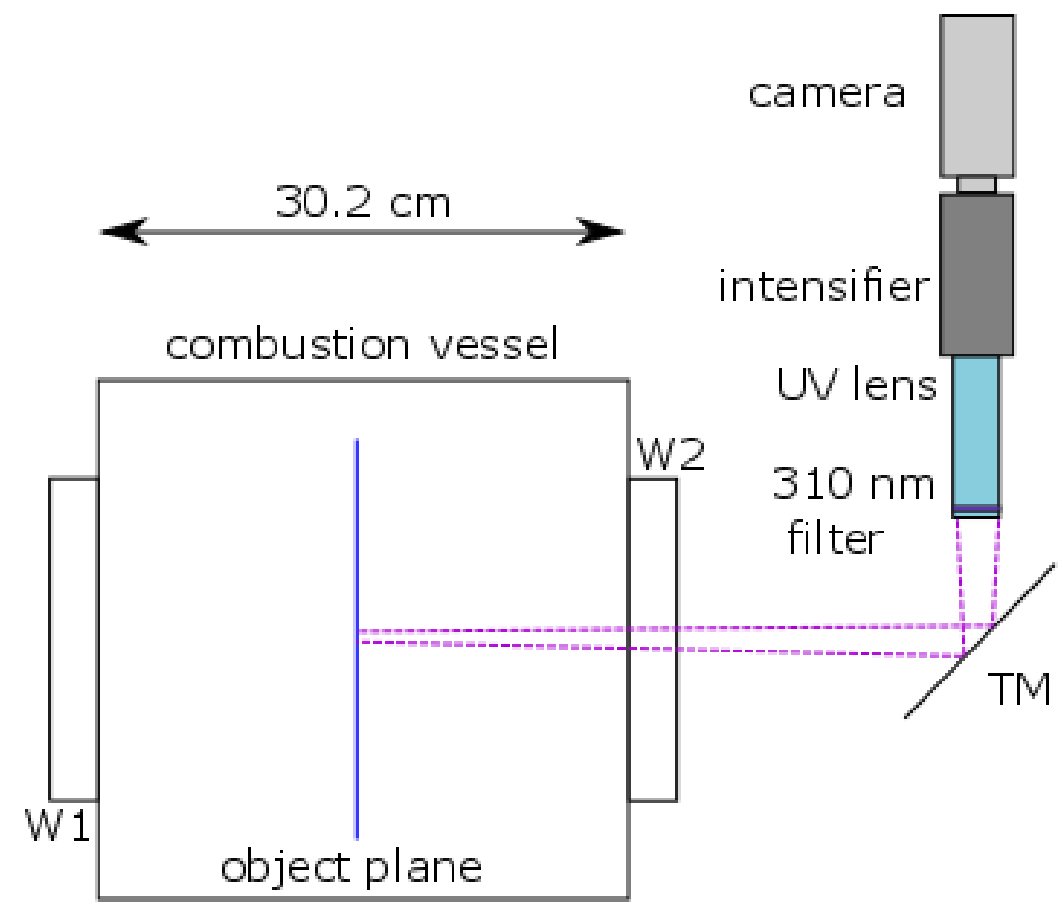

Figure 2.29: Schematic of $\mathrm{OH}^{*}$ chemiluminescence setup. $\mathrm{W} 1$ and $\mathrm{W} 2$ denote window 1 and 2 on the vessel, respectively, and TM denotes the enhanced aluminum turning mirror. The total distance rays travel between the object plane and the UV lens is approximately $50 \mathrm{~cm}$.

used to turn the radiation emitted from the vessel into the collection optics of the intensifier. The placement of this mirror blocks the beam path of the interferometer; for that reason, interferometry and $\mathrm{OH}^{*}$ chemilumninesce cannot be run simultaneously. The laser and camera for interferometry are turned off for shots where $\mathrm{OH}^{*}$ chemiluminescence is performed.

To collect the $\mathrm{OH}^{*}$ chemiluminescence images, the camera and intensifier must be collecting data synchronously, i.e. the gate on the intensifier must be open at the same time as the exposure on the camera is running to fully capture the $\mathrm{OH}^{*}$ radiation. To ensure synchronicity, the fsync port on the phantom camera is connected to the gate on the intensifier; this ensures the exposure time for both the camera and intensifier start at the same time. To ensure all of the radiation collected by the intensifier is passed on to the camera, the length of the intensifier gate must be no longer than the exposure time on the camera. Additionally, the intensifier is set to "internal" mode when running; this ensures that the intensifier gate is ready and collecting information, rather than waiting for a trigger signal. This operating mode is chosen for the intensifier because the trigger for ignition occurs off of a spike on 
the dynamic pressure gauge reading. The pressure increases dramatically only once the flame reaches the walls of the combustion vessel; the actual ignition event occurs at approximately constant pressure. This means that if the intensifier were run on "external mode", i.e. the gate opened once a trigger signal arrived, the ignition event would have already occurred. The phantom cameras can collect data from the moments prior to a trigger signal, but the intensifier does not have this capability. As a result, the intensifier must be operated continuously using "internal" mode during testing in order to fully capture the ignition event.

It is also important to select an appropriate gain for the intensifier; for too low a value, no $\mathrm{OH}^{*}$ chemiluminescence will appear, and for too high a value, the intensifier could be damaged. An approximate value for the gain was found by using the camera/intensifier setup with the $\mathrm{OH}^{*}$ fileter attached to observe a candle. The gain started at zero and was increased one step at a time until the radiation from the candle was observed. During an combustion event, there are more $\mathrm{OH}^{*}$ radicals present than for a small candle, but this is balanced by the chemilumenescence attenuating by approximately a factor of 2 due to the $2.54 \mathrm{~cm}$ thick N-BK7 windows used for visualization.

The intensifier settings typically used in these experiments are as follows: the delay was set to the lowest possible value (5 ns), the gain was set to $78 \%$, and the gate was set to $200 \mu$ s. These intensifier settings allow imaging at frame rates up to $5000 \mathrm{fps}$.

\section{References}

Adomeit, G. (1965). "Ignition of gases at hot surfaces under nonsteady-state conditions". In: Symposium (International) on Combustion. Tenth Symposium (International) on Combustion 10.1, pp. 237-243.

Boettcher, Philipp (2012). "Thermal Ignition". PhD thesis. California Institute of Technology.

Coronel, Stephanie A. (2016). "Thermal Ignition Using Moving Hot Particles". PhD thesis. California Institute of Technology.

Deleo, M. et al. (2007). "OH and CH luminescence in opposed flow methane oxyflames." In: Combustion and Flame 149.4, pp. 435-447. Dor: 10 . 1016/ j . combustflame.2007.01.008. 
Gardiner, W. C. Jr., Y. Hidaka, and T Tanzawa (1981). "Refractivity of Combustion Gases”. In: Combustion and Flame 40, pp. 213-219.

Guenther, Bob and Duncan Steel (2018). Encyclopedia of Modern Optics. 2nd ed. Elsevier.

Howes, Walton L. (1984). "Large-aperture interferometer with local reference beam". en. In: Applied Optics 10, p. 1467. (Visited on 05/09/2018).

Kemao, Qian (2013). Windowed Fringe Pattern Analysis. SPIE.

Kuchta, J., A. Bartkowiak, and M. Zabetakis (1965). "Hot Surface Ignition Temperatures of Hydrocarbon Fuel Vapor-Air Mixtures". In: Journal of Chemical Engineering Data 10.3, pp. 282-288.

Liu, Yao et al. (2020). "Quantitative Measurement of $\mathrm{OH}^{*}$ and $\mathrm{CH}^{*}$ Chemiluminescence in Jet Diffusion Flames". In: ACS Omega 5.26. Publisher: American Chemical Society, pp. 15922-15930. ISSN: 2470-1343. DOI: 10.1021/acsomega . Qc01093.

Melguizo-Gavilanes, J. et al. (2016). "Hot Surface Ignition of n-Hexane Mixtures using Simplified Kinetics". In: Combustion Science and Technology 188.11-12, pp. 2060-2076.

Merzkirch, Wolfgang (1987). Flow Visualization. Academic Press.

Michalski, Dariusz, Kinga Strąk, and Magdalena Piasecka (2017). "Comparison of two surface temperature measurement using thermocouples and infrared camera". In: EPJ Web of Conferences 143. Ed. by P. Dančová. (Visited on 11/12/2020).

Michalski, L, K. Eckersdorf, et al. (2001). Temperature Measurement. John Wiley \& Sons.

Nestor, O. H. and H. N. Olsen (1960). "Numerical methods for reducing line and surface probe data". In: SIAM Review 2.3, pp. 200-207.

Ono, S. et al. (1976). "Ignition in a Free Convection from Vertical Hot Plate". In: Bulletin of JSME 19.132, pp. 676-683.

Patterson, S (1940). "The ignition of inflammable gases by hot moving particles II'. In: The London, Edinburgh, and Dublin Philosophical Magazine and Journal of Science, 30.203, pp. 437-457.

Roth, David, Thomas Haber, and Henning Bockhorn (2017). "Experimental and numerical study on the ignition of fuel/air mixtures at laser heated silicon nitride particles". In: Proceedings of the Combustion Institute 36, pp. 1475-1484.

Sardeshmukh, Swanand, Michael Bedard, and William Anderson (2017). "The use of $\mathrm{OH}^{*}$ and $\mathrm{CH}^{*}$ as heat release markers in combustion dynamics". In: International Journal of Spray and Combustion Dynamics 9.4, pp. 409-423.

Silver, R.S. (1937). "The ignition of gaseous mixtures by hot particles". In: The London, Edinburgh, and Dublin Philosophical Magazine and Journal of Science, 23.156, pp. 633-657. 
Telescope astigmatism (2019). uRL: https : //wWW . telescope-optics . net/ astigmatism1.htm.

Tinaut, F. V. et al. (2011). "Measurements of $\mathrm{OH}^{*}$ and $\mathrm{CH}^{*}$ Chemiluminescence in Premixed Flames in a Constant Volume Combustion Bomb under Autoignition Conditions". In: Energy \& Fuels 25.1. Publisher: American Chemical Society, pp. 119-129. ISSN: 0887-0624. DOI: 10.1021/ef1013456.

Wyant, James C. (2016). Lecture notes in Basic Interferometry and Optical Testing. 
Chapter 3

\section{BOUNDARY LAYER MODELING}

\subsection{Similarity Arguments for Boundary Layer Development}

It was important when designing the experiment that we had an understanding of how the boundary layer would develop over the test cylinder. In order to gain such an understanding, a similarity solution was explored to describe the development of the boundary layer in a natural convection flow. For simplicity, the gas was considered to be inert air; no chemical reactions are considered. The temperature difference between the wall and the ambient gas is large enough that gas properties are considered a function of temperature. The following sections describe the similarity solution used to develop a prediction for boundary layers in a natural convection flow with variable properties.

\section{Boundary Layer Equations}

To perform this analysis, start with the steady, laminar, two-dimensional equations for a boundary layer flow of a fluid adjacent to a heated vertical plate. The fluid is assumed compressible, an ideal gas, with variable thermal properties, and the pressure work and viscous dissipation are assumed negligible. The equations of motion are:

$$
\begin{gathered}
\frac{\partial(\rho u)}{\partial x}+\frac{\partial(\rho v)}{\partial y}=0 \\
\rho\left(u \frac{\partial u}{\partial x}+v \frac{\partial u}{\partial y}\right)=-\rho g \sin (\alpha)-\frac{d p}{d x}+\frac{\partial}{\partial y}\left(\mu \frac{\partial u}{\partial y}\right), \\
\rho c_{p}\left(u \frac{\partial T}{\partial x}+v \frac{\partial T}{\partial y}\right)=\frac{\partial}{\partial y}\left(\lambda \frac{\partial T}{\partial y}\right)+\beta T u \frac{d p}{d x}+\mu\left(\frac{\partial u}{\partial y}\right)^{2} .
\end{gathered}
$$

Here, $\alpha$ is the angle the surface makes with the gravity vector, and $p=$ constant for constant pressure approximation. This means the gradient of pressure in the $\mathrm{x}$-direction is hydrostatic:

$$
-\rho g \sin (\alpha)-\frac{d p}{d x}=-\left(\rho-\rho_{\infty}\right) g \sin (\alpha) .
$$


Due to small velocities in natural convection, dissipation is neglected; this means the second two terms of the RHS of the energy equation can be neglected. Additionally, all surfaces in this work will be vertical, thus $\alpha=\frac{\pi}{2}$ and $\sin \alpha=1$. These are the equations we will work with moving forward.

\section{Boussinesq Approximation}

The traditional formulation of a similarity solution for naturally convecting flow uses the Boussinesq approximation. The Boussinesq approximation is used for small temperature differences; that is, density differences in the flow are neglected except where they would be multiplied by gravity in the governing equations. This means the governing equations are now represented by:

$$
\begin{gathered}
\frac{\partial u}{\partial x}+\frac{\partial v}{\partial y}=0 \\
u \frac{\partial u}{\partial x}+v \frac{\partial u}{\partial y}=g \beta_{\infty}\left(T-T_{\infty}\right)+v_{\infty} \frac{\partial^{2} u}{\partial y^{2}} \\
u \frac{\partial T}{\partial x}+v \frac{\partial T}{\partial y}=a_{\infty} \frac{\partial^{2} T}{\partial y^{2}}
\end{gathered}
$$

where $a_{\infty}=\frac{\lambda}{\rho c_{p}}$ and $\beta=1 / T$ for gases. However, this typical approximation for formulating the similarity solutions is not valid for high temperature differences between wall and ambient conditions, as will be shown in Fig. 3.4. Large temperature differences cause large changes in fluid properties including density. Accurate simulation requires developing a full variable property similarity solution.

\section{Full Variable Properties in Natural Convection, Vertical Isothermal Plate}

In order to develop a full variable property solution, we work with Equations 3.1 . 3.3. Following the analysis laid out by Sparrow and Gregg (1958), the assumptions of constant pressure, compressible flow, an ideal gas, and neglected pressure work and viscous dissipation are applied to the two-dimensional steady boundary layer equations:

$$
\begin{gathered}
\frac{\partial(\rho u)}{\partial x}+\frac{\partial(\rho v)}{\partial y}=0 \\
\rho\left(u \frac{\partial u}{\partial x}+v \frac{\partial u}{\partial y}\right)=g\left(\rho_{\infty}-\rho\right)+\frac{\partial}{\partial y}\left(\mu \frac{\partial u}{\partial y}\right),
\end{gathered}
$$




$$
\rho c_{p}\left(u \frac{\partial T}{\partial x}+v \frac{\partial T}{\partial y}\right)=\frac{\partial}{\partial y}\left(\lambda \frac{\partial T}{\partial y}\right)
$$

The approach by Sparrow and Gregg (1958) uses a stream function, $\psi$, that accounted for the variable density character of the flow. The equation for conservation of mass is automatically satisfied by using $\psi$, and the momentum and energy equations replace $u$ and $v$ with $\psi$. This yields complicated simultaneous partial differential equations for $\psi$ and $T$ as functions of $x$ and $y$. These coupled partial differential equations are computationally expensive to solve. It is much easier to instead transform the momentum and energy equations into ordinary differential equations by the use of what is traditionally called a similarity transformation. An independent variable is formed, $\eta$, called a similarity variable.

$$
\eta=\frac{c}{x^{\frac{1}{4}}} \int_{0}^{y} \frac{\rho}{\rho_{r}} d y, c=\left[\frac{g\left(\rho_{\infty}-\rho\right) / \rho_{r}}{4 v_{r}^{2}}\right]^{\frac{1}{4}},
$$

where $\eta$ is a Howarth-Dorodnitsyn type of variable. It is not the same as the traditional similarity variable, subsequently referred to as $\eta_{O}$, used for the constant property similarity solution as documented by Ostrarch (1953). New dependent variables $F$ and $\theta$ are formulated as:

$$
F(\eta)=\left(\frac{\psi}{x^{\frac{3}{4}}}\right)\left(\frac{1}{4 v_{r} c}\right), \theta=\frac{T-T_{\infty}}{T_{w}-T_{\infty}} .
$$

Note that with the perfect gas assumption, $P=\rho R T$ can be used to simplify the dimensionless buoyancy force:

$$
\frac{\left(\rho_{\infty} / \rho\right)-1}{\left(\rho_{\infty} / \rho_{w}\right)-1}=\frac{T-T_{\infty}}{T_{w}-T_{\infty}}=\theta
$$

Due to the assumption of isothermal boundary conditions, a similarity solution is allowed. That is, the momentum and energy equations are transformed into ordinary differential equations where $F$ and $\theta$ are functions of $\eta$ alone:

$$
\begin{gathered}
\frac{\partial}{\partial \eta}\left[\frac{\rho \mu}{\rho_{r} \mu_{r}} F^{\prime \prime}\right]+3 F F^{\prime \prime}-2\left(F^{\prime}\right)^{2}+\theta=0, \\
\frac{\partial}{\partial \eta}\left[\frac{\rho \lambda}{\rho_{r} \lambda_{r}} \theta^{\prime}\right]+3 P r_{r}\left[\frac{c p}{c p_{r}}\right] F \theta^{\prime}=0,
\end{gathered}
$$

with the boundary conditions: 


$$
F(0)=F^{\prime}(0)=0, \theta(0)=1, F^{\prime}(\infty)=\theta(\infty)=0 .
$$

This similarity solution is determined by the stationary two-point boundary value problem described above. Following the analysis laid out by Cairnie et al. (Cairnie and Harrison, 1982), we assume the fluid properties vary with temperature as follows:

$$
\begin{gathered}
M(\theta)=\frac{\rho \mu}{\rho_{r} \mu_{r}}, \\
\dot{M}(\theta)=\frac{d M}{d \theta}, \\
L(\theta)=\frac{1}{P r_{r}}\left(\frac{c_{p_{r}}}{c_{p}}\right) \frac{\rho \lambda}{\rho_{r} \lambda_{r}}, \\
Y(\theta)=\frac{1}{P r_{r}}\left(\frac{c_{p_{r}}}{c_{p}}\right) \frac{d}{d \theta}\left(\frac{\rho \lambda}{\rho_{r} \lambda_{r}}\right) .
\end{gathered}
$$

These functions for the fluid properties are found by fitting each property to a piecewise function of temperature. The value of various fluid properties are taken from tables showing the temperature variation of dry air properties. A piecewise function is fit to the numerical derivative of $M$ and $\rho \lambda$ to create a function for $\dot{M}$ and $Y$. In this way, functions for $M, \dot{M}, L$, and $Y$ are created.

The governing equations can be written as:

$$
\begin{gathered}
M F^{\prime \prime \prime}+\dot{M} \theta^{\prime} F^{\prime \prime}+3 F F^{\prime \prime}-2\left(F^{\prime}\right)^{2}+\theta=0, \\
L \theta^{\prime \prime}+Y\left(\theta^{\prime}\right)^{2}+3 F \theta^{\prime}=0,
\end{gathered}
$$

with the boundary conditions:

$$
F(0)=F^{\prime}(0)=0, \theta(0)=1, F^{\prime}(\infty)=\theta(\infty)=0 .
$$


The similarity solution transformed the governing equations into a system of simultaneous first-order ordinary differential equations which are solved as a boundaryvalue problem using MATLAB's bvp4c solver on a mesh:

$$
0=\eta_{0}<\eta_{1}<\ldots<\eta_{N}, \eta_{N}>10
$$

The results for u-velocity, v-velocity, and temperature are shown in Fig. 3.1
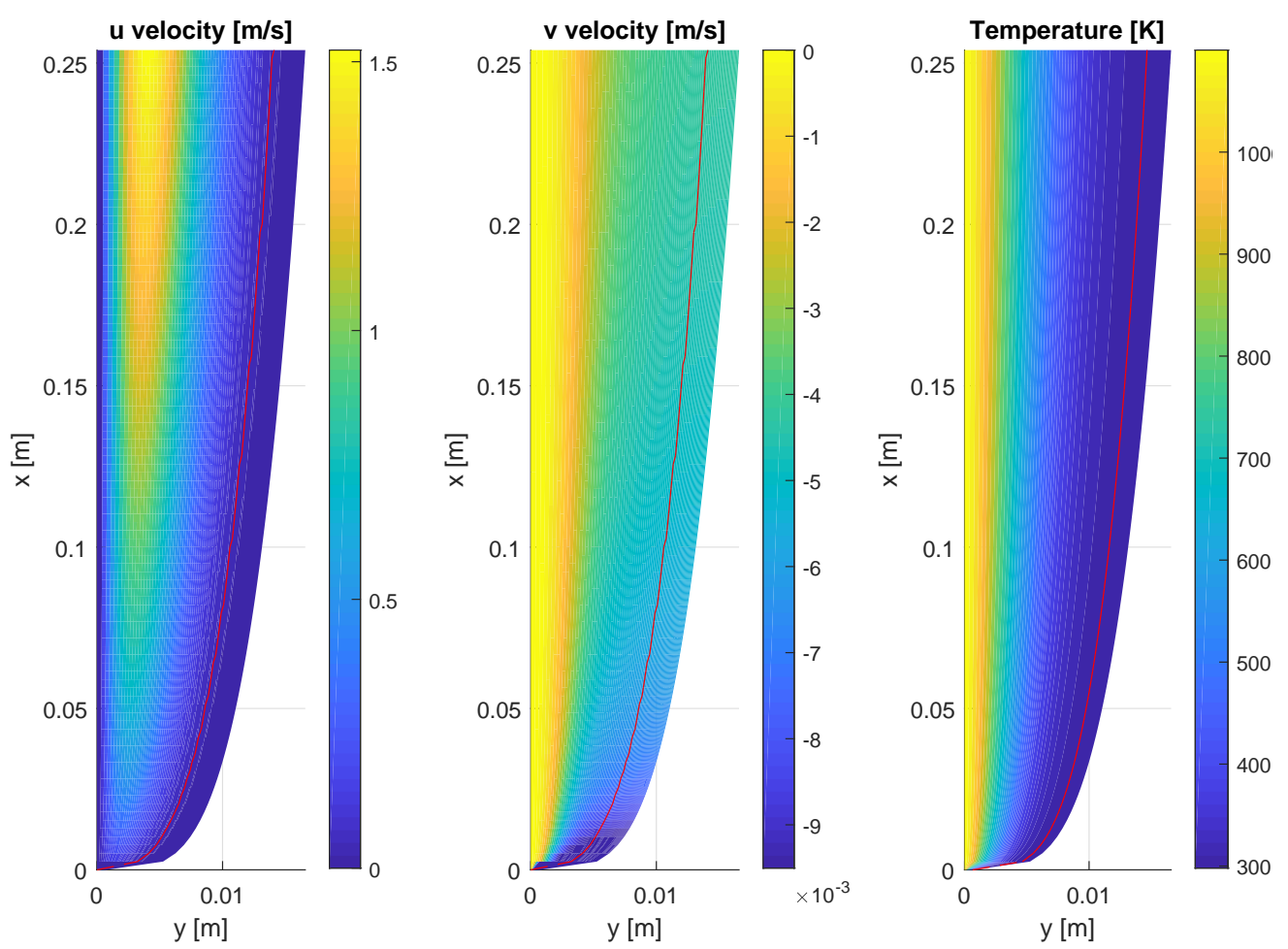

Figure 3.1: Results of similarity argument. Left: u-velocity field. Middle: vvelocity field. Right: temperature field. The wall temperature is $1100 \mathrm{~K}$, the gas is dry air, and the plate is $25.4 \mathrm{~cm}$ long.

The numerical results in Figure 3.1 are for a 25.4-cm-long plate. Results for a shorter plate can be obtained by truncating the numerical results at the desired total height of the plate. Velocity fields parallel (u-velocity) and perpendicular (v-velocity) to the wall and temperature fields are presented. The wall parallel velocity takes the expected structure for a natural convection flow: no-slip creates a velocity of zero next to the wall. Buoyancy forces increase the velocity as distance from the wall increases, reaching a maximum before velocity drops again to zero at the edge of 
the momentum layer to match ambient conditions. Similarly, wall perpendicular velocity appears as expected for a natural convection flow. The velocity is largest and negative at the leading edge of the cylinder as gas is entrained into the natural convection flow. The entrainment effect becomes less pronounced along the outer edge of the momentum layer as distance from the leading edge increases. The perpendicular velocity next to the wall is zero. Temperature results show the gas matches wall temperature immediately next to the surface, and then gas temperature drops off exponentially until ambient temperature is matched at the edge of the thermal layer. Additionally, the width of the momentum and thermal layers increase very quickly in approximately the first centimeter along the plate, after which there is a much more gradual increase in width as the distance from the leading edge increases.

\section{Verification and Validation of Natural Convection Simulation}

It is important to both verify and validate the natural convection simulations documented previously. Verification involves checking the results of the simulations against the original numerical results for this similarity solution by Cairnie and Harrison (1982). Validation requires checking that the results of this simulation reproduce the natural convection flow we are interested in. This can be done either by comparing the similarity results against a direct numerical simulation of a heated flat plate or results from experiments with a heated flat plate generating a natural convection flow. Here Cairnie and Harrison (1982) will be used for verification and validation as they report both the numerical results of the similarity solution and results from corresponding experiments with a heated vertical flat plate generating a buoyancy-driven flow.

First, we examine the results for temperature, $T$, plotted against wall-normal distance, $y$. Cairnie and Harrison (1982) report the numerical $T$ vs. $y$ profile at $x=0.1$ $\mathrm{m}$, as well as the experimental values of temperatures normal to a heated vertical plate as measured by a K-type thermocouple with a 0.13 -mm-diameter junction. The numerical method implemented in this work is compared with Cairnie and Harrison (1982) results for the four wall temperatures reported in Fig. 3.2. Inspection shows that at all four wall temperatures, there is very good comparison between the results from this work and those from Cairnie and Harrison (1982). Figure 3.3 shows the difference between the numerical results reported here and those from Cairnie and Harrison (1982) as a percent difference (blue) and absolute difference in K (red). 


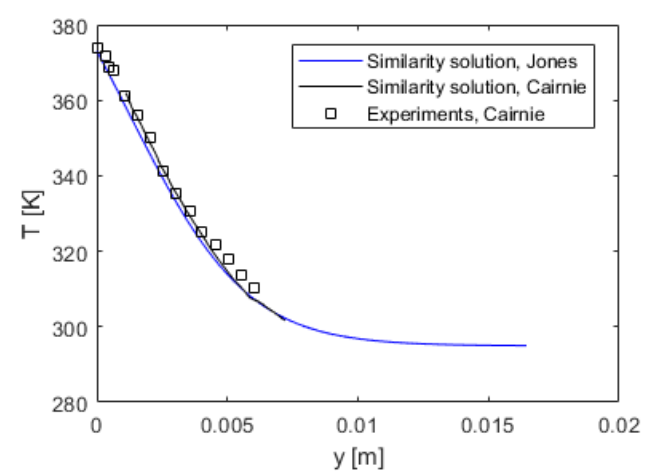

(a) $T_{w}=373 \mathrm{~K}$

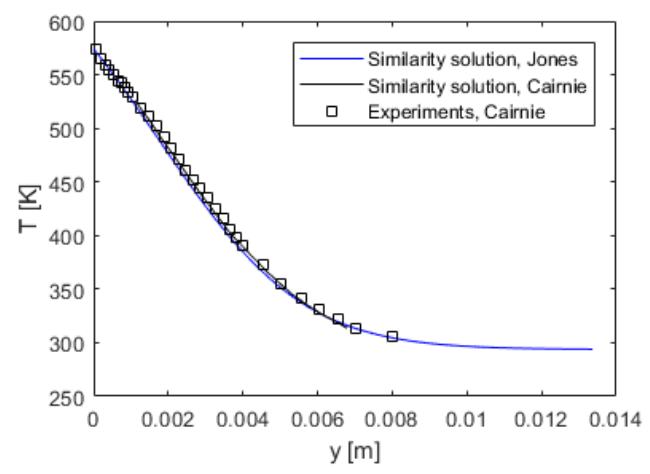

(c) $T_{w}=574 \mathrm{~K}$

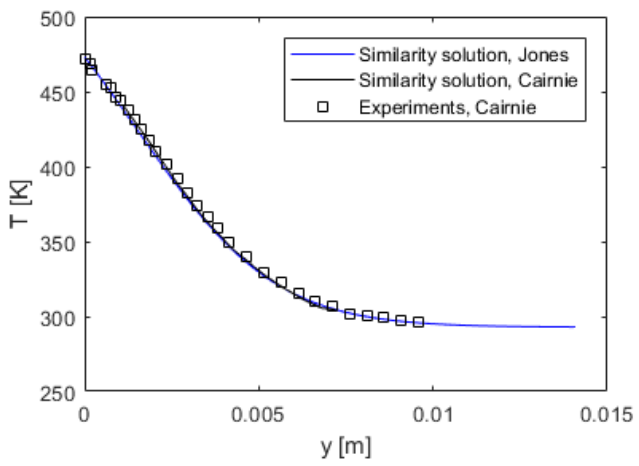

(b) $T_{w}=473 \mathrm{~K}$

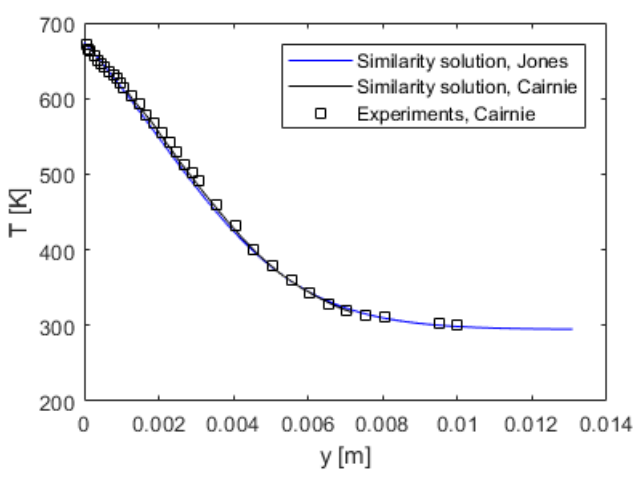

(d) $T_{w}=674 \mathrm{~K}$

Figure 3.2: Comparison of temperature profile along wall-normal direction for similarity solution. The blue line shows the numerical similarity solution. The black line shows the similarity solution results of Cairnie and Harrison (1982). The black squares show the experimental results of Cairnie and Harrison (1982) for a vertical flat plate. All comparisons made $0.1 \mathrm{~m}$ from leading edge $(x=0.1 \mathrm{~m})$.

This further demonstrates the agreement in numerical results, with errors for all four wall temperatures no more than $2 \%$ and mostly less than $1 \%$. This is excellent agreement and verifies the numerical method implemented here. Furthermore, since the numerical results of Cairnie and Harrison (1982) were validated against their experimental data, the numerical method in this work is also validated.

We can examine the results further by looking at the profile of non-dimensional temperature, $\theta$ in Fig. 3.4. Non-dimensional temperature is plotted against the similarity variable for the Boussinesq similarity solution presented by Ostrarch (1953), $\eta_{O}$, 


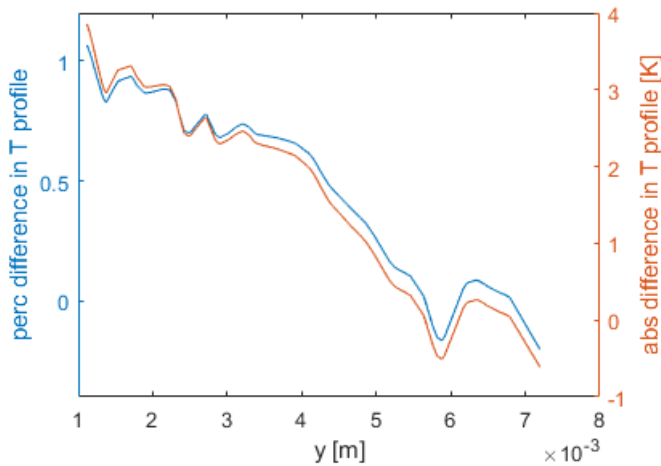

(a) $T_{w}=373 \mathrm{~K}$

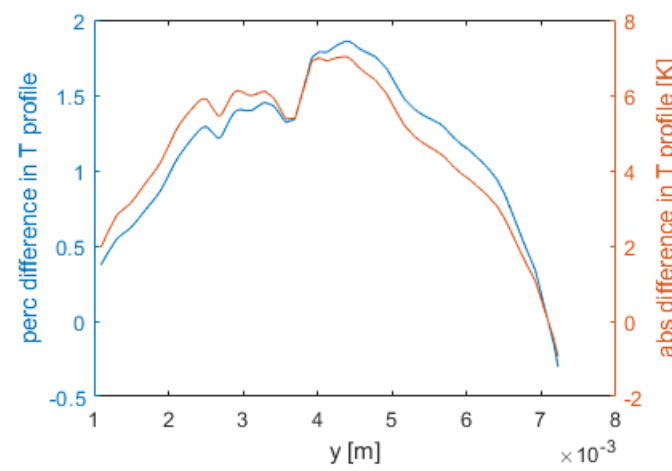

(c) $T_{w}=574 \mathrm{~K}$

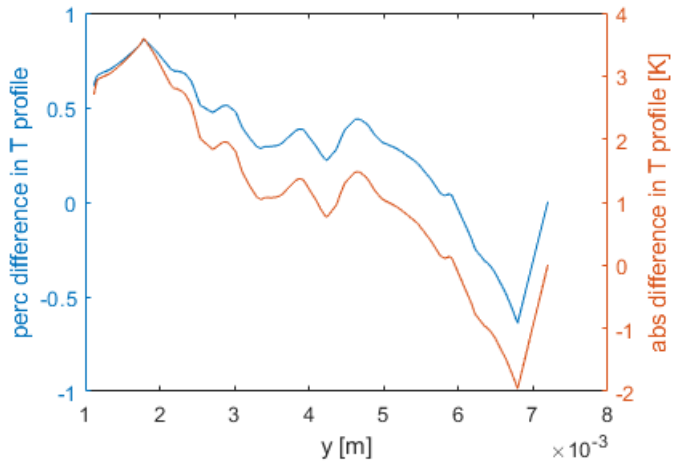

(b) $T_{w}=473 \mathrm{~K}$

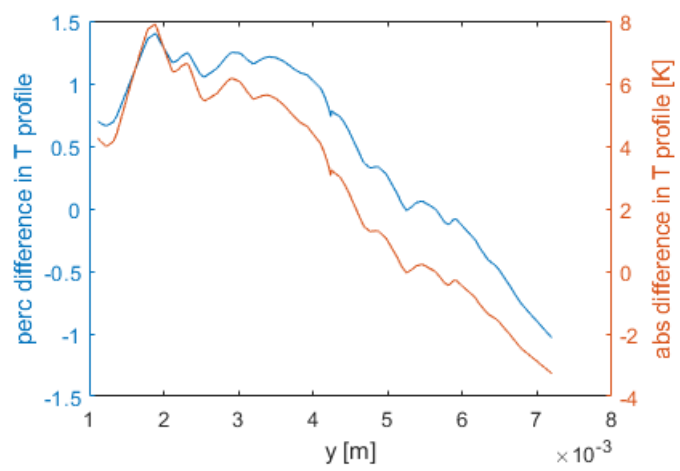

(d) $T_{w}=674 \mathrm{~K}$

Figure 3.3: Differences in numerical temperature profile along wall-normal direction for similarity solution presented here and in Cairnie and Harrison (1982) similarity solution results, $0.1 \mathrm{~m}$ from leading edge $(x=0.1 \mathrm{~m})$. The blue line represents percent difference in temperature profile, and the red line represents absolute difference in temperature profile in $\mathrm{K}$.

$$
\eta_{O}=\frac{y}{x}\left(\frac{\Delta T x^{3} g}{T_{\infty} 4 v_{\infty}^{2}}\right)^{0.25} .
$$

Once again, there is excellent comparison between the numerical results from this work and numerical and experimental results from Cairnie and Harrison (1982). This is further demonstrated in Fig. 3.5 as the differences between numerical results from this work and Cairnie and Harrison (1982) are shown as percent differences (blue line) and absolute differences (red line). The percent differences are quite low for $T_{W}=324 \mathrm{~K}$ up until $\eta_{O}>3$. At this point, the percent difference starts rising quite rapidly. This is due to $\theta$ tending to 0 far from the wall, and small errors in the digitization of the results from Cairnie and Harrison (1982) showing up as large percent differences since the absolute value (red line) is quite small far from the wall. A similar phenomenon occurs for $T_{w}=674 \mathrm{~K}$ with the large increase in 
percent difference while absolute difference remains small, starting at $\eta>5$.

Furthermore, the dashed line in Fig. 3.4 indicates the solution to the Boussinesq similarity solution developed by Ostrarch (1953). It is obvious that while the Boussinesq approximation is acceptable for small wall-ambient temperature differences $\left(T_{w}=324 \mathrm{~K}, \Delta T=29 \mathrm{~K}\right)$, it is not adequate for large temperature differences between wall and ambient conditions $\left(T_{w}=674 \mathrm{~K}, \Delta T=379 \mathrm{~K}\right)$. This demonstrates the necessity of implementing the more complicated but more appropriate variable property similarity solution for natural convection flows with large surface to ambient temperature differences.

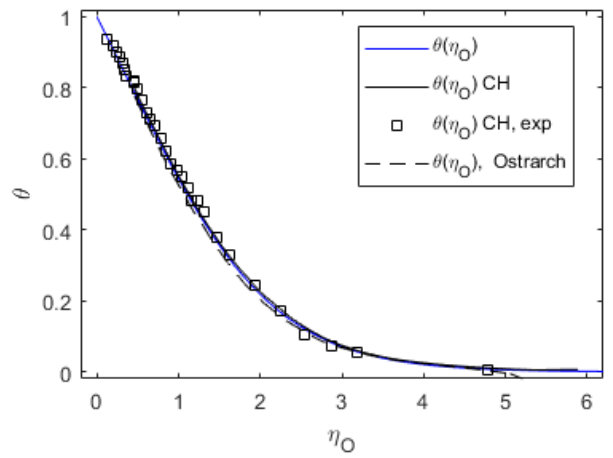

(a) $T_{w}=324 \mathrm{~K}$

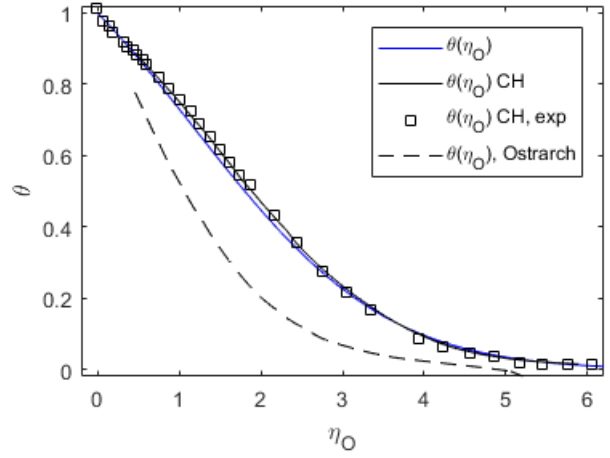

(b) $T_{w}=674 \mathrm{~K}$

Figure 3.4: Comparison of non-dimensional temperature, $\theta$ along Ostrach's similarity variable, $\eta_{O}$. The blue line represents numerical similarity solution. The black line represents the similarity solution results from Cairnie and Harrison (1982). The black squares represent experimental results from Cairnie and Harrison (1982) for a vertical flat plate. All comparisons made $0.1 \mathrm{~m}$ from leading edge $(x=0.1 \mathrm{~m})$.

Finally, we can examine the normalized wall parallel velocity profile for validation with the results of Cairnie and Harrison (1982). Figure 3.6a shows the numerical results for normalized $u$ velocity,

$$
s=\frac{u}{\sqrt{\frac{4 g\left(T_{w}-T_{\infty}\right) x}{T_{\text {infty }}}}}
$$

plotted against the similarity variable, $\eta_{O}$, from Ostrarch (1953) for a wall temperature of $T_{w}=688 \mathrm{~K}$. The black line shows the numerical results, and the black squares the experimental data obtained from real-fringe laser Doppler anemometry 


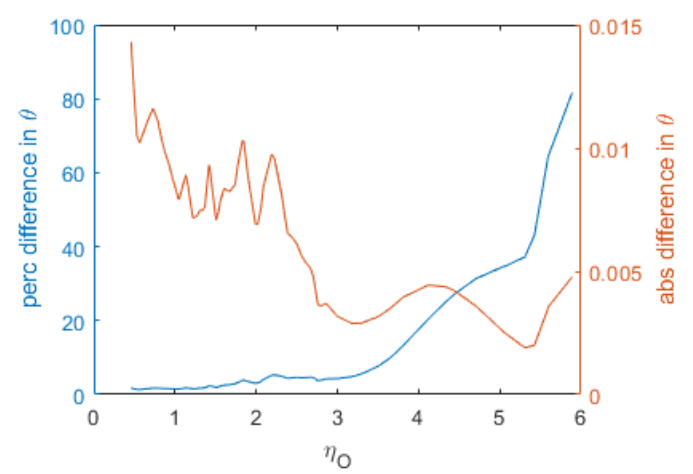

(a) $T_{w}=324 \mathrm{~K}$

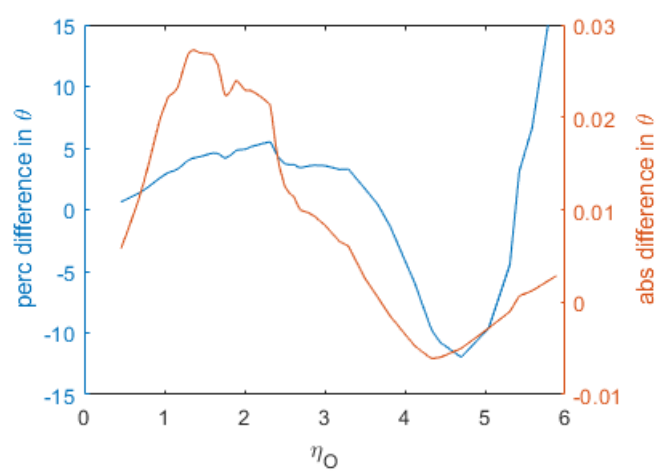

(b) $T_{w}=674 \mathrm{~K}$

Figure 3.5: Differences in non-dimensional temperature, $\theta$ along Ostrach's similarity variable, $\eta_{O}$ for current similarity solution and for Cairnie and Harrison (1982) similarity solution results, $0.1 \mathrm{~m}$ from leading edge $(x=0.1 \mathrm{~m})$. The blue line represents percent difference in non-dimensional temperature, and the red line represents absolute difference in non-dimensional temperature.

velocity measurements from Cairnie and Harrison (1982). The dashed line once again shows the constant property solution from Ostrarch (1953), and it is again obvious that the similarity solution with constant gas properties is inaccurate for large temperature differences.

The experimental data from Cairnie and Harrison (1982) shows a larger velocity than predicted at large $\eta_{O}$ (i.e. the edge of the boundary layer); the source of this discrepancy is unknown. The numerical results of Cairnie and Harrison (1982) are in good agreement with the normalized velocity profile calculated in this work; the differences are shown in Fig. 3.6b. The percent difference is mostly within approximately $10 \%$. The regions where the percent difference is greater than $10 \%$ are regions where the absolute difference is small, the value of normalized velocity is tending to 0 , and thus are likely due to small errors in digitizing the results from Cairnie and Harrison (1982).

The results of the similarity solution presented previously are compared with numerical results from Cairnie and Harrison (1982) for verification of the method and experimental results from the same authors for validation of the natural convection flow predictions. There is excellent agreement in the temperature, which is the quantity of greatest interest as it will be compared with experimental temperature profiles from interferometry. There are slightly larger discrepancies in the velocity profile, but they remain within about $10 \%$ and are assumed acceptable. This confirms that 


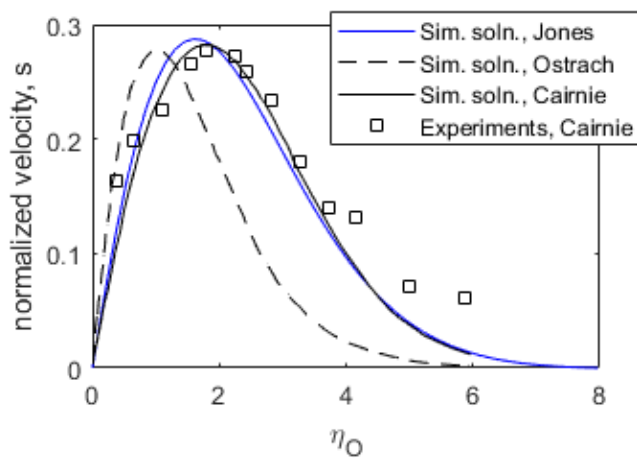

(a) $T_{w}=688 \mathrm{~K}$. Normalized $\mathrm{u}$

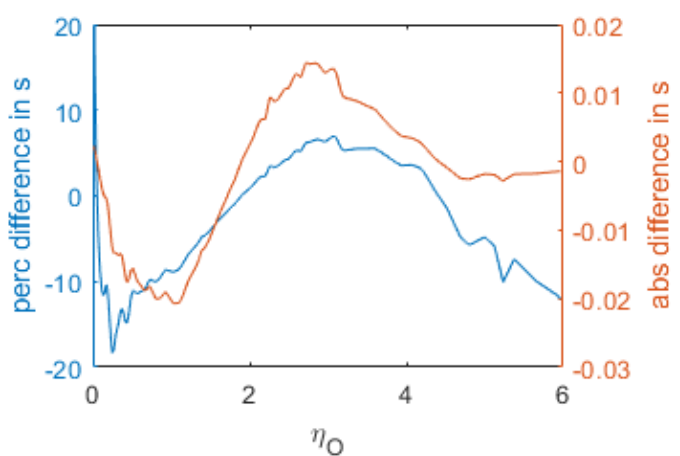

(b) $T_{w}=688 \mathrm{~K}$. Differences in normalized $\mathrm{u}$

Figure 3.6: Normalized wall-parallel velocity, $s$ along Ostrach's similarity variable, $\eta_{O}$. (a) The blue line represents the numerical similarity solution. The black line represents similarity solution results from Cairnie and Harrison (1982). The black squares represent experimental results from Cairnie and Harrison (1982) for a vertical flat plate. (b) The blue line represents percent difference in normalized velocity, and the red line represents absolute difference in normalized velocity. All comparisons made $0.1 \mathrm{~m}$ from leading edge $(x=0.1 \mathrm{~m})$.

the similarity solution implemented is useful for predicting natural convection flows over a vertical plate with large surface-to-wall temperature differences. The following sections will discuss some of the limitations of this numerical approach.

\section{Non-dimensional parameters}

There are several dimensionless parameters important to buoyancy flows that are relevant to discuss at this point. These dimensionless parameters describe the relationship between various phenomena at work in the flow. The Grashof number, for example, describes the ratio of the buoyancy force to the viscous force fluid flow. It is often used in natural convection flows as an analog to the Reynolds number $(\operatorname{Re}=\rho U L / \mu)$. The Grashof number is defined in Eq. 3.27.

$$
G r=\frac{g \beta\left(T_{s}-T_{\infty}\right) L^{3}}{v^{2}}
$$

where $g$ is gravitational acceleration, $\beta$ is the coefficient of thermal expansion, $T_{S}$ is the surface temperature, $T_{\infty}$ is ambient temperature, $L$ is length, and $v$ is the kinematic viscosity. Fluid properties are calculated at the film temperature unless otherwise stated. The Grashof number for for a surface temperature of $1100 \mathrm{~K}$ is $5.3 \times 10^{6}, 1.8 \times 10^{7}$, and $4.2 \times 10^{7}$ for cylinders where $L=12.7,19.1$, and $25.4 \mathrm{~cm}$ 
lengths, respectively. The longest cylinders are approaching $1 \times 10^{8}$, which is the lower end of the range at which transition to turbulence occurs (Bahrami, 2009), meaning the flow around the largest cylinders may exhibit some signs of a transitional flow.

Another important dimensionless parameter is the Nusselt number, $\mathrm{Nu}$, which is defined in Eq. 3.28, It is the ratio of convective to conductive heat transfer across a boundary.

$$
N u=\frac{h L}{\kappa},
$$

where $h$ is the convective heat transfer coefficient (see Eq. 3.29), $L$ is the characteristic length, and $\kappa$ is the thermal conductivity of the fluid. A Nusselt number of 1 indicates a purely conductive heat transfer mode. Larger Nusselt numbers indicated an enhancement of heat transfer due to convection, radiation, mass transport, etc.

For vertical flat plates with laminar flow, the convective heat transfer coefficient can be approximated through the empirical relation proposed by Churchill:etal:1975

$$
h=\frac{\kappa}{L}\left(0.68+\frac{0.67 R a^{1 / 4}}{\left(1+(0.492 / P r)^{9 / 16}\right)^{4 / 9}}\right), 10^{-1}<R a<10^{9},
$$

where $R a$ is the Rayleigh number, defined in Eq. 3.30, and $\operatorname{Pr}$ is the Prandlt number, $\operatorname{Pr}=v / \alpha$, where $\alpha$ is the thermal diffusivity of the fluid.

$$
R a=\frac{g \beta}{v \alpha}\left(T_{s}-T_{\infty}\right) L^{3}=G r P r,
$$

From these equations, the Nusselt number of the cylinders is estimated at 23.0 for $L=12.7 \mathrm{~cm}, 30.9$ for $L=19.1 \mathrm{~cm}$, and 38.2 for $L=25.4 \mathrm{~cm}$. The Nusselt number increases with increasing length. These values, like those found for the Grashof number, indicate that the flow regime for the cylinders (particularly the longest ones) may be transitional in nature.

\section{Limitations of Natural Convection Simulation}

There are a number of limitations inherent in the natural convection simulation presented in Section 3.1. Some are due to the boundary layer approximations made at 
the very beginning, while other limitations arise to simplifying assumptions about surface geometry.

The boundary layer approximation has limitations of course. By applying the approximation, we assume that the Reynolds number is sufficiently large but below the laminar-turbulent transition such that the flow remains laminar. We also assume that the pressure is constant across the boundary layer. These assumptions are reasonable and appear justified in the present situation. As a consequence of applying the boundary layer approximation, there is an infinite gradient (a mathematical singularity) at the leading edge that is obviously not physical. Therefore, the boundary layer approximation breaks down at the leading edge. We are interested in solutions far from the leading edge, however, where the the approximation is valid.

Further limitations are introduced by the assumption of a flat, isothermal surface. The cylinders are not isothermal, as demonstrated in Section 2.3. We tolerate this assumption since a large section of the surface is nearly isothermal. Additionally, the cylinders that we are trying to approximate with this numerical solution are not flat plates; the flow in the radial direction will be incorrect due to the divergence of the stream tubes in cylindrical geometry as compared to a planar flow. Sparrow and Gregg (1958) developed the following relation that defines when approximating a vertical cylinder as a vertical flat plate is valid such that there is less than $5 \%$ difference in $\mathrm{Nu}$ for a vertical flat plate and a vertical cylinder with the same length.

$$
\frac{D}{L}>\frac{35}{G r^{0.25}},
$$

where $D$ is the diameter of the cylinder, $L$ is the height of the cylinder, and $G r_{L}$ is the Grashof number based on the height of the cylinder. None of the cylinders satisfy this inequality, but for cylinders 50C, 100C, and 200A, the right and left hand sides of Eq. 3.31 are within the same order of magnitude. As shown in Section 3.2, experimental temperature fields from these cylinders in nitrogen compare well with the numerical results, with a maximum error of $10 \%$. Since there is an excellent comparison with the numerical prediction of the thermal layer in these cases, we conclude that these cylinders can still be approximated as a flat plate with reasonable accuracy despite not meeting the limit posed by Sparrow and Gregg (1958). 


\subsection{Interferometer Results on Cylinders in Nitrogen}

In Section 2.6, the interferometer has been validated against prior studies with a glow plug, a well-characterized heated surface. We now investigate interferometry results from the test cylinders. This consists of placing a test cylinder in the vessel and calibrating the thermocouples attached to it as described in Sections 2.1 and 2.4 respectively. The vessel is fully sealed and evacuated and then filled to atmospheric pressure with nitrogen gas. Nitrogen is used due to its inertness; the gas composition will not change with heating, and therefore its interferometry properties, like Gladstone-Dale constant and index of refraction, are known with high accuracy. This means the results from interferometry in nitrogen can be trusted with high confidence, which is important for examining the boundary layer formed over these cylinders for the first time.

The interferometry results shown in Figs. 3.8 through 3.10 are a time average over 100 seconds of the cylinder operating at a steady state temperature. Examples of the raw interferograms are shown in Fig. 3.7. This is done for a low temperature (700 $\mathrm{K})$ and a high temperature that is close to expected ignition temperatures $(1100 \mathrm{~K})$.
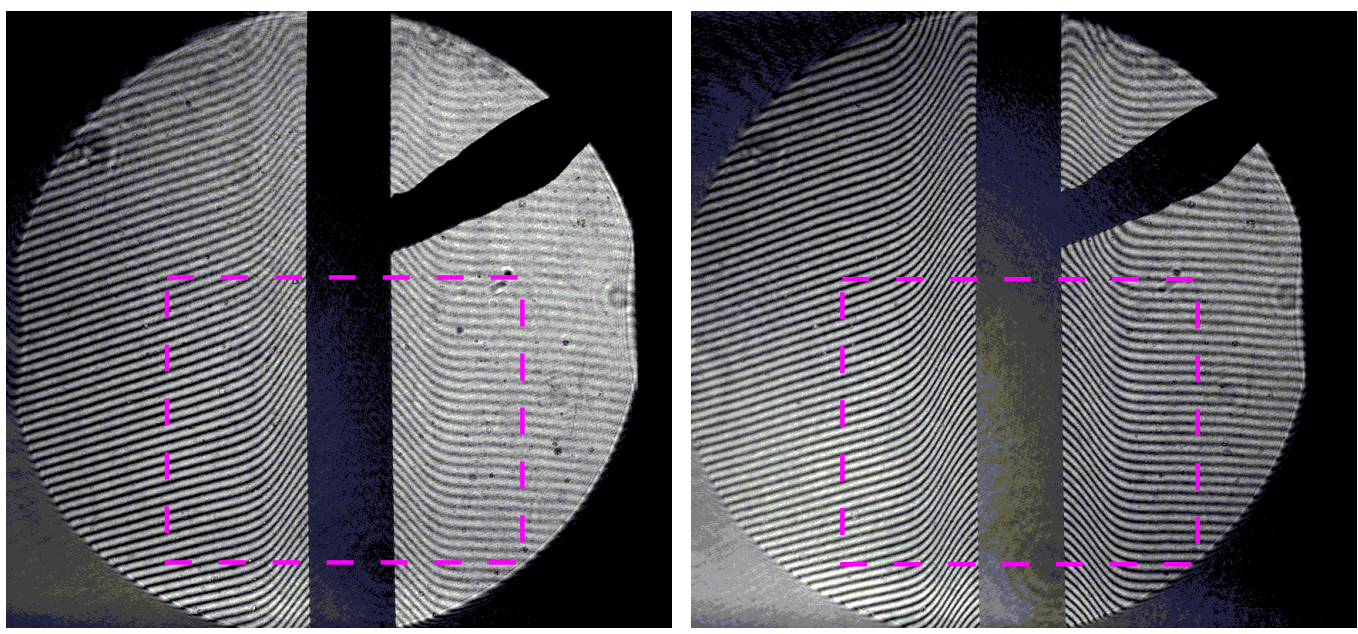

Figure 3.7: Raw interferograms for nitrogen tests at $700 \mathrm{~K}$ (shot 75) and $1100 \mathrm{~K}$ (shot 79) wall temperature for cylinder 75B. The pink box shows the field of view processed for interferometry results.

Both the results for a test at $700 \mathrm{~K}$ (left) and $1100 \mathrm{~K}$ (right) show good symmetry between the left and right halves of the temperature fields. Figure $3.10 \mathrm{c}$ shows less than 5\% difference between the left and right thermal boundary layer profiles for the test at $700 \mathrm{~K}$, and Fig. 3.10d shows the same for the $1100 \mathrm{~K}$ test. This amounts to a 

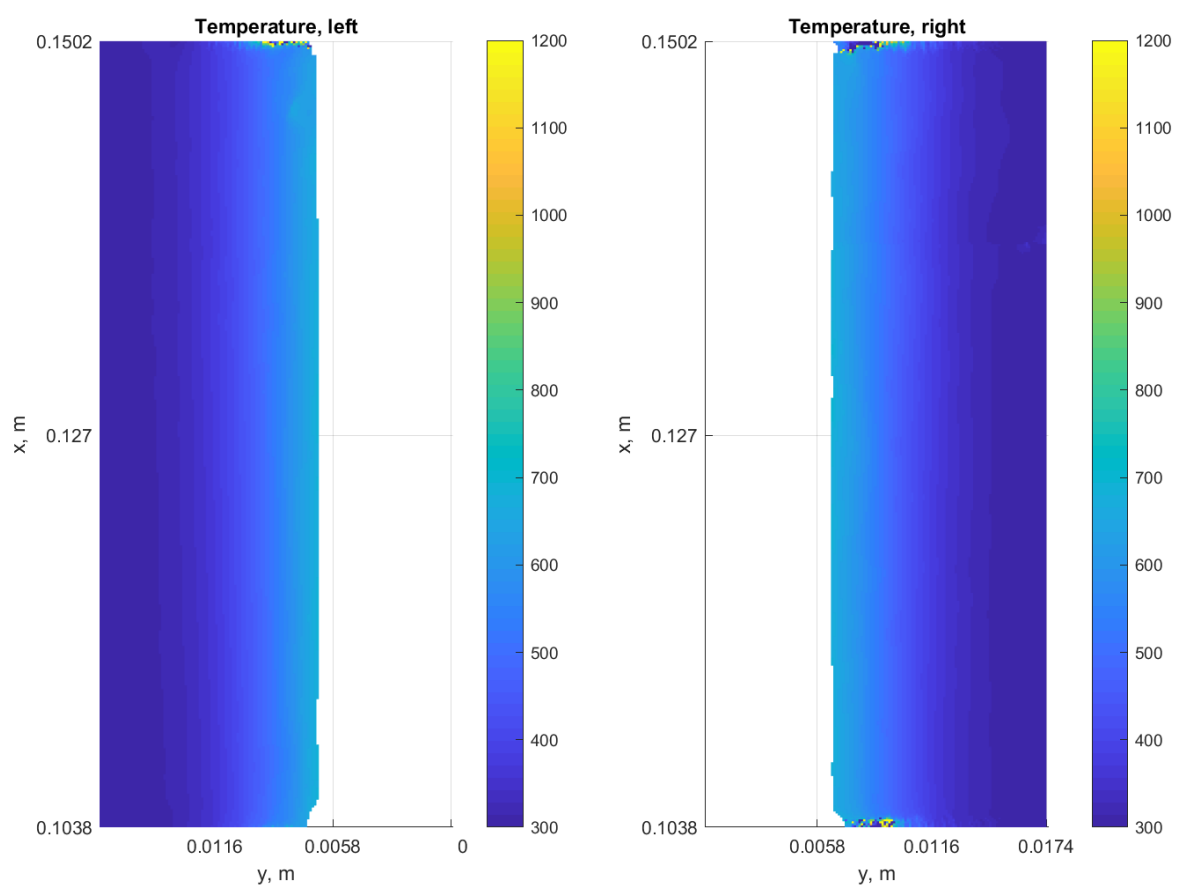

Figure 3.8: Interferometry temperature field results at $700 \mathrm{~K}$.
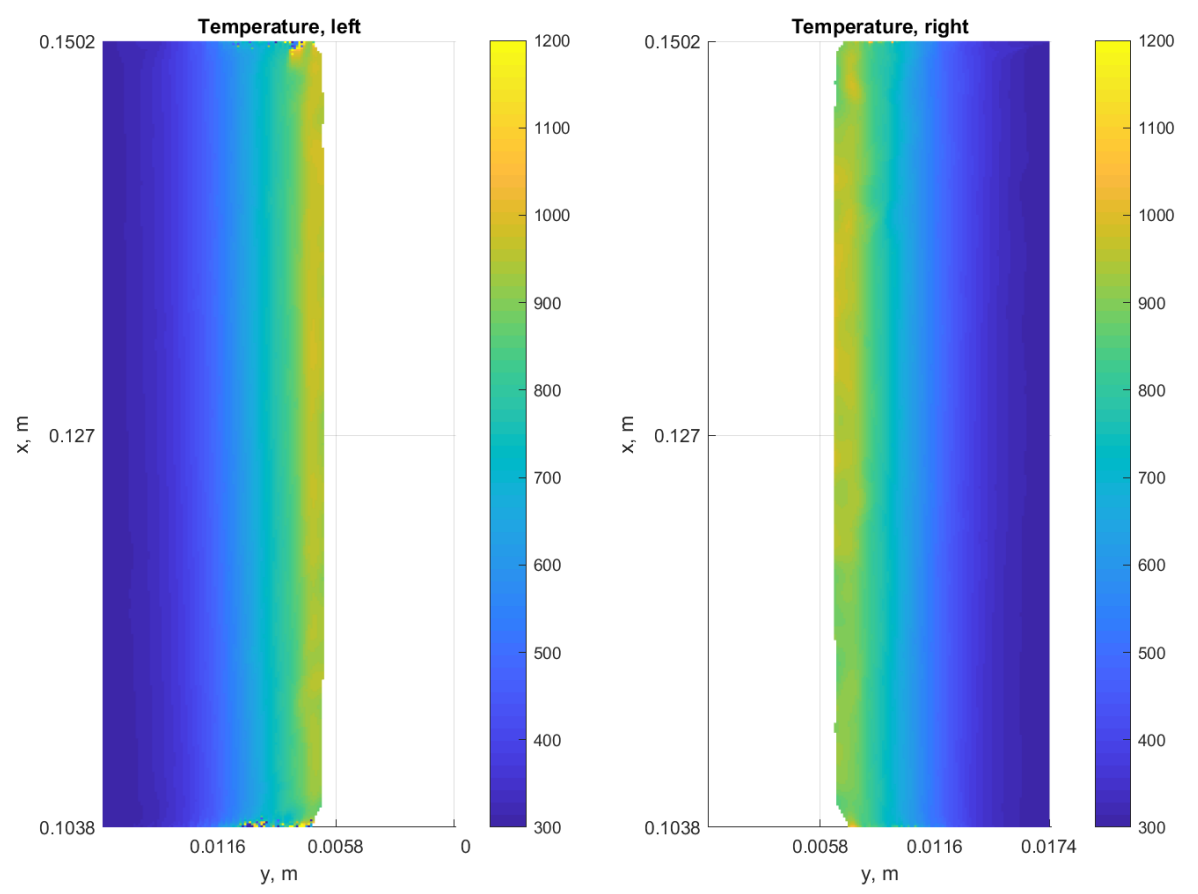

Figure 3.9: Interferometry temperature field results at $1100 \mathrm{~K}$ 


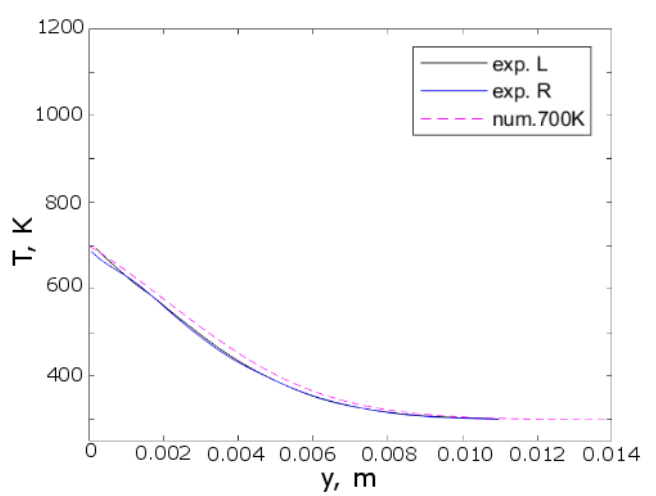

(a)

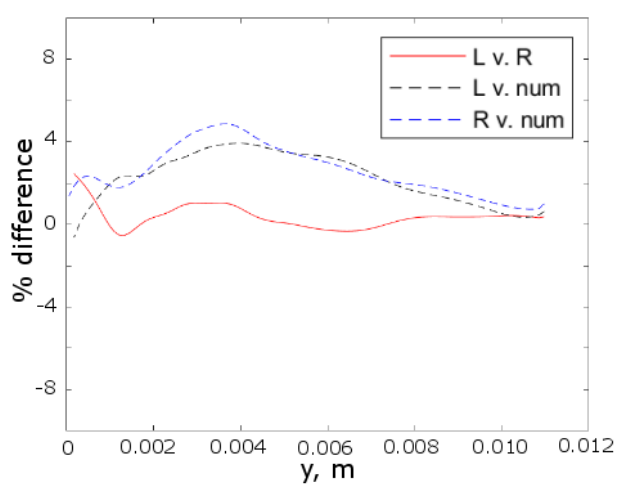

(c)

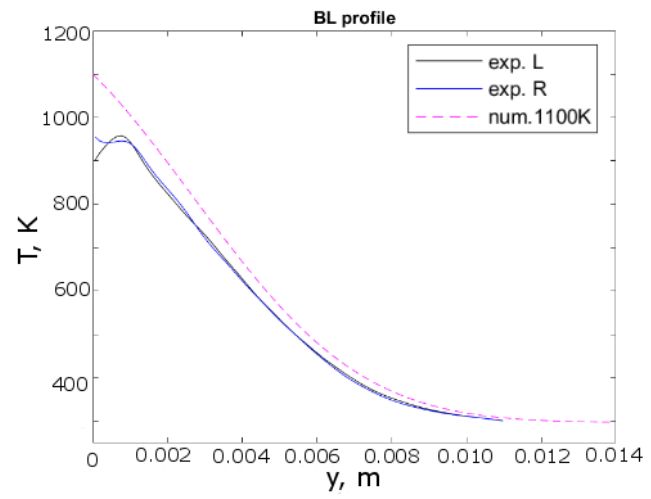

(b)

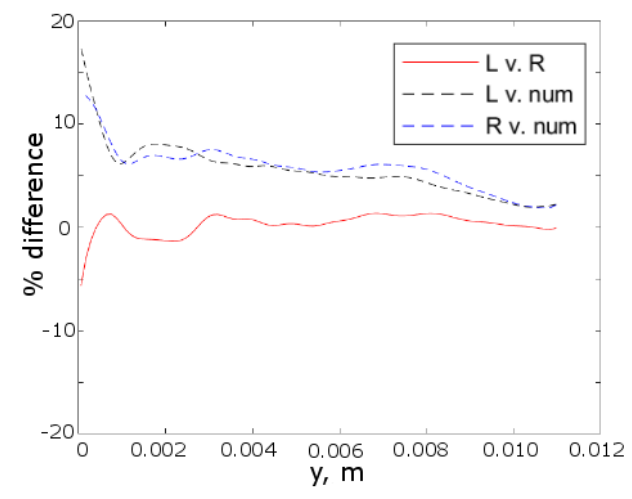

(d)

Figure 3.10: Interferometry results: left, $700 \mathrm{~K}$, right $1100 \mathrm{~K}$. Top row: profile of thermal layer for left and right sides compared with similarity solution; bottom row: percent error between profile of thermal layer for left vs. right sides, left vs. similarity solution, and right vs. similarity solution.

"very symmetrical" interferometry result and is a good indication of axisymmetry in the experiments. For the test at $700 \mathrm{~K}$, the experimental thermal boundary layer profiles are within about $5 \%$ of the numerical profile; at $1100 \mathrm{~K}$, the experimental profiles are within $10 \%$ of the numerical profile except in a narrow region very close to the cylinder surface (this limited region of high error will be discussed further in Chapter 4). The surface temperature is extrapolated from the interferometry results for the $1100 \mathrm{~K}$ case by fitting a linear function the the temperature profile of the boundary layer between $2 \mathrm{~mm}<y<6 \mathrm{~mm}$ (this process is described in more detail in Section 4.6 and is found to be approximately $1010 \mathrm{~K}$, or within $8.2 \%$ of the surface temperature measured via pyrometer. These results indicate the interferometer able to reconstruct temperature fields for the large cylinders accurately and the numerical prediction of the boundary layer profile is in good agreement with experimental observations. 


\section{References}

Bahrami, M. (2009). Natural Convection. uRL:https://www . sfu . ca/ mbahrami// ENSC\%5C\%20388/Notes/Natural\%5C\%20Convection.pdf

Cairnie, L. R. and A. J. Harrison (1982). "Natural Convection Adjacent to a Vertical Isothermal Hot Plate with a High Surface to Ambient Temperature Difference". In: International Journal of Heat and Mass Transfer 25.7, pp. 925-934. (Visited on $10 / 24 / 2018)$.

Ostrarch, S. (1953). An Analysis of Laminar Free Convection Flow and Heat Transfer About a Flat Plate Parallel to the Direction of the Generating Body Force. Tech. rep. NACA TR 1111. NASA, pp. 63-79.

Sparrow, E. M. and J. L. Gregg (1958). "The variable fluid property problem in free convection". In: Transactions of the ASME 80, pp. 879-886. 
Chapter 4

\section{HEXANE-AIR TESTS}

This chapter documents the results of testing cylinders of various geometries in a stoichiometric n-hexane and air mixture. Cylinders 25A, 50A, 50C, 75B, 100A, $100 \mathrm{C}$, and 200A were tested; the number of the cylinder denotes its surface area in $\mathrm{cm}^{2}$, the letter indicates the cylinder length $(\mathrm{A}: \mathrm{L}=25.4 \mathrm{~cm}, \mathrm{~B}: \mathrm{L}=19.1 \mathrm{~cm}, \mathrm{~A}: \mathrm{L}=$ $12.7 \mathrm{~cm}$ ). At least 20 individual experiments were performed for each cylinder, with the exception of cylinder $25 \mathrm{~A}$, which was tested five times before the cylinder failed due to buckling from thermal expansion during heating. The test matrix is reported in Appendix $\mathrm{F}$ and documents important test parameters like power supply settings, mixture composition, temperature set point, temperature at ignition, and time to ignition for each experiment. The test time for each experiment is limited to $300 \mathrm{~s}$.

The ignition temperatures from all tests are analyzed to produce a probabilistic model of ignition as a function of cylinder surface temperature. The ignition threshold is defined as the temperature at which there is a $50 \%$ probability of ignition. The ignition thresholds of various cylinders are compared to understand the influence of geometrical parameters like surface area and length on ignition behavior.

Optical diagnostics allow for visual inspection of ignition. Interferometry provides quantitative temperature fields of the gas surrounding the heated cylinder, although some limitations with hexane mixtures are found and discussed. The average ignition location is noted for each cylinder, and image sequences showing the dynamics of ignition and early flame propagation are presented. The flame speed of the hexane mixture is noted and compared with results from standard flame speed calculations.

\subsection{Experimental Conditions}

$\mathrm{N}$-hexane $\left(\mathrm{C}_{6} \mathrm{H}_{14}\right)$ is studied in the laboratory for a number of reasons. Previous predictions of aviation kerosene vapor composition by Coronel (2016) indicated that at low temperatures $(\approx 40 \operatorname{deg} C)$, hydrocarbons like hexane and heptane are reasonable surrogates for the vapor composition of aviation kerosene, of which hy- 
drocarbons with 6 or 7 carbon atoms make up the largest portion of the composition at low temperatures. Additionally, hexane fully vaporizes at room temperature unlike heavier hydrocarbon jet fuel surrogates. This makes hexane easy to work with, as no heating of the combustion vessel is needed to achieve full fuel vaporization and therefore accurate mixture composition control.

The hexane-air tests are performed at stoichiometry corresponding to unity equivalence ratio. Previous studies on hot surface ignition with hexane (Boeck et al., 2017) have shown that thermal ignition behavior is insensitive to equivalence ratio over a wide range away from the flammability limits. The hexane-air mixture is created in the laboratory for each individual test, regardless of if the mixture in the previous test ignited or not. This ensures that the initial conditions for each test are consistent and identical mixtures. The stoichiometric reaction for $n$-hexane and air in terms of major product species is

$$
\mathrm{C}_{6} \mathrm{H}_{14}+\frac{19}{2}\left(\mathrm{O}_{2}+3.76 \mathrm{~N}_{2}\right) \longrightarrow 7 \mathrm{H}_{2} \mathrm{O}+6 \mathrm{CO}_{2}+35.7 \mathrm{~N}_{2}
$$

When filling the vessel with the reactive mixture for experiments, we translate stoichiometry given by Eq. 4.1 into mole fractions and partial pressure. The results are given in Table 4.1

\begin{tabular}{llll}
\hline Gas & Mole Fraction & Partial Pressure (Torr) & Total Pressure (Torr) \\
\hline n-Hexane & 0.022 & 16.4 & 16.4 \\
$\mathrm{~N}_{2}$ & 0.773 & 587.5 & 603.9 \\
$\mathrm{O}_{2}$ & 0.205 & 156.1 & 760.0
\end{tabular}

Table 4.1: Composition of gas mixture for stoichiometric n-hexane and air tests.

\subsection{Experiment Procedure}

With all the equipment and diagnostics powered on, the experiment begins by evacuating the vessel to less than 100 mTorr. Then the vessel is filled with the n-hexane and air mixture, which is created inside the vessel by filling with fuel, nitrogen, and oxidizer in the proportions specified by Table 4.1. A Heise 901A manometer (sensitivity down to 0.075 Torr) is used to monitor the pressure as gases are added to the vessel. The digital pressure readout has a resolution of 0.1 Torr. The fuel is added first; liquid $n$-hexane is injected into the vessel via syringe and fully vaporizes. The vapor pressure of hexane, over 100 Torr at $293 \mathrm{~K}$, is greater than the partial 
pressure of hexane added to the vessel of 16.4 Torr. Then nitrogen gas is added to the target partial pressure of 587.5 Torr, and finally oxygen gas is added to a target partial pressure of 156.1 Torr. The total pressure in the vessel is 760 Torr (101 $\mathrm{kPa}$ ). This order of gas addition is chosen as a safety measure so that there is never a stoichiometric mixture of pure fuel and oxygen without a diluent (nitrogen) present.

After the reactive mixture is created inside the vessel, a mixing fan runs for 3 mins to ensure a homogenous mixture inside the vessel. The fan is then turned off and the gas is allowed to settle for 3 mins so that the mixture at the start of the ignition tests is quiescent. A reference measurement is taken for both the pyrometer and the interferometer. For the pyrometer, a dark current measurement is taken to measure current levels in the photodetectors before heating. For the interferometer, a reference image is taken of the fringe pattern before deflection due to heating. The test then begins by energizing the heating circuit and allowing the test cylinder to heat up. The test runs until either an ignition event occurs, or until $300 \mathrm{~s}$ have elapsed, at which point the test is ended manually by the operator. Power to the heating circuit is turned off, and the vessel is evacuated. If an ignition event occured, the vessel can directly be evacuated; otherwise, the reactive mixture must be diluted with nitrogen to a total pressure of 1520 Torr $(202 \mathrm{kPa})$ to take the mixture beyond the lower flammability limit for safe evacuation.

The data collection takes place in three parts. First, the pyrometer data is fed to a computer through a digital data acquisition system to provide feedback control for the heating system via LabView software. The pyrometer data is also collected by a Yokogawa oscilloscope, model DL850E with 12-bit input channels (4096 digitizer units). The pyrometer data collected from the oscilloscope is used to extract the temperatures reported later in this chapter. Additionally, the pressure transducer data is collected by the oscilloscope to monitor the pressure transient in the vessel. Finally, the interferometer images are collected by a Phantom high-speed camera and saved to the computer after the test.

During an ignition event, the oscilloscope is triggered by a rise in the pressure over 1520 Torr $(202 \mathrm{kPa})$. The Phantom camera is triggered by the trigger output of the oscilloscope. 
There are four ways of identifying an ignition event. The first is by a sharp increase in pressure as monitored by the pressure transducer. The second method is by a sudden increase in the ambient gas temperature in the vessel as monitored by a K-type thermocouple suspended inside the vessel but away from the test cylinder. The third method is by identifying a flame front on the high speed video (this is possible for all video types; interferometer, schlieren, and $\mathrm{OH}^{*}$ chemiluminescence). In interferometry, the flame front manifests as a sharp deflection of the fringes. In schlieren imaging, the flame front manifests as a dark wavefront. $\mathrm{OH}^{*}$ chemiluminescence is perhaps the most obvious of all the imaging techniques for identifying ignition: the image is dark until ignition occurs, at which point the flame front is directly observed by the presence of a bright expanding wave of emission from the electronically excited $\mathrm{OH}$ radicals. Finally, ignition can be identified via pyrometery due to the sharp increase in detector signal that occurs during an ignition event. The thermocouple and dynamic pressure gauge mounted at the wall of the combustion vessel will indicate an ignition event at approximately $50 \mathrm{~ms}$ after ignition is observed in the visualization techniques and via the spike in the pyrometer detector signal; this is due to the time it takes for the flame to travel from its origin near the heated surface to the inner walls of the combustion vessel.

\subsection{Ignition Temperatures}

From the pyrometer data, the cylinder surface temperature can be extracted. The cylinder surface temperature represents the independent variable in the experiments. The dependent variable for these tests is a binary outcome variable; a 0 represents a "no-go" or no ignition, a 1 represents a "go" ignition event. These data pairs of $\left(T_{\text {surf }}, 0 / 1\right)$ are particularly useful for analyzing the results across multiple tests with the same test cylinder via a logistic regression. The application to ignition data is described in detail in Bane et al. (2011), and the statistical basis is given by Hosmer, Lemeshow, and Sturdivant (1989) and Neter et al. (1996). The logistic regression provides an estimate (with appropriate confidence intervals) of the probability of achieving ignition as a function of the surface temperature of the test cylinder.

It takes approximately 20 to 25 individual hexane experiments to generate a highquality logistic regression. These tests must span a range of "go" and "no-go" results to generate reliable statistics. From the results of the logistic regression, we extract the ignition temperature, $T_{i g n}$, and the surface temperature at which there is a 50 
$\%$ chance of ignition. This is the ignition temperature reported in the rest of this chapter unless otherwise noted. Figures 4.1 through 4.6 show the ignition data and logistic regression results for cylinders 50A, 50C, 75B, 100A, 100C, and 200A.

Cylinder 25A failed after five experiments and therefore there was not enough data to generate a logistic regression. The five tests that were performed with $25 \mathrm{~A}$ did span a range of temperatures that included 3 "go" results and 2 "no-go" results, which are enough to form a deterministic estimate of the ignition temperature based on the lowest observed temperature at which $25 \mathrm{~A}$ achieved ignition. Figure 4.7 shows the ignition data for $25 \mathrm{~A}$.

The ignition temperature for each cylinder as well as the corresponding $95 \%$ confidence limits are shown is Table 4.2.

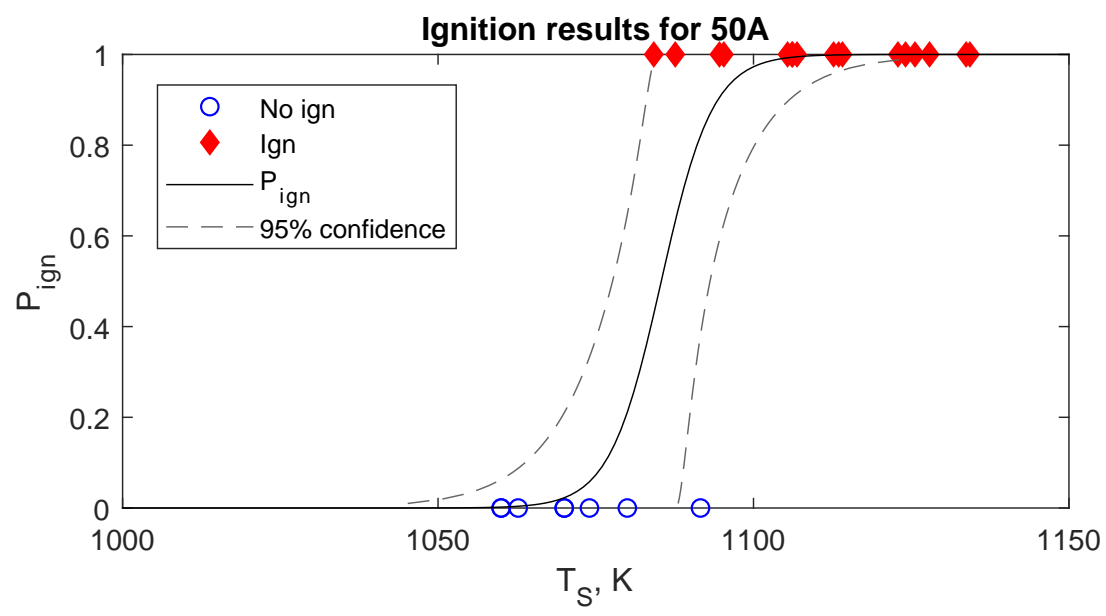

Figure 4.1: Ignition data ("go" red diamonds, "no-go" blue circles) and probabilistic model of ignition based on logistic regression (black line) with upper and lower 95\% confidence limits (gray dashed lines) for cylinder 50A.

The value of performing a large number of repeat tests at a given condition is to account for the variability of the outcome of ignition testing through statistical methods. Rather than reporting the igniton temperature as a single deterministic value, the probability of ignition is reported as a function of temperature. Furthermore, the variation in ignition temperature seen in the outcome of individual tests (see in particular the overlap in "go" and "no-go" temperatures for cylinder 100A as documented in Fig. 4.4) would not be captured by a single test. 


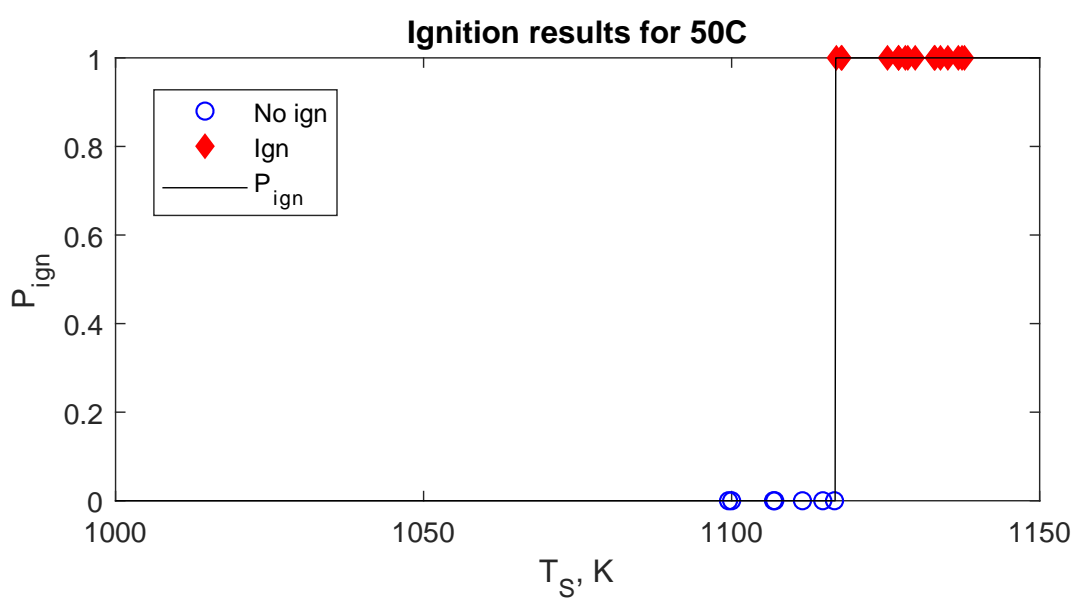

Figure 4.2: Ignition data and probabilistic model of ignition for cylinder 50C.

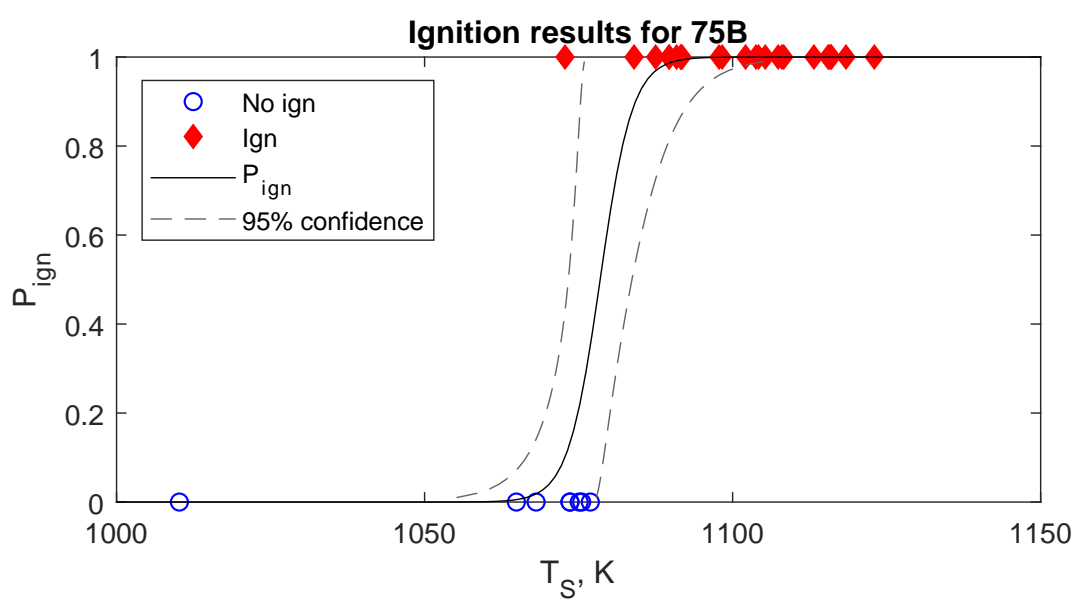

Figure 4.3: Ignition data and probabilistic model of ignition for cylinder 75B.

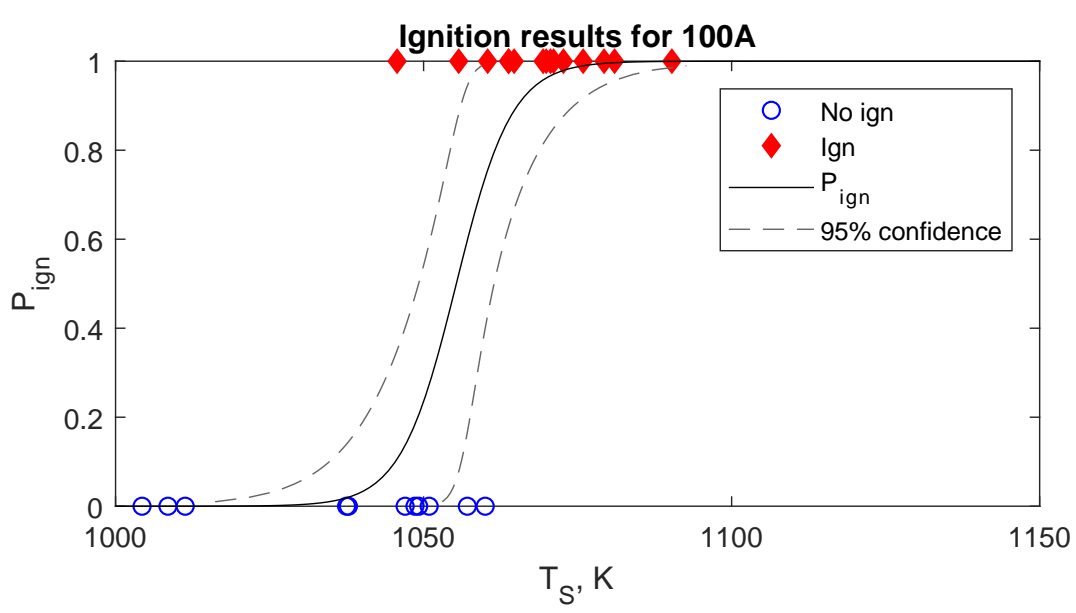

Figure 4.4: Ignition data and probabilistic model of ignition for cylinder 100A. 


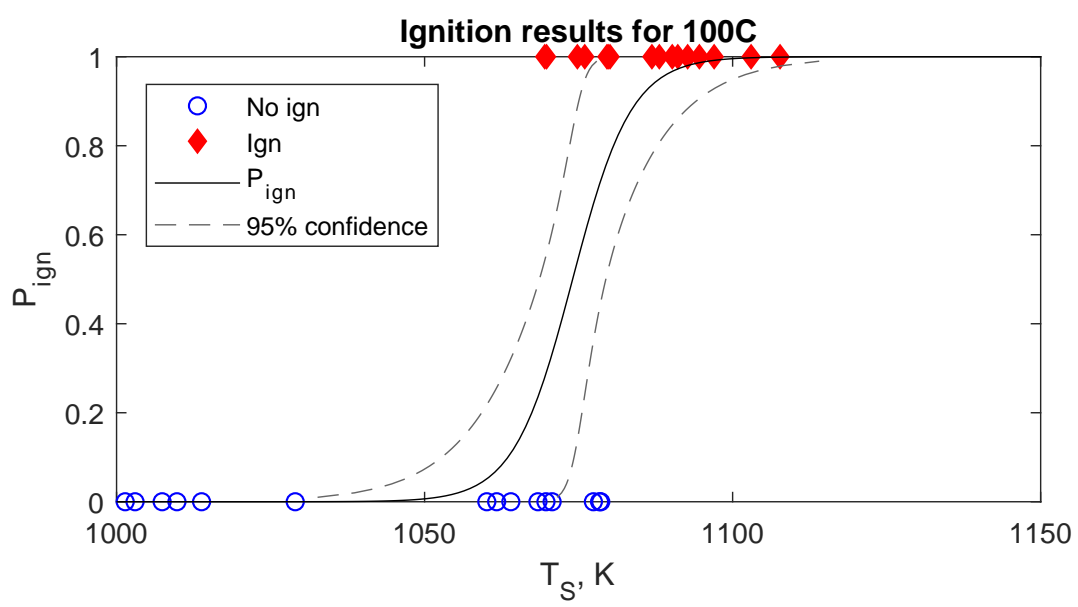

Figure 4.5: Ignition data and probabilistic model of ignition for cylinder 100C.

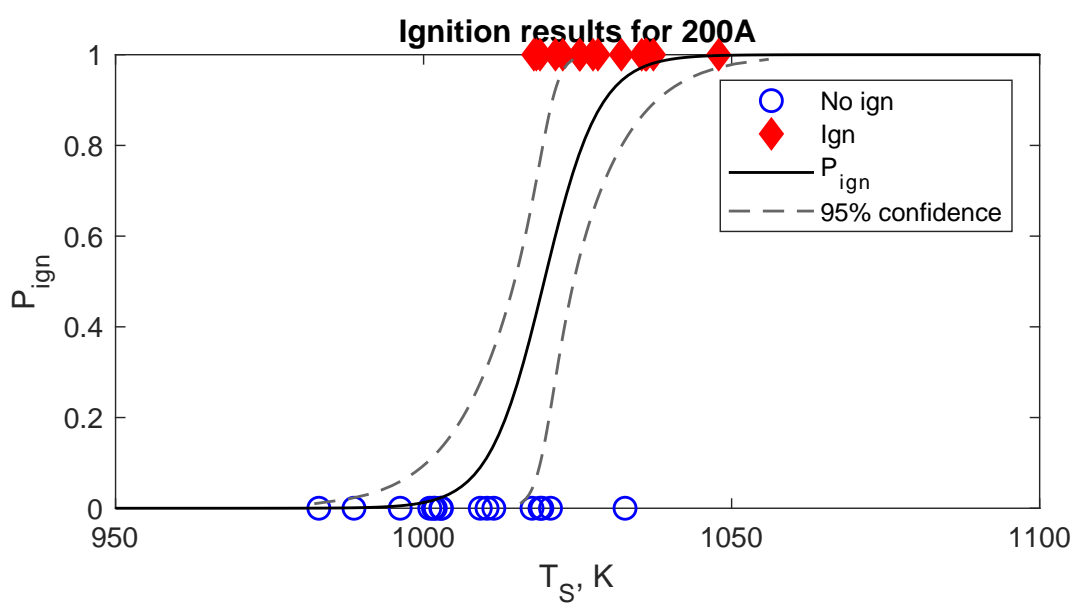

Figure 4.6: Ignition data and probabilistic model of ignition for cylinder 200A.

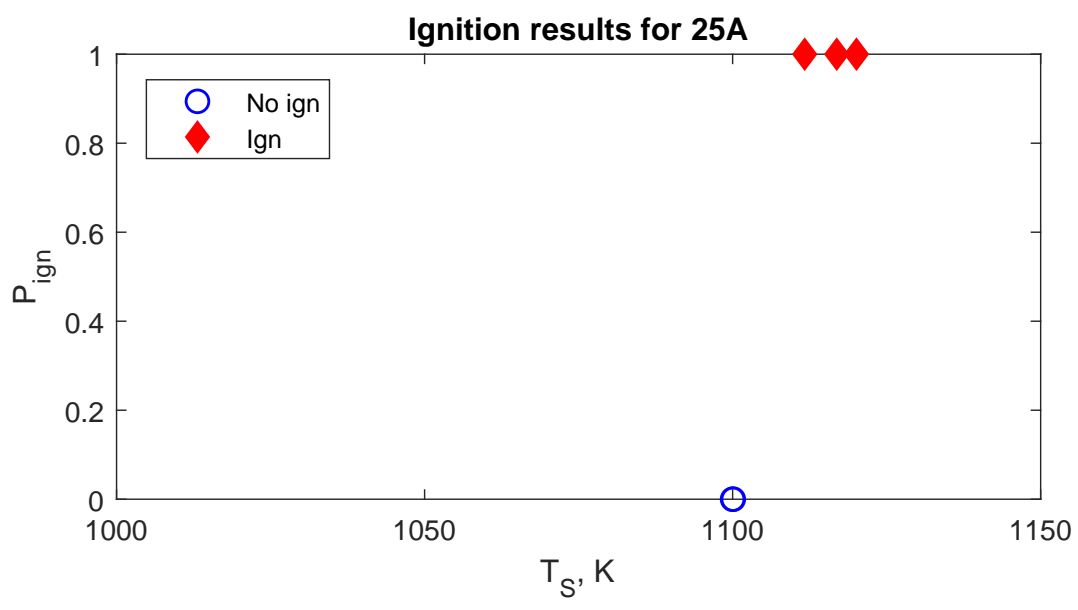

Figure 4.7: Ignition data for cylinder 25A. 


\begin{tabular}{ccc}
\hline Cylinder Tag & Ignition Temperature $(\mathrm{K})$ & $95 \%$ Confidence Limit $(\mathrm{K})$ \\
\hline $25 \mathrm{~A}$ & $1113^{*}$ & N/A \\
$50 \mathrm{~A}$ & 1085 & \pm 8 \\
$50 \mathrm{C}$ & 1117 & - \\
$75 \mathrm{~B}$ & 1078 & \pm 6 \\
$100 \mathrm{~A}$ & 1055 & \pm 6 \\
$100 \mathrm{C}$ & 1074 & \pm 5 \\
$200 \mathrm{~A}$ & 1019 & \pm 5 \\
\hline
\end{tabular}

Table 4.2: Ignition temperatures and confidence limits for all cylinders.

Note that the confidence limits are significantly smaller than the measurement uncertainty of $\pm 28 \mathrm{~K}$ reported for the pyrometer. As the discussion in Section 2.5 notes, this is a upper bound on the measurment uncertainty; comparing the pyrometer measurements to thermocouple measurements during the setup of each cylinder suggests a measurement uncertainty of approximately $\pm 10 \mathrm{~K}$, which is similar in magnitude to the confidence limits reported for the ignition thresholds.

We can also examine how long it takes for the mixture to ignite for each cylinder. The surface temperature of the cylinder ramps up during the early stage of the experiment towards the temperature set point. Depending on the cylinder and the set point, ignition can be achieved during the temperature ramp or the cylinder can reach the set point and settle into steady-state behavior for a significant time before ignition occurs. Examples of these two behaviors are shown in Fig. 4.8. Generally, when the mixture ignites during the heating ramp, this is a supercritical case, meaning that the temperature set point exceeds the critical ignition temperature. There is no significant delay in the ignition, because the mixture ignites as soon as the surface temperature exceeds the ignition threshold. Contrastingly, when the final surface temperature is set very near the critcal temperature (i.e. the tipping point between ignition and non-ignition), the surface can reach that temperature and remain there for a significant amount of time before ignition occurs.

If we classify reaching steady-state behavior as when the cylinder surface temperature is within $1 \%$ of the set point, we can find the time to reach steady state (where possible) and compare that to the time to reach igntion. We can therefore let the ignition delay for the experiments be the difference between the time to ignition and the time to steady state, where it exists. This allows examination of the ignition delay, $\tau_{i g n}$, to see if there is any correlation of the ignition delay with surface 


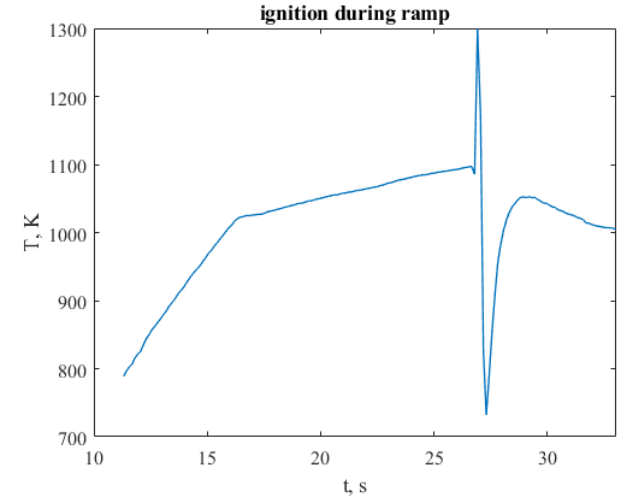

(a) Ign during ramp

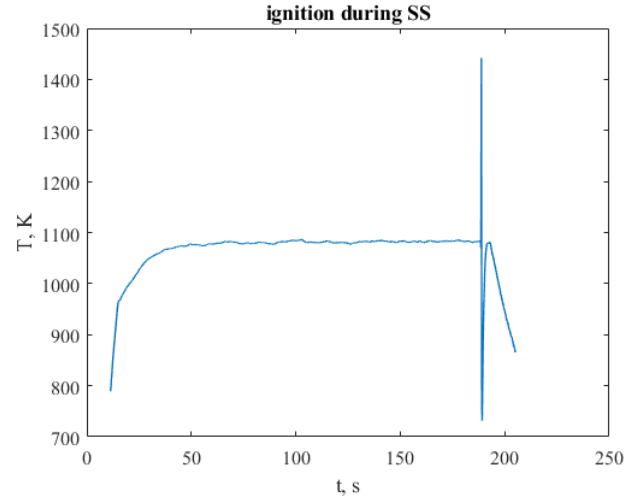

(b) Ign during SS

Figure 4.8: Side by side of ignition during the heating ramp (a) and ignition during steady-state behavior (b).

temperature at ignition. The results are shown in Fig. 4.9.

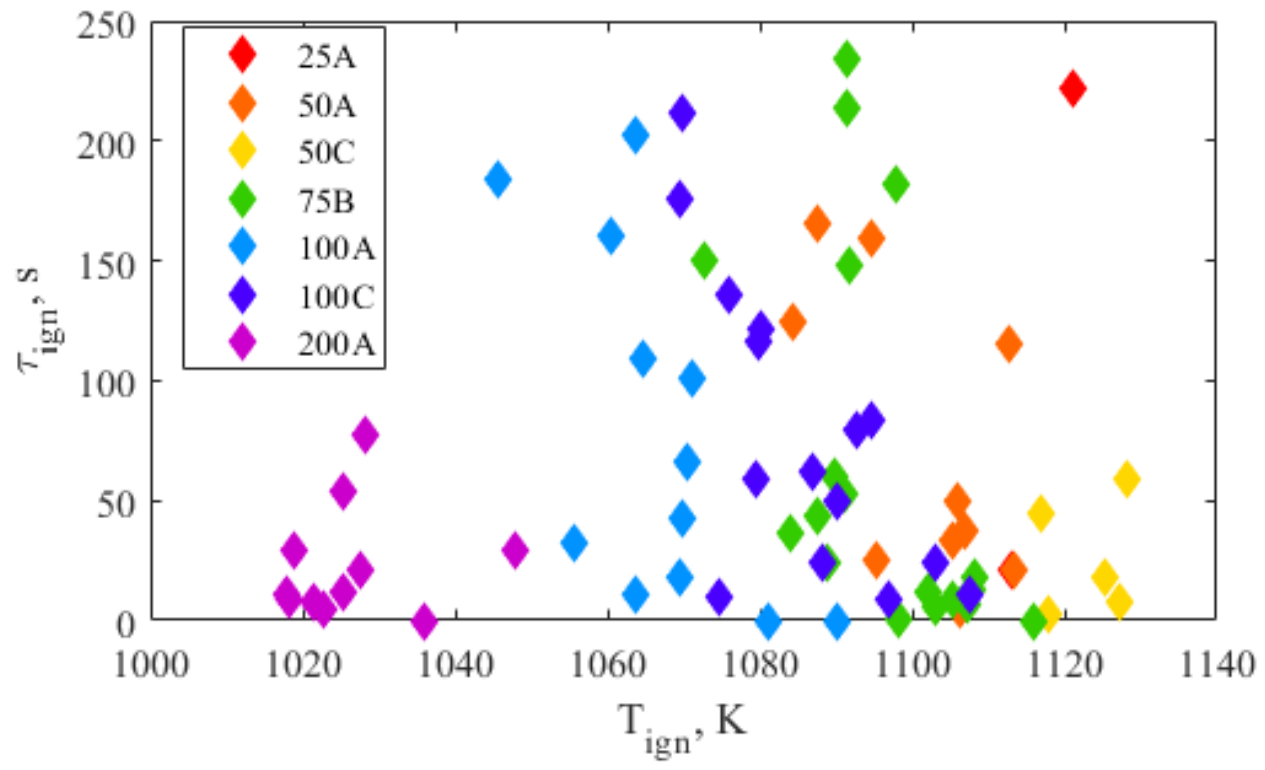

Figure 4.9: Scatter plot of $\tau_{i g n}$ vs $T_{i g n}$.

The scatter in the results of the ignition delay with ignition temperature is significant. It is difficult to draw any meaningful correlations from these results. Figure 4.9 instead serves as a visual indication of the variation inherent to the experimental results. Some of this comes from experimental and measurement uncertainty, but there is clearly a stochastic component that contributes substantially to the scatter in the ignition delay times of the experiments. This stands in contrast to the welldefined values obtained in ignition tests using rapid compression machines, shock 
tubes, or rapidly (millisecond heating timescale) heated cylinders (Adomeit, 1965). Those experiments all examine supercritical conditions and have a rapid increase from ambient to ignition temperature so that ignition delay is well defined. The present experiments are all carried out close to the ignition temperature threshold, and the ignition delay near the threshold is known to be exceedingly sensitive to tiny variations in conditions.

\subsection{Effects of Geometry}

The cylinders tested were designed and selected to allow a systematic study of the effects of various geometrical parameters on the ignition temperatures. In this section, we will examine how much of an effect a change in a particular geometrical parameter, namely surface area, length, and diameter, has on the ignition behavior. Figures ??, 4.10, and 4.11 plot the ignition temperature against surface area, length, and diameter respectively to allow for consideration of each parameter individually.

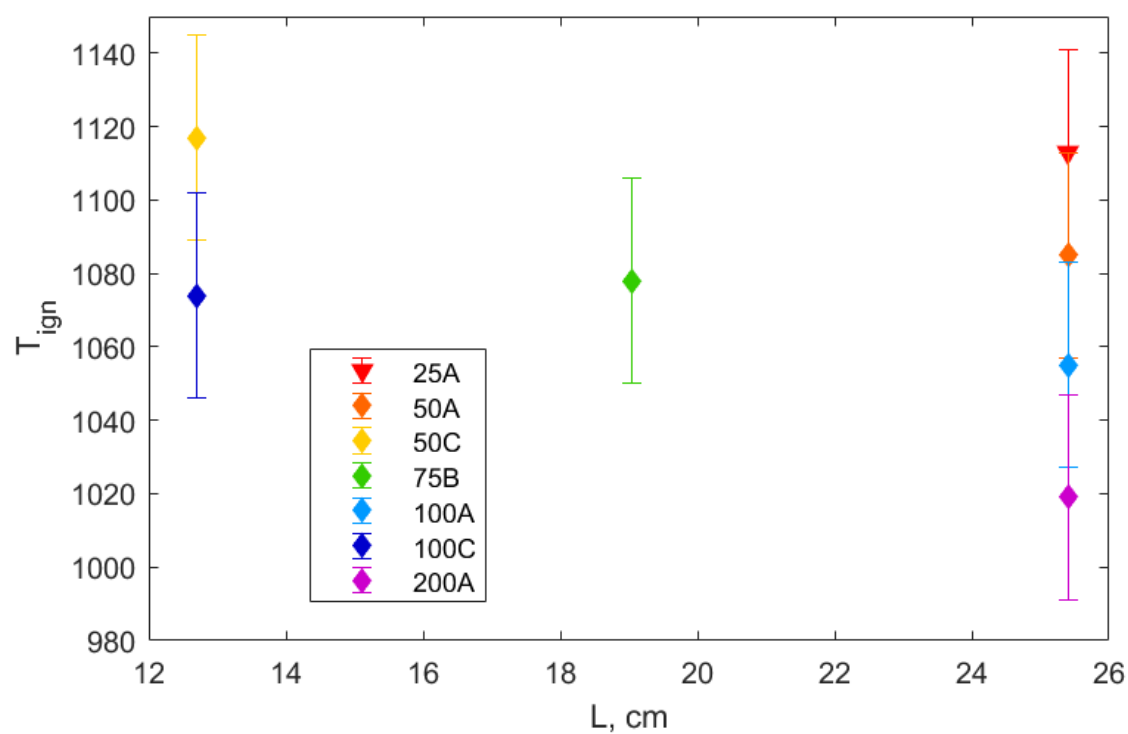

Figure 4.10: Plot of $\mathrm{T}_{i g n}$ vs length for all cylinders. Error bars represent measurement uncertainty for pyrometer.

In Fig. ??, we see that the ignition temperature gradually decreases as surface area increases, from $1113 \mathrm{~K}$ at $25 \mathrm{~cm}^{2}$ to $1019 \mathrm{~K}$ at $200 \mathrm{~cm}^{2}$. This amounts to a total decrease in the ignition temperature of approximately $100 \mathrm{~K}$ (less than 10\%) over a near order of magnitude change in the surface area. This is a very modest dependence of the ignition temperature on surface area. 


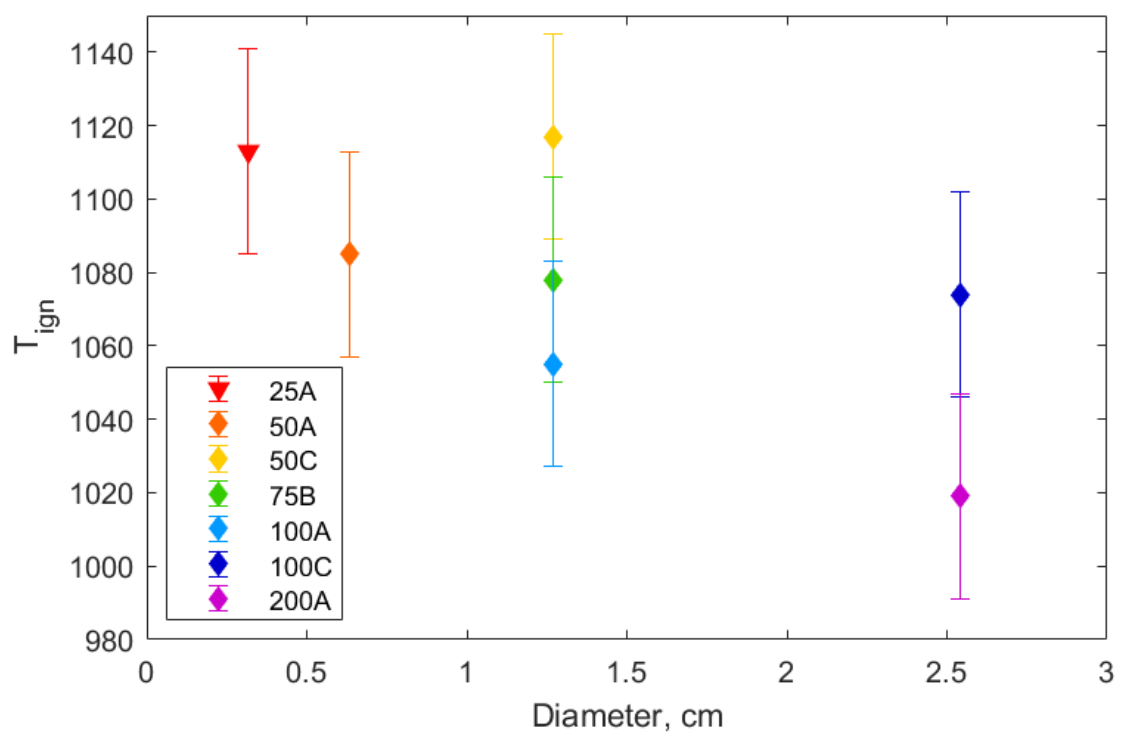

Figure 4.11: Plot of $\mathrm{T}_{i g n}$ vs diameter for all cylinders. Error bars represent measurement uncertainty for pyrometer.

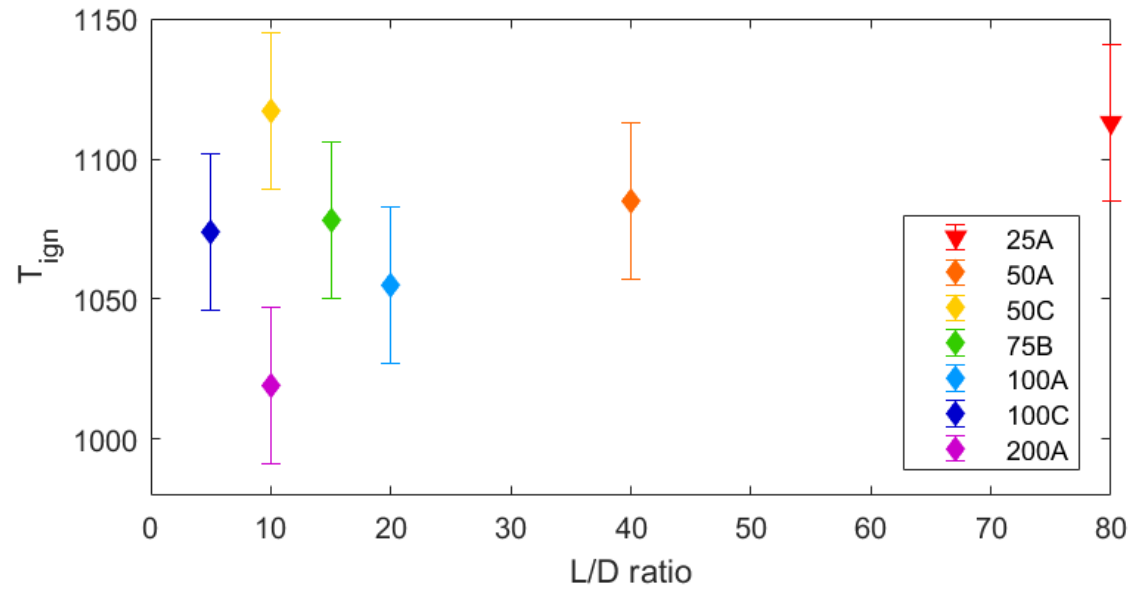

Figure 4.12: Plot of $\mathrm{T}_{i g n}$ vs L/D for all cylinders. Error bars represent measurement uncertainty for pyrometer.

The ignition temperature as a function of cylinder length is shown in Fig 4.10. At a fixed cylinder length of $25.4 \mathrm{~cm}$, the ignition temperature changes by $94 \mathrm{~K}$. The maximum change in ignition temperature observed with these cylinders was only slightly greater $(98 \mathrm{~K})$. Combined with the fact that Fig. ?? shows that a change in length at a fixed surface causes a change in the ignition temperature, this suggests that the ignition temperature has a very modest dependence on both surface area 
and length.

Finally, we consider the effect of cylinder diameter on the ignition temperature. The cylinders were designed to target certain surface area and length combinations, and as a result, the diameter would change to accommodate the choice of surface area and length. Additionally, the diameters had to be compatible with off-the-shelf tubing stock for construction. Due to the vertical orientation of the cylinders, we hypothesized that cylinder diameter would not directly be a significant parameter for ignition temperatures. Figure 4.11 plots the ignition temperatures against cylinder diameter. The maximum difference in ignition temperature is achieved by a change in the diameter from 1.27 to $2.54 \mathrm{~cm}$. However, it is challenging to determine from Fig. 4.11 alone how the diameter affects the ignition temperature.

We now consider how each parameter affects ignition temperatures while other values remain constant. First we examine the effect of changing length and diameter with constant surface area. Increasing the cylinder length leads to a decrease in ignition temperature; $\Delta T_{S A=50 \mathrm{~cm}^{2}}=-32 \mathrm{~K}$ and $\Delta T_{S A=100 \mathrm{~cm}^{2}}=-19 \mathrm{~K}$. When the surface area is constant, the ignition temperature rises with an increase of the diameter; $\Delta T_{S A=50 \mathrm{~cm}^{2}}=32 \mathrm{~K}$ with the diameter increasing from 0.64 to $1.27 \mathrm{~cm}$, and $\Delta T_{S A=100 \mathrm{~cm}^{2}}=19 \mathrm{~K}$ with the diameter increasing from 1.27 to $2.54 \mathrm{~cm}$. If we look at $\mathrm{D}=1.27 \mathrm{~cm}$, an increase in the surface area leads to a decrease in ignition temperature of $62 \mathrm{~K}$.

Next, the effects of changing surface area and diameter are examined while the length is fixed. At a fixed cylinder length, an increase in surface area is found to decrease the ignition temperature; $\Delta T_{L=12.7 \mathrm{~cm}}=-43 \mathrm{~K}$ and $\Delta T_{L=25.4 \mathrm{~cm}}=-30 \mathrm{~K}$ for when the cylinder surface area is increased from 50 to $100 \mathrm{~cm}^{2}$. When the length is constant, an increase in diameter decreases the ignition temperature; $\Delta T_{L=12.7 \mathrm{~cm}}=-43$ $\mathrm{K}$ with diameter increasing from 1.27 to $2.54 \mathrm{~cm}$, and $\Delta T_{L=25.4 \mathrm{~cm}}=-30 \mathrm{~K}$ with diameter increasing from $0.64 \mathrm{~cm}$ to $1.27 \mathrm{~cm}$.

Finally, we examine the effects of changing surface area and length while the diameter is constant. At a fixed diameter, an increase in surface area is found to decrease the ignition temperature; at $\mathrm{D}=1.27 \mathrm{~cm}$, increasing the surface area from 50 to $100 \mathrm{~cm}^{2}$ leads to a decrease in ignition temperature of $62 \mathrm{~K}$. For $\mathrm{D}=2.54$ 
$\mathrm{cm}$, increasing the surface area from 100 to $200 \mathrm{~cm}^{2}$ decreases the ignition temperature by $55 \mathrm{~K}$. When the length changes from 12.7 to $25.4 \mathrm{~cm}$ a fixed diameter, the igniton temperature decreases by $62 \mathrm{~K}$ for $\mathrm{D}=1.27 \mathrm{~cm}$ and $55 \mathrm{~K}$ for $\mathrm{D}=2.54 \mathrm{~cm}$.

We can also examine the effect of length to diameter ratio on the ignition threshold as plotted in Fig. 4.12. There does not appear to be any significant correlation of the ignition temperature with the L/D ratios investigated here.

The effect of changing surface area on ignition temperature proved to be quite modest. Doubling the surface area from 50 to $100 \mathrm{~cm}^{2}$ causes a decrease in ignition temperature of $43 \mathrm{~K}$ at $\mathrm{L}=12.7 \mathrm{~cm}$, and $30 \mathrm{~K}$ at $\mathrm{L}=25.4 \mathrm{~cm}$. In fact, the surface area is doubled three times at $\mathrm{L}=25.4 \mathrm{~cm}$, as shown in Fig. 4.13 . Each time the surface area doubles, the ignition temperature decreases by approximately $30 \mathrm{~K} ; 25$ to $50 \mathrm{~cm}^{2}$ results in a decrease of $28 \mathrm{~K}, 50$ to $100 \mathrm{~cm}^{2}$ results in a decrease of 30 $\mathrm{K}$, and 100 to $200 \mathrm{~cm}^{2}$ results in a decrease of $36 \mathrm{~K}$. From this we can conclude that doubling the surface area will only linearly decrease the ignition temperature. This quantitatively confirms that the ignition temperature of these cylinders is only weakly dependent on surface area.

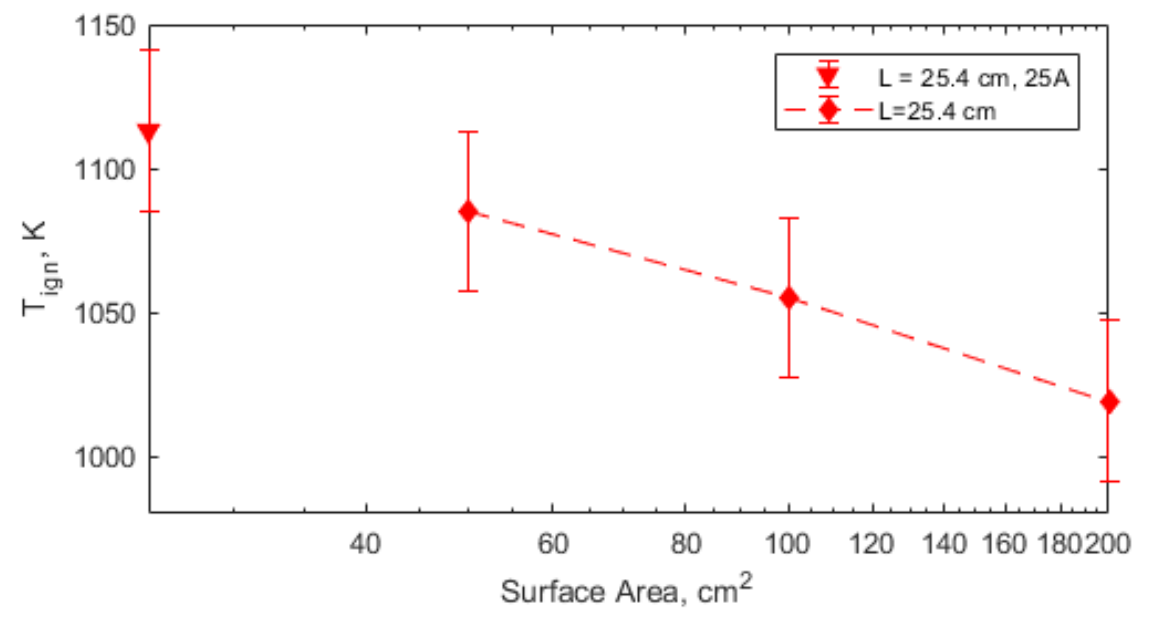

Figure 4.13: $\mathrm{T}_{i g n}$ vs surface area at a constant length of $\mathrm{L}=25.4 \mathrm{~cm}$, shown on a logarithmic x-axis. The surface area differs by a factor of two between cylinders, and the largest surface area is eight times the smallest.

Doubling the length from 12.7 to $25.4 \mathrm{~cm}$ causes a decrease in ignition temperature of $32 \mathrm{~K}$ at $50 \mathrm{~cm}^{2}$ and $19 \mathrm{~K}$ at $100 \mathrm{~cm}^{2}$. For the range of parameters investigated, 
doubling cylinder length causes a similar decrease in the ignition temperature as doubling the surface area of the cylinder; doubling surface area causes a mean decrease in ignition temperature of $34.3 \mathrm{~K}$, and doubling length causes a mean decrease in ignition temperature of $25.5 \mathrm{~K}$. We conclude that a doubling of either surface area or length of a vertical cylinder subject to external natural convection will produce approximately the same modest decrease in the ignition temperature. Ignition temperature therefore depends weakly on both.

Note that while an order of magnitude change in the surface area was achieved using the set of cylinders tested, it was only possible to double the length of the cylinders. A cylinder (25D) was designed to be an order of magnitude shorter than the longest cylinder tested, but the power supply at full capacity (600 A) was not able to heat the cylinder to likely ignition temperatures. The maximum temperature achieved when testing $25 \mathrm{D}$ was approximately $800 \mathrm{~K}$. A possible direction for future work could be using a more powerful power supply to enable ignition testing with 25D. This would present an order of magnitude change in the range of lengths in addition to the order of magnitude change in surface area already explored.

\subsection{Comparison with Literature Results}

The ignition results can be compared with results from the literature. First, the results are compared with those from Kuchta, Bartkowiak, and Zabetakis (1965) for internal natural convection of n-hexane mixtures with similar surface areas, as shown in Fig. 4.14. The mixture composition of the data points from Kuchta shown in Fig. 4.14 is not reported in the original text, but the range of mixture compositions investigated varied from 0.05 to 0.5 fuel-air weight ratio with little effect of composition. There is a significant change in ignition temperature between internal and external natural convection flows with similar surface areas. The ignition temperatures for external natural convection flows investigated here are approximately 200 to $500 \mathrm{~K}$ higher than the ignition temperatures for internal natural convection (Kuchta, Bartkowiak, and Zabetakis, 1965).

This can be explained by the difference in confinement between these two flow configurations. An internal natural convection flow is confined inside a heated vessel, and the reactive mixture continuously recirculates within that vessel, getting exposed to the heated surface many times. The significant confinement and recir- 


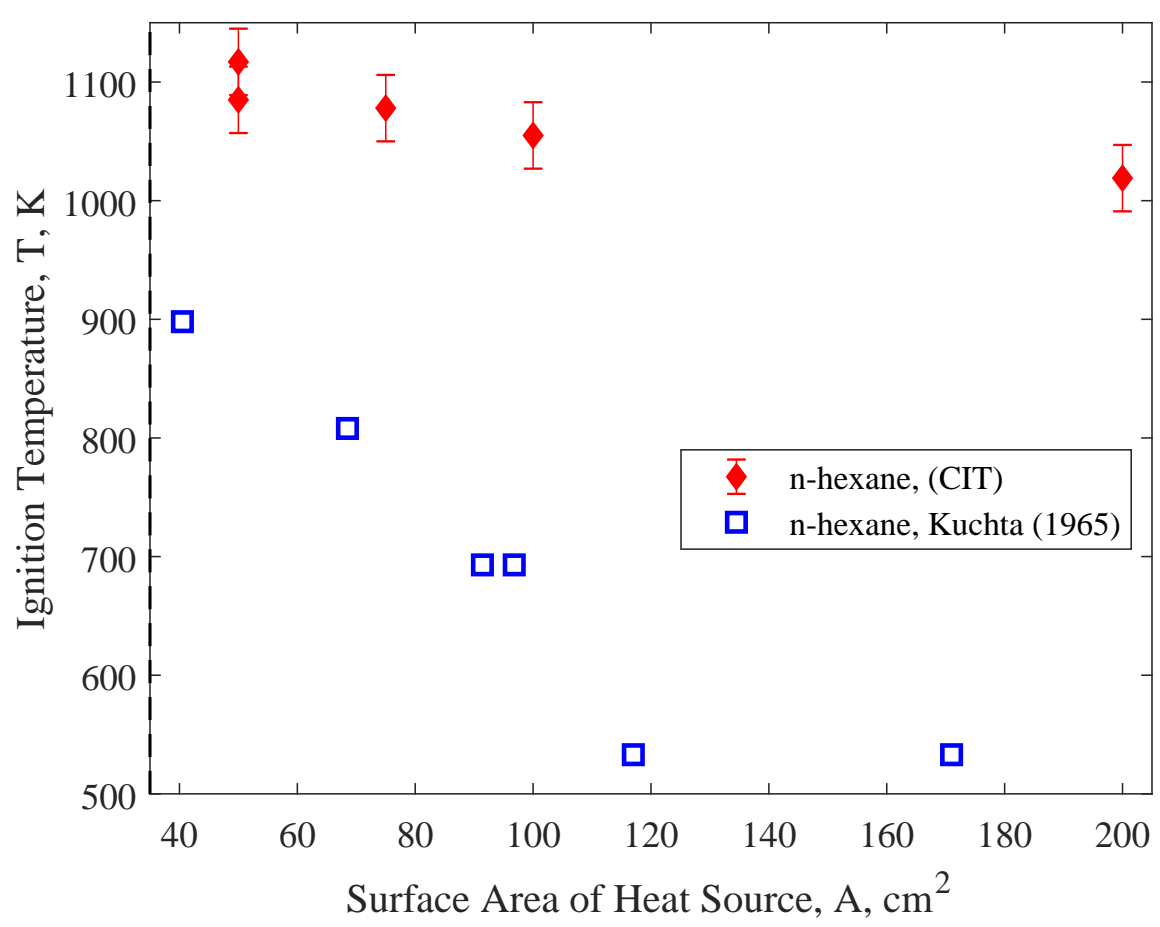

Figure 4.14: Ignition results from this work (red-filled diamonds) compared with those from Kuchta (1965) (empty blue squares) for a similar range of surface areas.

culation causes a long residence time. Long residence times generally lead to lower ignition temperatures; this idea is implicit in the concept of ignition being determined by a fixed value for the critical first Damkohler number (ratio of flow time to chemical reaction time) (Alkidas and Durbetaki, 1973). The characteristic flow time $(L / U)$ is interpreted as a residence time, and the reaction rate is assumed to have an Arrhenius dependence on temperature such that the characteristic reaction time is proportional to $\exp \left(E_{a} / R T\right)$. If these assumptions are applied to the first Damkohler number and ignition is assumed to be defined by a fixed value of $D a$, increasing the flow time will result in a lower ignition temperature. This concept holds until the model of a one-step Arrhenius reaction fails to be useful. Alkidas and Durbetaki (1973), Ono et al. (1976), and Law (1979) all present results that implicitly support this idea about the importance of residence time in determining ignition thresholds even though residence time is not explicitly discussed in those works. 
We must be cautious when discussing residence times in the context of boundary layer flows. While the concept of a residence time is useful when considering the Damkohler number, the residence time for flows in this work (and for boundary layer flows generally) is not well defined. The residence time is different for each streamline in the flow (see Section 6.1). This may be in part why residence time is not directly discussed by Alkidas and Durbetaki (1973), Ono et al. (1976), or Law (1979). Residence time is better defined in configurations like well-stirred reactors. Consequently, we do not present residence times for the flows considered in this work.

For external natural convection flows in unconfined geometries, the reactive mixture is only exposed to the hot surface once as it moves through the thermal layer. The gas does not recirculate. However, for flow within a natural convection boundary layer, the temperature-time history varies significantly depending on the streamline of interest, and time required to traverse the length of the cylinder varies significantly between streamlines. See Fig. 6.2 for example streamlines illustrating these points.

The temperature increases rapidly on streamlines originating close to the surface. These streamlines have very long residence times at high temperatures due to the no-slip condition at the surface. Streamlines originating in the outer part of the boundary layer take a much longer time to heat up, accelerate due to buoyancy, and then slow down as they approach the wall. The gas on these streamlines has much shorter residence times at lower temperatures. For these reasons, it is not possible to characterize the natural convection boundary layer flow with a single time scale or temperature.

As a brief aside, the first Damkohler number is a dimensionless parameter that represents the residence time of a fluid particle compared to the chemical timescale (Alkidas and Durbetaki, 1973); this has been used as an alternative to the Van't Hoff ignition criterion as a way to describe the criteria for ignition (Alkidas and Durbetaki, 1973; Law and Law, 1979; Song, Schmidt, and Aris, 1991). Law and Law (1979) found that with their choice of scaling a Damkohler number of unity was the appropriate ignition criterion, which they identified as a bifurcation point between two solutions: ignition and non-ignition. The Damkohler number is difficult to quantify from experimental data, but the idea of the Damkohler number as a 
way of identifying the ignition criterion will be revisited in Chapter 6 .

Because the hot cylinder in the present study is confined within a closed vessel, we estimated the potential for recirculation of gas through the thermal layer. We do this to justify the assumption made previously that there is no significant recirculation of the flows investigated in these experiments. The natural convection around the cylinder acts a pump with cold gas entering from the bottom, accelerating upward, and exiting the top of the thermal layer. The total mass flow rate induced by this process can be estimated from the model of natural convection presented in Section 3. Using the numerical results presented in Section 4.2, the wall parallel velocity and density are numerically integrated to estimate the mass flow rate. Wall perpendicular velocity is neglected as it is two orders of magnitude smaller than wall parallel velocity.

$$
\dot{m}=\int_{V} \rho u d V=\int_{0}^{L} \int_{0}^{2 \pi} \int_{R_{c}}^{R^{\prime}} \rho(r, z) u(r, z) r d r d \theta d z
$$

Where $L$ is the length of the cylinder, $R_{c}$ is the radius of the cylinder, and $R^{\prime}$ is the outer radius of the flow as determined by the point at which conditions are within one percent of ambient values. The shortest estimated recirculation time, estimated as $M / \dot{m}$ where $M$ is the total mass of gas in the vessel, is 460 seconds and corresponds to cylinder 200A. This is $50 \%$ more than the test time of the experiments. Therefore it is reasonable to assume there is no significant recirculation in these experiments, and any given portion of the reactive mixture is heated only once.

Comparisons can also be made with the wider body of literature. Starting with results on external natural convection, Melguizo-Gavilanes et al. (2016) report ignition temperatures of $n$-hexane with an equivalence ratio of 0.9 at $1275 \pm 45 \mathrm{~K}$ for a glowplug (approximately a vertical cylinder) with $1.5 \mathrm{~cm}^{2}$ surface area. Boeck et al. (2017) report ignition temperatures of $1270 \mathrm{~K}$ for stoichiometric n-hexane air mixtures for a vertical cylinder $3.14 \mathrm{~cm}^{2}$ in area, and Ono et al. (1976) report an ignition temperature of approximately $1250 \mathrm{~K}$ for stoichimetric propane-air and vertical plates $9 \mathrm{~cm}^{2}$ in area. Data from all cited studies are compared against results from this work in Fig. 4.15. Two conclusions are drawn when literature data are compared with the results found in this work. Ignition temperatures for external natural convection are consistently much higher than ignition temperatures for in- 


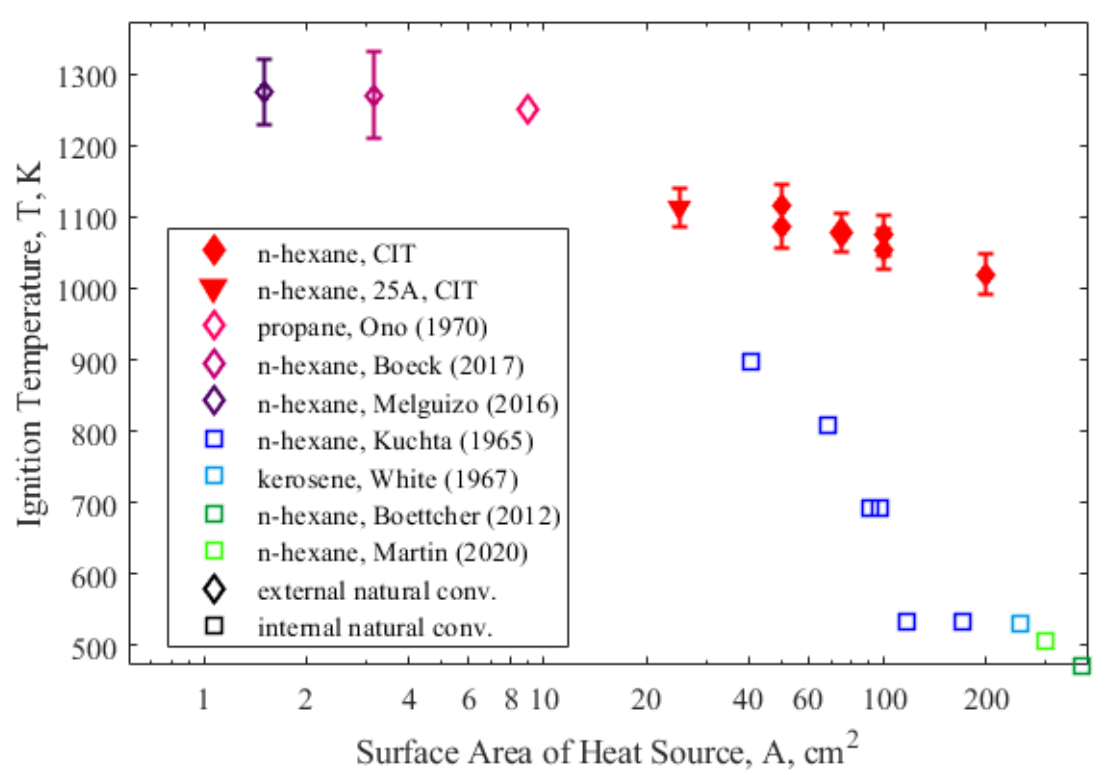

Figure 4.15: $\mathrm{T}_{i g n}$ versus surface area for all vertical cylinder data points (red-filled markers. Red-filled diamonds for ignition temperatures extracted from full logistic regression, red-filled triangles for ignition temperature from lowest ignition of 25A) compared with wider literature on thermal ignition. Empty diamonds represent literature on thermal ignition by external natural convection, empty squares represent literature on thermal ignition by internal natural convection.

ternal natural convection flows. Additionally, there is a significant difference in the trend of ignition temperature with surface area. Kuchta, Bartkowiak, and Zabetakis (1965) report ignition temperature dropping from $898 \mathrm{~K}$ at $40.5 \mathrm{~cm}^{2}$ to $533 \mathrm{~K}$ at $171 \mathrm{~cm}^{2}$ which amounts to a $365 \mathrm{~K}$ decrease in ignition temperature over $130 \mathrm{~cm}^{2}$ of area change. For a similar area change, the ignition temperature of the cylinders drops from $1117 \mathrm{~K}$ at $50 \mathrm{~cm}^{2}$ to $1019 \mathrm{~K}$ at $200 \mathrm{~cm}^{2}$. This is a difference of $98 \mathrm{~K}$ over a $150 \mathrm{~cm}^{2}$ area change. The precipitous drop in ignition temperature at around 75 $\mathrm{cm}^{2}$ that Kuchta, Bartkowiak, and Zabetakis (1965) observed does not take place. Instead, there is only a slight and gradual decrease in the ignition temperature with increasing surface area for the external flows.

Literature on thermal ignition from internal natural convection gives additional points of comparison for the results of Kuchta, Bartkowiak, and Zabetakis (1965), as shown in Fig. 4.15. White (1967) reports ignition temperatures of $530 \mathrm{~K}$ for a vessel approximately $250 \mathrm{~cm}^{2}$ in area with an air-fuel ratio from 0.85 to 40 for kerosene-air mixtures. Boettcher (2012) reports ignition temperatures of $473 \mathrm{~K}$ for 
a $380 \mathrm{~cm}^{2}$ vessel with $\mathrm{n}$-hexane fuel at an equivalence ratio of 1.2. Martin and Shepherd (2020) followed the autoignition testing procedure prescribed by ASTM E659 (ASTM International, 2015) and found an ignition temperature of $508 \pm 3 \mathrm{~K}$ for hexane in a $300 \mathrm{~cm}^{2}$ vessel. These results further demonstrate the large difference in ignition temperatures between internal and external natural convection flows. This indicates that a change from internal to external flow has much more impact on ignition temperature than a change in surface area for the range investigated here.

\subsection{Visualization Studies}

In this section, we will discuss the different techniques used during ignition testing to visualize the ignition event. These include interferometry, schlieren imaging, and excited $\mathrm{OH}$ chemiluminescence. Details about these techniques are presented in Section 2.6. Interferometry allows for quantitative analysis of gas temperature prior to the ignition event, and qualitative analysis of the ignition event. Schlieren imaging provides a very intuitive and easy to understand qualitative visualization of the ignition event. $\mathrm{OH}^{*}$ chemiluminescence provides the ability to directly observe the formation of the ignition kernel and subsequent propagation of the flame front.

\section{Interferometry Visualization of Ignition}

In this section, we will examine the results of using interferometry to extract quantitative temperature fields from heated cylinder experiments. There are challenges to working with reactive mixtures like the hexane mixture used for ignition testing, namely, the mixture composition can change as heat from the surface allows for some decomposition of the fuel. The change in mixture composition causes a change in index of refraction, and if the change in index of refraction is significant, it can cause inaccurate temperature measurements. For this reason, we will examine interferometry results in pure nitrogen first to eliminate the complications that arise with reactive mixtures. This is used to establish the accuracy of the interferometer. Then the results from hexane experiments will be examined, and the effect of changing mixture composition will be discussed. Finally, the results of experiments performed in a diluted hydrogen air mixture will be discussed as a point of comparison since the mixture is reactive but the composition changes less prior to ignition when compared with the hexane mixture.

The raw interferograms are collected and processed according to the procedure laid out in Section 2.6. In this section, results from post-processed interferometry 
videos of $30 \mathrm{~s}$ or longer are presented unless otherwise noted, either leading up to an ignition event or at the end of a test with no ignition. This allows for the time-averaging of the temperature fields to smooth out any temporary fluctuations in the boundary layer structure, and to smooth out any artifacts remaining from post-processing (Coronel, 2016). Interferometry results were processed for tests in pure nitrogen mixtures, stoichiometric hexane-air mixtures, and a limited number of diluted hydrogen-air mixtures. A few exemplary results will be shown directly in this section, and a summary of all processed results will be discussed. The results of every test that was processed can be found in Appendices $\mathrm{B}, \mathrm{C}$, and $\mathrm{D}$ for pure nitrogen, stoichiometric hexane-air, and diluted hydrogen-air mixtures, respectively.

The results that are presented in the following sections consist of three main components. First, there is a side-by-side comparison of the left and right half of the temperature field surrounding the cylinder, which is constructed from a time average over the length of the recording (30-100 s, typically). Second, the thermal boundary layer profile for each side is presented by taking a horizontal slice through the middle of the temperature field. These profiles are compared with the predicted boundary layer profile for a wall with the surface temperature measured by the pyrometer, and the interferometer surface temperature is extrapolated from the mean of the two experimental boundary layer profiles. The methodology for extrapolating surface temperature from interferometer temperature fields is explained below. Finally, the percent difference of the two experimental boundary layer profiles is quantified, as is the percent difference between the mean experimental boundary layer profile and the predicted boundary layer profile from similarity solutions. Good agreement is classified if the two sides of the interferometer are symmetrical (typically less than $10 \%$ difference between left and right sides) and the experimental profile and numerically predicted profile are also within $10 \%$ difference.

\section{Extrapolating Surface Temperature}

One goal of using interferometry is to compare the pyrometer surface temperature measurement to the surface temperature extrapolated from the interferometer temperature fields. This provides a way to corroborate the surface temperature measurement. However, as mentioned briefly in Section 2.6, a narrow region of the temperature fields very near the surface (within a milimeter) is prone to errors due to the nature of the the Nestor-Olson algorithm for the inverse Abel transform. 
This phenomenon will be discussed at greater length in the following sections, but it means extracting the surface temperature from interferometry is more complicated than simply reporting the temperature next to the surface due to this error-prone region.

In order to extrapolate the surface temperature from interferometry, we curve-fit to the middle portion of the thermal boundary layer profile. This usually means sampling the portion of the thermal boundary layer 0.002 to $0.006 \mathrm{~m}$ from the surface for a linear fit; this range is chosen because $0.002 \mathrm{~m}$ is typically outside the narrow region of larger error, and $0.006 \mathrm{~m}$ is still within the relatively linear portion of the boundary layer profile before it starts flattening as the temperature approaches ambient conditions. When tested on the similarity solution predictions of the boundary layer profile, a linear fit on this portion of the boundary layer produced an extrapolated surface temperature within approximately $2 \%$ of the surface temperature used in the similarity solution.

\section{Interferometry Results: Nitrogen}

We begin investigating the interferometer results by looking at the tests done in a pure nitrogen environment. This is the simplest gas mixture that we will investigate. In order to get accurate temperature fields from the interferometer, the index of refraction and Gladstone-Dale constant must be accurately known. Working with an inert gas like nitrogen ensures that the gas composition is constant, and as such, these two properties remain unaltered throughout the test time. For these reasons, an interferometer result in nitrogen can be thought of as a validation step before working with reactive mixtures, where compositions can change and result in significant uncertainties on the index of refraction and Gladstone-Dale constant.

Figures B.28 to B.30 show the interferometry results from shot 66 , a test with cylinder $100 \mathrm{C}$ run in pure nitrogen with a surface temperature of $1000 \mathrm{~K}$. All interferometry results in nitrogen are presented in Appendix B. Also, a summary of the nitrogen results is presented in Table 4.3 .

Figure 4.16 shows the temperature fields surrounding the cylinder from shot 66 , reconstructed from the left and right half of the interferometry images. These tem- 

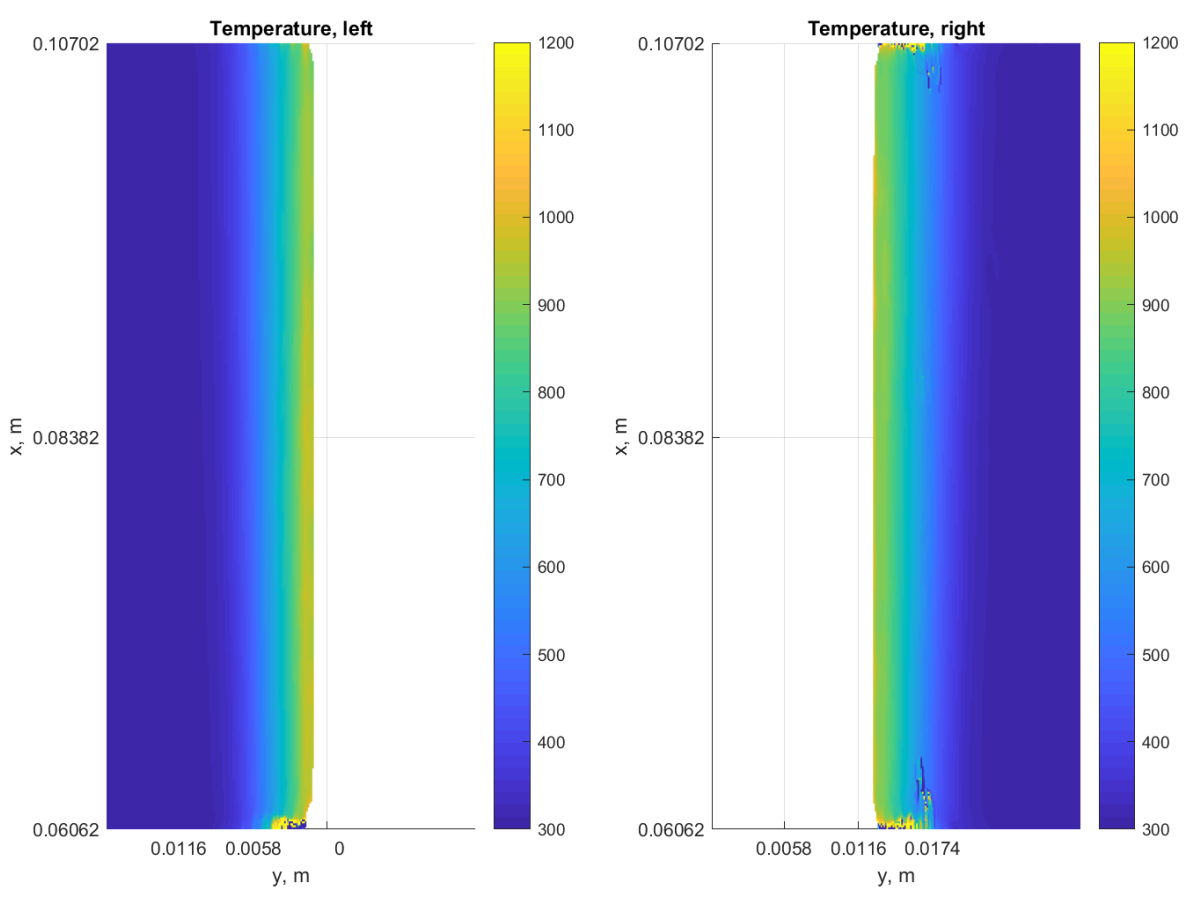

Figure 4.16: Shot 66. Temperature fields from interferometry for nitrogen test. Surface temperature measured by pyrometer $=1000 \mathrm{~K}$.

peratures fields are an average over the last $100 \mathrm{~s}$ of the $300 \mathrm{~s}$ test time for this nitrogen test. The two sides qualitatively look very similar; this is a good indication of symmetry. We expect symmetry in the two halves as the setup was designed to be axisymmetric. The thermal boundary layer thickness looks similar between the two sides, and the temperature near the surface of the cylinder (white space) approaches $1000 \mathrm{~K}$. We want to examine the structure of the boundary layer in a quantitative manner, as shown in Fig. B.29.

Figure 4.17 shows the thermal boundary layer profile from both sides (solid black and blue lines). This is done by taking a horizontal slice through the vertical center of the temperature field. The two experimental boundary profiles are compared to each other, and also to the numerical boundary layer profile predicted from similarity solutions (magenta dashed line), based on the wall temperatures measured via pyrometer. Finally, a linear fit (red dot-dashed line) is applied to the mean of the two experimental profiles to allow for surface temperature extrapolation from interferometry. Shot 66 exhibits excellent agreement among all of these measurements; except for the error-prone region within about one milimeter of the 


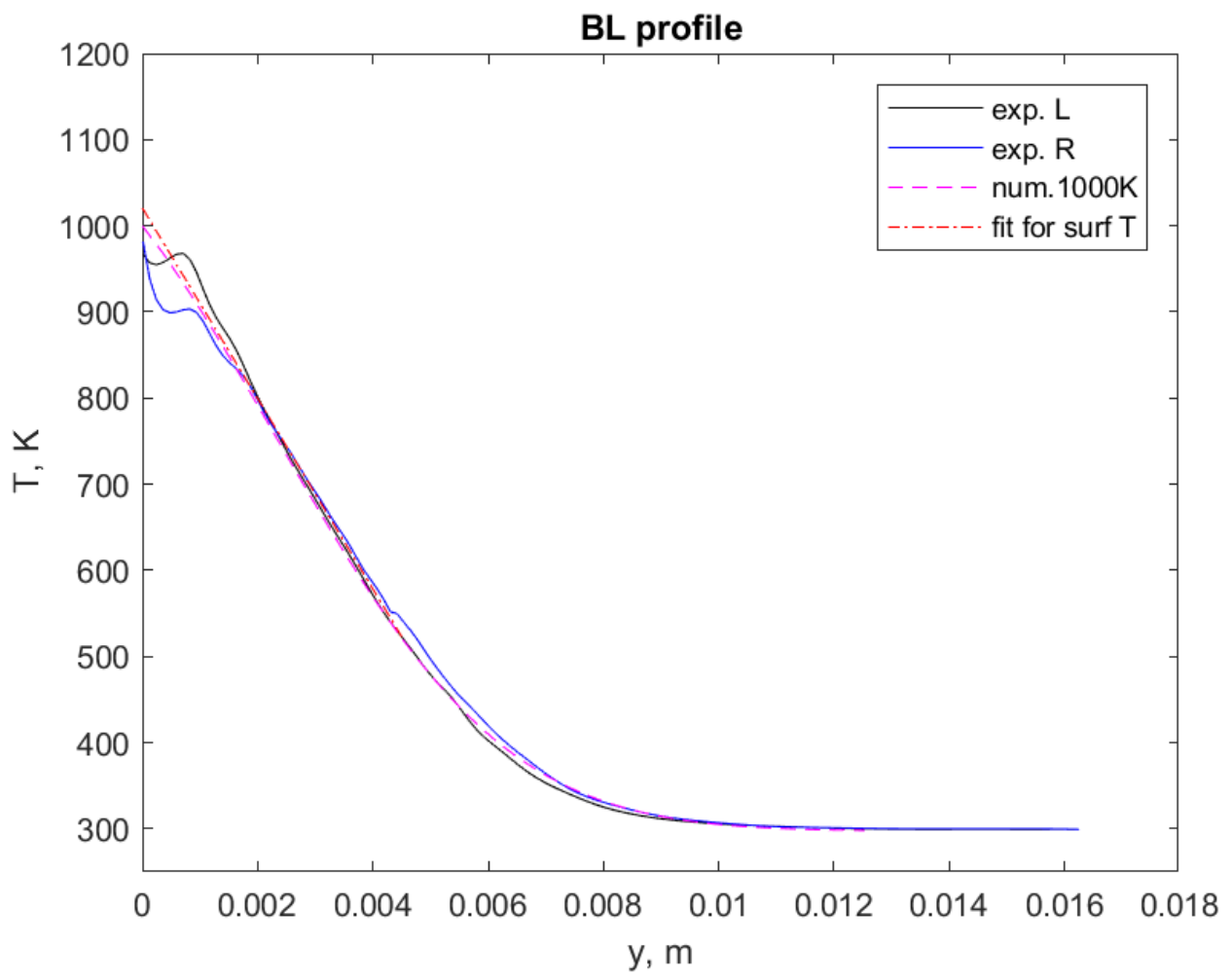

Figure 4.17: Shot 66. Thermal boundary layer profiles for left (black line) and right (blue line) sides of experimental results compared with prediction of boundary layer profile (dashed magenta line) based on similarity solution for pyrometer surface temperature. Linear fit to averaged experimental profile (red dot-dashed line) used to extrapolate surface temperature from interferometer post processing. Extrapolated interferometer surface temperature $=1020.7 \mathrm{~K}$.

cylinder surface, thermal boundary layer profiles from the left and right sides of the cylinder look nearly identical. Furthermore, the numerical boundary layer profile is nearly identical to the experimental one in shape and temperature distribution. Finally, the surface temperature extrapolated from interferometry is $1020 \mathrm{~K}$, about $2 \%$ difference from pyrometer measurement of $1000 \mathrm{~K}$.

Figure 4.18 further illustrates the agreement in the results of shot 66 . The left and right experimental boundary layer profiles are within 5\% difference of each other (except very near the surface) for shot 66, as shown by the solid blue line. This is what we will classify as a "very symmetrical" result. A 5-10\% difference between the two sides would constitute a "mostly symmetrical" result, and more than $10 \%$ difference would classify as an "asymmetrical" result. Symmetry between the two experimental profiles is a good result because it is an indication of axisymmetry in the experiment. Additionally, the red dashed line in Fig. 4.18 quantifies the 


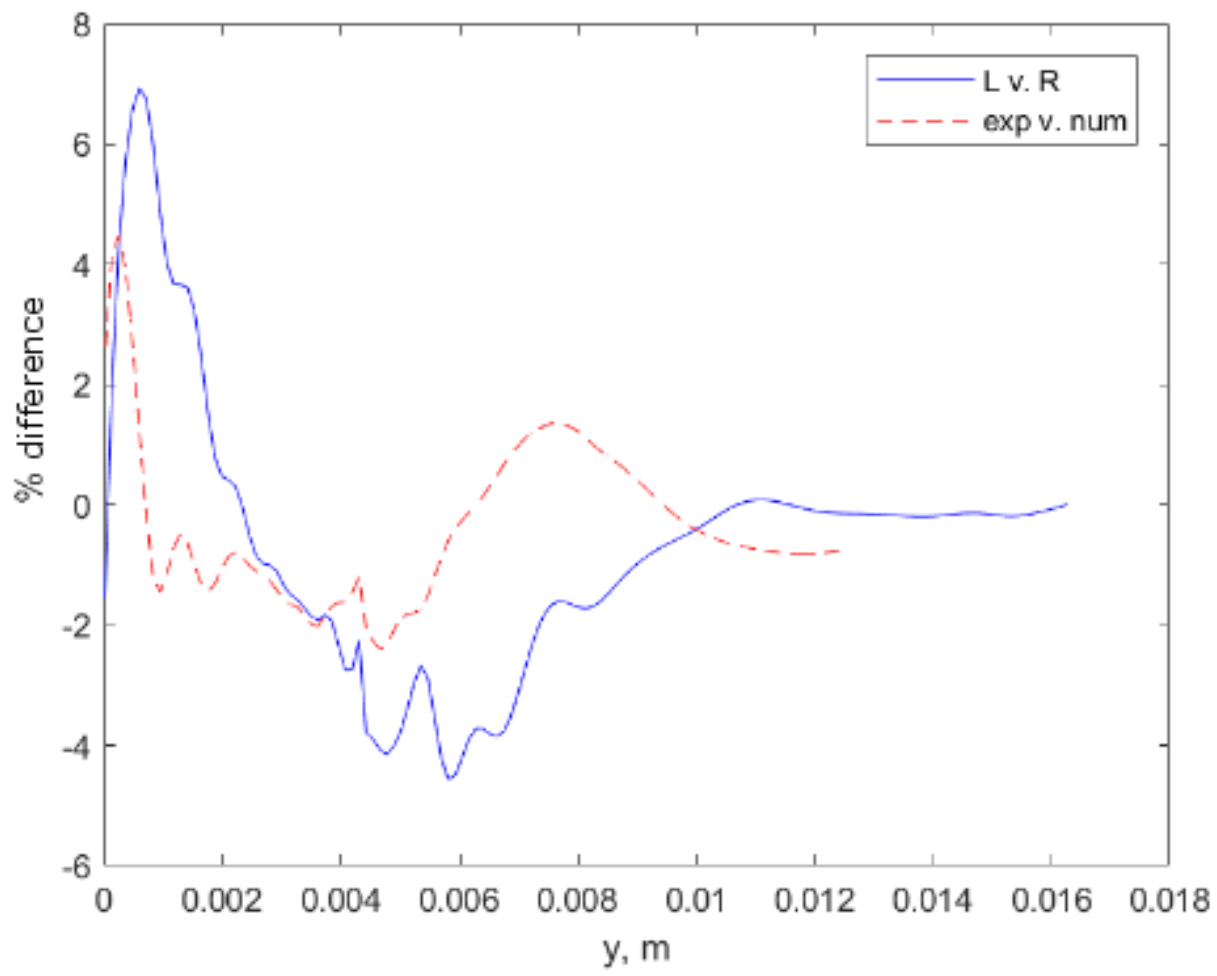

Figure 4.18: Shot 66. Percent difference in thermal boundary layer between both sides of experimental boundary layer profiles (red line) and percent difference between predicted boundary layer profile from similarity solution and averaged experimental boundary layer profile (blue dashed line).

percent difference between the mean of the experimental boundary layer profiles and the numerical boundary layer profile. There is excellent agreement between the experimental and numerical results here. Furthermore, the surface temperature extrapolated from interferometry is $2.1 \%$ higher than the surface temperature measured by the pyrometer. All of these results indicate excellent agreement between experimental results, numerical predictions, and the two main diagnostic tools.

The results of shot 66 are an example of the behavior observed in most of the nitrogen results; the percent difference between the surface temperature from pyrometry and interferometry is within 5\% for all cylinders (see Table 4.3) except for the outlier of 100A. Furthermore, there is excellent symmetry in the nitrogen results; all but one falls within the "very symmetrical" classification, and the one that is not "very symmetrical" is classified as "mostly symmetrical". These results indicate that the temperature fields extracted from interferometry are very accurate and reliable when 
using nitrogen gas.

\section{Interferometry Results: n-Hexane}

We continue examining the results of interferometry with results from testing done with the stoichiometric hexane-air mixture described in Section 4.1 . Briefly, processing this hexane mixture is more difficult than nitrogen not only because it is a more complicated mixture of different gases, but also because hexane is reactive and prone to decomposition before ignition. This will be discussed at length later in this section and in Chapter 6. The difficulty with processing hexane mixtures is documented in the results of this section. Here we will examine the results from two shots that exemplify two distinctly different behaviors observed when working with hexane: a "successful" processing and an "unsuccessful" processing of interferometry in a hexane mixture. A summary of the interferometry results is presented in Table 4.3. The results of all processed interferometer videos from hexane experiments are documented in Appendix C.

First we look at an example of a successful processing of the n-hexane mixture with the results of shot 31 as shown in Figs. 4.19 to 4.21. Shot 31 was performed using cylinder $75 \mathrm{~B}$ in a stoichiometric n-hexane/air mixture, with a surface temperature of $1104 \mathrm{~K}$.

Figure 4.19 shows the processed temperature fields on the left and right sides of the cylinder. A visual inspection shows the two sides look relatively similar. The left side looks like it might have a slightly higher temperature very near the surface, but the overall thickness of the thermal boundary layer looks relatively similar between the two sides. The temperature near the surface looks to be slightly higher than $1000 \mathrm{~K}$ and is examined in more detail in Fig. 4.20 .

Figure 4.20 shows the experimental thermal boundary layer profiles for shot 31 . The left and right sides look relatively similar (except within $1 \mathrm{~mm}$ of the surface) although not quite as similar as the experimental boundary layer profiles from Fig. 4.17(shot 66, Nitrogen). The experimental boundary layer profiles are cooler than the profile predicted by the similarity solution for a wall temperature of $1100 \mathrm{~K}$. The surface temperature from interferometry is extrapolated via linear fit and found to 

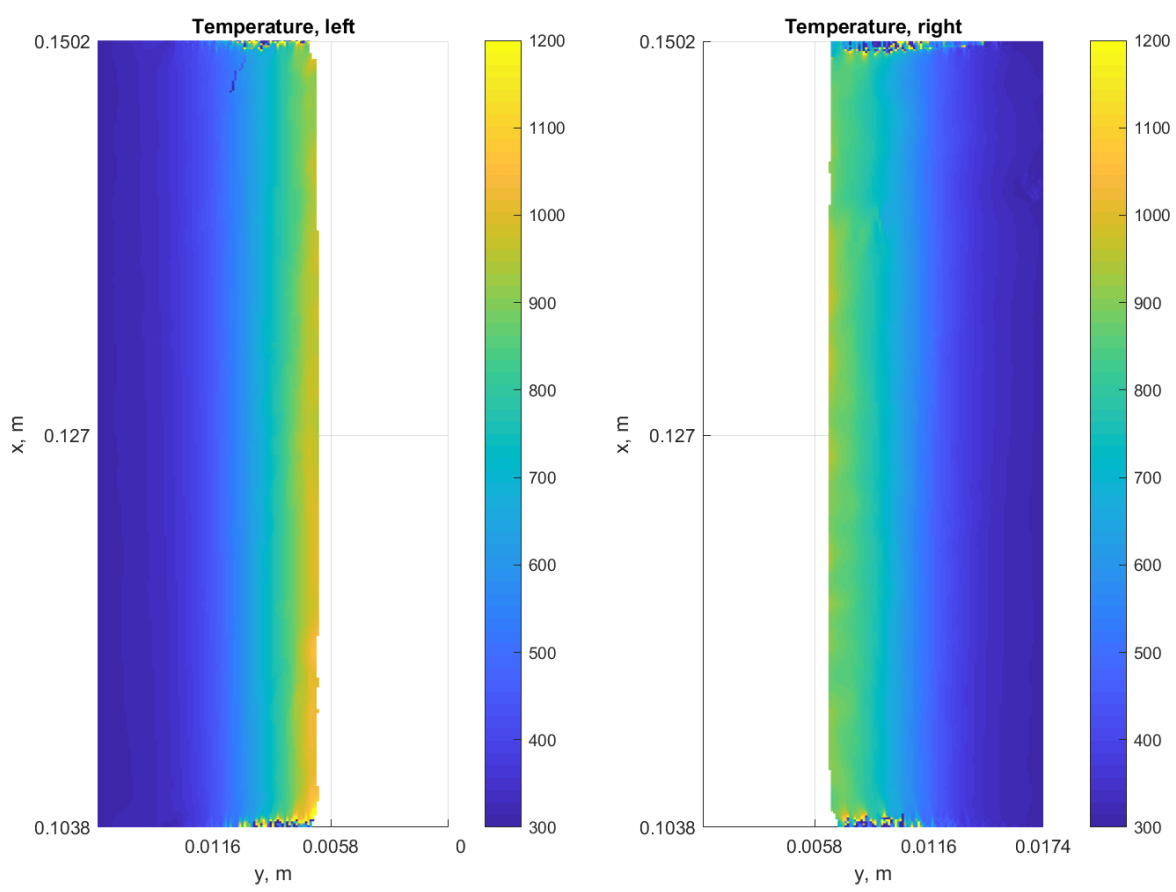

Figure 4.19: Shot 31. Temperature fields from interferometry for hexane test. Surface temperature measured by pyrometer $=1104 \mathrm{~K}$.

be $1007 \mathrm{~K}$.

Comparing these result with previous interferometry results in nitrogen indicates that the hexane mixture is the likely cause of increase in asymmetry. Results in nitrogen showed good symmetry and therefore demonstrated a successful axisymmetric setup of the cylinders. As will be discussed shortly, hexane is prone to decomposition prior to the ignition event. The decomposition of hexane can alter the index of refraction of the mixture and therefore the temperature measured by the interferometer. The decomposition may not occur evenly over the cylinder surface, but instead occur with some intermittent unsteady behavior (see Section 4.6 for other indications of unsteady behavior in the thermal layer). The results reported in Figs. 4.19 through 4.21 come from a $30 \mathrm{~s}$ time average of interferometry results in hexane; since the periodicity of disturbances in these flows is not well characterized (see Section 4.6), a longer time average may be required to eliminate asymmetry due to decomposition of hexane fuel. This is a possible direction for future investigations to explore more fully. 


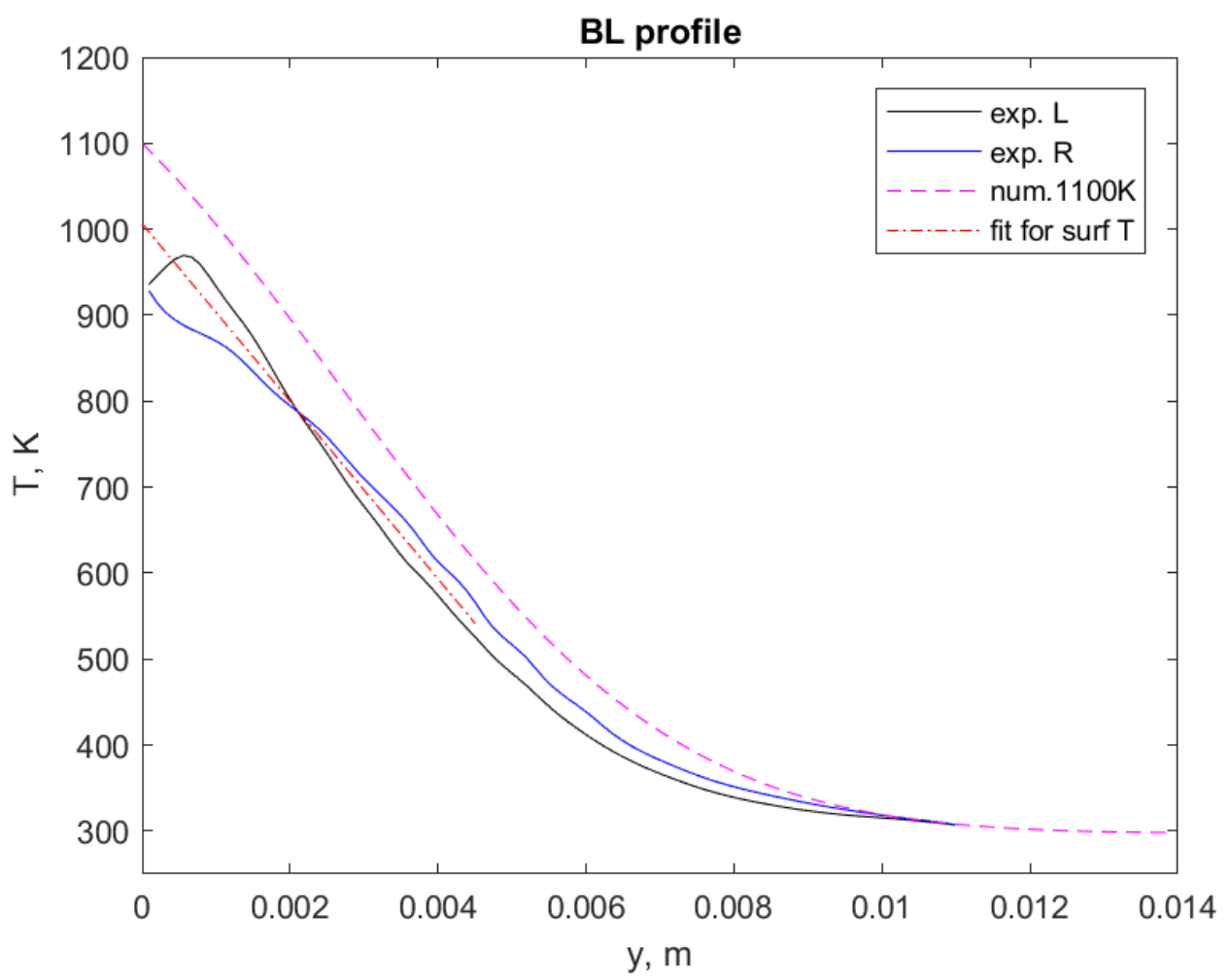

Figure 4.20: Shot 31. Thermal boundary layer profiles for left (black line) and right (blue line) sides of experimental results compared with prediction of boundary layer profile (dashed magenta line) based on similarity solution for pyrometer surface temperature. Linear fit to averaged experimental profile (red dot-dashed line) used to extrapolate surface temperature from interferometer post processing. Extrapolated interferometer surface temperature $=1006.5 \mathrm{~K}$.

The differences between the experimental profiles as well as the difference compared with the numerical boundary layer prediction are shown in Fig. 4.21. The percent difference between the two experimental boundary layer profiles is less than $10 \%$, so this would classify as a "mostly symmetrical" result. The percent difference between the mean experimental thermal boundary layer profile and the predicted numerical profile is higher, a little over $10 \%$. The experimental thermal profile is cooler than expected given the surface temperature. The surface temperature extrapolated from interferometry for shot 31 is $1007 \mathrm{~K}$, which is $8.8 \%$ less than the surface temperature measure by pyrometery.

In summary, shot 31 represents one of the more successful post-processed interferometry results for a stoichiometric hexane-air mixture. The percent difference between the pyrometer and interferometer surface temperatures is less than $10 \%$, 


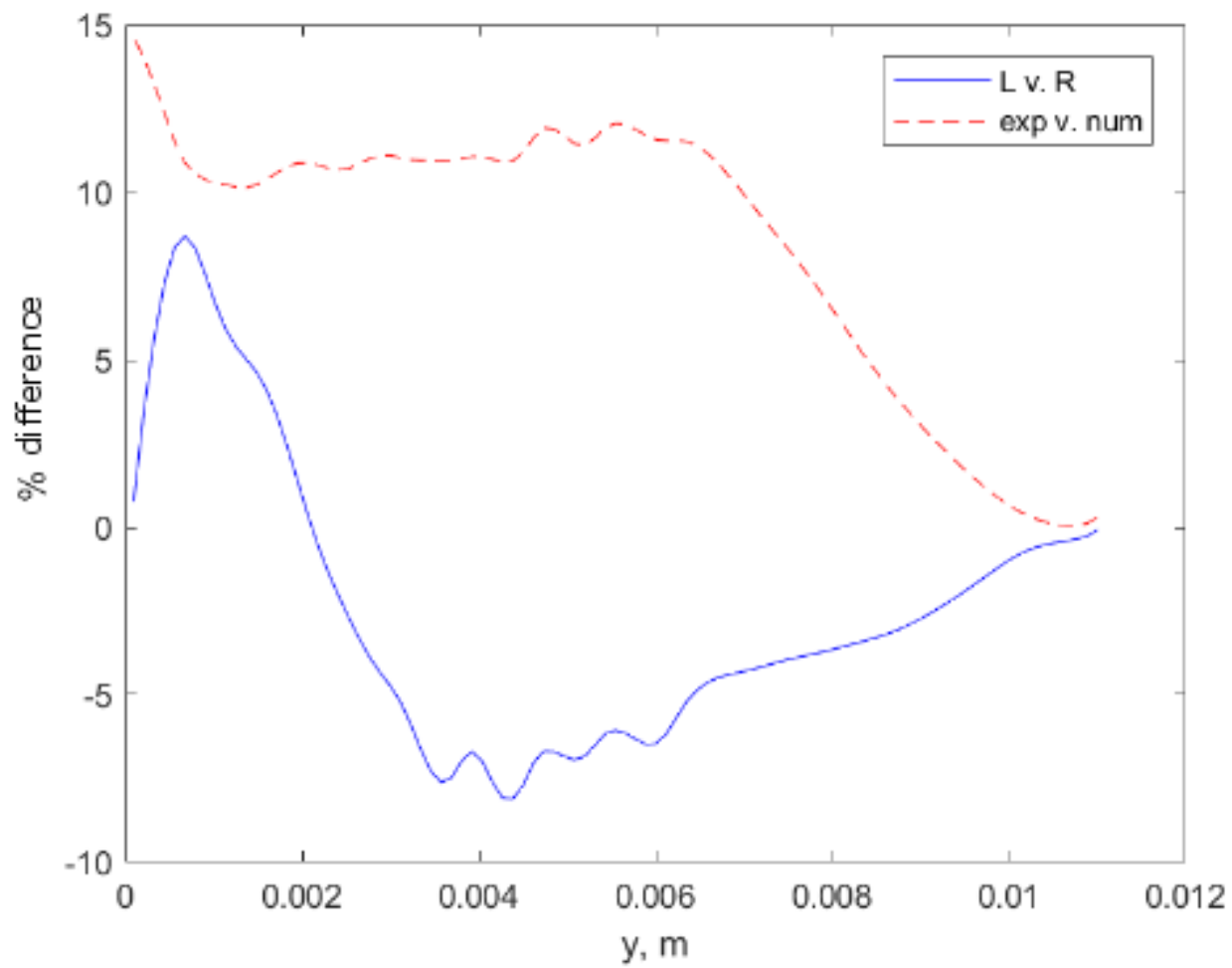

Figure 4.21: Shot 31: Percent difference in thermal boundary layer between both sides of experimental boundary layer profiles (red line) and percent difference between predicted boundary layer profile from similarity solution and averaged experimental boundary layer profile (blue dashed line).

but it is worth noting that the results for a "successful" hexane processing are not in as good of agreement with pyrometry and similarity results as those from a test in nitrogen. This becomes more evident as we document the results of an "unsuccessful" processing of a hexane test. Details about the difficulties of processing a hexane mixture are given in a following discussion.

As an example of "unsuccessful" hexane processing, we examine the results of shot 164 as shown in Figs. 4.19 to 4.21 . Shot 164 was performed using cylinder 100C in a stoichiometric n-hexane/air mixture, with a surface temperature of $1080 \mathrm{~K}$. Figures 4.22 to 4.24 document the interferometry results of this test.

Figure 4.22 shows the temperature fields found from shot 164. Examination of the two halves of the temperature field shows that the thermal boundary layers look similar. However, the temperature near the surface seems to be a little over $900 \mathrm{~K}$, 

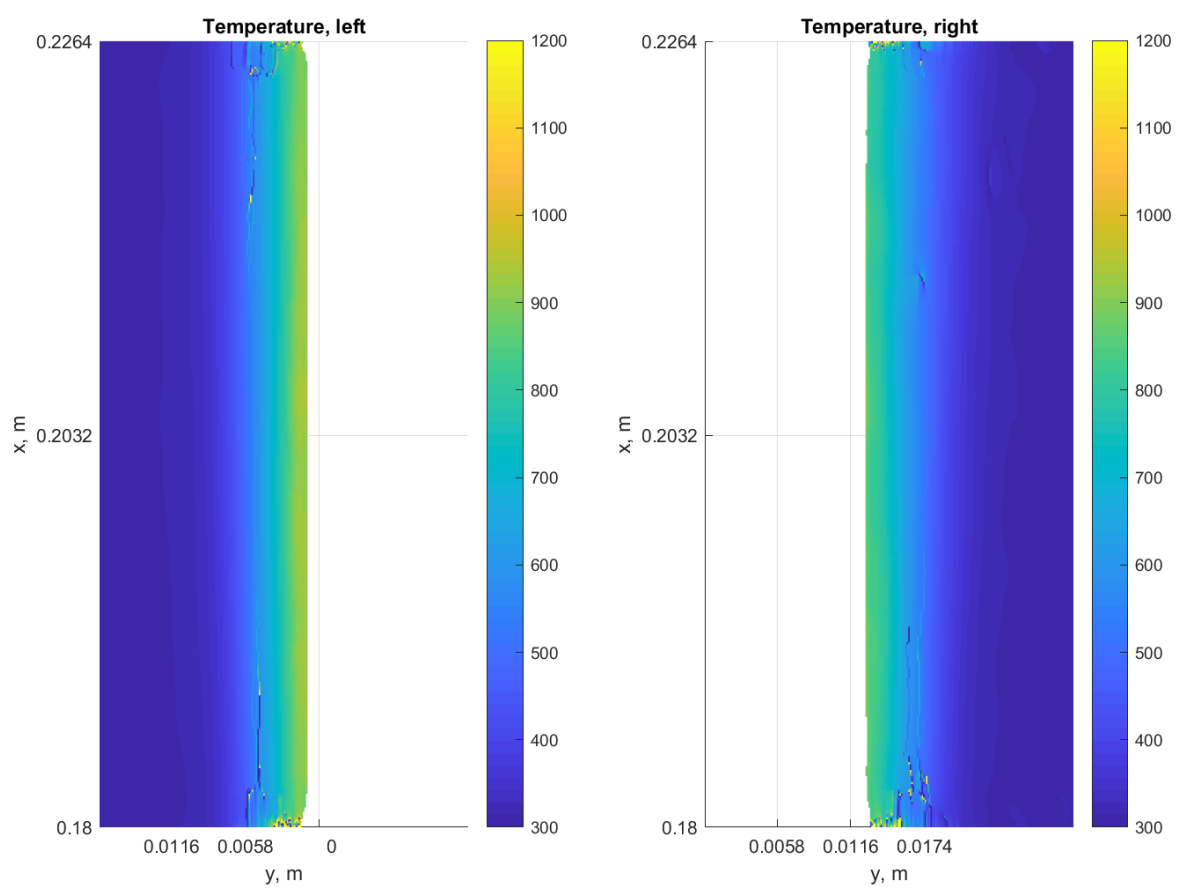

Figure 4.22: Shot 164. Temperature fields from interferometry for hexane test. Surface temperature measured by pyrometer $=1080 \mathrm{~K}$.

which is significantly lower than the surface temperature of $1080 \mathrm{~K}$ measured by pyrometer. We can examine these results more quantitatively in Fig. 4.23.

In Fig. 4.23, the two experimental thermal boundary layer profiles are shown as solid black and blue lines for the left and right sides, respectively. The two sides are relatively similar to one another and indicate good symmetry in the experiment. However, when compared the the predicted boundary layer profile (surface temperature of $1100 \mathrm{~K}$ ), the experimental results indicate a significantly lower temperature measured by interferometry as compared to the pyrometer measurement. The extrapolated surface temperature from interferometry is $940.2 \mathrm{~K}$.

Examination of Fig. 4.24 provides insight on the differences between experimental and numerical results. First, the solid blue line shows the percent difference between the two sides of the experimental boundary layer profile: outside of the narrow region near the surface, there is $10 \%$ difference or less between the left and right sides. This classifies as a "mostly symmetrical" result. The percent difference between the mean experimental boundary layer profile and the predicted numerical 


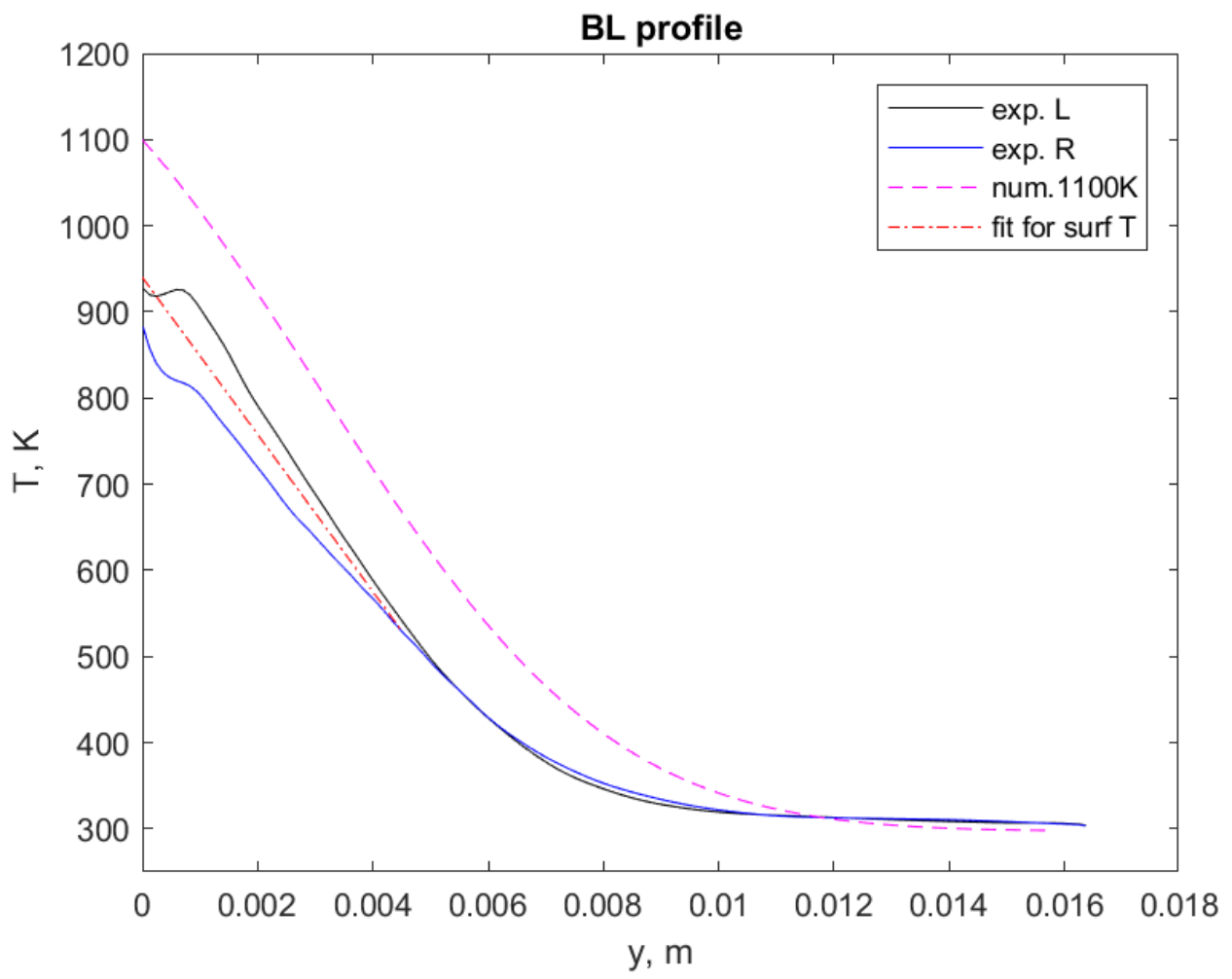

Figure 4.23: Shot 164. Thermal boundary layer profiles for left (black line) and right (blue line) sides of experimental results compared with prediction of boundary layer profile (dashed magenta line) based on similarity solution for pyrometer surface temperature. Linear fit to averaged experimental profile (red dot-dashed line) used to extrapolate surface temperature from interferometer post processing. Extrapolated interferometer surface temperature $=940.2 \mathrm{~K}$.

profile is shown as the dashed red line. The percent difference throughout most of the boundary layer is quite high, as much as $20 \%$, showing a significant challenge in processing the interferometry results. Further evidence of this processing difficulty comes from the extrapolated surface temperature, which is $13 \%$ lower than the temperature measured via pyrometery.

A summary of the results of processing interferometry with n-hexane air mixtures is presented in Table 4.3. Briefly, the interferometry results from nitrogen are accurate within about $5 \%$ for most cylinders as indicated by both agreement among the experimental results as well as agreement with numerical predictions based on similarity solutions. The flat plate approximation used in the similarity solution is found to be appropriate for most of the cylinders as seen by good agreement between the experimental and numerical temperature profiles in nitrogen (ie Fig. B.14, more examples 


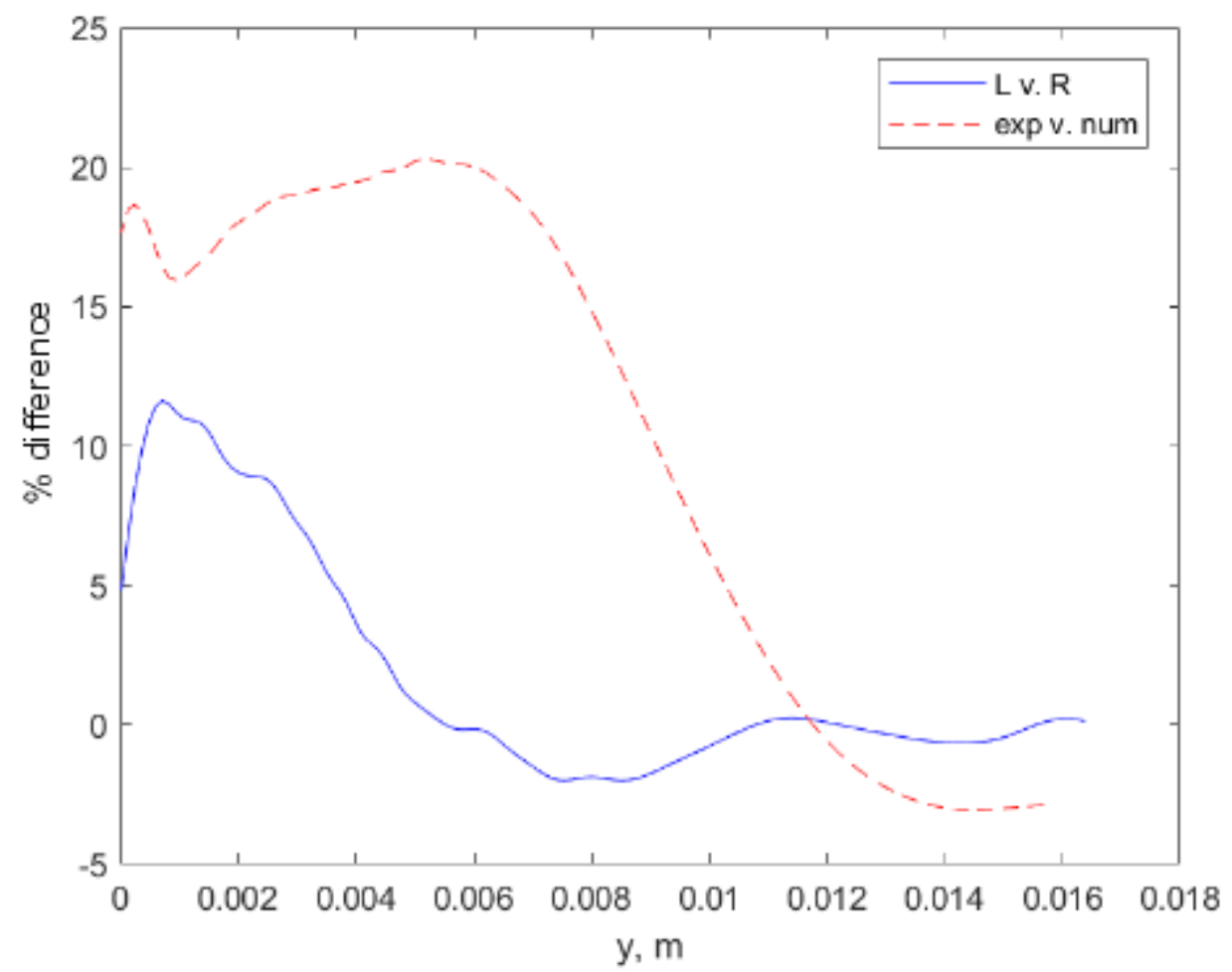

Figure 4.24: Shot 164. Percent difference in thermal boundary layer between both sides of experimental boundary layer profiles (red line) and percent difference between predicted boundary layer profile from similarity solution and averaged experimental boundary layer profile (blue dashed line).

given in Appendix $B$. The only cylinder for which there were significant issues with the flat plate approximation was $25 \mathrm{~A}$, which has the smallest diameter and thus the most pronounced effects of body curvature. There were other challenges associated with processing cylinder $25 \mathrm{~A}$, including great difficulty in accurately selecting the centerline of the cylinder for post-processing. The small diameter of $25 \mathrm{~A}$ meant any small inaccuracies in picking the centerline caused significant asymmetry in the results. For these reasons, interferometry results for cylinder $25 \mathrm{~A}$ are not presented here.

As for hexane mixtures, interferometry processing can be considered "successful" if results are within $10 \%$ of the pyrometer measurements and/or the numerical predictions. However, even the "successful" results from hexane appear less accurate than the tests run in a pure nitrogen environment. Furthermore, cylinders 100C and 200A show consistently "unsuccessful" interferometry processing results, with the percent 
difference between surface temperature measured by pyrometer and interferometer averaging to more than $15 \%$. This is likely due to decomposition of hexane in the boundary layer, which alters the index of refraction and Gladstone-Dale constant. These parameters are critical to know accurately in order to reconstruct the temperature fields with interferometry.

\begin{tabular}{|l||ll|ll|}
\hline \multirow{3}{*}{ Cyl Tag } & \multicolumn{3}{|c|}{ Pure $\mathrm{N}_{2}$} & \multicolumn{3}{c|}{ Stoich hexane-air } \\
& Avg. $\Delta T, \mathrm{~K}, \quad \%$ diff, (Pyr & Avg. $\Delta T, \mathrm{~K}, \quad$ Avg. \% diff, \\
(Pyr vs. Intf) & vs. Intf) & (Pyr vs. Intf) & (Pyr vs. Intf) \\
\hline 25A & - & - & - & - \\
50A & 54.9 & 5.0 & 32.9 & 3.1 \\
50C & 35.0 & 3.2 & 100.1 & 9.1 \\
75B & 44.3 & 4.0 & 71.9 & 6.6 \\
100A & -165.2 & -15.0 & 58.4 & 5.6 \\
100C & -20.7 & -2.1 & 161.7 & 15.7 \\
200A & 43.1 & 4.3 & 182.5 & 18.1 \\
\hline
\end{tabular}

Table 4.3: Summary of interferometry results in stoichiometric n-hexane and air mixtures.

Cylinders 100C and 200A likely have the least successful interferometry processing results in hexane most likely due to the diameter of these cylinders $(2.54 \mathrm{~cm})$ being the largest diameter tested. The largest diameter cylinders are effected by decomposition of hexane to the greatest extent. For two cylinders with different diameters $(D>d$ but the same boundary layer thickness $(\delta)$, the line of sight of the interferometer through the boundary layer, $z$, is greater in the case of the larger cylinder $\left(z_{D}>z_{d}\right)$. If we assume all decomposition products remain in the boundary layer, then the interferometer "sees" more of the decomposition products for a large cylinder diameter versus a smaller one, even for the same boundary layer thickness. This leads to the largest diameter cylinders having the largest inaccuracies in postprocessed interferometer temperature measurements. This explanation is illustrated in the schematic shown in Fig. 4.25.

Before discussing the limitations of these interferometry results in detail, we present the results of interferometry from a test of cylinder 100C in a diluted hydrogen mixture. The mixture is has a stoichiometric ratio of hydrogen to oxygen and is diluted with extra nitrogen to slow down the flame speed. The mixture is given in Eq. 4.3 . 


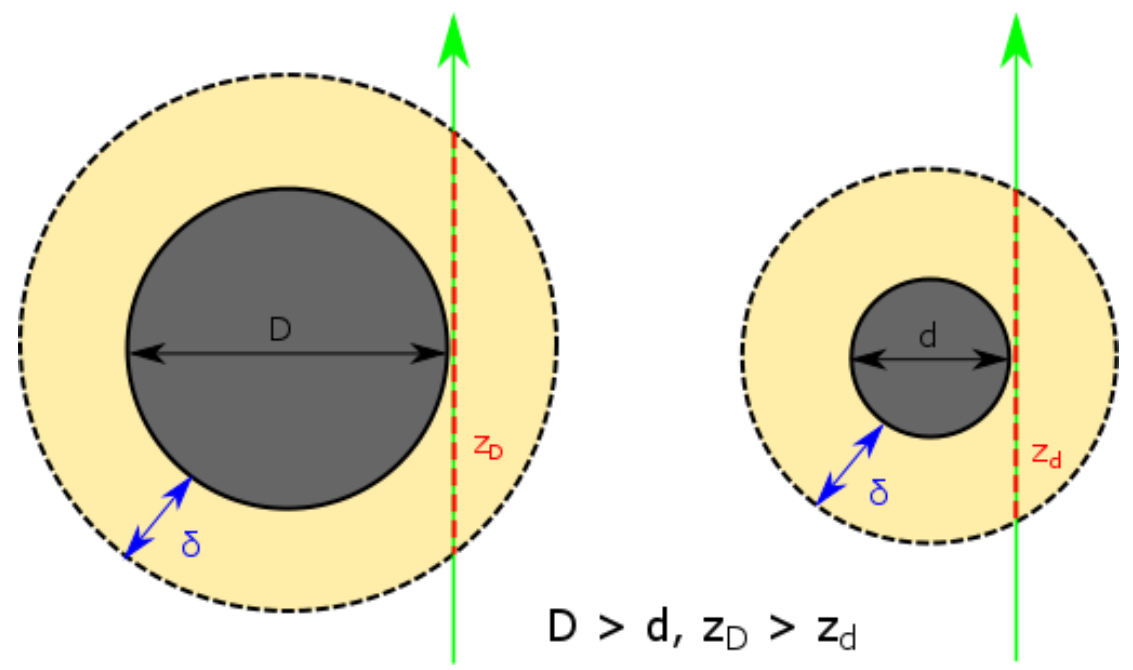

Figure 4.25: Line-of-sight depth, $z$, through boundary layer for cylinders of different diameters $(D>d)$ with the same boundary layer thickness, $\delta$.

$$
2 \mathrm{H}_{2}+1 \mathrm{O}_{2}+9 \mathrm{~N}_{2}
$$

This mixture is then tested for ignition with cylinder 100C in the same manner as the hexane mixtures. The goal of working with hydrogen is to identify whether the processing difficulties are specific to hexane as a fuel (like decomposition of hexane prior to ignition) or if there is some larger difficulty processing reactive mixtures in general (like having a multi-component mixture). Hydrogen is not as prone to significant decomposition prior to ignition, so if interferometry results in hydrogen can be processed successfully, that is an indication that the problems with processing hexane could be coming from significant fuel decomposition in the boundary layer prior to ignition.

Figures 4.26 to 4.28 document the results of processing shot 4 , a test in the diluted hydrogen mixture mentioned previously. The surface temperature measured by pyrometer was $963 \mathrm{~K}$, and the interferometry results for this test are time averaged over a duration of $10 \mathrm{~s}$. The temperature field presented in Fig. 4.26 includes the ignition location (lower half of left hand temperature field).

The temperature fields from shot 4 are shown in Fig. 4.26. The thermal boundary layers look quite similar to one another, and both sides approach a temperature of 

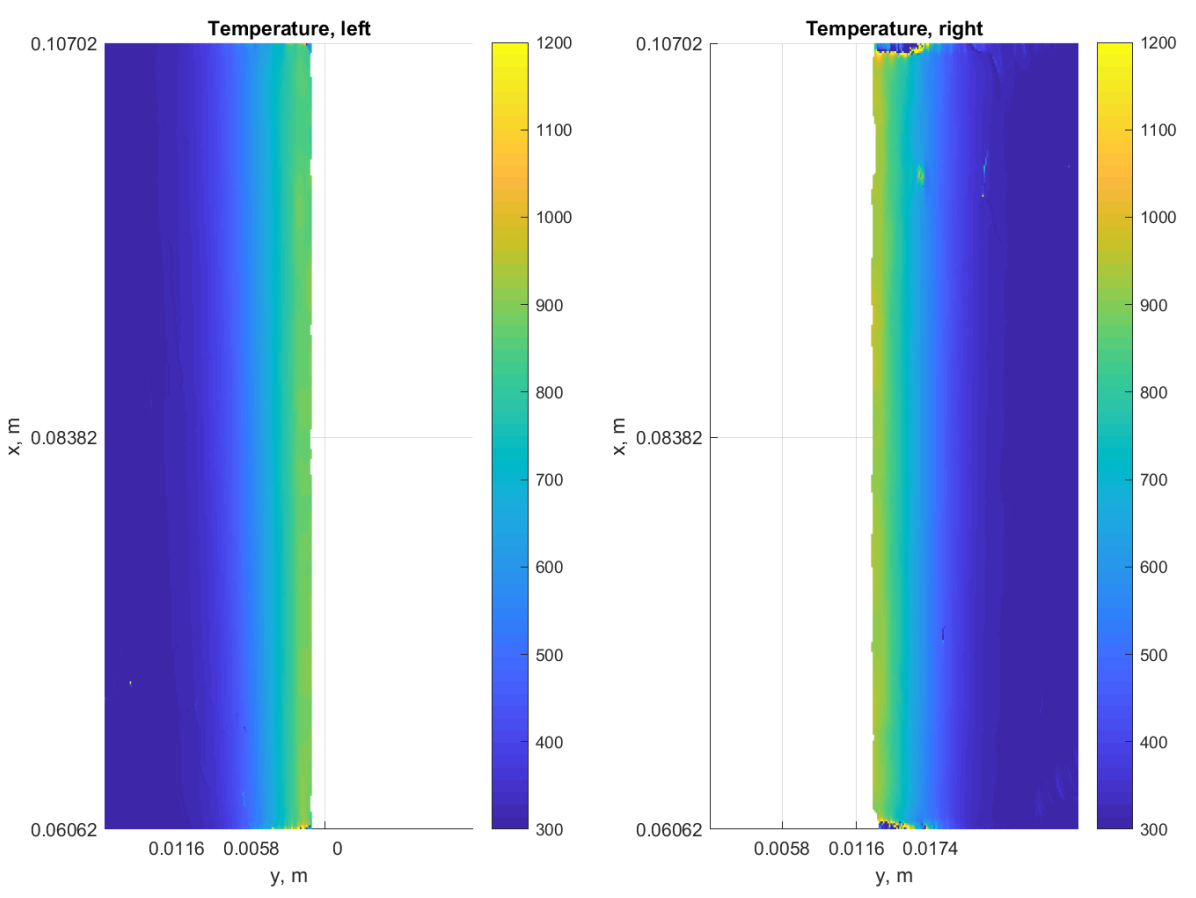

Figure 4.26: Shot 4. Temperature fields from interferometry for hydrogen-air test. Surface temperature measured by pyrometer $=963 \mathrm{~K}$.

approximately $950 \mathrm{~K}$ near the surface, which is in the right range for the surface temperature $(963 \mathrm{~K})$ as measured by pyrometer.

The experimental and numerical temperature profiles are shown in Fig. 4.27. The numerical temperature profile is taken from the nonreactive similarity solution for natural convection in air presented in Chapter 3 . The left and right sides of the experimental boundary layer profile are very similar to one another. Both experimental profiles are slightly hotter and wider than the numerical temperature profile predicted for a surface temperature of $950 \mathrm{~K}$, but overall there is good agreement between the experimental and numerical results for shot 4 . Finally, the surface temperature extrapolated from the interferometer is $976 \mathrm{~K}$, which is very close to the 963 measured via pyrometer. This indicates good processing of the hydrogen mixture. This could occur because hydrogen decomposes to a lesser extent than hexane prior to ignition, causing less of a mixture composition change. This possibility will be discussed in further detail shortly.

The percent difference between the experimental profiles and the numerical prediction is shown in Fig. 4.28. The two experimental profiles are within $10 \%$ difference of each other, which qualifies this result as "mostly symmetrical". The mean exper- 


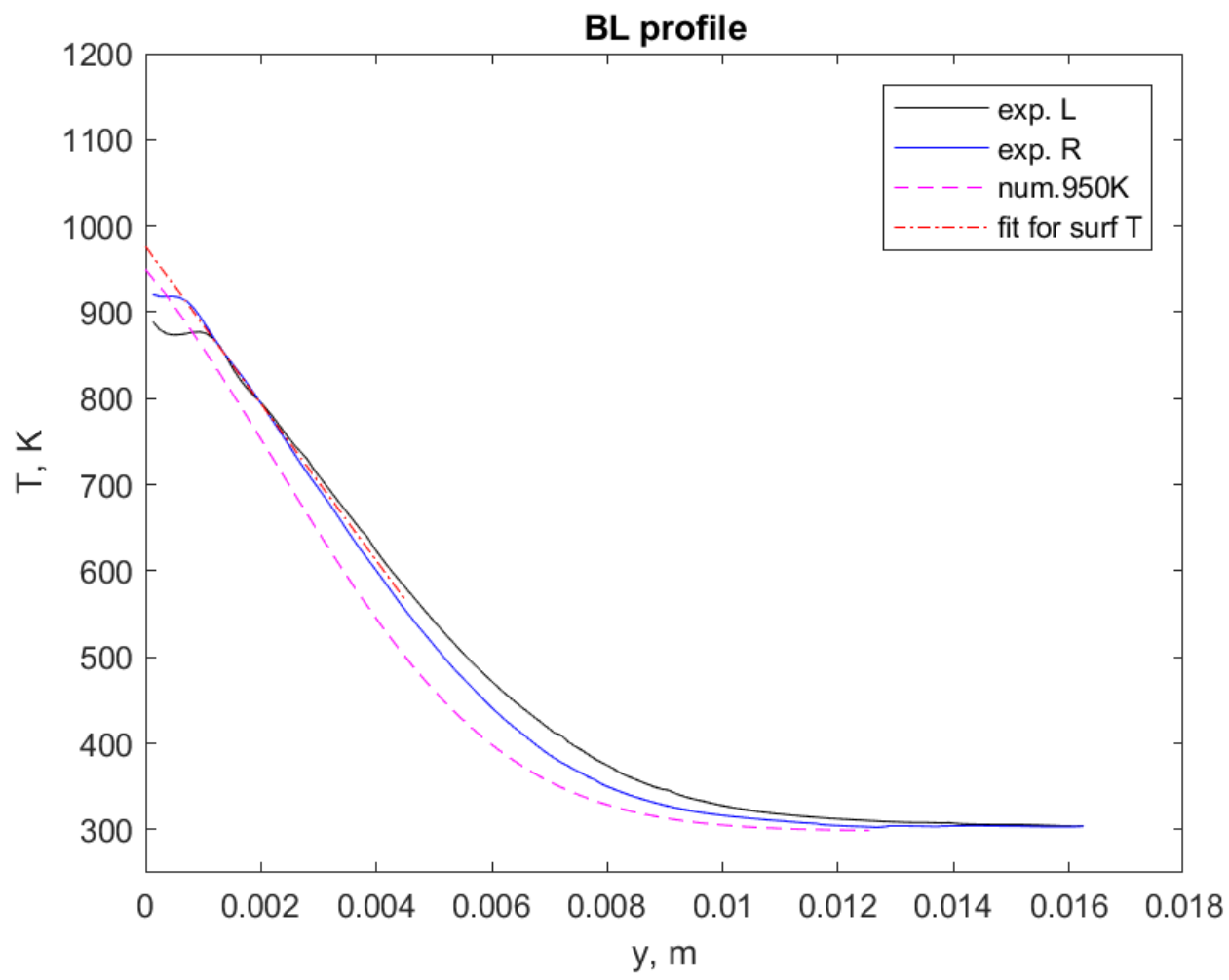

Figure 4.27: Shot 4. Thermal boundary layer profiles for left (black line) and right (blue line) sides of experimental results compared with prediction of boundary layer profile (dashed magenta line) based on similarity solution using pyrometer measurement as the wall temperature. Linear fit to averaged experimental profile (red dot-dashed line) used to extrapolate surface temperature from interferometer post processing. Extrapolated interferometer surface temperature $=976.2 \mathrm{~K}$.

imental profile is a little wider than the predicted boundary layer profile, leading to up to $15 \%$ difference in the outer portion of the boundary layer. The extrapolated surface temperature is within $1.4 \%$ difference of the pyrometer surface temperature, leading to excellent agreement in the measurements made via pyrometry and interferometry.

In conclusion, the processing of interferometry results from this hydrogen mixture were considered successful. This supports the idea that the difficulty with processing hexane due to the specific nature of that fuel. In other words, one reactive mixture is challenging to process successfully, and uses a fuel (hexane) which is known to decompose in significant quantities prior to ignition. Another reactive mixture uses a fuel (hydrogen) that does not decompose as much and does not exhibit the same difficulties in processing interferometry. 


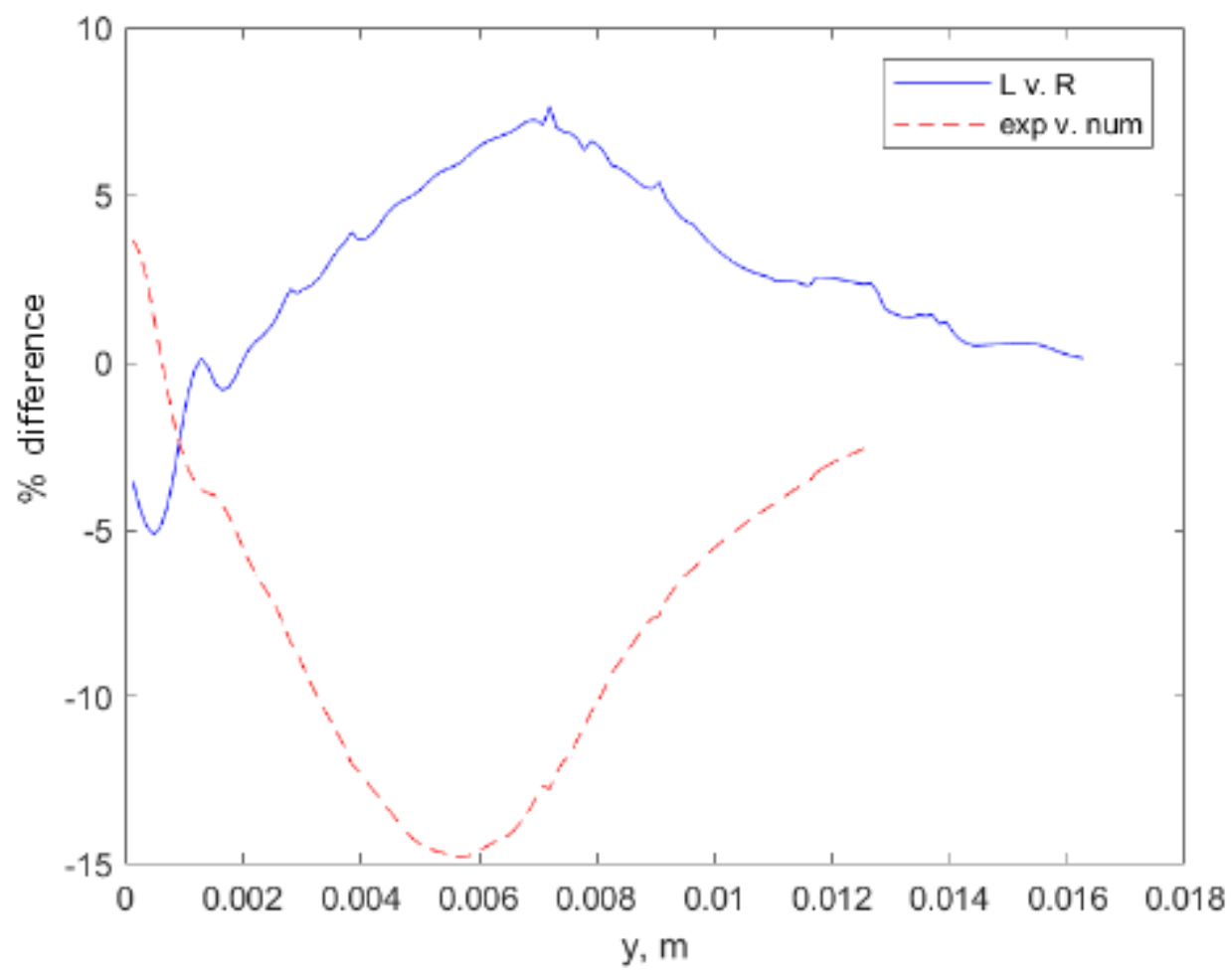

Figure 4.28: Shot 4. Percent difference in thermal boundary layer between both sides of experimental boundary layer profiles (red line) and percent difference between predicted boundary layer profile from similarity solution and averaged experimental boundary layer profile (blue dashed line).

One factor contributing to the difference in processing hexane versus hydrogen mixtures could be the extent of decomposition. It could be that hydrogen decomposes less than hexane before the ignition event, to the extent that it remains essentially unreactive until immediately before ignition. This possibility is examined by running zero-dimensional ignition delay time calculations for both mixtures: the temperature of the hydrogen mixture (same composition as the experiments) is set at $963 \mathrm{~K}$, the surface temperature at ignition observed for Shot 4, and the temperature of the hexane mixture (same composition as the hexane experiments) is set at $1080 \mathrm{~K}$, the surface temperature at ignition of Shot 164. The mass fraction of various species is plotted against time normalized by the ignition delay, $t / \tau_{i g n}$. The results are shown in Fig. 4.29

The ignition delay for the diluted hydrogen mixture at $963 \mathrm{~K}$ was $\tau_{i g n}=6.57 \times 10^{-4}$ $\mathrm{s}$, and the ignition delay for the stoichiometric hexane mixture at $1080 \mathrm{~K}$ was $\tau_{i g n}=1.39 \times 10^{-2} \mathrm{~s}$. The metric by which to determine if the mixture is reactive 

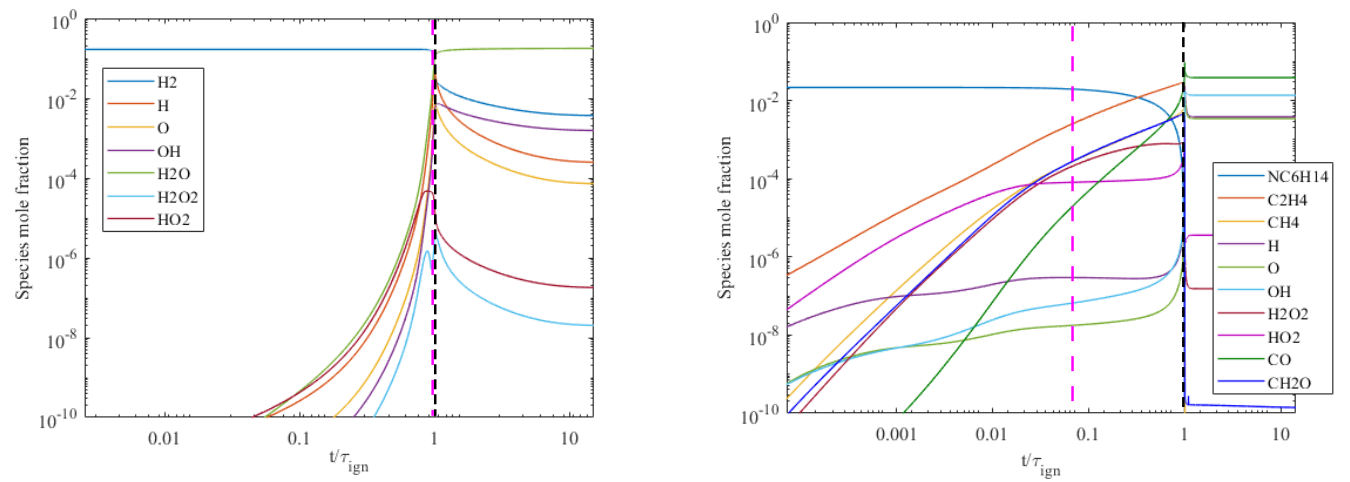

Figure 4.29: Mass fraction evolution for hydrogen (left) and hexane (right) mixture plotted against non-dimensional time, $t / \tau_{i g n}$. The magenta dashed vertical line indicates time of $10 \%$ mass fraction change in the fuel and the black dashed vertical line indicates the time of ignition.

is set as time at which the initial fuel mass fraction has changed by $10 \%$. This is a somewhat arbitrary criterion, but it enables comparison of the relative reactivity of the hydrogen and hexane mixtures before ignition. The mass fraction of hydrogen changes by $10 \%$ at $\mathrm{t}=6.28 \times 10^{-4} \mathrm{~s}, 96 \%$ of the ignition delay time. The mass fraction of hexane changes by $10 \%$ at $\mathrm{t}=9.82 \times 10^{-4} \mathrm{~s}$, only $7 \%$ of the ignition delay time. While these are very simple calculations and do not capture all the physics occurring in the experiments, these results do indicate that the hexane mixture is reactive and decomposing to a significant extent well before ignition. By the same metrics, hydrogen does not significantly decompose until just before ignition occurs. This supports the idea that hexane is more challenging to process than hydrogen because the mixture fraction changes significantly well before the ignition event.

We conclude that the hydrogen interferometry is less difficult to post-process than hexane because the hydrogen mixture is essentially non-reacting until immediately before ignition. The hexane mixture is reactive and decomposing in significant amounts well before the ignition time, and this leads to a change in mixture composition that significantly alters parameters like index of refraction, which must be precisely known to accurately reconstruct temperature fields via interferometry. These limitations are discussed at length in the next section.

We can therefore conclude, due to the success of processing inert gas (nitrogen) that the issues with processing hexane are not due to issues with the interferometer 
construction but due to the tendency of hexane to decompose into other hydrocarbon species prior to ignition. This decomposition alters the index of refraction and Gladstone-Dale constants in the boundary layer, while the post-processing assumes these remain constant throughout the field of view and leads to errors.

\section{Interferometry Limitations}

There are limitations to using quantitative interferometry results. The first limitation is that there is a narrow region near the surface or centerline of a processed interferometry image where errors accumulate. This occurs typically within one millimeter of the surface for this setup, and arises due to the nature of Nestor-Olsen algorithm used to compute the inverse Abel transform (Nestor and Olsen, 1960). This algorithm computes the transform by working toward the centerline from the outer edge of the domain. This means any errors from the outer edges compile into the centermost portion of the domain, causing the narrow region of larger error observed near the surface in many of the interferometry results. This concentrated error region near the surface prompted extrapolating the surface temperature from interferometry data (excluding points close to the surface) by applying a linear fit to the middle portion of the thermal boundary layer. The errors are confined to a limited region near the surface and do not appear to affect the outer part of the temperature field as shown by the comparison with the numerical solution of the thermal boundary layer.

The second limitation is the accuracy with which we can know the composition of the gas during an experiment. Gas composition must be known accurately in order to compute the proper index of refraction, Gladstone-Dale constant, and specific gas constant that are critical to accurate post-processing of interferometry results. These parameters can be calculated following the procedure laid out by Gardiner, Hidaka, and Tanzawa (1981), who tabulated the molar refractivity, $R_{L_{i}}$, of common combustion gases. The molar refractivity for the entire mixture is calculated by a molar weighted average:

$$
R_{L}=\sum_{i=1}^{n} R_{L_{i}} X_{i}
$$

The index of refraction, $\mathrm{n}_{0}$, and Gladstone-Dale constant, $K$, can be calculated as: 


$$
\begin{gathered}
n_{0}=\left(\frac{1+2 \rho_{m} R_{L}}{1-\rho_{m}}\right)^{\frac{1}{2}}, \\
K=\frac{n_{0}-1}{\rho},
\end{gathered}
$$

where $\rho_{m}$ is the molar density of the mixture.

Finally, the specific gas constant of the mixture is calculated from the universal gas constant and the mixture averaged molar mass:

$$
R_{s}=\frac{\tilde{R}}{M_{m i x}} .
$$

As discussed previously in this section, the composition of pure nitrogen or a diluted hydrogen-air mixture is known with enough accuracy to produce reliable results. However, when working with hexane as a fuel, there is an unknown alteration of the gas composition, especially near a hot surface, due to the decomposition of hexane.

Table 4.4 estimates the effects that different values of $\mathrm{n}_{0}, \mathrm{~K}$, and $\mathrm{R}_{s}$ have on the processed interferometry temperature. The calculation begins with a stoichiometric hexane-air mixture. Then, for each of the columns in the table, a portion of the initial hexane mass is decomposed into other hydrocarbon components. The first column is $100 \%$ hexane, essentially assuming no decomposition (this is the assumption made when processing the interferometer results). The second column assumes the entire mass of hexane is decomposed into ethylene (one of the major products of decomposing hexane). The third column assumes all of the hexane mass is decomposed into methane (another major product of decomposing hexane). The fourth column uses the mass fractions of hydrocarbons near the wall prior to ignition in the one-dimensional simulation presented by Coronel (2016) to inform a rough approximation of the mixture composition for the actual experiments (1:5:1 ratio of hexane:ethylene:methane by mass). These mixtures are not assumed to reflect the composition near the wall for experiments performed in hexane; rather, these mixtures are selected to provide bounds on how a change of mixture composition could affect inteferometry temperature measurements. 
The top half of the table documents the values of $\mathrm{n}_{0}, \mathrm{~K}$, and $\mathrm{R}_{s}$ for each of those mixtures. The bottom half calculates how each of these sets of parameters leads to a different final temperature, given the same optical phase. The start point is selected to be the inverse able transformation on optical phase, i.e. $\frac{\lambda}{2 \pi} f(r)$ from Eq. 2.41 , as up to this point there has been no use of any mixture-dependent parameters like $\mathrm{n}_{0}, \mathrm{~K}$, and $\mathrm{R}_{s}$. The value of $\frac{\lambda}{2 \pi} f(r)$ selected is $-2.0361 \mathrm{e}-5$, sampled next to cylinder surface of frame 3700 of shot 164 . Given this value of $\frac{\lambda}{2 \pi} f(r)$ to start from, the temperature is found for each mixture, reported in the last line of Table 4.4.

\begin{tabular}{|l||llll|}
\hline $\begin{array}{l}\text { fuel mix } \\
\text { by weight } \\
\%\end{array}$ & $100 \%$ hexane & $100 \%$ ethylene & $100 \%$ methane & $\begin{array}{l}14.3 \% \\
71.4 \% \\
14.3 \% \text { methane }\end{array}$ \\
\hline $\mathrm{n}_{0}$ & 1.00029769777 & 1.00028878070 & 1.00027863692 & 1.00028858974 \\
ethylene, \\
$\mathrm{K}$ & $2.46787492823 \mathrm{e}-4$ & $2.5011041371 \mathrm{e}-4$ & $2.52818786466 \mathrm{e}-4$ & $2.50093103736 \mathrm{e}-4$ \\
$\mathrm{R}_{s}$ & 276.328826446 & 288.696979420 & 302.447015727 & 282.868013410 \\
\hline$\frac{\Lambda}{2 \pi} f(r)$ & $-2.0361 \mathrm{e}-4$ & $-2.0361 \mathrm{e}-4$ & $-2.0361 \mathrm{e}-4$ & $-2.0361 \mathrm{e}-4$ \\
$\Delta \mathrm{n}$ & $9.409 \mathrm{e}-5$ & $8.517 \mathrm{e}-5$ & $7.503 \mathrm{e}-5$ & $8.498 \mathrm{e}-5$ \\
$\rho$ & 0.3813 & 0.3405 & 0.2968 & 0.3398 \\
$\mathrm{~T}$ & 949.2 & 1017.2 & 1114.1 & 1040.4 \\
\hline
\end{tabular}

Table 4.4: Effect of varying mixtures index of refraction $\left(\mathrm{n}_{0}\right)$, Gladstone-Dale constant $(\mathrm{K})$, and specific gas constant $\left(\mathrm{R}_{S}\right)$ on interferometry processing results. Bottom half demonstrates given the same inverted Abel optical phase the effect that selecting the proper $\mathrm{n}_{0}, \mathrm{~K}$, and $\mathrm{R}_{s}$ can have on temperature results.

The first column shows the interferometry temperature measurement using parameters for a stoichiometric hexane-air mixture where none of the hexane decomposes; these are the parameters used and the assumption made when processing the hexane results discussed previously. The second column shows the temperature if all of the hexane mass decomposes into ethylene; this is obviously not a realistic assumption but provides insight into what effect the presence of ethylene has on the resulting temperature reading. A similar principle applies to the third column; all of the hexane mass is assumed to decompose into methane. The final column is a 1:5:1 hexane:ethylene:methane mixture by mass. This mixture is a rough approximation of the major components of hexane decomposition near a heated wall based on the calculations of Coronel (2016). While it is the most realistic of the compositions investigated here, it is only meant to be an approximation to bound the effect that a decomposing hexane mixture might have on interferometer temperature reading.

The different temperatures reported in Table 4.4 provide some insight into why the processed hexane results (particularly for cylinders 100C and 200A) are lower 
than the pyrometer temperature. We assume in our post-processing that there is no hexane decomposition, leading to a temperature reading of approximately $950 \mathrm{~K}$ in the case of Shot 164. If, however, the initial mass of hexane has decomposed into a 1:5:1 mixture of hexane:ethylene:methane and we use the corresponding parameters for that mixture, the temperature near the surface reads approximately $1040 \mathrm{~K}$. This is much closer to the pyrometer reading of 1080 (within 4\%) for shot 164 . We can therefore conclude that if there is decomposition of the hexane fuel near the heated surface, the use of interferometry parameters that assume no decomposition of hexane will cause an artificially low temperature reading. In fact, this is exactly the behavior we observe in the processed hexane results; the interferometry temperatures are consistently lower than the pyrometer temperatures. In the cases of 100C and 200A, this discrepancy can be more than $100 \mathrm{~K}$ and classify the processing results as "unsuccessful".

To improve the interferometry processing for hexane, we would need to assume a radially varying composition that accounted for decomposition of hexane near the hot surface while the ambient mixture remained unaltered. This radial variation of the mixture composition could be informed by experimental measurement of species concentration or numerical simulation of the decomposition of hexane near a heated surface using a detailed chemical kinetic mechanism. The post-processing procedure would have to be altered to allow for radial variation of parameters $n_{0}, K$, and $\mathrm{R}_{s}$ whose values are informed by the radial variation of mixture composition. This is a complex undertaking and is left as a potential future direction for further research.

\section{Schlieren Visualization of Ignition}

As discussed in 2.6, the interferometer can be converted into a schlieren visualization system by blocking off the reference beam and putting a stop in the second focal point (depicted as FP2 in Fig. 2.18). For this work, the stop was chosen to be a "dot", an opaque circle $0.5 \mathrm{~mm}$ in diameter. This causes a type of image colloquially referred to as a "dot schlieren" image. The image is completely dark until a refractive disturbance is introduced; in this way, a dot schlieren acts like a background subtraction: the DC component of the image is removed and only changes in the field are visualized. 
Here we will present and discuss the visualization results for three tests with cylinder 100C. Results from two ignition tests and one non-ignition test are shown.

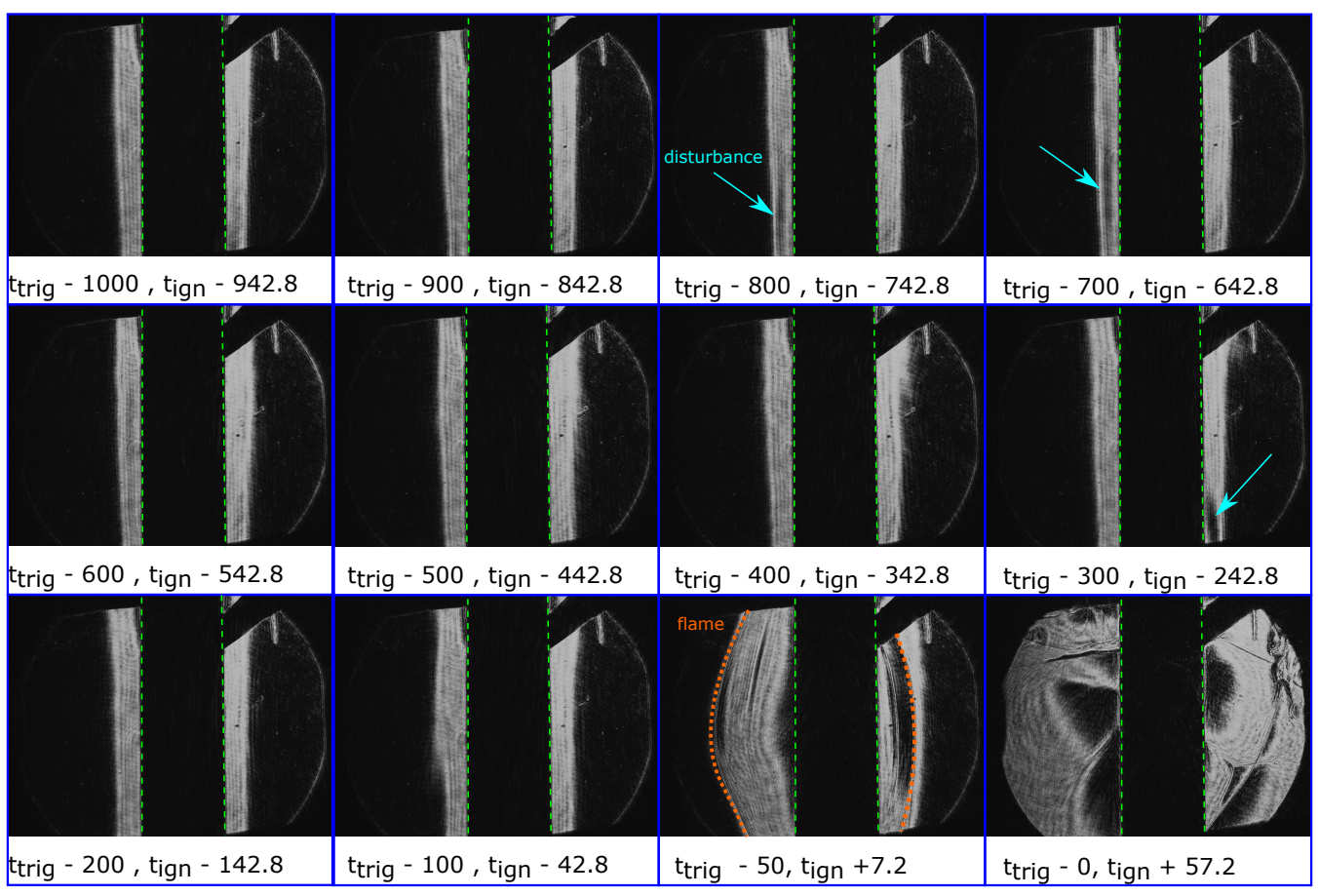

Figure 4.30: Dot schlieren visualization of pre-ignition from Shot 175. Camera frame rate at $5000 \mathrm{fps}$. Time given relative to trigger and to first sign of ignition for each frame in units of miliseconds. Visualization over $1000 \mathrm{~ms}$ leading up to trigger time. Surface temperature at ignition (at test time $103.4 \mathrm{~s}$ ) is $1097 \mathrm{~K}$.

Figures 4.30 to 4.32 show the dot schlieren images for tests 175 through 177, documenting the last second of test time pre-trigger. In each of these figures, we observe the thermal boundary layer as a bright region surrounding the dark cylinder, the edges of which are noted with dashed green lines. Intermittent disturbances pass through the thermal boundary layer; these present sometimes as dark regions within the thermal boundary layer, as seen in Figs. 4.30 and 4.32, or as significant distortion of the outer edge as seen in 4.31 . The disturbances are indicated by light blue arrows. These disturbances indicate that the boundary layer surrounding the cylinder is not perfectly steady. The disturbances occur in both ignition and non ignition tests; the presence of a disturbance does not guarantee an ignition event. Figure 4.33 shows a side-by-side comparison of three different shots; shot 175, where ignition occurs in a well-structured laminar thermal layer, shot 176, where ignition occurs in the proximity of a significant disturbance, and shot 177 , where a significant disturbance 


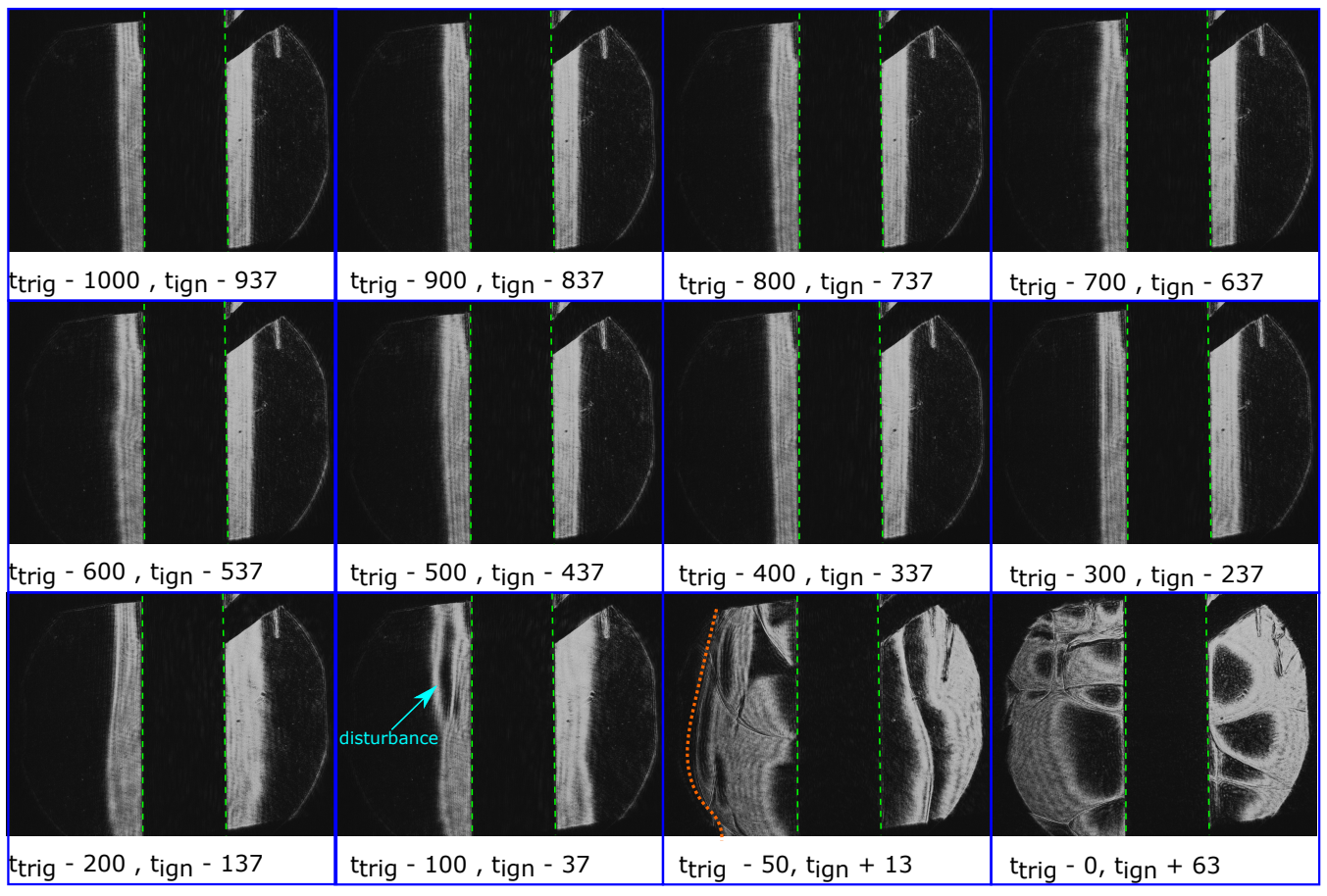

Figure 4.31: Dot schlieren visualization of pre-ignition from Shot 176. Camera frame rate at $5000 \mathrm{fps}$. Time given relative to trigger and to first sign of ignition for each frame in units of miliseconds. Visualization over $1000 \mathrm{~ms}$ leading up to trigger time. Surface temperature at ignition (at test time $258.7 \mathrm{~s}$ ) is $1069.5 \mathrm{~K}$.

occurs but no ignition event is observed. The frames from these tests are compared at similar times relative to ignition where applicable.

We now examine the ignition sequences for shots 175 and 176. Figure 4.34 shows the ignition sequence for shot 175. The first appearance of the ignition kernel occurs $57.0 \mathrm{~ms}$ before trigger time (103.4 s). The ignition kernel is located approximately $8.6 \mathrm{~cm}$ up from the bottom edge. The outline of the ignition kernel is highlighted in orange for easy identification. Figure 4.35 shows the ignition sequence for shot 176 in a similar manner.

Now, we consider what the location of the ignition kernel along the length of the cylinder reveals. For shot 175, the ignition kernel is up $8.6 \mathrm{~cm}$ from the bottom edge of the cylinder. For shot 176, the ignition kernel is up $7.7 \mathrm{~cm}$ from the bottom edge. The average distance between the bottom edge of the cylinder and the ignition kernel is $8.5 \mathrm{~cm}$ (standard deviation $0.6 \mathrm{~cm}$ ) for all ignition tests with a high enough frame rate to observe the formation of the ignition kernel. Referring back to Fig. 2.8, this 


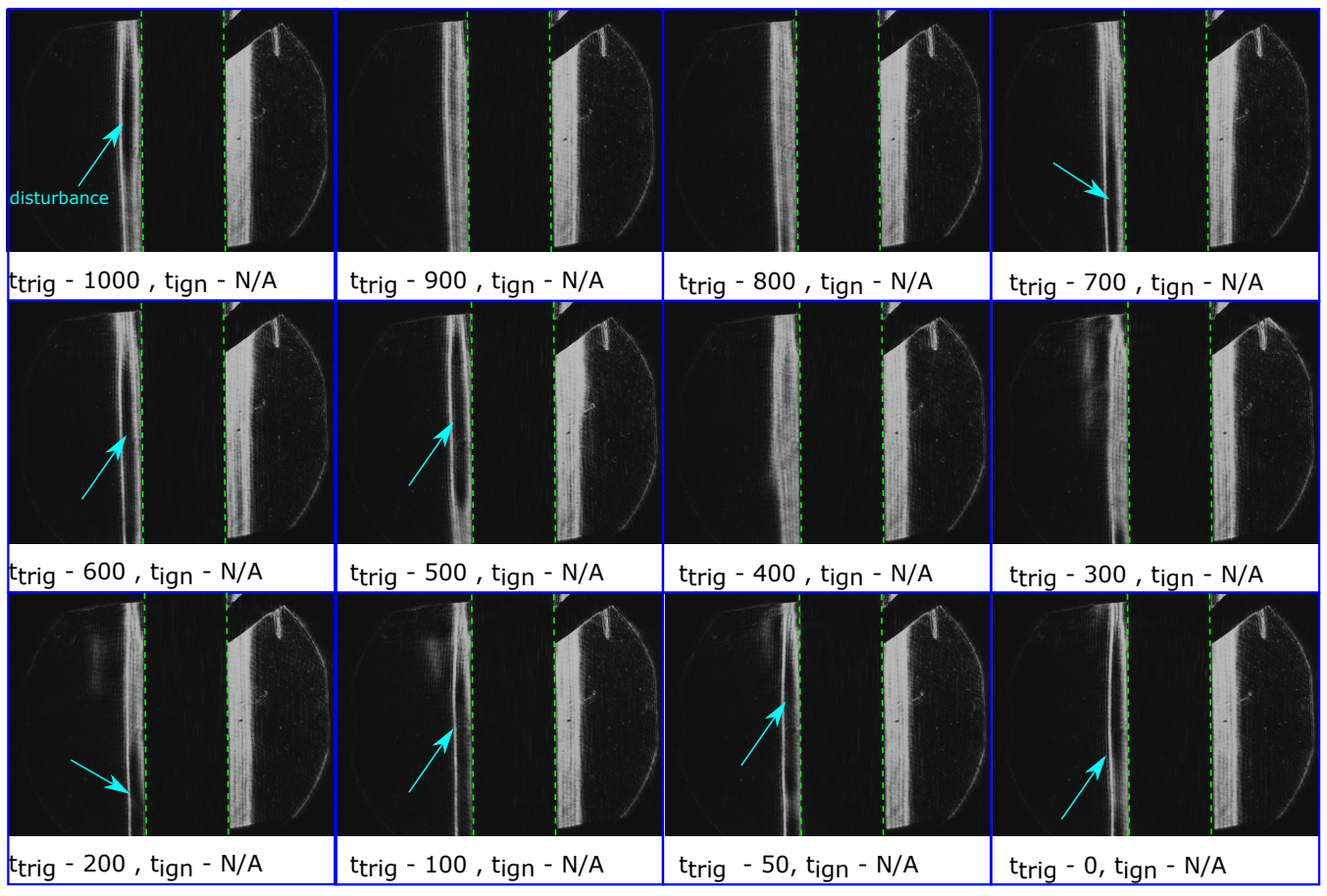

Figure 4.32: Dot schlieren visualization of pre-ignition from Shot 177. Camera frame rate at $1000 \mathrm{fps}$. Time given relative to trigger and to first sign of ignition for each frame in units of miliseconds. Visualization over $1000 \mathrm{~ms}$ leading up to trigger time. Surface temperature of $1060.1 \mathrm{~K}$ did not lead to an ignition event over 300 s test time.

is near the top edge of the uniform high-temperature region of the $12.7 \mathrm{~cm}$ length cylinder. From those results, the region that reaches at least $90 \%$ of the maximum surface temperature spans from 2.3 to $10.5 \mathrm{~cm}$; the region within $30 \mathrm{~K}$ (i.e. the measurement uncertainty of the pyrometer) of the maximum surface temperature spans from 3.6 to $9.6 \mathrm{~cm}$. The ignition kernel therefore occurs near the top of the highest temperature portion of the cylinder. This is exactly what we would expect to see for these tests, which are designed to identify the ignition threshold. Trying to find the ignition threshold (minimum ignition temperature) means ignition occurs at the location where a fluid particle has been exposed to elevated surface temperatures the longest. For the vertical cylinders, this location is at the top edge of the high temperature region.

Due to the vertical growth of the thermal layer, the thermal layer is at its widest at the top of the hot region of the cylinder and thus the thermal gradient is at its shallowest. As discussed in Boeck et al. (2017), there are arguments that support the 
(a) shot 175

trigger at $\mathrm{t}=103.4 \mathrm{~s}$ (b) shot 176 trigger at $\mathrm{t}=258.7 \mathrm{~s}$ (c) shot 177

trigger at $\mathrm{t}=300 \mathrm{~s}$
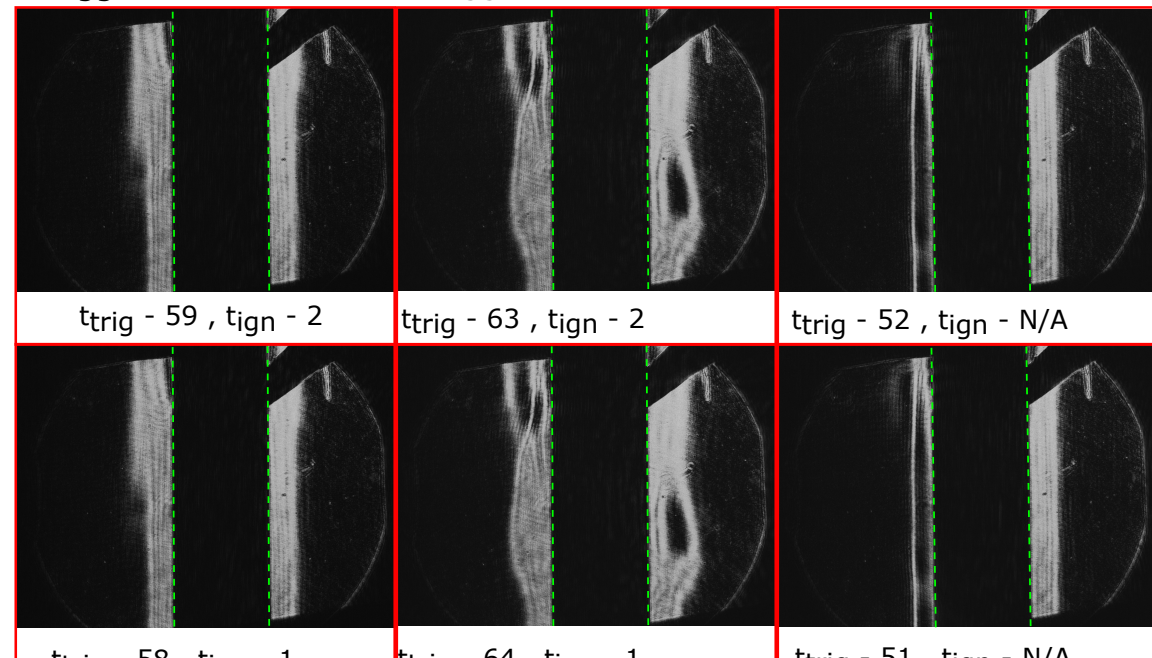

ttrig -63, tign -2

ttrig $-52, t_{i g n}-\mathrm{N} / \mathrm{A}$

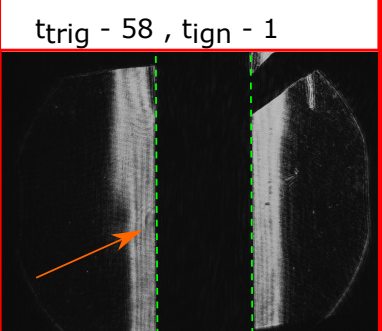

ttrig - 64, tign - 1

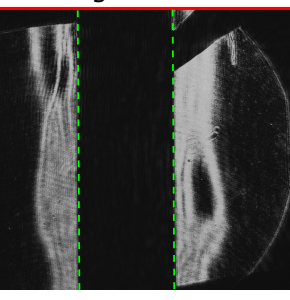

V

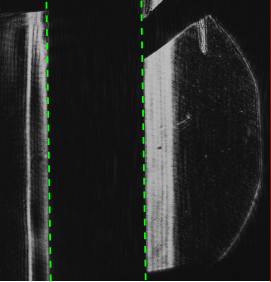

$t_{\text {trig }}-51$, tign $^{-N / A}$

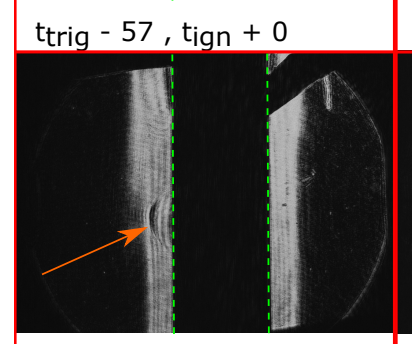

ttrig -63 , tign -0
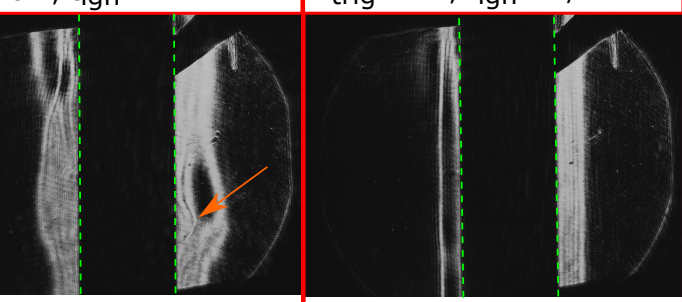

ttrig -56, tign +1

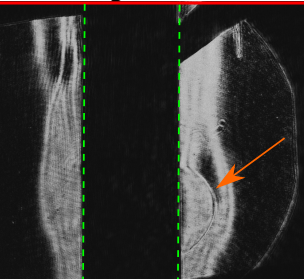

i

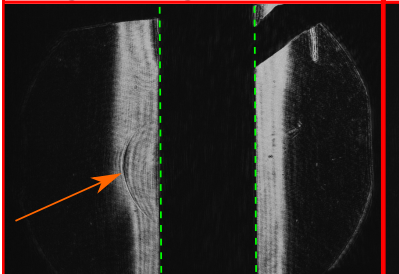

ttrig -62, tign +1

ttrig - 49, tign - N/A

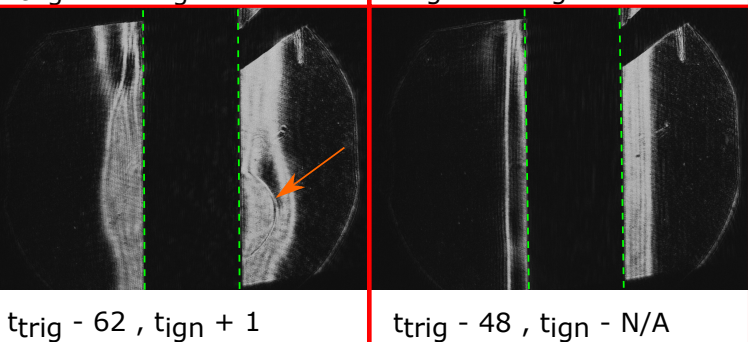

ttrig -55, tign +2

ttrig -62, tign +1

ttrig -48, tign $^{-N}$ /A

Figure 4.33: Dot schlieren visualization of shots 175, 176, and 177. Camera frame rate at $1000 \mathrm{fps}$. Time given relative to trigger and to first sign of ignition for each frame in units of miliseconds. Ignition kernel indicated by orange arrow.

idea that ignition is favored in regions with shallow thermal gradients. In particular, the scaling laws presented by Laurendeau (1982) suggest that ignition from free convection will occur first in the region with the smallest Nusselt number (Nusselt 


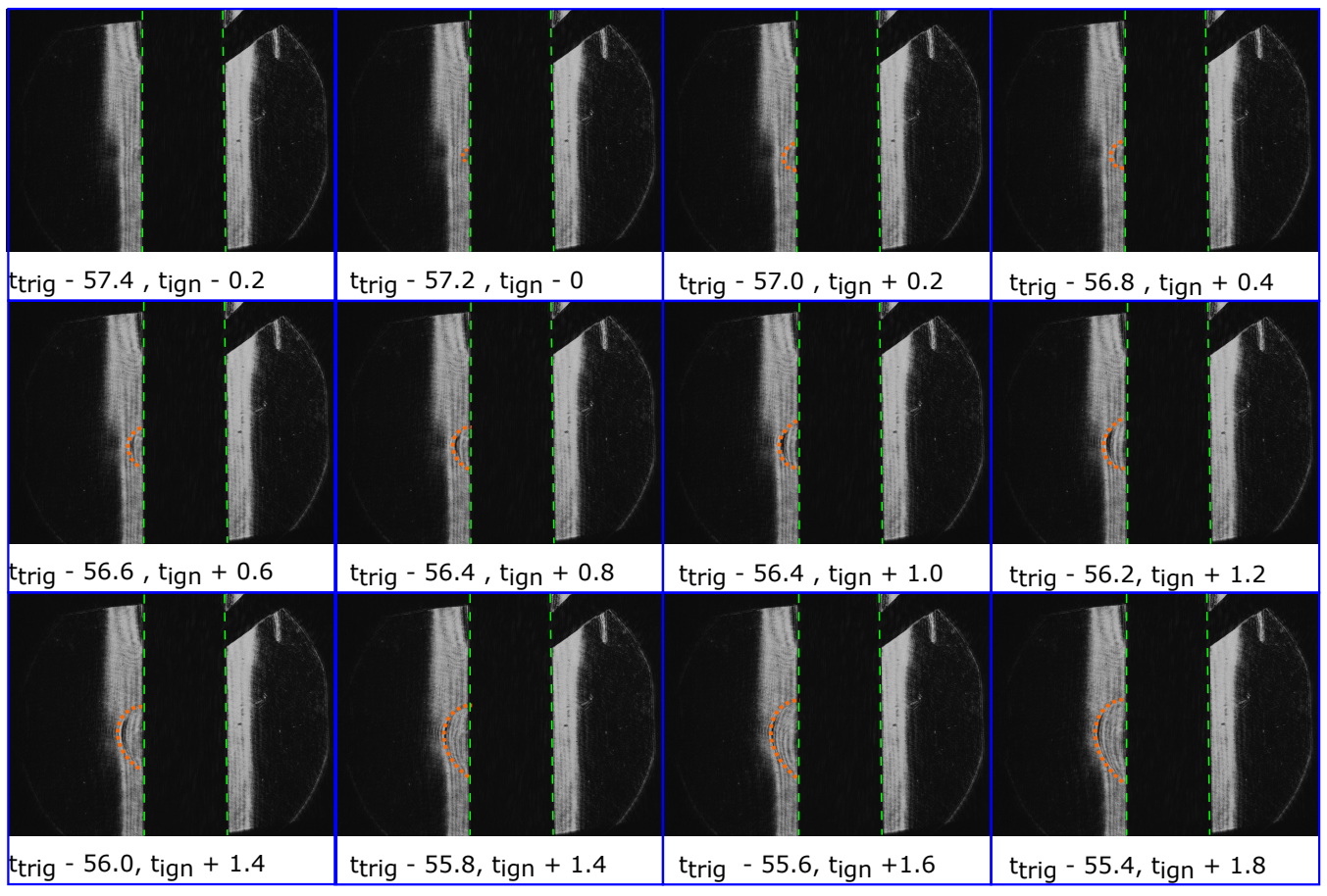

Figure 4.34: Dot schlieren visualization of ignition from Shot 175. Camera frame rate at $5000 \mathrm{fps}$. Time given relative to trigger and to first sign of ignition for each frame in units of miliseconds. Visualization over $2 \mathrm{~ms}$ leading up to trigger time. Surface temperature at ignition (at test time 103.4 s) is $1097 \mathrm{~K}$.

number defined in Chapter 3). The smallest Nusselt number corresponds with the shallowest thermal gradient; for a uniformly heated vertical cylinder, this occurs at its upper edge. There are limitations to the correlation of ignition location with Nusselt number; for example, in stagnation point flows, ignition is tied to residence time via the Damkohler number (Song, Schmidt, and Aris, 1991).

Table 4.5 documents the average ignition location (distance from bottom edge of cylinder) for all cylinders investigated. For cylinders $19.1 \mathrm{~cm}$ long, the region that reaches at least $90 \%$ of the maximum surface temperature spans from 3.2 to 16.9 $\mathrm{cm}$; the region within $30 \mathrm{~K}$ (i.e. the measurement uncertainty of the pyrometer) of the maximum surface temperature spans from 4.4 to $16.0 \mathrm{~cm}$. For cylinders 25.4 $\mathrm{cm}$ long, the region that reaches at least $90 \%$ of the maximum surface temperature spans from 3.0 to $22.8 \mathrm{~cm}$; the region within $30 \mathrm{~K}$ (i.e. the measurement uncertainty of the pyrometer) of the maximum surface temperature spans from 5.0 to $22.0 \mathrm{~cm}$. Due to the fact that the experiments are designed to find a minimum ignition temperature, we expect that for all cylinders, the ignition kernel forms near the top of the high temperature region of the cylinder. At this location, the thermal gradient is 


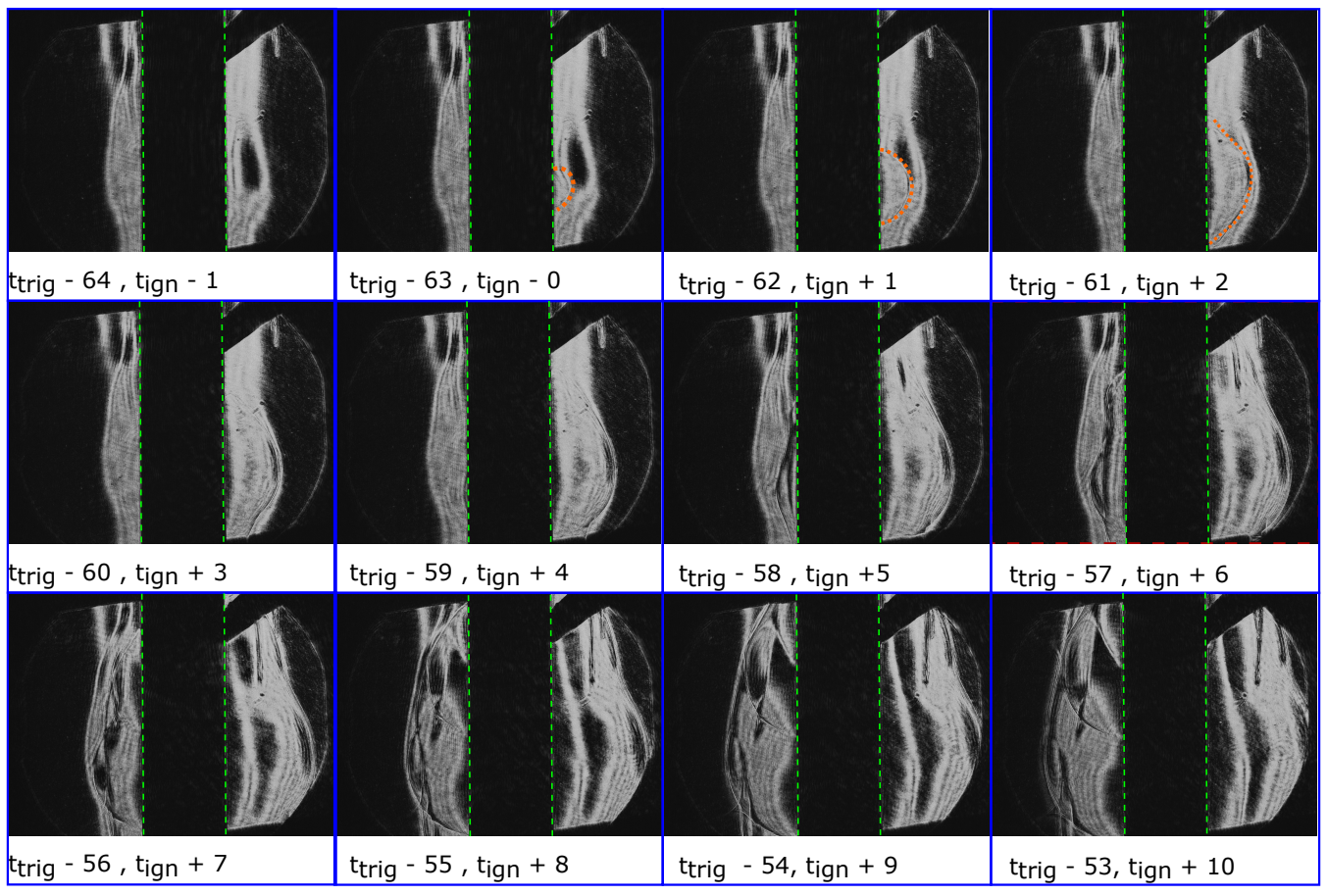

Figure 4.35: Dot schlieren visualization of ignition from Shot 176. Camera frame rate at $1000 \mathrm{fps}$. Time given relative to trigger and to first sign of ignition for each frame in units of miliseconds. Visualization over $11 \mathrm{~ms}$ leading up to trigger time. Surface temperature at ignition (at test time $258.7 \mathrm{~s}$ ) is $1069.5 \mathrm{~K}$.

shallowest and the Nusselt number is smallest.

\begin{tabular}{llllll}
\hline Cyl Tag & $\begin{array}{l}\text { Cyl Length } \\
(\mathrm{cm})\end{array}$ & $\begin{array}{l}\text { Avg. } \\
\text { Location } \\
(\mathrm{cm})\end{array}$ & $\begin{array}{l}\text { Agn. } \\
\text { Location } \\
(\% \quad \text { Cyl. } \\
\text { Length) }\end{array}$ & $\begin{array}{l}\text { Std. } \\
\text { Ign. } \\
\text { tion (cm) }\end{array}$ \\
\hline 25A & 25.4 & 22.7 & 98.4 & 0.6 \\
loca-
\end{tabular}

Table 4.5: Average ignition location for all cylinders.

The circumferential location of ignition varied randomly, which indicates a high temperature uniformity along the cylinder circumference. This supports the use of the simplifying assumption of one-dimensional temperature variation used in Sec- 
tion 2.1 to predict the temperature distribution along the cylinder.

Figure $4.36 \mathrm{a}$ shows a scatter plot of the ignition temperature versus the ignition location in terms of \% distance along the cylinder length. There is no significant correlation of the ignition temperature with the ignition location. The scatter in the results is likely a reflection of the stochastic nature of ignition. Figure $4.36 \mathrm{~b}$ shows a scatter plot of the ignition location versus the length of the cylinder. There is noticeable scatter in the results for a particular cylinder; once again likely a reflection of the stochastic nature of ignition. As the cylinder length increases, the ignition location increases as well. This is a reflection of the relatively fixed size of the cooled edge region of the cylinder (approximately $3 \mathrm{~cm}$ at each end); a larger percentage of the cylinder length is in the cooled edge region for a cylinder $12.7 \mathrm{~cm}$ long than for a cylinder $25.4 \mathrm{~cm}$ long. The ignition always occurs near the upper edge of the hot portion of the cylinder no matter its length. This is due to the fact that these ignition tests are designed to find minimum ignition temperatures; the mixture has the best chance of igniting at the top edge of the hot region of the cylinder, where the reactive mixture has been exposed to the heated surface for the longest time.

These results, as well as the results in Fig. 4.12, are examples of the challenges in formulating a non-dimensional scaling of the ignition results. Furthermore, we can consider the scaling of ignition temperature using the relation postulated by Ono et al. (1976) on the basis of work by Khitrin and Goldenberg (1957). Laurendeau (1982) extends this result to a variety of situations and discusses the flow scaling in terms of the Grashof or Rayleigh number and the parameters of one-step chemical reaction models. Ono et al. (1976) proposed that for free convection flows, $\ln \left(L^{1 / 2}\right) \propto \frac{E_{a}}{R T_{i g n}}$; $L$ is characteristic surface height for the case of natural convetion, $E_{a}$ is activation energy, $R$ is the universal gas constant, and $T_{i g n}$ is the ignition temperature. This can be rearranged to indicate the scaling of ignition temperature with characteristic size of the surface: $T_{i g n} \propto \frac{2 E_{a}}{R \ln (L)}$, which can be generalized to be a nondimensional correlation of the form,

$$
\frac{L}{L^{*}}=f\left(\frac{E_{a}}{R T_{i g n}}, G r, \ldots\right),
$$

Where $L^{*}$ is a reference length and there may be additional non-dimensional parameters to account for geometric factors and properties of the combustible gas. For a 


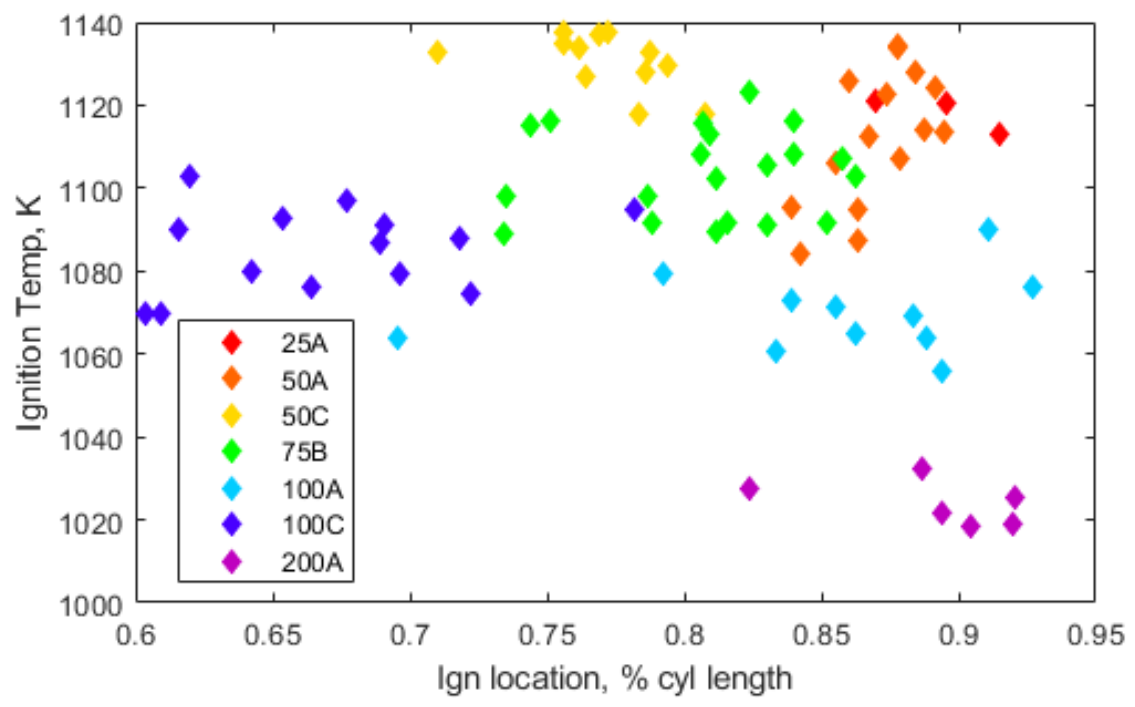

(a) $T_{i g n}$ vs ignition location in terms of \% cylinder length

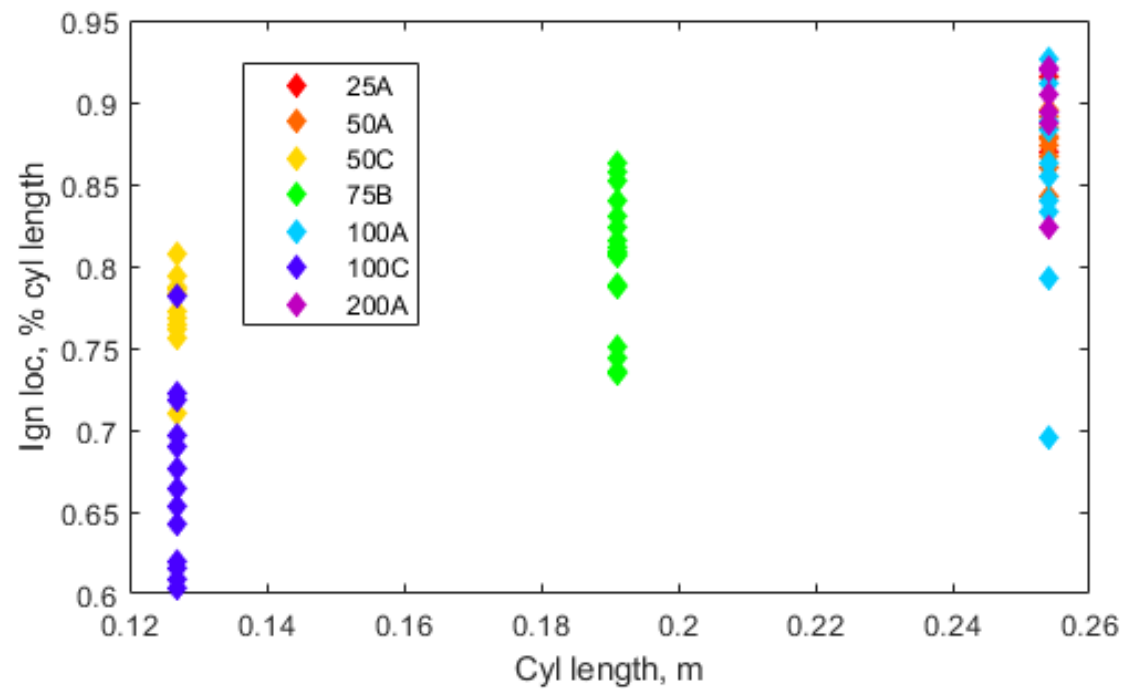

(b) ignition location in terms of \% cylinder length vs. cylinder length

Figure 4.36: Scatter plots of (a) $T_{i g n}$ vs ignition location in terms of \% cylinder length or (b) ignition location in terms of \% cylinder length vs. cylinder length.

given geometry, initial pressure, and fuel type, Ono et al. (1976) demonstrates that experimental data on ignition temperature and surface height are well correlated by,

$$
\ln (L)=\frac{C_{1}}{T_{i g n}}+C_{2}
$$


or

$$
T_{i g n}=\frac{C_{1}}{\ln (L)-C_{2}},
$$

$C_{1}$ and $C_{2}$ are empirical constants determined from fitting the experimental data. Ono et al. (1976) demonstrated the validity of this correlation on vertical heated plates with $0.5<L<3 \mathrm{~cm}$. The present data extends the range of $L$ up to 25 $\mathrm{cm}$ for vertical heated cylinders. All of these results are in the laminar flow regime $G r \leq 10^{7}$. In contrast to the very modest decrease in ignition temperature with increasing length, the Grashof number will increase as the cube of characteristic length, $G r=\frac{g \beta\left(T_{i g n}-T_{\infty}\right) L^{3}}{v^{2}}$ or $G r \propto L^{3}$. The comparison of the Ono correlation with data from our laboratory on vertical heated cylinders is shown on Fig. 4.37. Although the data is very sparse, the trend is reasonable and predicts that for laminar flow, continuing to increase the cylinder height will result in further decreases in $T_{i g n}$, below $900 \mathrm{~K}$ for $L=1000 \mathrm{~cm}$. However, as shown on the plot, the dramatic increase in $\mathrm{Gr}$ with length is predicted to result in transition to turbulence, which will likely have a significant impact on the ignition temperature and an altogether different scaling relationship than for laminar flow.

As shown in Fig. 4.37, the proximity of the Grashof number of these cylinders (as large as $\mathrm{Gr}=4.2 \times 10^{7}$ ) to numbers associated with a transition to turbulence $\left(\mathrm{Gr} \approx 10^{8}\right)$, we anticipate that increasing the characteristic lengths of the cylinders would quickly cause the external natural convection flow to transition to turbulence. We anticipate that transition to turbulence will dramatically change the ignition behavior, but the direction and magnitude of this effect on ignition temperature is at present not known. Analysis and experimentation in the turbulent flow regime will be required to resolve this issue.

\section{Chemiluminescence Visualization of Ignition}

$\mathrm{OH}^{*}$ chemiluminescence using the methodology described in Section 2.7 is observed for three experiments each with cylinders $100 \mathrm{C}$ and 200A. In this section, we present the results of shot 179, an ignition experiment performed with cylinder $100 \mathrm{C}$ in a stoichiometric hexane-air mixture with a surface temperature of $1090 \mathrm{~K}$ at ignition. Figure 4.38 documents the formation of the ignition kernel and beginnings 


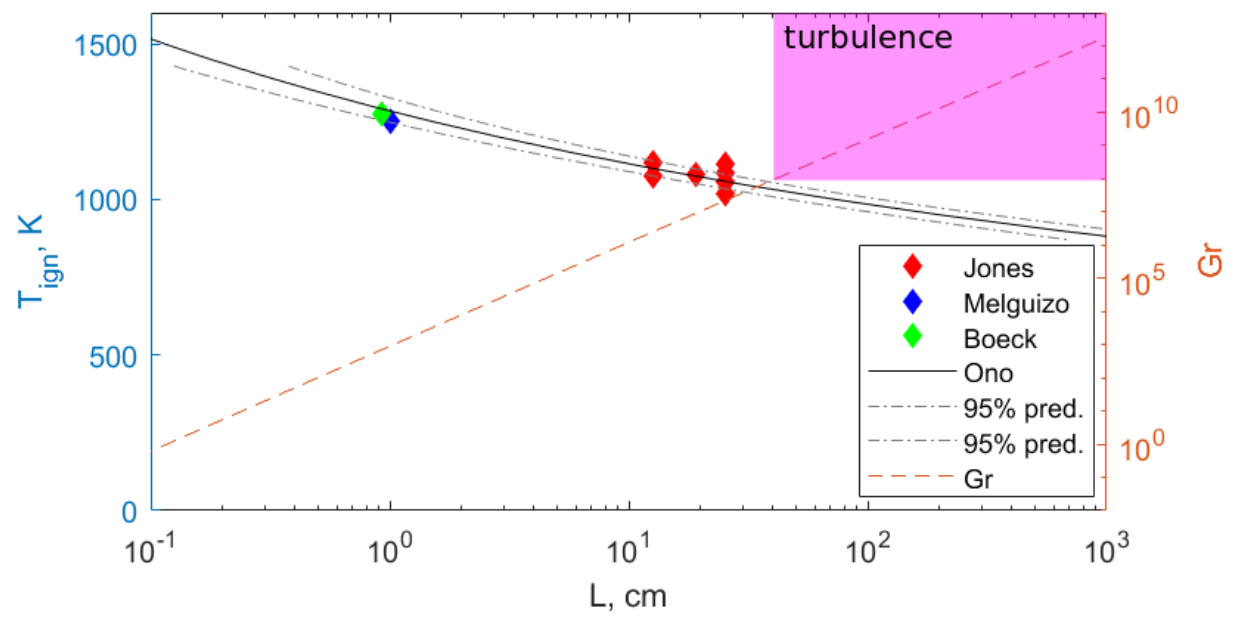

Figure 4.37: Comparison of the Caltech data with Ono correlation: $\ln (L)=$ $C_{1} / T_{i g n}+C_{2}$. Through a linear regression analysis of the data, we find $C_{1}=$ $19381 \pm 2869 \mathrm{~K}, C_{2}=-15.09 \pm 2.58$. Note $C_{1}$ is reasonably consistent with the value of $E_{a} / R=20740 \mathrm{~K}$ predicted independently from analysis of a detailed hexane mechanism by Boeck et al. (2017). The gray dot-dashed lines represent the $95 \%$ prediction limits (uncertainty associated with using this correlation to predict future measurements). Details on the linear regression analysis are given in Navidi (2007).

of flame propagation.

The ignition kernel forms on the side of the cylinder facing the camera, as seen at $609 \mathrm{~ms}$ prior to the trigger time. Careful visual inspection found a small, faint circle of bright pixel inside the red dashed region of the second frame of Fig. 4.38, Each bright pixel indicates emission from an excited $\mathrm{OH}$ radical; the field of view is completely dark until ignition occurs and $\mathrm{OH}$ radicals are produced in sufficiently large amounts that emission is captured by the $\mathrm{OH}^{*}$ chemiluminescence setup. The ignition kernel grows into larger and larger circular regions for the next $2 \mathrm{~ms}$, until $607 \mathrm{~ms}$ prior to the trigger time. At this time ( $2 \mathrm{~ms}$ after ignition), the circular flame front (as it is observed from the line of sight of the camera) starts to develop into an oblong shape with the long axis oriented vertically. This "barrel" shape becomes more oblong as time continues.

Figure 4.39 shows a side-by-side comparison of ignition sequences for cylinder 100C using all three ignition visualization techniques. Shot 160 is used for interferometry, shot 173 for dot schlieren, and shot 179 for $\mathrm{OH}^{*}$ chemiluminescence. The surface 


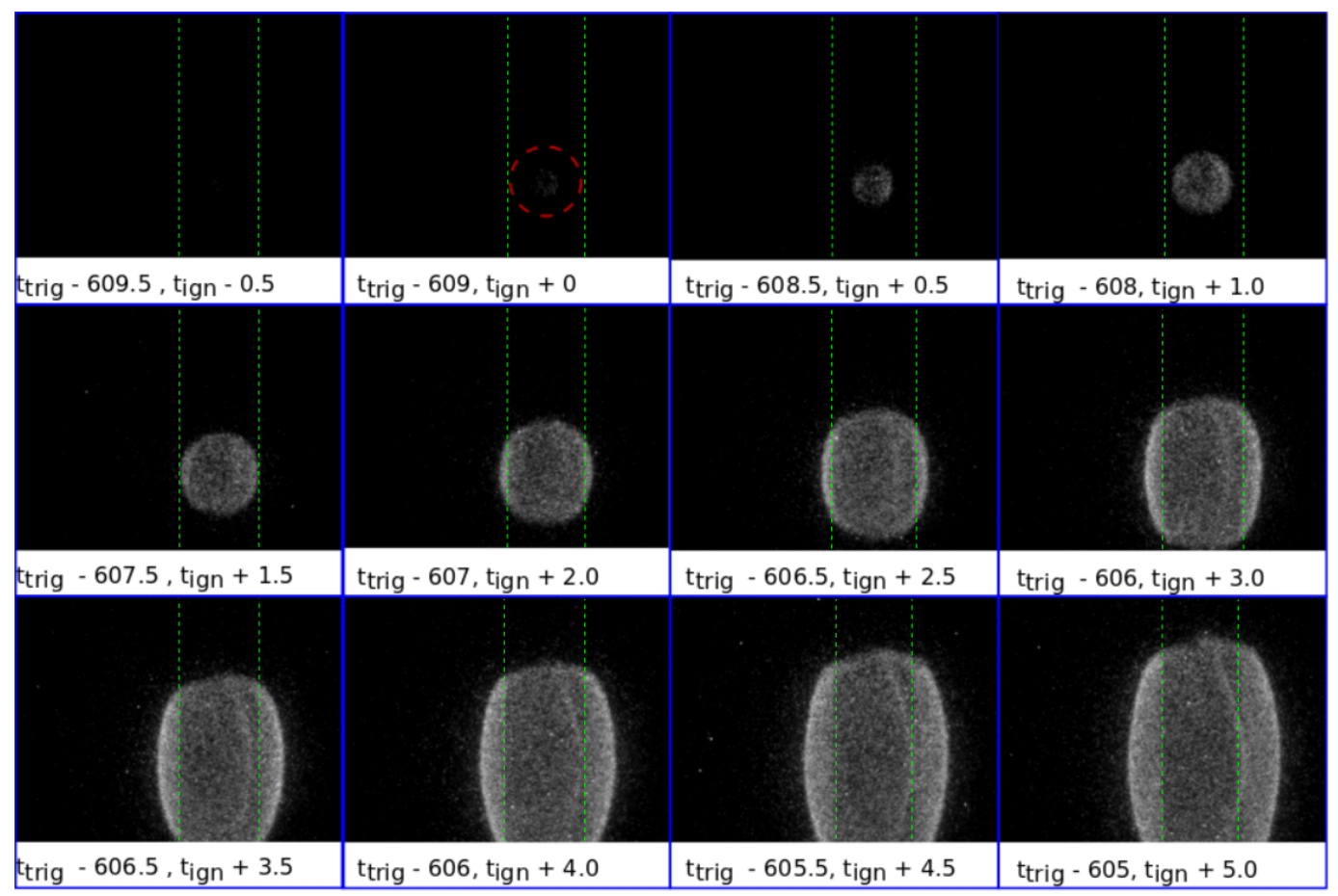

Figure 4.38: $\mathrm{OH}^{*}$ chemiluminescence visualization of ignition from shot 179 . Camera frame rate at $2000 \mathrm{fps}$. Time given relative to trigger and to first sign of ignition for each frame in units of miliseconds. Visualization over the first $5 \mathrm{~ms}$ after ignition. Surface temperature at ignition is $1090.2 \mathrm{~K}$.

temperature at ignition was 1093,1080 , and $1090 \mathrm{~K}$ for shot 160,173 , and 179, respectively. Frames are compared at similar times relative to ignition across all three visualization techniques. For uniformity, ignition is considered to be the point at which the flame is visible outside of the cylinder surface, as the cylinder itself blocks any information about the flame before it propagates outside the edges of the cylinder for interferometry and schlieren. The general "barrel" shape of the flame observed in Fig. 4.38, created as the flame propagates faster in the vertical direction than the horizontal direction, is also observed with interferomety and schlieren imaging. This confirms that each technique observes similar ignition dynamics and flame propagation.

\section{Flame Speed Measurements}

The $\mathrm{OH}^{*}$ chemiluminescence allows us to track the position of the flame front from the first sign of ignition. Using simple edge-finding image-processing techniques, we can estimate the speed of the flame in both horizontal and vertical directions. The results are shown in Fig. 4.40. The left, right, top, and bottom edges of the 
(a) shot 160

trigger at $\mathrm{t}=171.1 \mathrm{~s}$ (b) shot 173

trigger at $\mathrm{t}=227.4 \mathrm{~s}$ (c) shot 179

trigger at $\mathrm{t}=142.3 \mathrm{~s}$

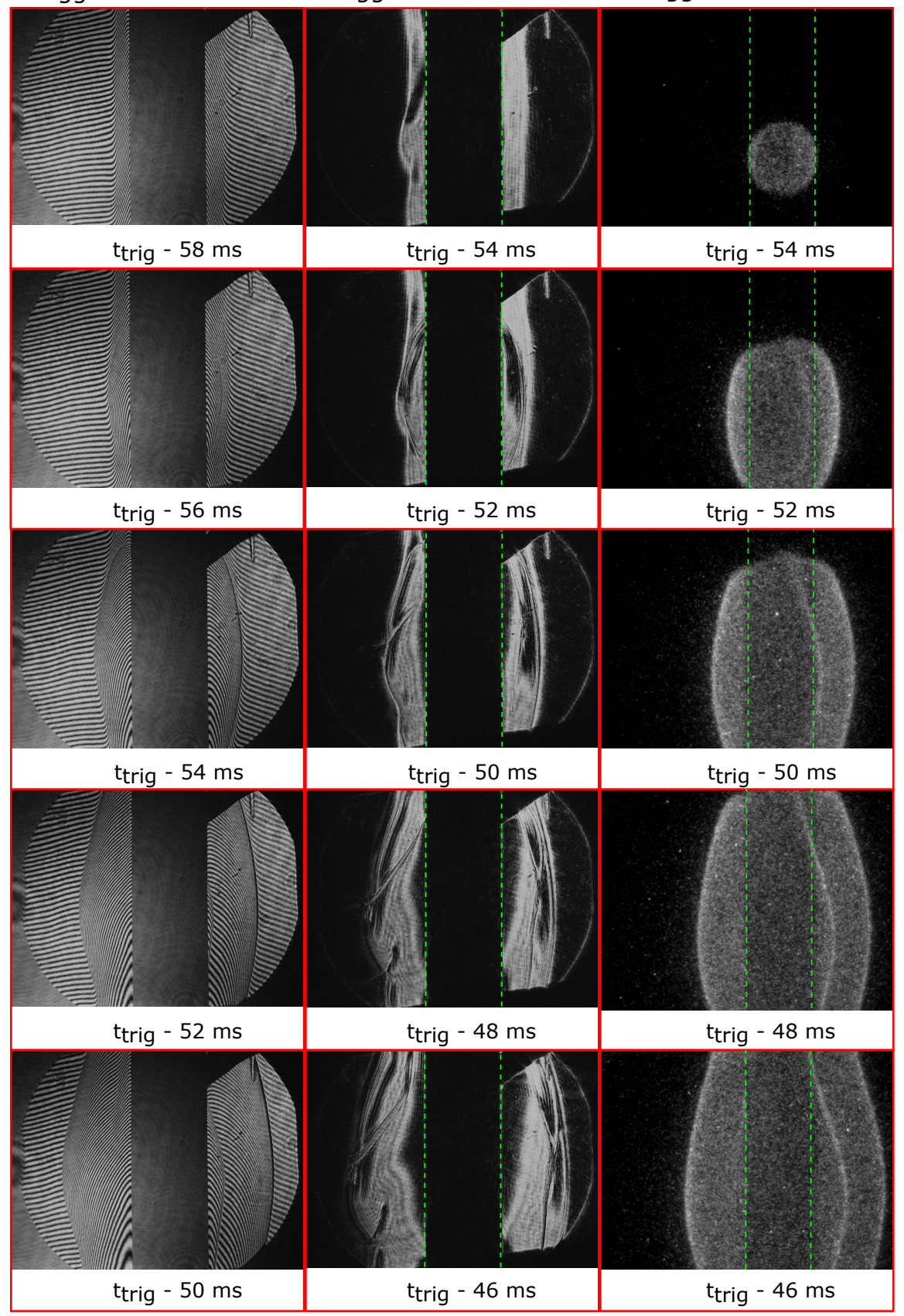

Figure 4.39: Side-by-side visualization of ignition from 100C for Shots 160, 173, and 179 using interferometry, schlieren imaging, and $\mathrm{OH}^{*}$ chemiluminescence. Time given relative to trigger for each frame in units of miliseconds. Visualization over the first $10 \mathrm{~ms}$ after ignition, defined here as the first sign of ignition appearing past the surface the cylinder. Ignition occured at surface temperatures of 1093, 1080 , and $1090 \mathrm{~K}$, respectively. 


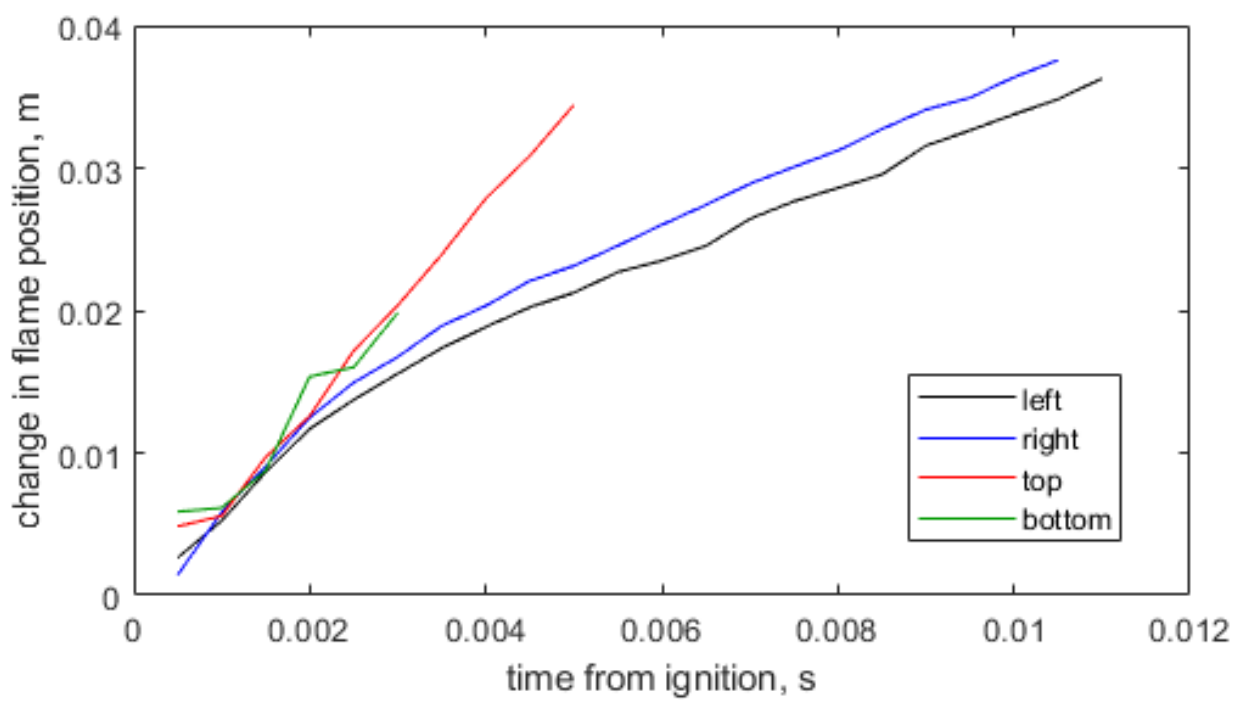

Figure 4.40: Tracking flame position relative to initial location ignition kernel for Shot 179. The position of the left edge (black), right edge (blue), top edge (red), and bottom edge (green) of the flame are tracked.

flame are tracked and plotted as black, blue, red, and green lines respectively. The bottom edge of the flame reaches the edge of the field of view first, followed by the top edge. The left- and right-most edges of the flame remain in the field of view longer. What we see in Fig. 4.40 is that the flame propagates at roughly the same speed in the first $2 \mathrm{~ms}$ after ignition. This matches the observation from Fig 4.38 that the flame front as viewed by the line of sight of the camera is circular during this early stage. The flame front is actually three-dimensional, and likely propagating as a sphere during this early stage of ignition, but the $\mathrm{OH}^{*}$ visualization only observes the motion in two dimensions. The flame speed is approximately $6.4 \mathrm{~m} / \mathrm{s}$ for the first $2 \mathrm{~ms}$ after ignition. After $2 \mathrm{~ms}$ have elapsed, the horizontal flame speed slows down noticeably, marked by a flattening of the slope of the position data shown in Fig. 4.40 for the left and right edges to a speed of approximately $2.7 \mathrm{~m} / \mathrm{s}$. It is difficult to tell from the limited data if the bottom of the flame front slows down in the same manner, but it is evident that the top edge of the flame does not slow down. Indeed, its flame speed remains constant at approximately $6.4 \mathrm{~m} / \mathrm{s}$ up until the point at which further movement of the top of the flame occurs outside of the field of view.

The observations in shot 179 are compared with flame speed extracted from following the flame front in raw interferograms. Examples from experiments with cylinder $100 \mathrm{C}$ are used, as are experiments with cylinder 75B. The velocity of the freely- 
propagating flame in all directions is tabulated in Table 4.6. Note that depending on the circumferential location of the ignition kernel, some information is lost about the early stages of ignition due to the cylinder surface blocking light.

\begin{tabular}{cc|cccc}
\hline Cyl Tag & Shot Number & $V_{s}$, left $(\mathrm{m} / \mathrm{s})$ & $V_{s}$, right $(\mathrm{m} / \mathrm{s})$ & $V_{s}$, top $(\mathrm{m} / \mathrm{s})$ & $V_{s}$, bottom $(\mathrm{m} / \mathrm{s})$ \\
\hline 100C & $179\left(\mathrm{OH}^{*}\right)$ & 2.7 & 2.8 & 6.6 & 5.6 \\
100C & 163 & 3.1 & 3.5 & 6.8 & 6.3 \\
$75 \mathrm{~B}$ & 23 & 2.9 & 3.3 & 5.5 & 4.7 \\
$75 \mathrm{~B}$ & 29 & 2.7 & 2.8 & 7.5 & 7.9 \\
\hline
\end{tabular}

Table 4.6: Flame speed of hexane-air mixture

As discussed previously, the flame propagates faster in the vertical direction, where it remains in the high temperature thermal boundary layer, than in the horizontal direction, where it propogates into the cool ambient conditions. To check the accuracy of the flame speeds found from experiments, we must calculate the flame speed under different temperature conditions. The flame speed, $V_{s}$, is calculated as the product of the laminar burning speed, $S_{L}$ and the expansion ratio, $\sigma$, where the expansion ratio is the ratio of the unburnt to burnt gas density:

$$
V_{s}=S_{L} \sigma=S_{L} \frac{\rho_{u}}{\rho_{b}} .
$$

The laminar burning speed is found via a calculation based on the Cantera routine for calculating adiabatic one-dimensional flame properties (adiabatic_flame.py). The expansion ratio is found by comparing the denisty of the initial gas mixture to the density after equilibration (at constant enthalpy and pressure), effectively creating a burned mixture. The theoretical values of laminar burning speed, expansion ratio, and flame speed are shown in Table 4.7 for temperatures of 300, 700, and $1100 \mathrm{~K}$. These temperatures correspond to ambient temperature, film temperature, and wall temperature, respectively.

\begin{tabular}{cccc}
\hline Temperature $(\mathrm{K})$ & $L_{S},(\mathrm{~m} / \mathrm{s})$ & $\sigma$ & $V_{s},(\mathrm{~m} / \mathrm{s})$ \\
\hline 300 & 0.37 & 8.08 & 3.05 \\
700 & 1.70 & 3.77 & 6.40 \\
1100 & 4.92 & 2.60 & 12.8 \\
\hline
\end{tabular}

Table 4.7: Flame speed calculations for hexane at varying temperatures.

From Table 4.7, we note that the components of flame speed scale approximately with temperature. The ratio of the laminar burning speed between any two temperatures 
scales as approximately the square of the temperature ratio (Dugger and Graab, 1953; Gaydon and Wolfhard, 1970; Konnov, Meuwissen, and Goey, 2011). The expansion ratio meanwhile scales as approximately the inverse of the temperature ratio. Using the ideal gas law we find that:

$$
\frac{\sigma_{1}}{\sigma_{2}}=\frac{\rho_{u_{1}} / \rho_{b_{1}}}{\rho_{u_{2}} / \rho_{b_{2}}} \approx \frac{T_{b_{1}} / T_{u_{1}}}{T_{b_{2}} / T_{u_{2}}} \approx \frac{T_{u_{2}}}{T_{u_{1}}} .
$$

This approximation assumed that the burned gas temperature remains constant even with changes in the initial temperature. This is not exact, the burned gas temperature increases slightly with increasing unburned gas temperature, but this is a slight change. The burned gas temperature at $1100 \mathrm{~K}$ increased by less than $10 \%$ compared to the burned gas temperature at $300 \mathrm{~K}$. This approximation is close enough to make some simple scaling arguments. When the laminar burning speed and expansion ratio scalings are combined, we find that the ratio of the flame speed scales approximately with the temperature ratio.

The flame speeds found in the experiments are in reasonable agreement with the computed flame speeds. In particular, the average horizontal flame speed $(2.98 \mathrm{~m} / \mathrm{s})$ from the experiments is within $2.5 \%$ of the flame speed calculated for $300 \mathrm{~K}$. That supports the assumption that the lower flame speed in the horizontal directions is due to the lower temperature of the gas outside the boundary layer. The average vertical flame speed $(6.36 \mathrm{~m} / \mathrm{s})$ meanwhile, is within $1 \%$ to the flame speed calculated for $700 \mathrm{~K}$, the film temperature of the boundary layer. Again, this supports the previous claim that the flame propagates faster in the vertical direction because it remains in the high temperature boundary layer where the flame speed is higher. While the circumfrential propagation speed of the flame cannot be observed directly due to the line of sight of the cameras, we hypothesize that the flame travels around the the circumference of the cylinder at a similar speed to its vertical propagation speed. Since the flame will remain near the heated surface as it travels circumfrentially around the cylinder, we expect to see flame speeds near $6 \mathrm{~m} / \mathrm{s}$ due to the increased flame speed in the high temperature boundary layer. Only when the flame moves away from the heated surface and out of the heated boundary layer do we expect to see the flame speed drop to about $3 \mathrm{~m} / \mathrm{s}$, the expected flame speed in the ambient mixture.

It is interesting to note the vertical flame speeds are observed at mixture temperatures much higher than traditional flame speed experiments can achieve. The highest 
mixture temperature reported in a broad review of work on flame speeds by Ranzi et al. (2012) was $470 \mathrm{~K}$, while the film temperature in this work is approximately 700 $\mathrm{K}$. While these experiments are not specifically designed to measure flame speeds, it is possible to take some measurements of flame speeds at unique high temperature and low pressure conditions.

It is interesting to note that there is no strong indication that the flame propagates significantly faster upward along the cylinder (aided by buoyancy and free convection) than downwards along the cylinder (opposed to buoyancy and free convection). There are too few frames with the bottom of the flame in view in shot 179 to draw firm conclusions about its speed, and the only other experimental result that indicates the speed of the top edge is significantly faster than the bottom edge is shot 23. This is an outlier that will be discussed shortly. We know that gravity is critically important in creating the buoyancy-driven natural convection flow that forms the thermal layer and initial conditions for ignition, but its influence during the ignition transient appears limited. We can estimate the additional velocity created by gravitational acceleration during the observation time as $\Delta V=\frac{\Delta \rho}{\rho} g \Delta t$, where $\Delta V$ is the change in velocity due to gravitational/buoyancy forces, $\Delta \rho$ is the change in density across the flame, and $\Delta t$ is the observation time. Even if $\frac{\Delta \rho}{\rho}=1$, the maximum possible value, $\Delta V$ remains small $(\approx 0.1 \mathrm{~m} / \mathrm{s})$ during the short observation time of the flame $(\Delta t \approx 10 \mathrm{~ms})$. The thermodynamics and chemistry are far more important drivers of the ignition dynamics and flame speed than buoyancy forces during the early stages of ignition and flame propagation.

As mentioned previously, out of the interferometry flame speed results, shot 23 is the only one that indicates a significantly slower flame speed at the bottom edge of the flame versus the top. Test 23 is also atypical in comparison to the other tests in Table 4.6 in that there is a significant disturbance to the thermal boundary layer structure immediately preceding ignition, and this could be a cause for the variation seen in the flame speed results of Shot 23. The moving disturbances, particularly those where there appears to be a ejection of hot fluid into cooler regions of the boundary layer, could convect the flame upwards in a manner that creates the observed asymmetry in the vertical velocities of Shot 23. 


\section{Disturbances to the Natural Convection Flow}

It is important to point out that the disturbances observed via visualization are intermittent, and the presence of one does not guarantee an ignition event. Some experiments ignite in what appears to be a well-structured, laminar boundary layer, while in other experiments, the ignition kernel forms within a disturbance. Some disturbances pass through the boundary layer without igniting the mixture; they are observed in experiments that never ignite. These disturbances seem to occur randomly, and may or may not be connected with ignition.

There are two potential explanations for the presence of the thermal layer disturbances observed in some tests; the first involves the flow regime transitioning out of a steady, laminar flow regime towards a more turbulent flow structure. We can use the Grashof number, Gr, to estimate which part of the flow regime the cylinder is in. The Grashof number approximates the ratio of buoyancy forces to viscous forces in a natural convection flow, analogous to the use of Reynolds number for forced convection flows. The Grashof number is:

$$
G r=\frac{g \beta\left(T-T_{\infty}\right) L^{3}}{v^{2}}
$$

where $g$ is gravitational acceleration, $\beta$ is the thermal expansion coefficient, com-

monly approximated as $\frac{1}{T}$ for gases, $T$ is the wall temperature, $T_{\infty}$ is the ambient temperature, $L$ is the characteristic length, and $v$ is the kinematic viscosity. For flat plates (an approximately analogous condition for the vertical cylinder), transition to turbulence occurs in the range from $10^{8}<G r<10^{9}$ (Smith, Inomata, and Peters, 2013), (Bahrami, 2009). The Grashof numbers for the cylinders used in testing, using a wall temperature of $1100 \mathrm{~K}$, can be as high as $4 \times 10^{7}$. This is close to the range of Grashof numbers at which transition to turbulence occurs, so it is not surprising to observe some unsteady features in the boundary layer.

Another potential explanation for the disturbances in the boundary layer is the possibility of significant decomposition of hexane near the hot wall. As will be discussed in detail in Chapter 6, hexane can exhibit two-stage ignition behavior; the first stage primarily consists of hexane breaking down into other hydrocarbon species. Significant reaction can occur without causing a thermal explosion (i.e. traditional ignition) event. Some of the disturbances observed in the boundary 
could arise due to the decomposition of hexane, which could significantly alter the index of refraction in the boundary layer or could alter the structure of the thermal boundary layer directly due to gas dynamics from reaction, such as ejection of hot decomposition products into the cooler regions of the natural convection flow.

The appearance of a "normally" structured laminar boundary layer over the cylinder surface is documented in Figs. 4.41 and 4.42 for schlieren and interferometry, respectively, in subfigure (a). This contrasts with two different types of disturbances typically seen in the ignition tests: the first type is a "vortical-appearing structure" near the very top of the cylinder. There is a certain periodicity at which these structures occur. This type of disturbance is shown in subfigure (b) in Figs. 4.41 and 4.42. Figure 4.43 shows a sequence of images in which the vortical-appearing structure propagates up the length of the cylinder. This type of vortical-appearing structure disturbance might be attributed to the influence of large Grashof numbers and the flow structure becoming unsteady as it approaches a transistion to turbulence. It is also possible these structures may be unique to these experiments due to the combination of large characteristic length and large temperature difference between surface and ambient mixture. It is observed prior to ignition in hexane tests as well as nitrogen tests, and consequently does not seem to be influenced by reactive versus non-reactive mixture. For this reason, it is possible that instability in the flow associated with incipient transition to turbulence at a high Grashof number could be responsible for this type of disturbance.

The other type of disturbance observed is shown in subfigure (c) in Figs. 4.41 and 4.42, this is referred to as a "puffing" disturbance. This type of disturbance does not look like the vortical-appearing structure disturbance. It generally is more random in shape and size and less structured than other disturbances. This puffing disturbance is difficult to characterize, and it is not clear what causes its appearance.

In general, the cause of either type of disturbance is not well understood. There is limited literature on the instabilities of natural convection flows, (Polymeropoulos and Gebhart, 1967), and the nature of unstable structures is not well identified (Hattori et al., 2006) compared with the large body of work on instabilities in forced convection boundary layer flows. A possible direction for future research would be efforts to better characterize and understand the causes of this intermittent distur- 
bance behavior in a reactive natural convection flow.

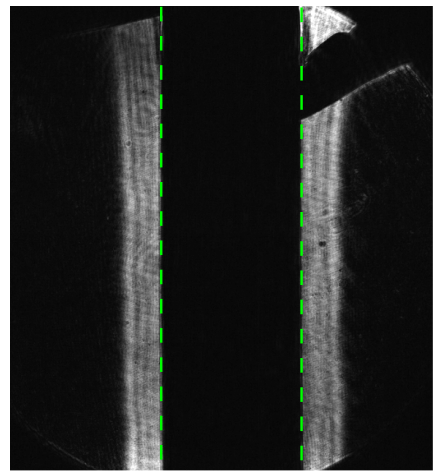

(a)

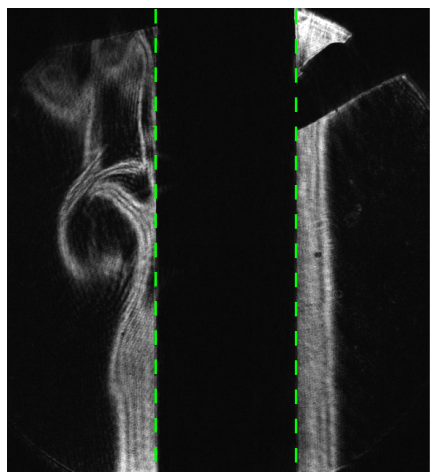

(b)

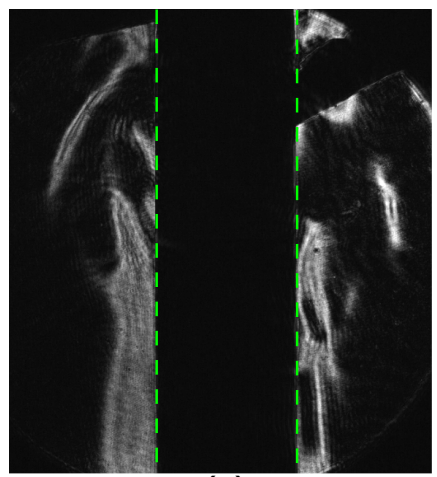

(c)

Figure 4.41: Schlieren images with examples of (a) a normally structured laminar boundary layer (shot 149, frame -2578), (b) a "vortical-appearing structures" disturbance (shot 149, frame -2990), and (c) a "puffing" disturbance (shot 155, frame -761).

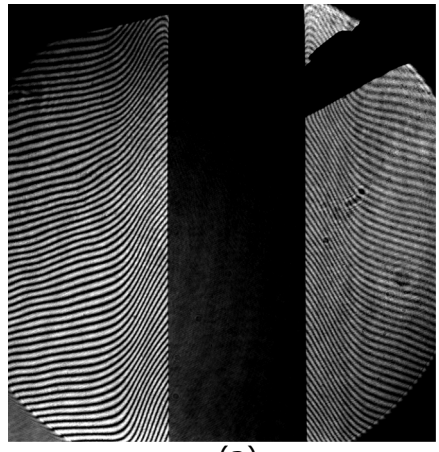

(a)

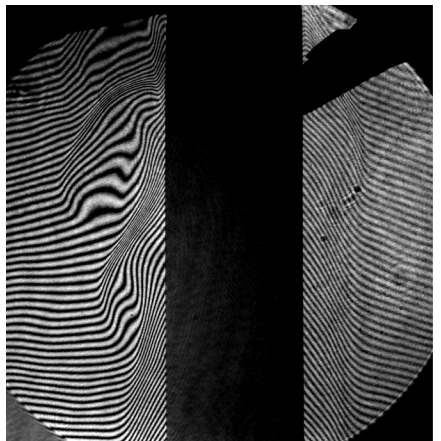

(b)

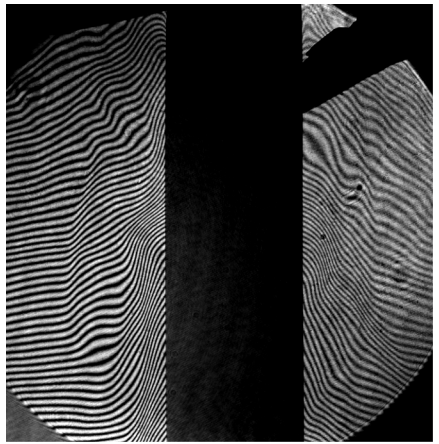

(c)

Figure 4.42: Raw interferogram images with examples of (a) a normally structured laminar boundary layer (shot 143, frame -3178), (b) a "vortical-appearing structure" disturbance (shot 143, frame-4904), and (c) a "puffing" disturbance (shot 143, frame -3674). 


\section{shot 154}

trigger at $\mathrm{t}=116.7 \mathrm{~s}$

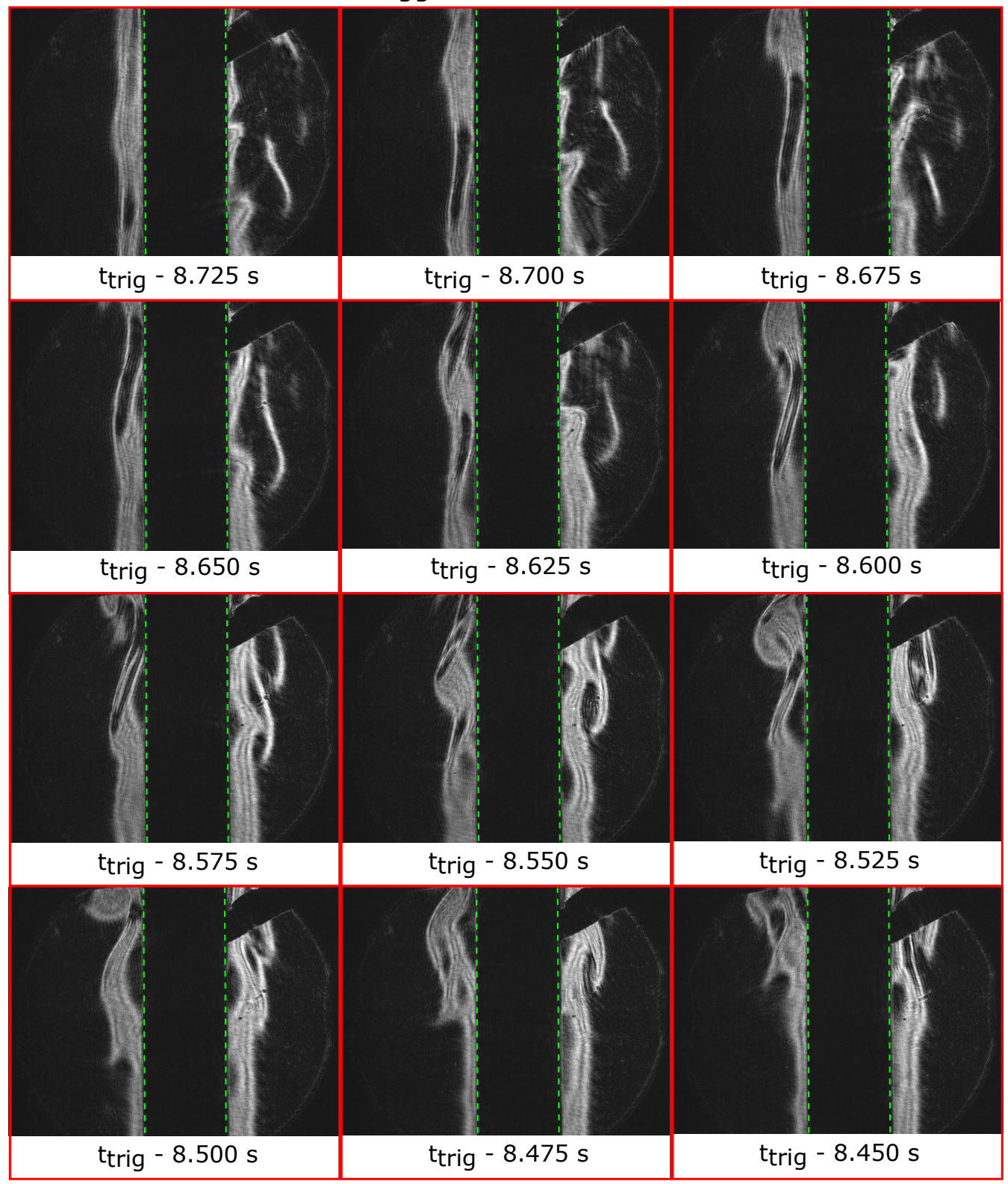

Figure 4.43: Dot schlieren images of a sequence of "vortical-appearing structures" disturbances propagating up the length of the cylinder. Shot 154. 


\section{BIBLIOGRAPHY}

Adomeit, G. (1965). "Ignition of gases at hot surfaces under nonsteady-state conditions". In: Symposium (International) on Combustion. Tenth Symposium (International) on Combustion 10.1, pp. 237-243.

Alkidas, A. and P. Durbetaki (1973). "A Simplified Model for Stagnation Heat Transfer and Ignition of a Gaseous Mixture”. In: Combustion Science and Technology 7, pp. 135-140.

ASTM International (2015). ASTM E659-15, Standard Test Method for Autoignition Temperature of Chemicals.

Bahrami, M. (2009). Natural Convection. uRL:https://www . sfu . ca/ mbahrami// ENSC\%5C\%20388/Notes/Natural\%5C\%20Convection.pdf

Bane, S et al. (2011). "Statistical analysis of electrostatic spark ignition of lean $\mathrm{H}_{2} / \mathrm{O}_{2}$ /Ar mixtures." In: International Journal of Hydrogen Energy 36, pp. 23442350 .

Boeck, Lorenz R. et al. (2017). "Ignition of fuel-air mixtures from a hot circular cylinder". In: Combustion and Flame 185, pp. 265-277.

Boettcher, Philipp (2012). “Thermal Ignition”. PhD thesis. California Institute of Technology.

Coronel, Stephanie A. (2016). "Thermal Ignition Using Moving Hot Particles". $\mathrm{PhD}$ thesis. California Institute of Technology.

Dugger, Gordon L. and Dorothy D. Graab (1953). "Flame velocities of hydrocarbonoxygen-nitrogen mixtures". In: Symposium (International) on Combustion 4.1, pp. 302-310.

Gardiner, W. C. Jr., Y. Hidaka, and T Tanzawa (1981). "Refractivity of Combustion Gases". In: Combustion and Flame 40, pp. 213-219.

Gaydon, A. G. and Hans G Wolfhard (1970). "Flames; their structure, radiation and temperature". In: Distributed in the U.S.A. by Barnes and Noble, 401 p. URL: http://caltech.tind.io/record/429665.

Hattori, Yasuo et al. (2006). "Turbulence characteristics of natural-convection boundary layer in air along a vertical plate heated at high temperatures". In: International Journal of Heat and Fluid Flow 27.3, pp. 445-455.

Hosmer, D., S. Lemeshow, and R. Sturdivant (1989). Applied Logistic Regression. John Wiley \& Sons.

Khitrin, L. and S. Goldenberg (1957). "The influence of the initial temperature of a combustible mixture and of the ambient pressure on the stabilization limits". In: Symposium (International) on Combustion 6.1, pp. 448-451. 
Konnov, A.A., R.J. Meuwissen, and L.P.H. de Goey (2011). "The temperature dependence of the laminar burning velocity of ethanol flames". In: Proceedings of the Combustion Institute 33.1, pp. 1011-1019.

Kuchta, J., A. Bartkowiak, and M. Zabetakis (1965). "Hot Surface Ignition Temperatures of Hydrocarbon Fuel Vapor-Air Mixtures". In: Journal of Chemical Engineering Data 10.3, pp. 282-288.

Laurendeau, Normand M. (1982). "Thermal ignition of methane-air mixtures by hot surfaces: A critical examination". In: Combustion and Flame 46, pp. 29-49.

Law, C. K. and H. K. Law (1979). "Thermal-ignition analysis in boundary-layer flows". In: Journal of Fluid Mechanics 92.1, pp. 97-108. (Visited on 04/01/2020).

Law, C.K. (1979). "Transient ignition of a combustible by stationary isothermal bodies". In: Combustion Science and Technology 19, pp. 237-242.

Martin, C. and J.E. Shepherd (2020). "Low Temperature Autoignition of Jet A and Surrogate Jet Fuels". In: 13th International Symposium on Hazards, Prevention, and Mitigation of Industrial Explosions.

Melguizo-Gavilanes, J. et al. (2016). "Hot Surface Ignition of n-Hexane Mixtures using Simplified Kinetics". In: Combustion Science and Technology 188.11-12, pp. 2060-2076.

Navidi, W. (2007). Statistics for Engineers and Scientists. McGraw-Hill Higher Education.

Nestor, O. H. and H. N. Olsen (1960). "Numerical methods for reducing line and surface probe data”. In: SIAM Review 2.3, pp. 200-207.

Neter, J. et al. (1996). Applied Linear Statistics Models. McGraw Hill.

Ono, S. et al. (1976). "Ignition in a Free Convection from Vertical Hot Plate". In: Bulletin of JSME 19.132, pp. 676-683.

Polymeropoulos, Constantine E. and Benjamin Gebhart (1967). "Incipient instability in free convection laminar boundary layers". In: Journal of Fluid Mechanics 30.2, pp. 225-239. IssN: 0022-1120, 1469-7645.

Ranzi, E. et al. (2012). "Hierarchical and comparative kinetic modeling of laminar flame speeds of hydrocarbon and oxygenated fuels". In: Progress in Energy and Combustion Science 38.4, pp. 468-501.

Smith, Richard, Hiroshi Inomata, and Cor Peters (2013). "Chapter 8 - Heat Transfer and Finite-Difference Methods". In: Introduction to Supercritical Fluids. Vol. 4. Supercritical Fluid Science and Technology. Elsevier, pp. 557-615.

Song, X., L.D. Schmidt, and R. Aris (1991). "The Ignition Criteria for StagnationPoint Flow: SemenovFrank-Kamenetski or van't Hoff'. In: Combustion Science and Technology 75.4-6, pp. 311-331. 
White, R.G. (1967). Spontaneous ignition of kerosene/AVTUR/vapour - the effect of the ratio, vessel surface area to volume (effect of vessel surface area on minimum spontaneous ignition temperature of kerosene). Tech. rep. 67107. Royal Aircraft Establishment. 


\section{Chapter 5}

\section{HEAVIER FUELS: JET A AND SURROGATES}

Jet fuel is a commodity product, and as such contains hundreds of different hydrocarbon species. The composition is imprecise and varies between different batches depending on the country of origin, refinery, and time of year. This makes it difficult to get reproducible results in the laboratory across different fuel batches. The complexity of jet fuel also makes it challenging to model the chemistry of reaction with a detailed chemical reaction mechanism. To address these issues, significant effort has gone into identifying simpler hydrocarbon mixtures that can reproduce important characteristics of commodity jet fuel. These are called surrogate fuels, and can be designed to reproduce ignition delay time, cetane number, and/or laminar flame speed while only using a small number of species. The composition of these surrogate fuels is well controlled, which allows reproducible experimental results. The relative simplicity of these fuels also enables numerical modeling of reaction pathways.

Several surrogate fuels have been developed in the literature to mimic the characteristics of Jet A ignition behavior at high pressures and temperatures $(\mathrm{P}=8.5-20 \mathrm{~atm}$, $\mathrm{T}=675-1750 \mathrm{~K})$. Work by Martin and Shepherd (2020) idenitified two multicomponent surrogate fuels, Aachen (Honnet et al., 2009) and JI Unified (Chen, Khani, and Chen, 2016), as suitable Jet A surrogates in the low pressure and temperature regime ( $\mathrm{P}=1 \mathrm{~atm}, \mathrm{~T}<675 \mathrm{~K}$ ) for autoignition testing. Based on the success of these surrogates in reproducing autoignition limits, we use Aachen and JI unified as surrogate fuels in our hot surface ignition tests. We will also work with a wellcontrolled and studied blend of Jet A (POSF-4658) that was also used by Martin and Shepherd (2020), and compare the ignition behavior of the two surrogate fuels to Jet A. It is worth noting that the two surrogates were formulated with different goals in mind; Aachen was designed to reduce to as few components as possible using one compound each for alkanes and aromatics (Honnet et al., 2009). JI was formulated with the vapor composition in mind; its distillation curve was matched to that of Jet A in order to mirror the droplet evaporation and ignition behaviors of Jet A (Chen, Khani, and Chen, 2016). The composition of each type of fuel in terms of alkanes and aromatics is shown in Table 5.1. The detailed composition of each fuel will be 
given in the following sections.

\begin{tabular}{|l|c|c|c|c|}
\hline & & \multicolumn{3}{|c|}{ \% by Weight } \\
\cline { 3 - 5 } & & Aachen Surrogate & JI Surrogate & POSF-4658 \\
\hline H/C ratio & & 2.016 & 2.019 & 1.935 \\
\hline $\begin{array}{l}\text { Avg. } \\
\text { Molecular } \\
\text { Formula }\end{array}$ & & $\mathrm{C}_{9.77} \mathrm{H}_{19.7}$ & $\mathrm{C}_{12.49} \mathrm{H}_{25.22}$ & $\mathrm{C}_{11.69} \mathrm{H}_{22.62}$ \\
\hline \multirow{4}{*}{ Alkanes } & & & & \\
& n-Alkanes & 80 & 29.17 & 19 \\
\cline { 2 - 5 } & iso-Alkanes & - & 46.53 & 31.34 \\
\cline { 2 - 5 } & cyclo-Alkanes & - & - & 28.42 \\
\cline { 2 - 5 } & Total & 80 & 75.7 & 78.76 \\
\hline \multirow{4}{*}{ Aromatics } & Alkylbenzenes & 20 & 4.94 & 13.69 \\
\cline { 2 - 5 } & Alkylnapthalenes & - & - & 1.76 \\
\cline { 2 - 5 } & Cycloaromatics & - & 24.35 & 21.24 \\
\cline { 2 - 5 } & Total & 20 & & \\
\hline
\end{tabular}

Table 5.1: Comparison of composition of jet fuel blend and surrogate fuels.

\subsection{Setup and Experimental Procedure}

The ignition tests planned involve the use of multi-component heavier hydrocarbon fuels. These fuels are not necessarily fully vaporized at room temperature, which presents a problem for measuring the gas composition via partial pressure measurements. With multi-component fuels, the heavier components will generally condense more readily than the light components, so if there is any condensation, the composition of the fuel vapor is not well known. To avoid these issues with condensation, the entire combustion vessel temperature must be raised to temperatures that allow for full vaporization of Aachen and JI surrogate fuels as well as Jet A fuel (Batch POSF-4658).

In previous work in the laboratory, the dew temperature of these fuels was calculated by Sund (2019), who found the dew temperature for a stoichiometric Aachen-air mixture was approximately $330 \mathrm{~K}$, and the dew temperature for a stoichiometric JI Unified-air mixture was approximately $365 \mathrm{~K}$. Jet A is handled in a different manner, as a large amount of fuel mass must be added to the vessel in order to ensure a reproducible gas composition. The details for calculating the conditions required to reach the desired composition for each fuel are discussed in the following sections. The preliminary dew temperatures from Sund (2019) present target temperatures 
for the heated vessel to reach.

In order to bring the entire vessel up to temperatures more than $80 \mathrm{~K}$ higher than ambient conditions, a heating system is installed. Power comes from two 3-phase, 208 V, 50 A supply lines, which are arranged in a wye configuration to create six single-phase lines. This creates six "zones" that can be separately powered and controlled, each with $120 \mathrm{~V}$ and $15 \mathrm{~A}$ for a power of $1.8 \mathrm{~kW}$ per zone. The total available power for the heating system is $10.8 \mathrm{~kW}$. Each zone has a number of flexible silicone-rubber heaters from Omega and a type E thermocouple arranged as shown in Fig. 5.1. The heaters are listed in Table 5.2. The heaters are arranged into zones such that there is a compromise between an even power split between zones, and keeping all heaters in a zone relatively close to one another enables accurate temperature monitoring by thermocouple.

Closed-loop control of temperature is achieved with Watlow 935A PID controllers. Each zone has a controller that is programmed to operate around a desired set point. The temperature for each zone is monitored via a type $\mathrm{E}$ thermocouple and fed into the PID controller, which provides either 0 or 5 VDC to a Watlow Dyn-A-Mite Solid State relay to turn supply power on or off to maintain the set point temperature. Thermal insulation blankets were installed around the vessel to minimize heat loss to the room.

The water cooling system was also modified for the heated vessel tests. The cooled plume plate was removed from the top of the vessel, and the NESLAB heat exchanger was not used. The heat exchanger has a cooling-only capacity and can achieve a maximum water temperature of $333 \mathrm{~K}$, which is the lowest target ambient temperature for these experiments. If we assume that the entire support structure will remain at the temperature of the water cooling system, there is a risk of the support structure being cool enough to cause some condensation of these multicomponent fuels. Therefore, the NESLAB cooling system was replaced with a small water pump in a 5-gallon bucket. Hot water was added to the bucket, and with the heat from operating the pump, the water temperature stabilized at about 353 $\mathrm{K}$. Vapor tests discussed in the following sections showed this to be a high enough water temperature to avoid condensation of the multicomponent fuels. Even at this higher temperature, the water is still able to remove enough heat from the support 

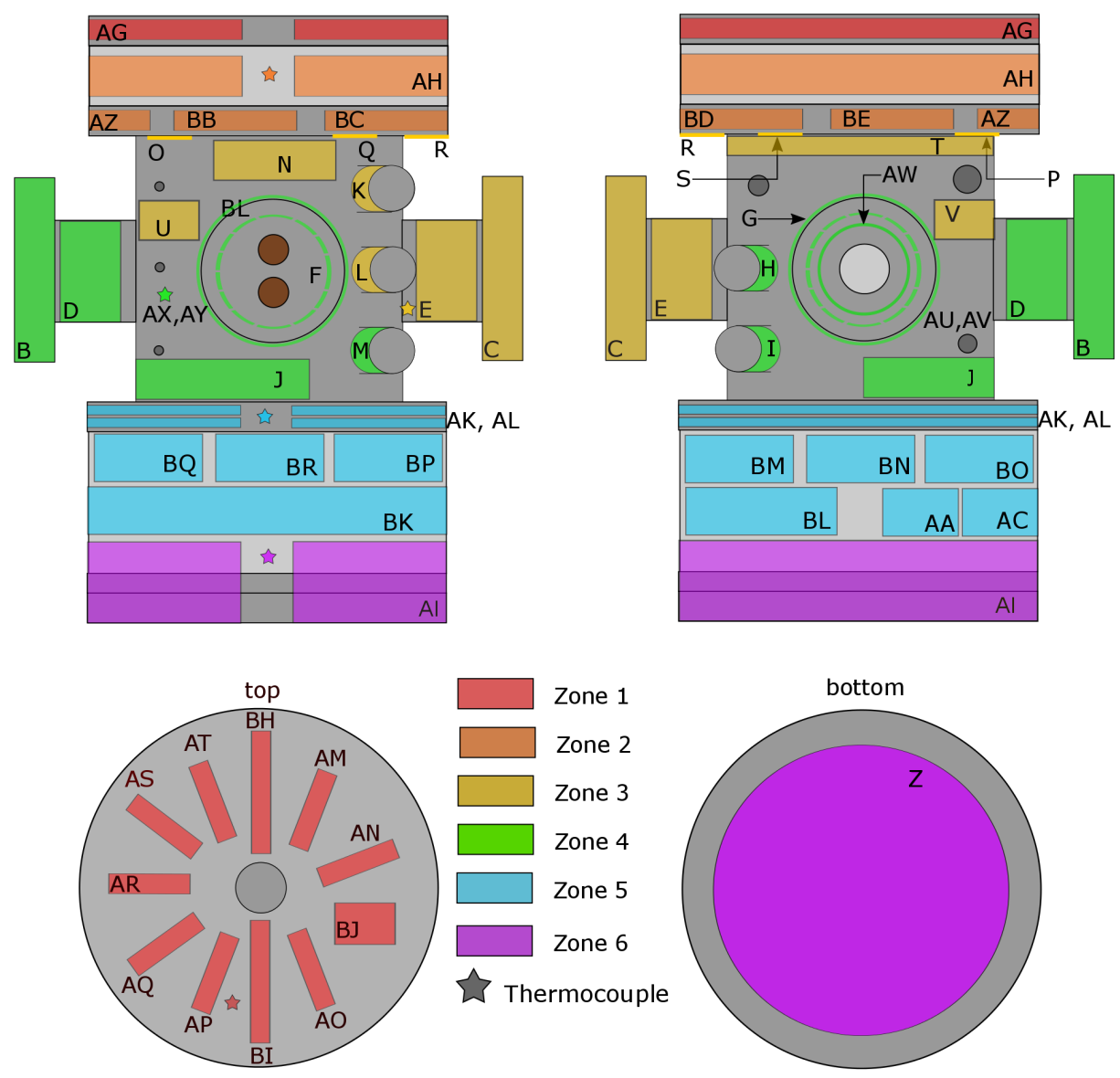

Figure 5.1: Schematic of heating system for vessel. Zones are color-coordinated, all heaters are labeled, and thermocouple placement for each zone is marked by an appropriately colored star.

structure during testing to avoid melting any components.

\subsection{Hexane with Heated Vessel}

The use of hexane as a fuel is repeated with an increased vessel temperature to identify what effect, if any, an increased ambient temperature has on ignition behavior. The vessel temperature used with hexane is $393 \mathrm{~K}$, to match the highest vessel temperature used in the surrogate experiments and set an upper bound on the effect of increased ambient temperature on ignition. The mixture composition used in the elevated temperature experiments is the same as for the room-temperature hexane experiments, specified in Table 4.1. Twenty individual experiments were performed with the elevated temperature hexane mixture, and a logistic regression was per- 


\begin{tabular}{|c|c|c|c|c|c|}
\hline Heater Tag & Zone No. & Power $(\mathrm{W})$ & Heater Tag & Zone No. & Power $(\mathrm{W})$ \\
\hline AM & 1 & 40 & $\mathrm{~L}$ & 3 & 90 \\
\hline $\mathrm{AN}$ & 1 & 40 & B & 4 & 240 \\
\hline $\mathrm{AO}$ & 1 & 40 & $\mathrm{D}$ & 4 & 160 \\
\hline $\mathrm{AP}$ & 1 & 40 & M & 4 & 90 \\
\hline AQ & 1 & 40 & $\mathbf{J}$ & 4 & 240 \\
\hline AR & 1 & 40 & $\mathrm{AU}$ & 4 & 80 \\
\hline AS & 1 & 40 & AV & 4 & 80 \\
\hline AT & 1 & 40 & AW & 4 & 80 \\
\hline $\mathrm{BH}$ & 1 & 60 & $\mathrm{AX}$ & 4 & 80 \\
\hline BI & 1 & 60 & AY & 4 & 80 \\
\hline BJ & 1 & 60 & $\mathrm{G}$ & 4 & 80 \\
\hline$A G$ & 1 & 480 & $\mathrm{AK}$ & 5 & 104 \\
\hline $\mathrm{AH}$ & 2 & 960 & $\mathrm{AL}$ & 5 & 104 \\
\hline $\mathrm{AZ}$ & 2 & 100 & BK & 5 & 300 \\
\hline BB & 2 & 100 & $\mathrm{BL}$ & 5 & 90 \\
\hline $\mathrm{BC}$ & 2 & 100 & $\mathrm{BM}$ & 5 & 80 \\
\hline $\mathrm{BD}$ & 2 & 100 & $\mathrm{BN}$ & 5 & 80 \\
\hline $\mathrm{BE}$ & 2 & 100 & $\mathrm{BO}$ & 5 & 80 \\
\hline $\mathrm{O}$ & 3 & 60 & $\mathrm{BP}$ & 5 & 80 \\
\hline $\mathrm{P}$ & 3 & 60 & BQ & 5 & 80 \\
\hline Q & 3 & 60 & $\mathrm{BR}$ & 5 & 80 \\
\hline $\mathrm{R}$ & 3 & 60 & AA & 5 & 80 \\
\hline S & 3 & 60 & $\mathrm{AC}$ & 5 & 80 \\
\hline $\mathrm{N}$ & 3 & 45 & AI & 6 & 480 \\
\hline $\mathrm{C}$ & 3 & 240 & Z & 6 & 600 \\
\hline E & 3 & 160 & Power total & 1 & 980 \\
\hline $\mathrm{T}$ & 3 & 200 & Power total & 2 & 1460 \\
\hline K & 3 & 90 & Power total & 3 & 1350 \\
\hline $\mathrm{U}$ & 3 & 15 & Power total & 4 & 1210 \\
\hline $\mathrm{BF}$ & 3 & 30 & Power total & 5 & 1238 \\
\hline $\mathrm{H}$ & 3 & 90 & Power total & 6 & 1080 \\
\hline I & 3 & 90 & Power total & all & 7158 \\
\hline
\end{tabular}

Table 5.2: Table of heaters with zone and power capacity labeled for each.

formed to model of the probability of ignition as a function of surface temperature. These results are shown in Fig. 5.2. The ignition threshold was found to be 933.3 $\mathrm{K}$, with $95 \%$ confidence limits of $\pm 12 \mathrm{~K}$.

Figure 5.3 documents an example of an ignition sequence in the high temperature hexane testing. The first image, immediately before ignition, is rather complicated. 


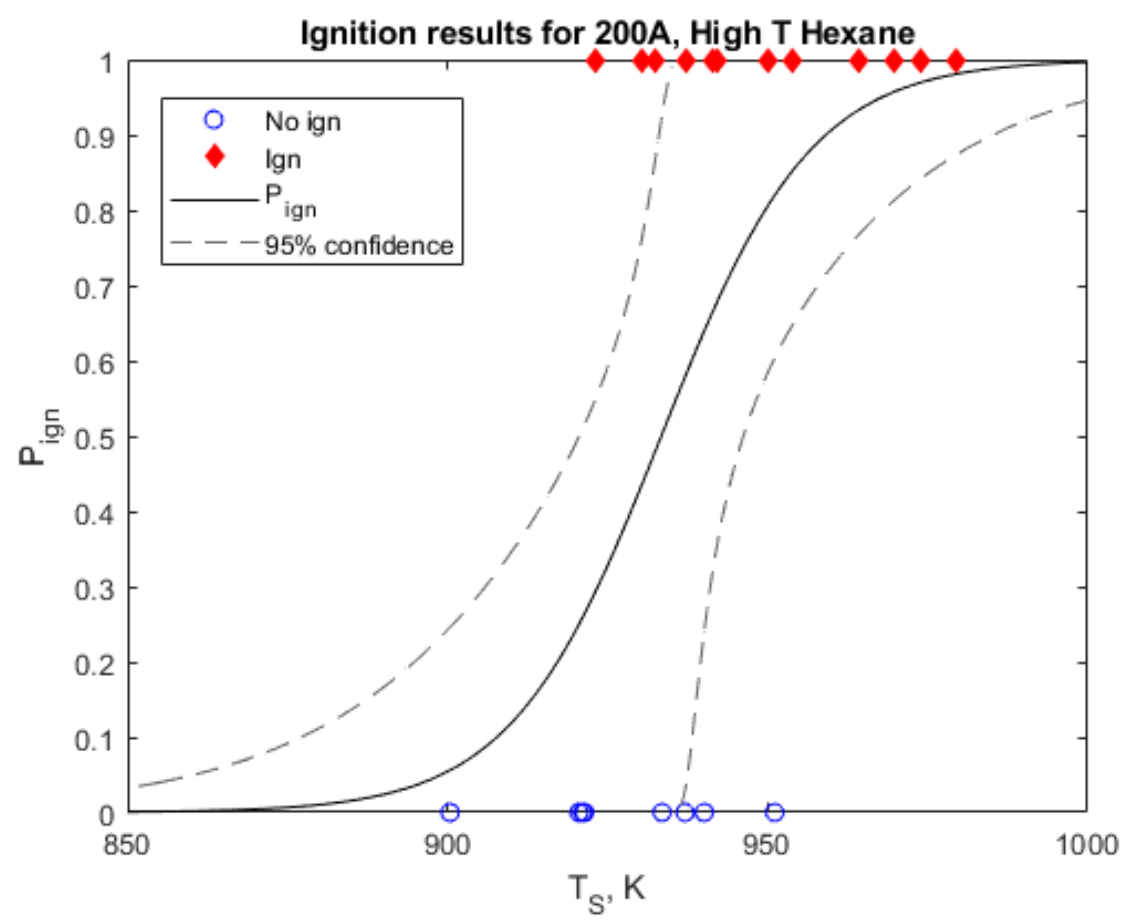

Figure 5.2: Ignition results for Hexane with a vessel temperature of $393 \mathrm{~K}$ using cylinder 200A.

The boundary layer can just be observed, but it is partially obscured by other density gradients randomly scattered across the field of view. These density gradients are likely due to the convection outside of the vessel (particularly near the windows where there is no insulation) due to density gradients from heating that obscure the gradients due to natural convection from the surface of the heated cylinder. However, the density gradient formed by the flame is intense enough to observe despite extra density gradients from outside of the vessel. Ignition occurs on the right side of the cylinder in this experiment (shot 239), with a cylinder temperature of 930.3 $\mathrm{K}$. The ignition dynamics are largely the same as those of the previous hexane tests with the vessel at room temperature. The flame structure assumes a similar "barrel" shape, although that shape is somewhat distorted in the ignition sequence shown here.

From the ignition sequence, we can track the position of the flame front and extract the flame speed in different directions. The position of the flame is plotted in Fig. 5.4. The flame speed of the left edge of the flame is $6.4 \mathrm{~m} / \mathrm{s}$, the right edge moves at $6.8 \mathrm{~m} / \mathrm{s}$, and the top and bottom edges of the flame move at 8.0 and $7.9 \mathrm{~m} / \mathrm{s}$, 


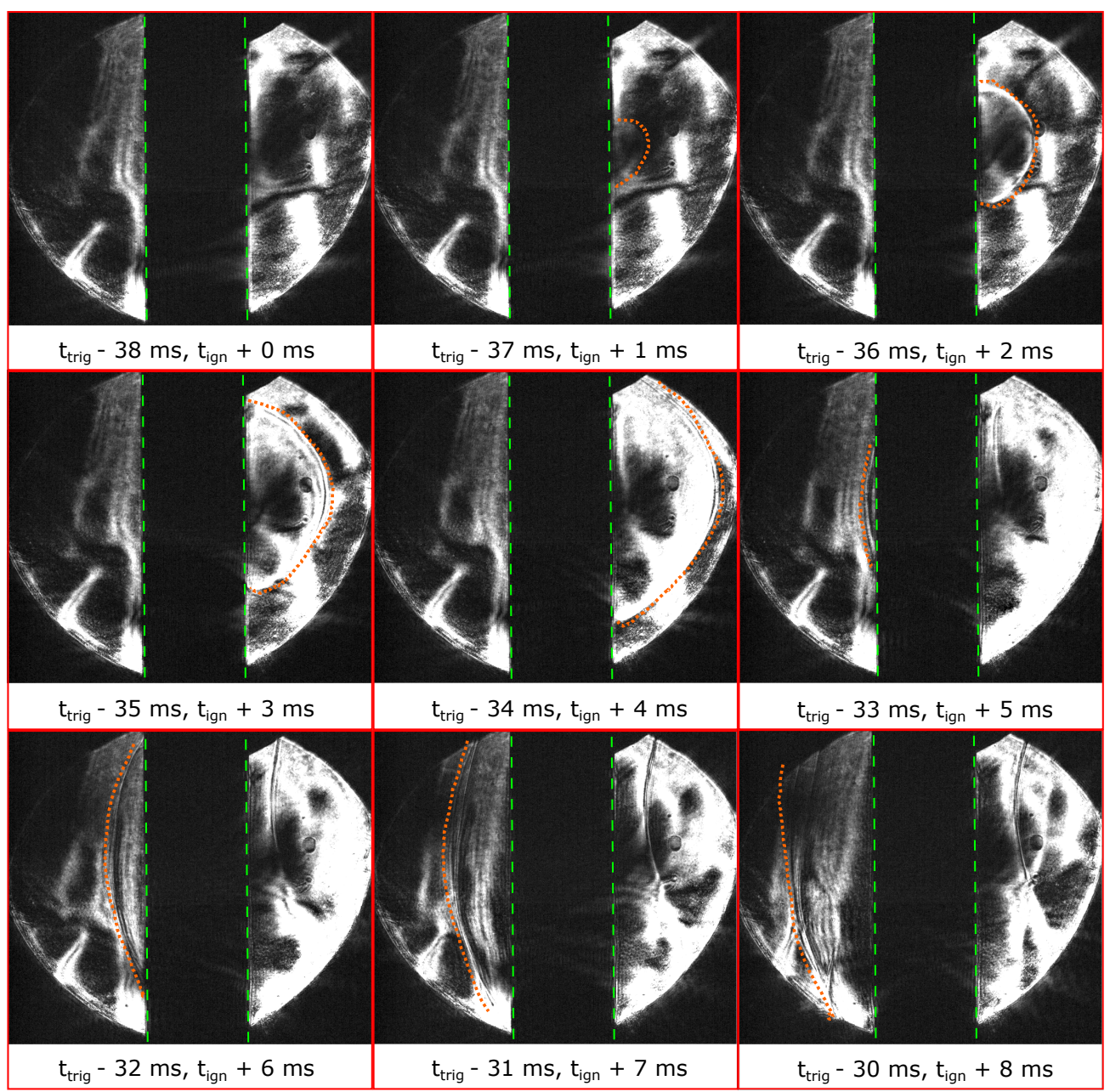

Figure 5.3: Ignition sequence for high temperature hexane with cylinder 200A. Flame front highlighted with dashed orange lines.

respectively. The horizontal flame speed has approximately doubled compared to the horizontal flame speed in Section 4.6. The vertical flame speed is faster than the previous hexane tests as well. The vertical flame speed has increased by $25 \%$ in the high temperature hexane tests compared to the tests in Chapter 4 . Both vertical and horizontal flame speed increase with the higher ambient temperature, but this increase is more pronouced $(\approx 100 \%$ ) for the horizontal flame speed versus the vertical flame speed. The computed flame speed for hexane at $393 \mathrm{~K}$ is $3.7 \mathrm{~m} / \mathrm{s}$, and at $750 \mathrm{~K}$ it is $7.1 \mathrm{~m} / \mathrm{s}$. The difference between computed and experimental flame speeds is $78 \%$ and $12 \%$ for the horizontal and vertical flame speeds, respectively. The discrepancy between the flame speeds at $393 \mathrm{~K}$ is rather large compared to the reasonable agreement seen in the low-temperature hexane tests. There are several 
possible causes for this discrepancy; additional convection in the vessel due to heating, unsteadiness in flame propagation, or decomposition of hexane prior to ignition due to the increased ambient temperature could all cause an increase in flame speed. The precise cause is not well understood and could be a subject of future work.

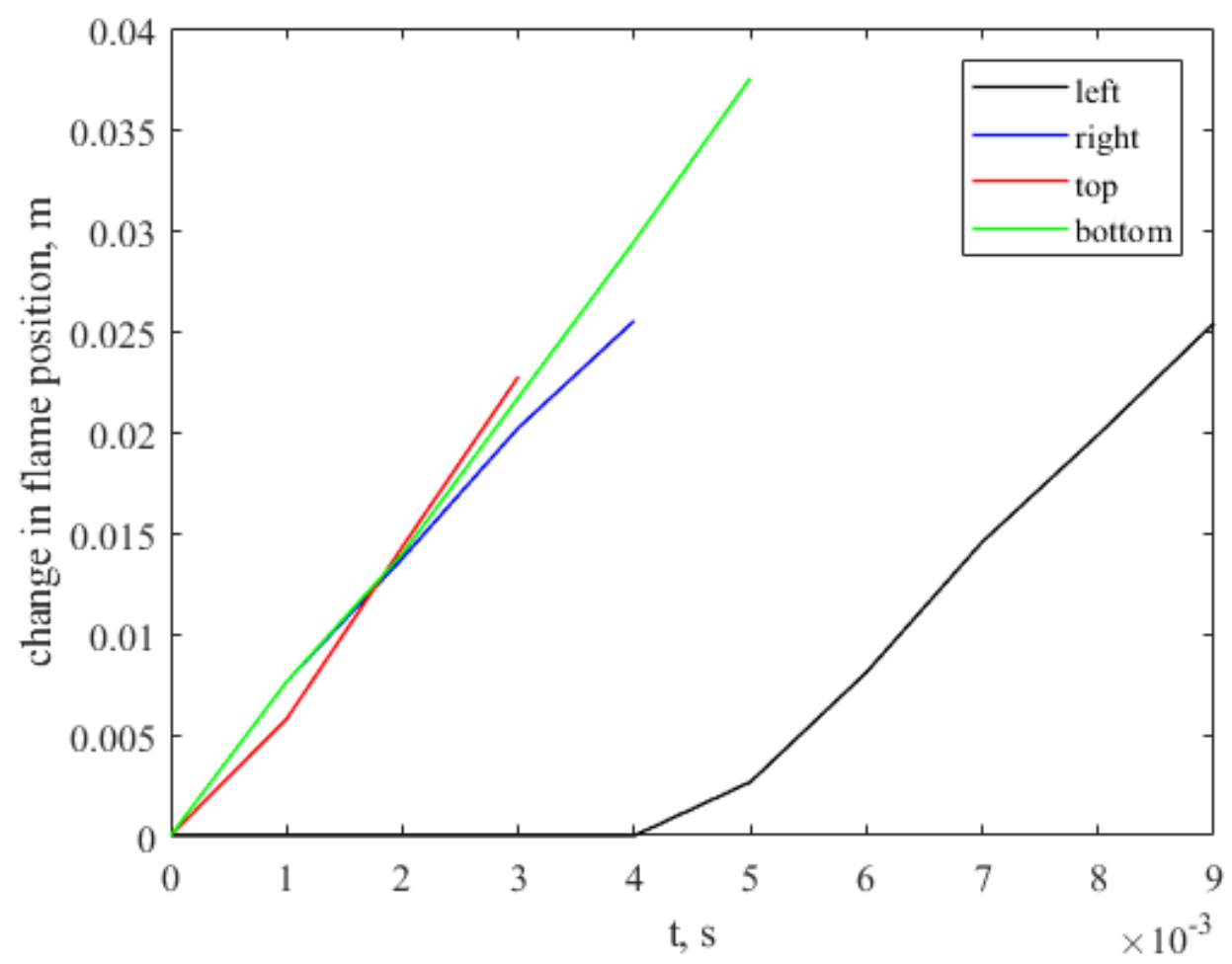

Figure 5.4: Flame position from shot 239, for high-temperature hexane mixture.

The ignition location was noted in all suitable visualization tests (ranging from 20.7 to $23.6 \mathrm{~cm}$ ), and the average location of ignition for the high temperature hexane experiments was 22.4 up from the bottom edge of the cylinder. This is similar to the average ignition location of $22.6 \mathrm{~cm}$ for the same cylinder (200A) with hexane experiments run in the room-temperature vessel (total range of 20.9 to $23.4 \mathrm{~cm}$ ). The ignition location has not been significantly altered by the increased ambient temperature. This is due to the experiments being designed to find minimum ignition temperatures; the ignition location is expected to be at the upper edge of the vertical cylinder when the temperature is close to the ignition threshold. For this reason, we observe a change in the ignition threshold while the ignition location remains unchanged with a change in ambient temperature. 
There are a few literature works reporting the effects of increased ambient temperature on thermal ignition. Mullen, Fenn, and Irby (1948) investigated thermal ignition by horizontal rods under forced convection and saw a slight decrease in ignition temperature with an increase in ambient temperature. The effect became more pronounced at higher flow velocities: a $5 \mathrm{~K}$ decrease in ignition temperature occurs at a flow rate of $24.4 \mathrm{~m} / \mathrm{s}$, and an $89 \mathrm{~K}$ decrease in ignition temperature occurs at a flow rate of $48.8 \mathrm{~m} / \mathrm{s}$ when the ambient temperature is increased by $61 \mathrm{~K}$. Ono et al. (1976) examine the effects of surface size on thermal ignition by vertical plates in free convection. They also noted a decrease in the ignition temperature as the ambient temperature was increased: the ignition temperature dropped by approximately $20 \mathrm{~K}$ corresponding to increasing the ambient temperature of $40 \mathrm{~K}$. Ono et al. note that for a given wall temperature, an increased ambient temperature reduces the convection velocity of natural convection flows, and cites the reduced velocity (leading to a longer time for the mixture to pass through the boundary layer) as the cause for a reduced ignition temperature.

We can investigate this claim by using the similarity solution presented in Section 3.1 to estimate how the boundary layer changes with a change in the ambient temperature. First, we calculate the similarity solution with a wall temperature of $1100 \mathrm{~K}$, and change the ambient temperature from 300 to $400 \mathrm{~K}$. With an ambient temperature of $300 \mathrm{~K}$, the maximum velocity in the momentum boundary layer is $1.52 \mathrm{~m} / \mathrm{s}$. With an increased ambient temperature of $400 \mathrm{~K}$, the maximum velocity in the momentum boundary layer is $1.22 \mathrm{~m} / \mathrm{s}$. We see exactly the sort of decrease in convection velocity that Ono et al. cite in their study of effects of ambient temperature on ignition.

We investigate the similarity solution further to examine how the boundary layer profile changes with an increase in the ambient temperature. The results are shown in Fig. 5.5. The red line shows the boundary layer profile at the top of a $25.4 \mathrm{~cm}$ long plate with a surface temperature of $1100 \mathrm{~K}$ and an ambient temperature of 300 $\mathrm{K}$. The blue line shows the boundary layer profile for a wall temperature of 1100 $\mathrm{K}$ and an ambient temperature of $400 \mathrm{~K}$. From these two profiles where the only variable changed is the ambient temperature, we can see that the thermal gradient near the wall is shallower in the case of the higher ambient temperature. This is a significant observation, as a common ignition criterion is the so-called Van't Hoff ignition criterion, which states that ignition occurs when the thermal gradient at the 
surface is zero. The Van't Hoff criterion can be represented as:

$$
\left(\frac{d T}{d y}\right)_{w}=0 .
$$

The Van't Hoff criterion implies that heat loss at the surface is balanced by heat transfer due to chemical reactions (Laurendeau, 1982). With the Van't Hoff criterion in mind, we return to the red and blue curves presented in Fig. 5.5. It is apparent that for the same wall temperature, an increase in the ambient temperature produces a significantly shallower thermal gradient. This effect is event more pronounced if the the wall temperature is reduced while at a higher ambient temperature, as seen for the green line in Fig. $5.5\left(T_{w}=1000 \mathrm{~K}, T_{\infty}=400 \mathrm{~K}\right)$. Starting with a shallower thermal gradient in the thermal boundary layer implies it will take less additional energy by chemical reaction to reach the Van't Hoff criterion, and therefore makes ignition possible at lower surface temperatures when the ambient temeprature is increased.

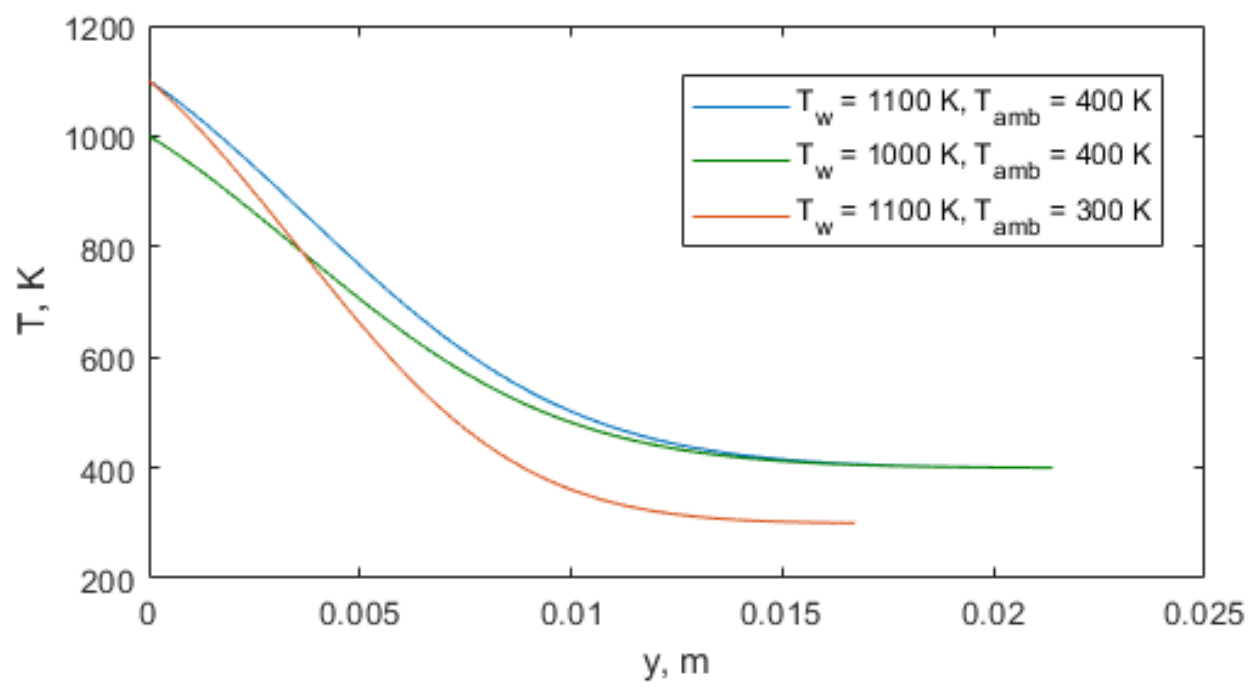

Figure 5.5: Effect of ambient temperature and wall temperature on boundary layer profiles as calculated via similarity solution.

We can approximate the difference in the thermal gradients seen for cylinder 200A with the room temperature and high temperature hexane tests using the similarity solution. We use the ignition threshold temperature from logistic regression as the surface temperature; for the room temperature hexane tests described in Chapter 4 . 
we use a wall temperature of $1019 \mathrm{~K}$ and and ambient temperature of $300 \mathrm{~K}$, and for the high temperature hexane tests described in this section, we use a wall temperature of $933 \mathrm{~K}$ and an ambient temperature of $400 \mathrm{~K}$ as the boundary conditions for the similarity solution. The resulting temperature profiles at the top of the 25.4-cm-long surface are shown in Fig. 5.6. We see that the blue thermal profile, representative of a high temperature hexane experiment, has a much shallower thermal gradient than the red profile, which represents a room temperature hexane experiment. From this we infer that the shallower gradient takes less additional energy to reach the Van't Hoff ignition criterion and is therefore easier to ignite. This is analogous to an increase in the ambient mixture resulting in a lower ignition threshold. This explanation obviously has its limitations; taken to the extreme, it implies a scenario where there is no thermal gradient (and thus no buoyancy-driven flow) because the wall temperature and the ambient temperature are the same. This amounts to a zero-dimensional ignition problem. The Van't Hoff ignition criterion breaks down in the zero-dimensional ignition problem. However, conceptualizing the effect of ambient temperature on ignition in terms of changes to the thermal gradient is at least a complementary explanation to the one offered by Ono et al. (1976). It offers another intuitive way to think about how a change in ambient temperature affects the buoyancy-driven flow that creates the conditions for an ignition event to occur.

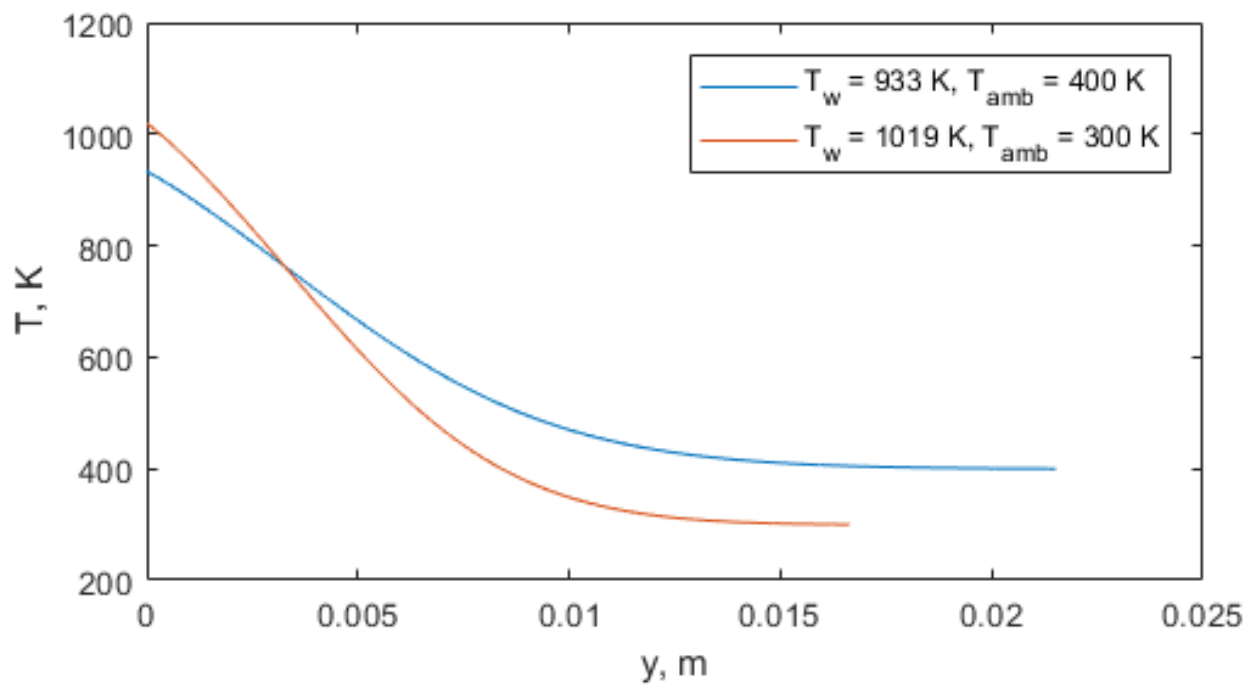

Figure 5.6: Effect of ambient temperature and wall temperature on boundary layer profiles as calculated via similarity solution using ignition temperatures from hexane testing as wall temperatures. 


\subsection{Aachen Surrogate}

The Aachen surrogate was developed by Honnet et al. (2009) to be a very simple surrogate fuel with one species each of an alkane and an aromatic. The composition is $80 \%$ n-Decane and $20 \%$ 1,2,4-Trimethylbenzene by weight. Table 5.3 shows the weight, mole, and volume percent of the Aachen surrogate.

\begin{tabular}{||c|ccc||}
\hline Aachen composition: & weight \% & mol \% & vol \% \\
\hline n-Decane & 80 & 77 & 82.7 \\
1,2,4-Trimethylbenzene & 20 & 23 & 17.3 \\
\hline
\end{tabular}

Table 5.3: Composition breakdown of Aachen surrogate by weight, mole, and volume percent.

The balanced chemical equation for Aachen surrogate and air combustion is:

$0.77 \mathrm{C}_{10} \mathrm{H}_{22}+0.23 \mathrm{C}_{9} \mathrm{H}_{12}+14.695 \mathrm{O}_{2}+55.25 \mathrm{~N}_{2} \longrightarrow 9.77 \mathrm{CO}_{2}+9.85 \mathrm{H}_{2} \mathrm{O}+55.25 \mathrm{~N}_{2}$.

Using a target final pressure of 760 Torr $(101 \mathrm{kPa})$, the target partial pressure of each component of the mixture is calculated to produce a stoichiometric mixture. The results are shown in Table 5.4 .

\begin{tabular}{||c|ccc||}
\hline & Aachen & $\mathrm{O}_{2}$ & $\mathrm{~N}_{2}$ \\
$X_{i}$ & 0.0141 & 0.2071 & 0.7788 \\
$P_{i}$ (Torr) & 10.7 & 157.4 & 591.9 \\
Liquid volume $(\mathrm{mL})$ & 4.5 & - & - \\
\hline
\end{tabular}

Table 5.4: Mixture composition for stoichiometric Aachen-air mixture.

As stated previously, the dew temperature for Aachen calculated by Sund (2019) is found to be $330 \mathrm{~K}$ for a stoichiometric mixture. Preliminary vaporization tests were performed with the vessel temperature set at $333 \mathrm{~K}$, where the liquid fuel volume was injected into the evacuated vessel. The vapor pressure of $4.5 \mathrm{~mL}$ of Aachen surrogate at this temperature was found to be 3.9 Torr. This is less than half of the desired target pressure of the Aachen surrogate, and is an indication of significant condensation inside the vessel. Additionally, the pressure of Aachen fuel slowly fell to this value over the period of five minutes, also indicating gradual condensation of the fuel. This is not surprising, as the temperature of the combustion vessel is only very slightly above the dew temperature, and it is difficult to get the vessel 
completely uniform in temperature. Regions like the visualization windows will often be slightly cooler than the set point as they are uninsulated and can act like cooling fins. To compensate for this, another vaporization test was performed with the vessel temperature set to $373 \mathrm{~K}$. This time, the vapor pressure stabilized at 9.6 Torr almost immediately, indicating that the temperature is high enough to avoid significant condensation of the fuel. While the pressure of Aachen vapor is slightly lower than the target value, this slight undershoot is consistent with the results of hexane injection into the room-temperature vessel. The hexane is able to fully vaporize at room temperature, so there is no danger of condensation, but the vapor pressure was often 1-2 Torr lower than the target partial pressure. This is attributed to a "virtual or false leak" by which some of the fuel vapor is absorbed by soot residue or by the plumbing insuration. It is a relatively small amount of fuel vapor that is lost for both the room-temperature hexane tests and for the fully vaporized Aachen tests and is considered satisfactory to proceed with testing.

Twenty-five individual tests were performed with the Aachen surrogate. The results were used to perform a logistic regression in order to find the probability of ignition as a function of the cylinder surface temperature. The ignition threshold, set as the surface temperature at which there is a $50 \%$ probability of ignition, is found to be $947.7 \mathrm{~K}$, with $95 \%$ confidence limits of $\pm 12 \mathrm{~K}$.

Figure 5.8 documents an example of an ignition sequence in the tests of the Aachen surrogate. Similar to Fig. 5.3, we see the density gradients caused by air currents outside the vessel impeding the visualization of the thermal boundary layer over the cylinder surface. It is possible to watch the evolution of the ignition kernel in spite of the extra disturbances. These images come from shot 202, and the temperature of the cylinder at ignition was $952.1 \mathrm{~K}$. Like with the hexane experiments performed with a room temperature vessel, the flame front evolves in to a barrel-like shape with the flame moving faster vertically than it does horizontally. This is most likely due to the increased flame speed in a higher temperature region near the heated cylinder surface, as was discussed in Section 4.6 .

The schlieren visualization of the ignition sequence can be used to track the position of the flame front. Fig. 5.9 shows the position of the flame front in time for the left, right, top, and bottom edges of the flame. The flame speed of the left, right, top, 


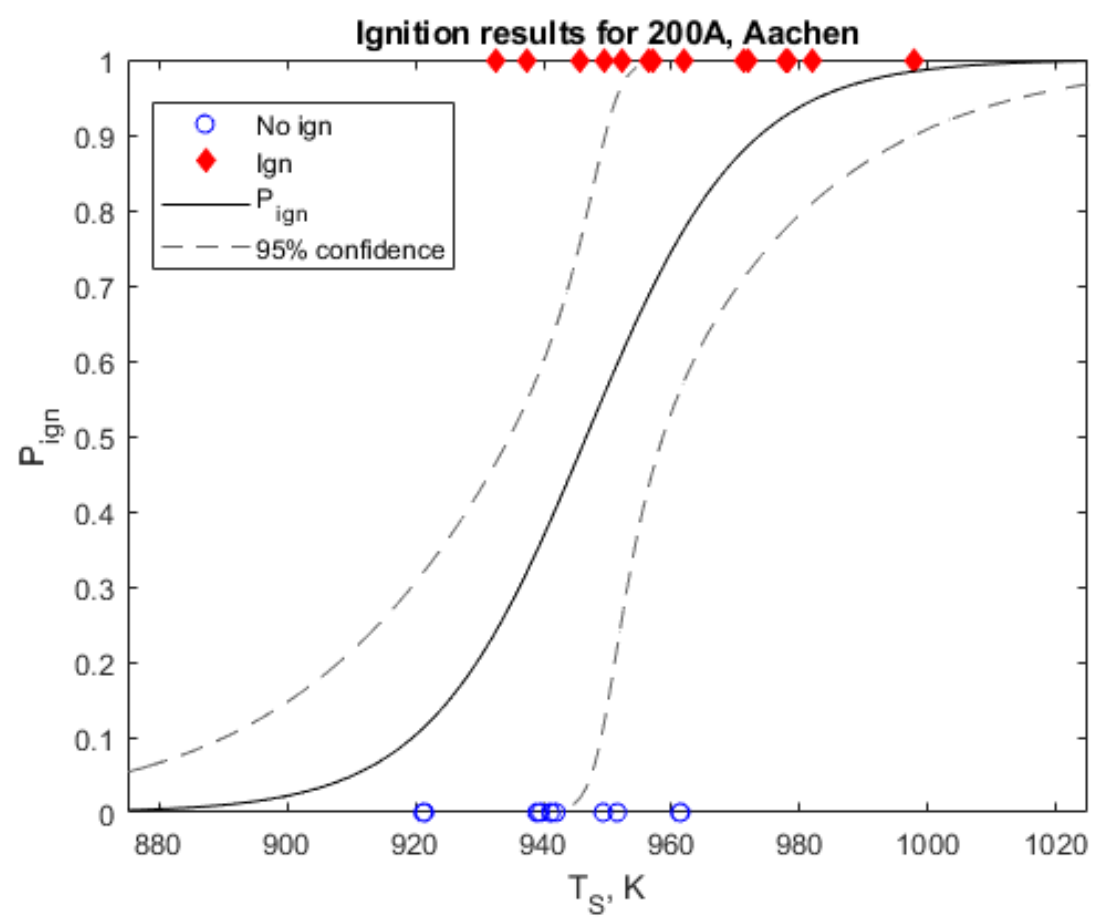

Figure 5.7: Ignition results for Aachen surrogate fuel with cylinder 200A.

and bottom edges are 3.5, 3.3, 6.9 and $6.3 \mathrm{~m} / \mathrm{s}$, respectively. The difference between the horizontal and vertical flame speeds is once again likely due to the difference in environmental temperature: horizontally, the flame propagates into the mixture at the ambient temperature of $373 \mathrm{~K}$, while the top and bottom edges of the flame remain quite close to the cylinder surface and in the high temperature boundary layer where the gas temperature can reach approximately $1100 \mathrm{~K}$. Using a skeleton mechanism (231 species, 5591 reactions) developed by Ranzi et al. (2014) for kerosene, we can calculate an estimate of the flame speed of Aachen. At an ambient temperature of $400 \mathrm{~K}$, the flame speed is predicted to be $3.7 \mathrm{~m} / \mathrm{s}$, and for the film temperature of $750 \mathrm{~K}$ the flame speed is calculated as $7.3 \mathrm{~m} / \mathrm{s}$. The experimental flame speeds are within $10 \%$ of the calculated flame speeds. This is a good result especially considering that the mechanism used in the calculations is for general kerosene mixtures and not developed specifically for the Aachen surrogate. As was observed for the hexane flame speeds, the Aachen flame speed is faster in the vertical direction as the flame remains in the higher temperature boundary layer and flame speed is seen to increase with increased temperature.

The schlieren visualization can also be used to track the ignition location. For the 


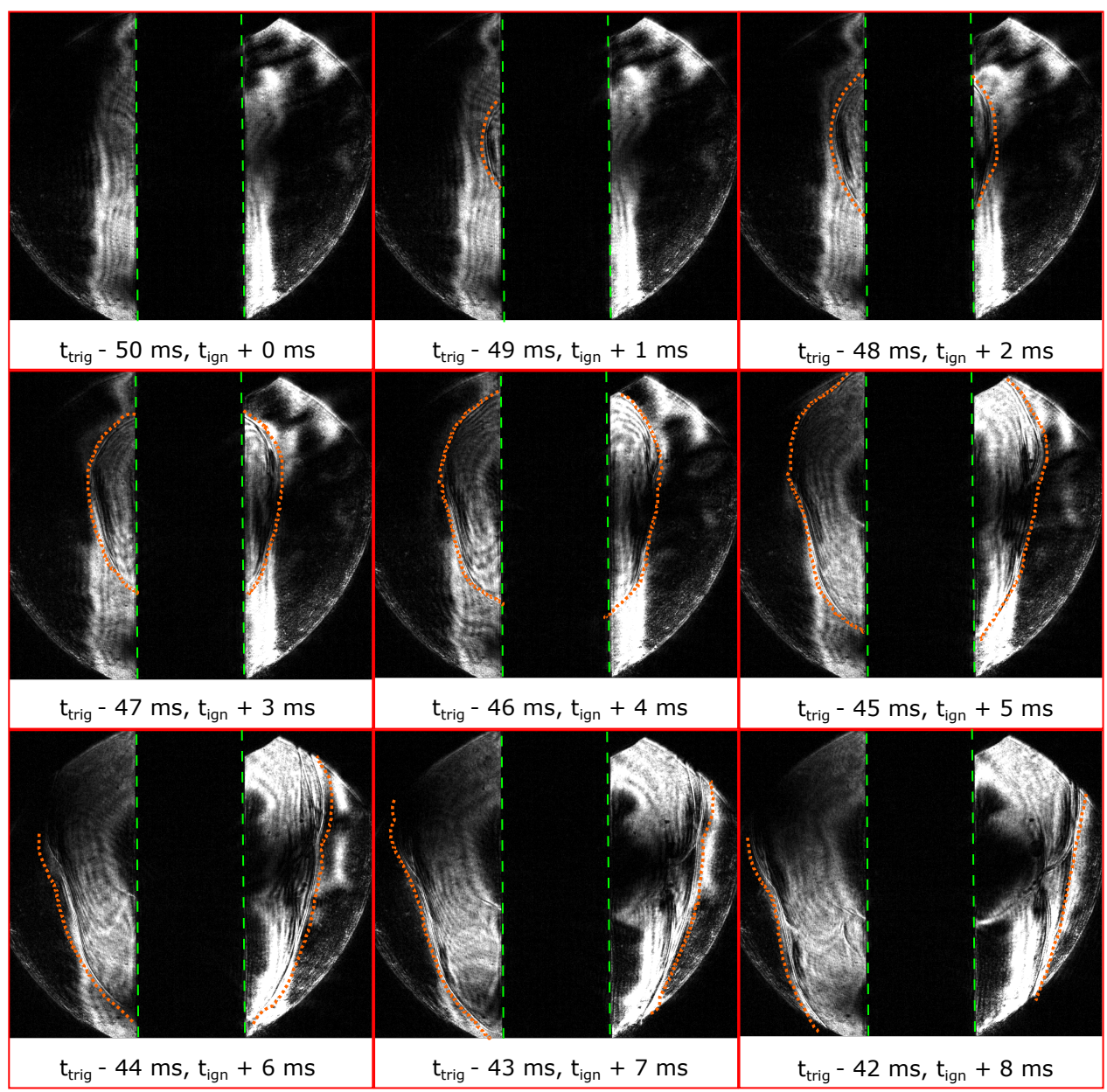

Figure 5.8: Ignition sequence for Aachen surrogate fuel with cylinder 200A. Flame front highlighted with dashed orange lines.

Aachen experiments, the average ignition height was $22.1 \mathrm{~cm}$. This is similar to the average ignition location of 22.6 and $22.4 \mathrm{~cm}$ for the room temperature and high temperature hexane tests, respectively. Overall, the ignition dynamics seem quite similar to previous ignition experiments.

\subsection{JI Unified Surrogate}

The JI surrogate was developed by Chen, Khani, and Chen (2016) to match the droplet evaporation characteristics of Jet A. In particular, this surrogate was developed to match the heating, evaporation, and shock tube and droplet ignition characteristics of Jet A as closely as possible. In formulation, JI targets chemical characteristics such as the cetane number, lower heating value, average molecular weight, and hydrogen-to-carbon ratio as well as physical characteristics like the 


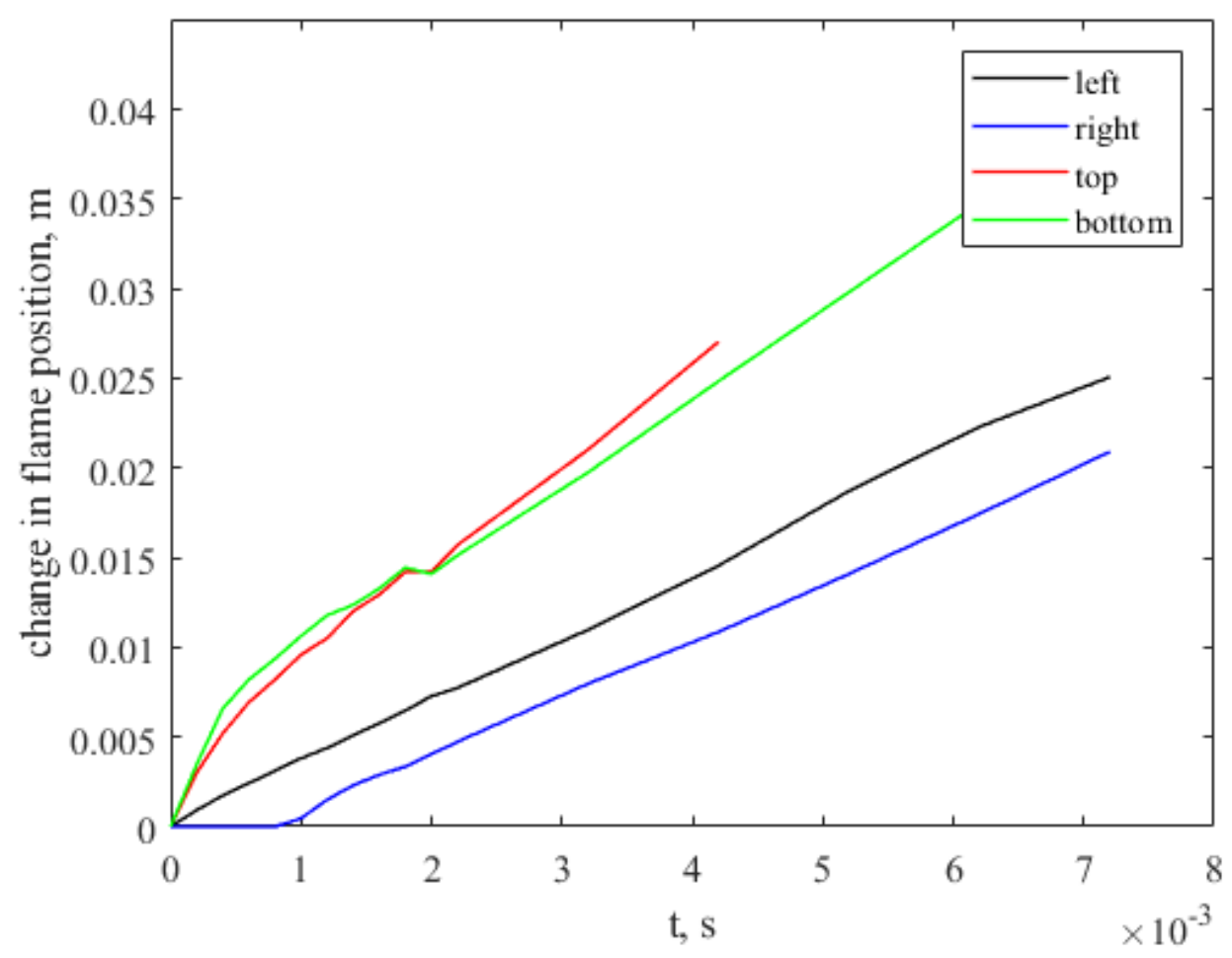

Figure 5.9: Flame position from shot 202, for the Aachen mixtures.

liquid density, surface tension, and kinematic viscosity. In this way, the formulation of JI is much more specifically targeted to emulate Jet A behavior than the Aachen surrogate. Consequently, it is also a more complex fuel, consisting of four components, as shown in Table 5.5 .

\begin{tabular}{||c|ccc||}
\hline JI composition: & weight \% & mol \% & vol \% \\
\hline Iso-cetane & 46.5 & 36.0 & 47.0 \\
n-Dodecane & 29.2 & 30.0 & 30.8 \\
trans-decalin & 19.4 & 24.6 & 17.7 \\
Toluene & 4.9 & 9.4 & 4.5 \\
\hline
\end{tabular}

Table 5.5: Composition breakdown of JI surrogate by weight, mole, and volume percent.

The balanced chemical equation for stoichiometric reaction to major products for JI surrogate and air combustion is: 


$$
\begin{array}{r}
0.36 \mathrm{C}_{16} \mathrm{H}_{34}+0.30 \mathrm{C}_{12} \mathrm{H}_{26}+0.246 \mathrm{C}_{10} \mathrm{H}_{18}+0.30 \mathrm{C}_{7} \mathrm{H}_{8}+18.783 \mathrm{O}_{2}+70.624 \mathrm{~N}_{2} \\
\longrightarrow 12.478 \mathrm{CO}_{2}+12.61 \mathrm{H}_{2} \mathrm{O}+70.624 \mathrm{~N}_{2} .
\end{array}
$$

\begin{tabular}{||c|ccc||}
\hline & $\mathrm{JI}$ & $\mathrm{O}_{2}$ & $\mathrm{~N}_{2}$ \\
\hline$X_{i}$ & 0.0111 & 0.2078 & 0.7812 \\
$P_{i}$ (Torr) & 8.4 & 157.9 & 593.7 \\
Liquid volume $(\mathrm{mL})$ & 4.4 & - & - \\
\hline
\end{tabular}

Table 5.6: Mixture composition for stoichiometric JI-air mixture.

Due to a typographic error in the initial composition calculations, a liquid fuel volume of $3.3 \mathrm{~mL}$ was used in the vaporization and ignition tests. Table 5.7 documents the mixture composition used in the experiments. This amounts to an equivalence ratio of 0.75 . While the actual equivalence ratio is leaner than the stoichiometric target, this is not expected to significantly alter the ignition behavior. Previous studies in the laboratory have shown that mixture composition has a minor effect on the ignition temperature away from flammability limits (Sund, 2019; Boeck et al., 2017; Coronel et al., 2018).

\begin{tabular}{||c|ccc||}
\hline & $\mathrm{JI}$ & $\mathrm{O}_{2}$ & $\mathrm{~N}_{2}$ \\
$X_{i}$ & 0.0083 & 0.2083 & 0.7834 \\
$P_{i}$ (Torr) & 6.3 & 158.3 & 595.4 \\
Liquid volume $(\mathrm{mL})$ & 3.3 & - & - \\
\hline
\end{tabular}

Table 5.7: Experimental mixture composition for JI-air mixture.

Vaporization tests were performed with $3.3 \mathrm{~mL}$ of JI fuel. The first vaporization test was performed with a vessel temperature of $373 \mathrm{~K}$. Immediately after the fuel was injected, the pressure of JI was 4.2 Torr, which decreased to 3.3 Torr over the course of five minutes. The fuel pressure only reaching about half of the target pressure, coupled with the gradual decrease of fuel pressure, indicates that the fuel is condensing and the vessel temperature must be increased further to achieve full vaporization of JI. This result is not surprising; like with the first vaporization test of Aachen, the vessel temperature for the the first vaporization test of JI is only a few Kelvin higher than the dew temperature for JI. For the second vaporization test of JI, the vessel temperature is increased to $393 \mathrm{~K}$. This time after the $3.3 \mathrm{~mL}$ of 
$\mathrm{JI}$ is injected, the vapor pressure almost immediately stabilizes at 6.1 Torr. This is very close to the target pressure of JI and ignition tests can therefore proceed with the vessel temperature set to $393 \mathrm{~K}$.

Twenty-two individual ignition tests were performed with JI. Applying a logistic regression to the data provides a model of the probability of ignition as a function of cylinder temperature. The ignition threshold is found to be $984.0 \mathrm{~K}$, with $95 \%$ confidence limits of $\pm 3 \mathrm{~K}$.

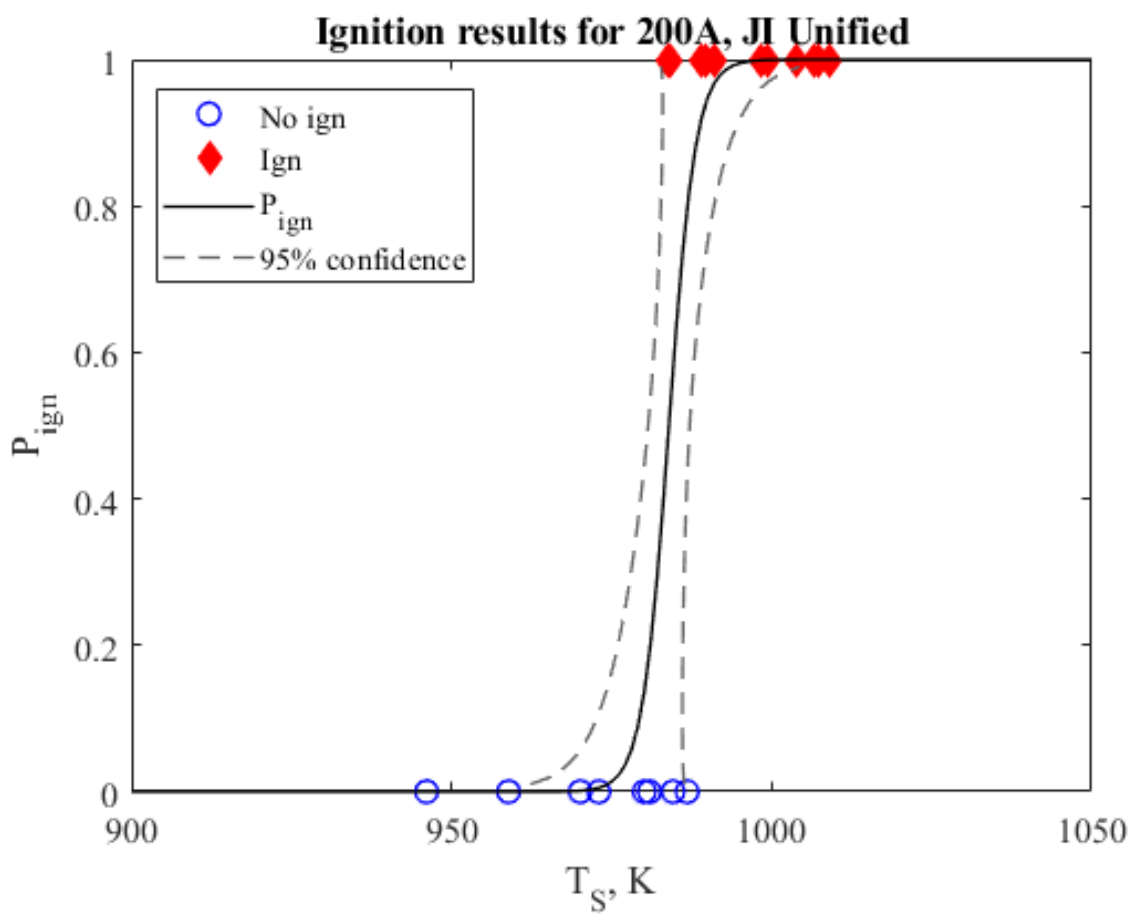

Figure 5.10: Ignition results for JI Unified surrogate fuel with cylinder 200A.

Figure 5.11 documents an example of an ignition sequence in the JI testing. The images are taken from Shot 231, and the temperature of the cylinder at ignition was 988.9 K. The first sign of ignition occurs on the right side of the cylinder, and the flame propogates outward and around the cylinder from there. The flame eventually develops a barrel shape, much like that seen in the Aachen testing and both types of hexane experiments. With the schlieren visualization, we can track the ignition location. For the JI experiments, the average height of ignition was $20.6 \mathrm{~cm}$ up from the bottom edge of the cylinder. This is a little bit lower than the ignition locations seen for hexane $(22.6 \mathrm{~cm})$, but still within $10 \%$ of the ignition location in room-temperature hexane experiments. Ignition still occurs in the upper portion of 
the high-temperature region of the cylinder, as expected.

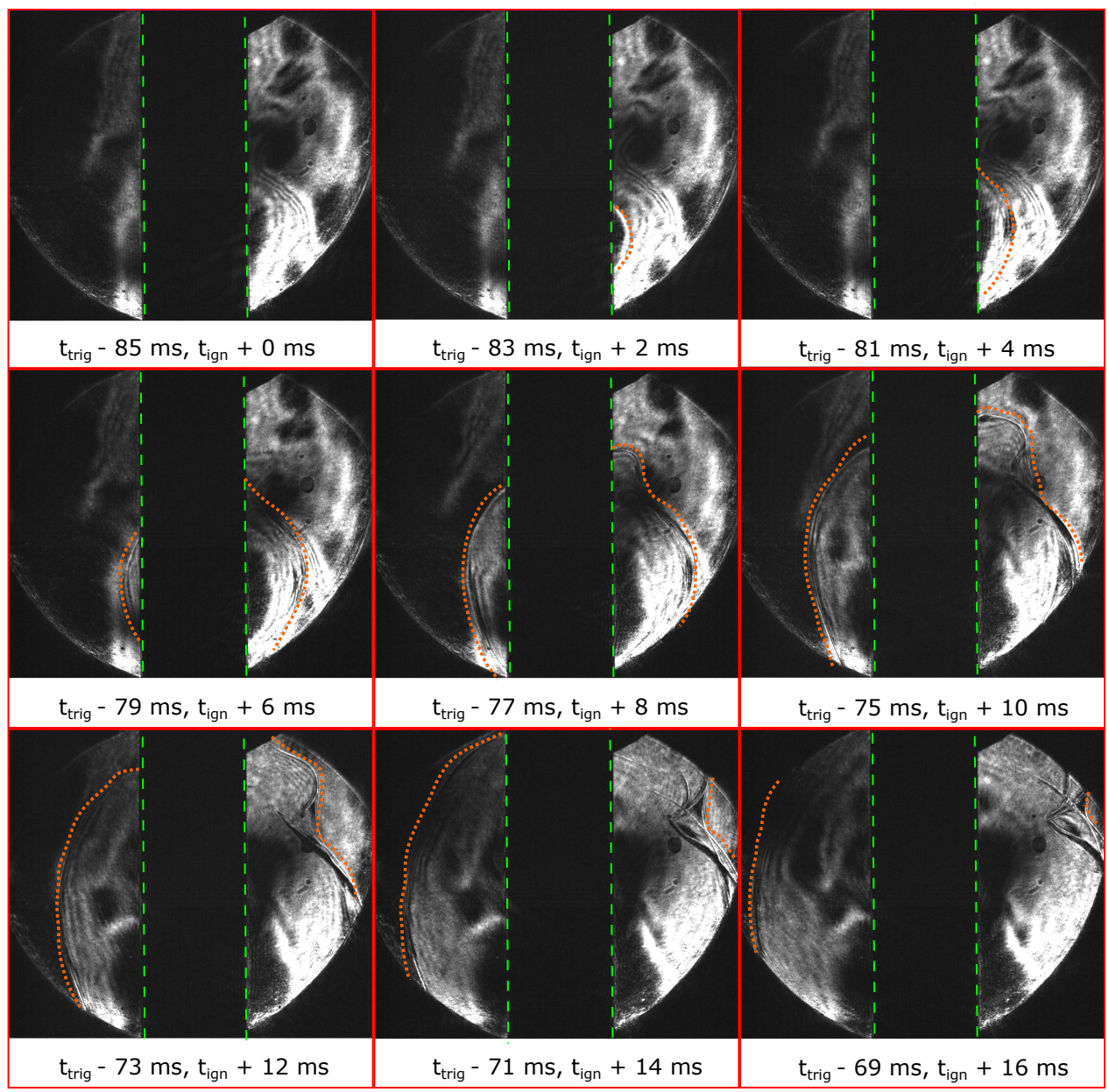

Figure 5.11: Ignition sequence for JI unified surrogate fuel with cylinder 200A. Flame front highlighted with dashed orange lines.

Figure 5.12 tracks the position of the flame front from the ignition sequence in Fig. 5.11. The flame speed of the left, right, top, and bottom edges of the flame are 2.7, 2.4, 6.0, and $6.0 \mathrm{~m} / \mathrm{s}$, respectively. These flame speeds are all slightly lower than those of Aachen; this could either be due to the slightly lean mixture of JI that was tested or due to the nature of the JI mixture itself, or a combination of those factors. We used the Cantera software to calculate the flame speed for this slightly lean mixture of JI using the mechanism of Ranzi et al. (2014). This is the same mechanism that was used in Chen, Khani, and Chen (2016) to calculate properties of the JI mixture. First, we calculate the laminar burning speed of a stoichiometric JI-air mixture to compare with the values reported in Chen, Khani, and Chen (2016) 


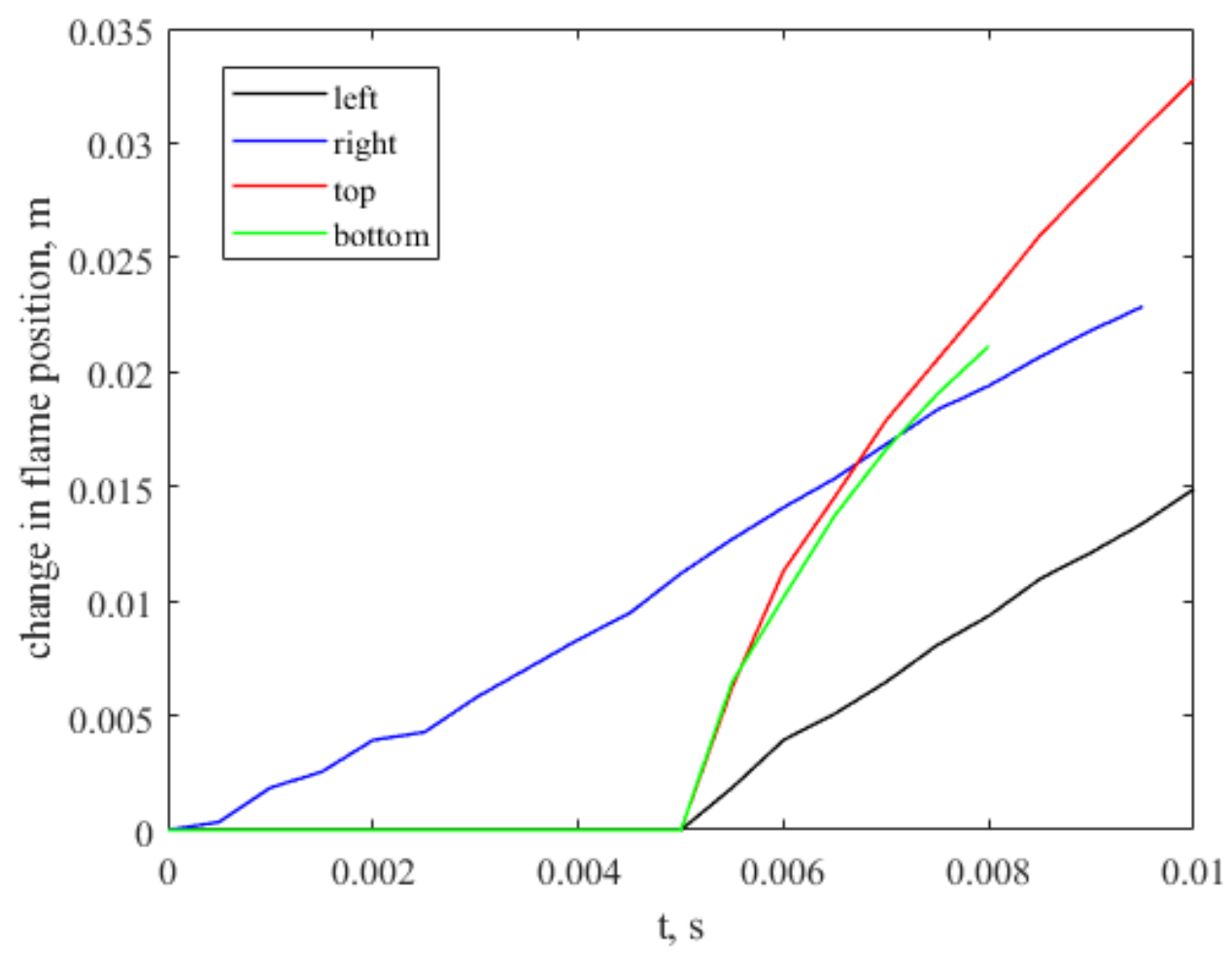

Figure 5.12: Flame position from shot 231, for the JI mixtures.

and validate our results. The laminar burning speed of a stoichiometric JI-air mixture is calculated to be $55.5 \mathrm{~cm} / \mathrm{s}$, very similar to the approximately $56 \mathrm{~cm} / \mathrm{s}$ reported by Chen, Khani, and Chen (2016) for a stoichiometric mixture at $400 \mathrm{~K}$ and 1 bar. Returning to the slightly lean JI mixture used in the experiments reported here, the flame speed calculated using the skeleton mechanism from Ranzi et al. (2014) is $2.1 \mathrm{~m} / \mathrm{s}$ at $400 \mathrm{~K}$ and $5.0 \mathrm{~m} / \mathrm{s}$ at the film temperature of $750 \mathrm{~K}$. We see that the flame speeds measured in experiments of this lean JI mixture are no more than $20 \%$ higher than those predicted by calculations with the mechanism, but the behavior of an increased flame speed at higher temperatures is matched.

\subsection{Jet A}

In order to understand the suitability of the surrogates tested in this section, it is necessary to run tests with Jet $\mathrm{A}$ as a fuel. The jet fuel blend used in these experiments is POSF-4658; this jet fuel blend has a well-characterized composition and the physical properties are documented by Edwards (2017). Its composition in terms of various alkanes and aromatics is documented in Table 5.1. The flash point of this blend is $324 \mathrm{~K}$ (Edwards, 2017), and its autoignition temperature is noted by 
Martin and Shepherd (2019) as $502.0 \pm 3.1 \mathrm{~K}$, very close to the value of $505 \mathrm{~K}$ used for the purposes of aircraft certification (Federal Aviation Administration, 2018).

One of the major challenges in performing experiments with Jet A as a fuel is potential differences between the liquid and vapor phase compositions due to differences in the component vapor pressure of the species in jet fuel. Previous studies have shown that a lower fuel mass loading, a measure of how many kilograms of fuel are loaded per unit of vessel volume, results in a lower total vapor pressure of the fuel (Lee and Shepherd, 2000; Shepherd, Nuyt, and Lee, 2000). Shepherd, Nuyt, and Lee (2000) find that with a lower fuel mass loading ( $3 \mathrm{vs} 400 \mathrm{~kg} / \mathrm{m}^{3}$ ) not only is the fuel vapor pressure lower, but the relative amount of lower molecular weight fuels is decreased as well. Lee and Shepherd (2000) suggest that at low fuel mass loading values (like $3 \mathrm{~kg} / \mathrm{m}^{3}$ ), vaporization results in the lower molecular weight components being preferentially depleted from the liquid fuel. This causes a shift in composition compared with large fuel mass loading values. Large fuel mass loadings provide enough of a liquid fuel reservoir within the combustion vessel to minimize depletion of light species. However, Shepherd, Krok, and Lee (1999) found a limited influence of the fuel mass loading between 3 and $400 \mathrm{~kg} / \mathrm{m}^{3}$ on the ignition energy in electrical spark ignition testing. Anticipating a similar limited influence on thermal ignition threshold and in order to keep the experimental procedure managable, we will investigate Jet A ignition using a fuel mass loading of 3 $\mathrm{kg} / \mathrm{m}^{3}$. This amounts to $150 \mathrm{~mL}$ of fuel added to the vessel for each experiment.

The vapor pressure of Jet A is controlled via the temperature of the vessel. Shepherd, Krok, and Lee (1997) measured the vapor pressure as a function of vessel temperature and provide functions to describe this relationship. For a particular fuel source and a fuel mass loading of $3 \mathrm{~kg} / \mathrm{m}^{3}$, they correlated the experimental data for vapor pressure as:

$$
P_{\sigma}=4.971 * 10^{4} e^{-2868 / T},
$$

where $P_{\sigma}$ is the vapor pressure in millibar and $T$ is the vessel temperature in Kelvin. Using the average molecular formula for POSF-4658, we find that the balanced global chemical equation is: 


$$
\mathrm{C}_{11.69} \mathrm{H}_{22.62}+17.34 \mathrm{O}_{2}+65.20 \mathrm{~N}_{2} \longrightarrow 11.69 \mathrm{CO}_{2}+11.31 \mathrm{H}_{2} \mathrm{O}+65.20 \mathrm{~N}_{2}
$$

This amounts to target parameters for partial pressure as shown in Table 5.8 .

\begin{tabular}{||c|ccc||}
\hline & Jet $\mathrm{A}$ & $\mathrm{O}_{2}$ & $\mathrm{~N}_{2}$ \\
\hline$X_{i}$ & 0.0120 & 0.2076 & 0.7805 \\
$P_{i}$ (Torr) & 9.1 & 157.8 & 593.1 \\
\hline
\end{tabular}

Table 5.8: Mixture composition for stoichiometric Jet A-air mixture.

Equation 5.4 predicts a vapor pressure of 9.04 mbar (6.8 Torr) for Jet A at $333 \mathrm{~K}$, a bit low for the target pressure of fuel. This is a consequence of the tests by Shepherd, Krok, and Lee (1997) perfomed with a fuel of different composition than the POSF4658 fuel used here. Edwards (2017) did not report vapor pressure measurements for POSF-4658; new measurements would have to be taken with POSF-4658 in order to improve the prediction of the vapor pressure. However, vaporization tests with Jet A blend POSF-4658 in the vessel at $333 \mathrm{~K}$ showed a vapor pressure of approximately 10.5 Torr, which is sufficiently close to the desired partial pressure to give confidence that the selected temperature for the vessel is suitable for proceeding with Jet A ignition tests.

Another issue addressed for Jet A is potential for dissolved air in the fuel. If there is enough air dissolved in the fuel, the partial pressure reading will include any dissolved air that comes out of solution instead of just the vapor pressure of the fuel. This is typically an issue with large fuel mass loading values, where there is a large amount of fuel (and therefore a large amount of dissolved air) and a relatively small vapor space. This is not the case in the present tests, but it is important to check the maximum level of dissolved air we can expect in the fuel. Using the discussion of dissolved air in Shepherd, Krok, and Lee (1997) as a guide, the maximum total mass of gas that can be dissolved is:

$$
M_{g}=\frac{\beta P_{g} V_{l}}{R T}, \text { and } R=\frac{\tilde{R}}{W_{g}},
$$

where $M_{g}$ is the mass of the gas dissolved in a liquid, $\beta$ is the Ostwald coefficient defined as defined as the maximum volume of gas (at a given gas pressure $P_{g}$ and temperature $T$ ) that can be dissolved in a unit volume of liquid, $V_{l}$ is the volume of 
the liquid, $\tilde{R}$ is the universal gas constant, and $W_{g}$ is the molar weight of the gas. A typical value of $\beta$ for Jet $A$ between temperatures of 293 and $313 \mathrm{~K}$ is $\beta \approx 0.17$ (Coordinating Research Council, 1983). Using a gas pressure of 1 atmosphere (101 $\mathrm{kPa}$ ), and a temperature of $303 \mathrm{~K}$, we estimate that there is approximately $30 \mathrm{mg}$ of air dissolved in the $150 \mathrm{~mL}$ of Jet A added to the vessel. We can estimate the partial pressure of air that emerges from the fuel volume using the equation for the partial pressure of air using the results of the analysis presented in Shepherd, Krok, and Lee (1997):

$$
P_{\text {air }}=\frac{\beta \frac{V_{l}}{V_{g}} P_{o}}{1+\beta \frac{V_{l}}{V_{g}}}
$$

where $V_{g}$ is the ullage volume (i.e. vapor space volume) and $P_{o}$ is the pressure at which gas was originally dissolved into the liquid. With a fuel volume of 150 $\mathrm{mL}$ and a total chamber volume of $40 \mathrm{~L}(40,000 \mathrm{~mL})$, the partial pressure of air originally dissolved into Jet A under atmospheric conditions is approximately 0.63 mbar or 0.5 Torr. This is at most $5 \%$ of the fuel vapor pressure, and less than $0.1 \%$ of the total pressure.

It is important to verify that these estimates are accurate. To do so, ignition experiments are conducted with a regular sample of Jet A and a degassed sample, and the results are compared. If the amount of dissolved air is negligible, we should observe similar fuel vapor pressures and peak combustion pressures in the ignition experiments run with degassed and unaltered fuel. First, however, one sample of fuel must be degassed.

A degassing procedure was formulated to remove dissolved air from a sample of Jet A. Approximately $1 \mathrm{~L}$ of POSF-4658, which had been stored in a $5^{\circ} \mathrm{C}$ flammables refrigerator to preserve the presence of light hydrocarbon fuels, was added to a stainless steel degassing vessel. This vessel had a lid with ports to a vacuum line, a Heise $901 \mathrm{~A}$ manometer with a digital readout sensitive to $0.01 \mathrm{kPa}$, and a type $\mathrm{K}$ thermocouple. The Heise gauge and vacuum lines could be sealed off via a hand valve. Once the fuel was added and the vessel was sealed, the degassing vessel was placed in an ice bath. The fuel was cooled to $5^{\circ} \mathrm{C}$, so that the vapor pressure of all fuel components would be very low and there would be minimal evacuation of the light components. Once at $5^{\circ} \mathrm{C}$, the degassing vessel was evacuated for one 
minute. This step marks the begining of the degassing cycle and is designed to pull dissolved air out of solution in the fuel. After a minute, the vacuum line is closed off and the pressure is allowed to stabilize in the ice bath for 5 minutes. The stabilized pressure after evacuation is noted, and the vessel is moved to a hot plate, where the fuel is heated to $30^{\circ} \mathrm{C}$. The pressure is allowed to stabilize for 5 minutes with the fuel temperature at $30^{\circ} \mathrm{C}$ before noting the pressure while the fuel is warm. Then the degassing vessel is returned to the ice bath until the fuel temperature drops once again to $5^{\circ} \mathrm{C}$. Once the fuel is cold again, the pressure after the warm-cool cycle is noted.

The pressure of the cold fuel immediately after evacuation is compared with the pressure of the cold fuel after the warm-cool cycle is completed. Based on degassing guidance from Shepherd, Krok, and Lee (1997) the difference between the initial pressure after evacuation and the pressure after a warm-cool cycle is an indication of the success of the degassing procedure. The degassing procedure laid out above is repeated until there is minimal difference in the pressures between two subsequent cycles. A single degassing cycle takes over an hour, and the pressures in the vessel are so low that even a very small leak can lead to differences in the pressure results before and after a warm-cool cycle. In these experiments, 6 degassing cycles were completed, and the differences in pressure was about 2.8 mbar (2.1 Torr) for cycles 5 and 6 . In cycle 5, the pressure after evacuation was 1.9 mbar (1.4 Torr), and after warm-cool, the pressure was 4.8 mbar (3.6 Torr). In cycle 6, the pressure after evacuation was 1.7 mbar (1.3 Torr), and after warm-cool, the pressure was 4.4 mbar (3.3 Torr). There was minimal change in pressure results between cycles 5 and 6 , so the degassing procedure was considered complete at this point.

With a sample of degassed fuel available, ignition tests were conducted to investigate if there was a significant difference in the behavior of the degassed fuel versus the normal fuel. In order to perform ignition tests with jet fuel, a further modification to the setup had to be made. Because of the large volume of fuel used for Jet A testing, the syringe port is moved to the bottom of the vessel. This prevents the potential for splattering of the fuel on windows during injection (this was not an issue for other fuels since they had a relatively small liquid fuel volume and were expected to fully vaporize prior to performing an ignition test). Additionally, the liquid Jet A leftover at the end of an experiment must be drained from the vessel to avoid fuel "weath- 
ering" effects. Shepherd, Nuyt, and Lee (2000) noted that fuel weathering refers to the change in composition of a single batch of fuel going through large changes in temperature and/or altitude. In the case of the ignition experiments, weathering can occur if a single sample of fuel is used for multiple ignition experiments, going through multiple heating, ignition, and evacuation cycles. Weathering primarily alters the composition of the fuel, often depleting the lighter hydrocarbon species in such a manner that the average molecular weight of the fuel mixture gets heavier with each subsequent test. To avoid the complications that weathering presents and to ensure reproducible results, the leftover Jet A liquid is drained after each ignition experiment into a portable vessel. This portable vessel can be removed from the experimental setup to drain the leftover Jet A into a waste jar. Coupled with the evacuation of the vessel after each experiment, the removal of leftover Jet A ensures that each experiment is run with a fresh batch of Jet A. A plumbing diagram in shown in Fig. 5.13, with the blue components representing features like the septum port and portable vessel that are specifically added or modified for the Jet A experiments.

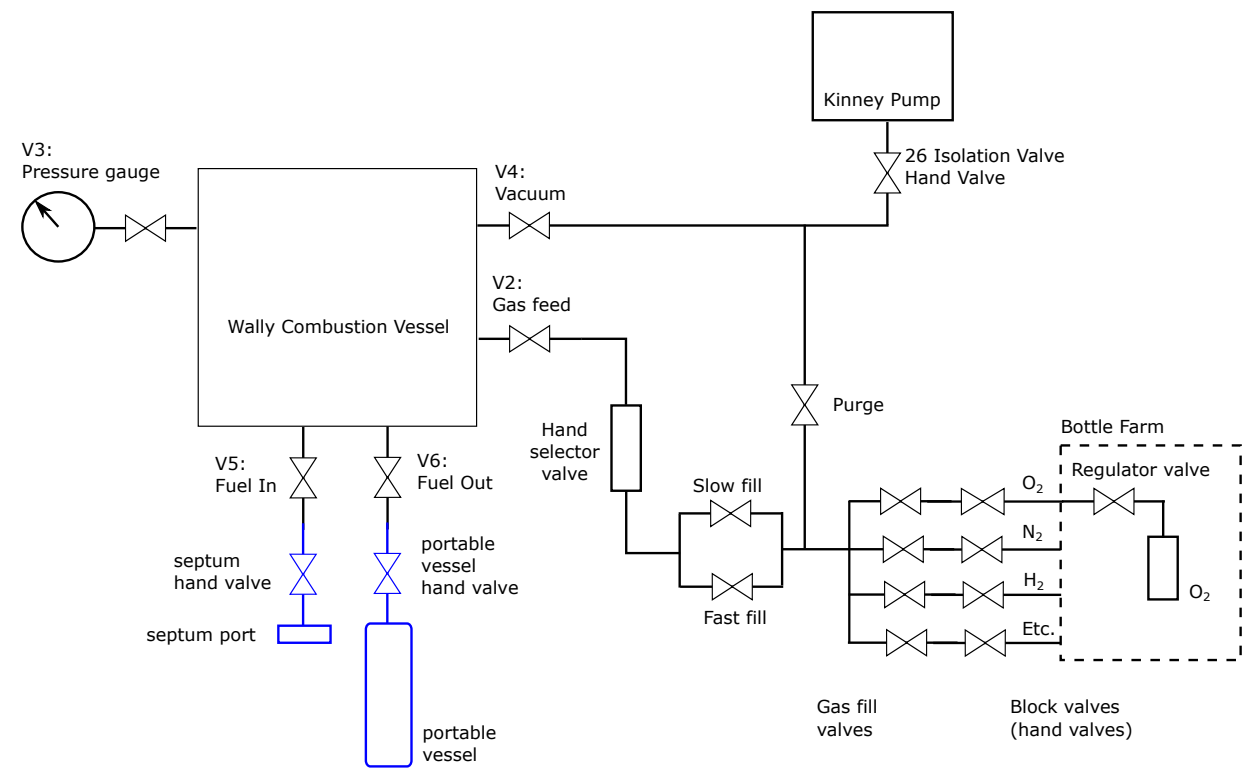

Figure 5.13: Plumbing schematic for combustion vessel. Blue areas represent plumbing components added for the Jet A ignition experiments.

With the plumbing modified, the ignition tests with degassed and normal Jet A can be conducted. The experiment was run according to the slightly modified procedure for working with Jet A (see the Jet A experiment checklist in Appx. $\mathrm{H}$ ) that accounts for the large fuel volume added for each experiment. The cylinder set temperature 
was $1050 \mathrm{~K}$ for both tests. A comparison of the fuel vapor pressure and of the peak pressure for normal and degassed fuel is shown in Table 5.9.

\begin{tabular}{|c|lll||}
\hline Fuel & $\begin{array}{l}\text { Fuel Vapor } \\
\text { Pressure } \\
\text { (Torr) }\end{array}$ & $\begin{array}{l}\text { Fuel Vapor } \\
\text { Pressure } \\
\text { (mbar) }\end{array}$ & $\begin{array}{l}\text { Peak Pres- } \\
\text { sure (bar) }\end{array}$ \\
\hline normal & 11.3 & 15.1 & 7.55 \\
degassed & 10.4 & 13.9 & 7.31 \\
\hline
\end{tabular}

Table 5.9: Results of ignition tests with normal and degassed fuel.

The variation in the results between the two fuel samples is within the expected range for the experiments. Some variation in both fuel vapor pressure and peak pressure occuring between shots is to be expected due to unavoidable slight differences in conditions and instrument drift. These results demonstrate that the difference between degassed Jet A and normal Jet A vapor pressures are less than 10\%, and the difference in peak combustion pressures are less than 3\%. These differences are consistent with the expected maximum amount of dissolved air and the potential shift in vapor composition due to the degassing procedure. We conclude that the influence of any dissolved air present in the normal fuel is minimal. With these results, full ignition testing can proceed. A total of 20 experiments were performed with Jet A (including the tests with normal and degassed Jet A), and logistic regression was performed on the ignition data. The results are shown in Fig. 5.14. The ignition temperature of Jet A was found to be $971.0 \mathrm{~K}$, with $95 \%$ confidence limits of $\pm 7 \mathrm{~K}$.

Figure 5.15 documents an example of an ignition sequence in the Jet A testing. These images come from shot 250, and the temperature of the cylinder at ignition was $960.6 \mathrm{~K}$. The ignition appears nearly symmetrically around the circumference of the cylinder with the flame appearing at nearly the same moment on both the left and right sides of the cylinder. Like with all the other fuels tested, the flame develops a barrel shape as the flame travels faster vertically, where it remains close to the hot surface. Using the schlieren visualization, we find that the average height of ignition was $21.6 \mathrm{~cm}$ from the bottom edge of the cylinder. This is similar to the ignition locations observed for the other fuels and confirms ignition is occuring at the top of the high-temperature region of the cylinder. Overall, the ignition dynamics of Jet A are very similar to those of other surrogate fuels. 


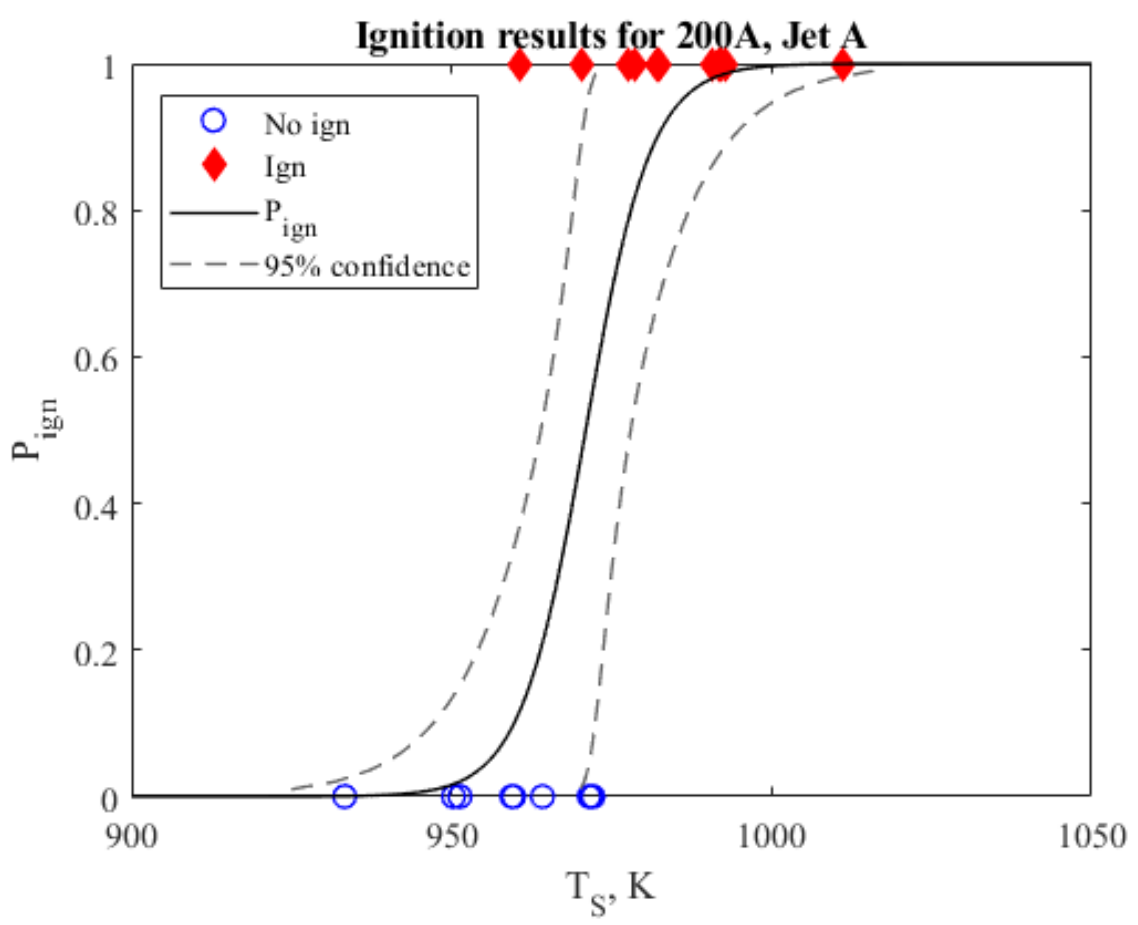

Figure 5.14: Ignition results for JI unified surrogate fuel with cylinder 200A.

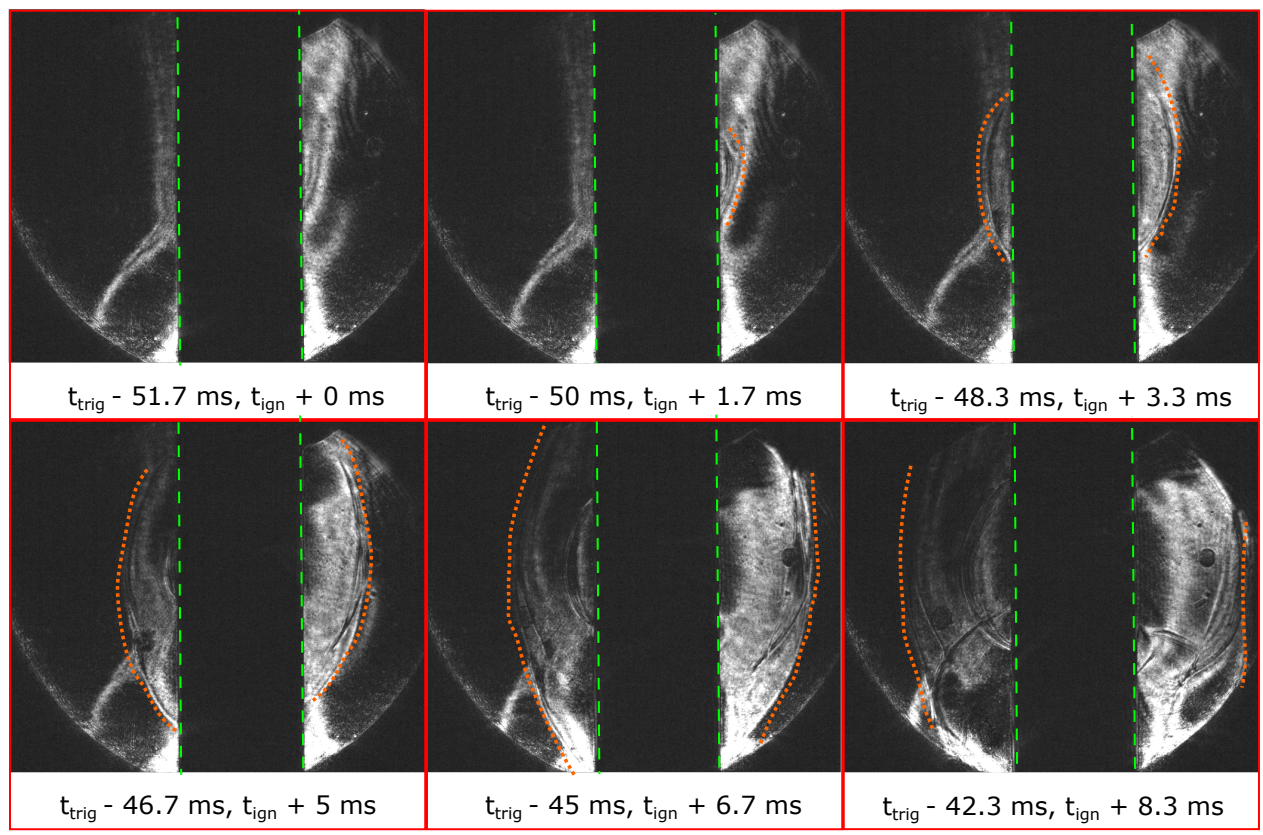

Figure 5.15: Ignition sequence for Jet A testing with cylinder 200A. Flame front highlighted with dashed orange lines.

The ignition sequence presented in Fig. 5.15 can be used to track the position of the flame front. The results are presented in Fig. 5.16. The flame speed of the left, 


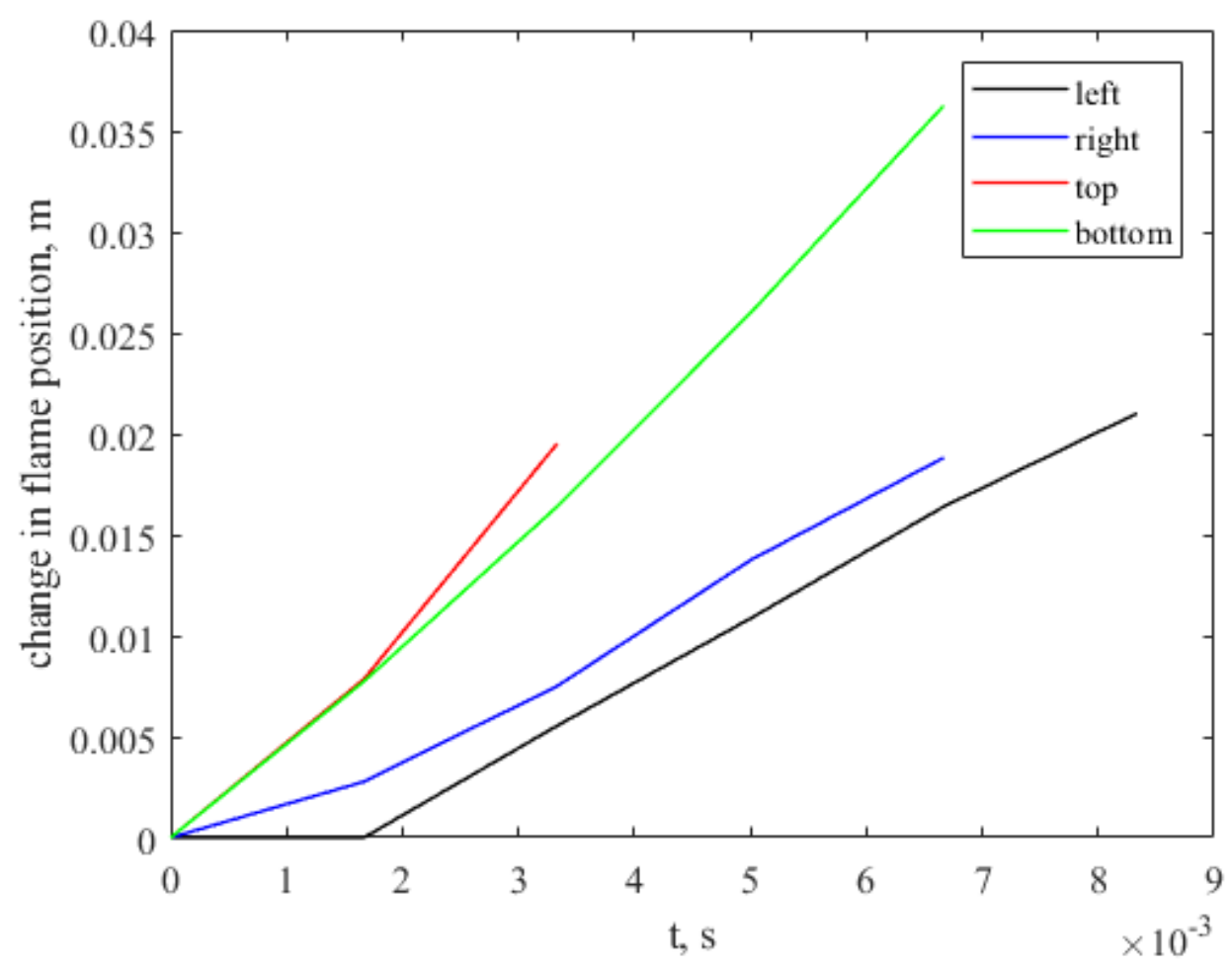

Figure 5.16: Flame position from shot 250, for the Jet A mixtures.

right, top, and bottom edges of the flame are 3.1, 2.8, 5.8, and $5.4 \mathrm{~m} / \mathrm{s}$, respectively. The flame speed of a stoichiometric JI mixture is computed for comparison with the Jet A flame speeds. For validation, the computed laminar burning speed of the JI mixture is compared to Jet A values from the literature. The computed laminar burning speed of the stoichiometric JI mixture at $400 \mathrm{~K}$ is $0.55 \mathrm{~m} / \mathrm{s}$, within $10 \%$ of experimental laminar burning speed of Jet $\mathrm{A}$ at 1 bar and $400 \mathrm{~K}$ measured by Kumar, Sung, and Hui (2011) to be approximately $0.59 \mathrm{~m} / \mathrm{s}$. The computed flame speed of the JI mixture at $400 \mathrm{~K}$ is $3.5 \mathrm{~m} / \mathrm{s}$ and at $750 \mathrm{~K}$ the flame speed is $7.0 \mathrm{~m} / \mathrm{s}$. This amounts to a maximum of $20 \%$ difference between the computed and experimental flame speeds. It is possible a more detailed mechanism and a better model for the Jet A mixture is needed in order to get more accurate estimates of Jet A flame speeds. However, the calculations confirm that the flame speed increases with increasing temperature, as has been observed in experiments with Jet A and other fuels. 


\subsection{Discussion and Summary}

The ignition temperatures for all experiments performed with cylinder 200A in the heated vessel (hexane, Aachen, JI, and Jet A) are reported in Table 5.10 along with the results of 200A tested with hexane in the room-temperature vessel.

\begin{tabular}{||c|ccc||}
\hline Fuel & Vessel Temp. (K) & Ign. Temp. (K) & Conf. Limits (K) \\
\hline Hexane (ambient temp) & 295 & 1019 & \pm 5 \\
Hexane (high temp) & 393 & 933.3 & \pm 12 \\
Aachen & 373 & 947.7 & \pm 12 \\
JI & 393 & 984.0 & \pm 3 \\
Jet A (POSF-4658) & 333 & 971.0 & \pm 7 \\
\hline
\end{tabular}

Table 5.10: Vessel temperature, ignition temperature, and confidence limits for all surrogate testing as well as regular and high ambient temperature testing of hexane.

The ignition temperatures can also be plotted against various parameters like molecular weight, hydrogen-to-carbon ratio, and vessel temperature. This is done in Figs. 5.17, 5.18, and 5.19, respectively. While the ignition temperature does have a general trend to increase with molecular weight, there are no obvious correlations between either ignition temperature and average hydrogen-to-carbon ratio of the fuel or ignition temperature and the vessel temperature.

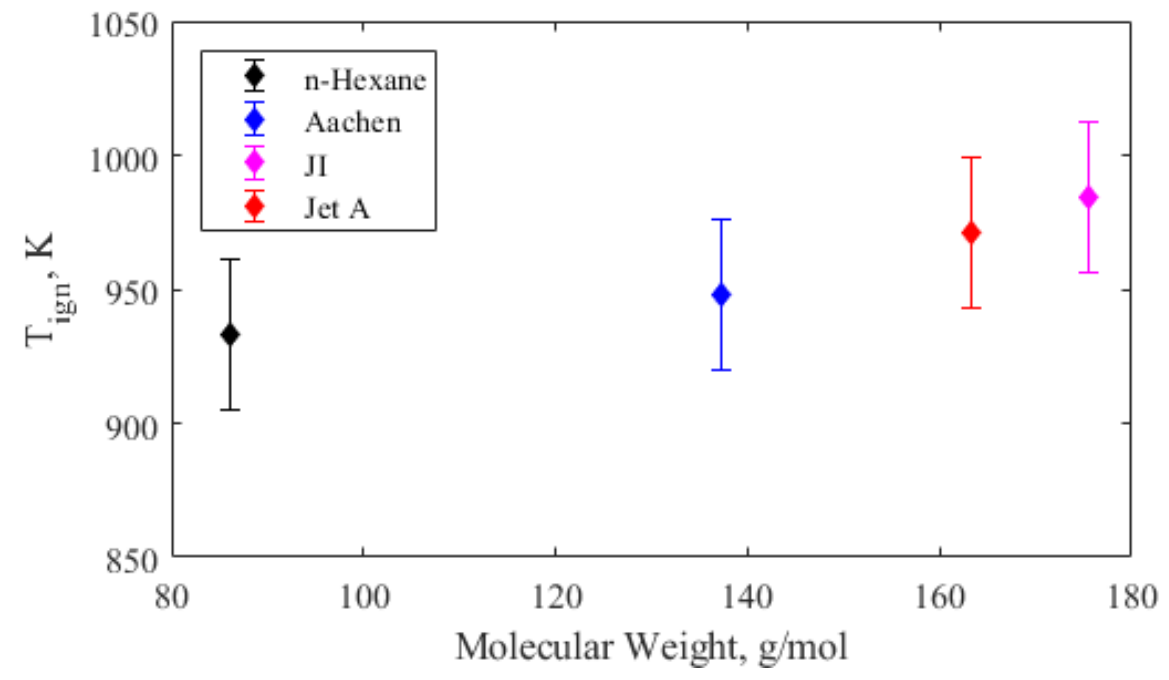

Figure 5.17: Ignition results for all surrogate testing.

Like in Chapter 4, we can extract the ignition delay times from the experiments where the surface temperature of the cylinder reached the steady-state set point prior to 


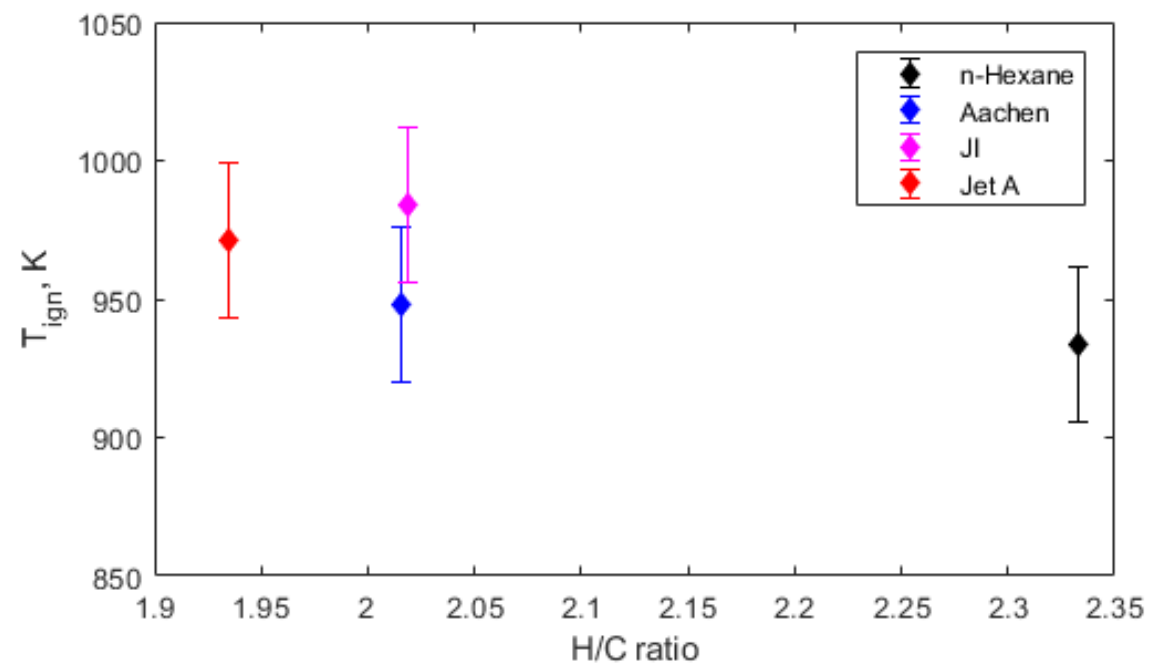

Figure 5.18: Ignition results for all surrogate testing.

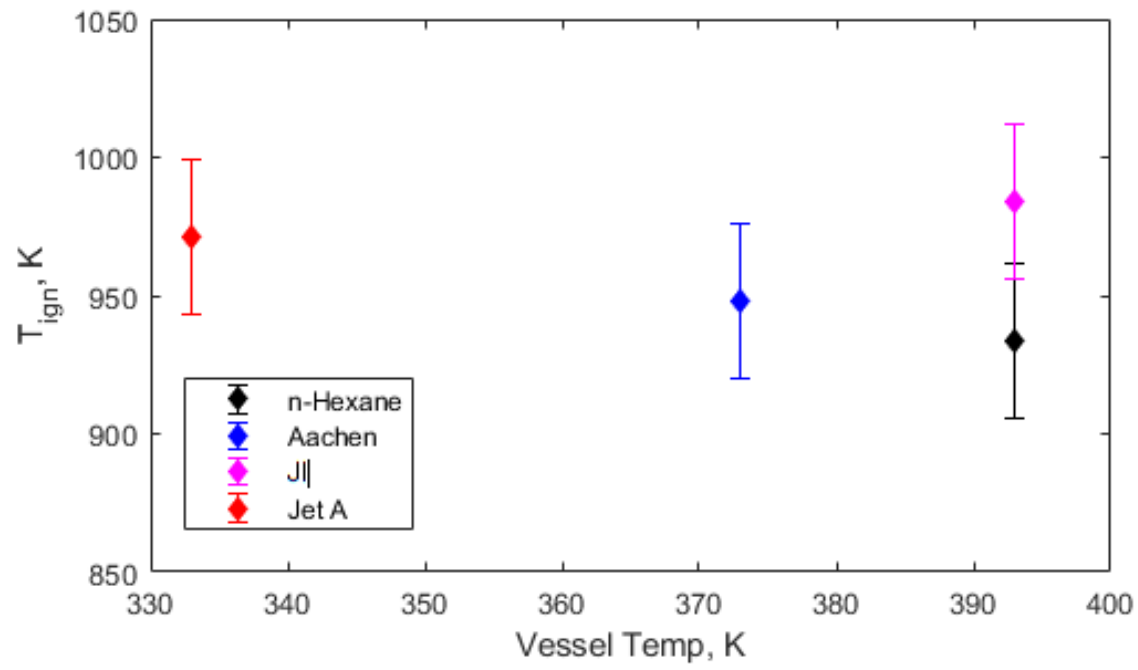

Figure 5.19: Ignition results for all surrogate testing.

ignition. A significant scatter in the results prevents any meaningful correlation between $\tau_{i g n}$ and $T_{i g n}$.

This work with the heated experimental setup and surrogate fuels reveals some interesting insights. We compare the results of each fuel to the Jet A results to discern which is the surrogate matches Jet A ignition behavior most closely. The JI ignition temperature is $13 \mathrm{~K}(1.3 \%)$ higher than the Jet A ignition ignition temperature, while the ignition temperature of Aachen is $23 \mathrm{~K}(2.4 \%)$ lower than that of Jet A. The high temperature hexane tests had an ignition temperature $38 \mathrm{~K}(3.9 \%)$ lower than Jet A, while the room temperature hexane tests had an ignition temperature $48 \mathrm{~K}(4.9 \%)$ 
higher than Jet A.

We also note that the ignition temperature for hexane is modestly different from Jet A. This difference is less than $5 \%$ as noted previously, but larger than the $50^{\circ} \mathrm{F}(28$ $\mathrm{K})$ difference required by the FAA between reported literature autoignition temperatures of Jet A and maximimum allowable temperatures in a flammable leakage zone (Federal Aviation Administration, 2018). Hexane is often used as a surrogate for Jet A due to its simplicity (vaporizes at room temperature, single-component fuel) and while it is capable of matching Jet A behavior to a certain extent, it is also important to consider more complex fuels that more closely match Jet A ignition behavior.

Flame speeds were measured for all of the fuels tested. The vertical flame speed was found to be approximately twice the horizontal flame speed due to the high temperature boundary layer. Flame speed computations for each fuel confirmed that an increase in temperature increased the flame speed. Hexane at $400 \mathrm{~K}$ was found to have a significantly higher flame speed than Aachen, JI, and Jet A under the same conditions. This observation is corroborated by Kumar, Sung, and Hui (2011), who note that laminar burning speeds of n-decane are significantly higher than those of Jet A under the same conditions. They note that the aromatic components are likely responsible for the reduced laminar burning speeds. While there is some variation in the flame speeds among Aachen, JI, and Jet A, on the whole, the two surrogates produce similar flame speed results to the Jet A that they are designed to emulate.

Of all the fuels investigated here, the JI surrogate matches the ignition behavior of Jet A best. This is similar to the results reported by Martin and Shepherd (2019) that $\mathrm{JI}$ is a more appropriate surrogate for Jet A than the Aachen mixture. This is expected due to the fact that the JI surrogate was formulated to match specific physical and chemical properties of Jet A (Chen, Khani, and Chen, 2016). The excellent agreement between Jet A and JI ignition temperatures is encouraging, as $\mathrm{JI}$ is significantly easier to perform ignition tests with than Jet A. There is no need to consider fuel mass loading, fuel weathering, or dissolved air effects when working with JI. Due to the specific formulation of the JI surrogate, reproduceable experiments are easier to achieve with JI than commodity Jet A batches. JI therefore presents a promising option for thermal ignition experiments that are simplified in a number of ways yet present very similar results to working with Jet A. 


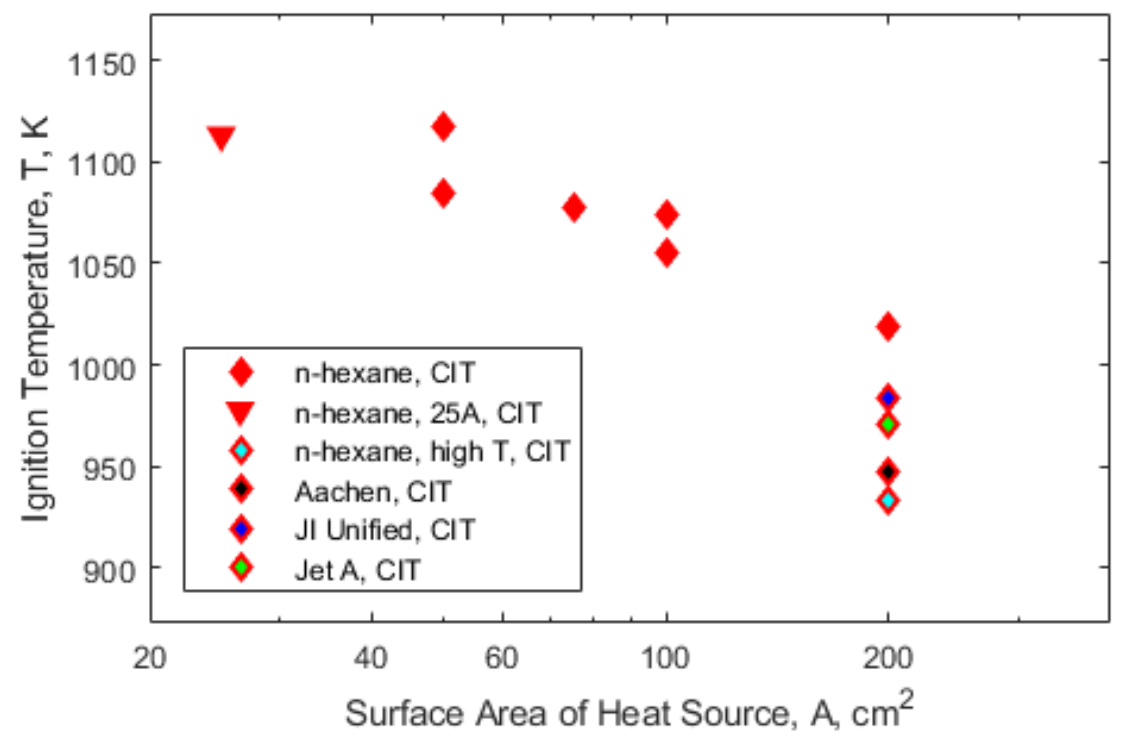

Figure 5.20: Ignition results for all ignition testing.

The results of testing surrogate fuels with a heated vessel are plotted with hexane tests reported in Chapter 4 in Figure 5.20. Error bars using the upper bound on pyrometer uncertainty $( \pm 28 \mathrm{~K})$ are not included in this figure so that each data point can be seen clearly. This data set is also plotted against thermal ignition results from the the literature in Fig. 5.21. This experimental campaign has revealed the modest dependence of ignition temperature on surface geometry for external natural convection, the key importance of flow configuration on ignition temperature, and the relevance of several surrogate fuels to reproducing Jet A behavior for thermal igntion from a concentrated hot surface. 


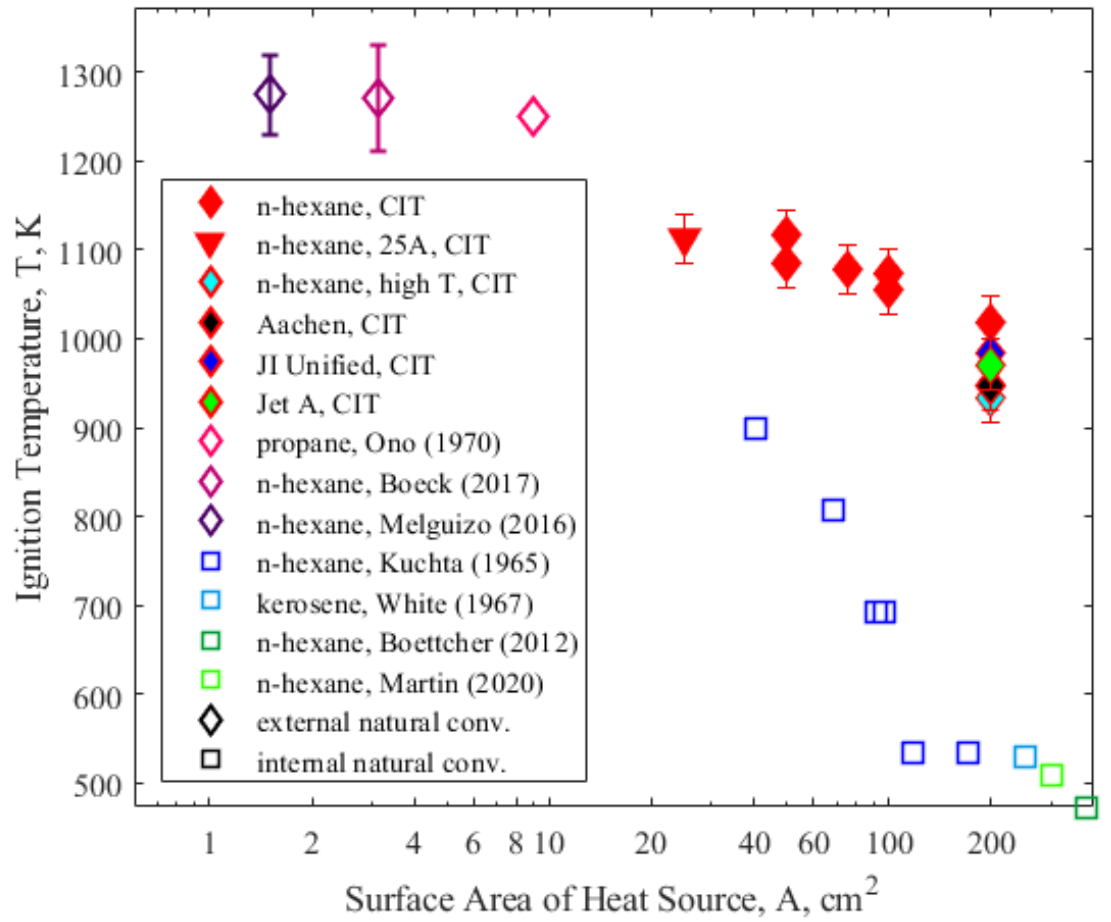

Figure 5.21: Ignition results for all ignition testing compared with literature values. 


\section{BIBLIOGRAPHY}

Boeck, Lorenz R. et al. (2017). "Ignition of fuel-air mixtures from a hot circular cylinder". In: Combustion and Flame 185, pp. 265-277.

Chen, X., E. Khani, and C. Chen (2016). "A unified jet fuel surrogate for droplet evaporation and ignition.” In: Fuel 182, pp. 284-291.

Coordinating Research Council (1983). Handbook of aviation fuel properties. English. Atlanta, Ga.; Warrendale, Pa.: Society of Automotive Engineers.

Coronel, Stephanie A. et al. (2018). "Experimental and numerical study on moving hot particle ignition”. In: Combustion and Flame 192, pp. 495-506.

Edwards, J. T. (2017). "Reference Jet Fuels for Combustion Testing”. In:

Federal Aviation Administration (2018). Advisory Circular 25.981-1D.

Honnet, S. et al. (2009). "A surrogate fuel for kerosene". In: Proceedings of the Combustion Institute 32.1, pp. 485-492.

Kumar, Kamal, Chih-Jen Sung, and Xin Hui (2011). "Laminar flame speeds and extinction limits of conventional and alternative jet fuels". In: Fuel 90.3, pp. 10041011. Dor: $10.1016 / \mathrm{j}$. fuel.2010.11.022.

Laurendeau, Normand M. (1982). "Thermal ignition of methane-air mixtures by hot surfaces: A critical examination”. In: Combustion and Flame 46, pp. 29-49.

Lee, J and J Shepherd (2000). Spark Ignition Measurements in Jet A: part II. Tech. rep. FM99-7. California Institute of Technology.

Martin, C. and J. Shepherd (2019). Autoignition testing of hydrocarbon fuels using the ASTM-E659 method. Tech. rep. EDL2019.002. Graduate Aeronautical Laboratories, California Institute of Technology.

Martin, C. and J.E. Shepherd (2020). "Low Temperature Autoignition of Jet A and Surrogate Jet Fuels". In: 13th International Symposium on Hazards, Prevention, and Mitigation of Industrial Explosions.

Mullen, James W., John B. Fenn, and Moreland R. Irby (1948). "The ignition of high velocity streams of combustible gases by heated cylindrical rods". In: Symposium on Combustion and Flame, and Explosion Phenomena 3.1, pp. 317-329.

Ono, S. et al. (1976). "Ignition in a Free Convection from Vertical Hot Plate". In: Bulletin of JSME 19.132, pp. 676-683.

Ranzi, E et al. (2014). "Reduced kinetic schemes of complex reaction systems: fossil and biomass-derived transportation fuels". In: Internatiomal Journal of Chemical Kinetics 46, pp. 512-542. 
Shepherd, J. E., C. D. Nuyt, and J. L. Lee (2000). Flash Point and Chemical Composition of Aviation Kerosene (Jet A). Tech. rep. FM99-4. California Institute of Technology.

Shepherd, J., J. Krok, and J. Lee (1997). Jet A Explosion Experiments: Laboratory Testing. Tech. rep. FM97-5. California Institute of Technology.

- (1999). Spark Ignition Energy Measurements in Jet A. Tech. rep. FM97-9. California Institute of Technology.

Sund, Sveinung M (2019). “Thermal Ignition of Surrogate Jet Fuels”. PhD thesis. University of South-Eastern Norway. 


\section{Chapter 6}

\section{MODELING}

In this chapter, two modeling approaches are carried out to gain insight into the ignition process. The first is an investigation of the ignition chemistry of hexane along streamlines. The goal of is to understand how the heating rate of a streamline affects chemical pathways to ignition. A parametric model of streamline temperature-time histories is used to prescribe the temperature increase to a zero-dimensional reactor model and a hexane kinetic mechanism models the fuel chemistry. The second modeling effort is a one-dimensional simulation of ignition adjacent to a suddenly heated cylinder. The governing equations include species and heat diffusion, and the chemistry is modeled with a kinetic mechanism for hydrogen. The goal of these simulations is to analyze the heat flux and energy balance to gain insight into the ignition dynamics near the heated surface at critical wall temperature conditions.

\subsection{Chemistry along Streamlines}

One way of gaining insight into the chemical processes of ignition is to take a simplified modeling approach. In this section, the temperature history of a streamline is used as input to a $0 \mathrm{D}$ reactor simulation to prescribe the temperature history in the reactor. The reactor model uses a detailed chemical kinetic mechanism to model the chemistry. This approach decouples the fluid mechanics from the chemistry, thereby making the modeling less computationally demanding. A similar method was explored by Mével et al. (2016) to model the chemical kinetics around a moving hot sphere in an $\mathrm{n}$-hexane environment.

While this model does not capture all the physics present in the experiments and therefore is not meant to be a predictive model, it does serve as a useful way to explore the chemistry that may be occurring in the experiments. In particular, Section 4.6 identified potential decomposition of hexane in the thermal layer as a source of error in the interferometry results. The model of chemistry along streamlines is used to further explore the decomposition process of hexane in a way that would be very challenging to observe with experiments alone. 


\section{Streamline Temperature History}

We carry out a simplified modeling of ignition over a cylinder by using the the similarity solution detailed in Chapter 3 to model streamlines of a natural convection flow. The stream function is computed from Eq. 3.12, originally from Sparrow and Gregg (1956). Rewriting this as:

$$
\psi=4 v r_{0} x^{3 / 4}\left(\frac{g \beta\left(T_{w}-T_{\infty}\right)}{4 v^{2}}\right)^{1 / 4} F(\xi, \eta) .
$$

The stream function, $\psi$, is then normalized by the maximum value of the stream function in the domain:

$$
\psi_{n}=\frac{\psi}{\psi_{\max }} .
$$

Streamlines are defined by a constant value of the stream function. Nine values of the normalized stream function, $\psi_{n}$, were used to define nine streamlines. A wide range of $\psi_{n}$ were chosen to allow the selection of streamlines close to the hot surface as well as streamlines that barely enter the thermal layer, as shown in Fig. 6.1. Once streamlines are selected, the $(x, y)$ position, the wall parallel and perpendicular velocities $u, v$, and the temperature, $T$ at every point along each streamline are stored.

The time history of the streamline can then be calculated by the following procedure: track the arc length, $s$, then integrate the reciprocal of arc velocity with respect to arc length. The change in arc length is defined as:

$$
d s^{2}=d x^{2}+d y^{2},
$$

and we define $s=0$ at the start of the streamline such that all subsequent arc lengths can be found by:

$$
s_{i}=s_{i-1}+\sqrt{d x_{i}^{2}+d y_{i}^{2}} .
$$

The total time to move along the streamline is given by:

$$
t_{s}=\int_{0}^{s} \frac{1}{\sqrt{u^{2}+v^{2}}} d s
$$




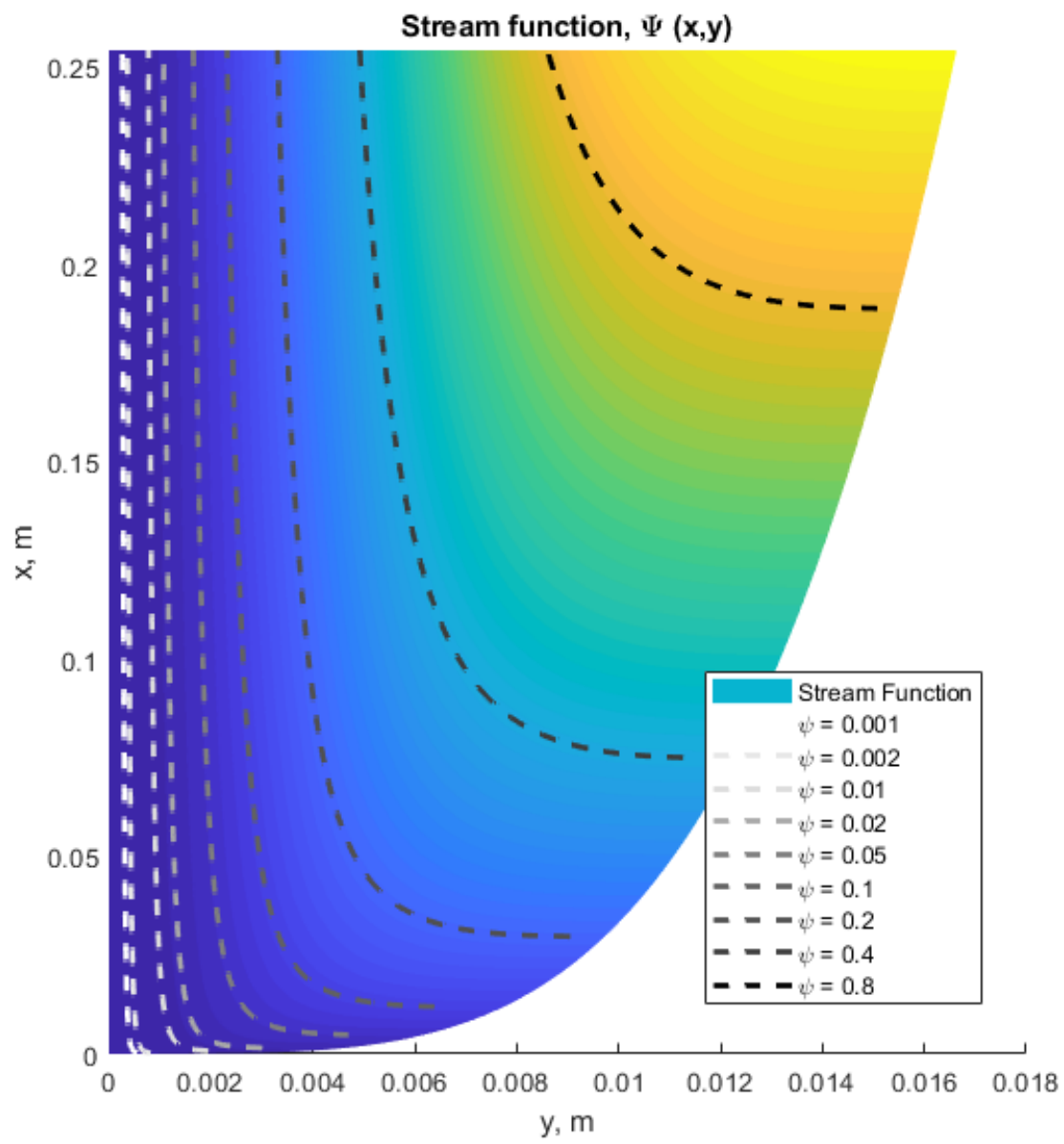

Figure 6.1: Normalized stream function, $\psi_{n}$, from similarity solutions. Selected streamlines for tracking highlighted with dashed gray lines.

The heat flux along each streamline is calculated:

$$
\rho c_{p} \frac{D T}{D t} \approx \rho c_{p} \frac{\Delta T}{\Delta t}
$$

and then $\rho c_{p} \frac{\Delta T}{\Delta t}$ is fit to a function by piecewise cubic Hermite interpolation. All streamline information is stored in a data structure and saved for use in a 0D reactor computation, including the temperature history of the streamline and the piecewise fit of that temperature history. The temperature history of the streamlines are shown in Fig. 6.2 , 


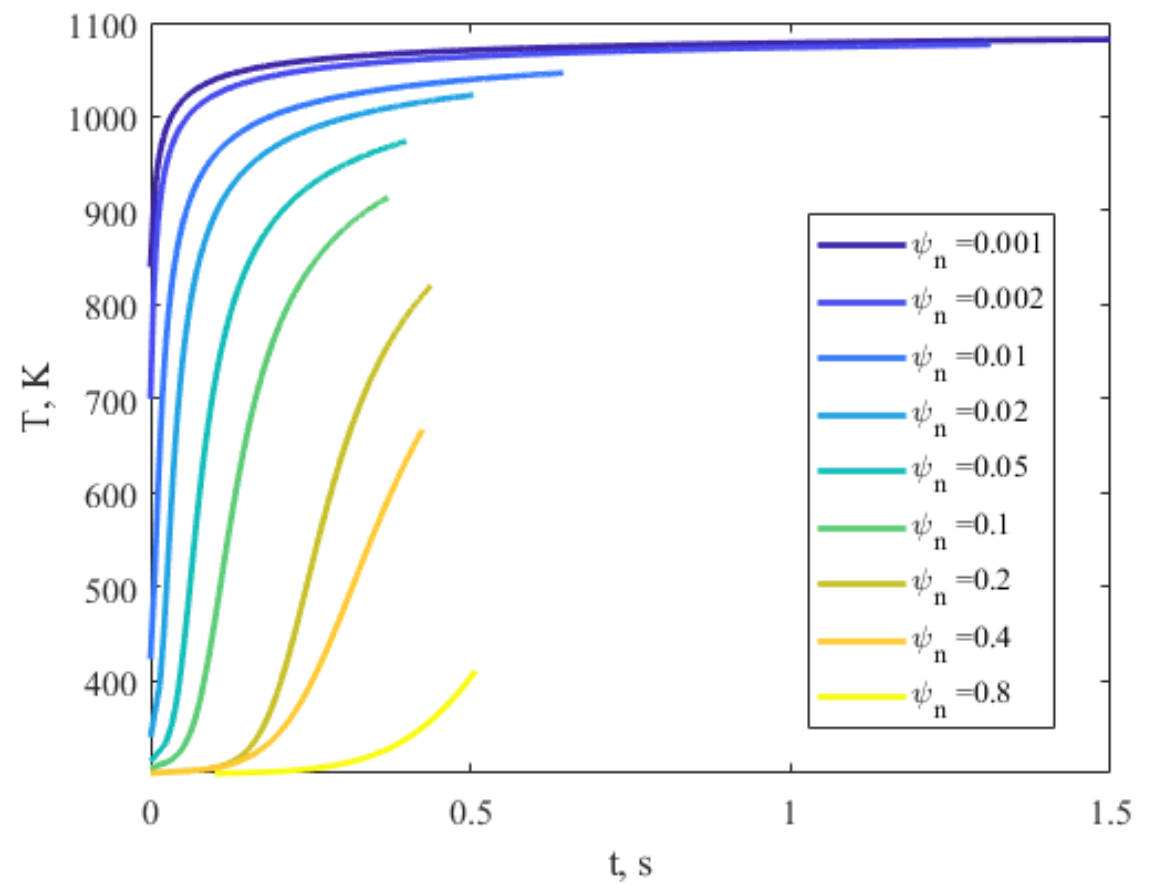

Figure 6.2: Stream function temperature history from similarity solutions.

\section{Zero Dimensional Reactor Calculation}

A 0D constant pressure reactor is used to approximately compute the chemistry that takes place along a streamline in the thermal layer. The kinetic mechanism used for this computation is hexaneReduced.cti, which was previously presented in Coronel, Melguizo-Gavilanes, Davidenko, et al. (2017). This is a reduced hexane mechanism with 63 species and 230 reactions.

The model for the zero-dimensional reactor starts from the energy and species transport equations:

$$
\begin{gathered}
\rho c_{p} \frac{D T}{D t}=-\rho \sum_{k=1}^{n} h_{k} \frac{D Y_{k}}{D t}-\nabla \cdot q-\rho \nabla T \cdot \sum_{k=1}^{n} Y_{k} c_{p, k} V_{k}, \\
\rho \frac{D Y_{k}}{D t}=-\nabla \cdot\left(\rho Y_{k} V_{k}\right)+M_{k} \dot{\omega}_{k},
\end{gathered}
$$

where $Y_{k}$ is the species mass fraction of species $\mathrm{k}, h_{k}$ is the enthalpy of species $\mathrm{k}, q$ is the heat flux, $c_{p, k}$ is the heat capacity of species $\mathrm{k}, V_{k}$ is the diffusion velocity of species k, $M_{k}$ is the molecular weight of species k, and $\dot{\omega}_{k}$ is the production rate of species k. 
To simplify the governing equations, the species diffusive transport is neglected:

$$
V_{k}=0 \text {. }
$$

Furthermore, the heat flux along a streamline is estimated from the non-reactive simulation of the boundary layer:

$$
-\nabla \cdot q=\left.\underline{\rho c_{p} \frac{\Delta T}{\Delta t}}\right|_{S L, \dot{\omega}_{k}=0} .
$$

Equations 6.9 and 6.10 are substituted into Eqs. 6.7 and 6.8.

$$
\begin{gathered}
\frac{d T}{d t}=-\frac{\sum_{i=1}^{n} M_{i} h_{i} \dot{\omega}_{i}}{\rho c_{p}}+\frac{\left.\rho c_{p} \frac{\Delta T}{\Delta t}\right|_{S L, \dot{\omega}_{k}=0}}{\rho c_{p}}, \\
\frac{d Y_{i}}{d t}=\frac{M_{i} \dot{\omega}_{i}}{\rho}
\end{gathered}
$$

where $\left.\rho c_{p} \frac{\Delta T}{\Delta t}\right|_{S L, \dot{\omega}_{k}=0}$ is the piecewise cubic Hermite fit of heat flux for each streamline of interest. This approximates the energy losses normal to the streamline. Equation 6.11 is the energy conservation equation, and 6.12 is the species conservation equation. The governing equations are integrated until the final time along each tracked streamline. The temperature, pressure, density, mole, and mass fraction of each species are stored for post-processing.

\section{Reactive Streamline Analysis without Diffusion}

The temperature history prescribed by the inert streamline computation and the temperature history output from the OD reactor computation are compared in Fig. 6.3 . Reaction is marked by an increase in the temperature compared to the inert streamline temperature profile. If no significant reaction takes place along a streamline, then the temperature history from the OD reactor should follow the prescribed temperature history of that streamline.

From this analysis, we see that streamlines $\psi_{n}=0.001,0.002$, and 0.01 ignite very quickly. This amounts to ignition at a distance along the surface of $\mathrm{x}=0.7,1.6$, and $14.2 \mathrm{~cm}$. The reactor computation predicts ignition well below the top edge $(x=25.4$ 

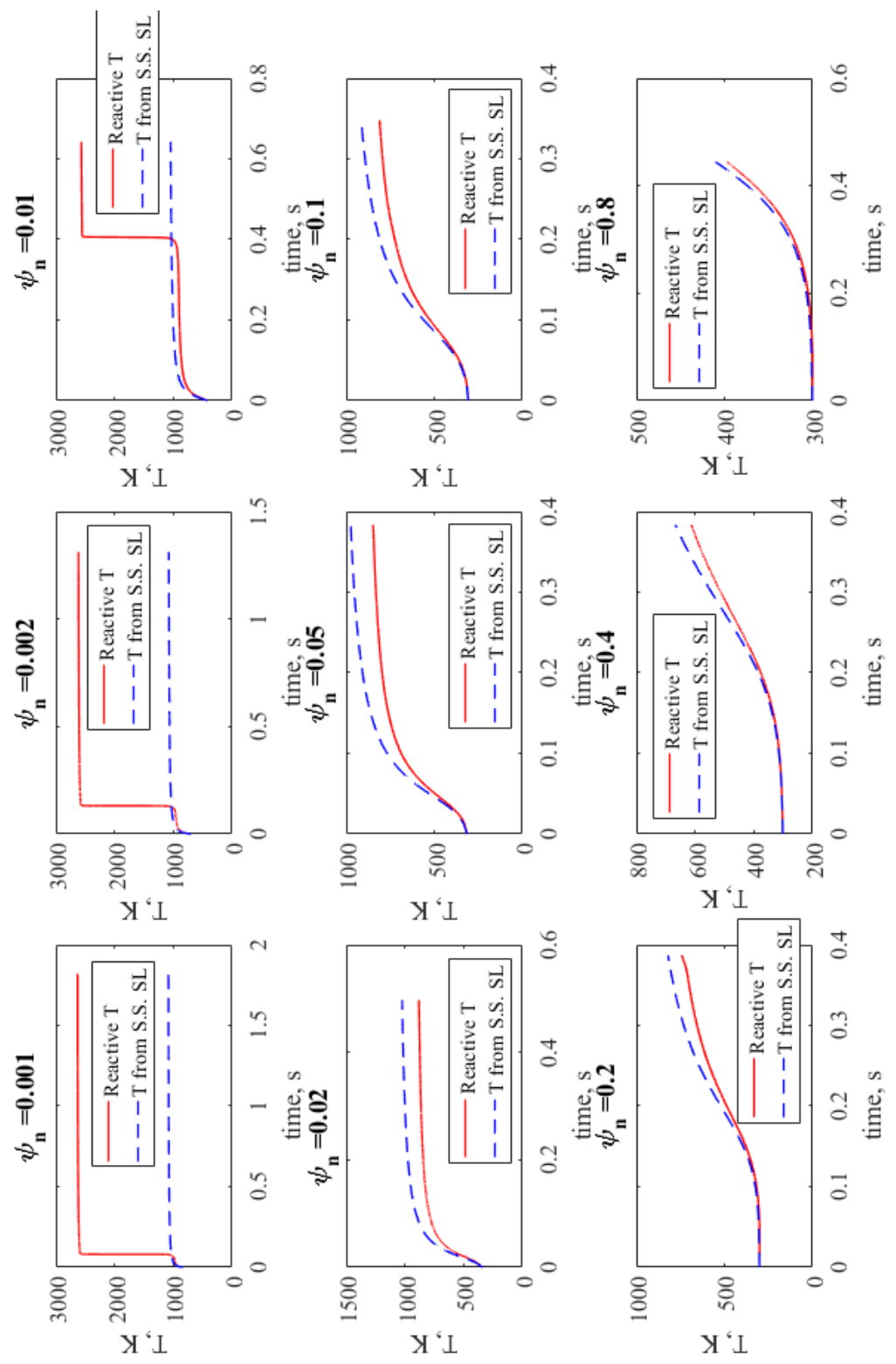

Figure 6.3: Temperature history comparison from inert streamlines taken from similarity solution (blue solid line) and reactive 0D reactor computation (red dashed line).

$\mathrm{cm})$ of the plate in contrast to the ignition location observed in the experiments. This is likely because this calculation does not include heat and species diffusion out of the fluid element, only the nonreactive heat transfer into the fluid element. 


\section{Parametric Analysis of Chemistry}

The advantage of the approximate streamline analysis is that the simplicity of the computation enables the use of chemical kinetic mechansims for hexane mixtures with very modest computing resources. A parametric study on the effect of rate of temperature increase of a streamline is performed to learn more about the chemical processes that occur for hexane. We will investigate how a change in timescale of heating of the streamline affects the chemical processes of hexane ignition. Informed by the $\mathrm{S}$-shape streamline temperature histories of seen in 6.2 , the following function is used to prescribe a temperature history for an artificial streamline:

$$
F=\frac{x^{2}}{1+x^{2}}
$$

where $F=\frac{T-T_{\infty}}{T_{s}-T_{\infty}}$, and $x=t / \tau * . \tau *$ is the time at which $F=1 / 2$. The derivative of $F$ is computed analytically.

$$
\frac{d F}{d x}=\frac{2 x}{\left(1+x^{2}\right)^{2}}
$$

Transforming this back into dimensional variables, the expression for the thermal energy source term is:

$$
\frac{d T}{d t}=\left(T_{s}-T_{\infty}\right) \frac{t / \tau *^{2}}{\left(1+(t / \tau *)^{2}\right)^{2}} .
$$

Changing the value of $\tau *$ changes the rate of the temperature increase for the artificial streamline. The prescribed temperature history for $\tau *=0.1,0.5$ is shown in Fig. 6.4 .

For these artificial streamlines, $T_{s}=1100 \mathrm{~K}$ and $T_{\infty}=300 \mathrm{~K}$, matching the temperature conditions in the streamline calculation. A value of $\tau *=0.1 \mathrm{~s}$ results in a very rapid temperature rise, while $\tau *=0.5 \mathrm{~s}$ causes a more gradual temperature rise. These two artificial streamlines will be used to run a $0 \mathrm{D}$ reactor caluculation using the system of equations in Eq. 6.11 and 6.12 to observe how rate of temperature increase affects ignition chemistry. The reactive temperature history of these streamlines is shown in Fig. 6.5 .

For $\tau *=0.1 \mathrm{~s}$, at approximately $\mathrm{t}=0.2 \mathrm{~s}$, there is a rapid and very large temperature increase relative to the inert streamline temperature history, on the order of 


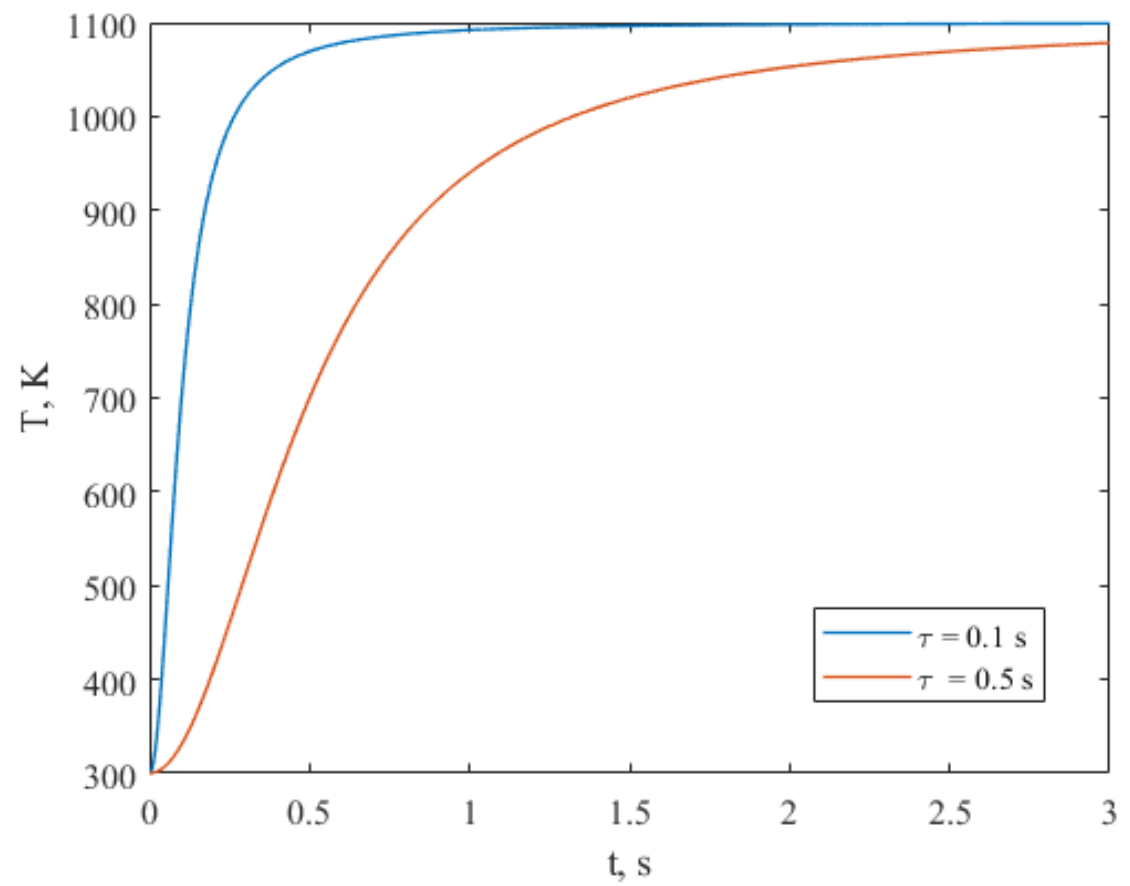

Figure 6.4: Temperature history of artificial streamlines.
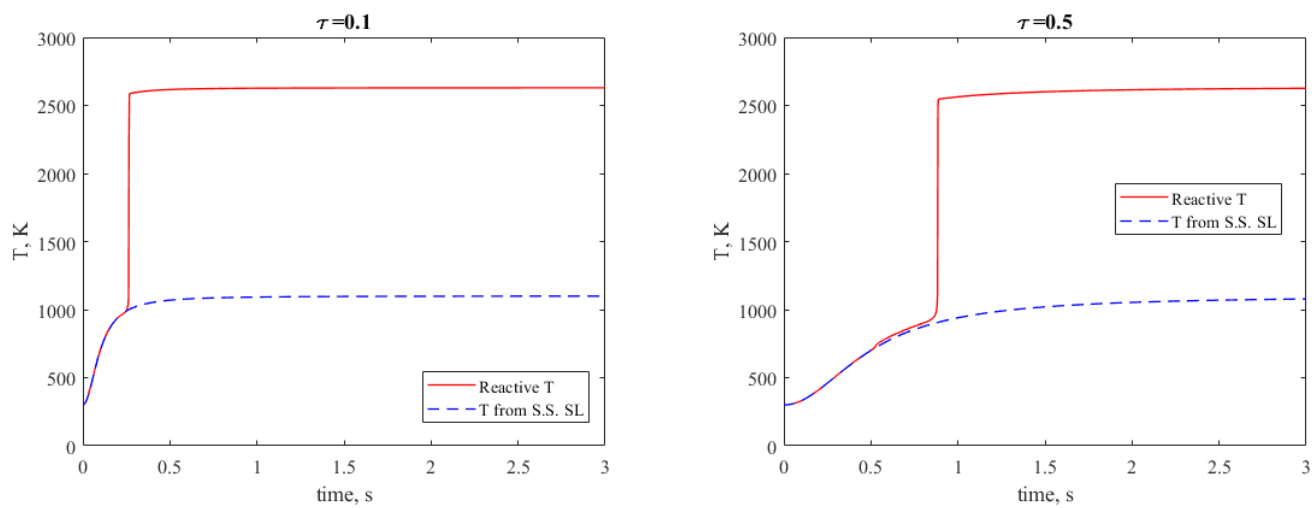

Figure 6.5: Reactive temperature history of artificial streamlines. Left: $\tau *=0.1 \mathrm{~s}$, Right: $\tau *=0.5 \mathrm{~s}$.

$\Delta T \approx 2000 \mathrm{~K}$. For $\tau *=0.5 \mathrm{~s}$, there is a small temperature increase, $\Delta T \approx 50 \mathrm{~K}$, at approximately $\mathrm{t}=0.5 \mathrm{~s}$, which is followed by a very large temperature increase on the order of $\Delta T \approx 2000 \mathrm{~K}$ at approximately $0.9 \mathrm{~s}$. The next section explores how these temperature increases correspond to chemical energy release. 


\section{Chemical Energy Analysis}

Another way to investigate the nature of the reaction taking place is to find peaks in the chemical energy term, that is, $-\frac{\sum_{i=1}^{n} h_{i} \dot{\omega}_{i}}{\rho c_{p}}$. A peak in the chemical energy term is identified by finding a local maxima of the energy term, given a minimum threshold value of $100 \mathrm{~K} / \mathrm{s}$. Figure 6.6 plots the chemical energy term $\tau *=0.1,0.5 \mathrm{~s}$.
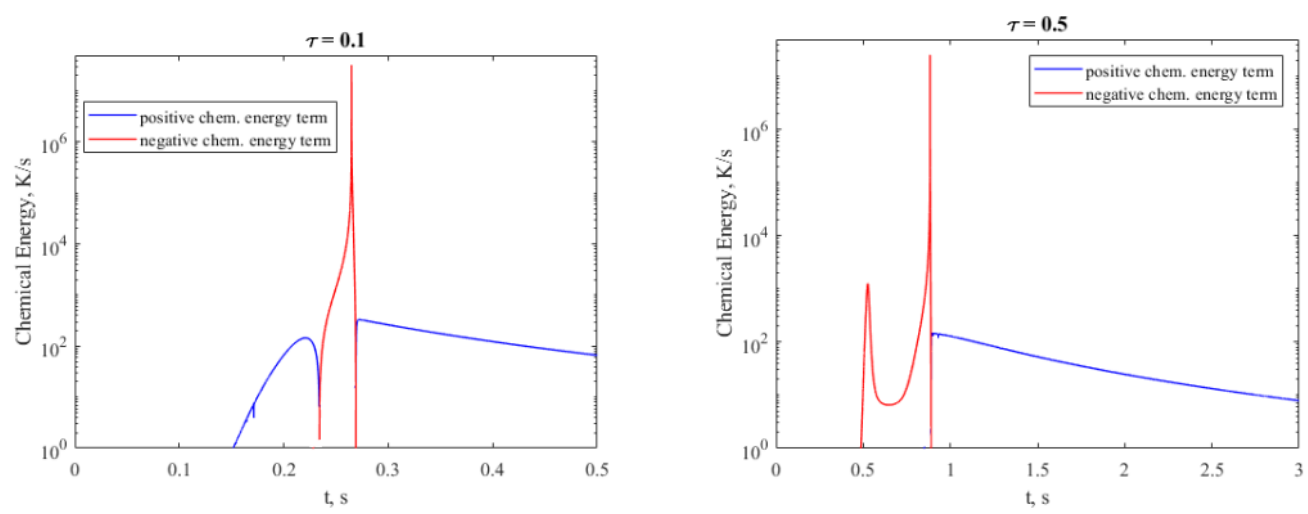

Figure 6.6: Chemical energy term for all $\tau *=0.1$ (left), $0.5 \mathrm{~s}$ (right). Red indicates the chemical energy term is negative, blue indicates the chemical energy is positive.

For the artificial stream line with $\tau *=0.1 \mathrm{~s}$, only one peak in the chemical energy term is observed, corresponding with the large temperature increase in Fig. 6.5 at $0.2 \mathrm{~s}$. The peak chemical energy release was on the order of $10^{7}$. For $\tau *=0.5 \mathrm{~s}$, two peaks in the chemical energy release occur. The first peak, of the order $10^{3}$, corresponded to the first temperature increase for around $t=0.5 \mathrm{~s}$. The second peak corresponded to the second very large temperature increase in the temperature history at $0.9 \mathrm{~s}$, and the peak energy release was on the order of $10^{7}$. These two distinctly different peak magnitudes in chemical energy release, coupled with the corresponding temperature increase for each peak, allude to two distinct stages of ignition taking place for $\tau *=0.5 \mathrm{~s}$. The first stage of ignition causes a small temperature increase of approximately $50 \mathrm{~K}$, and a peak chemical energy release of $10^{3}$. The second stage of ignition corresponds with a large temperature increase on the order of $2000 \mathrm{~K}$ and a peak chemical energy release of $10^{7}$.

The energy release in the first stage of ignition would likely alter the streamlines. The temperature increase and generation of first stage ignition products (discussed in the following sections) would likely cause expansion of the fluid element during the first stage of ignition compared to the inert streamlines shown in Fig. 6.1. This 
effect is challenging to estimate with the current model of ignition as the chemistry is decoupled from fluid mechanics. A two-dimensional model of the ignition problem would likely be required to calculate the effect of first stage ignition energy release on the streamline. This is a possible direction for future work.

\section{Literature on Two-Stage Ignition Behavior}

A survey of literature reveals explanations for this two-stage ignition phenomenon with hexane. Discussions in Menon et al. (2016) and Peters et al. (2002) investigate the two-stage ignition phenomenon in hydrocarbons, namely n-heptane. n-heptane has very similar ignition and flame propogation characteristics to n-hexane and is often used as an analog for $n$-hexane in computational work because its low temperature chemistry is better characterized than that of n-hexane.

Menon et al. (2016), Peters et al. (2002), and Boettcher et al. (2012) describe a twostage ignition process that occurs at the low to intermediate temperatures observed in the cylinder experiments. The first stage occurs due to the breakdown of fuel starting at temperatures around $650 \mathrm{~K}$ and is accompanied by a small temperature increase of 20 to $100 \mathrm{~K}$ (Menon et al., 2016). Peters et al. (2002) and Boettcher et al. (2012) describe in detail the dominant chemical chain branching process that occurs in the first stage of ignition:

- hydrogen abstraction of heptane fuel $\left(\mathrm{C}_{7} \mathrm{H}_{16}\right)$ by $\mathrm{OH}$ to form heptyl radical $\left(\mathrm{C}_{7} \mathrm{H}_{15}\right)$;

- $\mathrm{O} 2$ addition to heptyl radical forms heptyl peroxy radical $\left(\mathrm{C}_{7} \mathrm{H}_{15} \mathrm{O}_{2}\right)$;

- Intramolecular hydrogen abstraction of heptyl peroxy radical to form hydroperoxy heptyl $\left(\mathrm{C}_{7} \mathrm{H}_{14} \mathrm{O}_{2} \mathrm{H}\right)$;

- $\mathrm{O} 2$ addition to hydroperoxy heptyl to form hydroperoxyheptyl peroxy $\left(\mathrm{HO}_{2} \mathrm{C}_{7} \mathrm{H}_{13} \mathrm{O}_{2} \mathrm{H}\right)$;

- $\mathrm{OH}$ elimination of hydroperoxyheptyl peroxy to form ketohydroperoxide $\left(\mathrm{OC}_{7} \mathrm{H}_{13} \mathrm{O}_{2} \mathrm{H}\right)$;

- OH elimination of ketohydroperoxide.

Thus, two hydroxyl radicals are generated from this chain-branching reaction that begins with the consumption of one hydroxyl radical. This increase in the production 
of hydroxyl radicals is accompanied by a small temperature increase. This temperature increase eventually passes what Peters et al. (2002) describe as a crossover temperature, $T^{*}$. Once the temperature is greater than $T^{*}$, the low-temperature chain-branching reaction described above becomes chain-breaking, as the heptyl radicals are directly broken down into lighter hydrocarbon species. The production of hydroxyl radicals halts, and as a consequence, the temperature stops increasing as well. Menon et al. (2016) cites the same low-temperature chain-branching process, and notes that the fuel mass fraction can decrease by as much as $60 \%$ during the first stage of ignition. This concludes the first stage of ignition.

The second stage of ignition is what is normally thought of as a standard ignition event; there is a sharp increase in production of important radical species which react to form major products accompanied by a large temperature increase on the order of $2000 \mathrm{~K}$. Menon et al. (2016) and Peters et al. (2002) agree that the dissociation of $\mathrm{H}_{2} \mathrm{O}_{2}$ is the rate-limiting step of the second stage of ignition. In this stage, as fuel is depleted, $\mathrm{H}_{2} \mathrm{O}_{2}$ builds up. Relative to other important reactions in this stage, the hydrogen peroxide decomposes slowly. When it decomposes, the hydrogen peroxide produces two hydroxyl radicals, which are critical to the second "main" high-temperature ignition event.

Peters et al. (2002) mention that when the initial temperature of a reactor is greater than the crossover temperature $T^{*}$, only the second stage of ignition will occur. No first-stage ignition takes place since the chemical pathways that lead to the lowtemperature chain branching are out-competed by higher temperature reactions that directly break the fuel radical into lighter hydrocarbon species. This describes exactly the single ignition event observed for $\tau *=0.1 \mathrm{~s}$.

Menon et al. (2016) and Peters et al. (2002) come to different conclusions about the causes of the second-stage ignition event due to differences in the computational setup. The computations in Peters et al. (2002) were performed in a homogeneous reactor (zero-dimensional) with a prescribed initial temperature, and as such did not include any diffusion effects. Peters et al. (2002) concluded that second-stage ignition occurred when the fuel was fully depleted; the reaction of fuel and hydroxyl radical is fast so that as long as fuel is present, $\mathrm{OH}$ can be assumed to be in steadystate. When fuel is depleted, the hydroxyl radical builds up and leads to a traditional 
thermally explosive ignition event.

Menon et al. (2016), however, note different behavior leading up to second-stage ignition. The authors performed a combined numerical and experimental study of thermal ignition from a hot glowplug. The computations were two-dimensional and included diffusion effects. Instead of ignition occurring due to fuel depletion, Menon et al. (2016) notes that diffusion acts against local fuel depletion: fuel diffuses into regions near the hot surface where fuel is consumed quickly. $\mathrm{OH}$ buildup is observed before any local fuel depletion occurs, contradicting the conclusions drawn by Peters et al. (2002) that second-stage ignition occurs when fuel is depleted. In the tests modeled by Menon et al. (2016), the surface temperature of the glowplug continually increases, causes the gas temperature surrounding the glowplug to increase as well. The rise in gas temperature increases the rate of $\mathrm{H}_{2} \mathrm{O}_{2}$ decomposition which exothermically produces $\mathrm{OH}$ and further increases the gas temperature that then further accelerates $\mathrm{H}_{2} \mathrm{O}_{2}$ decomposition. This positive feedback loop leads to thermal runaway and explosion. Menon et al., 2016 notes this positive feedback loop occurs when temperatures exceed $900 \mathrm{~K}$.

\section{Major and Minor Species Analysis}

With these mechanisms for first- and second-stage ignition from the literature in mind, we can examine the results from the OD reactor computations of artificial streamlines in more detail. First, the major and minor species mole fractions are examined for the case with only a second-stage "main" ignition event ( $\tau *=0.1 \mathrm{~s}$ ) and for the case with first- and second-stage ignition events $(\tau *=0.5 \mathrm{~s})$. The minor species investigated in this process is informed by the important minor species noted in Menon et al. (2016) and Peters et al. (2002), namely, ethylene $\left(\mathrm{C}_{2} \mathrm{H}_{4}\right)$, hydrogen peroxide, hydroxyl radicals, the hexyl radical $\left(\mathrm{C}_{6} \mathrm{H}_{13}\right)$, hexyl peroxy $\left(\mathrm{C}_{6} \mathrm{H}_{13} \mathrm{O}_{2}\right)$, and ketohydroperoxide $\left(\mathrm{OC}_{6} \mathrm{H}_{11} \mathrm{O}_{2} \mathrm{H}\right)$.

First, we examine the results in terms of the mole fraction for the artificial streamline with $\tau *=0.1 \mathrm{~s}$ (rapid temperature increase) as an example of only the second-stage, high-temperature ignition process occurring. Figures $6.7 \mathrm{a}$ and $6.7 \mathrm{~b}$ show the evolution of the mole fraction over time. For the major species, there is a slow decrease in the mole fraction of hexane up to approximately $260 \mathrm{~ms}$, at which time there is a second-stage or "main" ignition event that causes rapid depletion of the fuel and a 


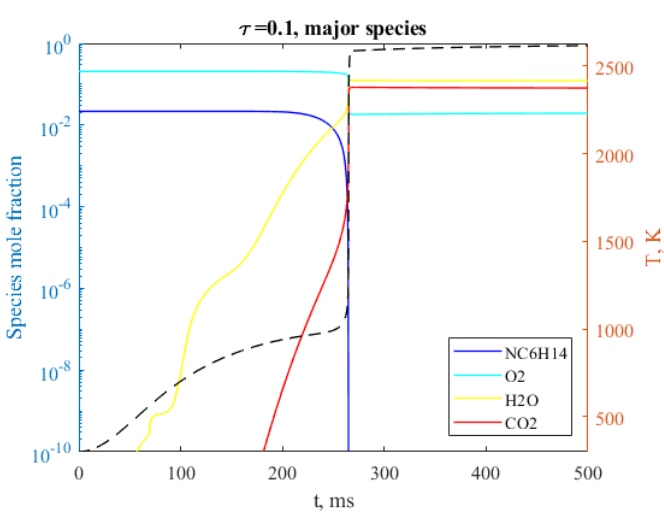

(a) Major species

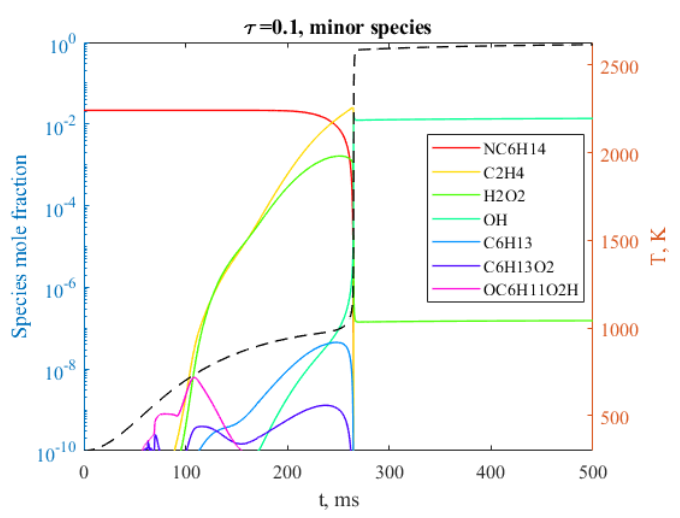

(b) Minor species

Figure 6.7: Major and minor species mole fraction for $\tau *=0.1 \mathrm{~s}$. Second-stage ignition only. (a) Major species mole fractions are shown as solid, colored lines. The temperature history is given as a black dashed line. (b) Minor species mole fractions are shown as solid, colored lines. The temperature history is given as a black dashed line.

large temperature rise. While the mole fraction of hexane gradually decreases prior to the ignition event, water and carbon dioxide are gradually increasing. This is due to the decomposition of hexane prior to ignition. Examining the minor species mole fractions presented in Fig. 6.7b confirms that as the hexane is gradually being consumed prior to ignition, lighter hydrocarbon species like ethylene are produced. However, there is no sharp peak in intermediate species or sudden increase in temperature prior to the "main" ignition event to indicate a first-stage ignition event. Indeed, the mole fraction of ketohydroperoxides ("OC6H1102H") remains small throughout the computation as the temperature of the artificial streamline rapidly increases to temperatures greater than $T^{*}$. Viable pathways to producing significant amounts of ketohydroperoxides are quickly made inactive, thus eliminating the potential for a first-stage ignition event.

We can contrast this with the ignition behavior of an artificial streamline with $\tau *=0.5 \mathrm{~s}$. First- and second-stage ignition events occur, and the major and minor species mole fractions are shown in Fig. 6.8a and 6.8b, respectively. The first stage ignition event occurs around $520 \mathrm{~ms}$ and causes a relatively small jump in temperature. This first stage ignition event is marked in Fig. 6.8a by a drop in hexane mole fraction and a jump in water and carbon dioxide. Prior to the first stage ignition event, the mole fraction of hexane was relatively constant. During the first stage ignition event, we see spikes in the minor species that are part of the low-temperature 


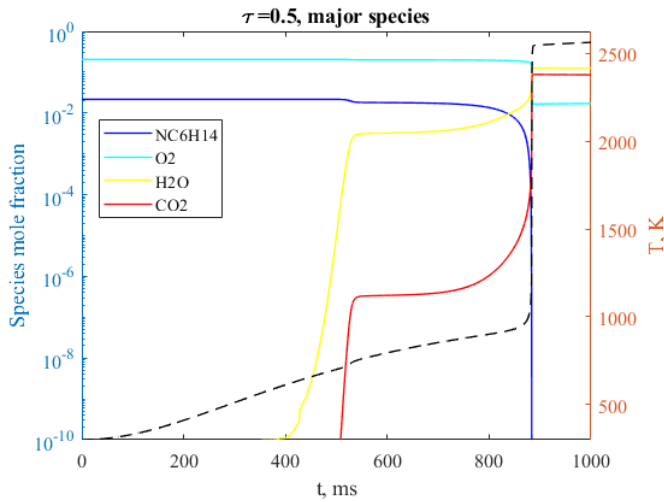

(a) Major species

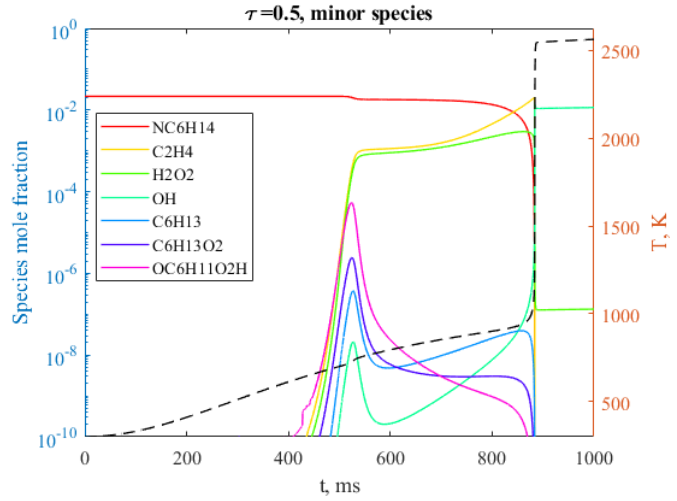

(b) Minor species

Figure 6.8: Major and minor species mole fraction for $\tau *=0.5 \mathrm{~s}$. First- and secondstage ignition behavior shown. (a) Major species mole fraction, $\mathrm{X}$, shown as solid, colored lines. The temperature history is given as a black dashed line. (b) Minor species mole fraction, $\mathrm{X}$, shown as solid, colored lines. The temperature history is given as a black dashed line.

chain branching pathway, namely, hexyl ("C6H13"), hexyl peroxide ("C6H13O2"), and ketohydroperoxide ("OC6H11O2H"). There is also a large jump in the mole fraction of ethylene and hydrogen peroxide during the first-stage ignition event, and a small spike in the hydroxyl radical mole fraction. The increase hydroxyl radical production causes a small jump in temperature. This brings the temperature of the gas over the crossover temperature $T^{*}$, at which point the low-temperature pathway becomes chain breaking and the first-stage ignition event ends.

The second-stage ignition event for $\tau *=0.5 \mathrm{~s}$ proceeds much like the ignition event for $\tau *=0.1 \mathrm{~s}$. Leading up to the second-stage ignition event, there is a gradual decrease of hexane mole fraction, accompanied by a gradual increase of ethylene and hydrogen peroxide mole fractions. During the second-stage ignition event, there is a large temperature increase and large spike in hydroxyl radical mole fraction.

\section{Reaction Pathway Diagrams}

We extract reaction pathway diagrams from various points during the streamline computation to learn more about how the chemical pathways evolve between the two stages of ignition. We use the case of the gradually heated streamline $(\tau *=0.5)$, as it involves both a first- and second-stage ignition event. Figure 6.9 shows the major chemical pathways at the peak energy release of the first stage of ignition. The 
chain-branching reaction described in Peters et al. (2002) and Menon et al. (2016) for the first stage of ignition is evident in Fig. 6.9. The dominant path is as follows: the n-hexane fuel (red) decomposes to a hexyl radical (orange) primarily via reaction with $\mathrm{OH}$. $\mathrm{O} 2$ addition to the hexyl radical forms hexyl peroxy radical (yellow), which then becomes hydroperoxy hexyl (green). This step shows no interaction with another species because this is an intramolecular hydrogen abstraction. The most prominent path of the hydroperoxy hexyl involves the addition of another $\mathrm{O}_{2}$ molecule to form the hydroperoxy hexyl peroxy radical (blue). $\mathrm{OH}$ elimination then transforms the hydroperoxy hexyl peroxy into ketohydroperoxide (purple). Ketohydroperoxide then breaks down into other hydrocarbon species via $\mathrm{OH}$ elimination. The key parts of the low temperature chain branching reaction have been color coded to make them easier to identify in Fig. 6.9.

Figure 6.10 shows the evolution of the chemical pathways as the first stage of ignition ends. The temperature has increased slightly due to the production of $\mathrm{OH}$ radicals from the low-temperature chain-branching pathway enough to start activating higher-temperature chain-terminating pathways. As we can see in Fig. 6.10. the low-temperature pathway that produces ketohydroperoxide is still active at this point, but alternative pathways are also active, siphoning off some of hexyl radicals that would have otherwise been used in the low-temperature pathway. Instead, these hexyl radicals break down directly into lighter hydrocarbon species (highlighted in various shades of pink).

Figure 6.11 shows the full termination of the low-temperature chain-branching pathways after the first stage of ignition. This path diagram is taken at the halfway point between the first- and second-stage ignition events. Hexane reacts primarily with $\mathrm{OH}$ but also increasingly with $\mathrm{HO}_{2}$ to form hexyl radicals. The low-temperature chain-branching pathway that led to the formation of ketohydroperoxide is entirely disrupted. Instead, the dominant pathway is the breakdown of hexyl radicals into lighter hydrocarbons (various shades of pink). This shows that the first stage of ignition is complete, and now we observe further decomposition of hexane into lighter hydrocarbon species.

Finally, we examine Fig. 6.12 to investigate the dominant chemical pathways during the peak of the second stage of ignition. The pathway starts with ethylene, as 
the hexane has mostly decomposed into ethylene at this point in the reaction. The ethylene is rapidly broken down until combustion products like carbon monoxide and carbon dioxide are formed. Particularly, the reaction of $\mathrm{CO}$ with $\mathrm{OH}$ is very exothermic and contributes to the thermal explosion that is a part of the second-stage ignition event. 


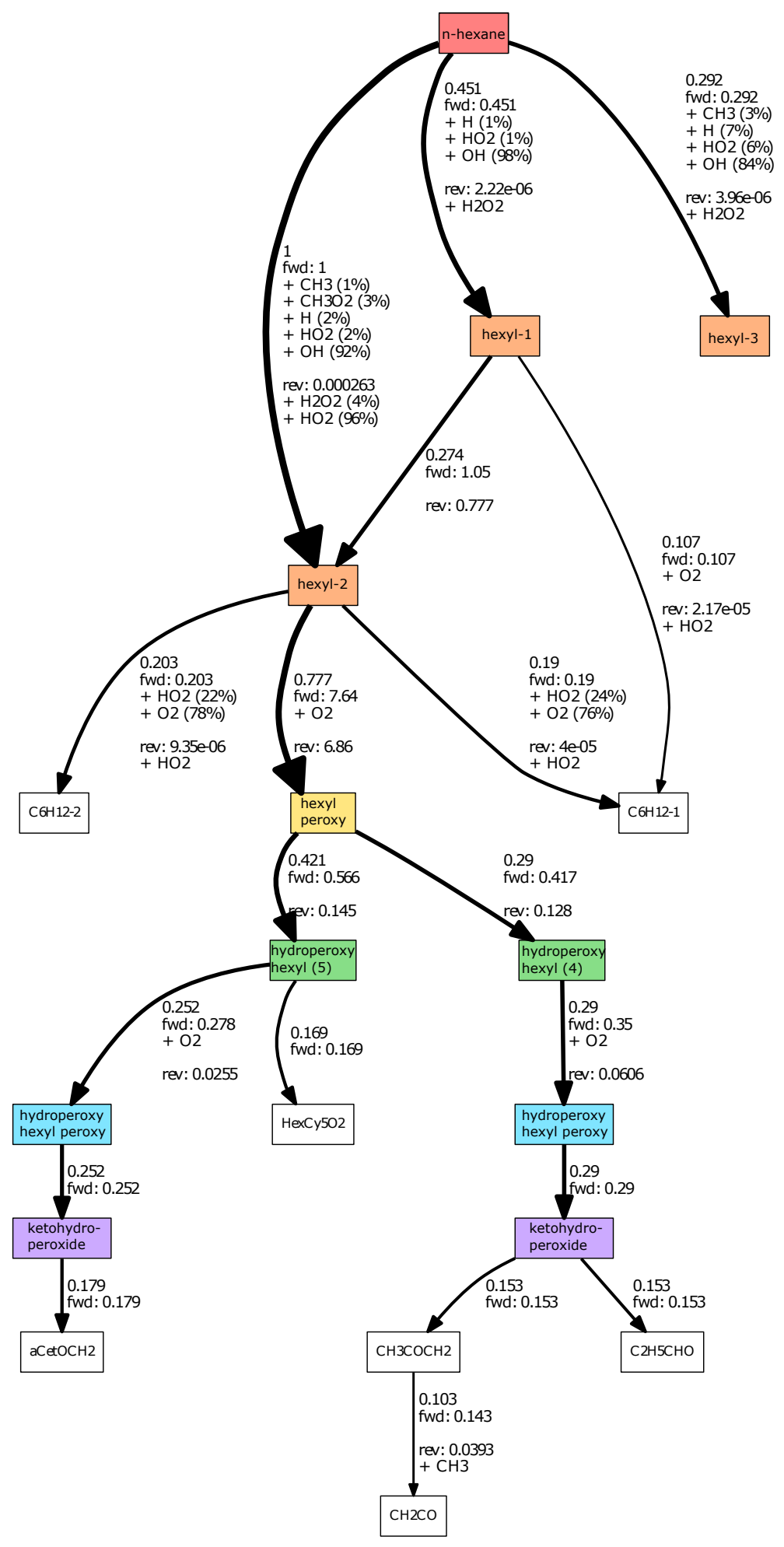

Figure 6.9: Reaction pathway diagram for peak of first stage of ignition. Taken from $\tau *=0.5$ during the peak chemical energy release of the first ignition stage. 


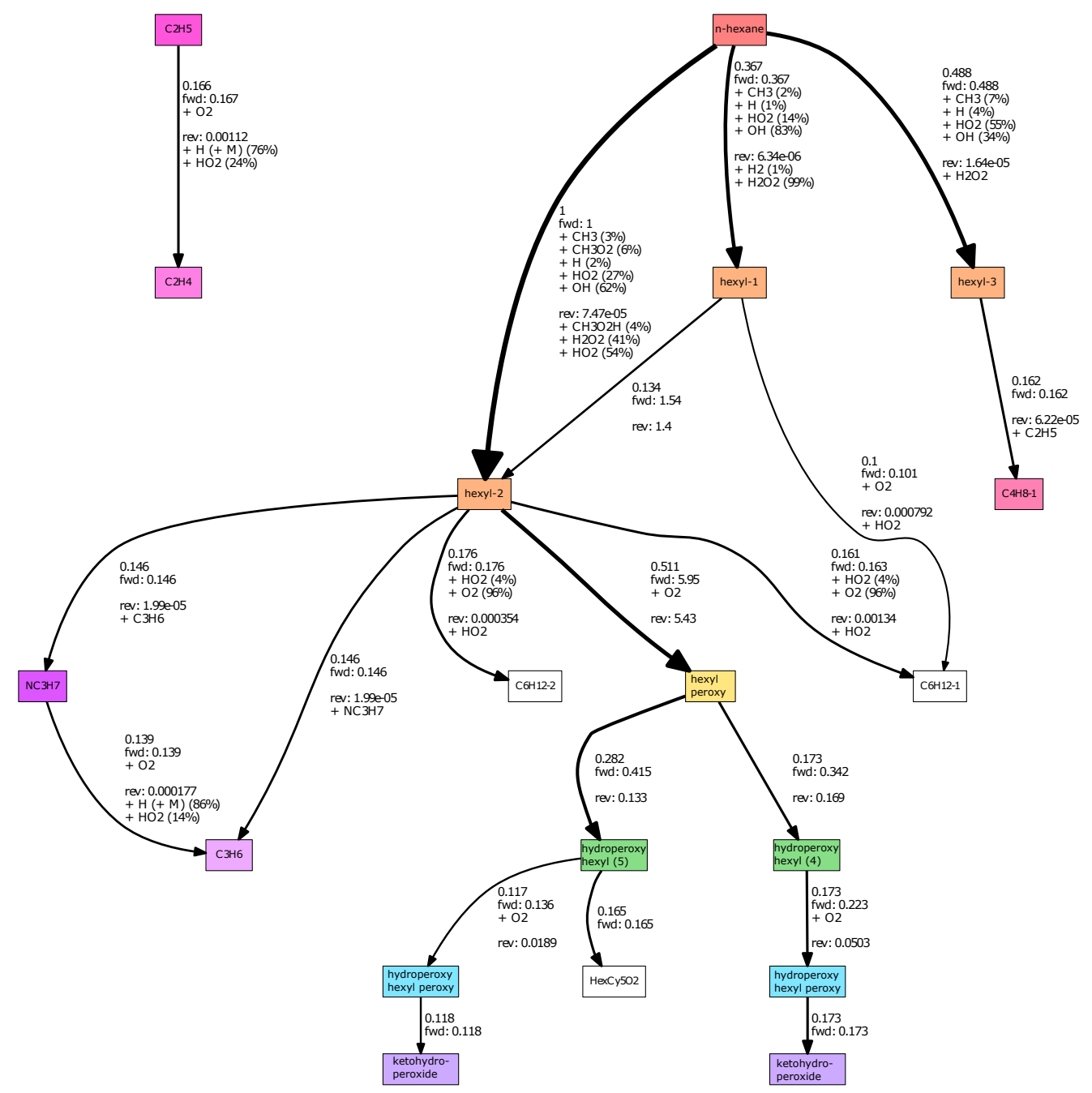

Figure 6.10: Reaction pathway diagram for end of first stage of ignition. Taken from $\tau *=0.5$ at the end of chemical energy release of the first ignition stage. 


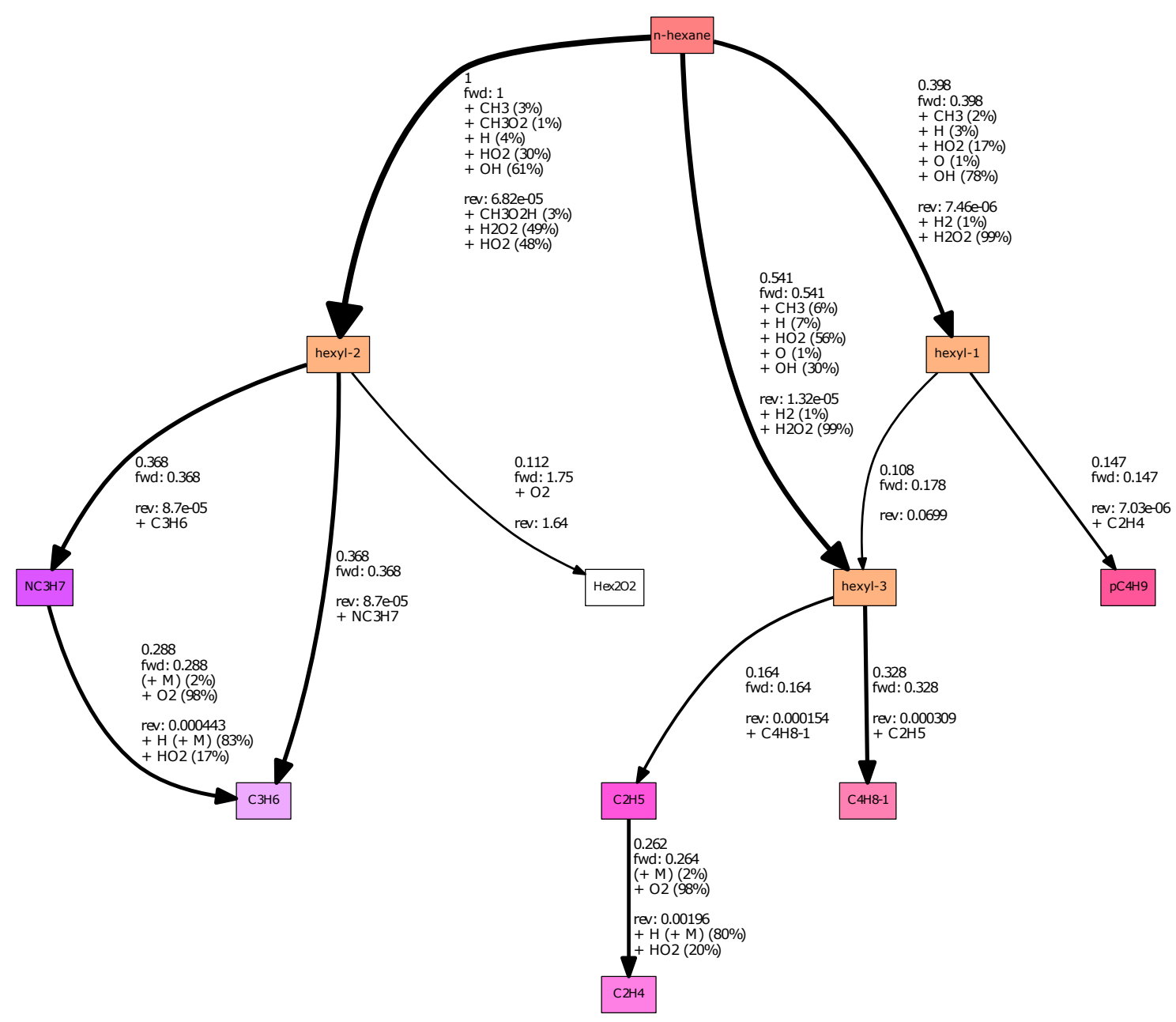

Figure 6.11: Reaction pathway diagram for transition between first- and secondstage ignition events. Taken from $\tau *=0.5$ halfway between first- and second-stage ignition events. 


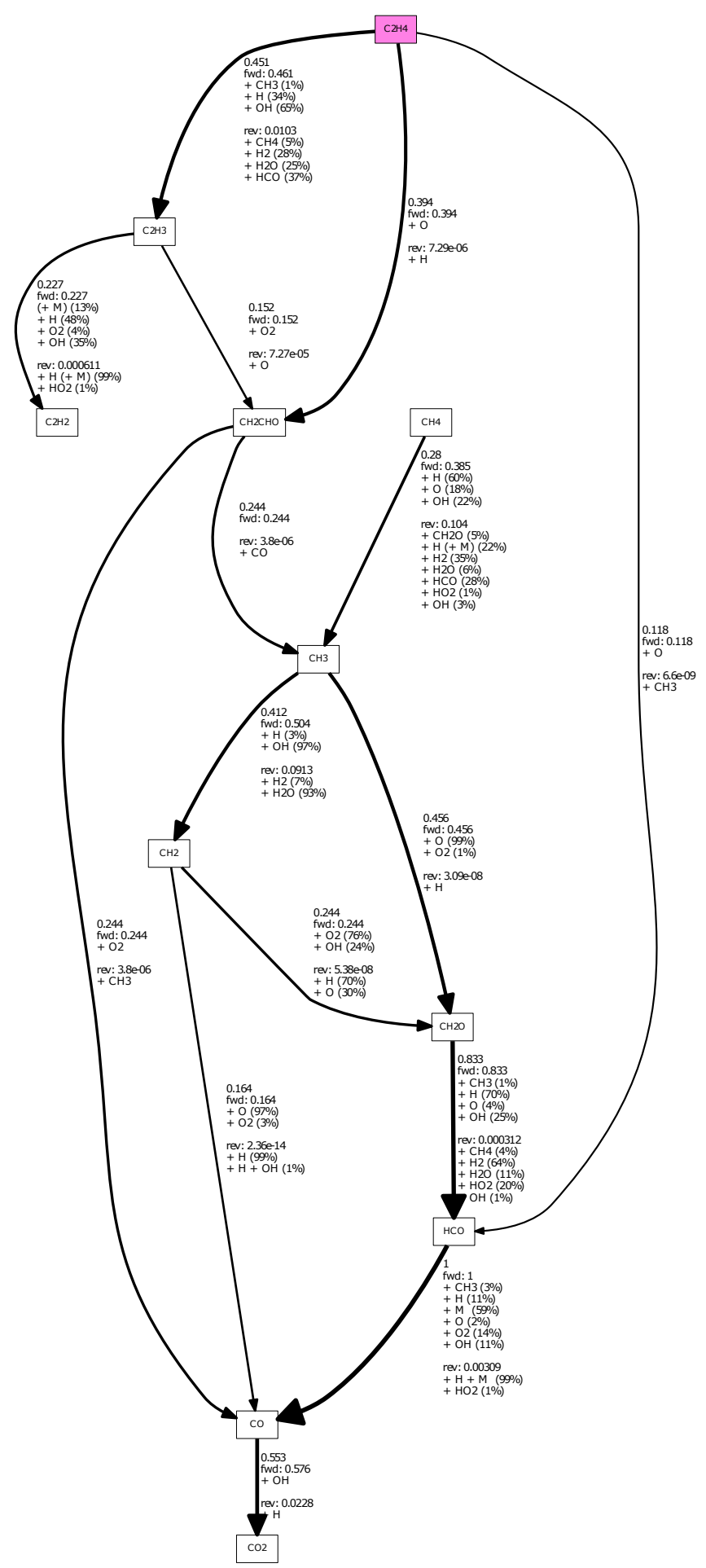

Figure 6.12: Reaction pathway diagram for second stage of ignition. Taken from $\tau *=0.5$ during the peak chemical energy release of the second ignition stage. 


\subsection{One-Dimensional Simulation in Lagrangian Coordinates}

The insights provided by the previous section are valuable but incomplete. In particular, the zero-dimensional reactor calculation does not account for the physical effects of diffusion by which species and energy are transferred across streamlines. As indicated by Menon et al. (2016), diffusion plays an important role in the ignition behavior. To explore the ignition dynamics near a heated surface, we solve a sample ignition problem that includes diffusion and considers only radial flow. This sample problem in one dimension is not as computationally intensive as a full multidimensional analysis of reactive free convection ignition.

Maas and Warnatz (1988) developed a one-dimensional reactive model in Lagrangian coordinates. The Lagrangian transformation follows a fluid particle and eliminates the convective terms from the governing equations. Maas restricts geometries to one-dimensional Cartesian, cylindrical, or spherical coordinates for simplicity. This model is compatible with a detailed chemical kinetics mechanism and multispecies transport models to capture the detailed physics of reaction and diffusion. We have followed their approach and implemented the model equations in cylindrical coordinates to treat the transient ignition around a suddenly heated cylinder in a hydrogen-air mixture. Numerical solutions of temperature and species are obtained as a function of radial distance and time using the method of lines to solve the initial value problem.

This one dimensional model of ignition bears some similarity to other investigations of ignition near a heated surface. Coronel (2016) studied a model problem of ignition in a thermal boundary layer. She extended the Rayleigh or Stokes problem of an impulsively started semi-infinite plate by including a elevated surface temperature of the semi-infinite plate such that both momentum and thermal boundary layers developed. Kinetic mechanisms of hydrogen and hexane were used such that ignition in this forced convection boundary layer could be studied. Coronel (2016) noted that heat and species diffusion played very significant roles in determining ignition delay time, and that ignition occurred at a finite distance from the wall. This model of ignition also has relevance to the experimental work of Adomeit (1965), who studied ignition of suddenly heated vertical cylinder in a hydrogen-air, pentane-air, and propane-air mixtures. 


\section{Untransformed Governing Equations}

The starting point of the Maas and Warnatz (1988) formulation is the governing equations of continuity, momentum, energy, and species mass conservation, which are simplified to one dimension in space and transformed to a Lagrangian coordinate system.

Continuity:

$$
\frac{\partial \rho}{\partial t}+\operatorname{div} \rho \mathbf{u}=0
$$

Momentum:

$$
\frac{\partial \rho \mathbf{u}}{\partial t}+\operatorname{grad} P+\operatorname{div} \bar{\Pi}+\operatorname{div}(\rho \mathbf{u} \circ \mathbf{u})=0
$$

Energy:

$$
\frac{\partial \rho h}{\partial t}-\frac{\partial P}{\partial t}+\operatorname{div}(\rho \mathbf{u} h)-v \operatorname{grad} P+\operatorname{div} \mathbf{j}_{q}+\bar{\Pi} \operatorname{grad} v=\dot{q}
$$

Species mass:

$$
\rho \frac{\partial Y_{k}}{\partial t}+\rho v \operatorname{grad} Y_{k}+\operatorname{div} \mathbf{j}_{\mathbf{k}}=\dot{\omega}_{k} M_{k}
$$

Where $\rho$ is density, $t$ is time, $\mathbf{u}$ is the velocity vector, $P$ is pressure, $\bar{\Pi}$ is the stretch tensor, $h$ is enthalpy, $\mathbf{j}_{q}$ is the heat flux vector, $v$ is the specific volume, $\dot{q}$ is energy source, $Y_{k}$ is mass fraction of species $k, \mathbf{j}_{\mathbf{k}}$ is the diffusion flux of vector of species $k$, $\dot{\omega}_{k}$ is the molar rate of formation of species $k$, and $M_{k}$ is the molar mass of species $k$.

\section{Lagrangian Transformation}

The transformation to Lagrangian coordinates in one-dimensional cylindrical coordinates is given by Stauch, Lipp, and Maas (2006) as:

$$
\psi(r, t)=\int_{0}^{r} \rho\left(r^{\prime}, t\right) r^{\prime} d r^{\prime}
$$

where $r$ is the spatial coordinate (radius) and $\psi$ is the Lagrangian-like coordinate. Differentiation of Eq. 6.20 and using Eq. 6.16 results in the following relationships 
(Raffel et al., 1986) for the time and space derivatives of the Lagrangian coordinate:

$$
\begin{gathered}
\left(\frac{\partial \psi}{\partial r}\right)_{t}=\rho r, \\
\left(\frac{\partial \psi}{\partial t}\right)_{r}=-\rho u r,
\end{gathered}
$$

where $u$ is velocity in the radial direction. With this definition, the governing equations can be transformed so the Lagrangian coordinate $\psi$ is the independent variable instead of the radial coordinate $r$. The resulting equations are for cylindrical symmetry.

Continuity:

$$
\frac{\partial \rho}{\partial t}+\rho^{2} \frac{\partial(r u)}{\partial \psi}=0
$$

Momentum:

$$
\frac{\partial u}{\partial t}+r \frac{\partial P}{\partial \psi}-\frac{4}{3} r \frac{\partial}{\partial \psi}\left(\rho u \frac{\partial}{\partial \psi}(u r)\right)+2 u \frac{\partial \mu}{\partial \psi}=0
$$

Energy:

$$
\begin{aligned}
\frac{\partial T}{\partial t}-\frac{1}{\rho c_{p}} \frac{\partial P}{\partial t}-\frac{1}{c_{p}} \frac{\partial}{\partial \psi}\left(\rho r^{2} \lambda \frac{\partial T}{\partial \psi}\right)+\frac{r}{c_{p}} \sum_{k=1}^{n_{s}} \rho Y_{k} V_{k} c_{p k} \frac{\partial T}{\partial \psi}+\frac{1}{\rho c_{p}} \sum_{k=1}^{n_{s}} \dot{\omega}_{k} h_{k} M_{k} \\
-\frac{4 \rho \mu}{3 c_{p}}\left(\frac{\partial(u r)}{\partial \psi}\right)^{2}+\frac{2 \mu}{c_{p}} \frac{\partial}{\partial \psi}\left(u^{2}\right)=\frac{\dot{q}}{\rho c_{p}}
\end{aligned}
$$

Species mass:

$$
\frac{\partial Y_{k}}{\partial t}+\frac{\partial}{\partial \psi}\left(\rho r Y_{k} V_{k}\right)-\frac{\dot{\omega}_{k} M_{k}}{\rho}=0
$$

where $\mu$ is kinematic viscosity, $\lambda$ is thermal conductivity, $n_{s}$ is number of species, $V_{k}$ is diffusion velocity of species $k, c_{p, k}$ is heat capacity of species $k$, and $h_{k}$ is specific enthalpy of species $k$.

Next, we assume uniform, constant pressure, $P=P_{0}$. This simplifies the momentum equation to: 


$$
\frac{\partial P}{\partial \psi}=0
$$

The assumption of constant pressure and the gas law $\rho=P / R T$ enable direct computation of the density from the temperature and species mass fractions, eliminating the need to solve 6.23. Equation 6.21 can be integrated to find the radial distance as a function of $\psi$ once the density $\rho(\psi, t)$ has been computed. Additionally, we assume there is no external energy addition, $\dot{q}=0$, to simplify the governing equations. Finally, by assuming a low-speed flow, the last two terms on the left-hand side of Eq. 6.25 can be dropped due to negligible viscous energy dissipation for low-speed flows. The energy equation thus simplifies to:

$$
\frac{\partial T}{\partial t}=\frac{1}{c_{p}} \frac{\partial}{\partial \psi}\left(\rho r^{2} \lambda \frac{\partial T}{\partial \psi}\right)-\frac{r}{c_{p}} \sum_{k=1}^{n_{s}} \rho Y_{k} V_{k} c_{p k} \frac{\partial T}{\partial \psi}-\frac{1}{\rho c_{p}} \sum_{i=1}^{n_{s}} \dot{\omega}_{k} h_{k} M_{k}
$$

Simple rearrangement of the species mass equation yields:

$$
\frac{\partial Y_{k}}{\partial t}=-\frac{\partial}{\partial \psi}\left(\rho r Y_{k} V_{k}\right)+\frac{\dot{\omega}_{k} M_{k}}{\rho}
$$

We make a further assumption when dealing with the diffusion velocity of species $k$. We use the mass-based approximation of diffusion velocity based off of Fick's law for a binary mixture:

$$
V_{k}=-\frac{D_{k}}{Y_{k}} \nabla Y_{K}=-\frac{D_{k}}{Y_{k}} \frac{\partial Y_{K}}{\partial r}=-\frac{D_{k}}{Y_{k}} \frac{\partial \psi}{\partial r} \frac{\partial Y_{K}}{\partial \psi}=-\rho r \frac{D_{k}}{Y_{k}} \frac{\partial Y_{K}}{\partial \psi}
$$

where $D_{k}$ is the mixture-averaged mass-based diffusion coefficient for species $k$. Since this model is formulated for a multi-component gas mixture, it is important to note that this is an approximation and is not exact as it is for a binary mixture. There will be a discussion in the following sections on how to handle the equations such that any errors due to this approximation lead to minimal errors in species mass fractions (which can be very small!).

Substituting Eq. 6.30 into Eqs. 6.28 and 6.29leads to the following forms for energy and species mass conservation: 


$$
\begin{gathered}
\frac{\partial T}{\partial t}=\frac{1}{c_{p}} \frac{\partial}{\partial \psi}\left(\rho r^{2} \lambda \frac{\partial T}{\partial \psi}\right)+\frac{\rho^{2} r^{2}}{c_{p}} \frac{\partial T}{\partial \psi} \sum_{k=1}^{n_{s}} D_{k} c_{p k} \frac{\partial Y_{k}}{\partial \psi}-\frac{1}{\rho c_{p}} \sum_{k=1}^{n_{s}} \dot{\omega}_{k} h_{k} M_{k}, \\
\frac{\partial Y_{k}}{\partial t}=\frac{\partial}{\partial \psi}\left(\rho^{2} r^{2} D_{k} \frac{\partial Y_{k}}{\partial \psi}\right)+\frac{\dot{\omega}_{k} M_{k}}{\rho} .
\end{gathered}
$$

Equations 6.31 and 6.32 are the final versions of the governing equations for the one-dimensional Lagrangian model of a reacting flow.

\section{Numerical Solution: Method of Lines}

To solve the governing equations for this model, we use the method of lines to discritize the spatial dimension. This reduces the two governing partial differential equations to a system of ordinary differential equations. We discritize all quantities on a non-uniform grid with $\mathrm{N}$ nodes. The value of a particular quantity, $g$, at node $j$ is represented as $g_{j}$. In this way, all terms on the right hand side of Eqs. 6.31 and 6.32 are discrete:

$$
\begin{gathered}
\left.\frac{\partial T}{\partial t}\right|_{j}=\left.\frac{1}{c_{p_{j}}} \frac{\partial}{\partial \psi}\left(\rho r^{2} \lambda \frac{\partial T}{\partial \psi}\right)\right|_{j}+\left.\left.\frac{\rho_{j}^{2} r_{j}^{2}}{c_{p_{j}}} \frac{\partial T}{\partial \psi}\right|_{j} \sum_{k=1}^{n_{s}} D_{k, j} c_{p_{k, j}} \frac{\partial Y_{k}}{\partial \psi}\right|_{j}-\frac{1}{\rho_{j} c_{p_{j}}} \sum_{k=1}^{n_{s}} \dot{\omega}_{k, j} h_{k, j} M_{k} \\
\left.\frac{\partial Y_{k}}{\partial t}\right|_{j}=\left.\frac{\partial}{\partial \psi}\left(\rho^{2} r^{2} D_{k} \frac{\partial Y_{k}}{\partial \psi}\right)\right|_{j}+\frac{\dot{\omega}_{k, j} M_{k}}{\rho_{j}}
\end{gathered}
$$

The solution takes the form:

$$
y_{j}=\left[\begin{array}{lllll}
T_{j} & Y_{1, j} & Y_{2, j} & \ldots & Y_{n_{s}-1, j}
\end{array}\right],
$$

and is of size $N$ by $n_{s}$. The governing ODEs are represented by:

$$
\left.\frac{d y}{d t}\right|_{j}=\left[\begin{array}{lllll}
\left.\frac{d T}{d t}\right|_{j} & \left.\frac{d Y_{1}}{d t}\right|_{j} & \left.\frac{d Y_{2}}{d t}\right|_{j} & \ldots & \left.\frac{d Y_{n_{s}-1}}{d t}\right|_{j}
\end{array}\right]
$$

The terms in Eqs. 6.34 and 6.33 that include gradients must be discussed a bit further. Following the work of Sundqvist and Veronis (1970), gradients are represented via central differences on a non-uniform grid as: 


$$
\left.\frac{d f}{d \psi}\right|_{j}=\frac{f_{j+1}-\left(\frac{\Delta \psi_{j}}{\Delta \psi_{j-1}}\right)^{2} f_{j-1}-\left(1-\left(\frac{\Delta \psi_{j}}{\Delta \psi_{j-1}}\right)^{2}\right) f_{j}}{\Delta \psi_{j}\left(1+\left(\frac{\Delta \psi_{j}}{\Delta \psi_{j-1}}\right)\right)} .
$$

We also need a formulation for discritizing the flux terms, which take the general form $\frac{d}{d \psi}\left(a \frac{d f}{d \psi}\right)$.

$$
\begin{gathered}
\left(\frac{\partial}{\partial \psi}\left(a \frac{\partial f}{\partial \psi}\right)\right) l_{j}=\left(\frac{\left(a \frac{\partial f}{\partial \psi}\right)_{j+1 / 2}-\left(a \frac{\partial f}{\partial \psi}\right)_{j-1 / 2}}{\psi_{j+1 / 2}-\psi_{j-1 / 2}}\right) \\
=\left(\frac{\left(\frac{\left(a_{j+1}+a_{j}\right)}{2} \frac{f_{j+1}-f_{j}}{\psi_{j+1}-\psi_{j}}\right)-\left(\frac{\left(a_{j}+a_{j-1}\right)}{2} \frac{f_{j}-f_{j-1}}{\psi_{j}-\psi_{j-1}}\right)}{\frac{1}{2}\left(\left(\psi_{j+1}-\psi_{j}\right)-\left(\psi_{j}-\psi_{j-1}\right)\right)}\right) .
\end{gathered}
$$

The conversion between a radial spatial coordinate and the Lagrangian coordinate can be approximated through use of the trapezoidal rule to integrate Eq. 6.20.

$$
\psi_{j+1}-\psi_{j}=\frac{1}{2}\left[(\rho r)_{j+1}+(\rho r)_{j}\right]\left(r_{j+1}-r_{j}\right)
$$

Note that in the solution formulation, there are only $n_{s}-1$ species mass fractions directly solved for. This is done to compensate for the mass approximation made for the diffusion velocity. To ensure all species mass fractions sum to one despite the inexact formulation for diffusion velocity, $n_{s}$ th species mass fraction is found via:

$$
Y_{n_{s}}=1-\sum_{k=1}^{n_{s}-1} Y_{k}
$$

The $n_{s}$ th species is chosen to be a species that is relatively inert and exists in large quantities, such that any errors are negligible. In this case, the $n_{s}$ th species is chosen to be nitrogen, as it has a large mass fraction that does not change significantly during reaction. 
The mixture-averaged transport properties such as dynamic viscosity and thermal diffusivity are calculated for ideal gas mixtures following the standard mixture theories of Kee, Coltrin, and Glarborg (2003) and evaluated by Cantera (Goodwin et al., 2018). The formulas used by Cantera for computing these properties are given below.

The mixture averaged viscosity is:

$$
\mu=\sum_{k} \frac{\mu_{k} X_{k}}{\sum_{j} \Phi_{k, j} X_{j}},
$$

where $\mu_{k}$ is the viscosity of pure species $\mathrm{k}, X_{k}$ is the mole fraction of species $\mathrm{k}$, and

$$
\Phi_{k, j}=\frac{\left[1+\sqrt{\left(\frac{\mu_{k}}{\mu_{j}} \sqrt{\frac{M_{j}}{M_{k}}}\right)}\right]^{2}}{\sqrt{8} \sqrt{1+M_{k} / M_{j}}},
$$

where $M_{k}$ is the molecular weight of species $\mathrm{k}$.

The mixture-averaged thermal conductivity is:

$$
\lambda=0.5\left(\sum_{k} X_{k} \lambda_{k}+\frac{1}{\sum_{k} X_{k} / \lambda_{k}}\right) .
$$

where $\lambda_{k}$ is the thermal conductivity of species $\mathrm{k}$.

No approximations are made regarding the Lewis numbers. The diffusion coefficients are calculated on a mass-basis using the methodology of Kee, Coltrin, and Glarborg (2003):

$$
\frac{1}{D_{k m}}=\sum_{j \neq k}^{K} \frac{X_{j}}{\mathcal{D}_{k j}}+\frac{X_{k}}{1-Y_{k}} \sum_{j \neq k}^{K} \frac{Y_{j}}{\mathcal{D}_{k j}} .
$$

All other fluid properties are also evaluated by Cantera. 


\section{Domain and Boundary Conditions}

The governing equations are set up for cylindrical symmetry to mimic the cylinders used in the experiments. The domain is a radial slice of an annulus, with the inner boundary set at $r_{i}=0.0127 \mathrm{~m}$ to match the radius of cylinders 100C and 200A. The outer boundary, $r_{o}$ is varied depending on the simulation, and is typically meant to represent the abmient mixture.

The boundary conditions at the inner (left-hand) boundary $r_{i}$ must match wall conditions. This means a Dirichlet boundary condition for temperature and a Neumann boundary condition for species mass fraction:

$$
\begin{gathered}
T\left(r_{i}\right)=T_{\text {wall }}, \\
\frac{d Y_{k}}{d r}\left(r_{i}\right)=0 .
\end{gathered}
$$

The boundary conditions at the outer (right-hand) boundary $r_{o}$ are meant to match the ambient gas condition. This can be done either through Dirichlet or Neumann boundary conditions if the domain is large enough. The Dirichlet boundary condition for the outer boundary is:

$$
\begin{gathered}
T\left(r_{o}\right)=T_{\infty}, \\
Y_{k}\left(r_{o}\right)=Y_{k, \infty},
\end{gathered}
$$

and the Neumann boundary condition for the outer boundary is:

$$
\begin{gathered}
\frac{d T}{d r}\left(r_{o}\right)=0, \\
\frac{d Y_{k}}{d r}\left(r_{o}\right)=0 .
\end{gathered}
$$

\section{Fomulating a Dirichlet Boundary Condition}

A Dirichlet boundary condition means that the variable in question is constant in time at that boundary. The simplest way to implement this boundary condition for 
the model discussed here is to set the time derivative of that variable equal to zero at the boundary. This amounts to

$$
\frac{d T}{d t}\left(r_{i}\right)=0
$$

for the inner boundary condition on temperature and

$$
\frac{d T}{d t}\left(r_{o}\right)=0, \text { and } \frac{d Y_{k}}{d t}\left(r_{o}\right)=0,
$$

at the outer boundary if we choose to use Dirichlet boundary conditions at $r_{o}$.

\section{Fomulating a Neumann Boundary Condition}

Implementing a Neumann boundary condition is more complicated for this model due to the use of the central differencing scheme in Eq. 6.37. For example, let us discuss the Neumann boundary condition on species mass fractions at the inner boundary given by Eq. 6.46 . We need to solve for $\left.\frac{d Y_{k}}{d t}\right|_{j=1}$, where $j=1$ denotes the first node, placed at the inner boundary $r_{j=1}=r_{i}$.

$$
\left.\frac{\partial Y_{k}}{\partial t}\right|_{1}=\left.\frac{\partial}{\partial \psi}\left(\rho^{2} r^{2} D_{k} \frac{\partial Y_{k}}{\partial \psi}\right)\right|_{1}+\frac{\dot{\omega}_{k, 1} M_{k}}{\rho_{1}}
$$

Substituting Eq. 6.38 into Eq. 6.53:

$$
\left.\frac{\partial Y_{k}}{\partial t}\right|_{1}=\left(\frac{\left(\frac{\left(\rho_{2}^{2} r_{2}^{2} D_{k, 2}+\rho_{1}^{2} r_{1}^{2} D_{k, 1}\right)}{2} \frac{Y_{k, 2}-Y_{k, 1}}{\psi_{2}-\psi_{1}}\right)-\left(\frac{\left(\rho_{1}^{2} r_{1}^{2} D_{k, 1}+\rho_{0}^{2} r_{0}^{2} D_{k, 0}\right)}{2} \frac{Y_{k, 1}-Y_{k, 0}}{\psi_{1}-\psi_{0}}\right)}{\frac{1}{2}\left(\left(\psi_{2}-\psi_{1}\right)-\left(\psi_{1}-\psi_{0}\right)\right)}\right)+\frac{\dot{\omega}_{k, 1} M_{k}}{\rho_{1}}
$$

The issue with this is that the node at $j=0$ doesn't exist; it is outside of the domain. However, it is necessary to find the value of various gas properties at this "ghost node" in order to solve for the time rate of change of species at the boundary. We use a typical ghost node approach to move forward. First, we let $r_{0}=r_{1}-\left(r_{2}-r_{1}\right)$, that is, the ghost node is the same distance from the boundary node as the first internal node. This is an arbitrary choice; any other selection would also work. Then we solve for $Y_{k, 0}$ by applying the central difference formula from Eq. 6.37 to the Neumann boundary condition on species given in Eq. 6.46 . 


$$
Y_{k, 0}=\frac{Y_{k, 2}-\left(1-\left(\frac{\Delta \psi_{1}}{\Delta \psi_{0}}\right)^{2}\right) Y_{k, 1}}{\Delta \psi_{1}\left(1+\frac{\Delta \psi_{1}}{\Delta \psi_{0}}\right)}
$$

We also let $T_{0}=T_{1}$ for simplicity, and with that it is possible to find all necessary gas properties like $\rho_{0}, c_{p, 0}, \lambda_{0}$, etc. With that, $\psi_{0}$ can be calculated from Eq. 6.39. and now all quantities in Eq. 6.54 are known and $\left.\frac{d Y_{k}}{d t}\right|_{1}$ can be solved for.

If the Neumann boundary condition is used on the right-hand boundary, a similar procedure will be followed for both species and temperature in that case.

The implementation of the numerical simulation is verified in several ways. The governing equations were implemented one term at a time; conduction, then diffusion, then reaction, etc. At each step, the results of an example problem were checked against an analytical solution where applicable. These example problems also presented a chance to check for proper implementation of the boundary conditions. When an appropriate analytical solution was not available, grid convergence studies were performed to ensure the numerical solution was converging. The effect of domain size was studied, and a domain was selected such that a compromise between resolution, domain size, and computational time was struck.

\subsection{One-Dimensional Simulation Results}

In this section, we will be presenting the results of the one-dimensional simulations with an initial mixture that is composed of stoichiometric hydrogen-air. The kinetic mechanism used is the hydrogen-air mechanism used in Melguizo-Gavilanes et al. (2017) and contains 9 species and 21 reactions. A hydrogen-air is used for the relative simplicity of the chemistry: the small size of the mechanism means it is less computationally demanding to use than a simplified mechanism for n-hexane.

The numerical system presented in Section 6.2 is implemented in MATLAB, and Cantera (Goodwin et al., 2018) is used to compute all thermochemical and transport properties as well as calculate the chemical reaction rates. Unless otherwise stated, the domain contains 200 grid points geometrically spaced in the radial dimension, with the inner boundary placed at $r_{i}=0.0127 \mathrm{~m}$ and the outer boundary at $r_{o}=0.0135 \mathrm{~m}$. The smallest grid spacing is next to the heated surface and has a width of $4.4 \times 10^{-6} \mathrm{~m}$. The number of grid points and the size of the domain was a 
compromise between the need to have a fine resolution in order to accurately resolve the flame while also having a domain large enough to allow for flame propagation (domain size at least $3 \mathrm{x}$ the flame thickness). The inner boundary, $r_{i}=0.0127 \mathrm{~m}$, has a Dirichlet boundary condition on temperature, $T\left(r_{i}\right)=T_{w}$, and a Neumann

boundary condition on species, $\frac{d Y_{k}}{d t}\left(r_{i}\right)=0$. The outer boundary has a Dirichlet boundary condition on both temperature and species: $T\left(r_{o}\right)=T_{\infty}, Y_{k}\left(r_{o}\right)=Y_{k, \infty}$.

The results from these simulations will be used to provide insight into the important chemical and physical processes governing ignition. Generally speaking, they will not be used to predict ignition thresholds, ignition delay times, or other quantitative values pertaining to the ignition behavior. Of particular interest for these purposes are the evolution of temperature and species over time, the heat flux next to the surface, and the change in energy balance during an ignition event. We will also examine the differences between supercritical (wall temperature well above threshold ignition temperature), critical (wall temperature just at threshold ignition temperature), and subcritical (wall temperature below threshold ignition temperature) ignition cases.

\section{Ignition Delay Times}

The ignition delay times are extracted from the simulations using two different criteria to define ignition delay. First, the ignition delay time based on the time rate of change of temperature is defined as:

$$
\tau_{i g n, T}=t\left(\left.\frac{d T}{d t}\right|_{\max }\right)
$$

That is, the ignition delay based on temperature, $\tau_{i g n, T}$, is the time at which the maximum time rate of change of temperature occurs.

The second criterion for ignition is $\tau_{i g n, O H}$. It is the ignition delay based on the time at which the maximum time rate of change of $\mathrm{OH}$ mass fraction occurs, defined similarly to $\tau_{\text {ign,T }}$ :

$$
\tau_{i g n, O H}=t\left(\left.\frac{d Y_{O H}}{d t}\right|_{\max }\right) .
$$

The simulations are run for a number of wall temperatures, while the ambient temperature remains fixed at $300 \mathrm{~K}$, and the initial mixture composition remains 
a stoichiometric hydrogen-air mixture in order to observe how the ignition delay changes with changing wall temperature. The results are plotted for $\tau_{i g n, T}$ in Fig. 6.13 and $\tau_{\text {ign,OH }}$ in Fig. 6.14 .

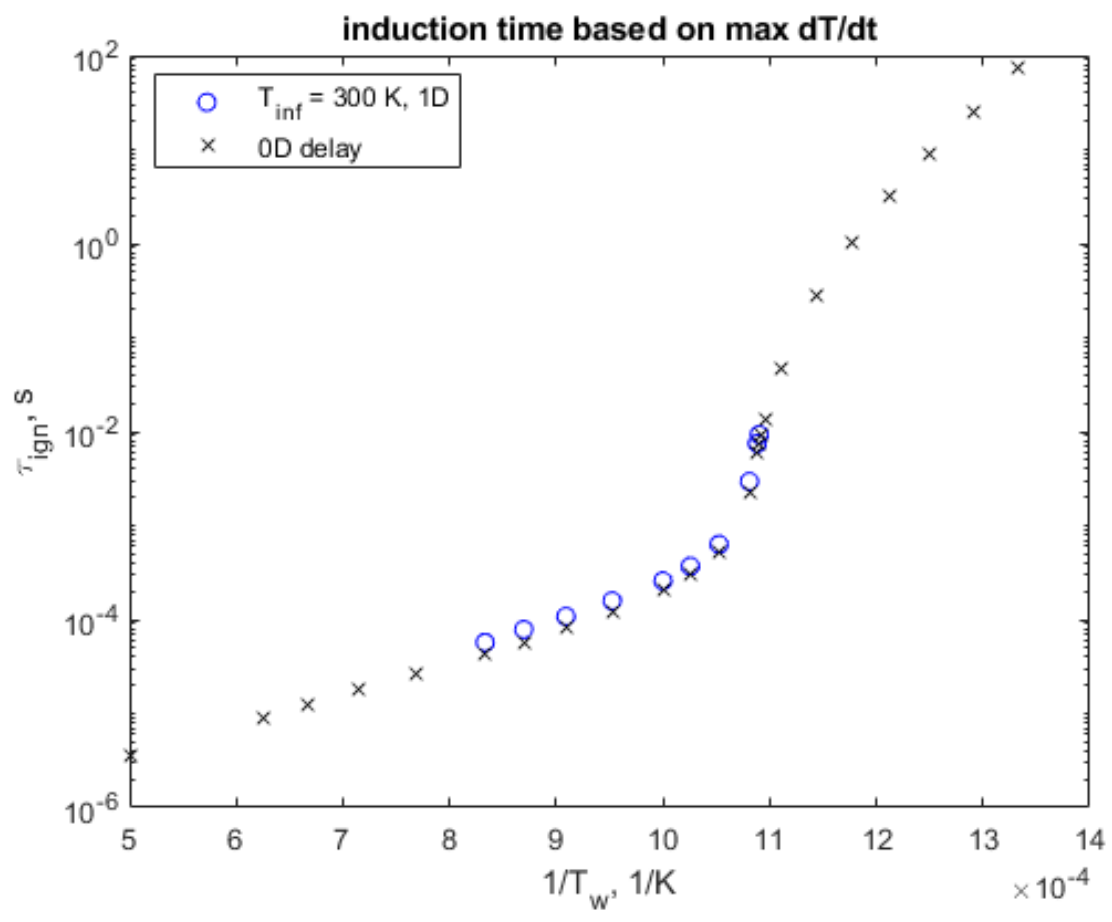

Figure 6.13: Ignition delay, $\tau_{i g n, T}$, based on maximum $\frac{d T}{d t}$, plotted against the reciprocal of wall temperature.

Figure 6.13 shows the difference in ignition delay time, $\tau_{i g n, T}$, between the onedimensional simulation and zero-dimensional, adiabatic constant pressure reactor calculations (see Eqns 6.11 and 6.12 with the heat flux term set to zero). The ignition delay time is approximately 20 to $35 \%$ longer in the one-dimensional simulations as a result of several factors. In the one-dimensional model, a thermal layer of sufficient thickness has to be created in the gas in order to not have the diffusion of thermal energy and species to the surface quench the reactions within the layer. The development of this layer takes some time. In addition, the diffusion of thermal energy and species out of the reactive thermal layer will delay the ignition process as compared to adiabatic explosion without species diffusion.

Simulations were run with the same range of wall temperatures but an an increased ambient temperature of $400 \mathrm{~K}$. The results are not documented here because the difference between ignition delay times with an ambient temperature of 300 versus 
$400 \mathrm{~K}$ were negligible, approximately $3 \%$ or less, for this hydrogen-air system. We see no significant effect of ambient temperature on ignition delay in these onedimensional simulations, indicating that the effects observed in testing and discussed in Chapter 5 do not have an explanation in transient ignition dynamics.

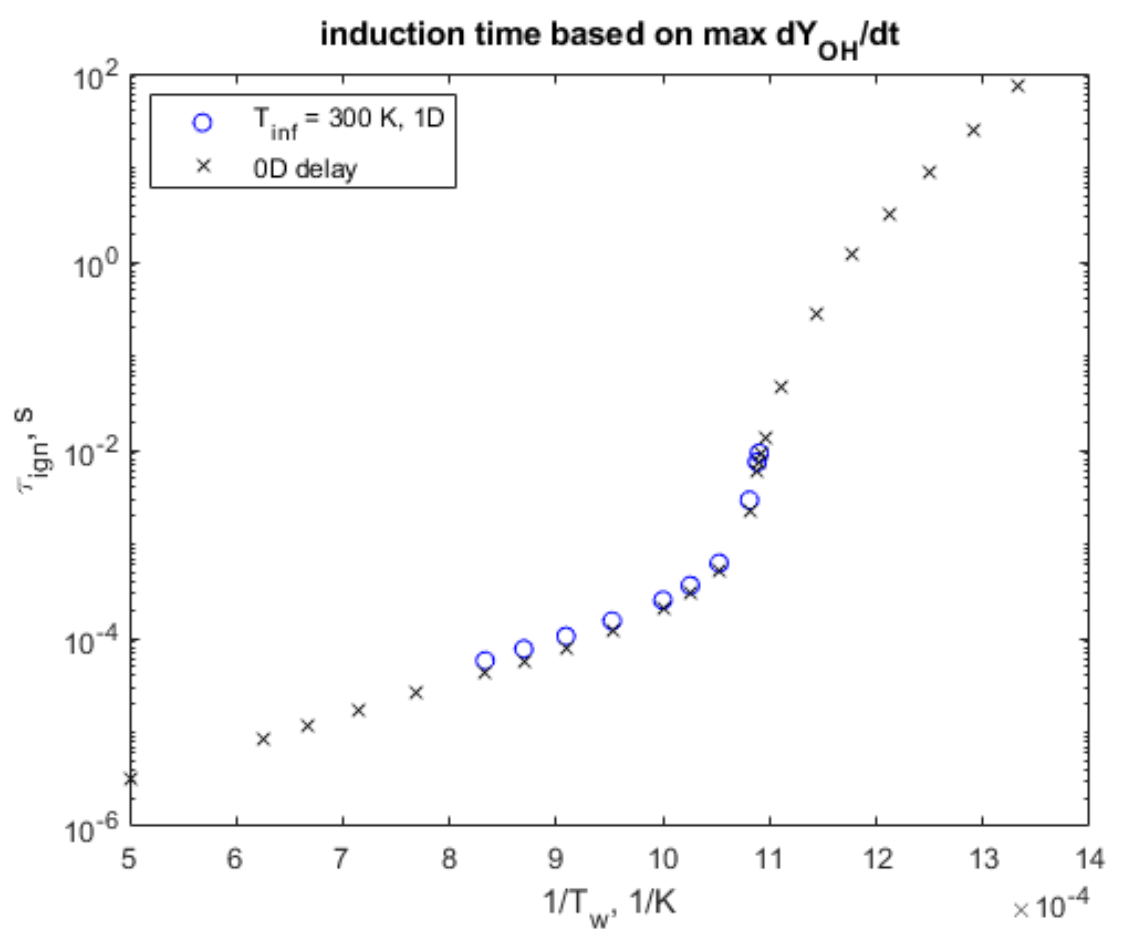

Figure 6.14: Ignition delay, $\tau_{i g n, O H}$, based on maximum $\frac{d Y_{O H}}{d t}$, plotted against the reciprocal of wall temperature.

Figure 6.14 compares ignition delay time, $\tau_{i g n, O H}$, between the one-dimensional simulation and the zero-dimensional calculations. These results are consistent with using the maximum temperature time derivative; the ignition delay is about 20-35\% longer than the zero-dimensional results. For the one-dimensional simulations, the difference in ignition delay time between $\tau_{i g n, T}$ and $\tau_{i g n, O H}$ is about $3 \%$ or less, making the two methods of tracking ignition delay quite similar. For the zerodimensional calculations, $\tau_{i g n, T}$ and $\tau_{i g n, O H}$ result in a difference in ignition delay that is about $1 \%$ or less.

Figures 6.13 and 6.14 plot the logarithm of ignition delay against the reciprocal of wall temperature in an Arrhenius-style plot. The black x's represent the ignition delay from zero-dimensional calculations and the blue o's represent ignition delays from the one-dimensional simulations. At large wall temperatures $\left(T_{w} \geq 950 \mathrm{~K}\right.$, 
$\left.1 / T_{w} \leq 10.5 \times 10^{-4} 1 / \mathrm{K}\right)$, the ignition times form a roughly straight line with a slope that corresponds to the activation energy of the chain-branching reaction. Around about $T_{w}=950 \mathrm{~K}$, the ignition delay time increases rapidly, as we approach the crossover temperature below which ignition time rises sharply. This temperature corresponds to the extended second explosion limit for which the three-body reaction $\mathrm{H}+\mathrm{OH}+\mathrm{M} \longrightarrow \mathrm{HO}_{2}+\mathrm{M}$ competes on equal footing with the chain-branching reaction $\mathrm{H}+\mathrm{OH} \longrightarrow \mathrm{H}_{2}+\mathrm{O}$ for the $\mathrm{OH}$ radical (Sánchez, Fernández-Tarrazo, and Williams, 2014). For lower temperatures, the reaction proceeds through the formation of $\mathrm{H}_{2} \mathrm{O}_{2}$ into $\mathrm{OH}$ and the re-initiation of the classical chain branching process. The slope of the low-temperature ignition delay time is consistent with an effective activation energy for $\mathrm{H}_{2} \mathrm{O}_{2}$ dissociation.

Below a critical temperature of about $917 \mathrm{~K}$, ignition in the one-dimensional solution was never observed while the zero-dimensional solution still ignites, albeit at increasingly large time with decreasing temperature. The computation of this ignition temperature threshold and the processes responsible for this are discussed in the following sections.

A binary search technique was used to approach the critical temperature until temperature differences in the wall temperature were approximately the same size as the accuracy on the numerical solver $(0.1 \%)$. The lowest wall temperature at which ignition was observed in the one-dimensional simulations was 917.2 K. The next lowest wall temperature used, $915.6 \mathrm{~K}$, did not ignite. As the ignition delay time rapidly increases near the critical temperature, the amount of time the simulation must be integrated over in order to observe an ignition also rapidly increases. In order to keep the run time of the simulations tractable, the integration time of the simulations near the critical temperature was limited to $1 \times 10^{-2} \mathrm{~s}$.

We examine specific cases in more detail for supercritical (1200 K), critical (917.2 $\mathrm{K})$, and subcritical $(915.6 \mathrm{~K})$ temperatures in the following sections.

\section{Temperature and Species Mass Fraction Fields Supercritical Ignition}

We begin with the results of a supercritical ignition simulation in which the mixture ignited promptly. The wall temperature was set to $1200 \mathrm{~K}$, and the ambient tem- 
perature was set to $300 \mathrm{~K}$. The mixture was premixed and uniform with an initial mass fraction, $Y_{\mathrm{H}_{2}}=0.0283, Y_{\mathrm{O}_{2}}=0.2265, Y_{\mathrm{N}_{2}}=0.7452$. The temperature field is plotted as a function of $\psi$ and time in Fig. 6.15.

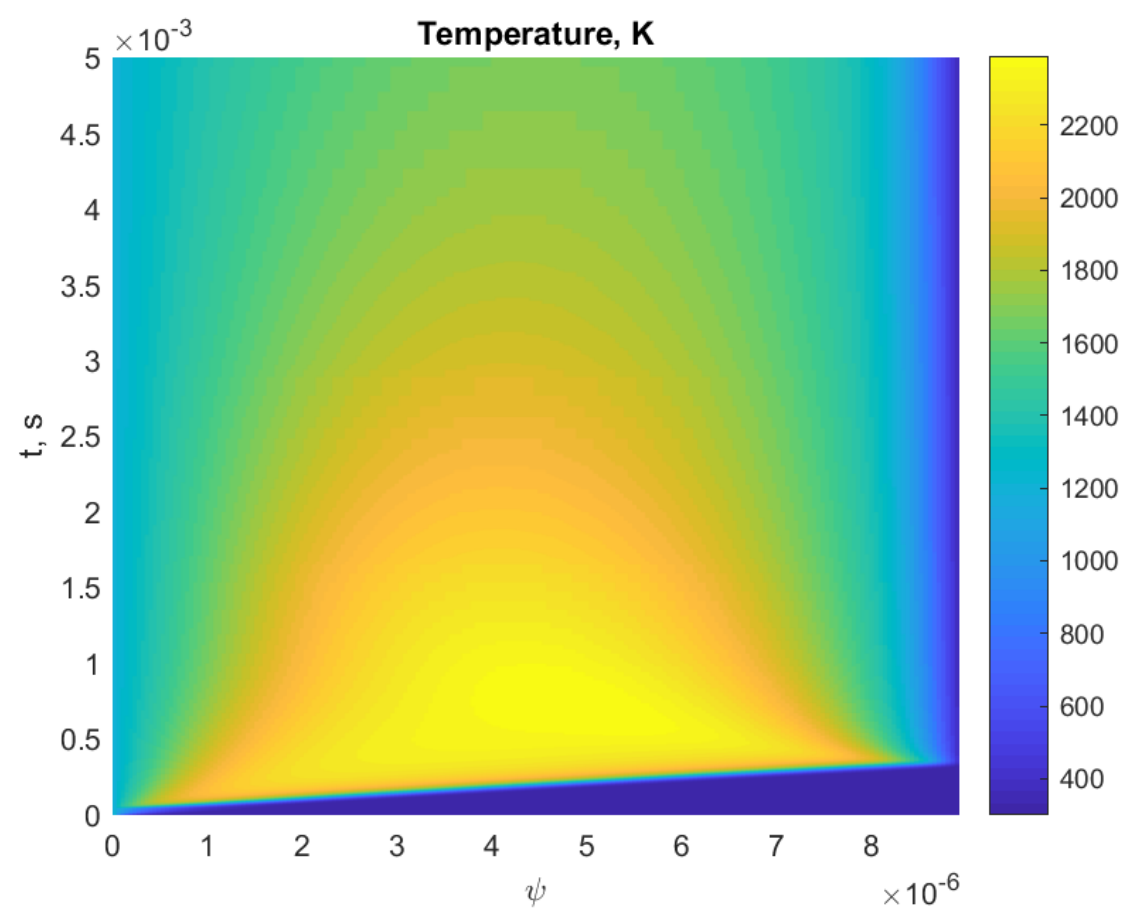

Figure 6.15: Temperature field plotted against Lagrangian coordinate, $\psi$, and time for stoichiometric hydrogen-air simulation with $T_{w}=1200 \mathrm{~K}, T_{\infty}=300 \mathrm{~K}$.

The mixture ignites at $5.7135 \times 10^{-5} \mathrm{~s}$ by the maximum time rate of change in temperature and $5.7700 \times 10^{-5} \mathrm{~s}$ by the maximum time rate of change in $\mathrm{OH}$ mass fraction. This is slightly slower than the zero-dimensional ignition delay prediction of $4.3331 \times 10^{-5} \mathrm{~s}$ and $4.2835 \times 10^{-5} \mathrm{~s}$, respectively, for maximum rate of change in temperature and $\mathrm{OH}$ mass fraction. This slight increase in ignition delay for the one-dimensional results makes sense, as the one-dimension simulation is non-ideal in a number of ways. The difference in ignition time produced by looking at the maximum time rate of change of temperature versus $\mathrm{OH}$ mass fraction is quite small, approximately $1 \%$ for both the zero-dimensional and one-dimensional results. From this we can conclude that either of these criteria can be used to define ignition time without significant difference in the results.

Note that the initial domain is specified in $r$, but the computations are carried out 
in the Lagrangian coordinate $\psi$. The boundaries in $\psi$ are computed via Eq. 6.39. Because the Lagrangian coordinates are mass based, they remain fixed throughout the computation even as expansion occurs in the spatial coordinate, $r$. This means that the final results, when transformed back into the original spatial coordinates (displayed in the following plots as $\Delta r$, the distance from the wall), show a domain of changing size as the spatial dimension is allowed to expand first due to heat conduction from the wall and then due to the ignition and propagation of the flame. After the flame reaches the cool outer boundary and dies, we can observe the domain contracting as heat is lost due to the fixed temperature imposed by the Dirichlet outer boundary condition. This is precisely what is observed in Fig. 6.16. The fields of temperature and species reported for times immediately surrounding the ignition event and flame propagation are shown in Figs. 6.17 through 6.19.

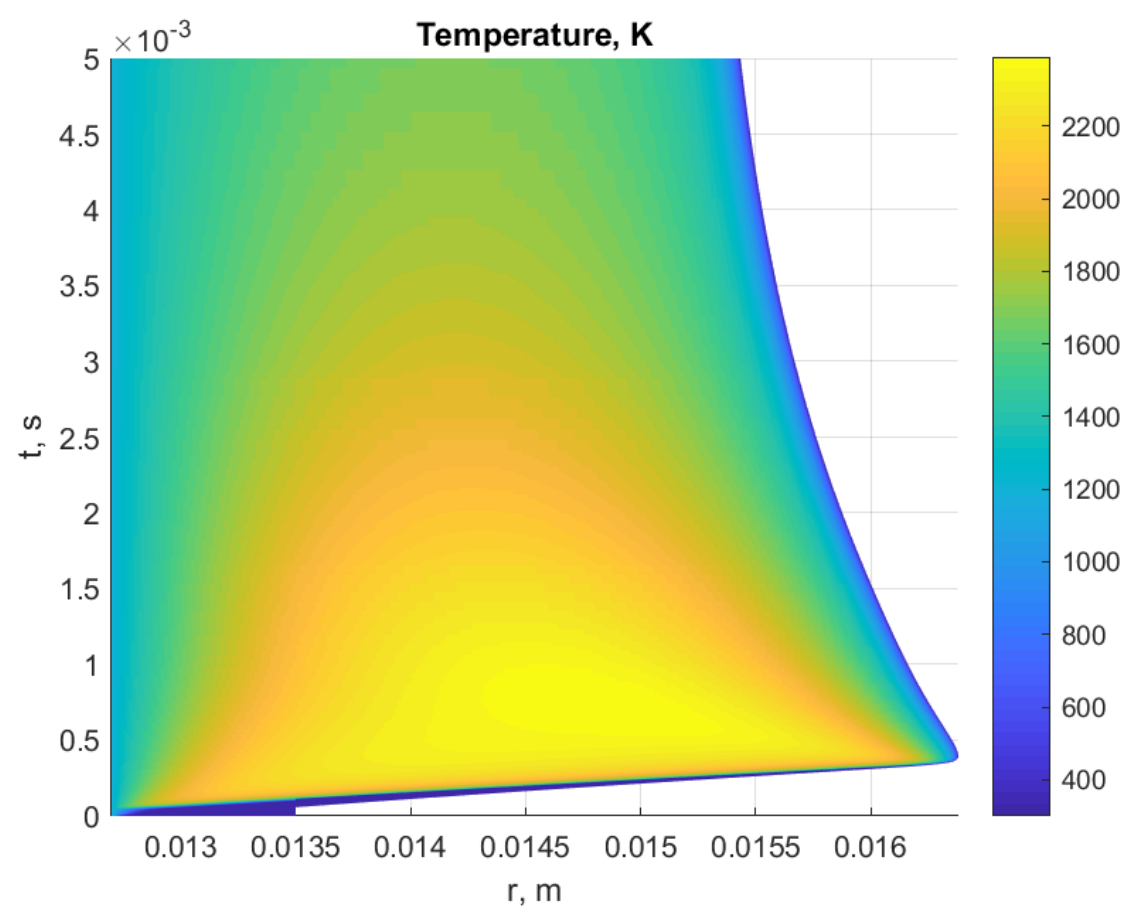

Figure 6.16: Temperature field plotted against radial distance from wall and time for stoichiometric hydrogen-air simulation with $T_{w}=1200 \mathrm{~K}, T_{\infty}=300 \mathrm{~K}$.

The ignition occurs very quickly, before much heat conduction from the wall has taken place. Ignition also occurs at a distance from the wall of $\Delta r=0.15 \mathrm{~mm}$. This can be attributed to two factors relating to the wall temperature: first, the temperature is high enough that sufficient energy for reaction is transferred into the gas quickly, 


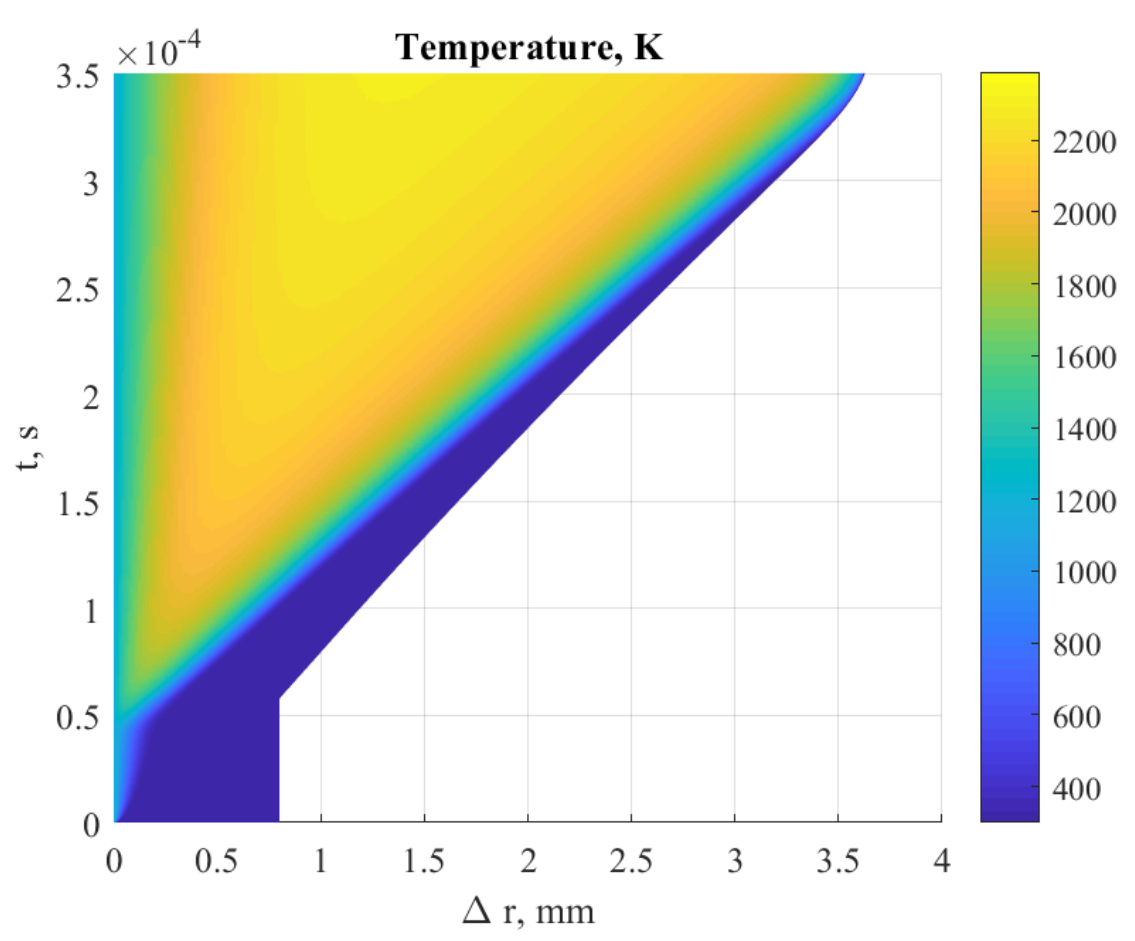

Figure 6.17: Temperature field plotted against radial distance from wall and time for stoichiometric hydrogen-air simulation with $T_{w}=1200 \mathrm{~K}, T_{\infty}=300 \mathrm{~K}$.

well before steady state behavior is established. Second, the wall temperature is "supercritical", that is, the wall temperature is sufficiently higher than the minimum temperature for ignition that ignition can occur in the early stages of heat conduction where a relatively small region of gas has been heated by the wall. Ignition occurs close to the wall; from this it is inferred that the wall temperature is high enough that it does not act as a heat sink in the early stages of reaction. By the time the reaction has strengthened enough to produce temperatures higher than the wall temperature and change the role of the wall to a heat sink rather than a heat source, the reaction is strong enough to sustain itself and develops into a propagating flame.

The fields of $\mathrm{H}_{2}$ (Fig. 6.18) and $\mathrm{HO}_{2}$ (Fig. 6.19) are plotted against radial distance from the wall and time in a similar manner to Fig. 6.16. The above explanations of changing domain size still apply.

The mass fraction of $\mathrm{H}_{2}$ is almost entirely depleted in most of the domain once the flame appears, due to the strong reaction from a supercritical case. The outer Dirichlet boundary condition on species allows for a sort of supply of reactants diffusing in from the outer boundary in the aftermath of the flame. The mass fraction of $\mathrm{HO}_{2}$ 


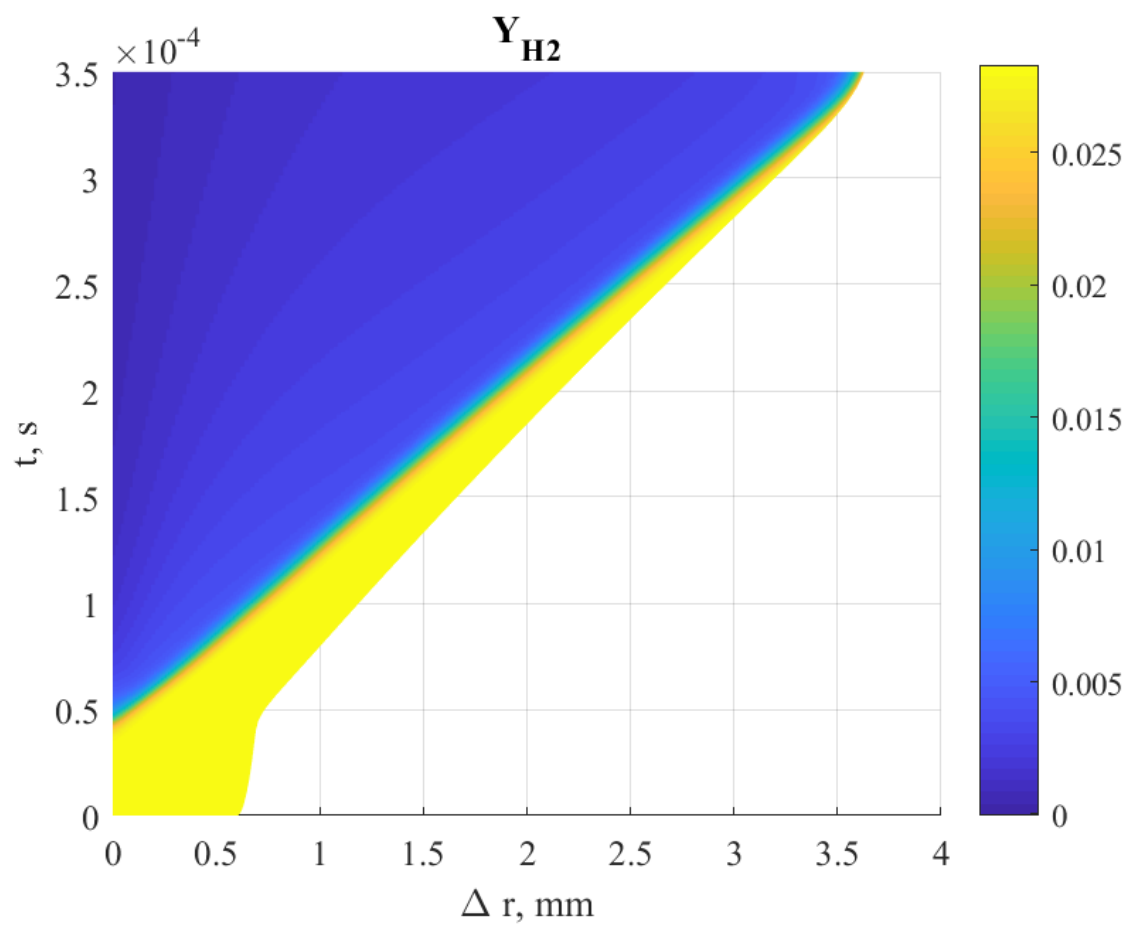

Figure 6.18: $\mathrm{H}_{2}$ mass fraction field plotted against radius and time for stoichiometric hydrogen-air simulation with $T_{w}=1200 \mathrm{~K}, T_{\infty}=300 \mathrm{~K}$.

is shown in Fig. 6.19. $\mathrm{HO}_{2}$ only occurs in significant amounts in a very narrow strip of the domain and acts as a marker for the flame front. Figure 6.20 magnifies the region surrounding the formation of the ignition kernel for the mass fraction of $\mathrm{HO}_{2}$.

An increase of $\mathrm{HO}_{2}$ mass fraction is seen in a region next to the wall prior to ignition $(\Delta r<0.2 \mathrm{~mm})$. As noted before, the ignition location (as found from the location of maximum time rate of change of temperature) is at $\Delta r=0.15 \mathrm{~mm}$. This matches the location in Fig. 6.20 where the flame forms as seen by a significant increase in the mass fraction of $\mathrm{HO}_{2}$.

For comparison, we estimate the flame thickness of this mixture, $l_{f}$ as:

$$
l_{f}=\frac{D}{S_{L}}=\frac{\lambda_{b}}{c_{p} \rho_{u} S_{L}}
$$

where $D$ is thermal diffusivity, $\lambda_{b}$ is the thermal conductivity of the burned gas, $c_{p}$ is the heat capacity of the mixture, $\rho_{u}$ is the density of the unburned mixture, and $S_{L}$ is the laminar burning speed of the mixture (Pitsch, 2014). For this hydrogen-air 


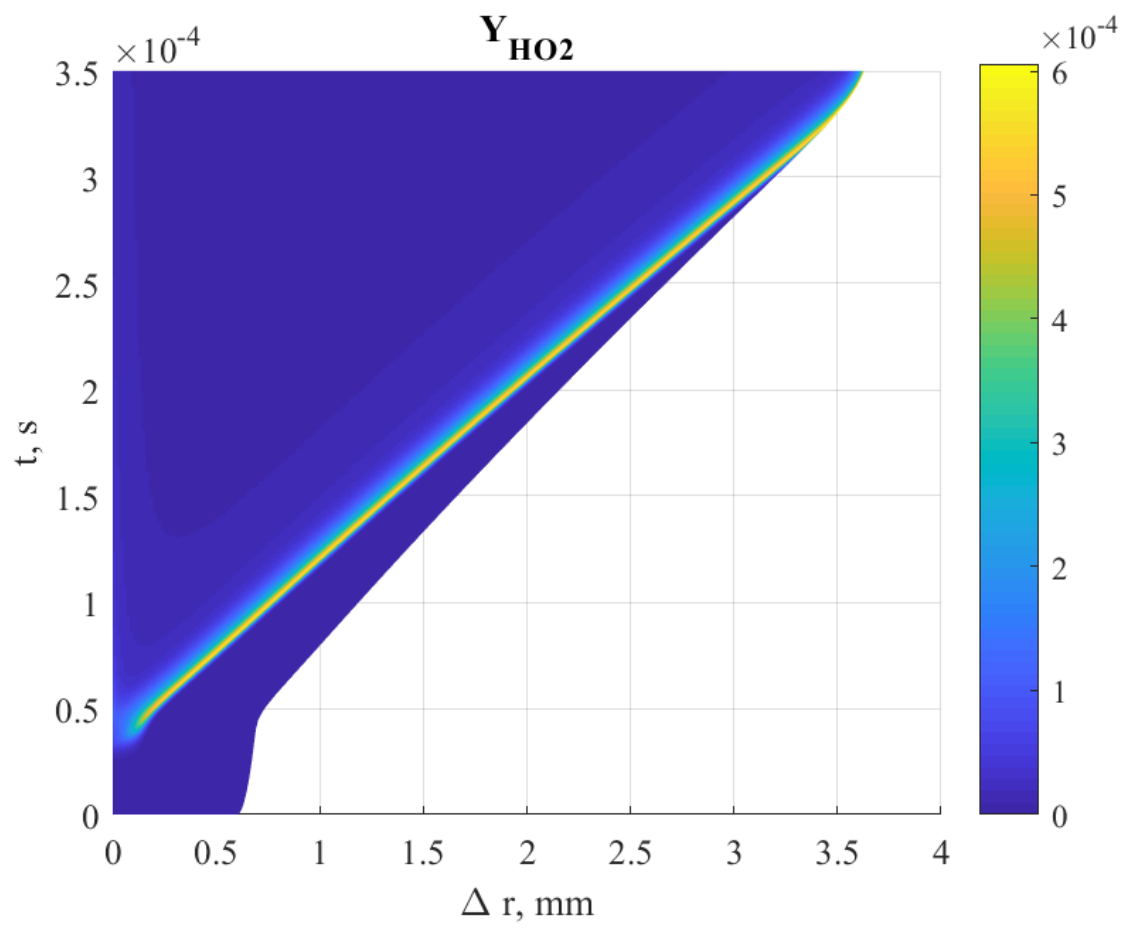

Figure 6.19: $\mathrm{HO}_{2}$ mass fraction field plotted against radial distance from wall and time for stoichiometric hydrogen-air simulation with $T_{w}=1200 \mathrm{~K}, T_{\infty}=300 \mathrm{~K}$.

mixture, $\lambda_{b} \approx 0.22 \mathrm{~W} / \mathrm{m}-\mathrm{K}, \rho_{u} \approx 0.27 \mathrm{~kg} / \mathrm{m}^{3}$, and $c_{p} \approx 1.5 \mathrm{~kJ} / \mathrm{kg}-\mathrm{K}$. At an ambient temperature of $300 \mathrm{~K}, S_{L}$ of a stoichiometric hydrogen-air mixture is calulated at $2.2 \mathrm{~m} / \mathrm{s}$. This produces an estimate of $l_{f} \approx 0.2 \mathrm{~mm}$. The flame thickness also be can be calculated by finding where a line whose slope is the maximum gradient of $\mathrm{OH}$ mass fraction intercepts the minimum and maximum $\mathrm{OH}$ mass fractions (see Fig. 6.21). This also yields a flame thickness of $l_{f}=0.2 \mathrm{~mm}$. For the supercritical case where $T_{w}=1200 \mathrm{~K}$, the ignition location is slightly less than one flame thickness away from the wall. 


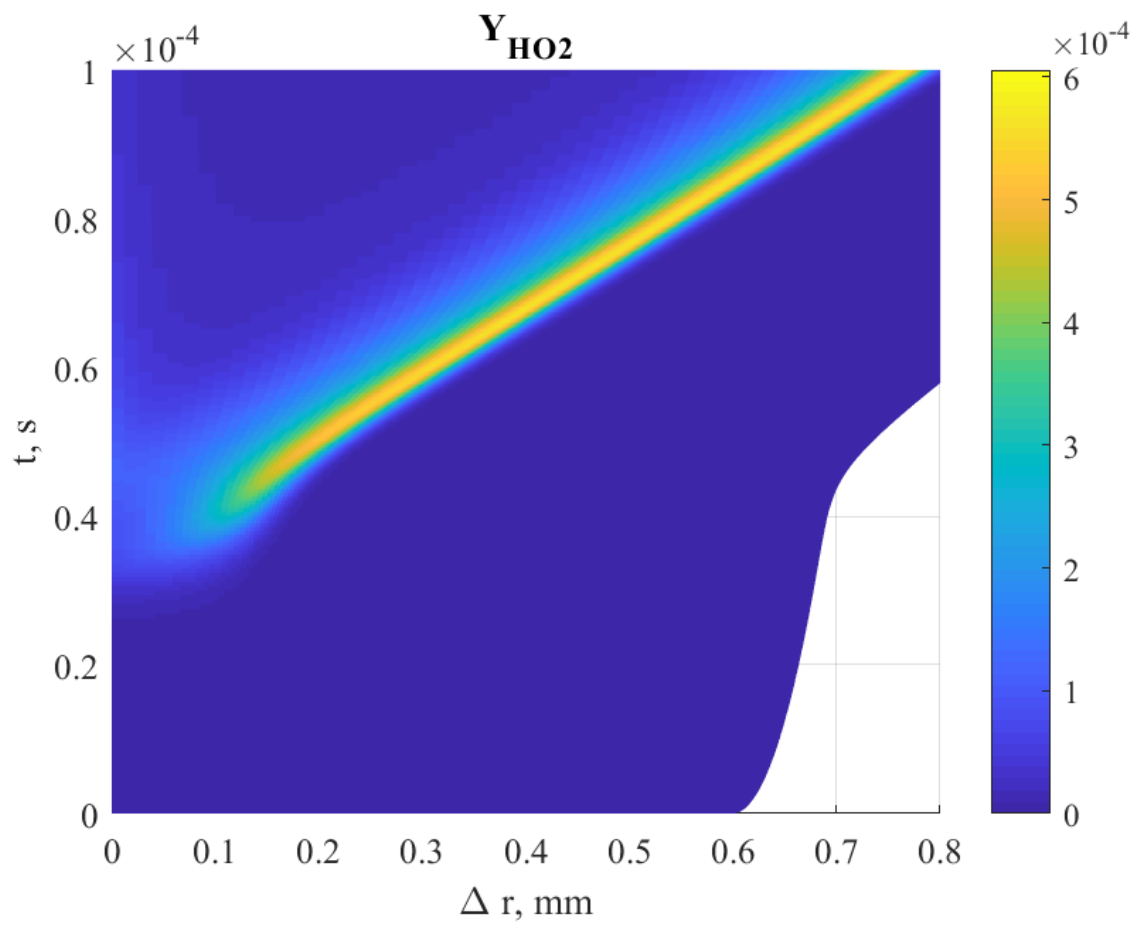

Figure 6.20: $\mathrm{HO}_{2}$ mass fraction field plotted against radial distance from wall and time for stoichiometric hydrogen-air simulation with $T_{w}=1200 \mathrm{~K}, T_{\infty}=300 \mathrm{~K}$. The region immediately surrounding the ignition kernel is examined in detail.

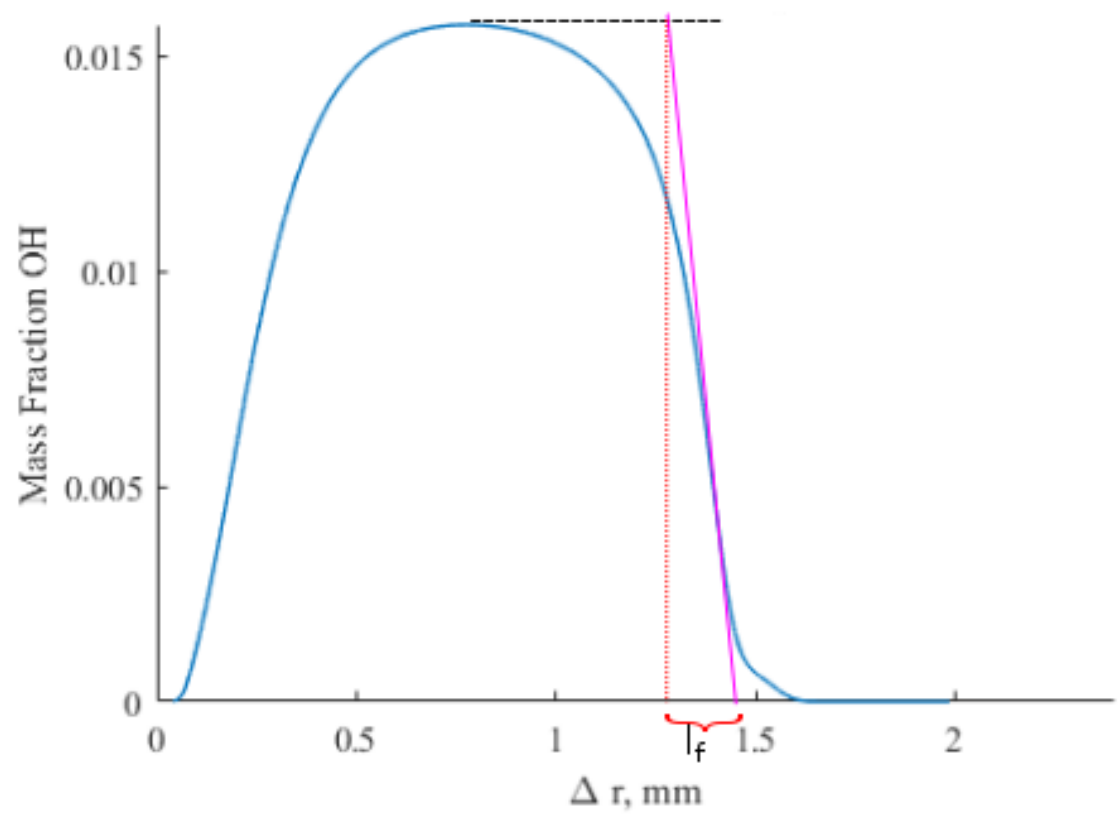

Figure 6.21: Graphical representation of flame thickness. 


\section{Critical Ignition Case}

Next, we present the results of a critical case. The wall temperature was set to $917.1875 \mathrm{~K}$, the ambient temperature to $300 \mathrm{~K}$, and the same initial gas composition was used for the hydrogen-air mixture. Here we present the temperature, mass fraction of $\mathrm{H}_{2}$, and mass fraction of $\mathrm{OH}$ fields as function of radius and time, as these provide a sufficient overview of the results for this section. Specific phenomena like heat flux at the wall and energy balance will be examined in detail later.

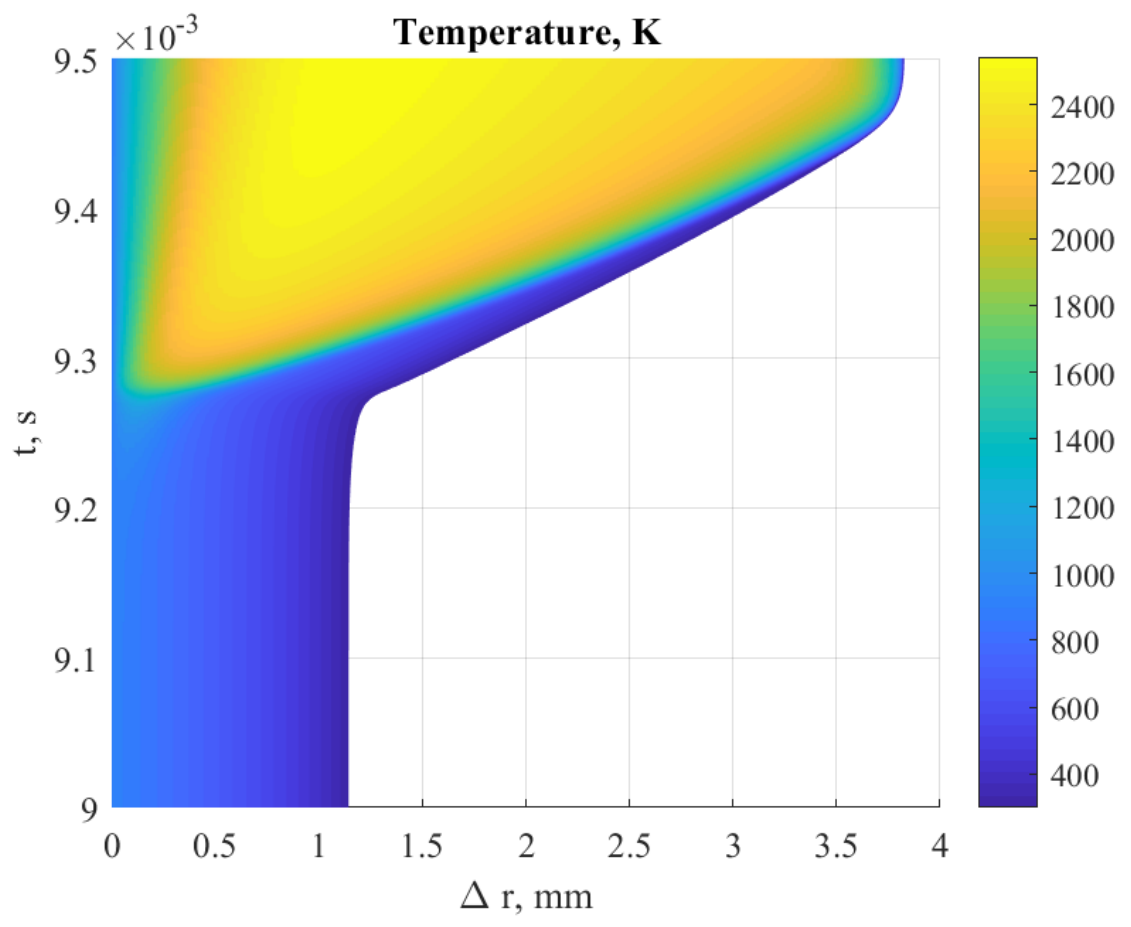

Figure 6.22: Temperature field plotted against radial distance from wall and time for stoichiometric hydrogen-air simulation with $T_{w}=917.1875 \mathrm{~K}, T_{\infty}=300 \mathrm{~K}$.

As can be observed in Fig. 6.22, the ignition occurs much later in the critical case than the supercritical case, at $9.2855 \times 10^{-3} \mathrm{~s}$ and $9.2779 \times 10^{-3} \mathrm{~s}$ using maximum time rate of change of temperature and $\mathrm{OH}$ mass fraction as the criterion for ignition, respectively. Once again, two ignition criteria produce ignition times that differ by less than $1 \%$. The ignition delay time predicted by zero-dimensional constant pressure calculations is $5.9444 \times 10^{-3} \mathrm{~s}$ and $5.9442 \times 10^{-3} \mathrm{~s}$ using maximum time rate of change of temperature and $\mathrm{OH}$ mass fraction as the criterion for ignition, respectively. Here the ignition delay time has increased significantly in the onedimensional simulations compared to the zero-dimensional ignition delay; in fact, it has nearly doubled. This is likely due to the increased effect of losses (like heat 
lost to the constant temperature wall at the inner boundary) in the one-dimensional simulation when the wall temperature approaches the critical temperature. These increased losses cause the ignition event to take longer to occur and place the location of the ignition event further from the wall, at $\Delta \mathrm{r}=0.53 \mathrm{~mm}$ using the maximum time rate of change of temperature to indicate ignition.

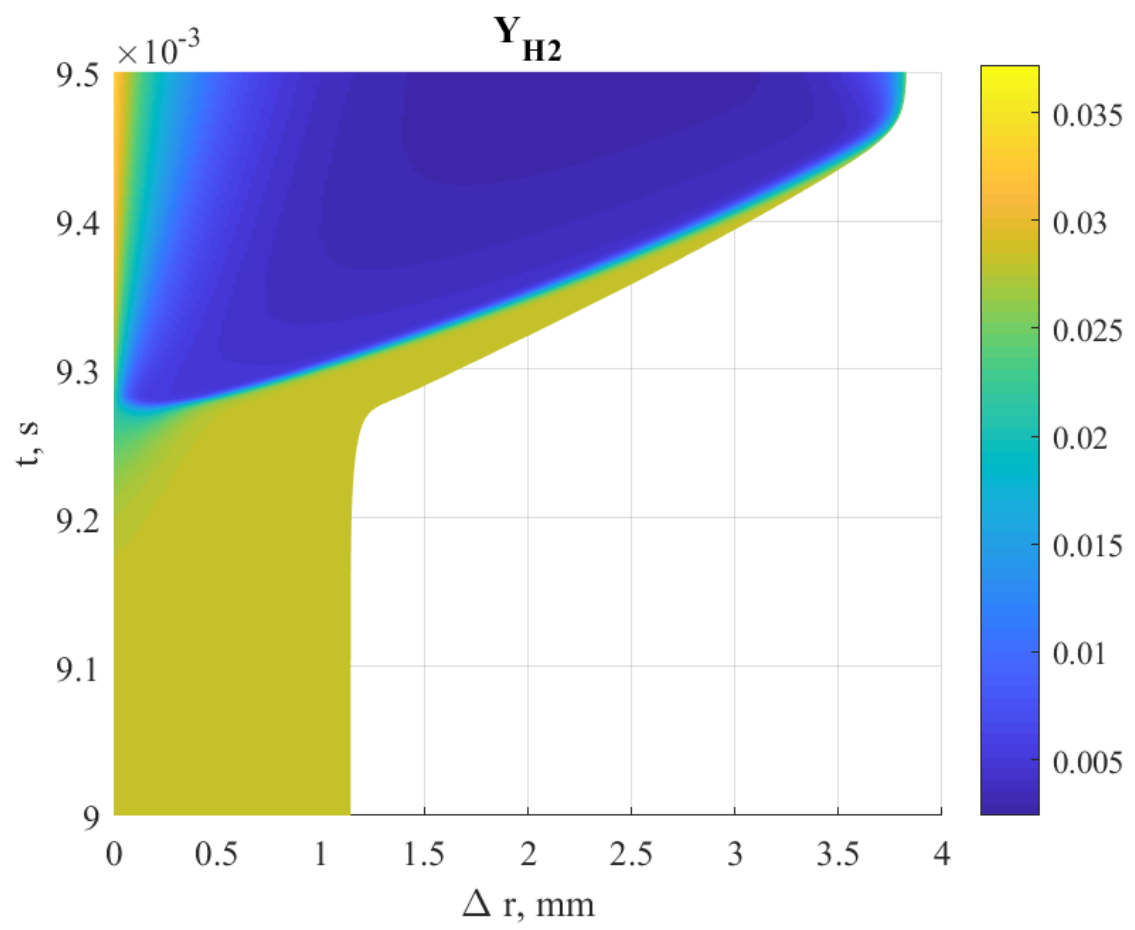

Figure 6.23: $\mathrm{H}_{2}$ mass fraction field plotted against radial distance from the wall and time for stoichiometric hydrogen-air simulation with $T_{w}=917.1875 \mathrm{~K}, T_{\infty}=300$ $\mathrm{K}$.

Figures 6.23 and 6.24 show the mass fractions of $\mathrm{H}_{2}$ and $\mathrm{HO}_{2}$, respectively, for the critical ignition case. While we can see a flame form, consuming reactants and producing radicals in the middle part of the domain, that consumption is limited near the wall, where heat losses to the wall prevent significant reaction from occurring. Figure 6.25 focuses on the $\mathrm{HO}_{2}$ mass fraction field in the immediate proximity of ignition.

A significant amount of $\mathrm{HO}_{2}$ has accumulated in proximity of the wall prior to the wall due to the long induction time of the critical case. The maximum mass fraction of $\mathrm{HO}_{2}$ during the early stages of ignition shown in Fig. 6.25 is approximately half 


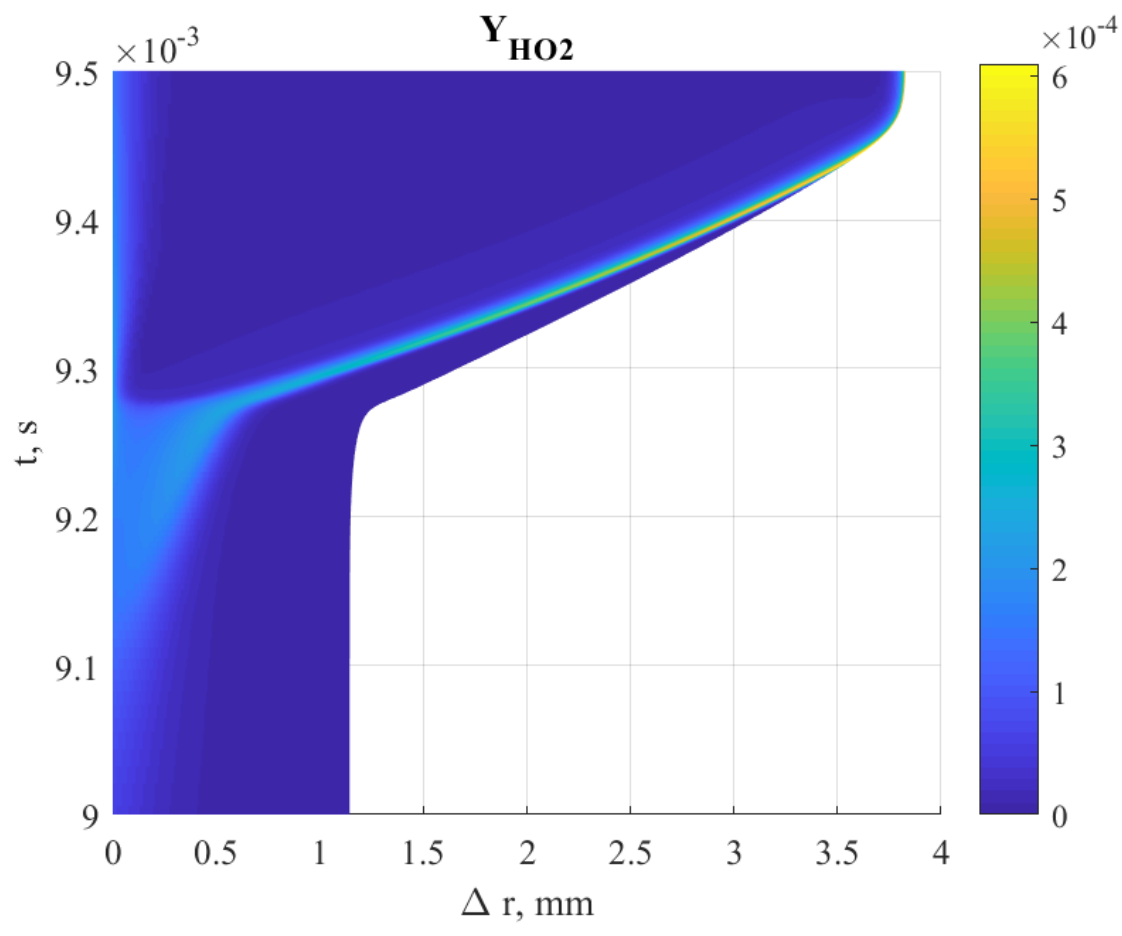

Figure 6.24: $\mathrm{HO}_{2}$ mass fraction field plotted against radius and time for stoichiometric hydrogen-air simulation with $T_{w}=917.1875 \mathrm{~K}, T_{\infty}=300 \mathrm{~K}$.

$\left(Y_{O H, \text { max }} \approx 3 \times 10^{-4}\right)$ that for the early stages of ignition in the supercritical case $\left(Y_{O H, \text { max }} \approx 6 \times 10^{-4}\right)$. As noted previously, the ignition location is $\Delta r=0.53 \mathrm{~mm}$ using the maximum time rate of change of temperature as the ignition criterion. This is approximately 2.5 flame thicknesses away from the wall, a greater distance than is observed for the supercritical case. We conclude that as the wall temperature approaches the critical condition, the ignition location moves further from the wall in order to balance the increase heat losses to the wall due to the lower temperature. We will return to a more detailed examination of heat transfer and energy balance for the critical case in the following sections. 


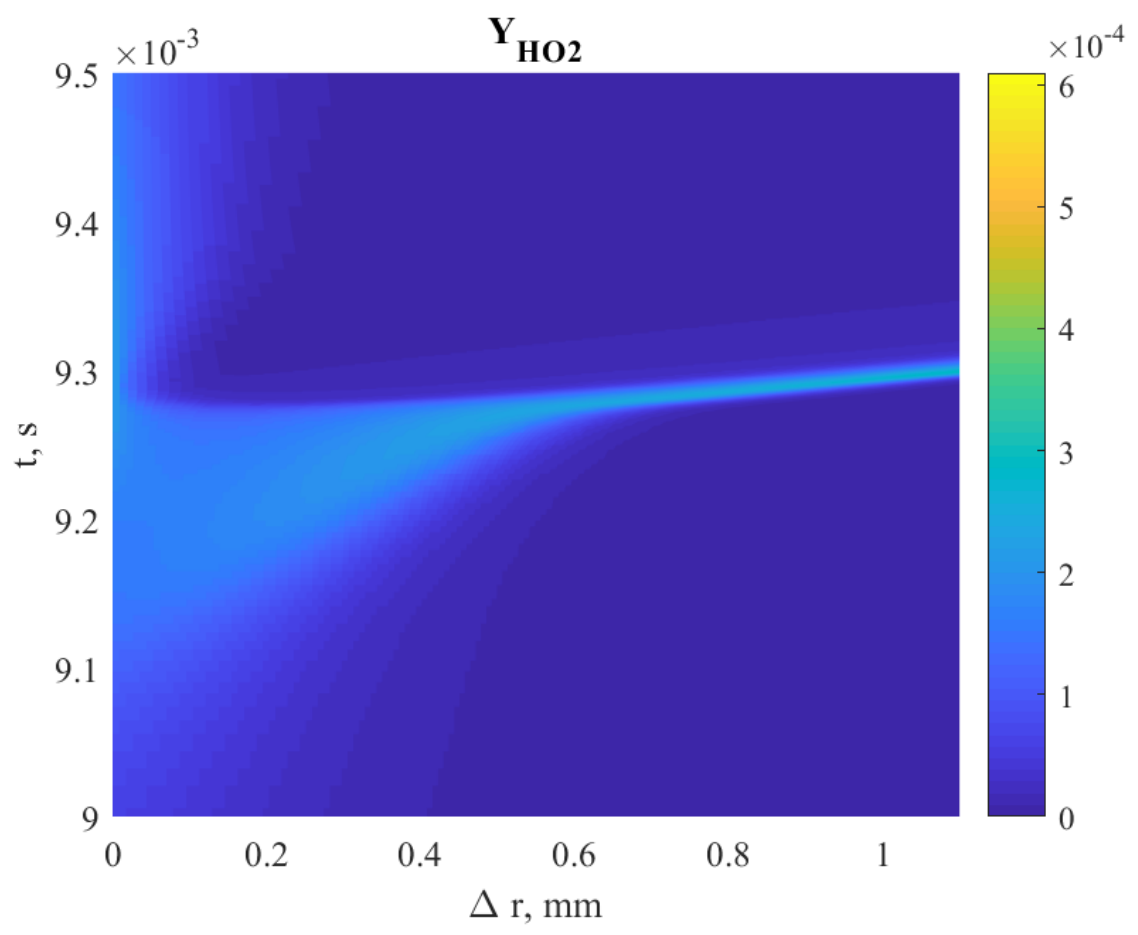

Figure 6.25: Magnified $\mathrm{HO}_{2}$ mass fraction field plotted against radius and time for stoichiometric hydrogen-air simulation with $T_{w}=917.1875 \mathrm{~K}, T_{\infty}=300 \mathrm{~K}$. 


\section{Subcritical Ignition Case}

Next, we present the results of a subcritical case. The wall temperature was set to $915.625 \mathrm{~K}$, just barely less than the wall temperature of the critical case, the ambient temperature to $300 \mathrm{~K}$, and the same initial gas composition was used for the hydrogen-air mixture. Here only the temperature, mass fraction of $\mathrm{H}_{2}$, and mass fraction of $\mathrm{OH}$ fields as function of radius and time are presented, as these provide a sufficient overview of the results for this section. Specific phenomena like heat flux at the wall and energy balance will be examined in detail later.

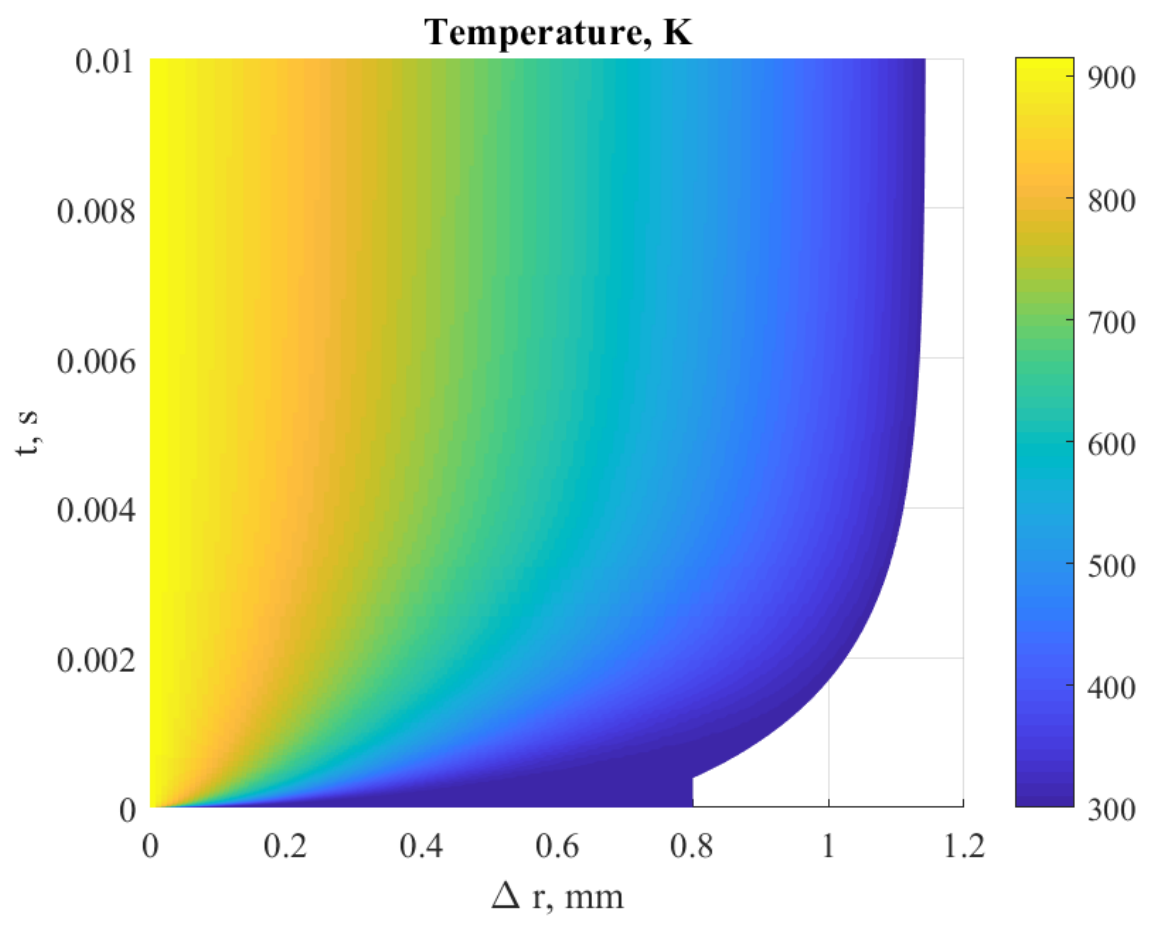

Figure 6.26: Temperature field plotted against radial distance from the wall and time for stoichiometric hydrogen-air simulation with $T_{w}=915.625 \mathrm{~K}, T_{\infty}=300 \mathrm{~K}$.

Fig. 6.26 shows the temperature field for the subcritical case. The heat transfer is dominated by conduction, and no significant increase in the temperature indicative of a flame is observed. The simulation is terminated at $\mathrm{t}=1 \times 10^{-2} \mathrm{~s}$ (the same end time as for the critical case) to limit the computation time.

Figures 6.27 and 6.28 show the mass fractions of $\mathrm{H} 2$ and $\mathrm{OH}$, respectively. They both show very little chemical activity, confirming that this is a subcritical case with no ignition given the integration time of the simulation. 


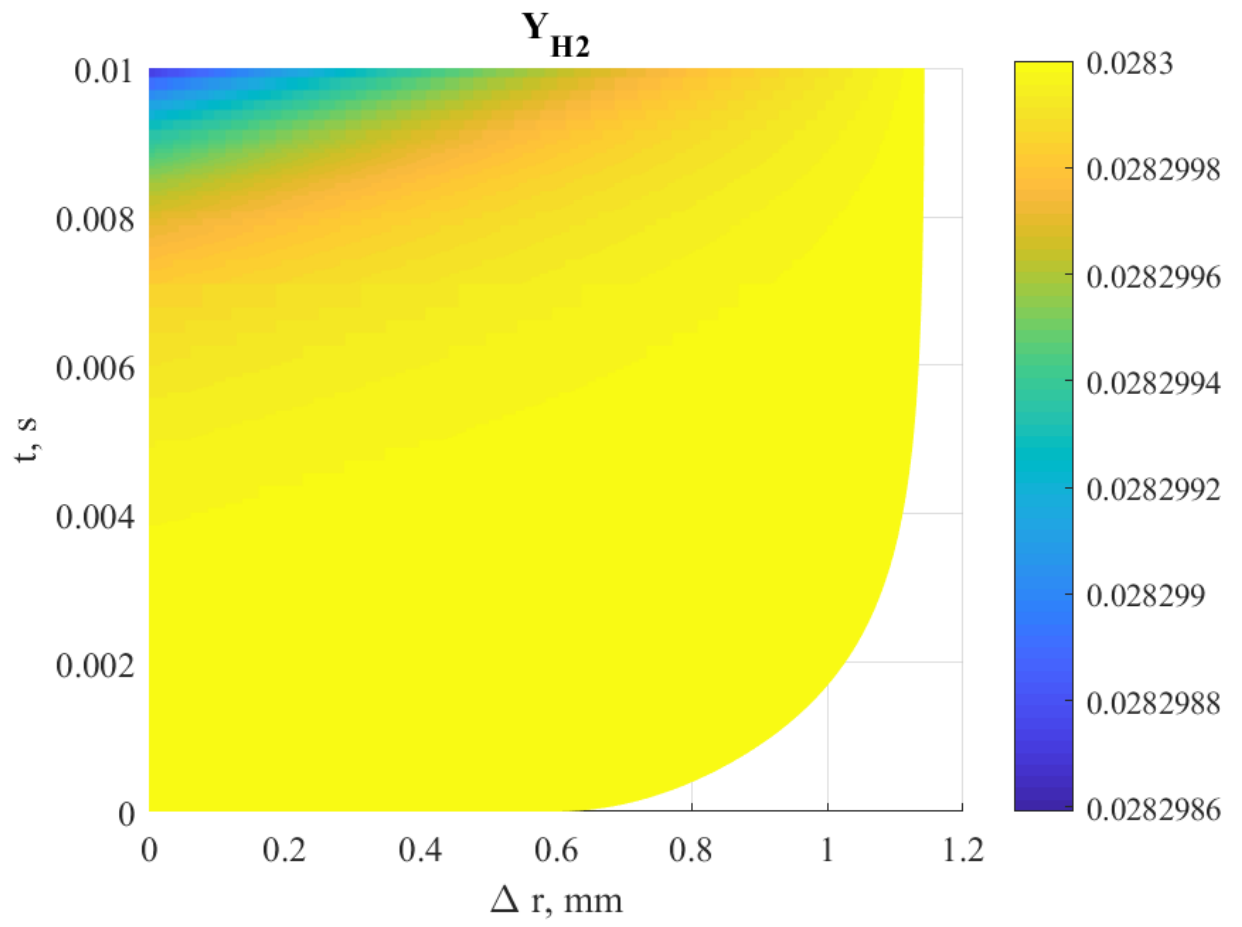

Figure 6.27: $\mathrm{H}_{2}$ mass fraction field plotted against radial distance from the wall and time for stoichiometric hydrogen-air simulation with $T_{w}=915.625 \mathrm{~K}, T_{\infty}=300 \mathrm{~K}$.

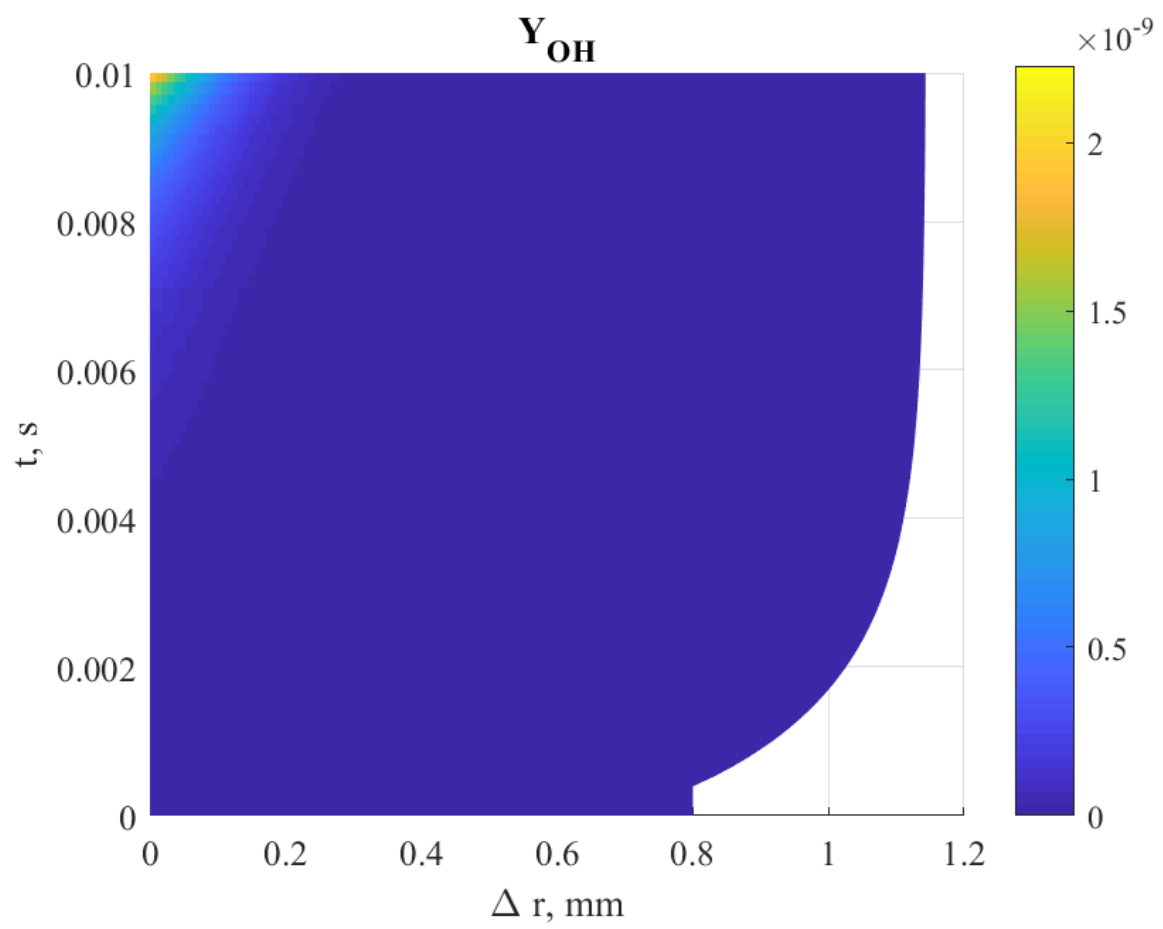

Figure 6.28: $\mathrm{OH}$ mass fraction field plotted against radial distance from the wall and time for stoichiometric hydrogen-air simulation with $T_{w}=915.625 \mathrm{~K}, T_{\infty}=300 \mathrm{~K}$. 


\section{Temperature and Species Mass Profiles}

\section{Supercritical Case: $T_{w}=1200 \mathrm{~K}$}

We next display the evolution in time of radial profiles of temperature and species for a supercritical ignition case, $T_{w}=1200 \mathrm{~K}$. We can see via Fig. 6.29 that the early stage of the simulation (blue lines) is dominated by heating of the boundary layer via conduction. At approximately 5e-5 s, it appears the Van't Hoff ignition criterion has been met at the wall. At this same time, we can see a significant mass fraction of $\mathrm{OH}$ has been produced from Fig. $6.30\left(\mathrm{Y}_{O} H \approx 5 \times 10^{-3}\right)$, which will increase to a constant peak value of approximately $1 \times 10^{-2}$ shortly after ignition. Figure 6.31 shows that at this time, the mass fraction of $\mathrm{HO}_{2}$ has reached a maximum value (approximately $5 \times 10^{-4}$ ), which will remain constant throughout the flame propogation portion of the region.

The green through red lines in Figs. 6.29, 6.30, and 6.31 show the development of the ignition kernel and subseqent flame propogation through the domain. As the flame develops and starts to propagate, the maximum temperature in the domain increases. The plots of $\mathrm{OH}$ and $\mathrm{HO}_{2}$ mass fraction profiles can be used to track the flame front: in Fig. 6.30, the sharp increase in $\mathrm{OH}$ mass fraction is indicative of the flame front, while in Fig. 6.31, the peak $\mathrm{HO}_{2}$ mass fraction indicates the location of the flame front.

We can track both the maximum gradient of $\mathrm{OH}$ mass fraction and the peak $\mathrm{HO}_{2}$ mass fraction to extract the flame speed from the simulations. The flame speed estimated by both quantities is $11.8 \mathrm{~m} / \mathrm{s}$. We can compare this to the standard calculation of flame speed (described previously in Chapters. 4) and 5) of a stoichiometric hydrogen air mixture, setting the ambient temperature to $300 \mathrm{~K}$. This ambient temperature is used because the supercritical case ignites quickly, meaning very little of the domain has been heated by conduction prior to ignition and therefore flame propagation in the one-dimensional simulation occurs in a roughly $300 \mathrm{~K}$ environment. The flame speed estimated for this mixture is $15.4 \mathrm{~m} / \mathrm{s}$. The one-dimensional simulation flame speed is $23.4 \%$ slower than the standard flame speed calculation estimate; this is still relatively good agreement given the many non-ideal aspects of the one-dimensional simulation. 


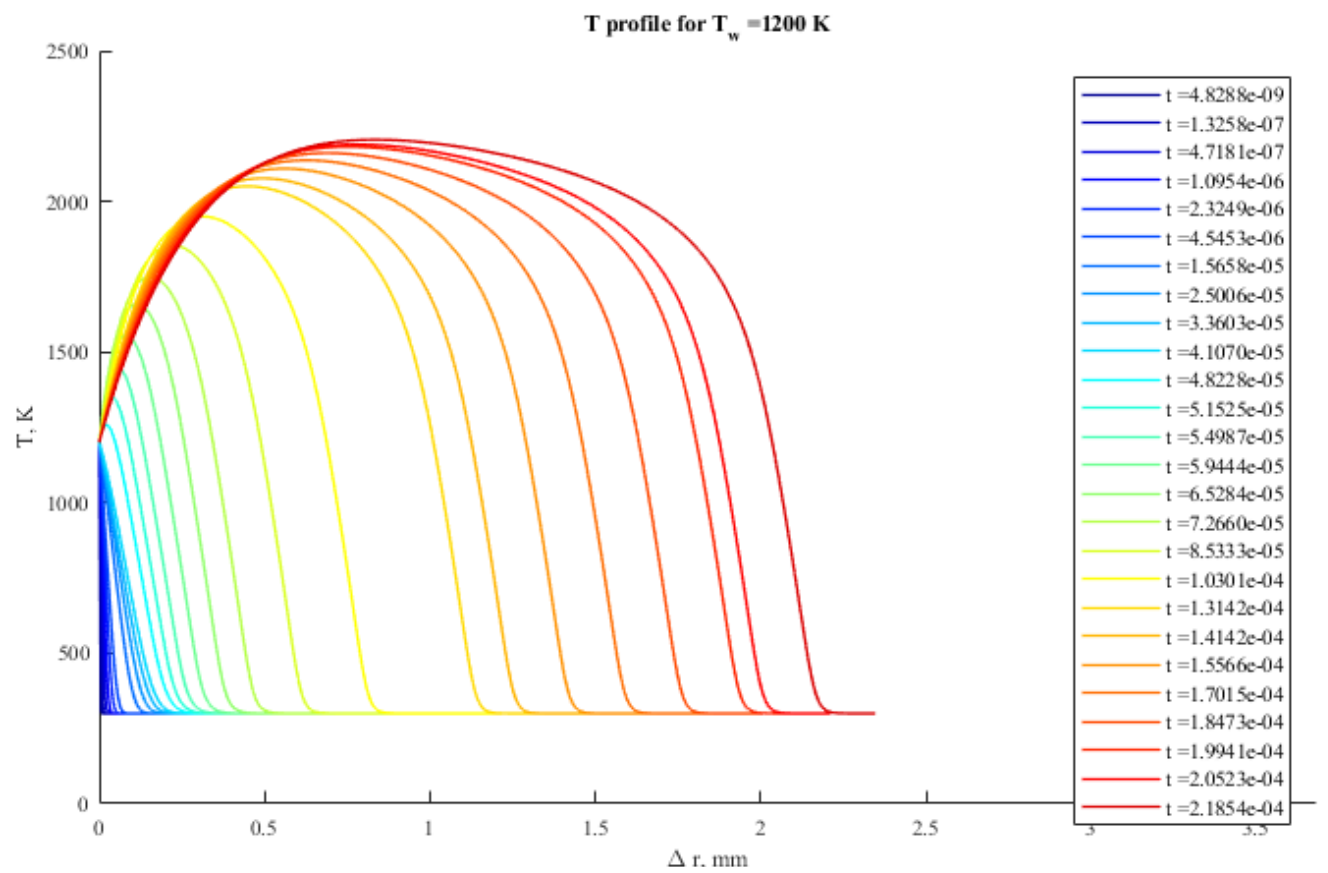

Figure 6.29: Radial profiles of temperature over time, supercritical case. $T_{w}=1200$ $\mathrm{K}, T_{\infty}=300 \mathrm{~K}$.

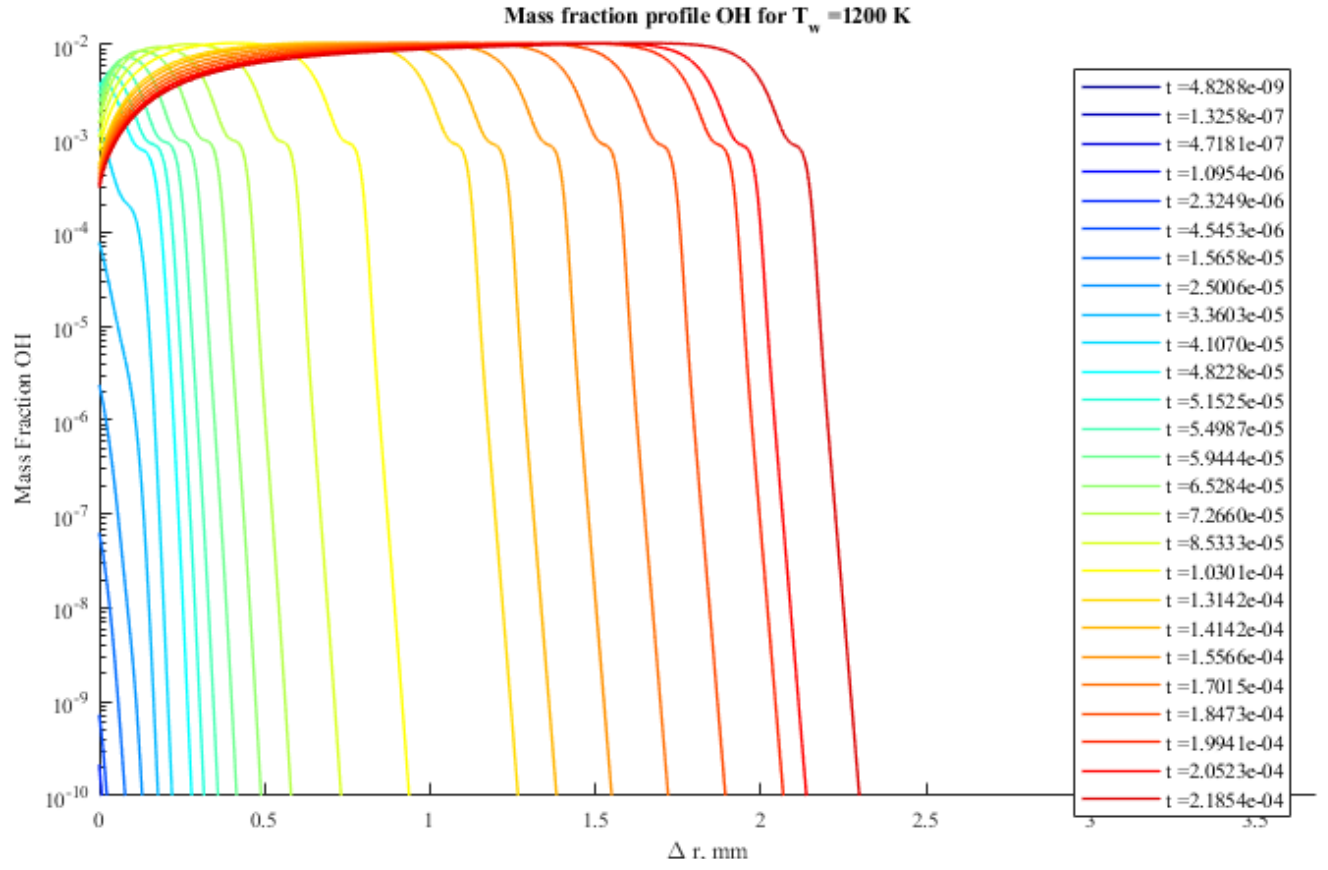

Figure 6.30: Radial profiles of $\mathrm{OH}$ mass fraction over time, supercritical case. $T_{w}=1200 \mathrm{~K}, T_{\infty}=300 \mathrm{~K}$. 


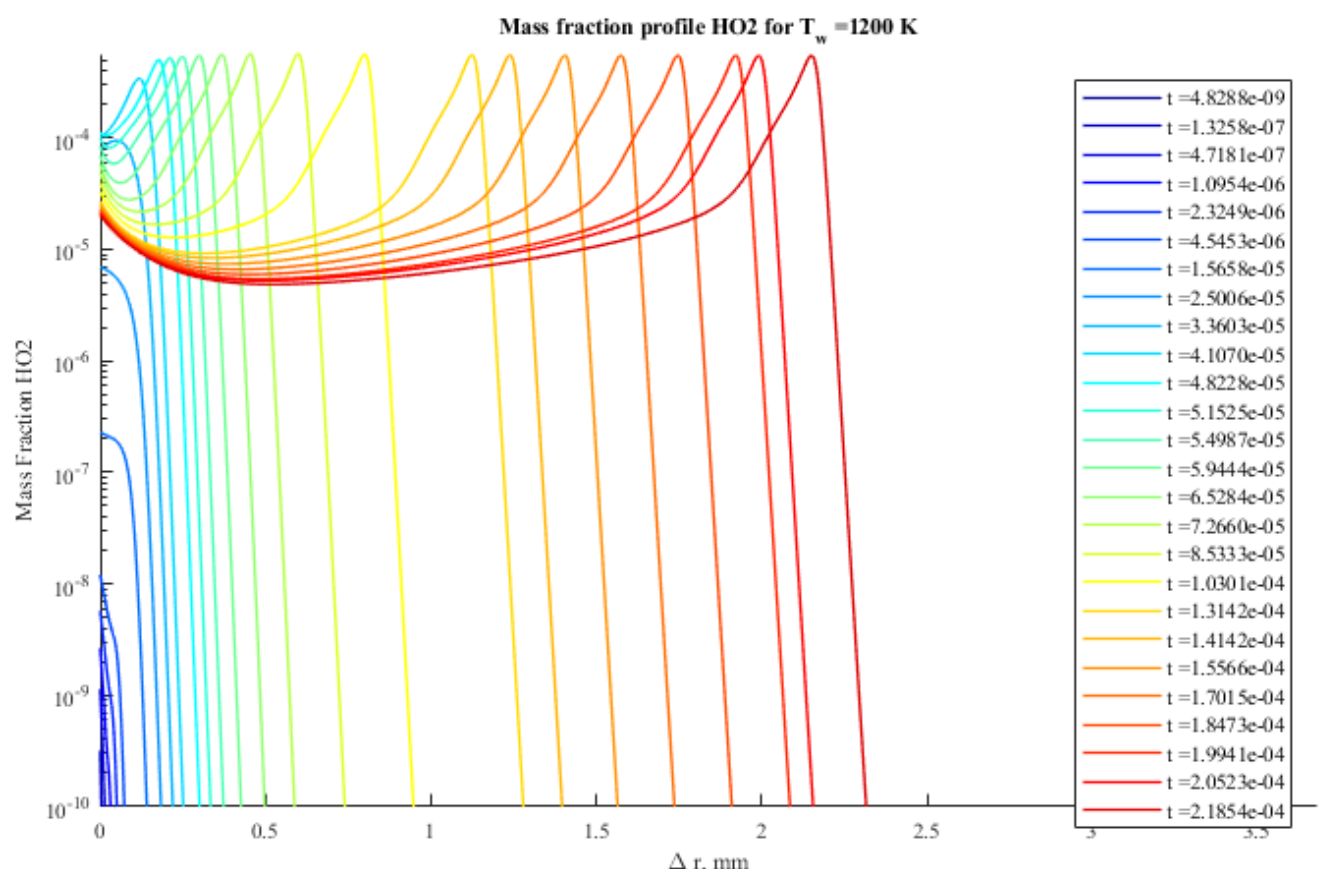

Figure 6.31: Radial profiles of $\mathrm{HO}_{2}$ mass fraction over time, supercritical case. $T_{w}=1200 \mathrm{~K}, T_{\infty}=300 \mathrm{~K}$. 


\section{Critical Case: $T_{w}=917.2 \mathbf{K}$}

The critical ignition case is next examined in terms of the evolution of radial profiles of temperature and radical mass fractions. The wall temperature is set to $917.2 \mathrm{~K}$, and the much lower wall temperature causes a significant increase in the ignition delay time, $\tau_{i g n, T}=9.29 \times 10^{-3} \mathrm{~s}$, compared to the supercritical case. Figure 6.32 shows the evolution of radial profiles of temperature. The early stages of the simulation (blue lines) show the progression of what is primarily an unsteady conduction problem: the majority of the boundary layer growth occurs by about $3 \times 10^{-3} \mathrm{~s}$, after which the temperature profile remains relatively unchanged up until about $9.2 \times 10^{-3}$ (bright blue line) at which the Van't Hoff criterion appears to be satisfied. After this point, reaction occurs, and the temperature profiles evolve rapidly with time as the exothermic reaction occurs.

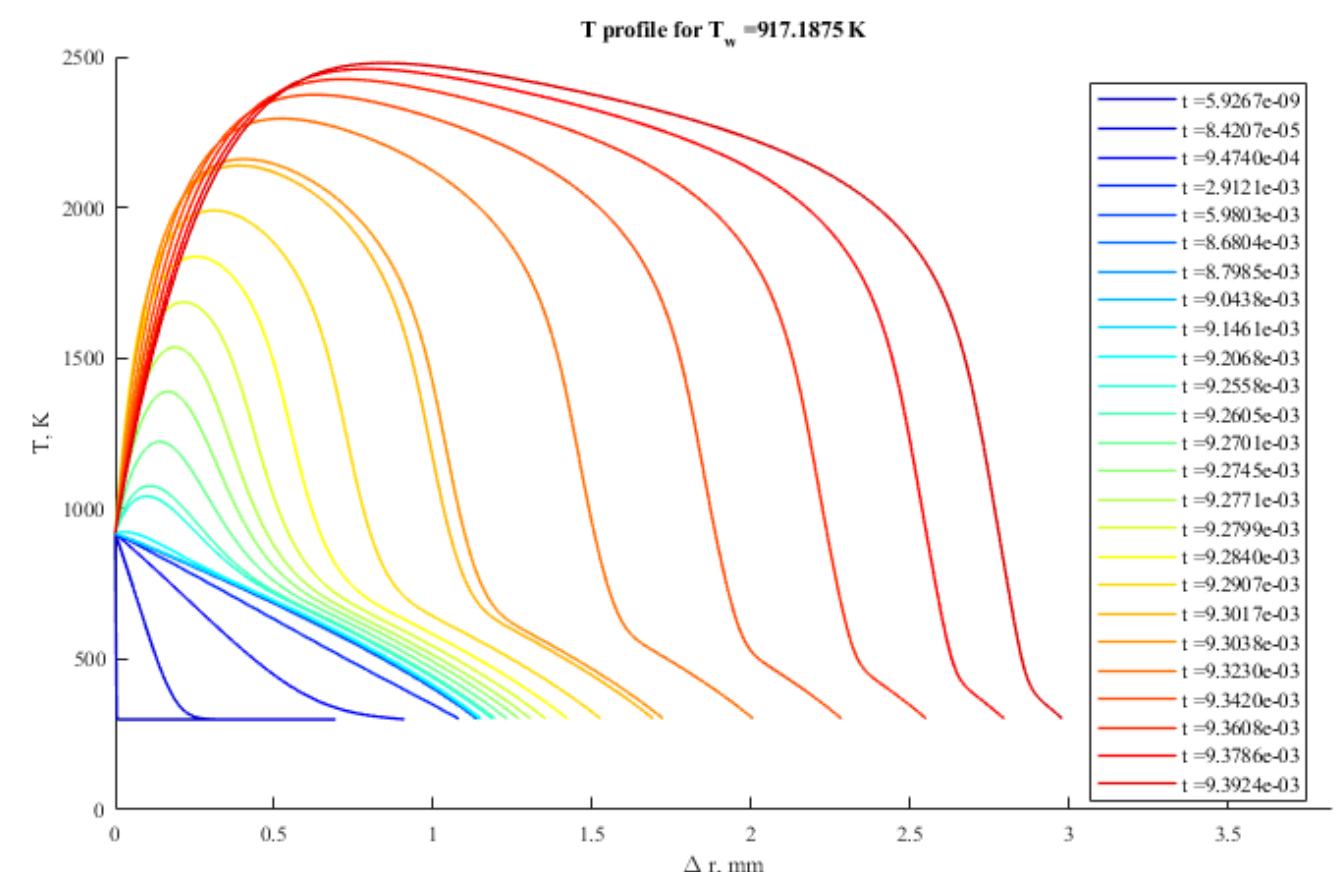

Figure 6.32: Radial profiles of temperature over time for critical case. $T_{w}=917.2$ $\mathrm{K}, T_{\infty}=300 \mathrm{~K}$.

Figures 6.33 plots the evolution of the radial profile of $\mathrm{OH}$ for the critical ignition case. Interestingly, at the time at which the Van't Hoff ignition criterion is satisfied (about $9.2 \times 10^{-3} \mathrm{~s}$ ), the mass fraction of $\mathrm{OH}$ is significantly less, $Y_{O H} \approx 1 \times 10^{-4}$, than at the point the Van't Hoff criteron is satisfied for the supercritical case, $Y_{O H} \approx$ $4 \times 10^{-3}$. It also takes longer to reach the peak value of the $\mathrm{OH}$ mass fraction 
(approximately $1 \times 10^{-2}$ ) in the critical ignition case. This suggests the heated surface in the critical ignition case is signficantly inhibiting reaction by acting as a heat sink. It is worth noting that the maximum value of the $\mathrm{OH}$ mass fraction achieved in both the critical and supercritical cases is approximately $1 \times 10^{-2}$, suggesting both cases eventually develop fully formed flames.

Figure 6.34 reveals a similar story: the peak mass fraction of $\mathrm{HO}_{2}$ around $\mathrm{t}=$ $9.2 \times 10^{-3} \mathrm{~s}$ is roughly $2 \times 10^{-4}$ and has not yet reached the maximum value. In the supercritical case, when the Van't Hoff ignition criterion was satisfied, the peak mass fraction of $\mathrm{HO}_{2}$ was not only higher, but had already reached the maximum $\mathrm{HO} 2$ mass fraction of $5 \times 10^{-4}$. While the peak mass fraction of $\mathrm{OH}$ will eventually reach this maximum value, it takes relatively longer in the critical ignition case compared to the supercritical case, evidence that the lower wall temperature in the critical ignition case inhibits the early stages of ignition. Using the peak of $\mathrm{HO}_{2}$ mass fraction as a rough tracker of the flame front, we see it is not until the flame moves further away from the wall that the peak in $\mathrm{HO}_{2}$ mass fraction reaches its maximum value.

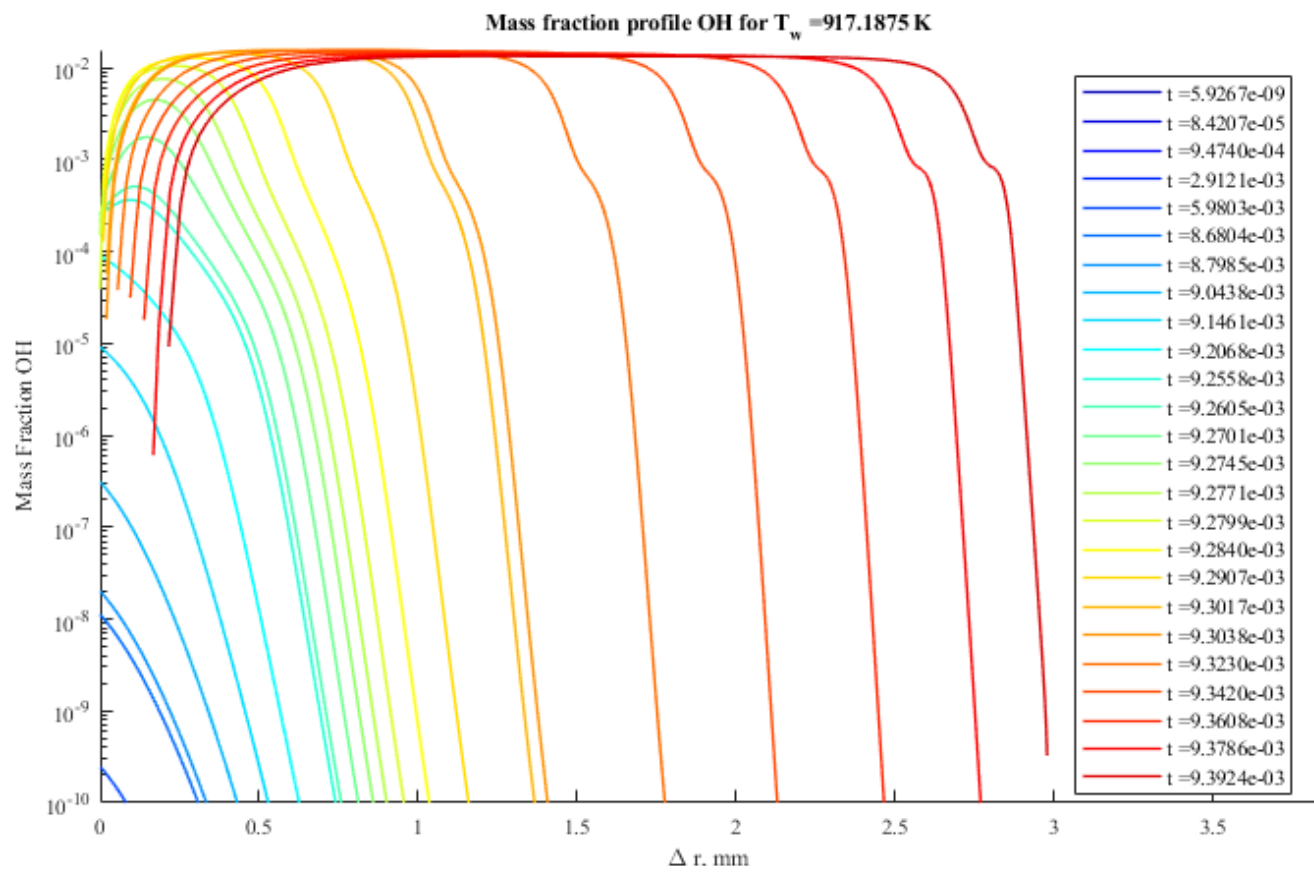

Figure 6.33: Radial profiles of $\mathrm{OH}$ mass fraction over time for critical case. $T_{w}=$ $917.2 \mathrm{~K}, T_{\infty}=300 \mathrm{~K}$.

We can once again extract the flame speed from the simulation results. In the critical case, a significant amount of heat conduction has occurred, meaning that 


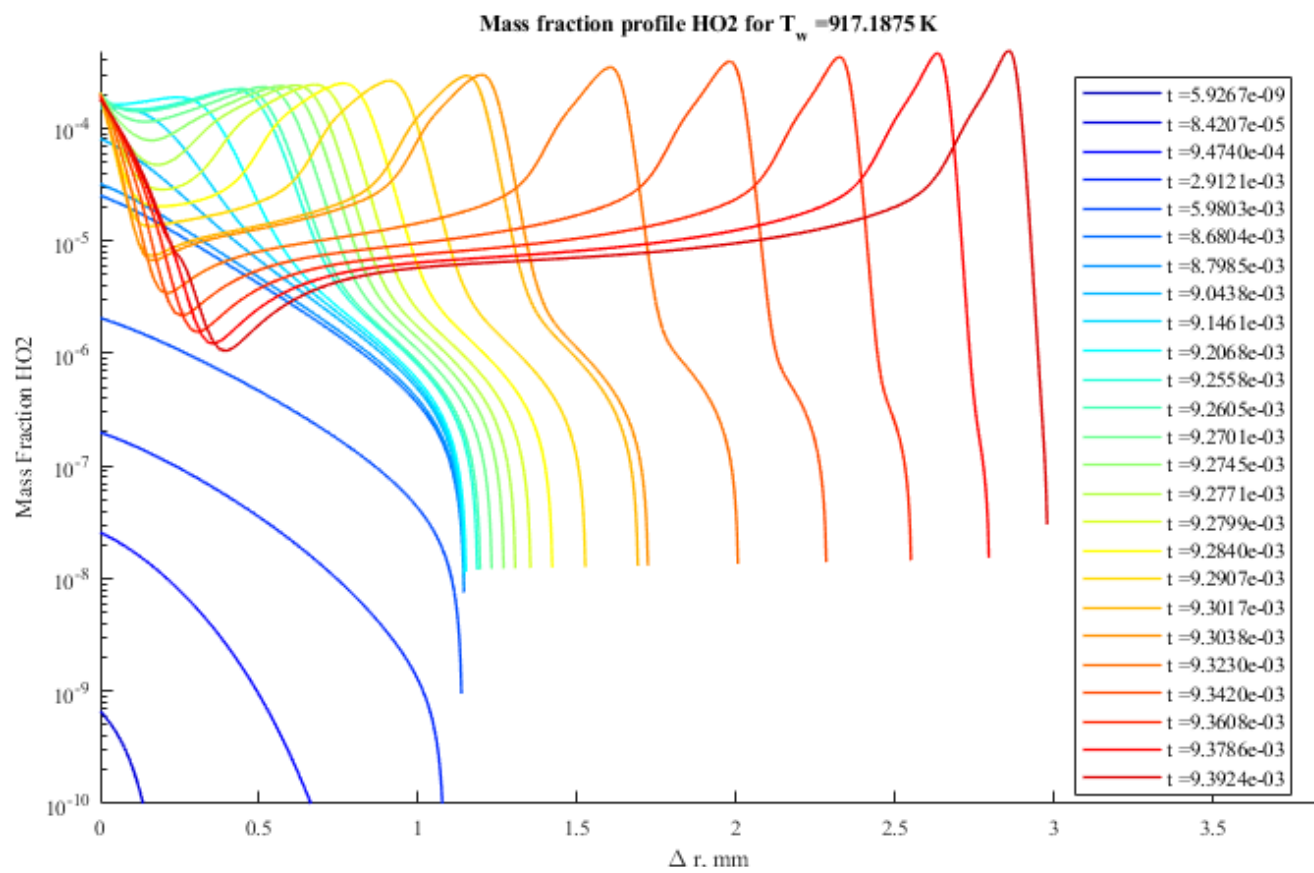

Figure 6.34: Radial profiles of $\mathrm{HO}_{2}$ mass fraction over time for the critical case. $T_{w}=917.2 \mathrm{~K}, T_{\infty}=300 \mathrm{~K}$.

the flame is propagating into a mixture of varying temperature. This makes comparison with a calculated flame speed value more challenging: the flame speed in the one-dimensional simulation will vary over time depending on the mixture temperature, and the flame speed calculation is performed with a constant mixture temperature. Still, we can at least get a rough estimate. We take the average flame speed over the entire flame propagation time, which come out to $18.8 \mathrm{~m} / \mathrm{s}$. The flame speed is then calculated using a freely propagating flame calculation with a mixture temperature of $600 \mathrm{~K}$, roughly the film temperature of the boundary layer in the critical one-dimensional simulation. The flame speed of the hydrogen-air mixutre at $600 \mathrm{~K}$ is calculated to be $26.9 \mathrm{~m} / \mathrm{s}$. The flame speed from the one-dimensional simulation is $30 \%$ slower than the predicted flame speed. This is a good agreement considering not only the non-ideal nature of the one-dimensional simulation, but also the approximations made in extracting a flame speed from the simulation and in the initial conditions for predicting flame speed. 
Subcritical Case: $T_{w}=915.6 \mathbf{K}$

The subcritical case has a wall temperature of $915.6 \mathrm{~K}$. The integration time is limited to $1 \times 10^{-2} \mathrm{~s}$ for the sake of limiting computational time. Within $1 \times 10^{-2} \mathrm{~s}$ of integration time, this simulation does not ignite. The temperature profiles are plotted in Fig. 6.35, extracted at the same times as the profiles from the critical case for comparison. The wall temperatures of the critical and subcritical cases are so similar that until ignition occurs in the critical case, they should be very similar. The early stage of the simulation (temperature profiles shown in shades of dark blue) is dominated by conduction; the boundary layer grows as heat is conducted from the wall to the gas. At times greater than approximately $3 \times 10^{-3} \mathrm{~s}$, the temperature profiles are very similar; no ignition occurs to cause an interesting further evolution of the temperature profiles.

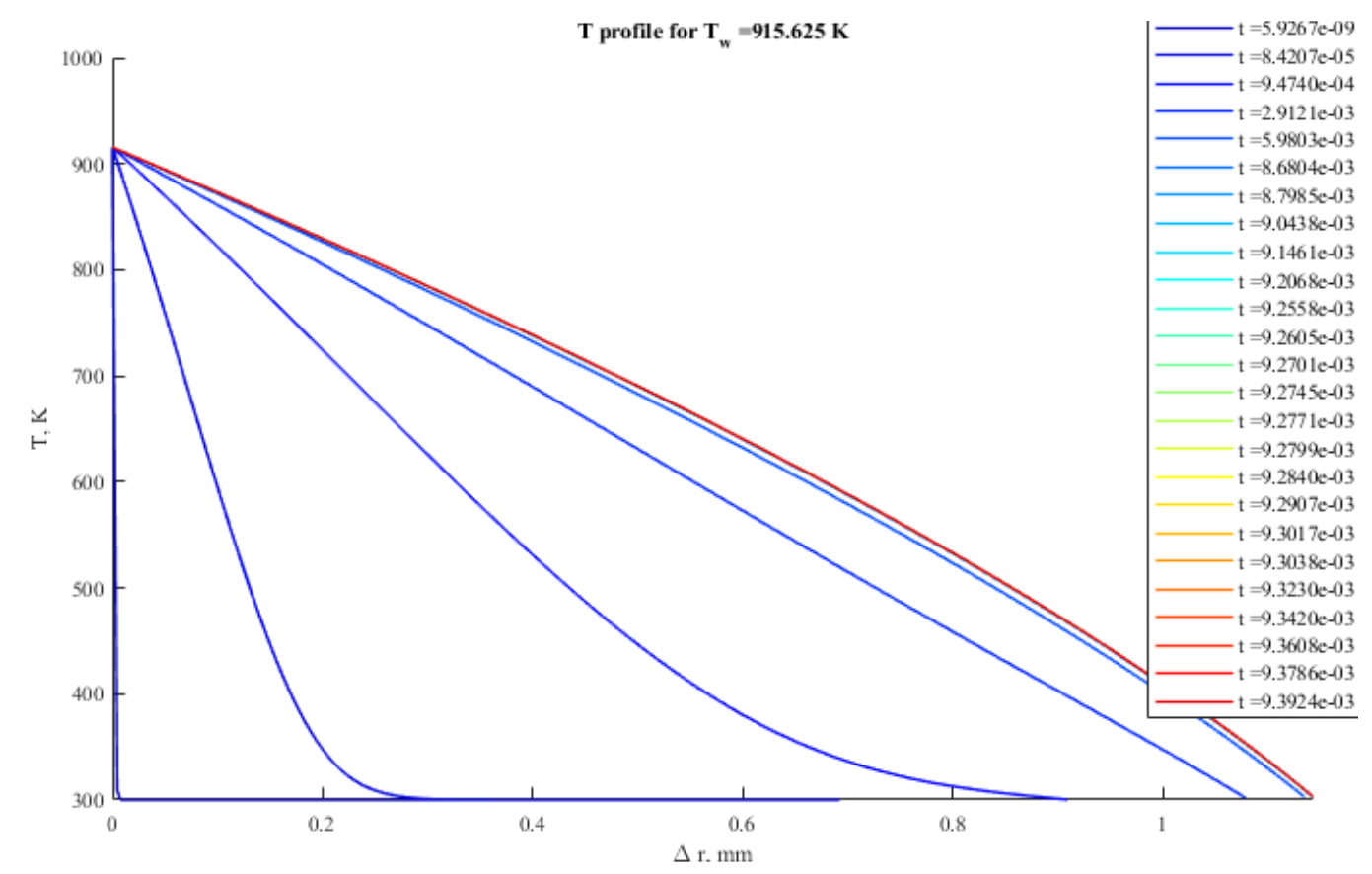

Figure 6.35: Radial profiles of temperature over time, subcritical case. $T_{w}=915.6$ $\mathrm{K}, T_{\infty}=300 \mathrm{~K}$.

Figure 6.36 shows the profiles of $\mathrm{OH}$ mass fraction over the course of the simulation. There is very little $\mathrm{OH}$ present; maximum mass fraction of $\mathrm{OH}$ in this simulation is approximately $1 \times 10^{-9}$. This indicates no significant activity of the chain branching process that leads to a thermal explosion. Interestingly, Fig. 6.37 shows a more significant mass fraction of $\mathrm{HO}_{2}$, with a maximum value of nearly $1 \times 10^{-5}$ by the 
end of the simulation. This indicates that at this subcritical condition, the linear chain that produces $\mathrm{HO}_{2}$ is much more active than the branching chain that leads to thermal explosion. This makes sense, as Shepherd (2020) notes that during the induction period before ignition, the chemistry is dominated by peroxides $\left(\mathrm{HO}_{2}\right.$ and $\mathrm{H}_{2} \mathrm{O}_{2}$ ). This simulation is terminated without any indications of transition towards thermal explosion and is thus considered a subcritical (non-ignition) case.

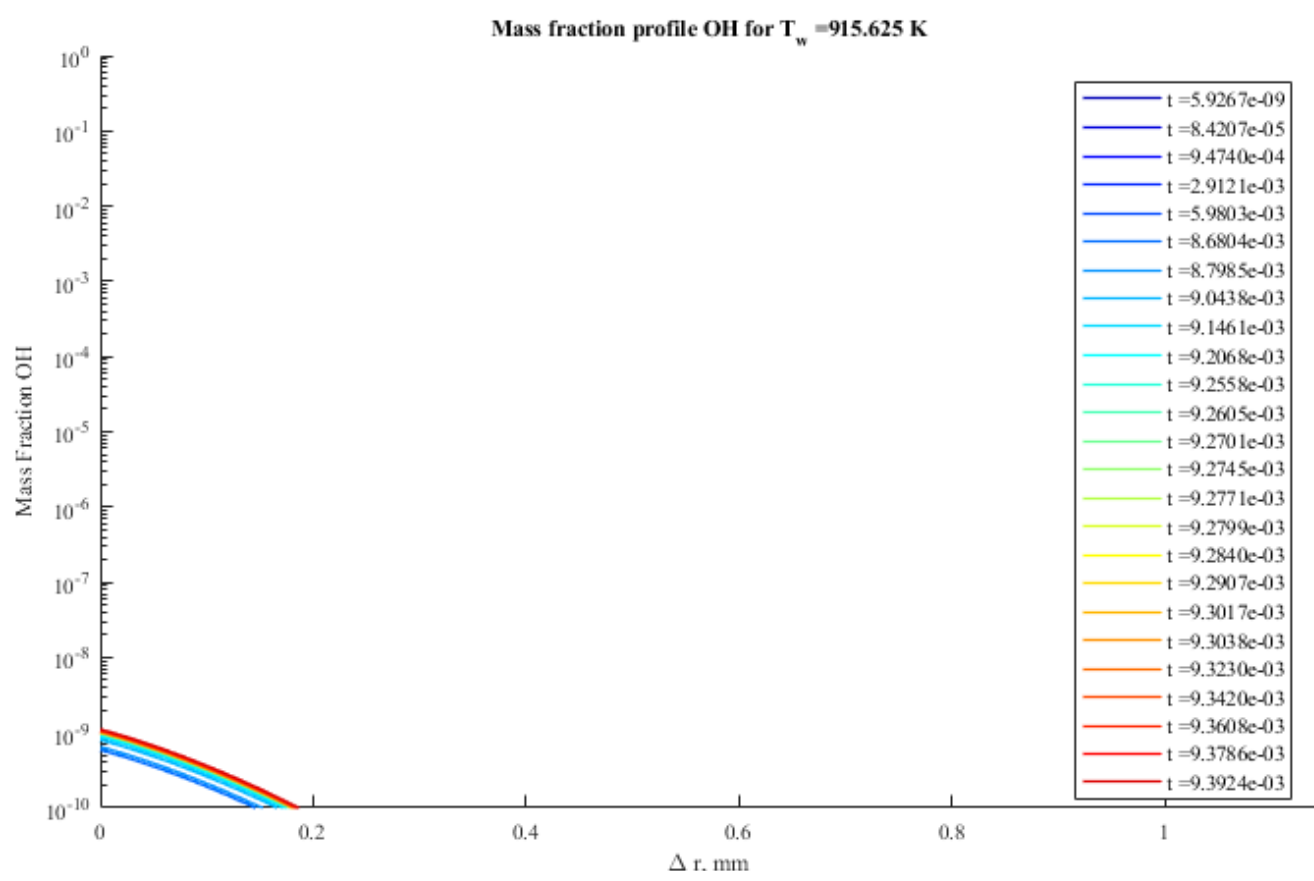

Figure 6.36: Radial profiles of $\mathrm{OH}$ mass fraction over time, subcritical case. $T_{w}=$ $915.6 \mathrm{~K}, T_{\infty}=300 \mathrm{~K}$. 


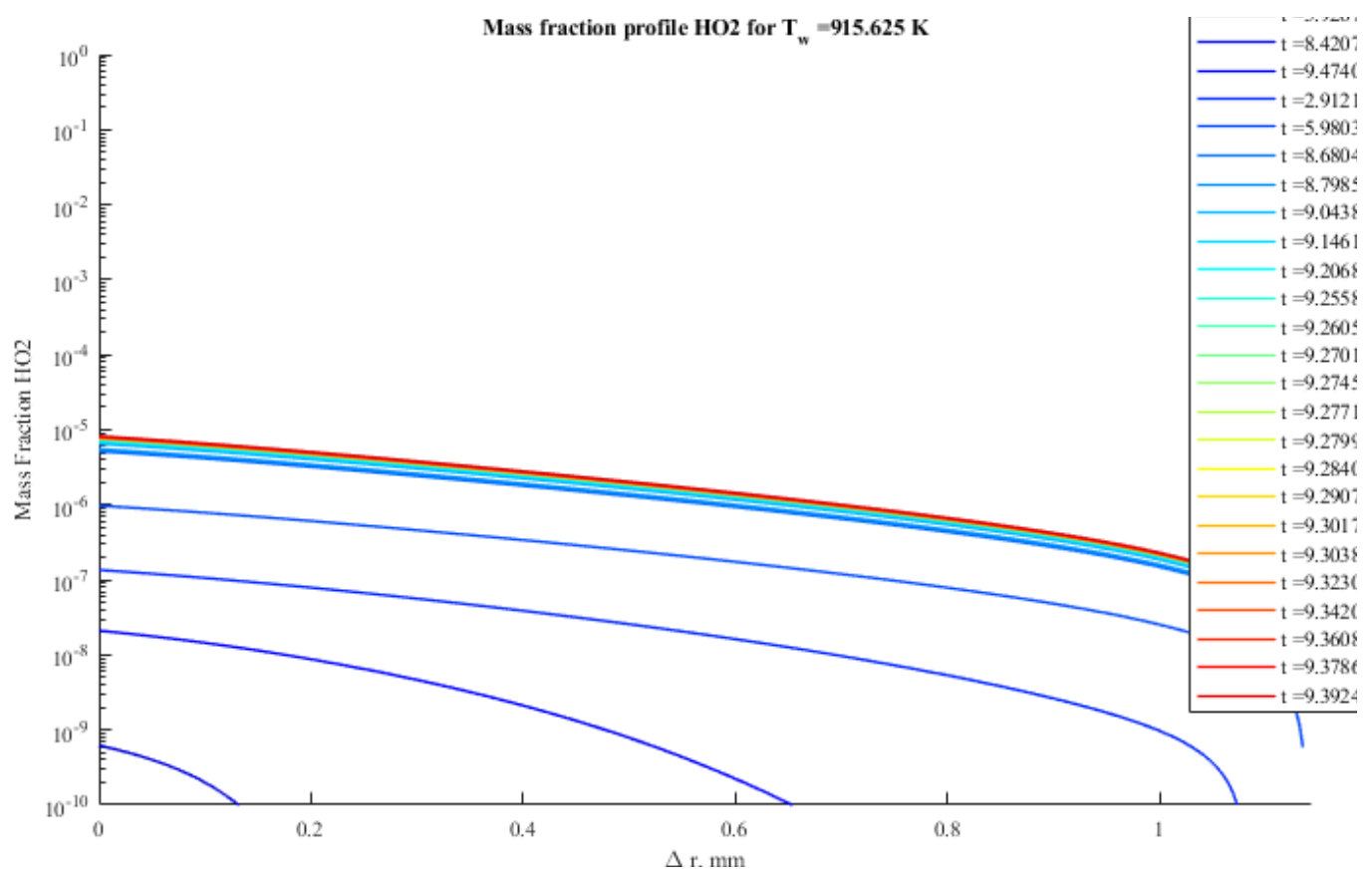

Figure 6.37: Radial profiles of $\mathrm{HO}_{2}$ mass fraction over time, subcritical case. $T_{w}=915.6 \mathrm{~K}, T_{\infty}=300 \mathrm{~K}$. 


\section{Energy Balance}

The energy conservation equation in Lagrangian coordinates is given in Eq. 6.33. It is rearranged so that all terms on on the same side of the equation and the various terms are highlighted in color to indicate the different terms considered in the energy balance analysis:

$0=\left.\frac{1}{c_{p_{j}}} \frac{\partial}{\partial \psi}\left(\rho r^{2} \lambda \frac{\partial T}{\partial \psi}\right)\right|_{j}+\left.\left.\frac{\rho_{j}^{2} r_{j}^{2}}{c_{p_{j}}} \frac{\partial T}{\partial \psi}\right|_{j} \sum_{k=1}^{n_{s}} D_{k, j} c_{p_{k, j}} \frac{\partial Y_{k}}{\partial \psi}\right|_{j}-\frac{1}{\rho_{j} c_{p_{j}}} \sum_{k=1}^{n_{s}} \dot{\omega}_{k, j} h_{k, j} M_{k}-\left.\frac{\partial T}{\partial t}\right|_{j}$,

where blue represents the conduction term, green represents the diffusion term, red represents the reaction term, and purple represents the unsteady term. As the equation indicates, these terms always sum to zero, so we can examine how the balance between these terms changes during the ignition process for a supercritical, critical, and subcritical ignition case to gain further insight into the ignition process.

\section{Supercritical Case: 1200 K}

The examination of energy balance during ignition starts with the supercritical case, $T_{w}=1200 \mathrm{~K}$. Figure 6.38 shows the evolution of each term in the energy equation over time in a separate plot. The lines are color-coded to reveal the time at which the balance was calculated. This figure reveals a few interesting points. First, in the early stage of the simulation before reaction occurs, the energy balance is primarily between conduction and diffusion. This makes sense as the early part of the simulation is an unsteady conduction problem, and no significant reaction or diffusion takes place. As time moves forward and the gas heats up due to heat transfer from the wall, reaction takes off, and the balance shifts to primarily a balance between reaction and unsteady terms, although conduction still plays a role in heating up the gas in front of the reaction zone. The diffusion term is most active once significant reaction occurs, but the diffusive term remains an order of magnitude smaller than the other three terms throughout the simulation.

Figure 6.38 provides a useful overview of the balance between the terms of the energy equation. Figures 6.39 through 6.43 provide a direct comparison of the four energy equation terms at times of particular interest to allow for a more detailed examination. 

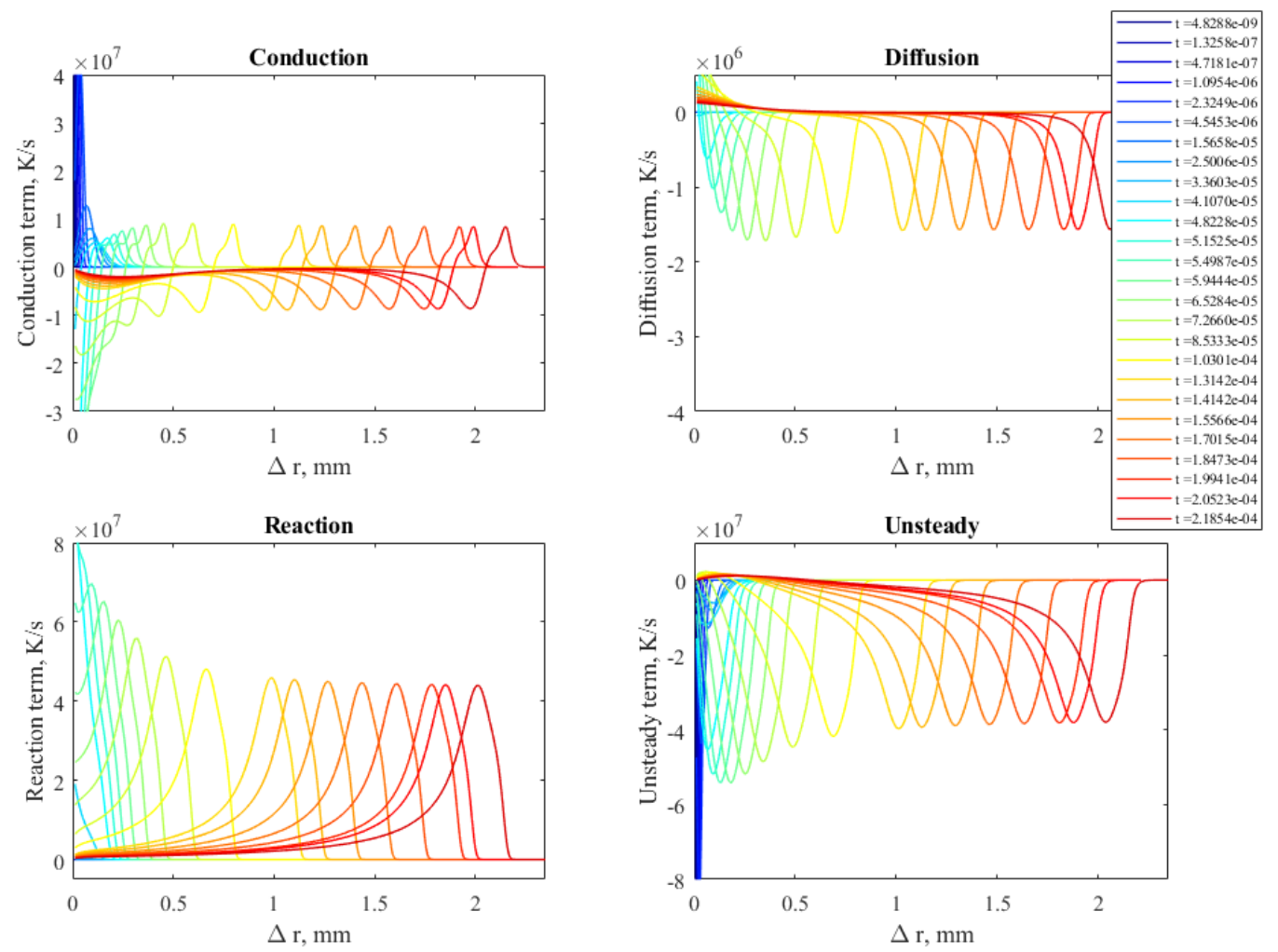

Figure 6.38: All energy equation terms, supercritical case. Top left, conduction term; top right, diffusion term; bottom left, reaction term; bottom right, unsteady term.

Figure 6.39 shows the four energy terms early in the simulation, before any ignition occurs. The conduction term is quite large and positive, indicating that the problem is dominated by heat transfer from the wall into the gas at this point. The conduction is balanced by the unsteady term, indicating the highly transient nature of conduction in this early stage. No significant activity is seen from the reaction or diffusion terms.

Figure 6.40 shows the four energy terms shortly before ignition. The balance is still dominated by the conduction and unsteady terms, but the magnitude of those has decreased by roughly an order of magnitude as the problem becomes less highly transient as the boundary layer grows (see the decreasing slope in the heat flux with increasing time in Fig. 6.54, prior to ignition). Additionally, some activity in the reaction term is observed, although it is still relatively small. The reaction is not yet strong enough to drive the conduction term negative, indicating that while the simulation is approaching the point at which heat is transferred from the gas back to 


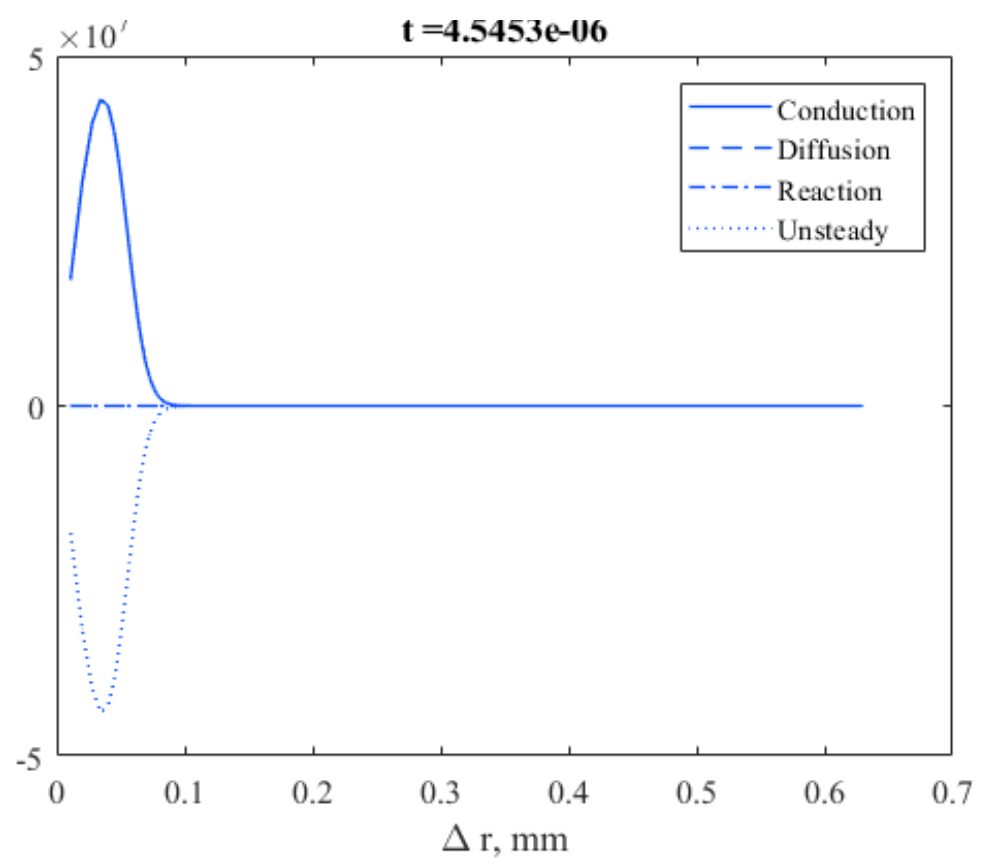

Figure 6.39: Energy balance terms during conduction stage, supercritical case.

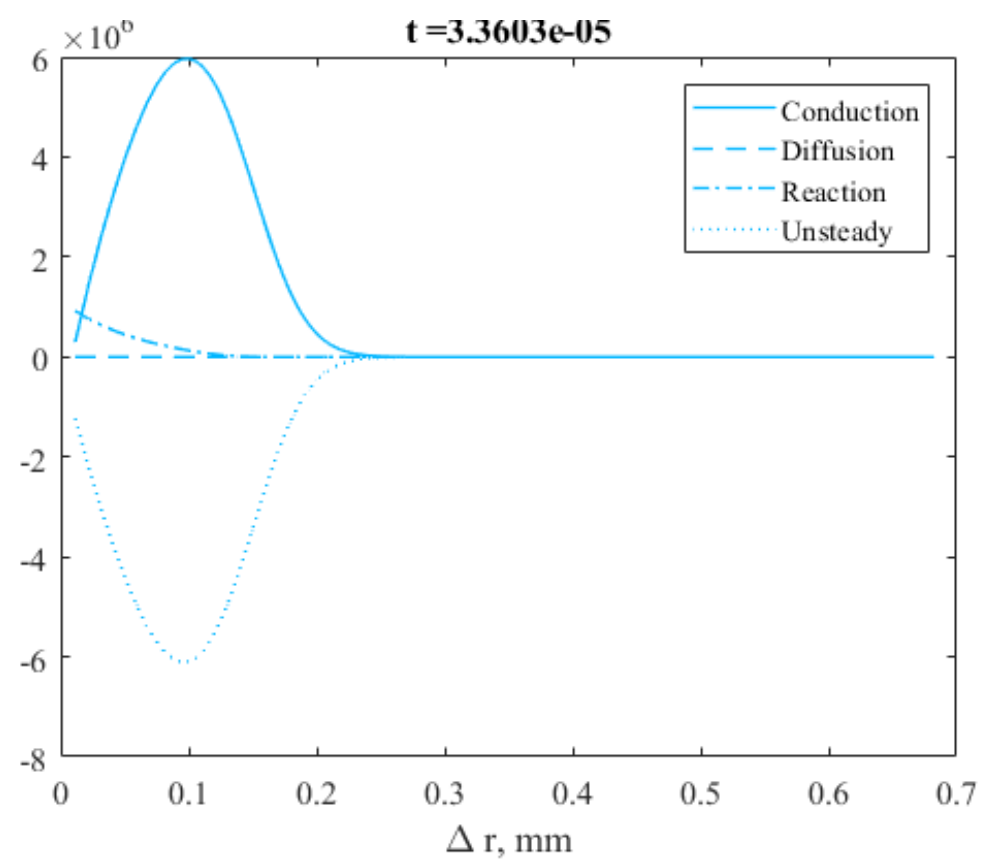

Figure 6.40: Energy balance terms during early reaction, supercritical case.

the wall due to reaction, that threshold is just shy of being crossed at $t=3.36 \times 10^{-5}$ s. There is still no significant activity from diffusion. 


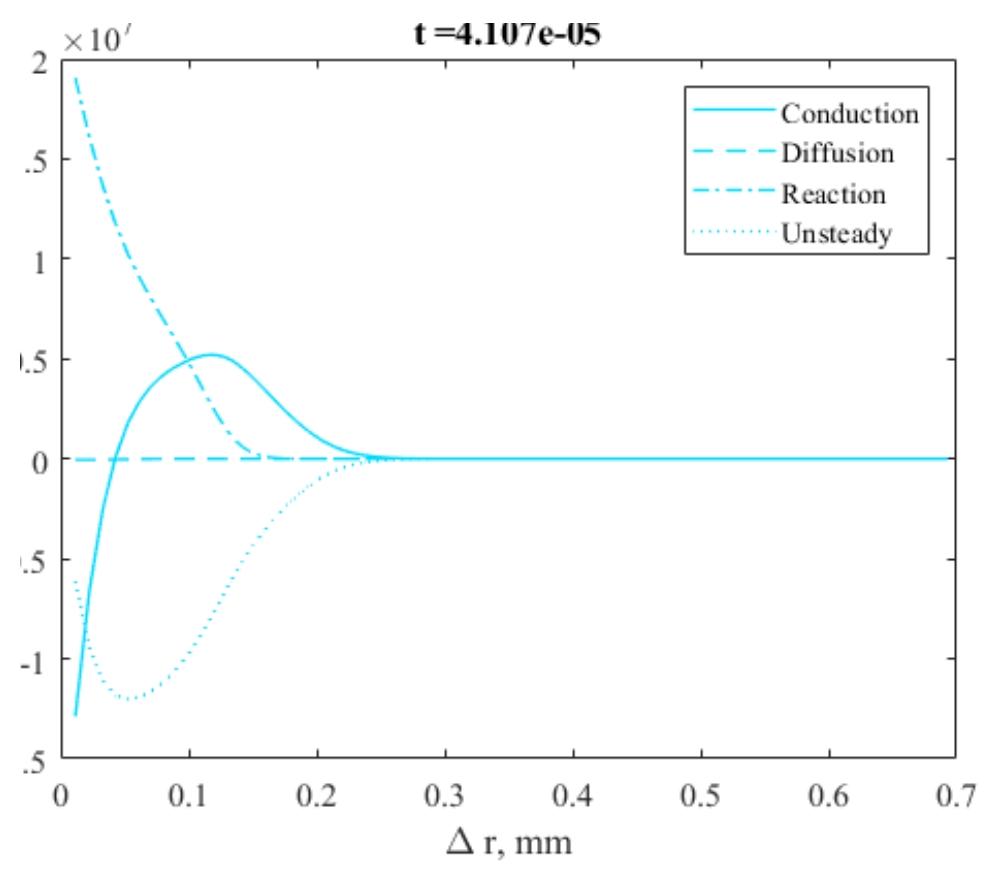

Figure 6.41: Energy balance terms during strengthening reaction, supercritical case.

Figure 6.41 shows the four energy terms just after $\tau_{i g n, j_{q}}$. The balance is now between reaction and conduction terms very near the wall and primarily between reaction and unsteady terms further from the wall. The reaction term is significantly larger and has driven the conduction term negative as heat is now transferred from the gas back into the wall and the very start of ignition has begun. Immediately next to the wall, the reaction term $\left(\approx 10 \times 10^{7}\right)$ is larger than the conduction term $(\approx$ $\left.-8 \times 10^{7}\right)$, indicating the reaction has strengthened enough to overcome the heat lost to the fixed-temperature wall. The remainder of the balance next to the wall is due to the unsteady term $\left(\approx-2 \times 10^{7}\right)$. The reaction will now be the driver of the unsteady nature of the simulation from this point onward as ignition and flame propagation occurs.

Figure 6.42 shows the energy balance as the reaction strengthens and moves away from the wall. The reaction has significantly strengthened and begun to move away from the wall as seen by the location of the reaction peak. The energy balance has shifted to being a balance primarily between the reaction and unsteady terms; however, conduction does still play a role. Conduction and the unsteady terms balance one another ahead of the peak in reaction; the positive conduction term indicated conduction of heat outward radially. The conduction term is negative behind the 


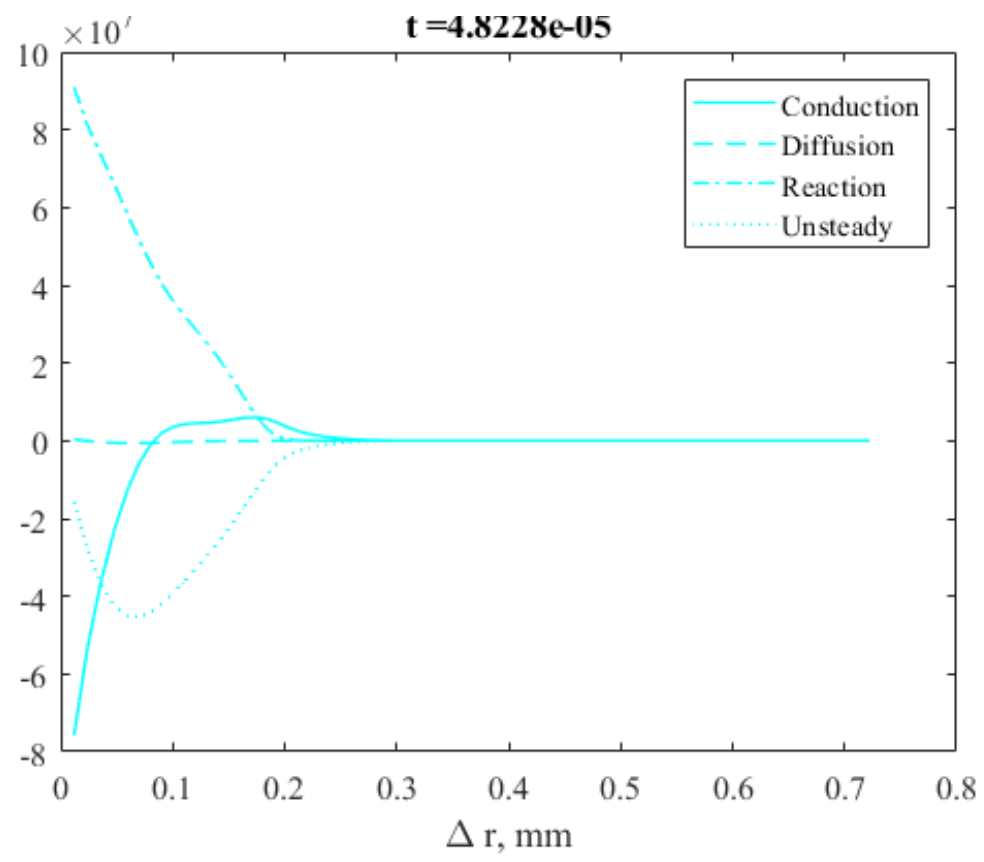

Figure 6.42: Energy balance terms during flame formation stage, supercritical case.

peak in reaction, indicating heat is conducted inward radially as heat is transferred from the strong reaction zone to areas behind it. Near the wall, the balance is largely achieved between conduction and reaction terms. The diffusion term is active now as well; although relatively small compared to the other terms in the energy equation, it is most active in the highly reactive region indicated by the peak in the reaction term.

Figure 6.43 shows the energy terms towards the end of flame propagation. In the highly reactive region indicated by the peak in the reaction term, the balance is mostly between reaction and unsteady terms. This makes sense, given the transient flame propagation taking place. The conduction term is smaller than reaction or unsteady terms but is still important; it is positive ahead of the peak reaction and negative behind it. This indicates conduction of heat away from the reaction zone. Diffusion is once again the smallest term, but still active in the reactive zone. Near the wall, conduction is balanced by unsteady and reaction terms, but all are smaller in the region near the wall than seen in Fig. 6.42 as activity dies down due to the flame moving away from the wall. 


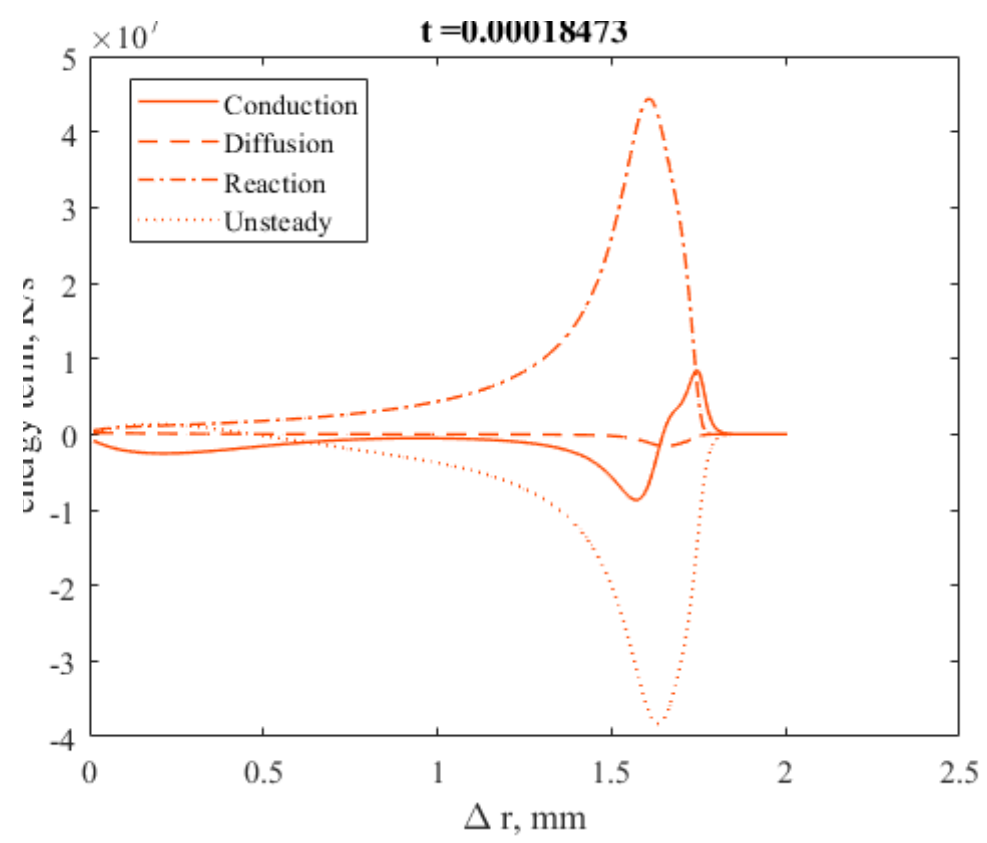

Figure 6.43: Energy balance terms during flame propagation stage, supercritical case.

\section{Critical Case: 917.2 K}

Figure 6.44 shows the evolution of all four terms of the energy equation over time in the critical ignition case $\left(T_{w}=917.2 \mathrm{~K}\right)$. This provides a good overview of how the energy balances evolves over the course of the simulation. At the start of the simulation, energy balance primarily occurs between conduction and unsteady terms. Ignition takes longer to reach in the critical case than the supercritical case, but once ignition starts, the reaction term becomes very active and the balance shifts to primarily one between the reaction and unsteady terms. Diffusion only comes into play once the reaction is established, and remains the smallest of the terms throughout the simulation.

We examine the evolution of the energy equation balance in more detail with Figs. 6.45 through 6.48 . Figure 6.45 shows the energy balance well before ignition. The balance is primarily between conduction and unsteady terms, indicating the transient conduction problem that occurs during the early stages of the simulation. There is no significant activity from either reaction or diffusion at this point.

Figure 6.46 shows the energy balance in the early stages of reaction activity. The 

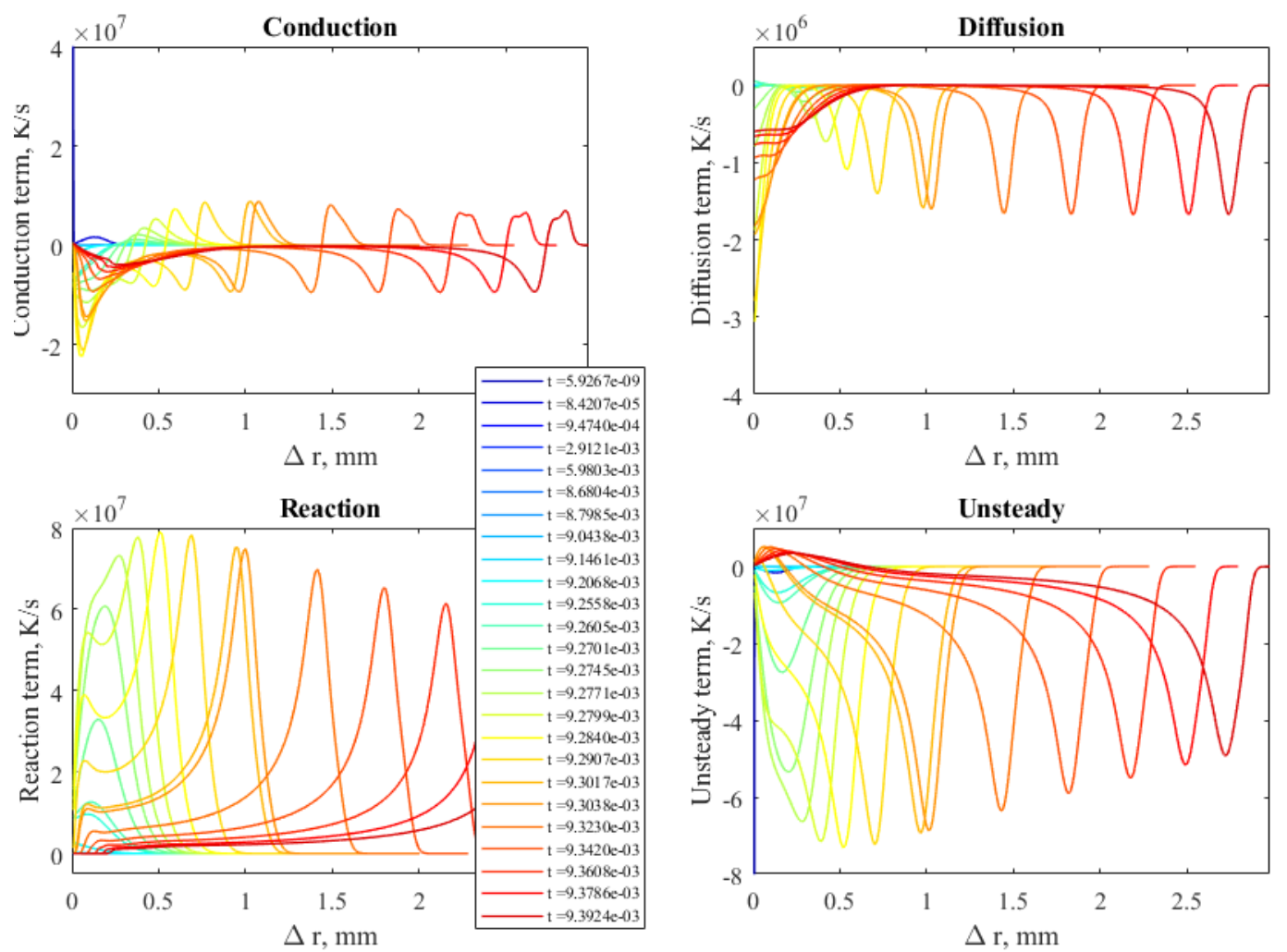

Figure 6.44: All energy equation terms, critical case. Top left, conduction term; top right, diffusion term; bottom left, reaction term; bottom right, unsteady term.

conduction and unsteady terms are about four orders of magnitude smaller than they were in the early conduction stages of the critical case. Interestingly, when the reaction term is the same order of magnitude as the unsteady and conduction terms, all three of these terms are also about four orders of magnitude smaller than in the early stages of reaction for the supercritical case shown in Fig. 6.40. This indicates the simulation is approaching steady-state behavior shortly before ignition occurs (See Fig. 6.54). The reaction is strong enough at this point to drive conduction negative, indicating heat is transferred from the gas back into the wall. This suggests that ignition (as defined by heat flux at the wall) has just begun. At this time, the energy balance is mostly between conduction and diffusion. However, near the wall, the balance is between conduction and reaction and the unsteady term. This indicates the energy balance is starting to shift as ignition occurs.

Figure 6.47 shows the energy balance as a flame is established and the reaction moves away from the wall. Again, we see the energy balance during the estab- 


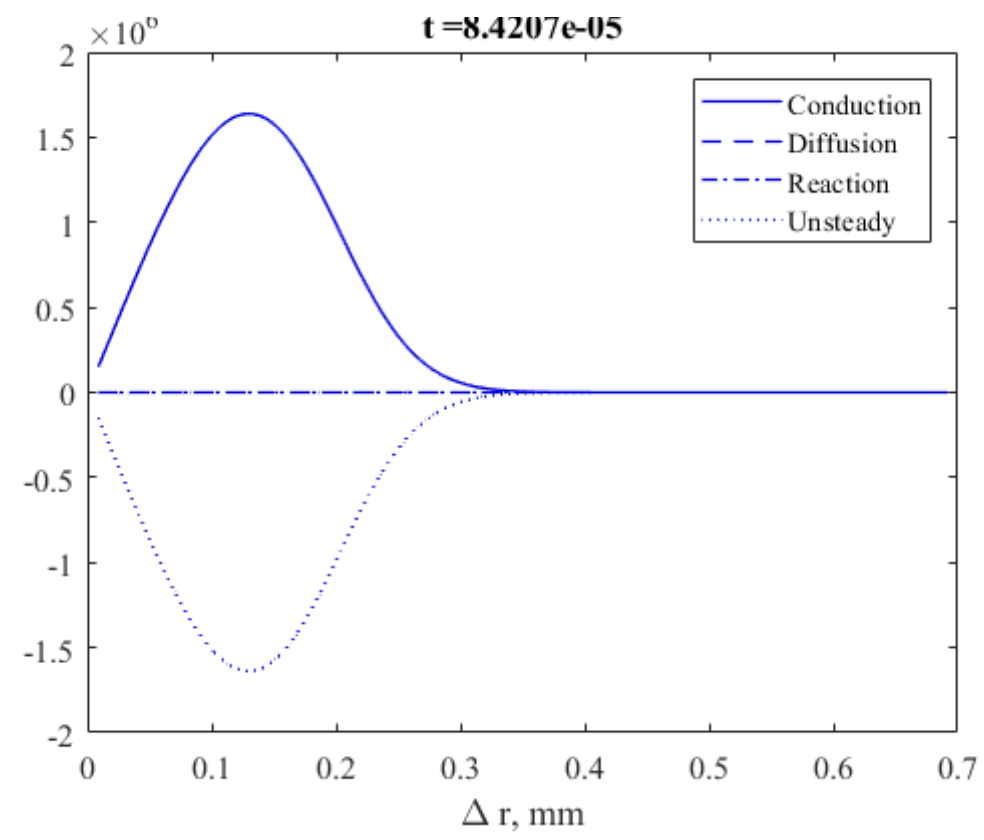

Figure 6.45: Energy balance terms during conduction stage, critical case.

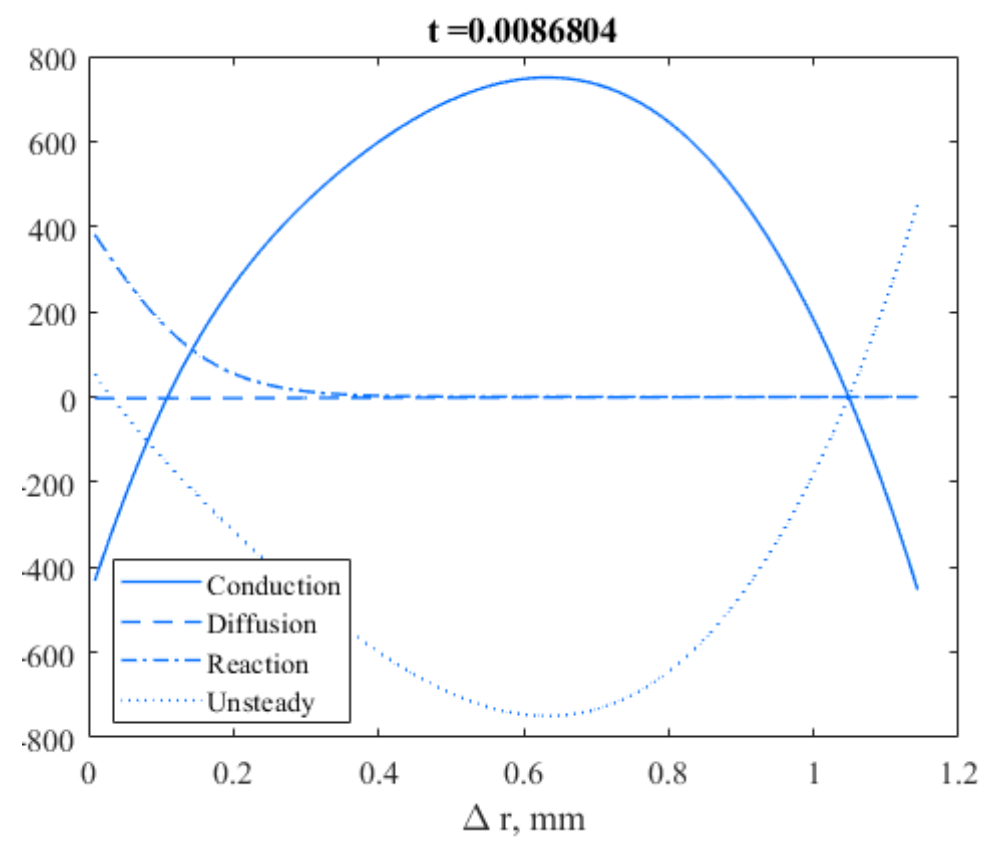

Figure 6.46: Energy balance terms during early reaction, critical case.

lishment of the flame is primarily between the reaction and unsteady terms. The transient nature of the simulation is now driven by reaction (flame propagation). Conduction is much smaller than the reaction or unsteady terms at this point and primarily acts to transfer energy away from the high-temperature reaction zone. 


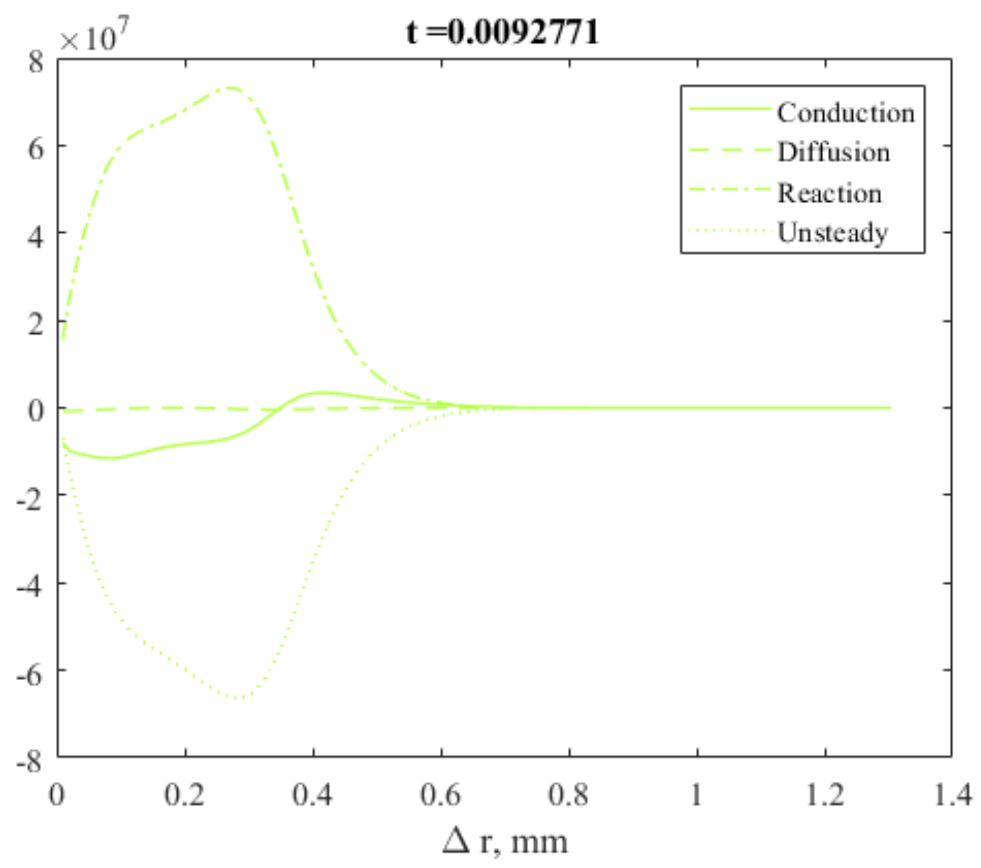

Figure 6.47: Energy balance terms during flame formation stage, critical case.

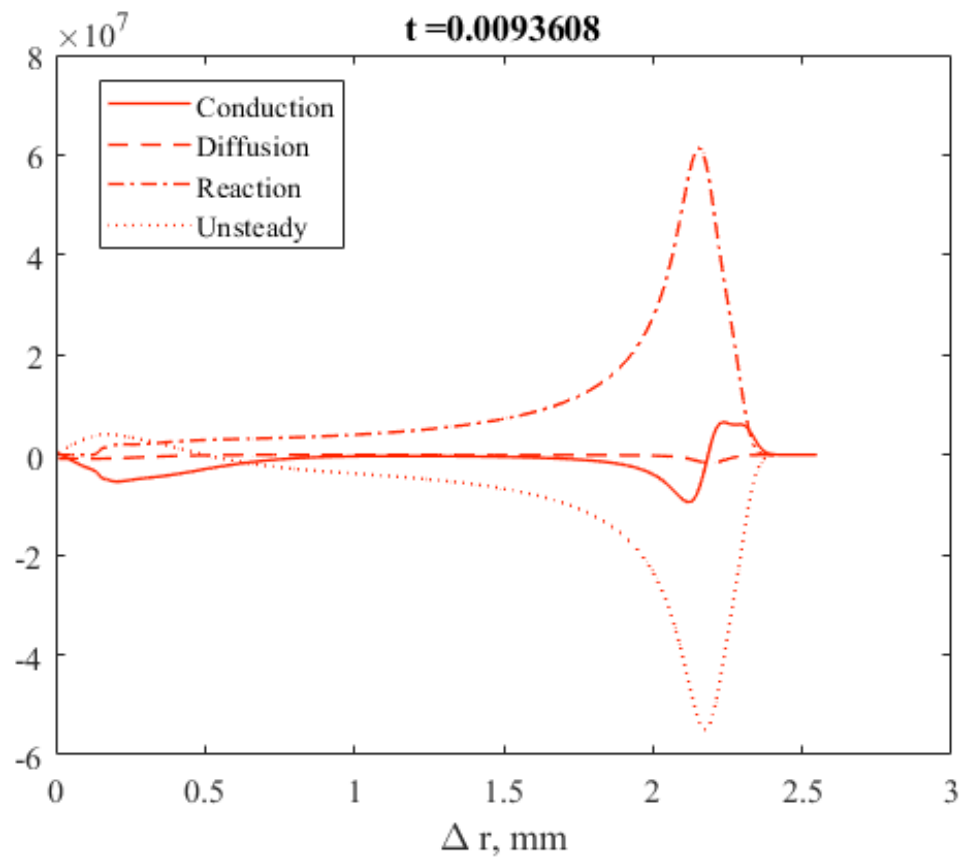

Figure 6.48: Energy balance terms during flame propagation stage, critical case.

Figure 6.48 shows the energy balance at the end of the flame propogation portion 
of the simulation. We see a well-established flame indicated by a large, sharp spike in the reaction term. The energy balance is dominated by reaction and unsteady terms, although conduction and diffusion play a role near the reaction zone. Immediately next to the wall and with the flame far away, the balance is only between conduction and unsteadiness; reaction seems to be quenched near the cooler critical wall temperature compared to a similar point in the supercritical case (Fig. 6.43). Indeed, near the wall, the reaction term is actually slightly negative. At a small distance from the wall ( $\Delta r \approx 0.14 \mathrm{~mm}$ ), reaction becomes more active, presumably far enough from the wall to avoid quenching heat loss.

\section{Subcritical Case: 915.6 K}

The evolution over time of each term in the energy equation is shown in Fig. 6.49 for the subcritical case $\left(T_{w}=915.6 \mathrm{~K}\right)$. The times at which these terms are shown are the same times used for the critical case. The difference in wall temperature between the critical and subcritical case is small enough that prior to ignition, the energy terms between the two cases should look quite similar at the same moment in time. The very earliest times are not displayed in Fig. 6.49 for the sake of clarity in the plots, as the conduction and unsteady terms are quite large and including them would obscure the later (arguably more interesting) evolution of the energy terms.

The $y$-axes of the plots in Fig. 6.49 are much smaller than the corresponding plots for the critical and supercritical cases. This is because no ignition occurs, and the simulation of the subcritical case remains essentially a conduction problem that is approaching a steady-state solution, as indicated by the peak magnitude of the conduction and unsteady terms growing smaller as time advances.

The evolution of the energy balance is examined in greater detail for the subcritical case in Figs. 6.50 through 6.53. Figure 6.50 shows the energy equation terms early in the simulation when the highly transient conduction problem is dominant. This plot is nearly identical to Fig 6.45, the energy balance at the same moment in time for the critical case. Conduction is balanced by the unsteady term; no significant activity in reaction or diffusion is observed.

Figure 6.51 shows the energy balance at $8.6 \times 10^{-3} \mathrm{~s}$, the same time at which the 

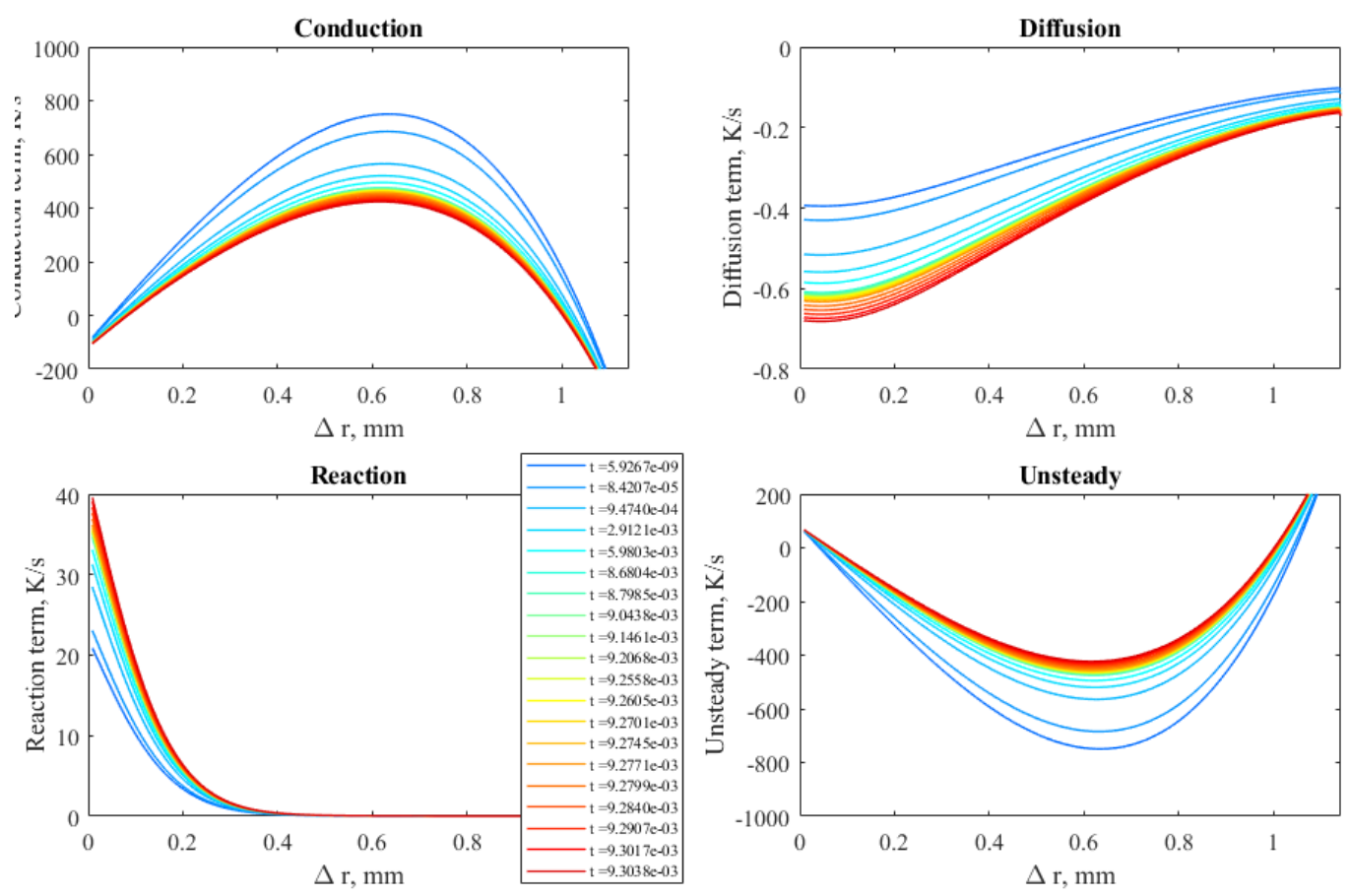

Figure 6.49: All energy equation terms, subcritical case. Top left, conduction term; top right, diffusion term; bottom left, reaction term; bottom right, unsteady term.

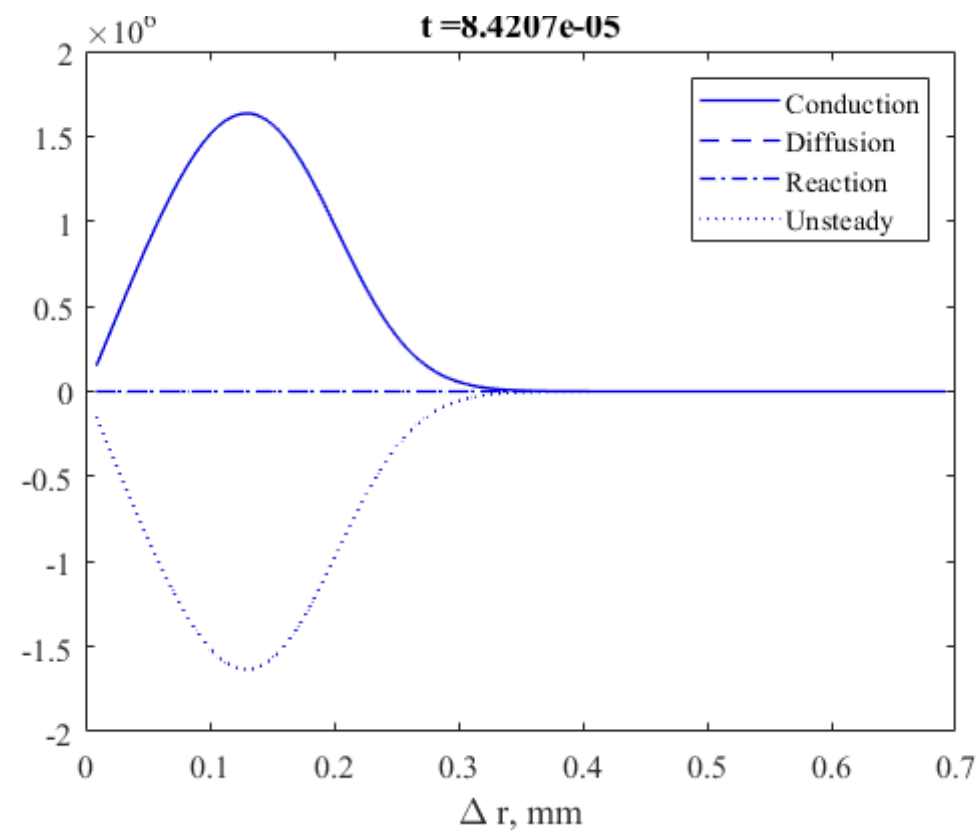

Figure 6.50: Energy balance terms during conduction stage, supercritical case.

early stage of reaction is observed for the critical case. Far from the wall, the plot is almost identical to Fig. 6.46, however, there are significant differences near the 


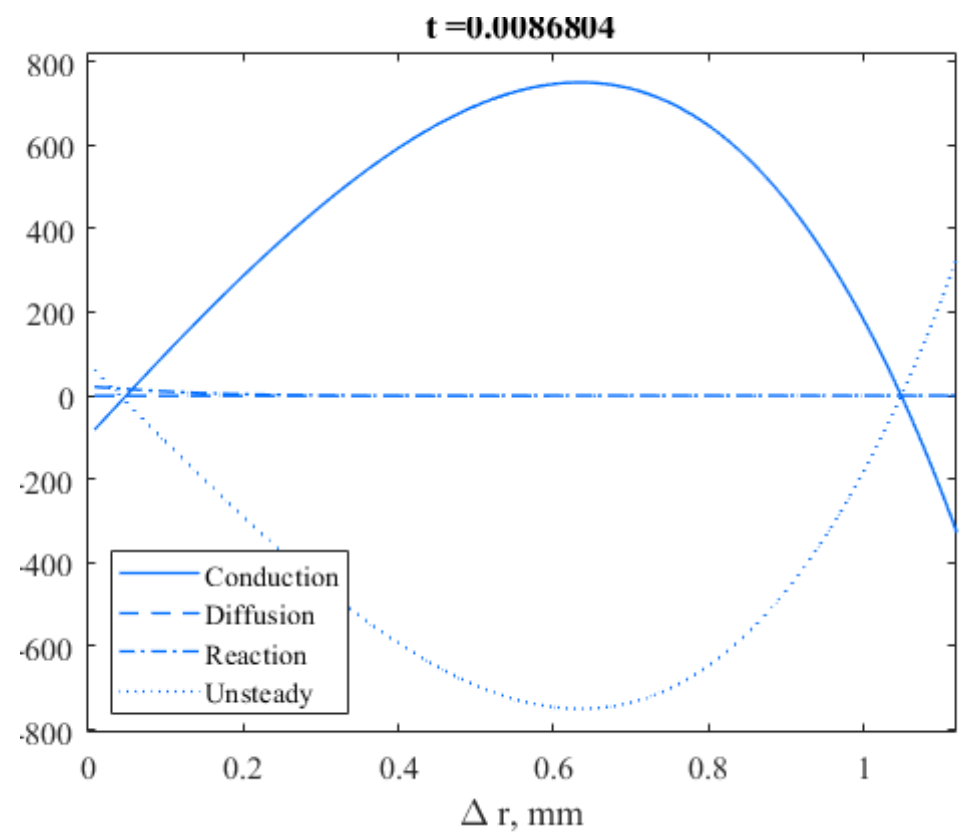

Figure 6.51: Energy balance terms during early reaction, subcritical case.

wall. Namely, the reaction term is less active at this time for the subcritical case than the ignition case. Reaction is not strong enough to cause an ignition event.

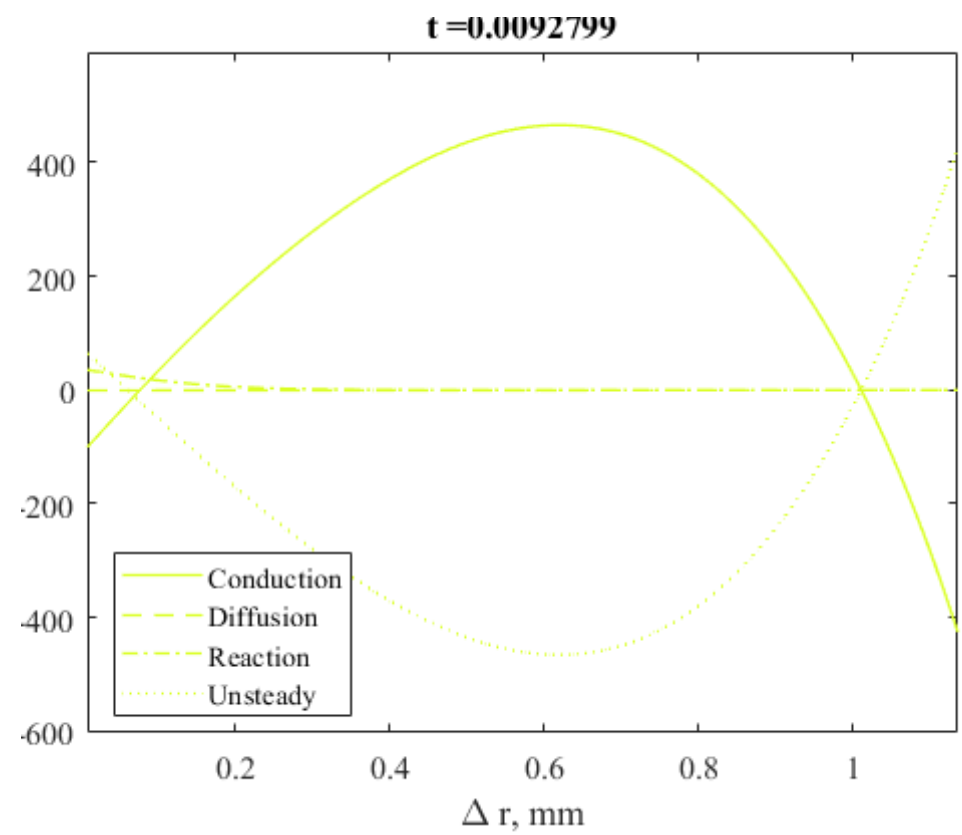

Figure 6.52: Energy balance terms during flame formation stage, subcritical case.

Figure 6.52 plots the energy terms at $t=9.3 \times 10^{-3} \mathrm{~s}$, the same time at which 
the flame begins to form and move away from the wall in the critical case. For the subcritical case, no significant change in the energy balance has occured; this remains primarily a balance between conduction and unsteadiness. The peak in the conduction and unsteady terms has slightly decreased compared to 6.51 as the conduction problem approaches a steady solution. There is slight activity in the reaction term immediately next to the wall, however, the conduction term is larger in magnitude than the reaction term, indicating that conduction transfers the slight amount of heat produced by the weak reaction away into the wall faster than energy can be produced by reaction, preventing a thermal explosion.

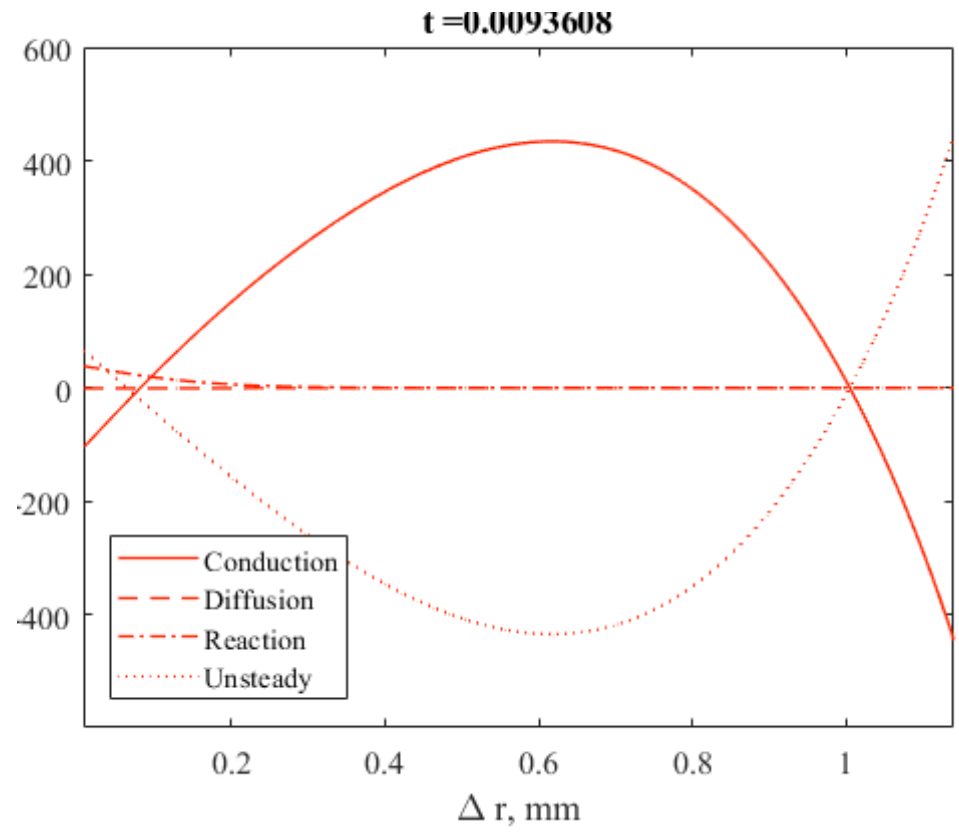

Figure 6.53: Energy balance terms during flame propagation stage, subcritical case.

Figure 6.53 shows the energy balance for the subcritical case at the same time at which the critical case reaches the end of flame propagation. The plot is very similar to 6.52 ; the balance remains between conduction and unsteady terms. There is some activity in the reaction term, but it is not enough to overcome conduction of heat back into the wall, and no reaction occurs.

\section{Heat Flux at Wall}

The heat flux at the wall is one phenomenon of particular interest. We define the heat flux in the manner laid out by Coronel, Melguizo-Gavilanes, Mével, et al. (2018): 


$$
\hat{j}_{q}=-\frac{\kappa}{c_{p}}\left(\nabla h_{s}-\sum_{i=1}^{N-1} h_{s, i} \nabla Y_{i}\right)+\sum_{i=1}^{N-1} \hat{j}_{i} h_{s, i},
$$

where $h_{s}$ is the sensible enthalpy. Sensible enthalpy is the absolute enthalpy minus the heat of formation, $h_{s}=h-\Delta h_{f}=\int_{T^{0}}^{T} c_{p} d T . \hat{j}_{i}$ is the species flux, defined as $\hat{j}_{i}=-\rho D_{i, m} \nabla Y_{i}$.

Applying Eq. 6.60 to the dimensions of the one-dimensional simulation (radial, 1D), we get:

$$
\begin{gathered}
\hat{j}_{q}=-\frac{\kappa}{c_{p}}\left(\frac{d h_{s}}{d r}-\sum_{i=1}^{N-1} h_{s, i} \frac{d Y_{i}}{d r}\right)+\sum_{i=1}^{N-1}-\rho D_{i, m} \frac{d Y_{i}}{d r} h_{s, i}, \\
\hat{j}_{q}=-\frac{\kappa}{c_{p}}\left(c_{p} \frac{d T}{d r}-\sum_{i=1}^{N-1}\left(h_{i}-\Delta h_{f, i}\right) \frac{d Y_{i}}{d r}\right)+\sum_{i=1}^{N-1}-\rho D_{i, m} \frac{d Y_{i}}{d r}\left(h_{i}-\Delta h_{f, i}\right) .
\end{gathered}
$$

Figure 6.54 plots the temporal evolution of the heat flux at the wall over time for all surface temperatures. The color-coded lines display the results for each unique wall temperature. The highest wall temperature, $1200 \mathrm{~K}$, is displayed in dark blue and the lowest wall temperature, $900 \mathrm{~K}$, is shown in red. At first, heat is transferred from the wall to the gas, which is reflected in the plot as a positive heat flux. Also, Fig. 6.54 shows that a higher wall temperature results in a higher initial heat flux.

As the gas continues to heat up, the exothermic reaction starts and produces heat within the gas. When the reaction is sufficiently active, enough heat is produced such that the gas is now hotter than the wall, and begins transferring heat back into the wall. At this point, the heat flux $\hat{j}_{q}$ is negative, and the time at which $\hat{j}_{q}=0$ marks the start of ignition. For a supercritical case, $T_{w}=1200 \mathrm{~K}$, this transition to ignition occurs very early while the heat flux is changing rapidly due to the transient conduction problem. For the critical case, $T_{w}=917.2 \mathrm{~K}$, ignition occurs much later as the heat flux is settling to a constant, positive value. For subcritical cases like $T_{w}=915.6$ or $900 \mathrm{~K}$, the heat flux remains positive; the exothermic reaction is not sufficiently activated to cause ignition.

The ignition delay based on heat flux is defined as the time at which the heat flux equals zero: 


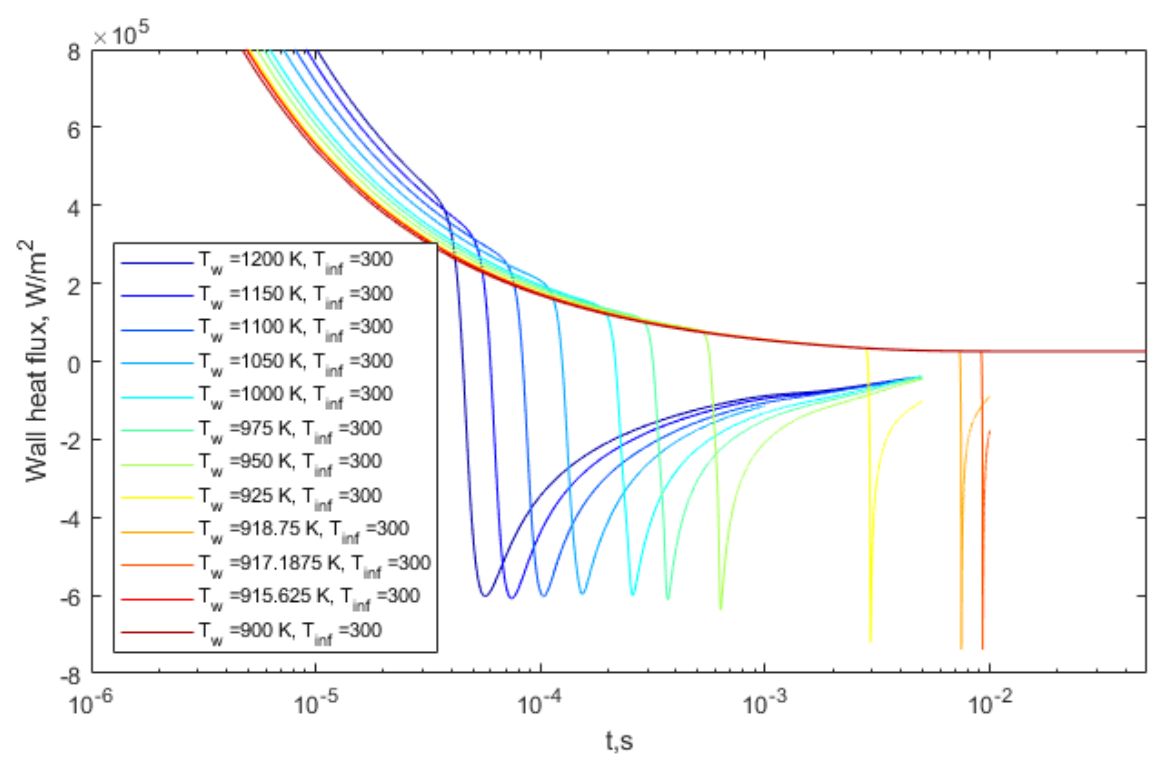

Figure 6.54: Heat flux next to wall for all $T_{w}$.

$$
\tau_{i g n, j_{q}}=t\left(\hat{j}_{q}=0\right)
$$

The ignition delay times based on heat flux are reported in Table 6.1, along with $\tau_{i g n, T}$ for comparison. We see that the ignition delay time based on zero heat flux at the wall tends to be faster than the ignition delay time based on maximum time rate of change of temperature. This is likely because zero heat flux at the wall is the very earliest indication of ignition while it takes a bit of time for the reaction to strengthen such that the maximum time rate of change in temperature is observed. The two methods of calculating ignition delay serve different but equally useful purposes. The ignition delay based on the maximum time rate of change of temperature is useful for comparison with ignition delay times from zero-dimensional calculations, as there is no wall at which to calculate heat flux. However, the ignition delay time based on heat flux is particularly useful to indicate the very first moments of ignition for a dimensional simulation.

We now examine the differences between the heat flux of the critical and subcritical cases in more detail. Figure 6.55 plots the heat flux of the critical $\left(T_{w}=917.2 \mathrm{~K}\right)$ and subcritical cases $\left(T_{w}=915.6 \mathrm{~K}\right)$. The heat flux between the two cases is essentially identical up to ignition, at which point the heat flux of the critical case suddenly shifts negative as the reaction generates enough heat to reverse the direction of heat 


\begin{tabular}{||c|cc||}
\hline $\mathrm{T}_{w}(\mathrm{~K})$ & $\tau_{\text {ign,T }}(\mathrm{s})$ & $\tau_{\text {ign, } j_{q}}(\mathrm{~s})$ \\
\hline 1200 & $5.71 \mathrm{e}-5$ & $4.51 \mathrm{e}-5$ \\
1150 & $7.84 \mathrm{e}-5$ & $6.04 \mathrm{e}-5$ \\
1100 & $1.08 \mathrm{e}-4$ & $8.35 \mathrm{e}-5$ \\
1050 & $1.58 \mathrm{e}-4$ & $1.26 \mathrm{e}-4$ \\
1000 & $2.59 \mathrm{e}-4$ & $2.17 \mathrm{e}-4$ \\
975 & $3.69 \mathrm{e}-4$ & $3.17 \mathrm{e}-4$ \\
950 & $6.32 \mathrm{e}-4$ & $5.70 \mathrm{e}-4$ \\
925 & $2.94 \mathrm{e}-3$ & $2.86 \mathrm{e}-3$ \\
918.75 & $7.47 \mathrm{e}-3$ & $7.38 \mathrm{e}-3$ \\
917.1875 & $9.29 \mathrm{e}-3$ & $9.20 \mathrm{e}-3$ \\
\hline
\end{tabular}

Table 6.1: Ignition delay time comparison using $\tau_{i g n, T}$ and $\tau_{i g n, j_{q}}$.

flux back into the wall. The subcritical case remains at a small positive value as it approaches a quasi-steady state solution without significant reaction.

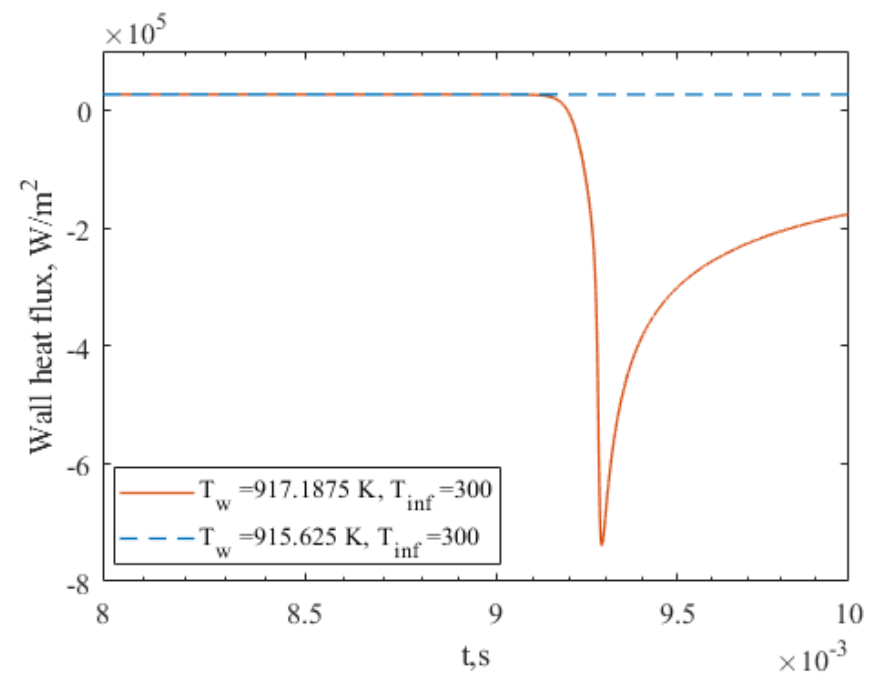

Figure 6.55: Heat flux next to wall for critical, subcritical cases.

The temperature profiles are plotted for the critical case up to the point at which the heat flux at the wall goes negative in Fig. 6.56. We can see that for the supercritical case, the temperature gradient at the wall gets continually shallower as time advances and as energy release from reaction strengthens. Eventually, the temperature gradient at the wall is zero; at $\mathrm{t}=9.20 \times 10^{-3} \mathrm{~s}$, the temperature profile has just advanced beyond this point. From this plot, it is apparent that the criteria of zero heat flux at the wall is equivalent to the Van't Hoff ignition criterion. The subcritical case (Fig. 6.57) is never satisfied; a positive temperature gradient and positive heat 


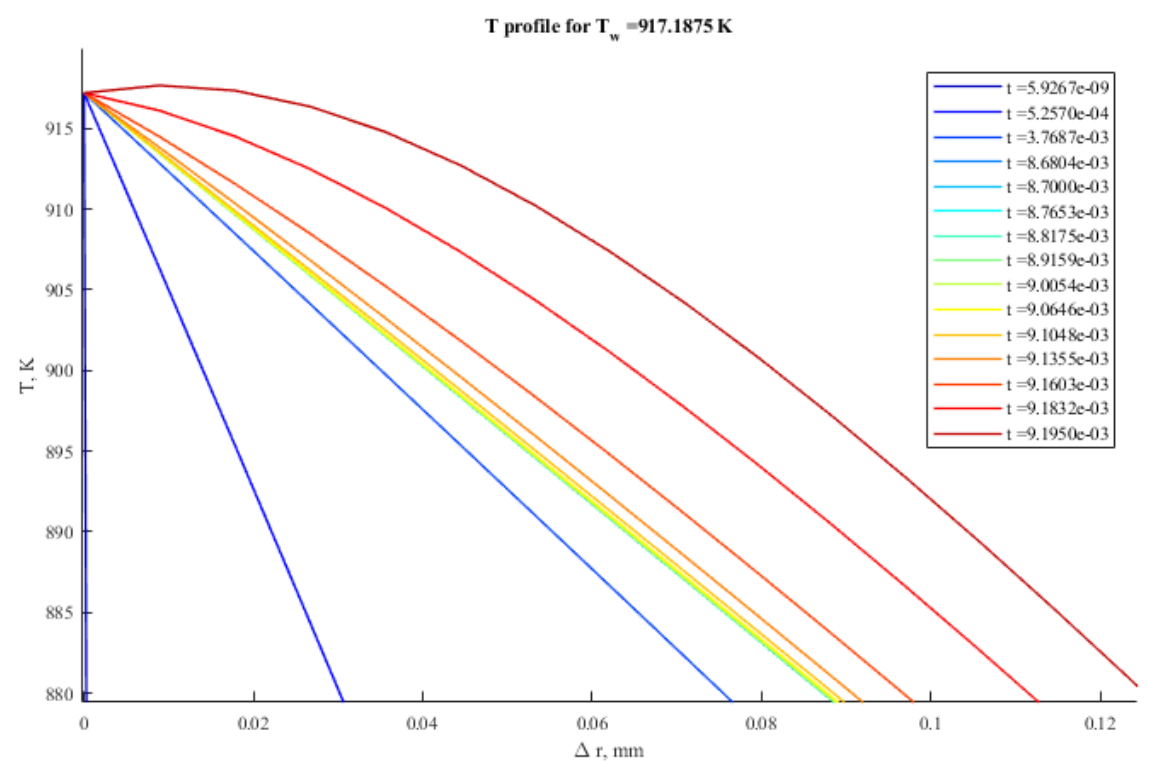

Figure 6.56: Temperature profiles of critical case, up to $\mathrm{t}=\tau_{i g n, j_{q}}$.

flux at the wall remain throughout the run time of the subcritical case.

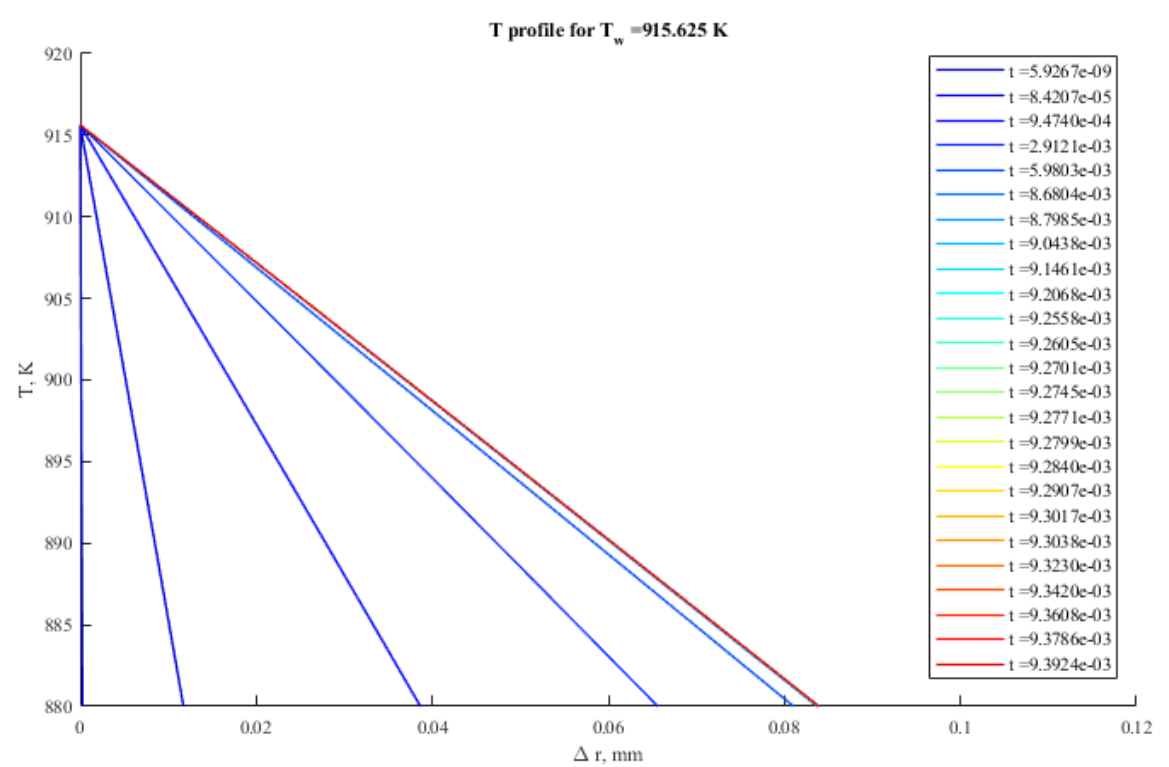

Figure 6.57: Temperature profiles of subcritical case.

A limitation of using zero heat flux at the wall to identify ignition is that it by definition can only define ignition time. Regardless, we can estimate the ignition 
location of the critical case using this ignition criterion by finding where the peak in $\mathrm{HO}_{2}$ is located when $\mathrm{t}=\tau_{i g n, j_{q}}$. The mass fraction of $\mathrm{HO}_{2}$ has previously been used to identify the ignition kernel. Figure 6.58 shows the evolution of the $\mathrm{HO}_{2}$ mass fraction in the lead up to $\mathrm{t}=\tau_{\text {ign, } j_{q}}$. The peak in mass fraction remains next to the wall until $0.2 \times 10^{-3} \mathrm{~s}$ before $\mathrm{t}=\tau_{i g n, j_{q}}$, but slowly evolves into a peak that moves away from the wall in the last $0.1 \times 10^{-3} \mathrm{~s}$ before ignition. At $\mathrm{t}=\tau_{i g n, j_{q}}=$ $9.20 \times 10^{-3} \mathrm{~s}$, the peak is located at $\Delta r=0.2 \mathrm{~mm}$ from the wall or approximately one flame thickness from the wall. By the same method, the supercritical case ignites at slightly less than one flame thickness $\Delta r=0.15 \mathrm{~mm}, 0.75 l_{f}$ (see Appendix E).

The subcritical case never develops enough reaction activity to allow the peak $\mathrm{HO}_{2}$ mass fraction to move away from the wall. At the time when the critical case is reaching $\mathrm{t}=\tau_{i g n, j_{q}}$, the $\mathrm{HO}_{2}$ mass fraction is approximately an order of magnitude smaller in the subcritical case than the critical case.

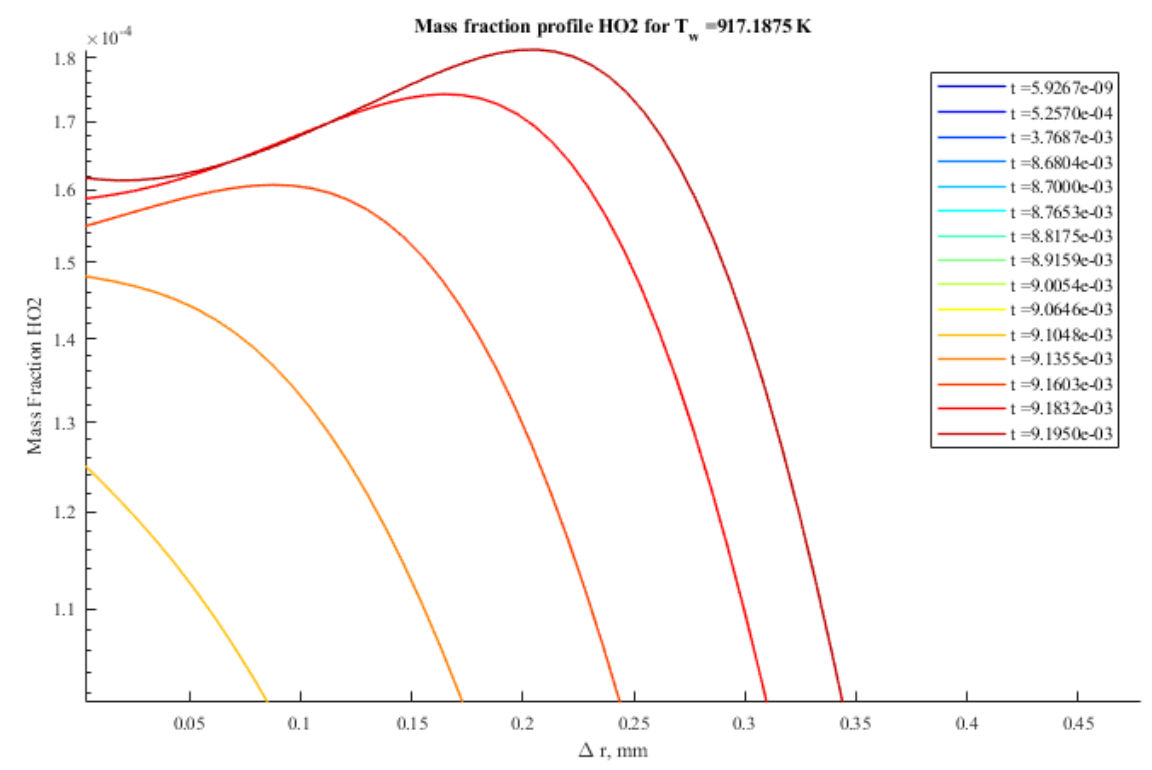

Figure 6.58: $\mathrm{YHO}_{2}$ profiles of critical case, up to $\mathrm{t}=\tau_{i g n, j_{q}}$.

\section{Relevance of One-Dimensional Problem}

We want to consider the relevance of this model one-dimensional ignition problem to the experiments discussed previously. Table 6.2 compares some relevant timescales between the one-dimensional model and the ignition experiments with vertical cylinders. 


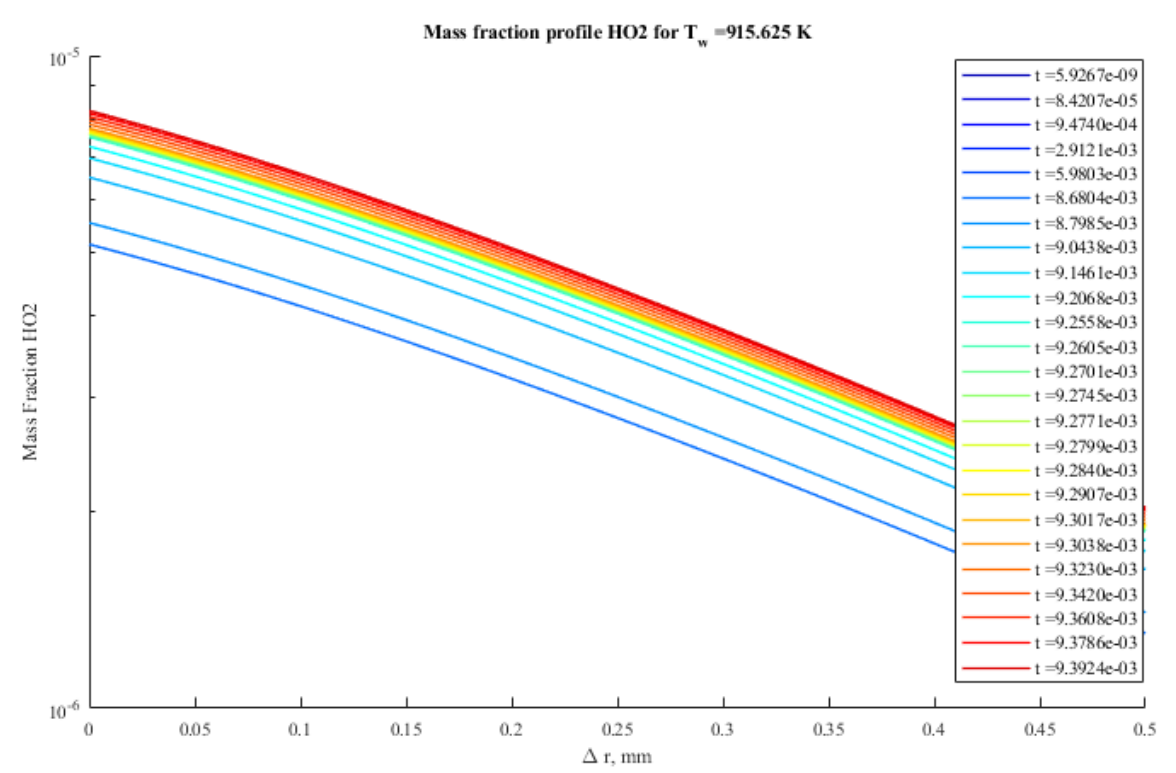

Figure 6.59: $Y_{\mathrm{HO}_{2}}$ profiles of subcritical case, up to $\mathrm{t}=\tau_{i g n, j_{q}}$.

$\tau_{\text {ign }}$ represents the time it takes to achieve ignition, starting from the initiation of the experiment or the simulation. Ignition in the experiments is determined by the trigger signal of the Yokogawa, which occurs when the pressure on the dynamic pressure gauge is approximately $2 \mathrm{~atm}$. Ignition in the simulations is determined by the time at which the heat flux next to the wall is zero, $\tau_{i g n, j_{q}}$, for the critical case. $\tau_{\text {heat }}$ is the characteristic heating time; in the experiments, this is the time it takes for the cylinder surface temperature to reach $99 \%$ of the set point temperature. In the simulations, the cylinder surface is fully heated instantaneously.

$\tau_{\text {diff }}$ represents the characteristic time it takes for heat to diffuse to the edge of the boundary layer. This can also be thought of as a characteristic time for development of the thermal layer. $\delta_{B L}$ is the thermal layer thickness, and $\alpha_{f}$ is the thermal diffusivity at the film temperature. For the experiments, we use the thermal boundary layer thickness of the similarity solution (see Chapter 3) at a wall temperature of $1100 \mathrm{~K}$ to estimate the experimental thermal layer thickness, $\delta_{B L_{\text {exp }}} \approx 0.01 \mathrm{~m}$. The thermal diffusivity of air at the experimental film temperature $(700 \mathrm{~K})$ is used to approximate $\alpha_{f}=9.7 \times 10^{-5} \mathrm{~m}^{2} / \mathrm{s}$ in the experiments. For the simulations, the thermal layer thickness is calculated by finding the distance from the heated surface at which the temperature is within $1 \%$ of the ambient (outer boundary) temperature at $t=\tau_{i g n, j_{q}}: \delta_{B L_{1 D}}=1.1 \times 10^{-3} \mathrm{~m} . \alpha_{f_{1 D}}$ is calculated at the same instant in time 


\begin{tabular}{|c|c|c|c|c|}
\hline \multirow[t]{2}{*}{ Timescale } & \multicolumn{2}{|l|}{ Experiments } & \multicolumn{2}{|c|}{$\begin{array}{l}\text { 1D simulations (917.2 K, } \\
\text { critical case) }\end{array}$} \\
\hline & formula & value (s) & formula & value (s) \\
\hline $\begin{array}{l}\text { heating, } \\
\tau_{\text {heat }}\end{array}$ & $t\left(0.99 T_{\text {set }}\right)$ & $20-60$ & N/A & 0 \\
\hline $\begin{array}{l}\text { ignition, } \\
\tau_{\text {ign }}\end{array}$ & $t_{\text {trig }}$ & $\approx 10^{1}-10^{2}$ & $\tau_{i g n, j_{q}}$ & $9.2 \times 10^{-3}$ \\
\hline $\begin{array}{l}\text { thermal } \\
\text { diffusion, } \\
\tau_{\text {diff }}\end{array}$ & $\frac{\delta_{B L_{\exp }}^{2}}{\alpha_{f_{\exp }}}$ & 1.0 & $\frac{\delta_{B L_{1 D}}^{2}}{\alpha_{f_{1 D}}}$ & $8.6 \times 10^{-3}$ \\
\hline $\begin{array}{l}\text { ignition } \\
\text { kernel } \\
\text { formation, } \\
\tau_{\text {kernel }} \\
\end{array}$ & - & - & $\tau_{i g n, T}-\tau_{i g n, j_{q}}$ & $9 \times 10^{-5}$ \\
\hline $\begin{array}{l}\text { flame, } \\
\tau_{\text {flame }}\end{array}$ & $\frac{\delta_{B L_{\text {exp }}}}{S_{L_{\text {exp }}}}$ & $3.3 \times 10^{-3}$ & $\frac{\delta_{B L_{1 D}}}{S_{L_{1 D}}}$ & $7.4 \times 10^{-5}$ \\
\hline $\begin{array}{l}\text { convection } \\
\text { during } \\
\text { ignition, } \\
\tau_{\text {conv }_{\text {kernel }}}\end{array}$ & $\frac{\Delta r_{i g n}}{u_{i g n}}$ & $1.4 \times 10^{-3}$ & - & - \\
\hline $\begin{array}{l}\text { convection } \\
\text { during } \\
\text { flame } \\
\text { prop., } \\
\tau_{\text {conv }_{\text {flame }}} \\
\end{array}$ & $\frac{\delta_{B L_{\exp }}}{u_{\max }}$ & $6.6 \times 10^{-3}$ & - & - \\
\hline $\begin{array}{l}\text { boundary } \\
\text { layer } \\
\text { thickness, } \\
\delta_{B L}\end{array}$ & $\begin{array}{l}\text { within } 1 \% \\
\text { ambient } \\
\text { temp }\end{array}$ & $0.01 \mathrm{~m}$ & $\begin{array}{ll}\text { within } & 1 \% \\
\text { ambient } & \\
\text { temp } & \end{array}$ & $1.1 \times 10^{-3} \mathrm{~m}$ \\
\hline $\begin{array}{l}\text { convection } \\
\text { velocity } \\
\text { at ign. } \\
\text { location, } \\
u_{\text {ign }} \\
\end{array}$ & $\begin{array}{l}u \text { at ign. } \\
\text { kernel loca- } \\
\text { tion }\end{array}$ & $0.14 \mathrm{~m} / \mathrm{s}$ & - & - \\
\hline $\begin{array}{l}\text { maximum } \\
\text { convection } \\
\text { velocity, } \\
u_{\max }\end{array}$ & $\max u$ & $1.5 \mathrm{~m} / \mathrm{s}$ & - & - \\
\hline
\end{tabular}

Table 6.2: Timescale comparison between experiments and 1D simulation. 
by using Cantera to evaluate the gas properties at the location in the domain where $T=T_{f} \approx 610 \mathrm{~K}, \alpha_{f_{1 D}}=1.5 \times 10^{-4} \mathrm{~m}^{2} / \mathrm{s}$.

The thermal boundary layer profiles are shown in Fig. 6.60. The blue line is the thermal boundary layer profile estimate for the experiments, taken from the similarity solution in Chapter 3. The red line is the thermal boundary layer profile from the one-dimensional simulations at $t=\tau_{i g n, j_{q}}$. Figure 6.60 provides a visual representation of the difference in boundary layer thickness between the experiments and the one-dimensional simulations: the experimental boundary layer is about ten times thicker than the boundary layer of the one-dimensional simulations.

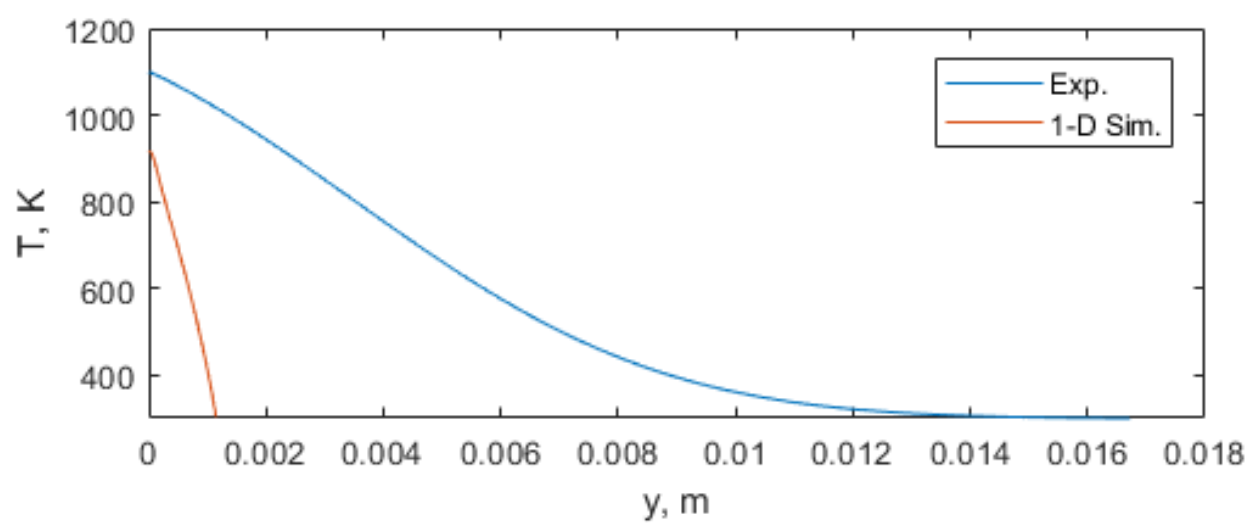

Figure 6.60: Boundary layer profile comparison between experiments and simulation.

$\tau_{\text {kernel }}$ is the time it takes for the ignition kernel to establish into a flame. This timescale can only be estimated from the one-dimensional simulations, as the spatial and temporal resolution of the cameras used in the experiments is not sufficient to observe $\tau_{\text {kernel }}$ for the experiments. For the one-dimensional simulations, we estimate $\tau_{\text {kernel }}=\tau_{i g n, T}-\tau_{i g n, j_{q}}$. That is, we estimate that the timescale for the ignition kernel to establish into a flame is the difference between the time at which the maximum temperature gradient is observed, $\tau_{i g n, T}=9.29 \times 10^{-3} \mathrm{~s}$, and the time at which the first indication of ignition has occurred, $\tau_{i g n, j_{q}}=9.20 \times 10^{-3} \mathrm{~s}$.

$\tau_{\text {flame }}$ represents the time it takes for the flame to emerge from the thermal layer. $S_{L}$ is the flame speed. $S_{L_{e x p}} \mathrm{~m} / \mathrm{s}$ is the flame speed measured via visualization in the experiments ( $\approx 3 \mathrm{~m} / \mathrm{s}$ ), and $S_{L_{1 D}}$ is the flame speed found in the simulation by 
tracking the movement of the peak in $\mathrm{HO}_{2}$ mass fraction $(\approx 15 \mathrm{~m} / \mathrm{s})$.

$\tau_{\text {conv }}$ represents the characteristic timescale of convection parallel to the plate. We have two different convection timescales: $\tau_{\text {conv }}{ }_{\text {kernel }}$, which is a characteristic convection timescale where the ignition kernel is first observed, and $\tau_{\text {convflame }}$, the convection timescale during flame propagation through the boundary layer. As noted in the previous section, the ignition kernel forms at a distance of $\Delta r_{i g n} \approx 0.2$ $\mathrm{mm}$ from the wall in the one-dimensional simulations. Since this distance can not be directly observed in the experiments, we assume that the ignition kernel is located a similar distance from the wall for the vertical cylinder tests. $u_{i g n}=0.14 \mathrm{~m} / \mathrm{s}$ is the estimated wall-parallel convection velocity at the ignition location, taken from the similarity solution presented in Chapter 3 . These estimates of $\Delta r_{i g n}$ and $u_{i g n}$ are used to estimate the convection timescale at the location of the ignition kernel in the experiments.

To estimate the convection timescale during flame propagation through the boundary layer, we use $\tau_{\text {conv flame }}=\frac{\delta_{B L_{\text {exp }}}}{u_{\max }} \cdot u_{\max }$ is an estimate of the maximum wall parallel velocity in the experiments, taken from the velocity calculated via similarity solutions in Chapter 3 . Figure 6.61 shows the wall-parallel velocity profile from the similarity solution results presented in Chapter 3 . The black diamond indicates the velocity at the ignition kernel location, and the red diamond indicates the maximum wall-parallel velocity in the boundary layer.

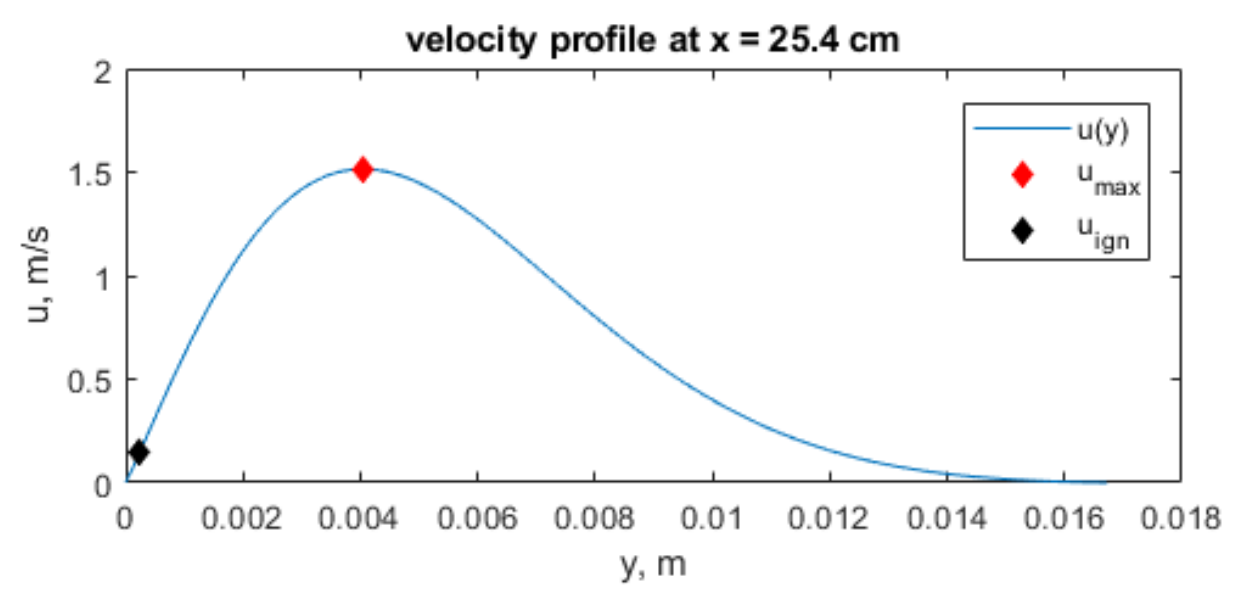

Figure 6.61: Wall-parallel velocity profile from similarity solution. 
Table 6.2 makes some important comparisons of timescales between the experiments and the one dimensional simulation that indicate the relevance (or lack thereof) of this one-dimensional model problem to the ignition seen in experiments. First, we note that for the experiments, $\tau_{\text {diff }} \ll \tau_{\text {heat }}$. This implies the boundary layer in the experiments is in quasi-steady state, as heat is able to diffuse through the boundary layer much faster than the cylinder itself is brought to a steady-state temperature. This thermal layer is able to adjust to the increasing surface temperature relatively quickly such that at any particular moment, the thermal layer is approximately in steady-state. The same is not true for the simulations; the time scale for thermal diffusion is longer than the instantaneous heating of the wall. This implies the model problem explored in the simulations has highly transient heating in contrast to the quasi-steady state heating observed in the experiments. This is one of the major differences between the simulations and experiments. We see a similar results when comparing $\tau_{\text {diff }}$ to $\tau_{\text {ign }}$; in the experiments $\tau_{\text {diff }} \ll \tau_{\text {ign }}$ such that ignition occurs in a quasi-steady state thermal layer, while in the simulations $\tau_{\text {diff }} \sim \tau_{\text {ign }}$ such that ignition occurs in a transient thermal layer.

We also compare the timescale for ignition kernel formation, $\tau_{k e r n e l}$, with the timescale for convection at the location of the ignition kernel, $\tau_{\text {conv }_{k e r n e l}}$. This comparison allows us to estimate if the development of the ignition kernel into a flame is a one- or two-dimensional phenomenon. If $\tau_{\text {conv }_{\text {kernel }}}$ is similar in magnitude to $\tau_{\text {kernel }}$, then significant convection occurs parallel to the plate during the early stage of ignition when the ignition kernel is developing into a flame. If instead, $\tau_{\text {conv }_{\text {kernel }}}$ is significantly larger than $\tau_{\text {kernel }}$, very little convection happens during the early ignition transient, and ignition can be considered as a solely radial phenomenon. From one-dimensional simulations, we estimate $\tau_{\text {kernel }} \approx 9 \times 10^{-5}$, and we assume this timescale is similar for the experiments. From this, we find that $\tau_{\text {kernel }} \ll \tau_{\text {conv }_{\text {kernel }}}$, and so there is no significant effect of convection as the ignition kernel develops into a flame. This means that the early ignition transient can be considered one-dimensional.

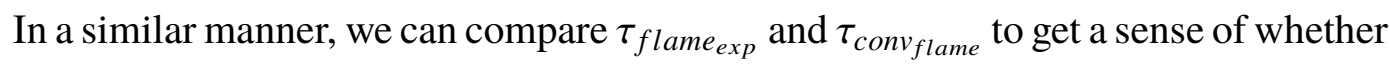
the flow is truly one- or two-dimensional during flame propagation. In other words, if $\tau_{\text {conv }_{\text {flame }}}$ is similar in magnitude to $\tau_{\text {flame }_{\text {exp }}}$, then a significant amount of convection is occurring parallel to the surface in the time it takes for the flame to 
propagate out of the boundary layer. This implies that the wall parallel motion is significant to the flame propagation and thus this portion of the experiments should be considered in two dimensions. However, if $\tau_{\text {conv flame }}$ significantly larger than $\tau_{\text {flame }_{\text {exp }}}$, very little convection happens parallel to the wall during flame propagation out of the boundary layer, and flame propagation can be considered as a solely

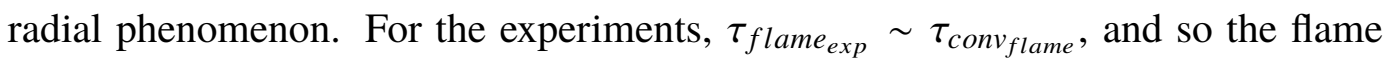
propagation stage of the experiments should be considered to be two-dimensional. In the simulations, the model is explicitly designed to be one-dimensional, and no convection parallel to the hot surface is considered.

From this we conclude that the simulations explored here have limited applicability to the ignition experiments discussed in Chapters 4 and 5 . In particular, ignition in the experiments occurs in a quasi-steady state thermal layer while in the simulations ignition occurs in a highly transient thermal layer. During the early stage of ignition in the experiments, we estimate that convection does not play a significant role in the initial development of the ignition kernel, and therefore this process can be considered one-dimensional. However, the flame propagation stage of the experiments should be considered to be two-dimensional, as significant convection can occur while the flame propagates through the boundary layer. For this reason, to accurately simulate the full ignition process in the experiments, a two-dimensional simulation would need to be performed.

Additionally, the simulations are different than the experiments because of fuel choice. The one-dimensional simulations use a reaction mechanism for hydrogen, while hexane was used in the experiments presented in Chapter 4. Hydrogen has a much higher flame speed and shorter ignition delay than hexane. For the onedimensional simulations, the stoichiometric hydrogen-air mixture was found to have a flame speed of $\approx 15 \mathrm{~m} / \mathrm{s}$, and the ignition delay was $\tau_{i g n} \approx 1 \times 10^{-2} \mathrm{~s}$. For the experiments with a stoichiometric hexane-air mixture, $V_{s} \approx 3 \mathrm{~m} / \mathrm{s}$ and $\tau_{\text {ign }} \approx 1 \times 10^{2} \mathrm{~s}$. Additionally, the ignition temperature for hydrogen is lower than that of hexane. This is seen in the one-dimensional simulations, which find a critical ignition temperature of 917.2 K for hydrogen, but can also be observed through the experiments. Cylinder 100C was tested in both hexane and hydrogen mixtures, and was found to ignite around $950 \mathrm{~K}$ for hydrogen and at $1055 \mathrm{~K}$ for hexane. The difference in ignition behavior between these fuels is another reason why the timescales discussed previ- 
ously are so different between the experiments and the one-dimensional simulations.

The key value of the one-dimensional simulations is in appreciating the proximity of the ignition kernel to the wall. The ignition kernel forms approximately one laminar flame thickness from the wall, close enough to the wall that convection is negligible at this location. This implies that wall parallel convection, while important in developing the thermal layer in the experiments, is not relevant to the early ignition process. This finding supports the approach of Ono et al. (1976), Laurendeau (1982), Law and Law (1979), and others in modeling the very earliest stages of the ignition process as one-dimensional, such that only wall normal variations in species and temperature are considered. Clearly, as observed in experiments presented in Chapter 4, the subsequent flame propagation through the thermal layer into the surround cold atmosphere is multi-dimensional and has to be treated as such.

Formulating a fully two-dimensional model of ignition for the cylinders investigated in Chapter 4 presents significant challenges. The size of the domain would need to be up to $25.4 \mathrm{~cm}$ long in the axial dimension and several centimeters long in the radial dimension to match the dimensions of the cylinders, but this large domain would need to have a mesh fine enough to resolve the flame front accurately. To ensure at least 10 grid points in the flame front, a minimum element size of approximately $2 \times 10^{-3} \mathrm{~cm}$ or less would be required: the total domain size could consist of millions of elements based on resolution considerations alone. Additionally, even a reduced hexane kinetic mechanism includes dozens of species and hundreds of reactions needed to compute chemistry and transport. A two-dimensional model of ignition that includes the relatively large domain while maintaining a suitable resolution and includes a kinetic mechanism for hexane would be enormously computationally demanding to solve and would likely require the use of super-computing resources. Such a model of ignition is outside the scope of this work and is left as a potential exploration for future research.

\section{Conclusions}

In this section, we examined the results of the one-dimensional ignition simulation in Lagrangian coordinates. A slight increase in ignition delay times was found compared with those from zero-dimensional reactor calculations, which is expected 
given the non-ideal aspects of the simulation. Profiles of temperature and species mass fractions were reported for supercritical, critical, and subcritical ignition simulations. The balance of the energy terms was considered for supercritical, critical, and subcritical ignition cases. A build-up of $\mathrm{HO}_{2}$ through low-temperature pathways appears to be necessary for ignition to occur. This implies that analogous low-temperature reaction pathways will be important for modeling ignition of hydrocarbons.

The heat flux at the wall tracked for all simulations. The condition of zero heat flux at the wall was used to define ignition. The ignition location was estimated by finding the location of peak $\mathrm{HO}_{2}$ mass fraction at the time when the heat flux at the wall is zero; the ignition location of the critical case is approximately one flame thickness away from the wall. Other methods of identifying ignition location found similar estimates of the ignition location. The ignition location for all cases ranged from 0.75 to $2.5 l_{f}$.

Differences in the energy balance between the critical and subcritical cases were observed at the same moments in time. Before ignition, each term in the energy equation was nearly identical between the critical and subcritical cases. This similarity prior to ignition, when the problem is largely a conduction problem, is attributed to the very small difference in wall temperature between the critical $(917.2 \mathrm{~K})$ and subcritical $(915.6 \mathrm{~K})$ cases. However, as ignition occurs, the energy terms diverge significantly between the critical and subcritical cases. This can be thought of as a sort of bifurcation in the solution at the critical wall temperature, akin to the sort of bifurcating solution discussed by Law and Law (1979) and Law (1979). The same concept of bifurcation is seen in the heat flux of a critical and subcritical case.

These numerical results agree well with some of the conclusions reached by Adomeit (1965) in their experimental study of ignition of suddenly heated cylinders. In particular, they note that the temperature distribution is initially determined by heat conduction and only later does heat generation due to reaction dominate. These results align with the findings from our consideration of the energy balance, where heat conduction dominated in the early stage of the simulation and ignition was marked by a transition to heat generation from reaction dominating the energy balance. Additionally, Adomeit (1965) notes that ignition can only occur when the 
temperature gradient at the wall is small such that heat generation is greater than heat losses at the wall. This is similar to our finding that at the time when the heat flux next to the wall is zero, the temperature gradient next to the wall is small and very close to satisfying the Van't Hoff ignition criterion. We find that the model problem of ignition investigated here is closely related to the experimental work of Adomeit (1965).

There are of course limitations on the usefulness of the solutions investigated here in terms of their applicability to the experiments discussed in previous chapters. The flame propagation process observed in the experiments presented in Chapters 4 and 5 is two-dimensional, and occurs in a quasi-steady state thermal layer, while the simulations model ignition in a one-dimensional and highly transient thermal layer. However, the initial development of the ignition kernel in the experiments is estimated to be mostly one-dimensional, and as a result, the one-dimensional simulations are thought to capture the early ignition dynamics well. A hydrogen mechanism was used instead of hexane to reduce the computational demand of running the one-dimensional simultion, but hydrogen has ignition behavior that is significantly different than the ignition behavior of hexane. The boundary conditions of the simulation do not reflect those of the experiments. In reality, the heated surface is not fixed in temperature, surface reactions can occur, and the temperature and mass fractions are not fixed at the outer boundary. Furthermore, the pressure is not uniform in the experiments, and the mixture does not behave as a perfect gas. In the experiments, the volume is fixed and the domain cannot expand as it does in these simulations. The domain of the simulations is much smaller than that of the experiments to due limited computational resources.

Despite the limitations of the one-dimensional simulations, they have proved useful in providing insights into the ignition process, particularly in identifying the initial location of the ignition kernel, and in tracking the evolution of temperature, species, heat flux, and energy balance during the very early stages of ignition. This serves as a complement to the zero-dimensional chemistry computations along streamlines, which provided insight into important chemical pathways and processes for ignition of $n$-hexane mixtures. 


\section{BIBLIOGRAPHY}

Adomeit, G. (1965). "Ignition of gases at hot surfaces under nonsteady-state conditions". In: Symposium (International) on Combustion. Tenth Symposium (International) on Combustion 10.1, pp. 237-243.

Boettcher, P.A. et al. (2012). "The effect of heating rates on low temperature hexane air combustion". In: Fuel 96, pp. 392-403.

Coronel, S, J Melguizo-Gavilanes, D Davidenko, et al. (2017). "Reduction Methodology for Detailed Kinetic Mechanisms: Application to n-Hexane-Air Hot Surface Ignition”. In: p. 4.

Coronel, Stephanie A. (2016). "Thermal Ignition Using Moving Hot Particles". $\mathrm{PhD}$ thesis. California Institute of Technology.

Coronel, Stephanie A., Josué Melguizo-Gavilanes, Rémy Mével, et al. (2018). "Experimental and numerical study on moving hot particle ignition". In: Combustion and Flame 192, pp. 495-506.

Goodwin, David G. et al. (2018). Cantera: An Object-oriented Software Toolkit for Chemical Kinetics, Thermodynamics, and Transport Processes. https://www. cantera.org. Version 2.4.0. Dor: $10.5281 /$ zenodo.1174508.

Kee, R., M. Coltrin, and P. Glarborg (2003). Chemically Reacting Flow: Theory \& Practice. John Wiley \& Sons.

Laurendeau, Normand M. (1982). "Thermal ignition of methane-air mixtures by hot surfaces: A critical examination". In: Combustion and Flame 46, pp. 29-49.

Law, C. K. and H. K. Law (1979). "Thermal-ignition analysis in boundary-layer flows". In: Journal of Fluid Mechanics 92.1, pp. 97-108. (Visited on 04/01/2020).

Law, C.K. (1979). "Transient ignition of a combustible by stationary isothermal bodies". In: Combustion Science and Technology 19, pp. 237-242.

Maas, U and J Warnatz (1988). "Ignition processes in hydrogen-oxygen mixtures". In: Combustion and Flame 74, pp. 53-69.

Melguizo-Gavilanes, J. et al. (2017). "Hot surface ignition of stoichiometric hydrogenair mixtures". In: International Journal of Hydrogen Energy 42.11, pp. 73937403.

Menon, Shyam K. et al. (2016). "Hot surface ignition of n-hexane in air". In: Combustion and Flame 163, pp. 42-53.

Mével, Rémy et al. (2016). "Chemical Kinetics of $n$-Hexane-Air Atmospheres in the Boundary Layer of a Moving Hot Sphere". In: Combustion Science and Technology 188.11-12, pp. 2267-2283. 
Ono, S. et al. (1976). "Ignition in a Free Convection from Vertical Hot Plate". In: Bulletin of JSME 19.132, pp. 676-683.

Peters, N. et al. (2002). "Temperature cross-over and non-thermal runaway at twostage ignition of n-heptane". In: Combustion and Flame 128.1-2, pp. 38-59.

Pitsch, Heinz (2014). Laminar Premixed Flames: Flame Structure. Princeton.

Raffel, B. et al. (1986). "Thermal ignition and minimum ignition energy in oxygenozone mixture”. In: Dynamics of Reactive Systems Part II. AIAA, p. 335.

Sánchez, Antonio L., Eduardo Fernández-Tarrazo, and Forman A. Williams (2014). "The chemistry involved in the third explosion limit of $\mathrm{H} 2-\mathrm{O} 2$ mixtures". In: Combustion and Flame 161.1, pp. 111-117.

Shepherd, J E (2020). Ignition Modeling and the Critical Decay Rate Concept. en. Tech. rep. EDL2019.002. GALCIT, p. 53.

Sparrow, E. M. and J. L. Gregg (1956). "Laminar-Free-Convection Heat Transfer From the Outer Surface of a Vertical Circular Cylinder". In: Transactions of the ASME 78, pp. 1823-1829.

Stauch, R., S. Lipp, and U. Maas (2006). "Detailed numerical simulations of the autoignition of single n-heptane droplets in air". In: Combustion and Flame 145.3, pp. 533-542.

Sundqvist, Hilding and George Veronis (1970). "A simple finite-difference grid with non-constant intervals". In: Tellus 22.1. 
Chapter 7

\section{CONCLUSIONS AND FUTURE WORK}

The goal of this study was to investigate thermal ignition by external natural convection flows to supplement the limited work on ignition by external natural convection over heated surfaces. To achieve this goal, an experimental system was designed to create an external natural convection flow over vertical cylinders with systematic variations in the length and surface area to examine the influence of geometrical parameters on ignition. Another goal of this work was to find ignition thresholds of Jet A and surrogate fuels and identify a surrogate for Jet A that can be used in future thermal ignition studies. To achieve this goal, ignition tests were carried out on Jet A and surrogate fuels.

An extensive series of tests demonstrated that over the range of parameters examined, surface area and length of the cylinder have a minor effect on ignition temperature. In contrast to the results found by Kuchta, Bartkowiak, and Zabetakis (1965) for internal natural convection flows, no drastic change of the ignition temperature was observed for the vertical cylinders with surface areas between 25 and $200 \mathrm{~cm}^{2}$. Additionally, the ignition threshold of the external natural convection flows was 500-600 K higher than ignition thresholds of internal natural convection flows of the same surface area.

Investigation of fuel type showed that hexane is a reasonable surrogate for Jet A ( $38 \mathrm{~K}$ difference in ignition thresholds) in external natural convection ignition tests. JI, a multicomponent surrogate, has an ignition threshold closest to Jet A of all the fuels tested ( $13 \mathrm{~K}$ difference in ignition thresholds). All the fuels investigated had ignition temperatures differed from that of Jet A by $40 \mathrm{~K}$ or less.

Ignition modeling revealed hexane exhibits two-stage ignition behavior on sufficiently gradually heated streamlines. One-dimensional simulation of ignition demonstrated the importance of species and heat diffusion in the ignition process; initially heat transfer occurs from the heated wall to the gas but as the reaction begins to release energy, the heat flux reverses such that it is from the gas to the 


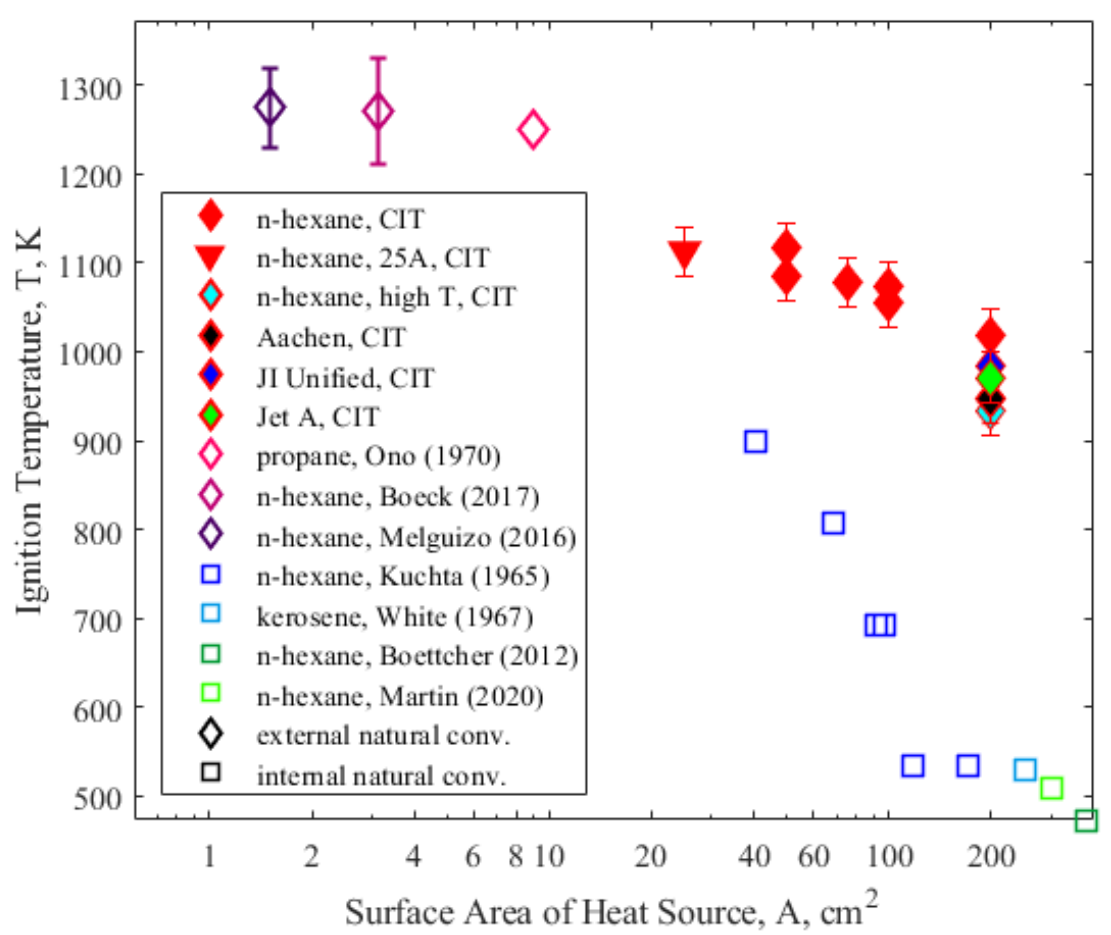

Figure 7.1: Ignition results.

wall. Ignition is found to occur approximately one flame thickness away from the heated surface.

This study has significant implications for safety assessments involving hot surface ignition. The data of Fig. 7.1 are widely quoted to support the assumptions used in safety assessments and regulations. The present results make it clear that the purported correlation with surface area is actually a consequence of flow geometry. This provides a new direction for safety engineers and regulators, focusing attention on the fluid mechanics, extent of confinement, and the distinction between internal (recirculating) flows and external (free convection) flows rather than surface area alone. In the case of external natural convection, the present study demonstrates that significantly (200-500 K) higher surface temperatures (on surfaces up to $200 \mathrm{~cm}^{2}$ in area) may be present without causing ignition in flammable atmospheres containing Jet A or surrogate fuels. 


\subsection{Experimental Development}

An experimental setup was developed and characterized for the purpose of creating large vertical cylinders capable of reaching surface temperatures in excess of 1100 $\mathrm{K}$. The test surfaces were designed such that a large portion of the surface reached a uniform temperature distribution. The design was successful for a wide range of geometrical parameters; surface areas from 25 to $200 \mathrm{~cm}^{2}$ and lengths between 12.7 and $25.4 \mathrm{~cm}$ were successfully tested. The experiment was also designed to provide optical access to two diagnostics used to measure temperature. A pyrometer takes surface temperature measurements of the test cylinders while an interferometer was used to take quantitative measurements of the gas temperature surrounding the heated cylinder. The pyrometer was improved such that the instrument is stable and only requires infrequent calibration. A new design for the interferometer was developed such that the field of view was as large as $10.6 \mathrm{~cm}$ in diameter in order to monitor the development of the thermal boundary layer of the large cylinders. The use of a fiber optic cable to transmit the reference beam of the interferometer made the design relatively compact despite the large field of view. The new interferometer design was shown to operate successfully through tests with pure nitrogen gas. An alternative visualization technique was used to capture the $\mathrm{OH}^{*}$ chemiluminescence during reaction in order to directly observe the formation of the ignition kernel and subsequent flame propagation.

\subsection{Geometry Effects for External Natural Convection}

Cylinders with a variety of different surface areas and lengths were tested in stoichiometric hexane-air mixtures. There existed variation in cylinder geometry such that cylinders with the same surface area had the different lengths and cylinders with the same lengths had different surface areas. This allowed investigation of the effect of surface area and length separately. The ignition temperature of the vertical cylinders was found to have a minor dependence on both surface area and length.

Surface areas from 25 to $200 \mathrm{~cm}^{2}$ were investigated; this range of surface areas was chosen to cover the range of surface areas at which Kuchta, Bartkowiak, and Zabetakis (1965) observed a sharp drop in ignition temperature for internal natural convection flows. For the cylinders tested, no sudden change in the ignition temperature was observed with increasing surface area. This is in contrast to the findings of Kuchta, Bartkowiak, and Zabetakis (1965). At the same surface area, the ignition temperatures of the vertical cylinders were significantly higher than 
the ignition temperatures Kuchta found for internal vessels by as much as $400 \mathrm{~K}$. From this we conclude that the flow configuration an extremely important ignition parameter. This is thought to be a result of the difference in residence time between and internal and external flow configuration due to recirculation. The scaling correlation between surface height and ignition temperature presented by Ono et al. (1976), $\ln (L) \propto T_{i g n}^{-1}$, is found to apply to the cylinders investigated in this work.

Visualization studies indicated when that the location of ignition was always at the top edge of the hot portion of the cylinder when testing for ignition thresholds. This remained true regardless of cylinder size. The interferometer was successfully used to produce temperature fields of pure nitrogen and diluted hydrogen mixtures. Some difficulty was discovered in processing the interferometry results of hexane mixtures, and the source of this difficulty is attributed to the decomposition of hexane in the high-temperature thermal boundary layer. Visualization also enabled the measurement of flame speed during the early stages of flame propagation. It was found that the horizontal flame speed was lower than the vertical flame speed. This is due to the temperature difference between the ambient mixture and the boundary layer. The top and bottom edges of the flame remain in the high temperature boundary layer where the flame speed is almost double that of the lower-temperature ambient mixture. Visualization also revealed unsteady disturbances to the natural convection boundary layer. The disturbances were not consistently tied to ignition and seemed to be of two distinct types.

\subsection{Jet Fuel and Surrogates}

A heating system was designed to elevate the temperature of the entire experimental setup such that multicomponent fuels with heavier hydrocarbon species could be tested. These included working with Jet A as well as two simplified surrogate fuels, Aachen and JI. Hexane was also tested with the heated vessel for comparison. The goal was to find the ignition threshold of Jet A and to identify a surrogate fuel that closely matched the ignition behavior of Jet A.

The ignition threshold of hexane decreased with an increased ambient temperature. Increasing the ambient temperature decreases the velocity of the natural convection flow and effectively increases the residence time of a fluid particle, leading to a lower 
ignition threshold. An alternative explanation for the effect ambient temperature is proposed; an increase in ambient temperature creates a shallower thermal gradient in the boundary layer, and as a result, less additional energy is required to satisfy the Van't Hoff ignition criterion.

Among the fuels tested, the ignition threshold of JI is closest to that of Jet A. Additionally, the flame speeds of Aachen, JI, and Jet A were very similar, while the flame speed of hexane in the heated vessel was approximately double that of the other fuels. From this, we conclude JI is a suitable surrogate for Jet A in the context of ignition testing by concentrated hot surfaces.

\subsection{Modeling Efforts}

Two modeling approaches were undertaken to gain further insight into the ignition process. The first is a technique used to model the chemistry of a hexane mixture. A parametric study of the effect of rate of temperature increase on the chemistry was performed. The temperature increase along a streamline was modeled via an s-shaped function, based on the temperature history of streamlines found via modeling the boundary layer via similarity solution. This artificial streamline temperature history is used to prescribe a temperature history to a zero-dimensional reactor calculation. A fast temperature increase led to a single ignition event, while a more gradual temperature increase caused the hexane mixture to undergo a twostage ignition event. The chemical pathways of the two stages of ignition were found to match the processes of a two-stage ignition of $\mathrm{n}$-heptane described in the literature.

The second modeling technique is a one-dimensional simulation of ignition in Lagrangian coordinates. A cylindrical geometry is chosen. The governing equations include diffusion of heat and species, key physical phenomena missing in the zerodimensional reactor model. A kinetic mechanism for hydrogen is used to reduce computational demand. Ignition and flame propagation are observed in the onedimensional simulation. The balance of the energy equation is also considered. The simulation begins as a balance between the conduction and unsteady terms of the equation. As time advances, ignition cases show an increase in the reactive term of the energy equation, which eventually drives the conduction term negative. At this point, ignition has begun, and the energy balance is primarily between reaction and 
unsteadiness as the flame forms and propagates through the domain. Conduction and diffusion terms show significant activity in proximity of the flame as they transport energy away from the reaction zone. The heat flux at the wall is considered; heat flux at the wall is initially positive as heat is transferred from the wall to the gas. Ignition occurs when the wall heat flux is zero, indicating that enough heat is generated by reaction to transfer heat from the gas back into the wall. The location of the ignition kernel is identified by the peak in $\mathrm{HO}_{2}$ mass fraction at the time when the heat flux at the wall is zero, and found to be approximately one laminar flame thickness from the wall.

Both modeling techniques have limitations: the reactor calculations do not include the physics of diffusion, and the streamlines are modeled parametrically rather than based on fluid phenomena. The boundary conditions in the one-dimensional simulation do not accurately match the conditions of the experiments. The one-dimensional simulation also does not capture the two-dimensional effects of buoyant fluid travel along a surface: analysis of timescales for ignition kernel development, flame propagation, and convection reveal that while the early ignition kernel development is a locally radial phenomenon, the flame propagation through the boundary layer is a two-dimensional process. As such, a two-dimensional simulation would be required to fully capture the physics of flame propagation through the boundary layer.

Neither of the two simulation techniques explored in this work are meant to be used as predictive tools. However, they do provide insight on the chemical and physical processes that are key to ignition, such as a two-stage ignition event, the loss of heat to the heated surface during the early stages of ignition, the location of the ignition kernel relative to the wall, and the shift in energy balance over the course of an ignition event.

\subsection{Future Work}

There are a number of possible directions for future studies of ignition by large surfaces. The range of cylinder lengths investigated could be expanded if cylinder 25D was used. Performing ignition tests with cylinder 25D would provide an order of magnitude change in cylinder length. Most of the supplies for this test are already procured. Cylinder 25D and its support structure are assembled, and water-cooled 
power feedthroughs capable of handling up to $1000 \mathrm{~A}$ are available in the laboratory. The equipment needed for testing with $25 \mathrm{D}$ is a more powerful power supply for resistive heating; the existing power supply can provide a maximum of $600 \mathrm{~A}$, which raises the cylinder to approximately $800 \mathrm{~K}$. The current needed to resistively heat cylinder $25 \mathrm{D}$ to ignition temperatures is approximately $1000 \mathrm{~A}$.

The use of interferometry to reconstruct temperature fields of n-hexane mixtures could be improved if the interferometry constants (index of refraction, GladstoneDale constant, specific gas constant) reflected the decomposition of hexane near the hot surface. The decomposition of hexane as a function of radial distance from the wall would need to be modeled numerically. The radial variation of mixture composition could be used to inform the value of interferometry post-processing constants based on distance from the surface. This would require altering the interferometry post-processing procedure to allow for spatial variation of the index of refraction, Gladstone-Dale constant, and specific gas constants. If implemented properly, this would significantly improve the accuracy of interferometry temperature fields for mixtures that contain fuels like hexane.

The cause of the intermittent disturbances in the boundary requires further investigation. A technique like particle imaging velocimetry would be useful in characterizing the flow behavior at the high Grashof numbers of the experiments. A technique for seeding particles in the reactive mixture would need to be developed, and the experiment would require modification to allow optical access for the laser sheet and PIV camera. PIV measurements would provide valuable insight on the structure of the momentum boundary layer, an aspect of the natural convection flow that cannot be directly observed with the current experimental setup.

It would also be useful to investigate the effects of turbulence on ignition behavior. In particular, it would be interesting to see if the scaling relationship provided by Ono et al. (1976), $\ln (L) \propto T_{i g n}^{-1}$, changes drastically once a turbulent flow develops over a heated surface. This could be investigated by testing cylinders with longer lengths. The longest cylinders investigated here, $L=25.4 \mathrm{~cm}$, are already approaching transitional flows, and as $G r \propto L^{3}$, relatively modest increases in cylinder length would likely be enough to develop a fully turbulent flow over the cylinder surface. 
The identification of JI as an appropriate surrogate for Jet A in the context of thermal ignition provides an opportunity to study ignition with a Jet A-like fuel while signficantly simplifing the experimental procedure. JI fully vaporizes at $393 \mathrm{~K}$ and there is no need to consider mass loading effects, fuel weathering, or the role of dissolved air when testing with JI. Its composition is well known, and the limited number of species present in the fuel makes the modeling of JI simpler compared to commodity jet fuel. This makes ignition testing with JI a promising alternative to working with Jet A in future ignition tests, as the JI can be tested so long as an ambient temperature of approximately $393 \mathrm{~K}$ is reached.

The modeling of ignition could be improved by using CFD software to model ignition. In particular, a two-dimensional transient simulation that includes diffusion and detailed hexane chemical kinetics would be useful in developing accurate predictions of ignition thresholds for the external natural convection flows investigated in the experiments. A sophisticated CFD model of the natural convection flow would also be useful in identifying the nature of the intermittent disturbances observed in the experiments. In particular, it would be useful to identify if a disturbance is caused by fluid dynamics (transition to turbulence), reaction, or a coupling between the two phenomena.

Finally, the role of confinement on ignition behavior requires further exploration. In this work, ignition by external natural convection flows has been studied in the case of no significant confinement; the recirculation time was estimated and found to be approximately 50\% larger than the test time, leading to the conclusion that there is no significant recirculation in these experiments. If the volume of the combustion vessel is decreased, the confinement becomes more significant and the recirculation time will decrease until a situation is reached where there is an external natural convection flow with significant recirculation due to confinement. This situation is a sort of bridge between the external flows investigated here and the internal natural convection flows presented by Kuchta, Bartkowiak, and Zabetakis (1965). Very little literature exists on the role of confinement in hot surface ignition studies, so this is one promising opportunity for further research. 


\section{BIBLIOGRAPHY}

Kuchta, J., A. Bartkowiak, and M. Zabetakis (1965). "Hot Surface Ignition Temperatures of Hydrocarbon Fuel Vapor-Air Mixtures". In: Journal of Chemical Engineering Data 10.3, pp. 282-288.

Ono, S. et al. (1976). "Ignition in a Free Convection from Vertical Hot Plate". In: Bulletin of JSME 19.132, pp. 676-683. 
Appendix A

\section{COMPONENT LISTS}

\section{A.1 Pyrometer Components}

\begin{tabular}{|c|c|c|c|c|c|}
\hline Part name & Manufacturer Part Number & Material & $\begin{array}{l}\text { Focal } \\
\text { Length } \\
(\mathrm{mm})\end{array}$ & $\begin{array}{l}\text { Wavelength } \\
(\mathrm{nm})\end{array}$ & $\begin{array}{l}\text { FWHM } \\
(\mathrm{nm})\end{array}$ \\
\hline $\begin{array}{l}\text { collection } \\
\text { lens } 1\end{array}$ & ThorLabs & BK-7 & $60 \mathrm{~mm}$ & $\overline{-}$ & - \\
\hline $\begin{array}{l}\text { collection } \\
\text { lens } 2\end{array}$ & ThorLabs & BK-7 & $35 \mathrm{~mm}$ & - & - \\
\hline $\begin{array}{l}\text { fiber optic } \\
\text { cable }\end{array}$ & FT1000EMT & silica & - & $400-2200$ & - \\
\hline body lens 1 & ThorLabs & BK-7 & $75 \mathrm{~mm}$ & - & - \\
\hline $\begin{array}{l}\text { dichroic mir- } \\
\text { ror }\end{array}$ & DMLP1800 & silica & - & $\begin{array}{l}1800 \text { long- } \\
\text { pass }\end{array}$ & - \\
\hline $\begin{array}{l}\text { bandpass fil- } \\
\text { ter short }\end{array}$ & $\begin{array}{l}\text { Infrared } \\
\text { Optical } \\
\text { Products }\end{array}$ & silica & - & 1705 & 97 \\
\hline $\begin{array}{l}\text { bandpass fil- } \\
\text { ter long }\end{array}$ & $\begin{array}{l}\text { Infrared } \\
\text { Optical } \\
\text { Products }\end{array}$ & silica & - & 1940 & 105 \\
\hline $\begin{array}{l}\text { photodetector } \\
(2 \mathrm{x})\end{array}$ & PDA10DT & - & - & $900-2570$ & - \\
\hline
\end{tabular}

Table A.1: Pyrometer optical components.

\section{A.2 Interferometer Components}




\begin{tabular}{|c|c|c|c|c|}
\hline Part name & Manufacturer & Part Number & Focal Length (mm) & Diameter (mm) \\
\hline Optical Isolator & Newport & ISO-04-532-MP & - & 4 \\
\hline $\begin{array}{l}\text { Beam Expander } 1 \\
(5 \mathrm{x})\end{array}$ & ThorLabs & BE05M-A & - & $4.6 / 28.0$ \\
\hline Mirror 1, 2, 6 & ThorLabs & $43-408-533$ & - & 25.4 \\
\hline $\begin{array}{l}\text { Beam Splitter 1, } 2 \\
(50: 50)\end{array}$ & Edmund & 47-009 & - & 25.4 cube \\
\hline $\begin{array}{l}\text { Beam Expander } \\
2,3(5 x)\end{array}$ & Edmund & $35-102$ & - & $7.5 / 26.0$ \\
\hline $\begin{array}{l}\text { Focusing Lens 1, } \\
2\end{array}$ & Edmund & SPX043 & 100 & 50.8 \\
\hline Cylindrical Lens & ThorLabs & LJ1267RM & 250 & 50.8 \\
\hline $\begin{array}{l}\text { Focusing Mirror } \\
1,2\end{array}$ & Edmund & $32-067-533$ & 863.6 & 108.0 \\
\hline Mirror 3, 4 & Edmund & $84-417$ & - & 101.6 \\
\hline Mirror 5 & Edmund & $43-416-533$ & - & 50.8 \\
\hline $\begin{array}{l}\text { Fiber Collimator } \\
1,2\end{array}$ & ThorLabs & TC18FC-543 & 18 & 14.9 \\
\hline $\begin{array}{l}\text { Single Mode } \\
\text { Polarization } \\
\text { Preserving Fiber }\end{array}$ & ThorLabs & PM460-HP & - & 0.0033 \\
\hline Focusing Lens 3 & Edmund & SPX046AR.14 & 150 & 50.8 \\
\hline
\end{tabular}

Table A.2: Interferometer optical components. 
Appendix B

\section{INTERFEROMETER POST-PROCESSED RESULTS, NITROGEN}

\begin{tabular}{|c||ccccccc|}
\hline Cyl Tag & shot & pyr T $(\mathrm{K})$ & intf T $(\mathrm{K})$ & $\Delta \mathrm{T}(\mathrm{K})$ & \% diff. T & length $(\mathrm{s})$ & symmetry \\
\hline 50A & 69 & 700 & 663.3 & 36.7 & 5.2 & 100 & very symm. \\
$50 \mathrm{~A}$ & 70 & 1100 & 1045.1 & 54.9 & 5.0 & 100 & very symm. \\
$50 \mathrm{C}$ & 61 & 700 & 674.1 & 25.9 & 3.7 & 100 & very symm. \\
$50 \mathrm{C}$ & 62 & 1100 & 1065.0 & 35.0 & 3.2 & 100 & very symm. \\
$75 \mathrm{~B}$ & 75 & 700 & 698.5 & 1.5 & 0.2 & 100 & very symm. \\
$75 \mathrm{~B}$ & 79 & 1100 & 1055.7 & 44.3 & 4.0 & 100 & very symm. \\
$100 \mathrm{~A}$ & 71 & 700 & 731.3 & -31.3 & -4.5 & 100 & mostly symm. \\
$100 \mathrm{~A}$ & 72 & 1100 & 1265.2 & -165.2 & -15.0 & 100 & very symm. \\
$100 \mathrm{C}$ & 65 & 700 & 700.6 & -0.6 & -0.1 & 100 & very symm. \\
$100 \mathrm{C}$ & 66 & 1100 & 1020.7 & -20.7 & -2.1 & 100 & very symm. \\
200A & 44 & 700 & 680.6 & 19.4 & 2.8 & 100 & very symm. \\
200A & 45 & 1100 & 956.9 & 43.1 & 4.3 & 100 & very symm. \\
\hline
\end{tabular}

Table B.1: All processed interferometry results in pure nitrogen.

\section{$50 \mathrm{~A}$}

Shot 69: 

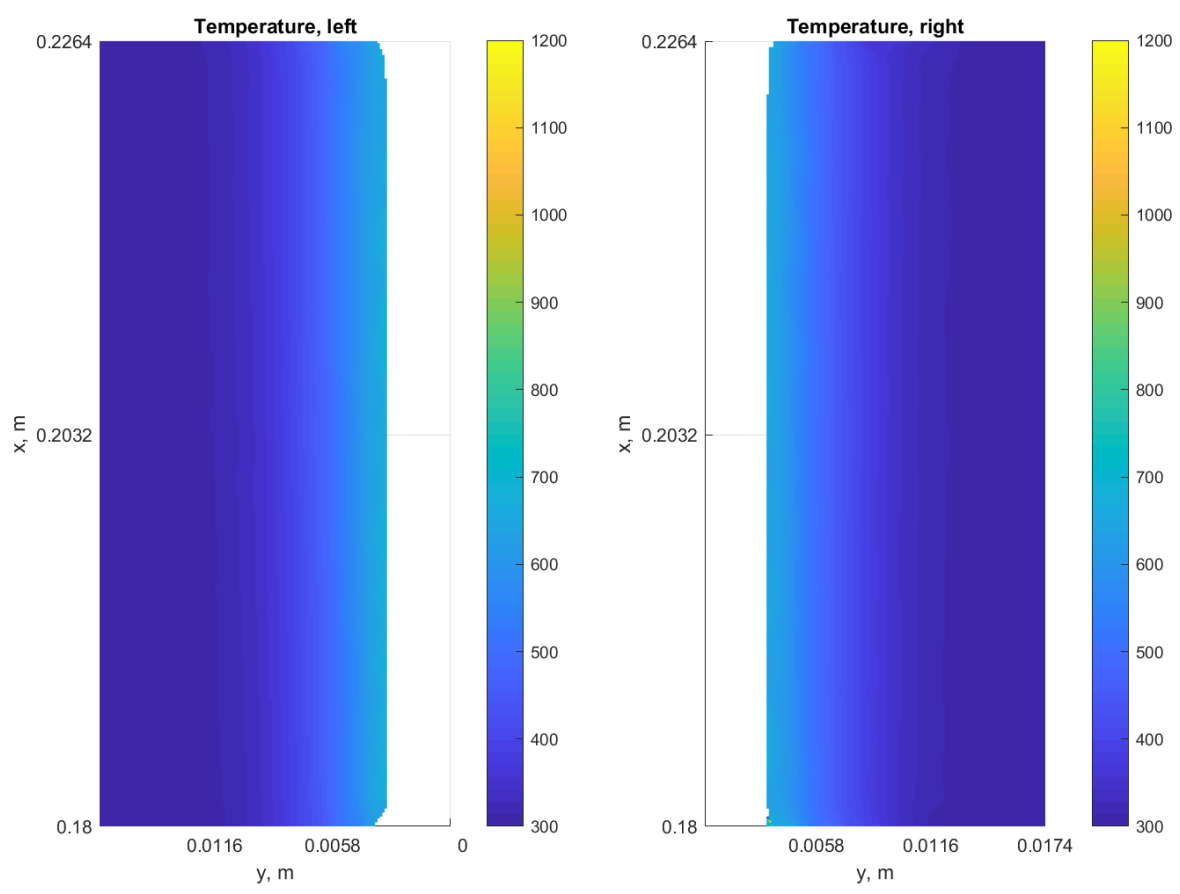

Figure B.1: Shot 69. Temperature fields from interferometry for nitrogen test. Surface temperature measured by pyrometer $=700 \mathrm{~K}$. 


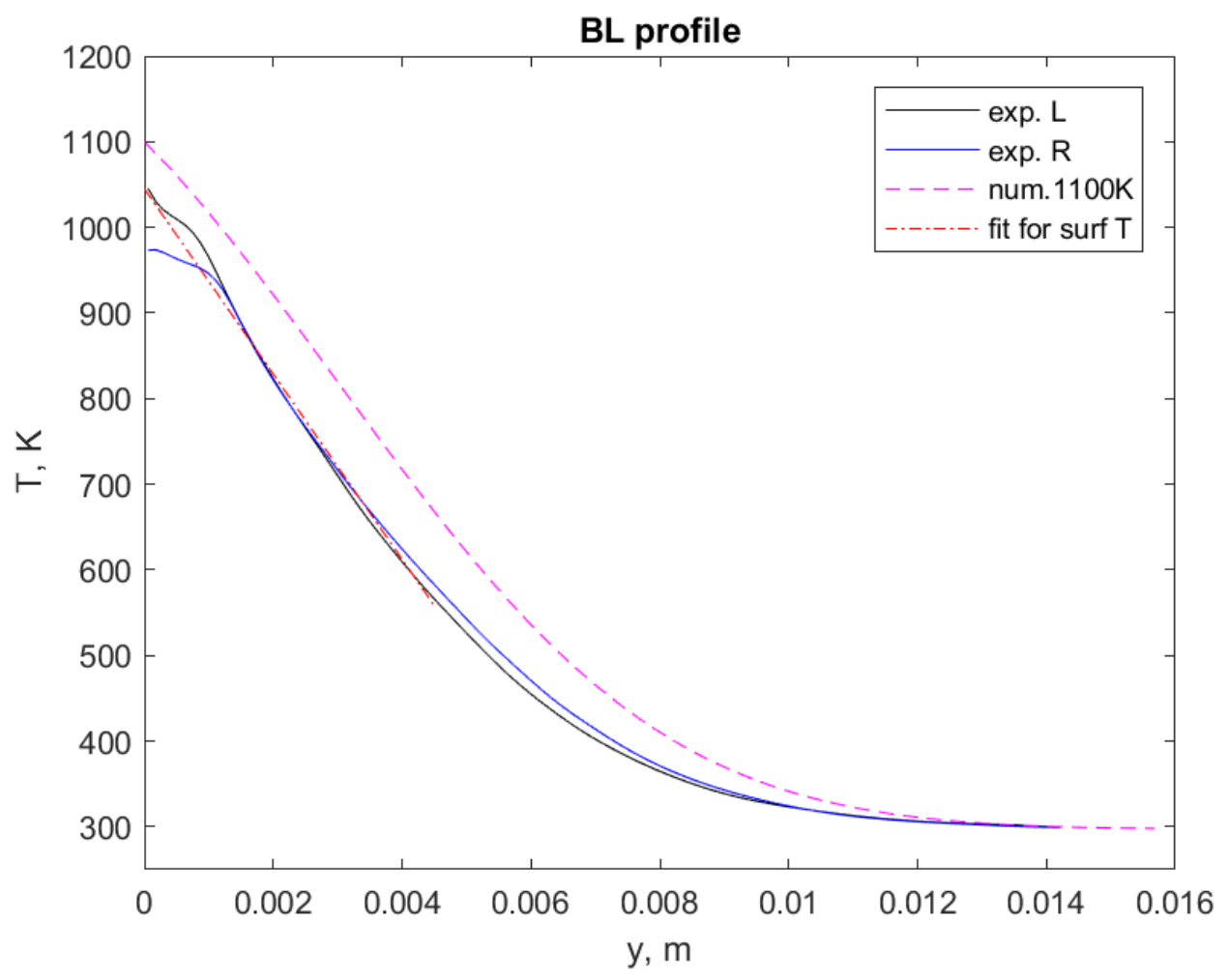

Figure B.2: Shot 69. Thermal boundary layer profiles for left (black line) and right (blue line) sides of experimental results compared with prediction of boundary layer profile (dashed magenta line) based on similarity solution for pyrometer surface temperature. Linear fit to averaged experimental profile (red dot-dashed line) used to extrapolate surface temperature from interferometer post processing. Extrapolated interferometer surface temperature $=663.3 \mathrm{~K}$. 


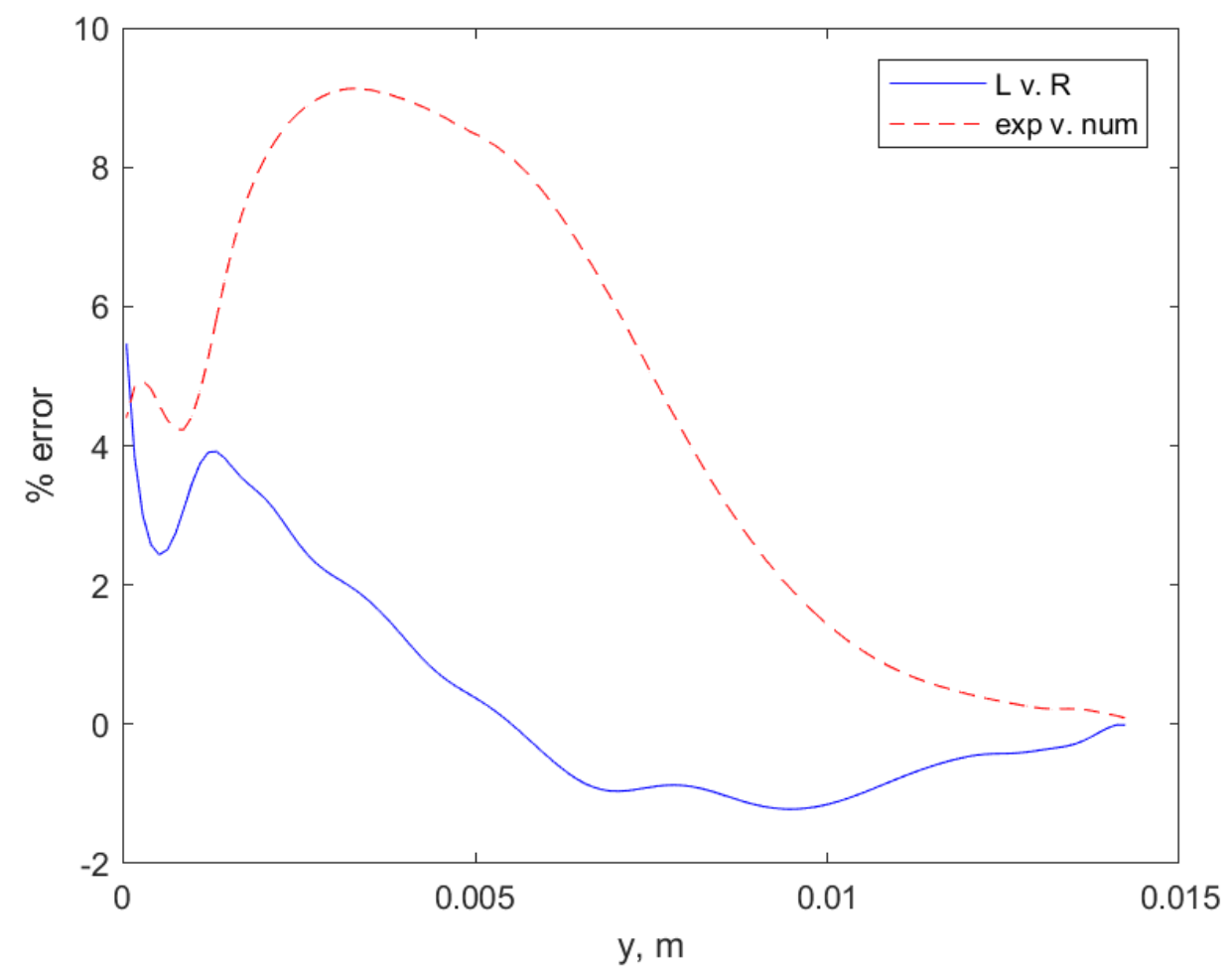

Figure B.3: Shot 69. Percent error in thermal boundary layer between both sides of experimental boundary layer profiles (red line) and percent error between predicted boundary layer profile from similarity solution and averaged experimental boundary layer profile (blue dashed line). 
Shot 70:
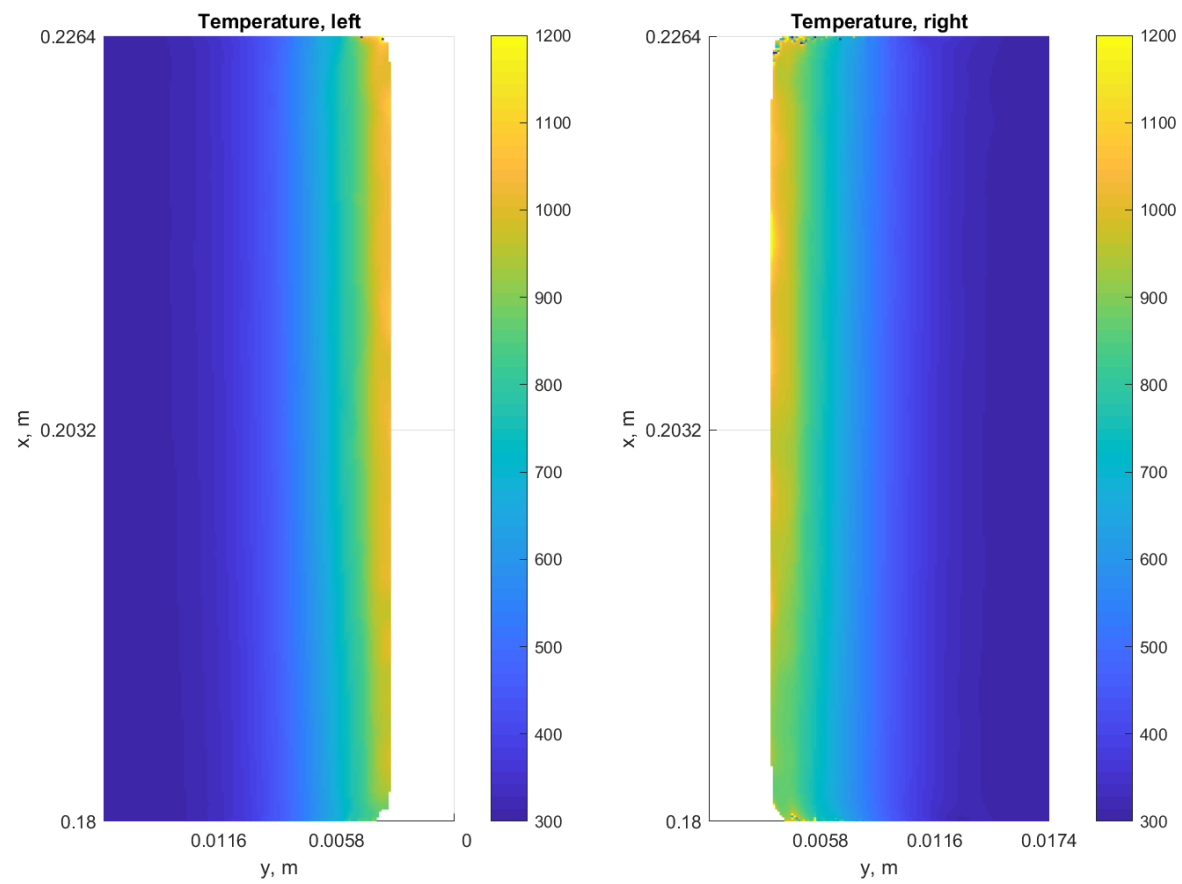

Figure B.4: Shot 70. Temperature fields from interferometry for nitrogen test. Surface temperature measured by pyrometer $=1100 \mathrm{~K}$. 


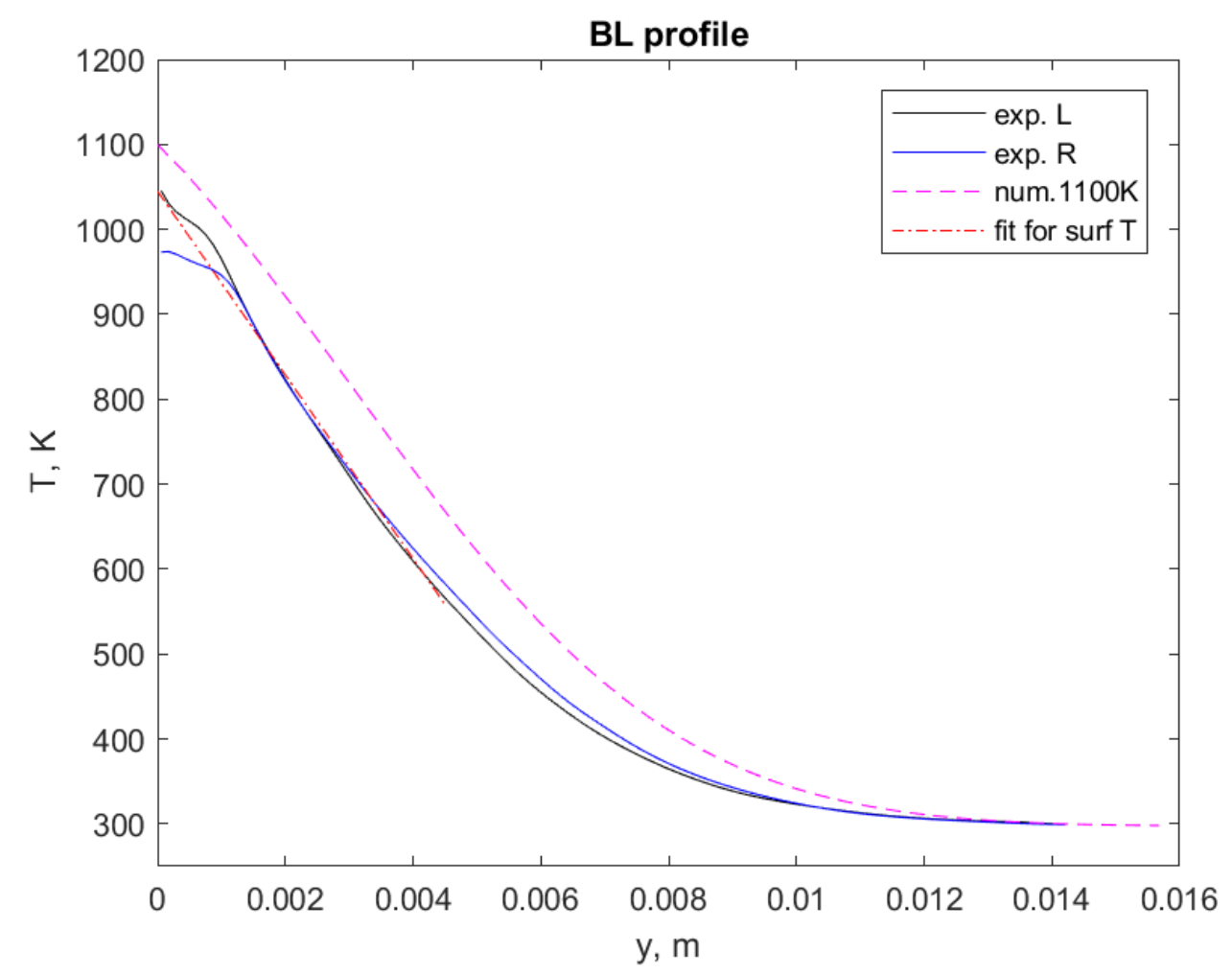

Figure B.5: Shot 70. Thermal boundary layer profiles for left (black line) and right (blue line) sides of experimental results compared with prediction of boundary layer profile (dashed magenta line) based on similarity solution for pyrometer surface temperature. Linear fit to averaged experimental profile (red dot-dashed line) used to extrapolate surface temperature from interferometer post processing. Extrapolated interferometer surface temperature $=1045.1 \mathrm{~K}$. 


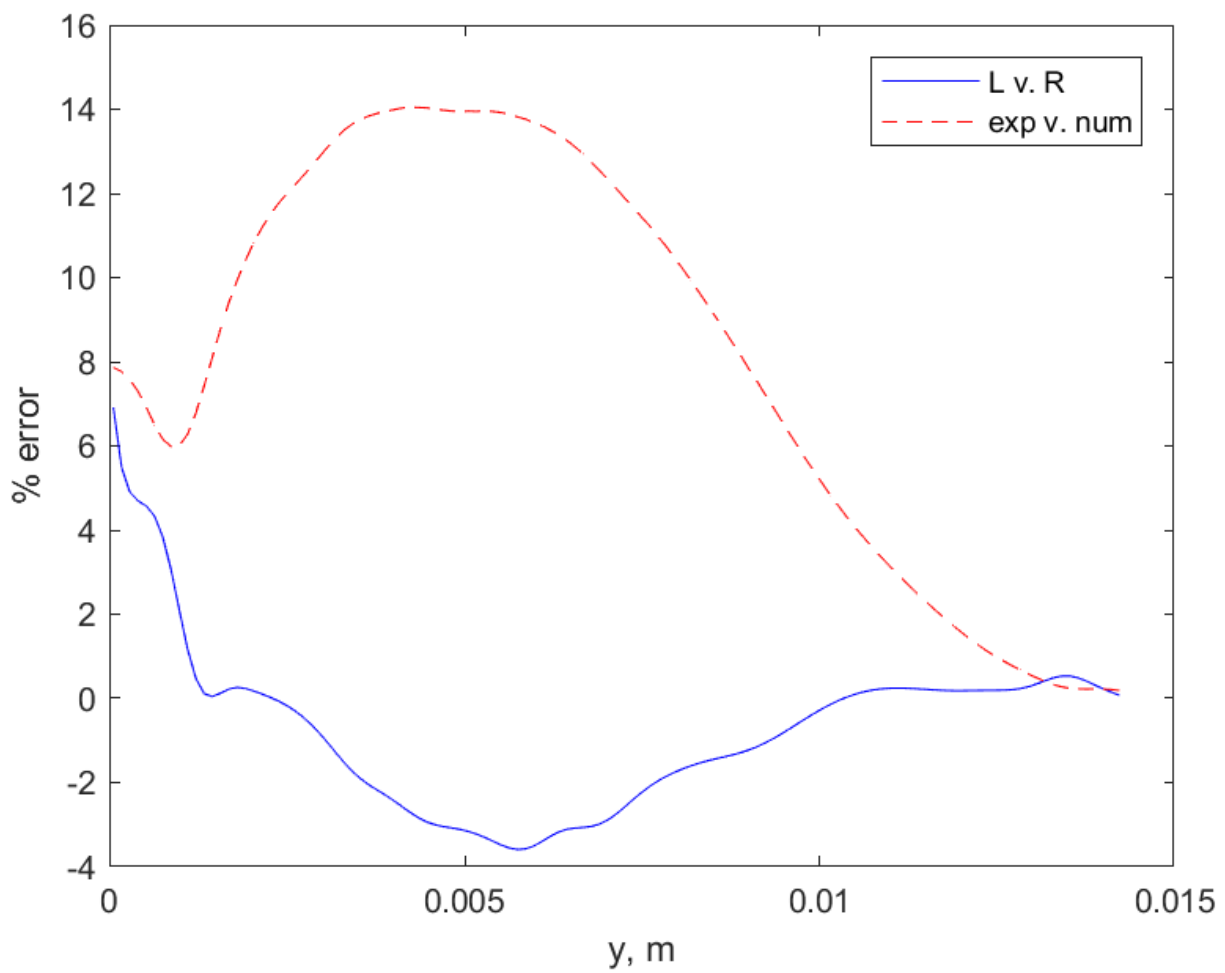

Figure B.6: Shot 70. Percent error in thermal boundary layer between both sides of experimental boundary layer profiles (red line) and percent error between predicted boundary layer profile from similarity solution and averaged experimental boundary layer profile (blue dashed line). 
50C

Shot 61:

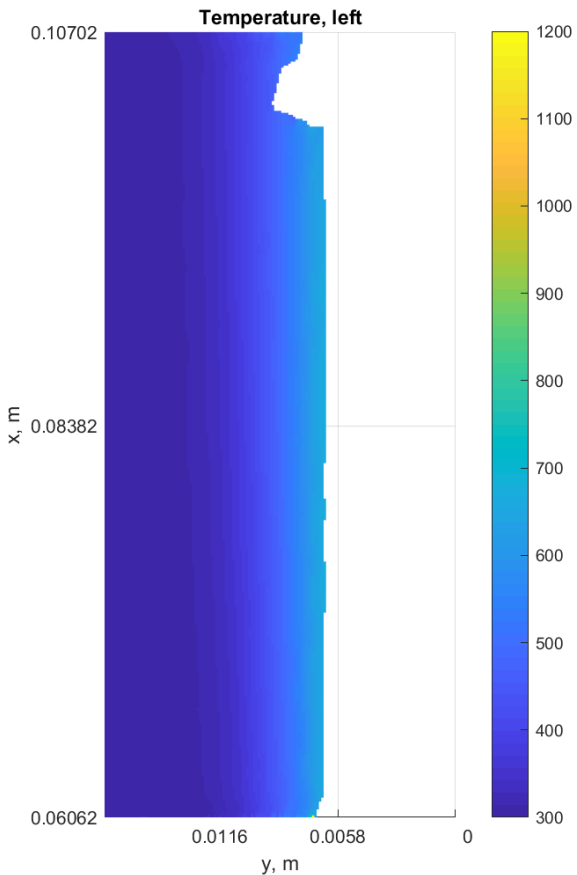

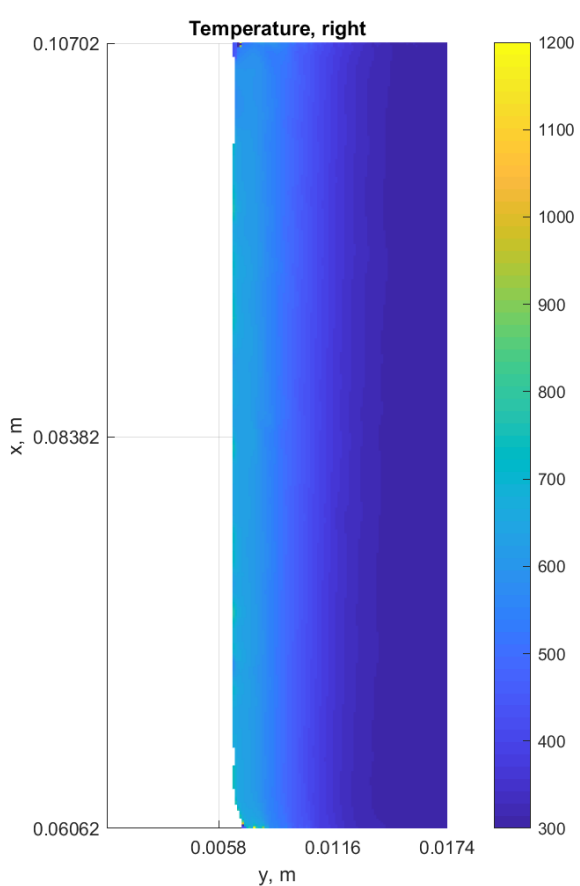

Figure B.7: Shot 61. Temperature fields from interferometry for nitrogen test. Surface temperature measured by pyrometer $=700 \mathrm{~K}$. 


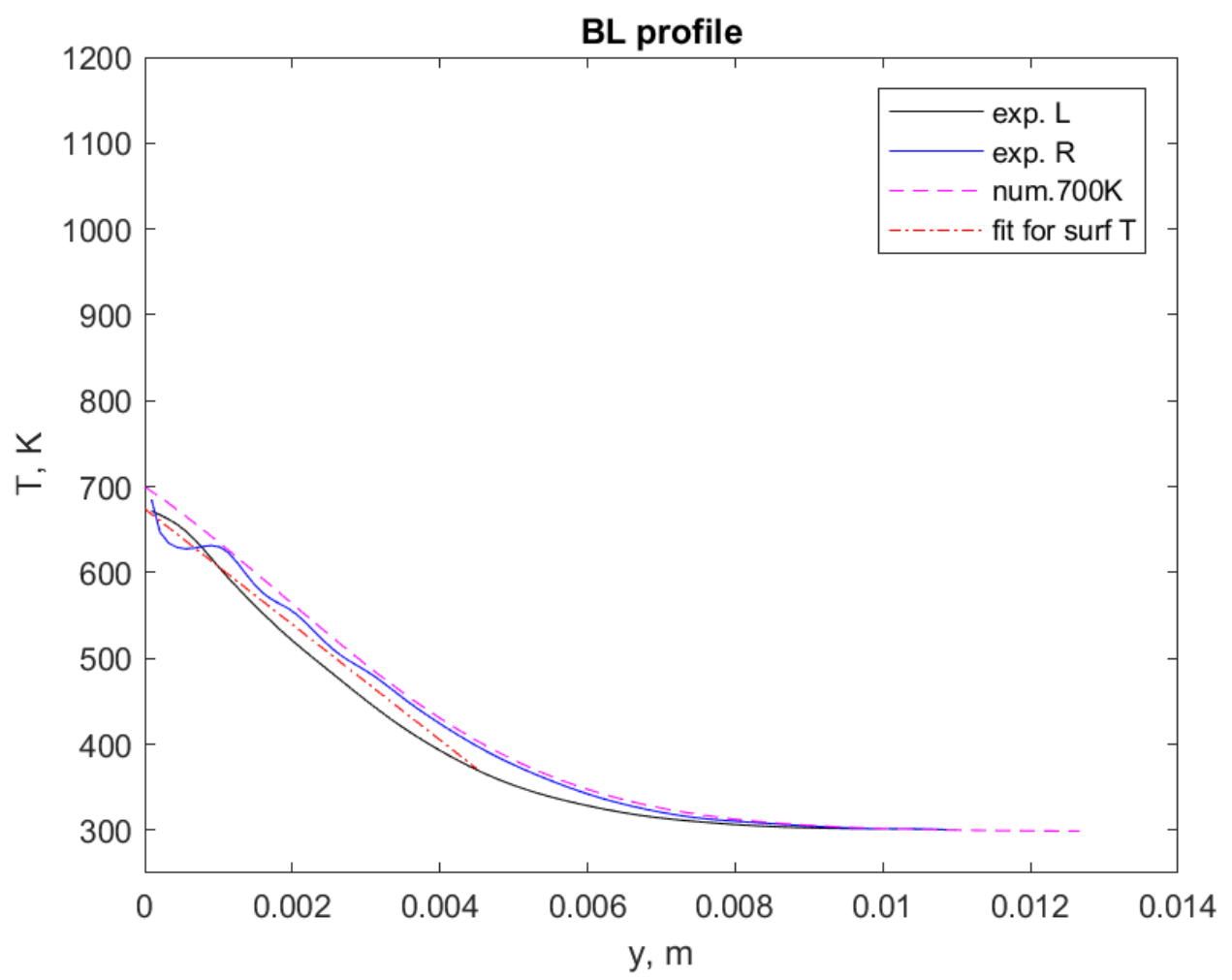

Figure B.8: Shot 61. Thermal boundary layer profiles for left (black line) and right (blue line) sides of experimental results compared with prediction of boundary layer profile (dashed magenta line) based on similarity solution for pyrometer surface temperature. Linear fit to averaged experimental profile (red dot-dashed line) used to extrapolate surface temperature from interferometer post processing. Extrapolated interferometer surface temperature $=674.1 \mathrm{~K}$. 


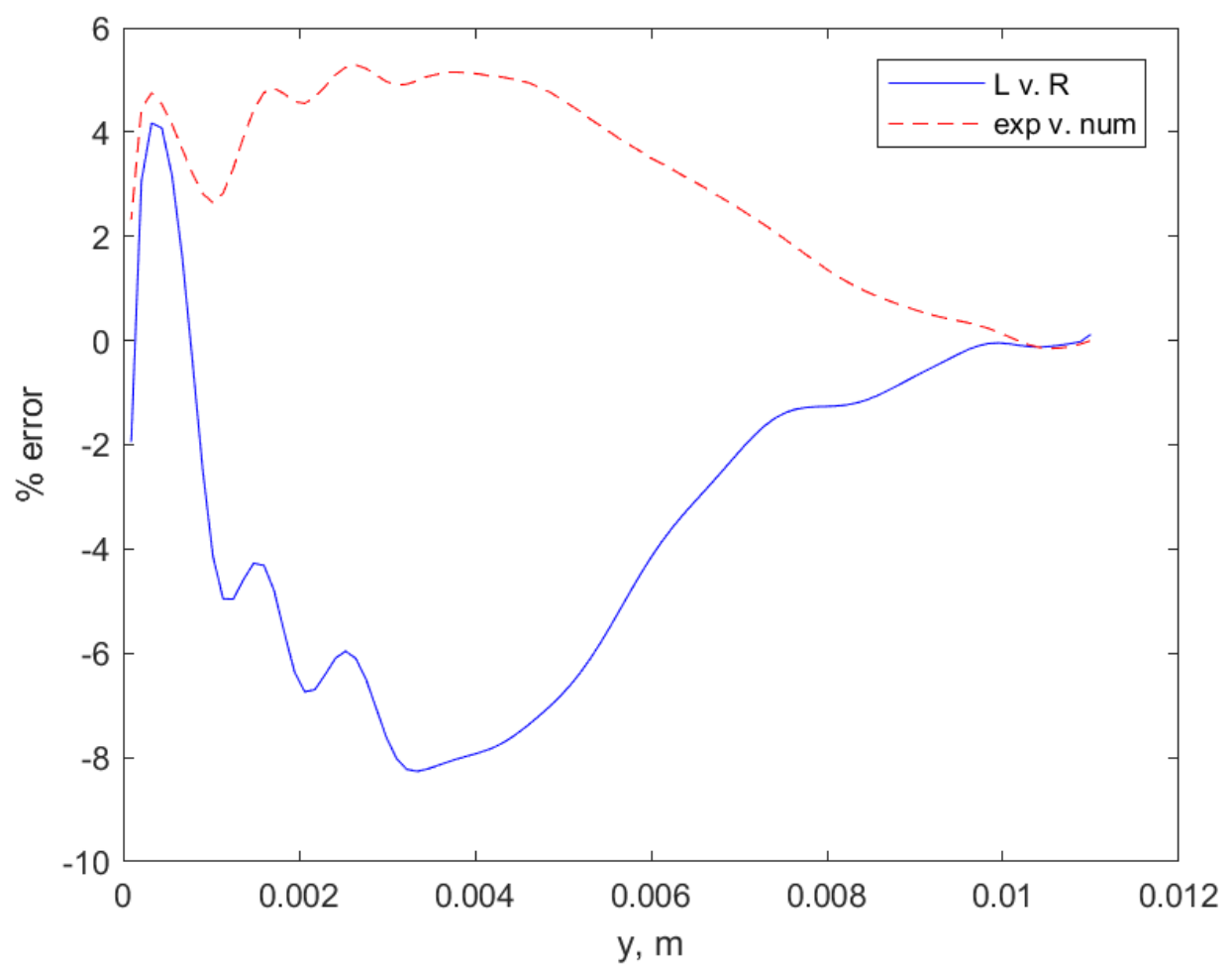

Figure B.9: Shot 61. Percent error in thermal boundary layer between both sides of experimental boundary layer profiles (red line) and percent error between predicted boundary layer profile from similarity solution and averaged experimental boundary layer profile (blue dashed line). 
Shot 62:
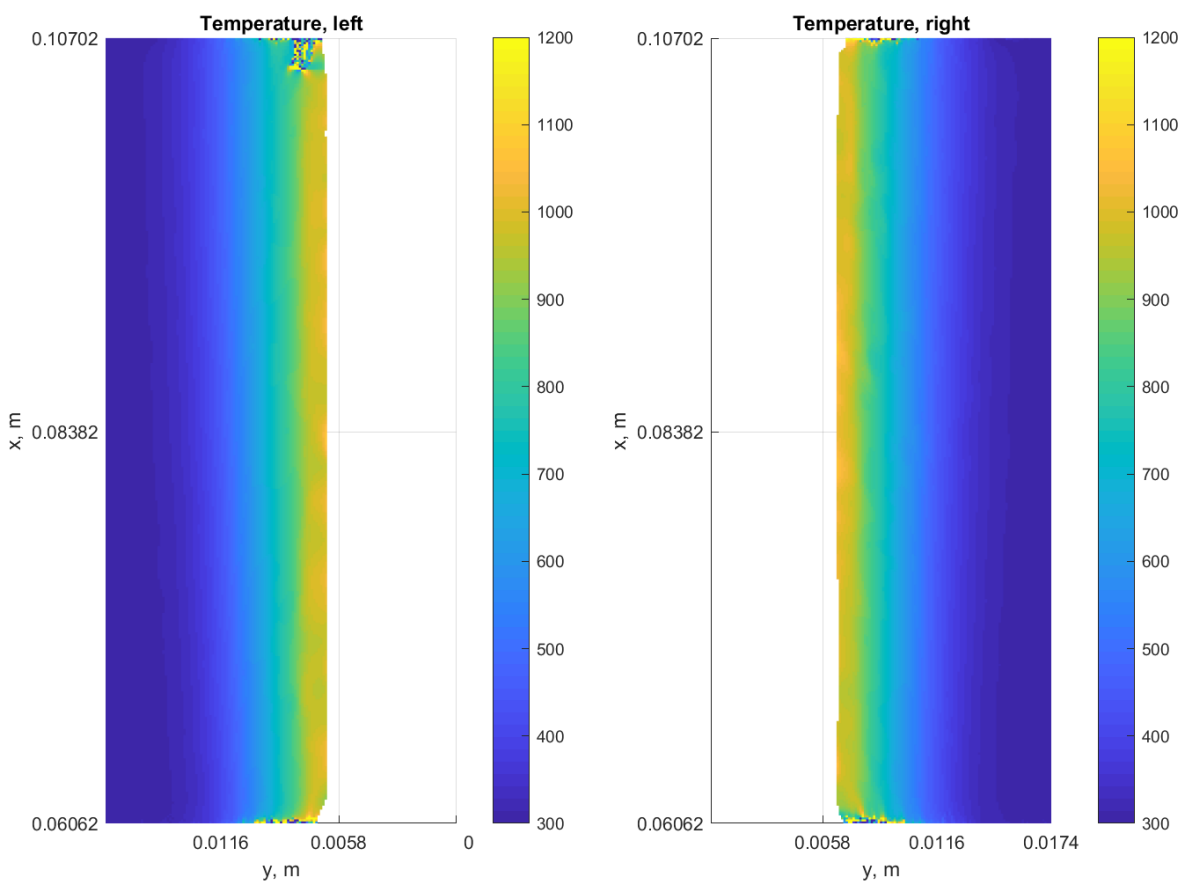

Figure B.10: Shot 62. Temperature fields from interferometry for nitrogen test. Surface temperature measured by pyrometer $=1100 \mathrm{~K}$. 


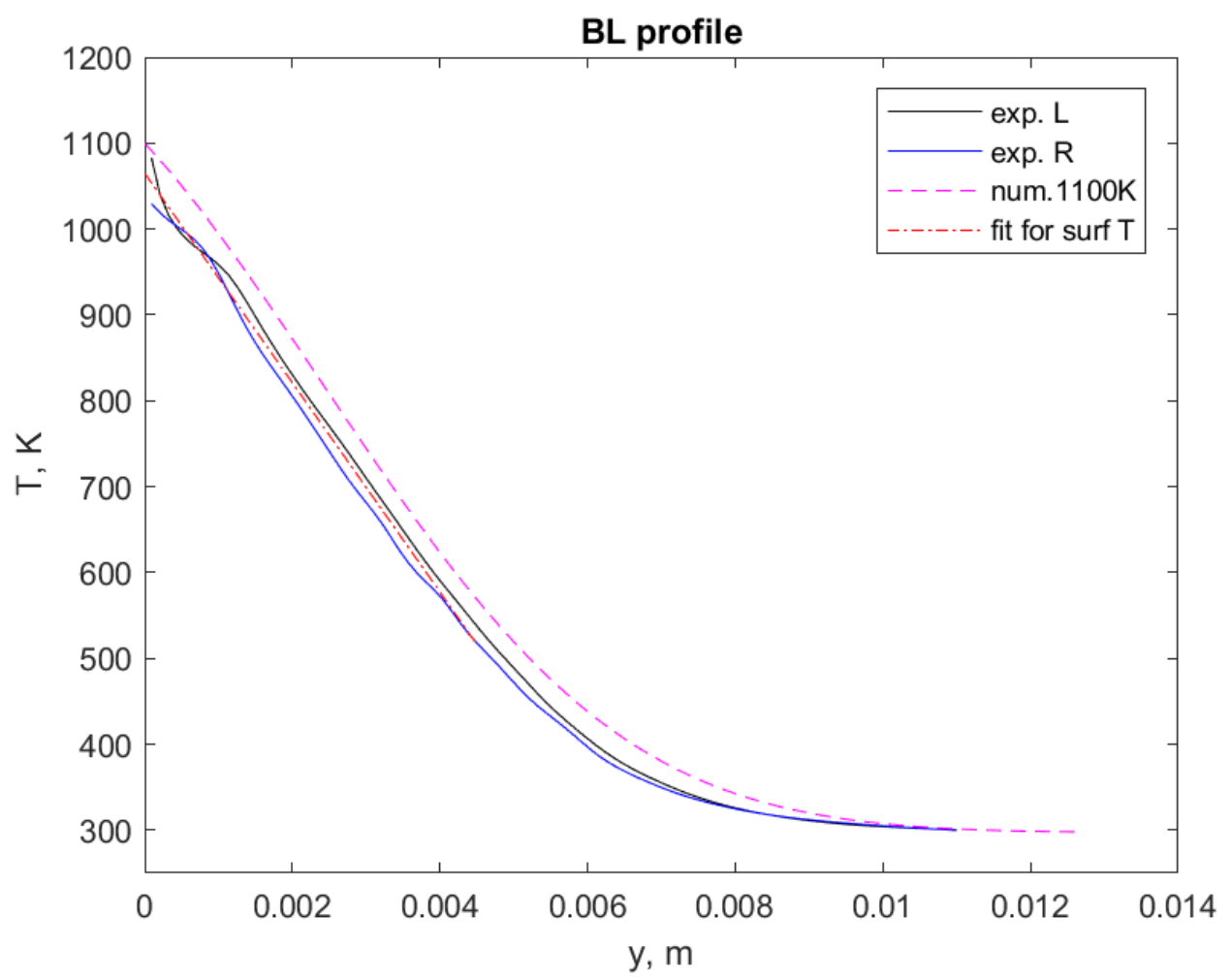

Figure B.11: Shot 62. Thermal boundary layer profiles for left (black line) and right (blue line) sides of experimental results compared with prediction of boundary layer profile (dashed magenta line) based on similarity solution for pyrometer surface temperature. Linear fit to averaged experimental profile (red dot-dashed line) used to extrapolate surface temperature from interferometer post processing. Extrapolated interferometer surface temperature $=1065.0 \mathrm{~K}$. 


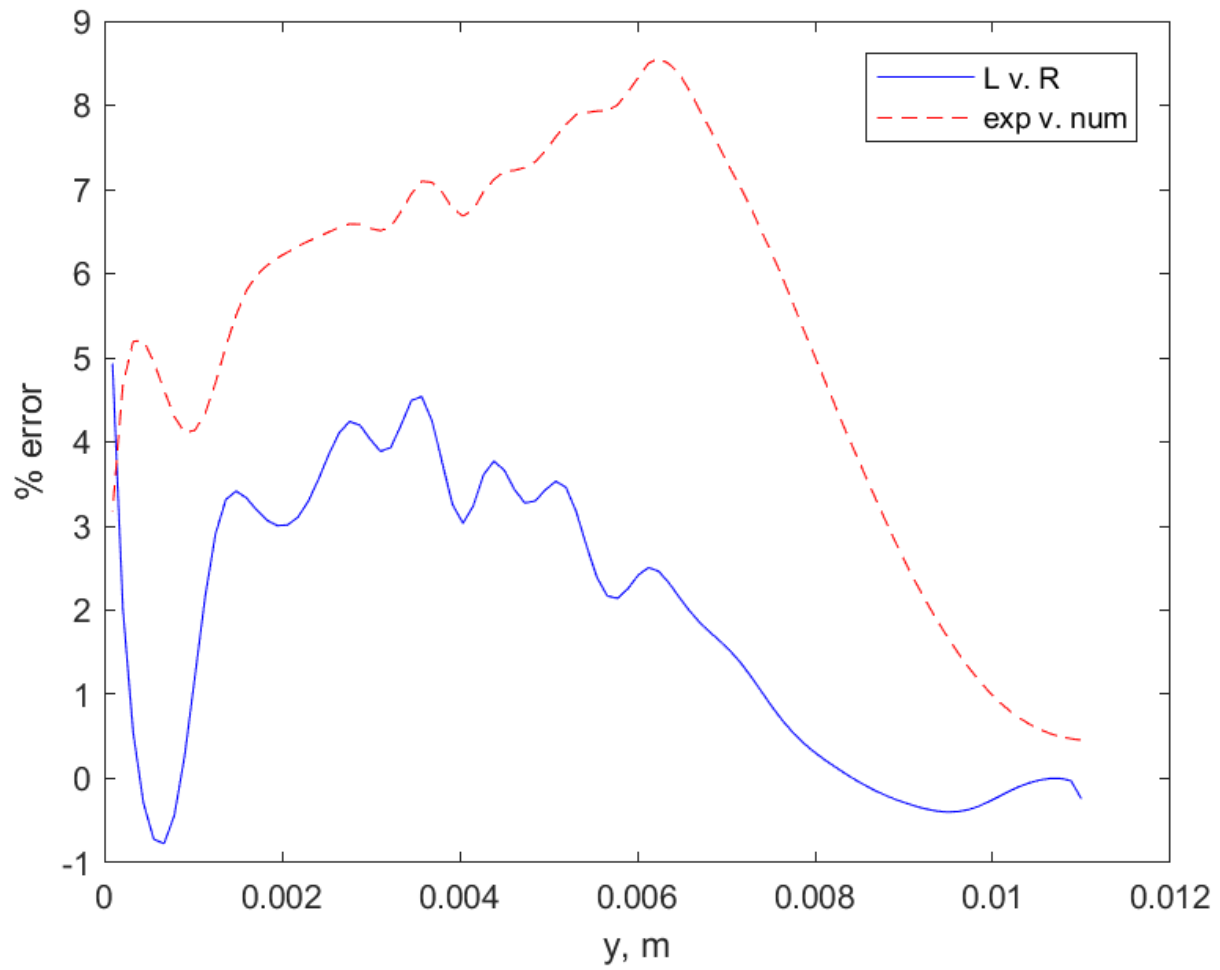

Figure B.12: Shot 62. Percent error in thermal boundary layer between both sides of experimental boundary layer profiles (red line) and percent error between predicted boundary layer profile from similarity solution and averaged experimental boundary layer profile (blue dashed line). 
75B

Shot 75:
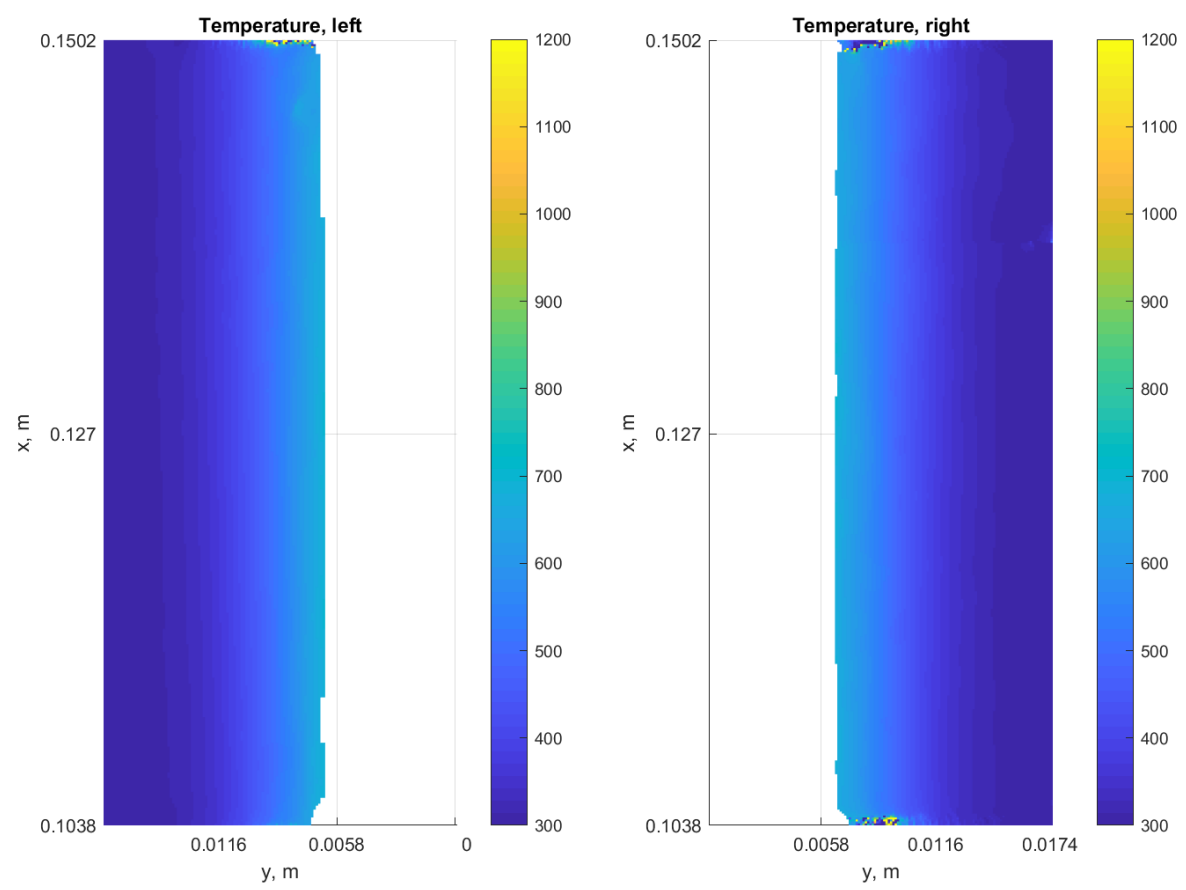

Figure B.13: Shot 75. Temperature fields from interferometry for nitrogen test. Surface temperature measured by pyrometer $=700 \mathrm{~K}$. 


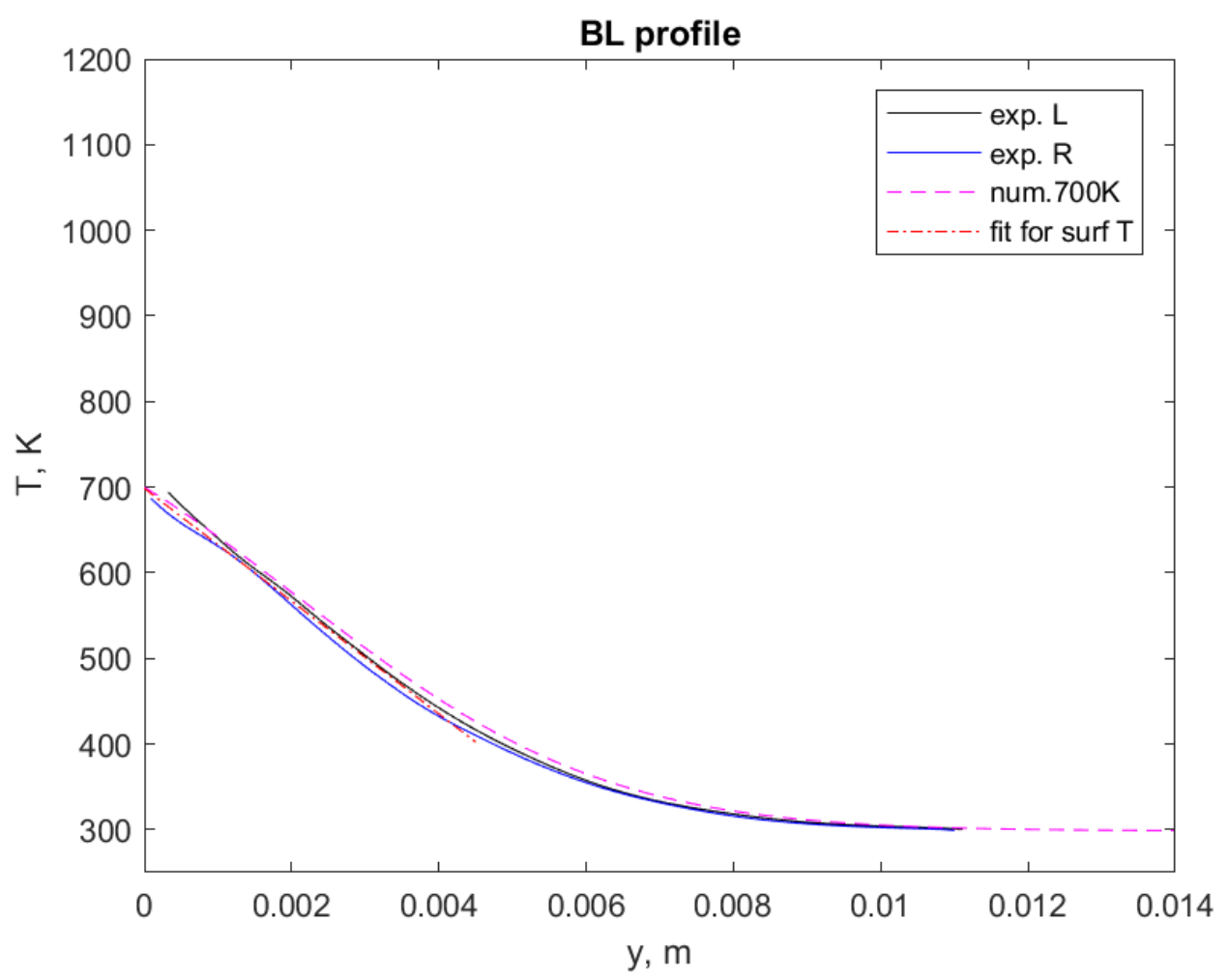

Figure B.14: Shot 75. Thermal boundary layer profiles for left (black line) and right (blue line) sides of experimental results compared with prediction of boundary layer profile (dashed magenta line) based on similarity solution for pyrometer surface temperature. Linear fit to averaged experimental profile (red dot-dashed line) used to extrapolate surface temperature from interferometer post processing. Extrapolated interferometer surface temperature $=698.5 \mathrm{~K}$. 


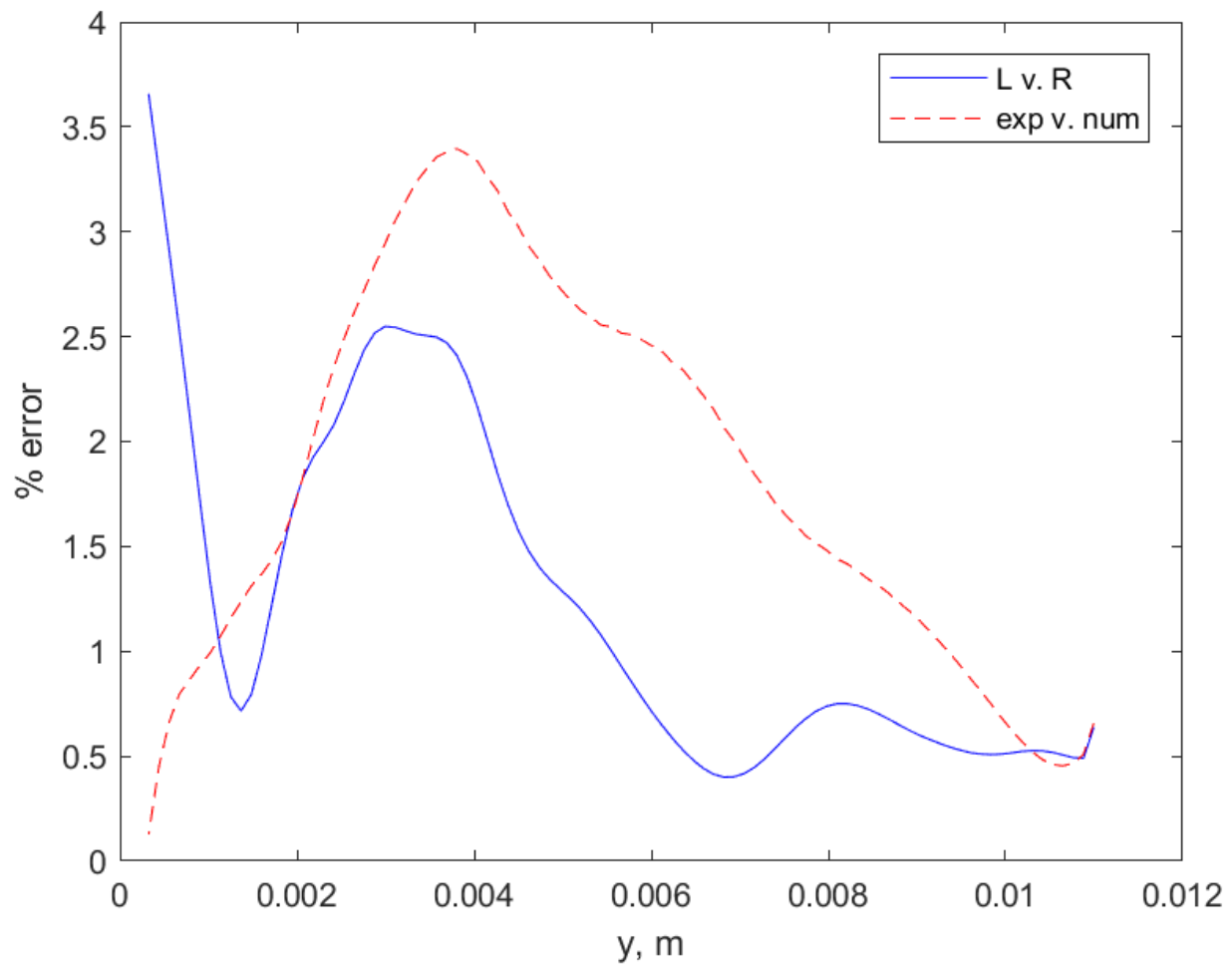

Figure B.15: Shot 75. Percent error in thermal boundary layer between both sides experimental boundary layer profiles (red line) and percent error between predicted boundary layer profile from similarity solution and averaged experimental boundary layer profile (blue dashed line). 
Shot 79:
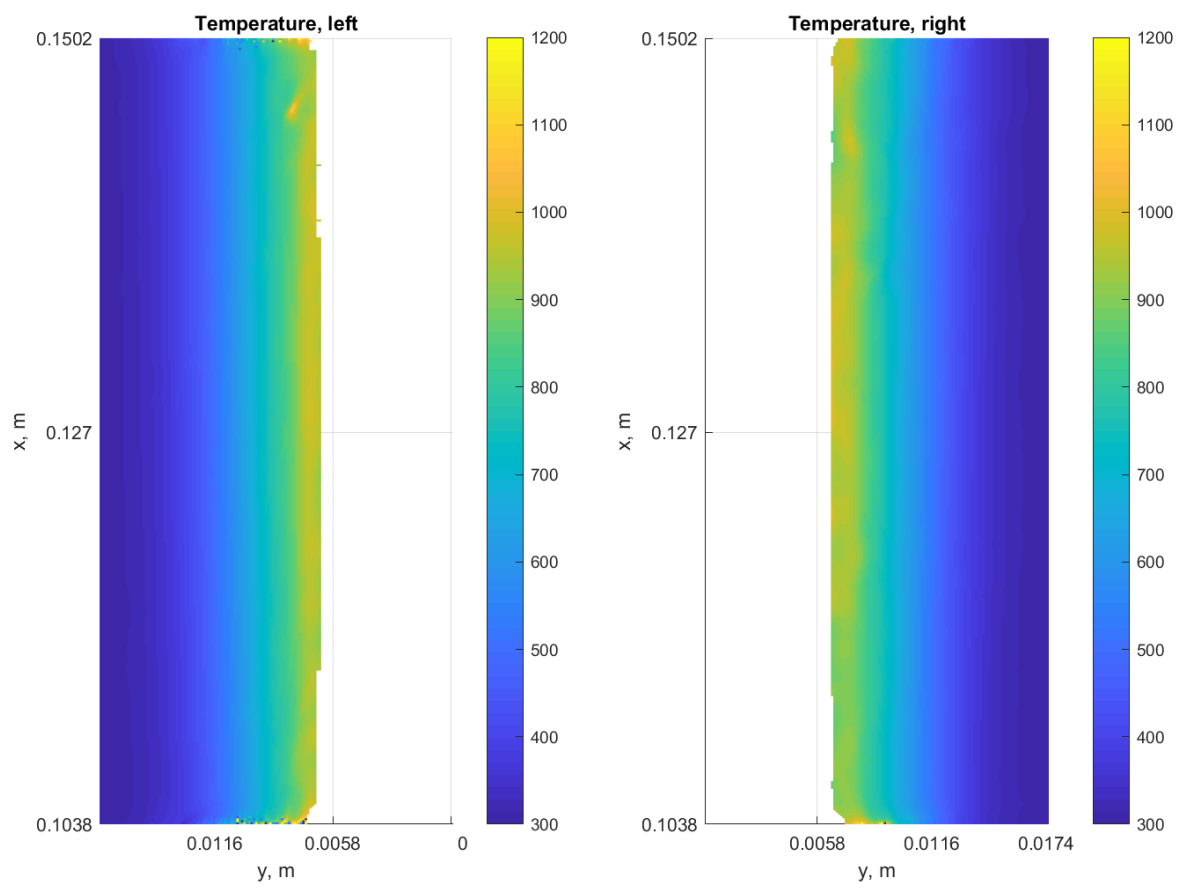

Figure B.16: Shot 79. Temperature fields from interferometry for nitrogen test. Surface temperature measured by pyrometer $=1100 \mathrm{~K}$. 


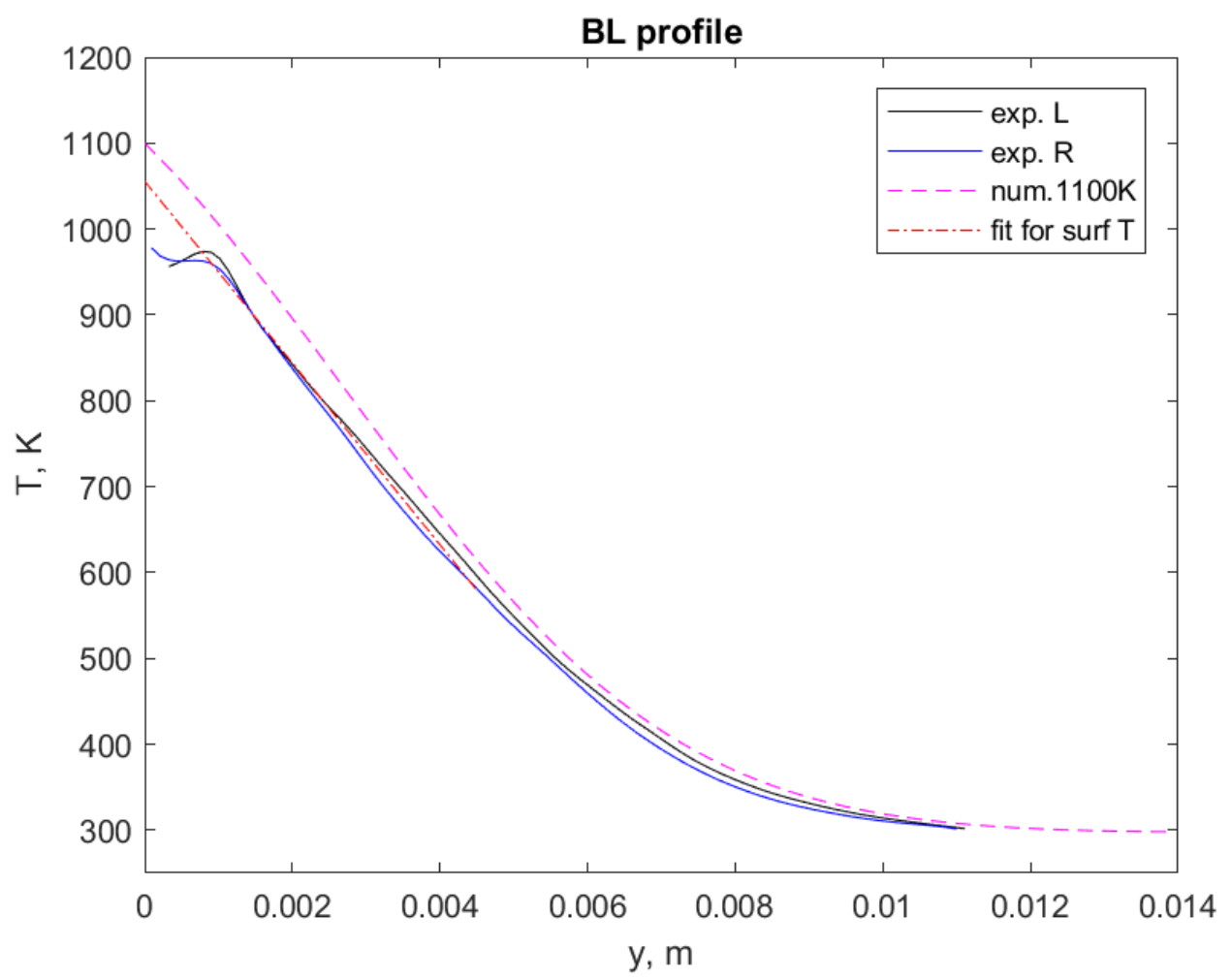

Figure B.17: Shot 79. Thermal boundary layer profiles for left (black line) and right (blue line) sides of experimental results compared with prediction of boundary layer profile (dashed magenta line) based on similarity solution for pyrometer surface temperature. Linear fit to averaged experimental profile (red dot-dashed line) used to extrapolate surface temperature from interferometer post processing. Extrapolated interferometer surface temperature $=1055.7 \mathrm{~K}$. 


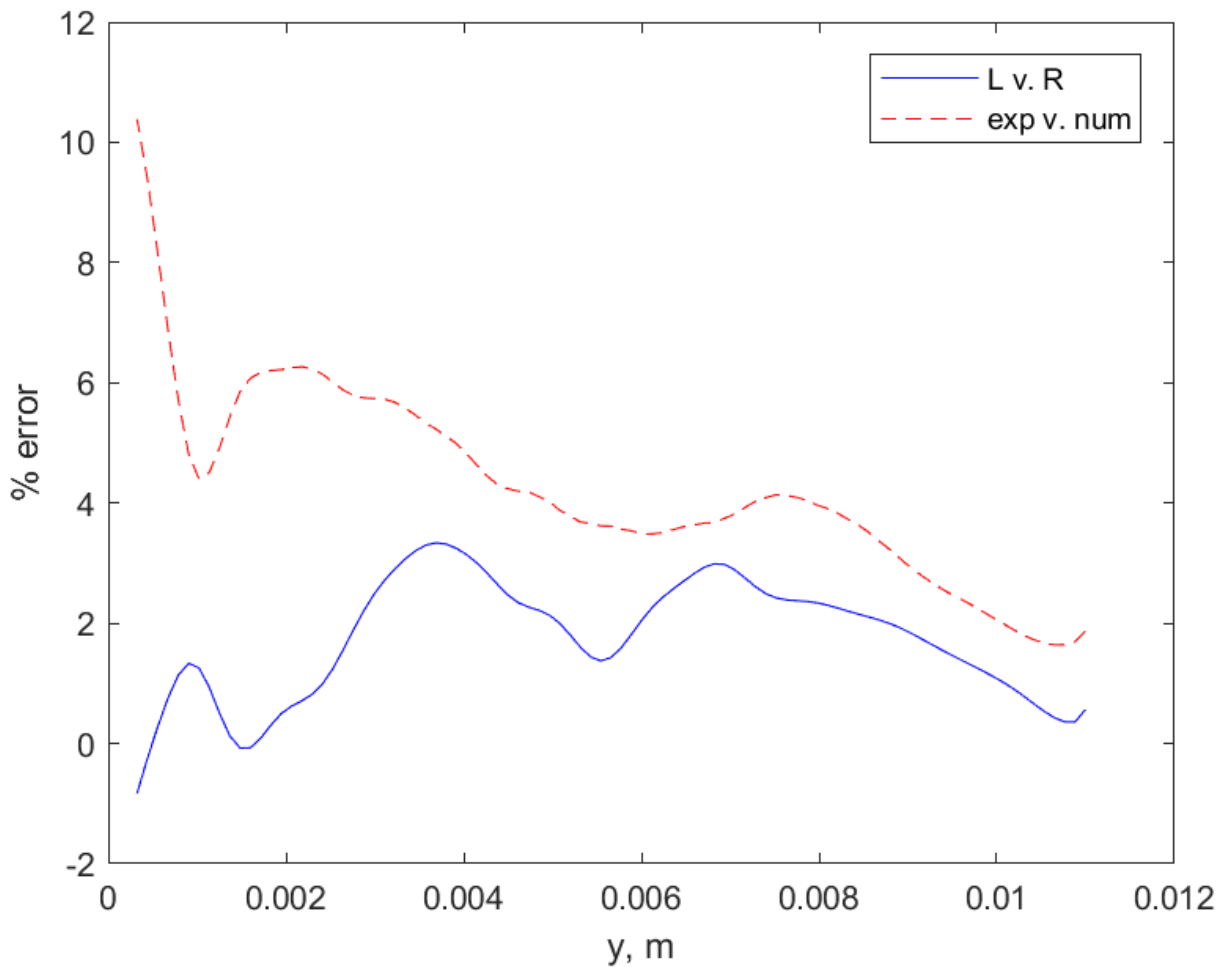

Figure B.18: Shot 79. Percent error in thermal boundary layer between both sides of experimental boundary layer profiles (red line) and percent error between predicted boundary layer profile from similarity solution and averaged experimental boundary layer profile (blue dashed line). 
100A

Shot 71:
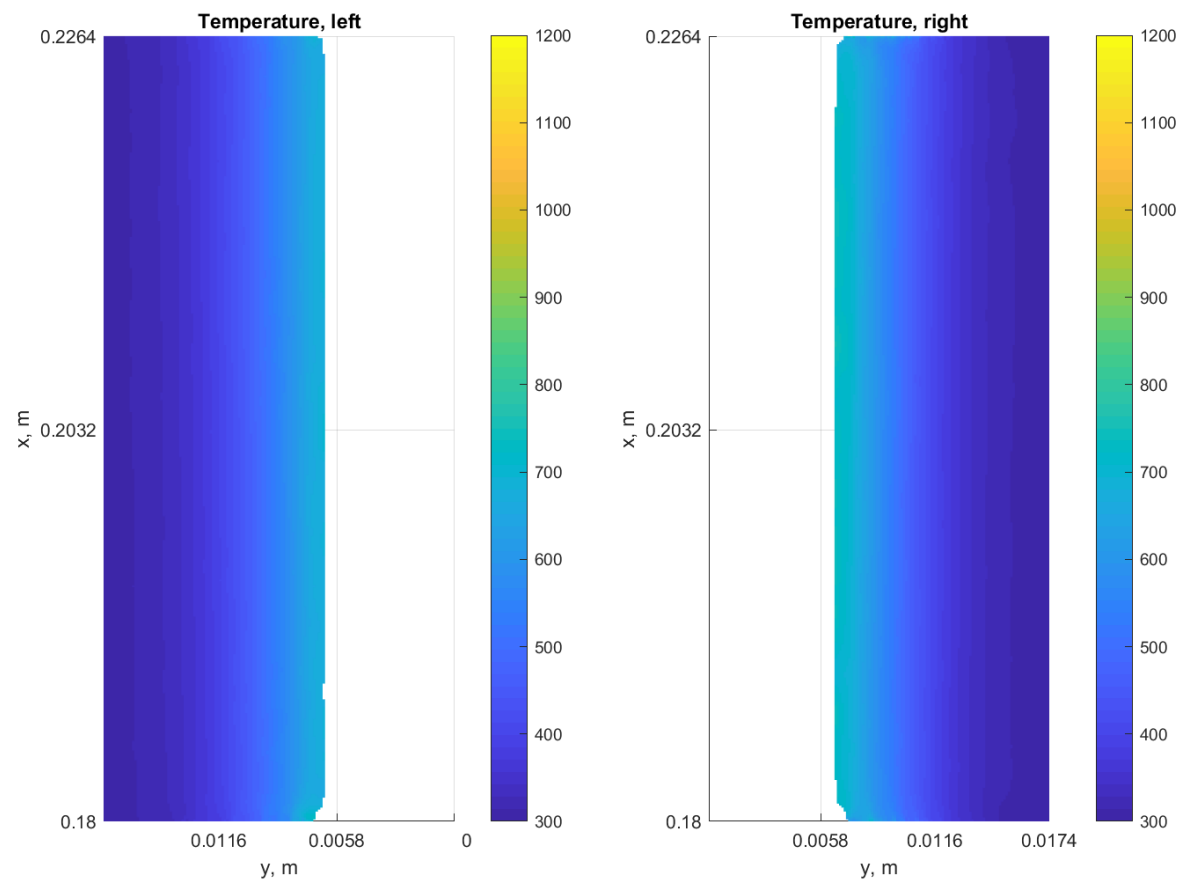

Figure B.19: Shot 71. Temperature fields from interferometry for nitrogen test. Surface temperature measured by pyrometer $=700 \mathrm{~K}$. 


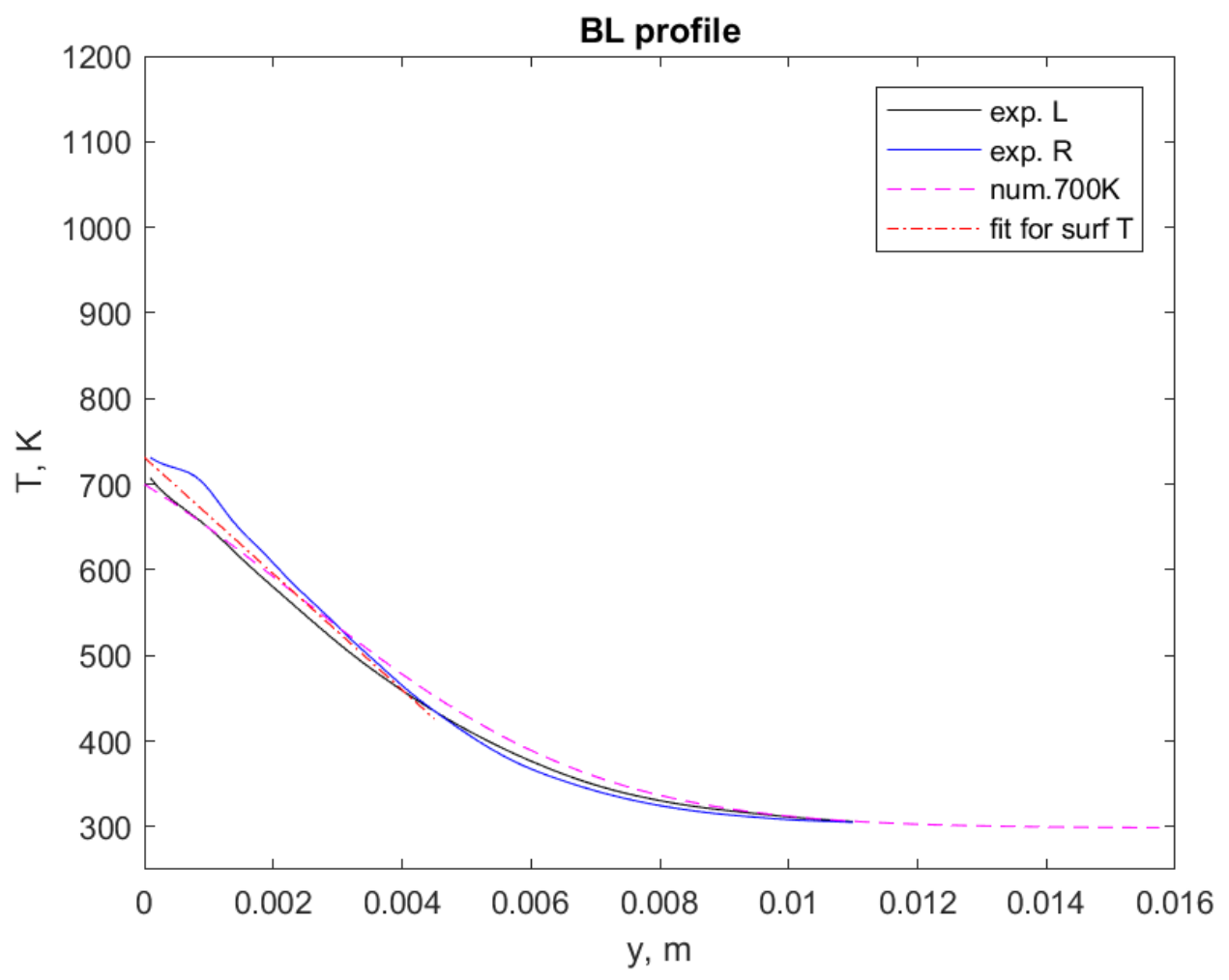

Figure B.20: Shot 71. Thermal boundary layer profiles for left (black line) and right (blue line) sides of experimental results compared with prediction of boundary layer profile (dashed magenta line) based on similarity solution for pyrometer surface temperature. Linear fit to averaged experimental profile (red dot-dashed line) used to extrapolate surface temperature from interferometer post processing. Extrapolated interferometer surface temperature $=731.3 \mathrm{~K}$. 


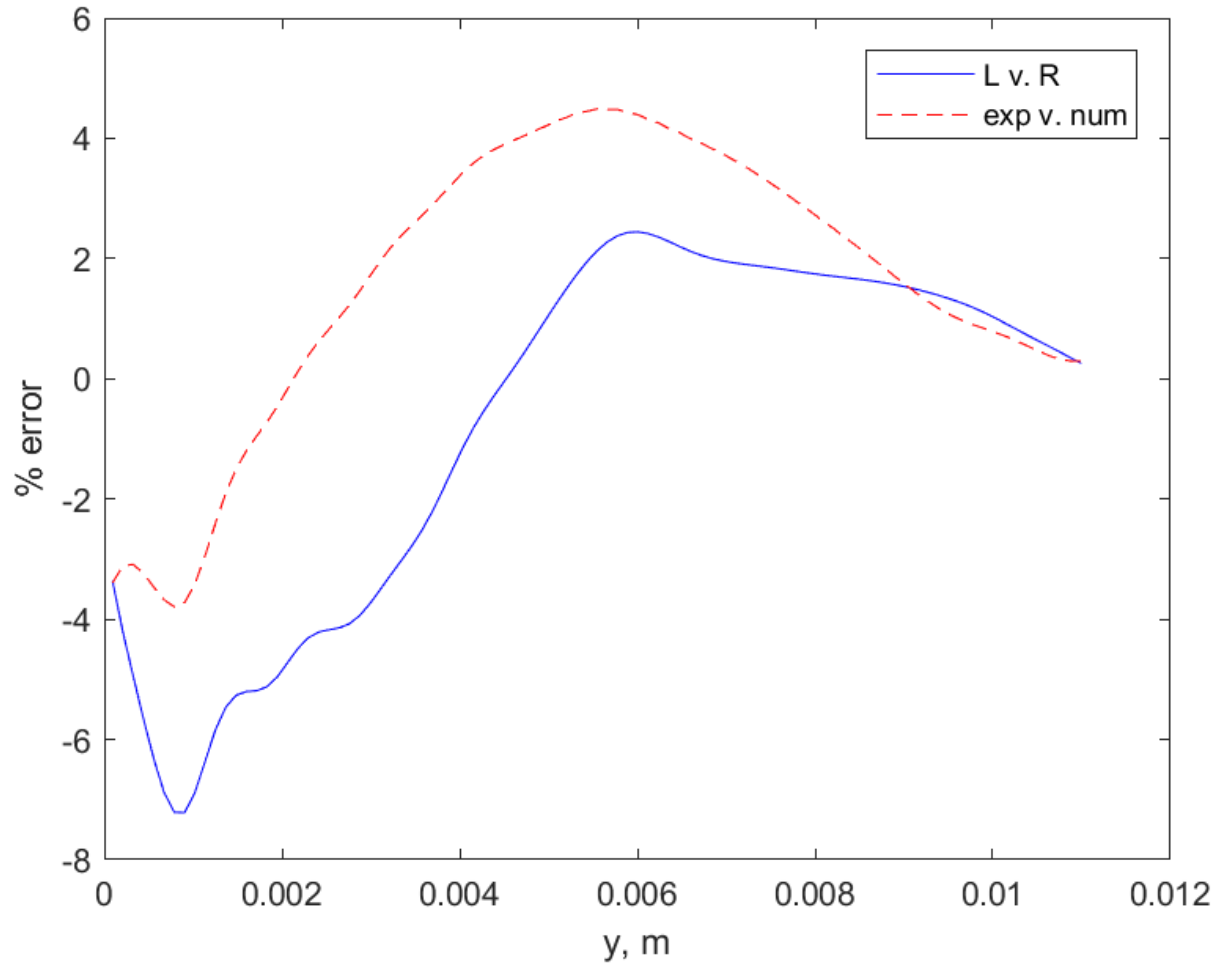

Figure B.21: Shot 71. Percent error in thermal boundary layer between both sides of experimental boundary layer profiles (red line) and percent error between predicted boundary layer profile from similarity solution and averaged experimental boundary layer profile (blue dashed line). 
Shot 72:
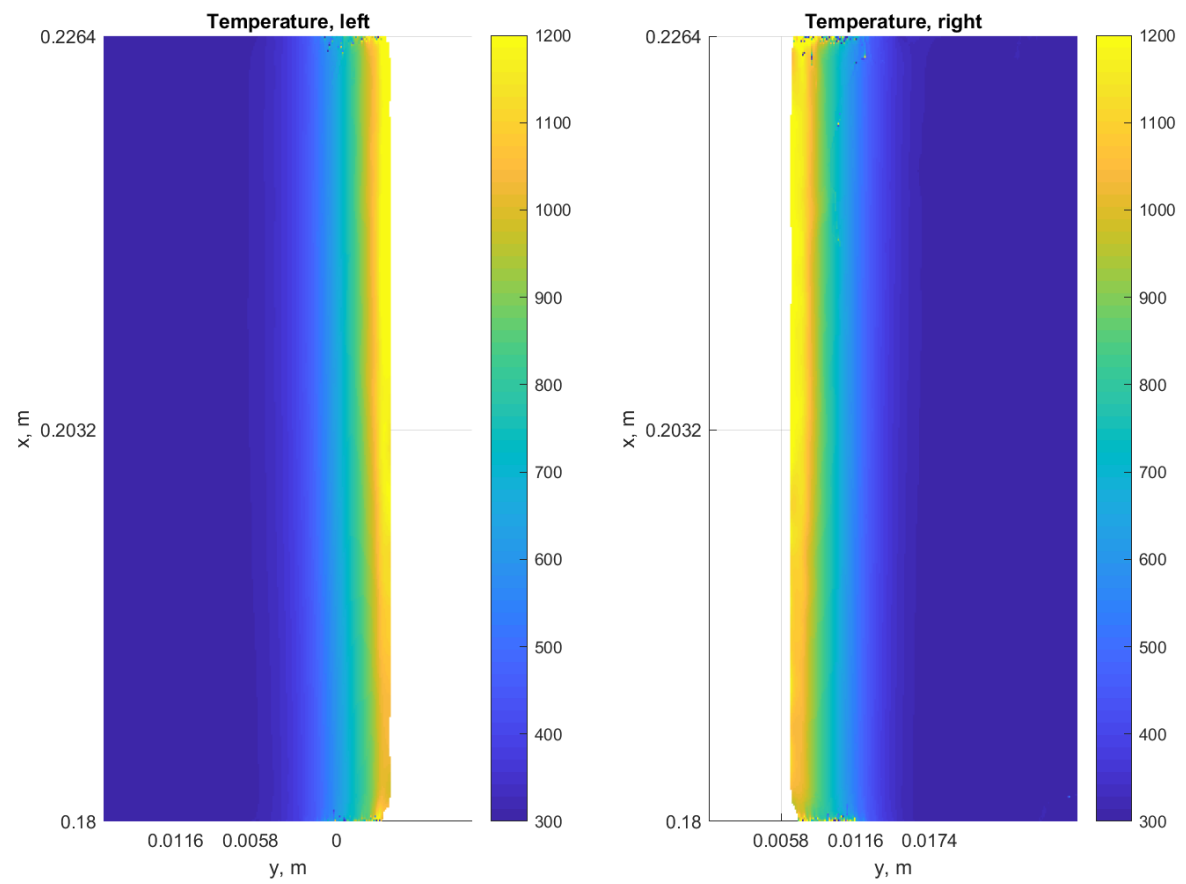

Figure B.22: Shot 72. Temperature fields from interferometry for nitrogen test. Surface temperature measured by pyrometer $=1100 \mathrm{~K}$. 


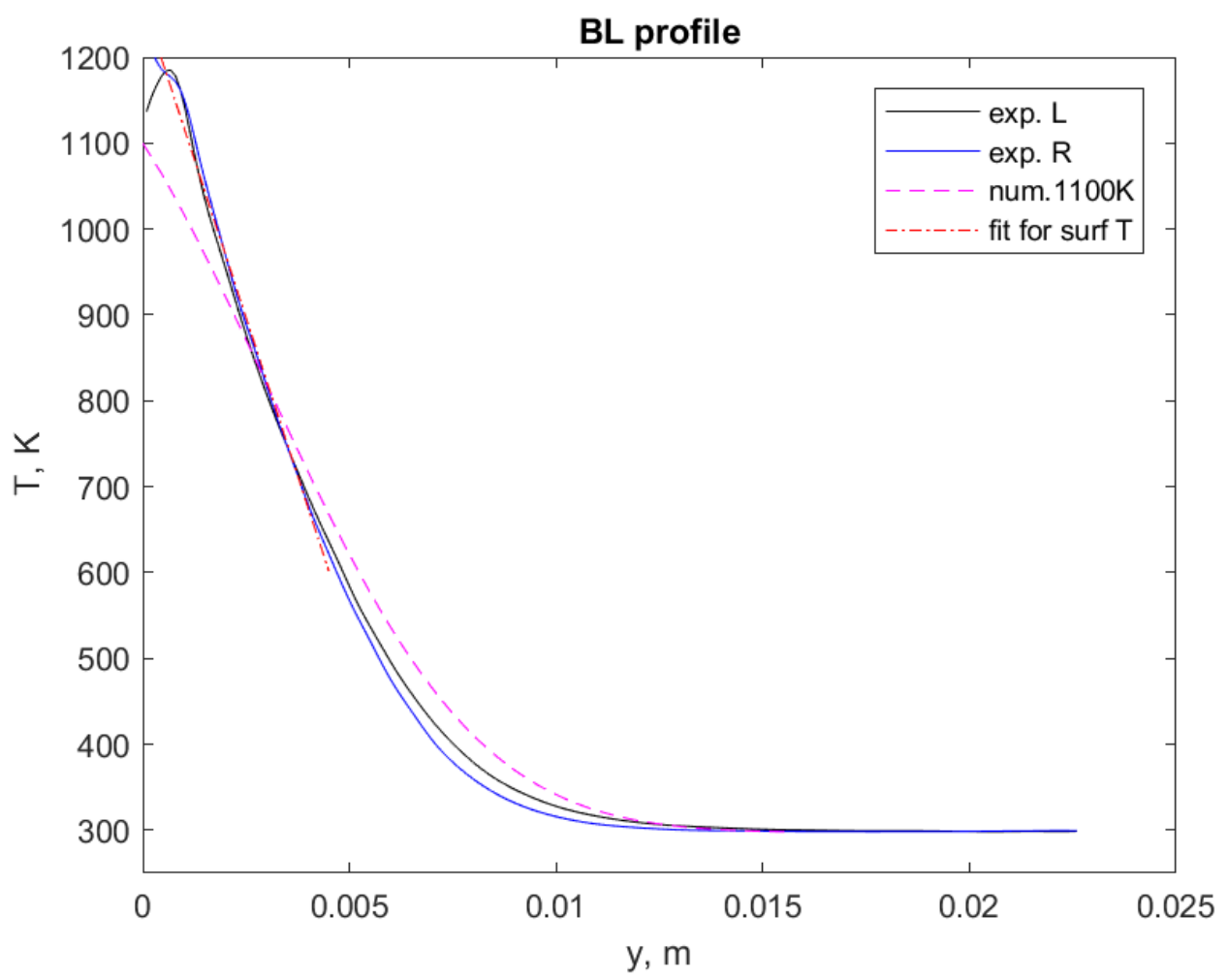

Figure B.23: Shot 72. Thermal boundary layer profiles for left (black line) and right (blue line) sides of experimental results compared with prediction of boundary layer profile (dashed magenta line) based on similarity solution for pyrometer surface temperature. Linear fit to averaged experimental profile (red dot-dashed line) used to extrapolate surface temperature from interferometer post processing. Extrapolated interferometer surface temperature $=1265.2 \mathrm{~K}$. 


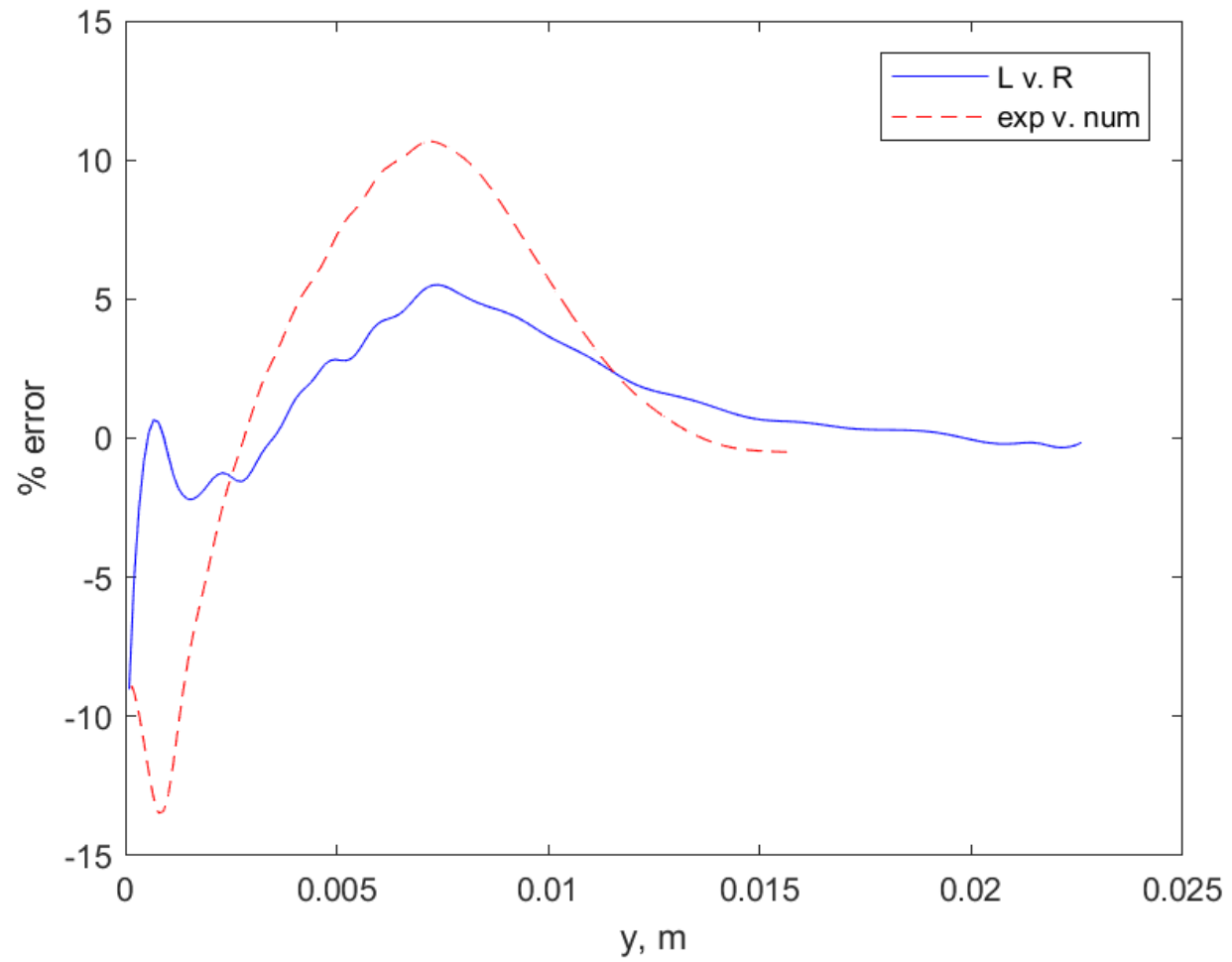

Figure B.24: Shot 72. Percent error in thermal boundary layer between both sides of experimental boundary layer profiles (red line) and percent error between predicted boundary layer profile from similarity solution and averaged experimental boundary layer profile (blue dashed line). 
100C

Shot 65:

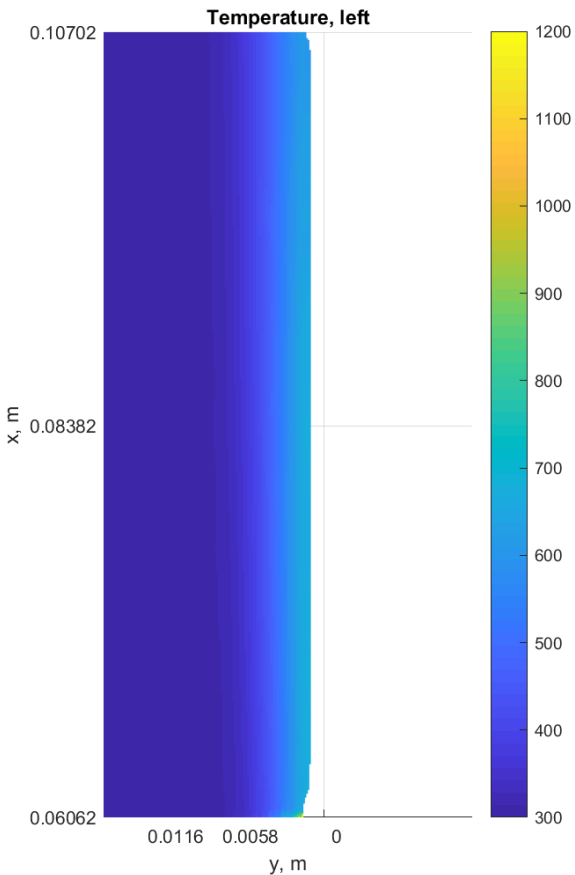

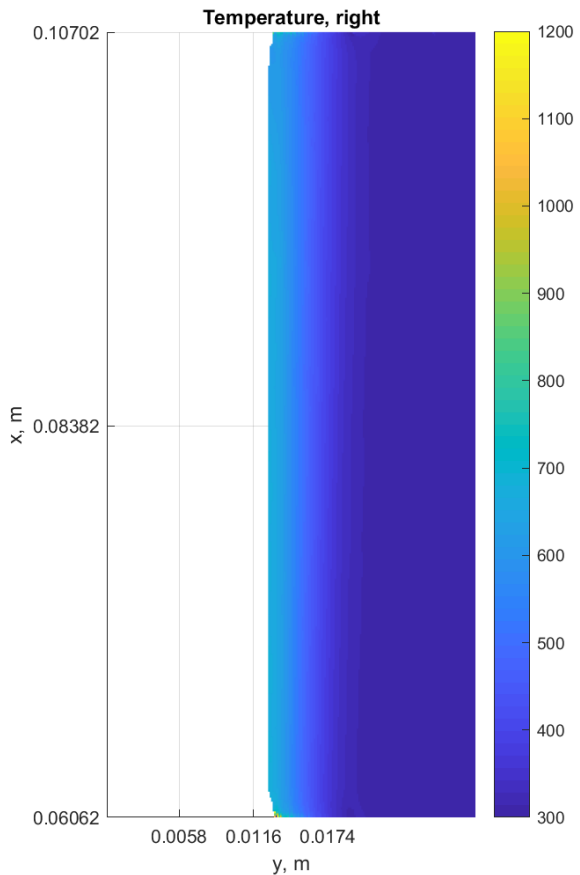

Figure B.25: Shot 65. Temperature fields from interferometry for nitrogen test. Surface temperature measured by pyrometer $=700 \mathrm{~K}$. 


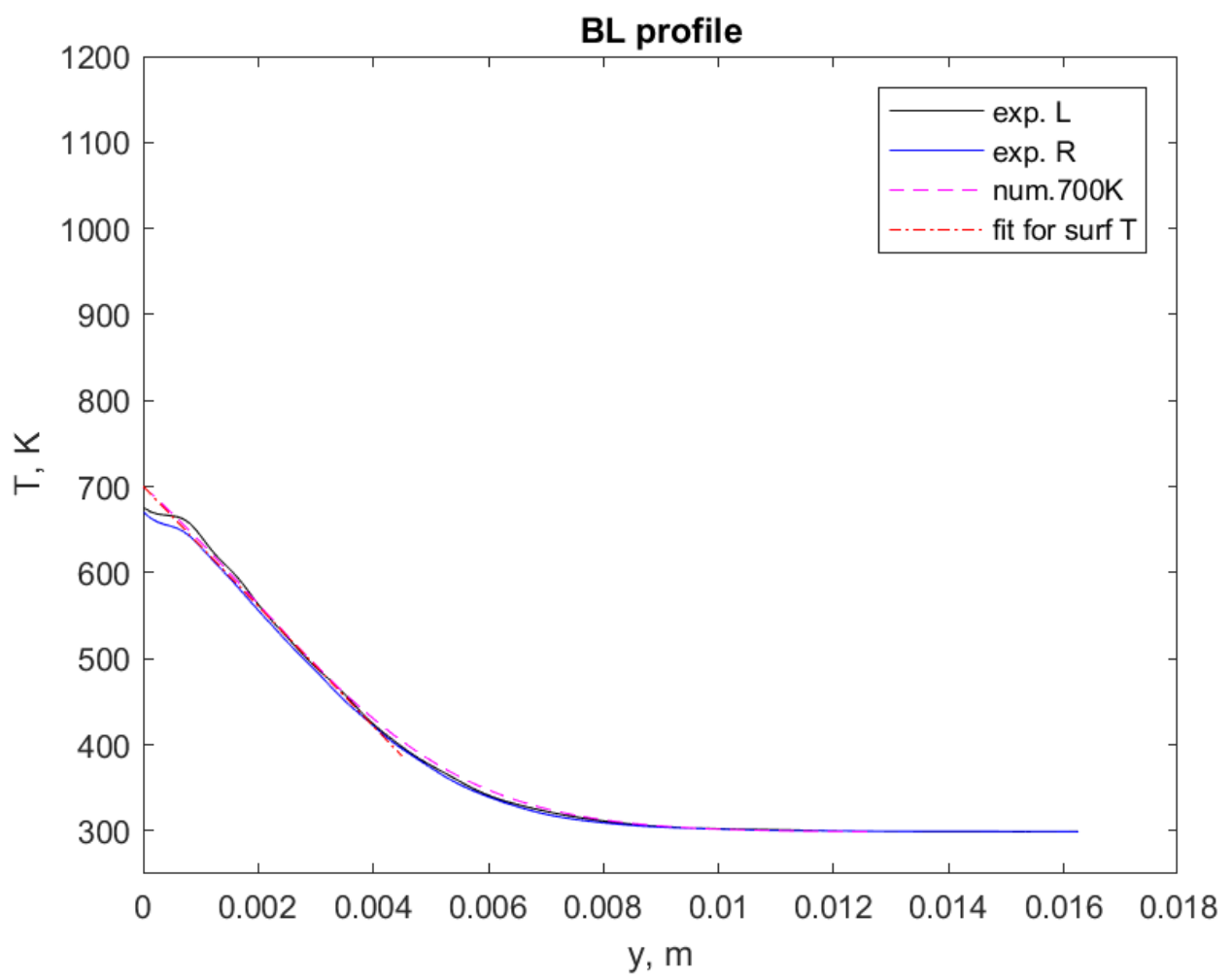

Figure B.26: Shot 65. Thermal boundary layer profiles for left (black line) and right (blue line) sides of experimental results compared with prediction of boundary layer profile (dashed magenta line) based on similarity solution for pyrometer surface temperature. Linear fit to averaged experimental profile (red dot-dashed line) used to extrapolate surface temperature from interferometer post processing. Extrapolated interferometer surface temperature $=700.6 \mathrm{~K}$. 


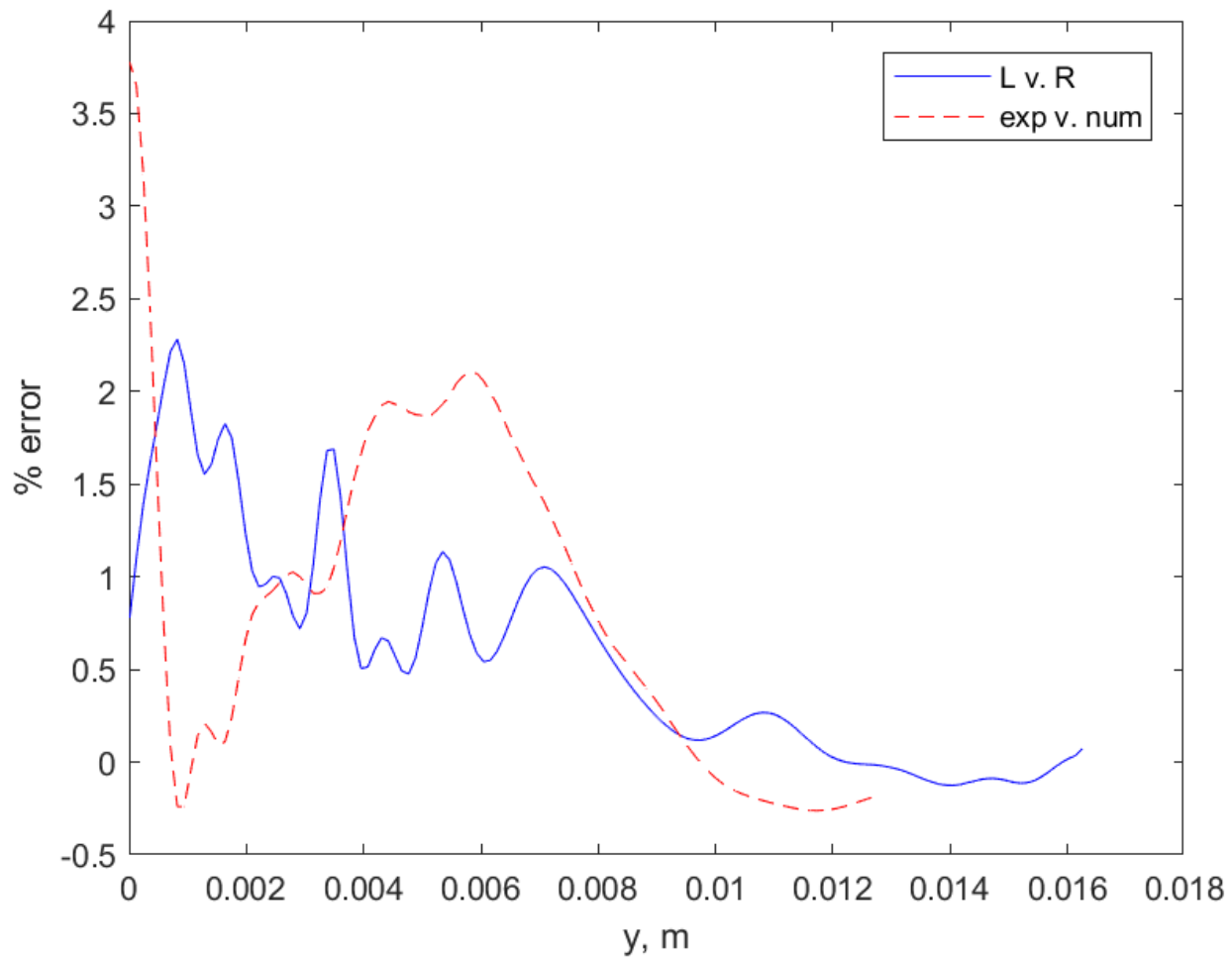

Figure B.27: Shot 65. Percent error in thermal boundary layer between both sides of experimental boundary layer profiles (red line) and percent error between predicted boundary layer profile from similarity solution and averaged experimental boundary layer profile (blue dashed line). 
Shot 66:
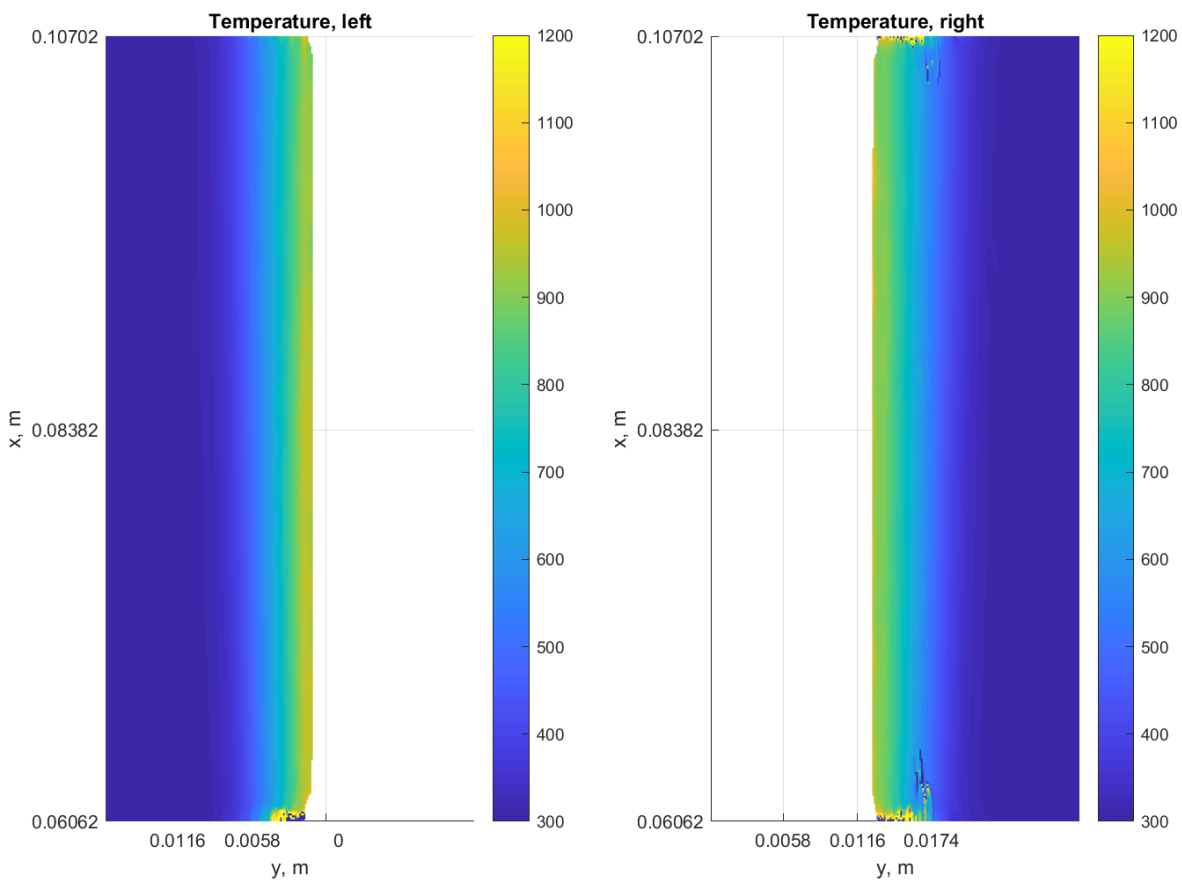

Figure B.28: Shot 66. Temperature fields from interferometry for nitrogen test. Surface temperature measured by pyrometer $=1000 \mathrm{~K}$. 


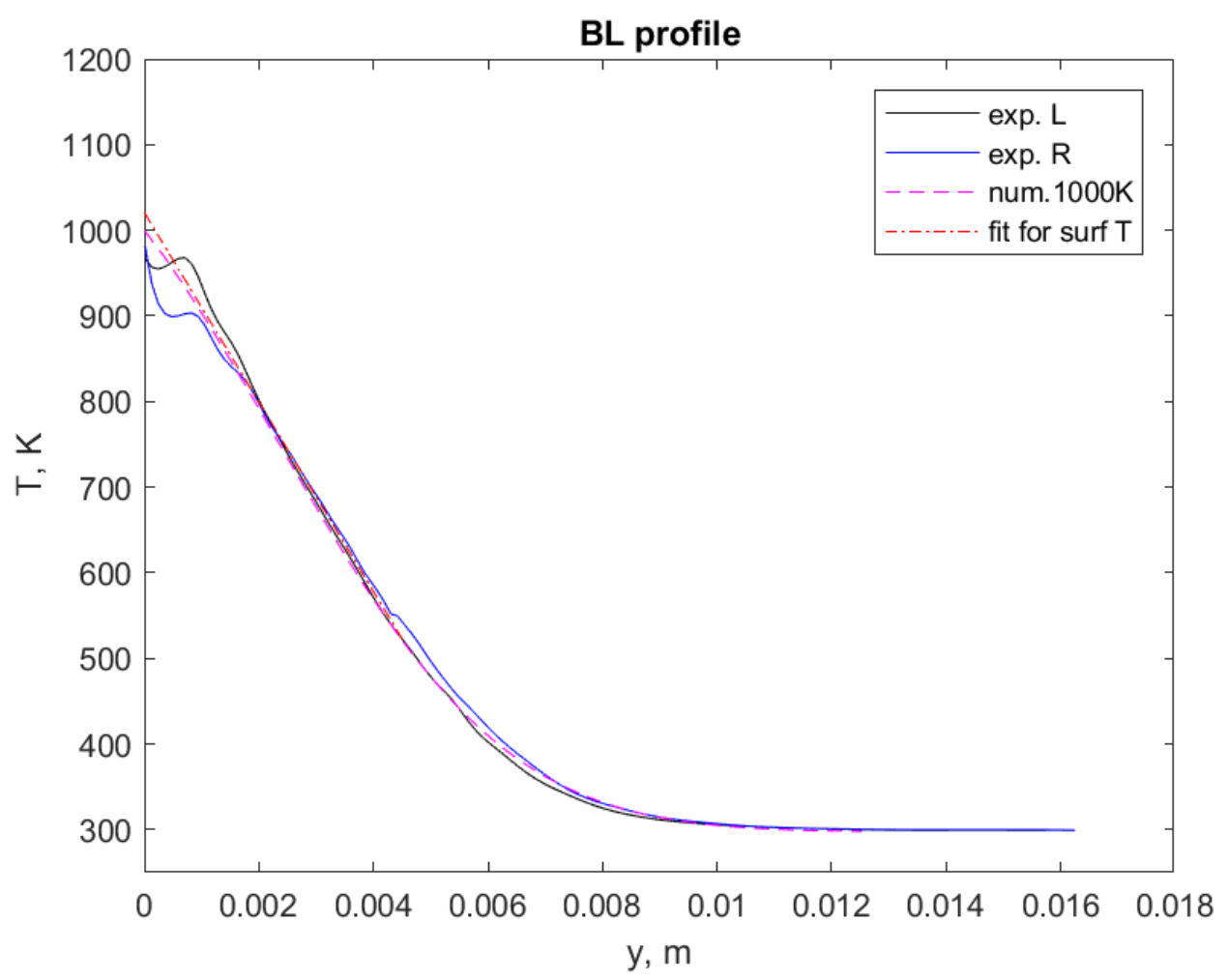

Figure B.29: Shot 66. Thermal boundary layer profiles for left (black line) and right (blue line) sides of experimental results compared with prediction of boundary layer profile (dashed magenta line) based on similarity solution for pyrometer surface temperature. Linear fit to averaged experimental profile (red dot-dashed line) used to extrapolate surface temperature from interferometer post processing. Extrapolated interferometer surface temperature $=1020.7 \mathrm{~K}$. 


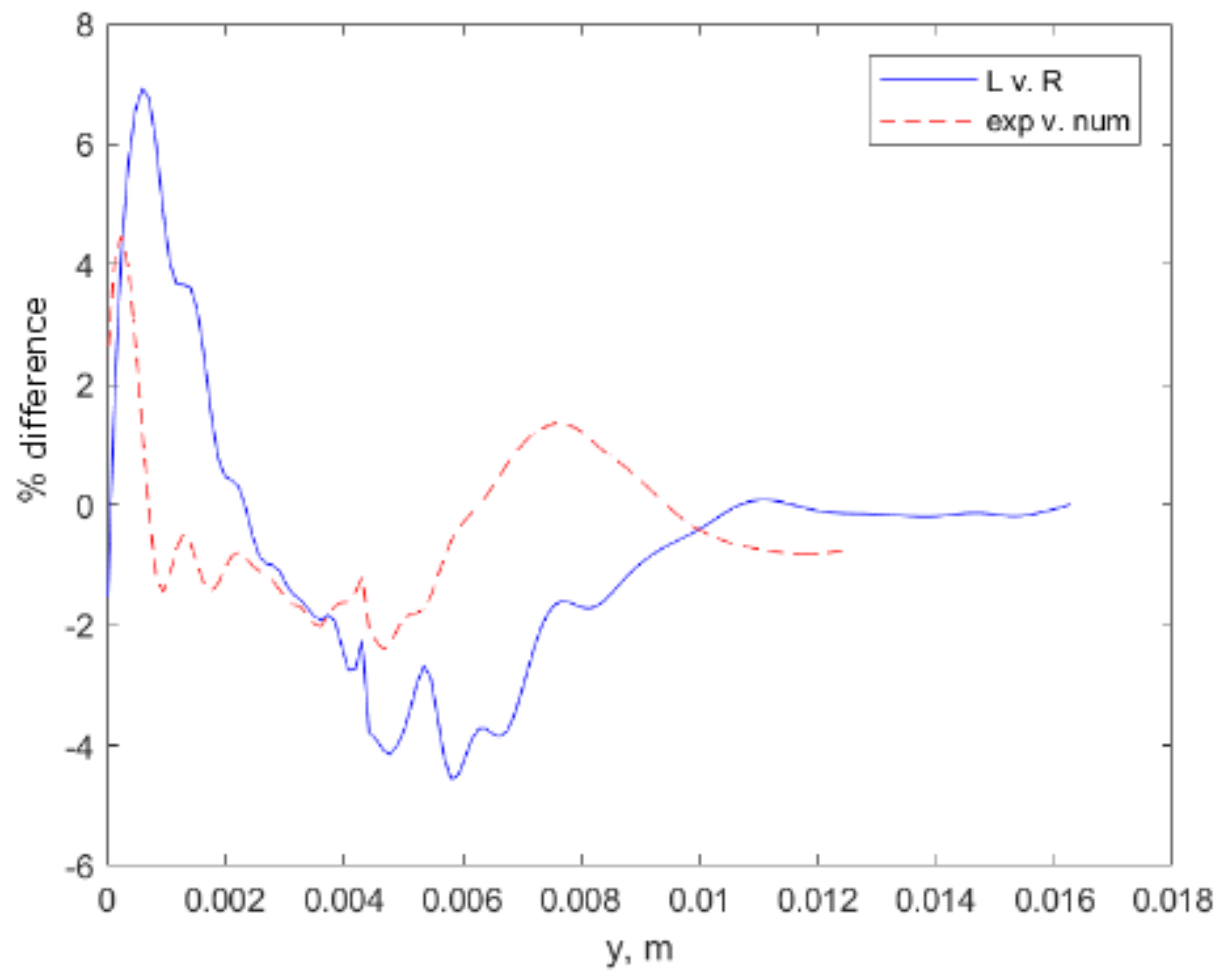

Figure B.30: Shot 66. Percent error in thermal boundary layer between both sides of experimental boundary layer profiles (red line) and percent error between predicted boundary layer profile from similarity solution and averaged experimental boundary layer profile (blue dashed line). 
200A

Shot 44:

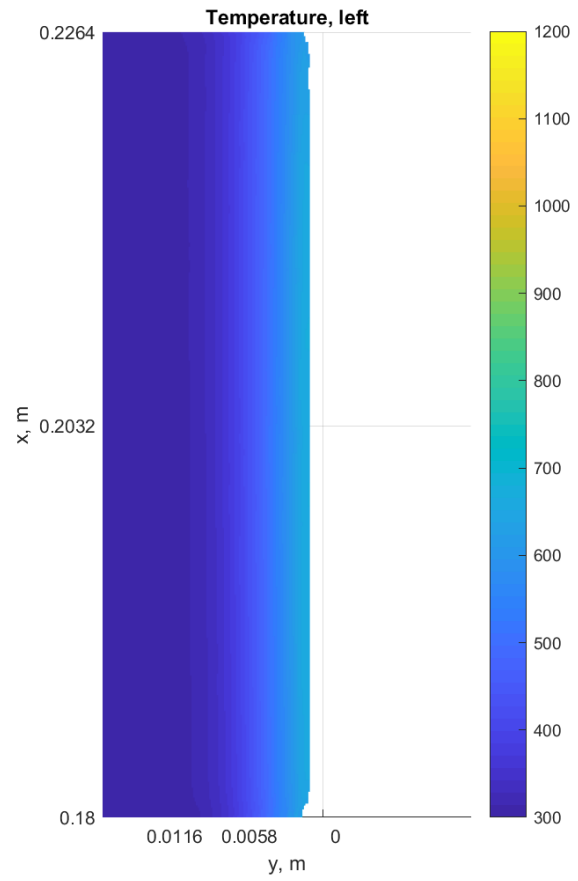

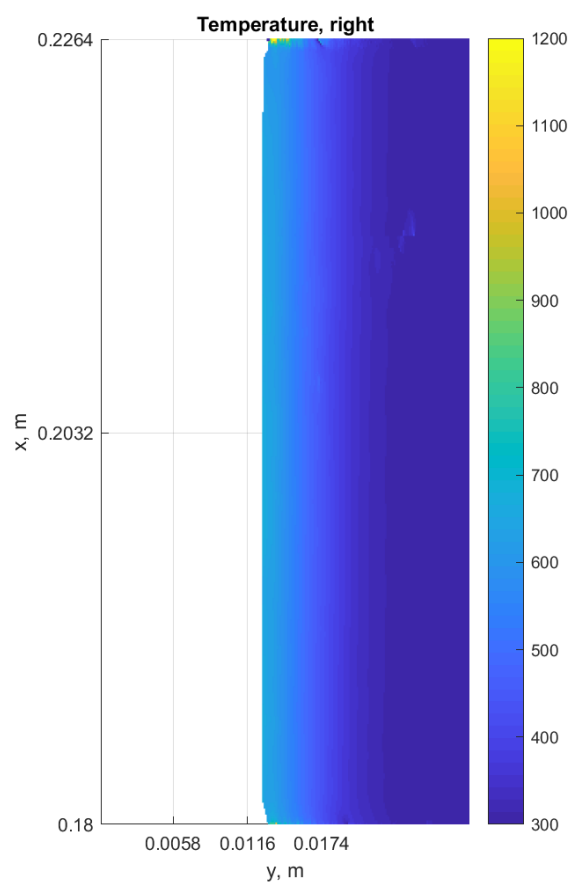

Figure B.31: Shot 44. Temperature fields from interferometry for nitrogen test. Surface temperature measured by pyrometer $=700 \mathrm{~K}$. 


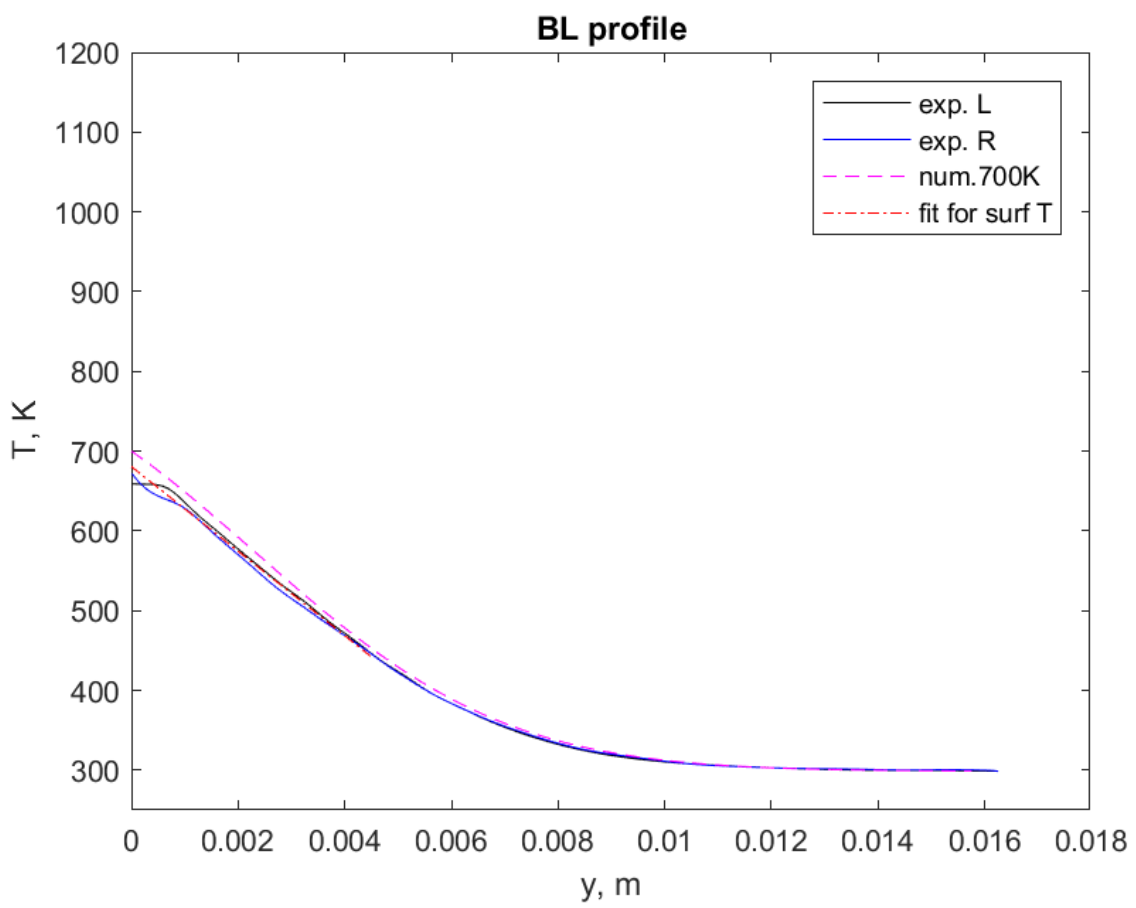

Figure B.32: Shot 44. Thermal boundary layer profiles for left (black line) and right (blue line) sides of experimental results compared with prediction of boundary layer profile (dashed magenta line) based on similarity solution for pyrometer surface temperature. Linear fit to averaged experimental profile (red dot-dashed line) used to extrapolate surface temperature from interferometer post processing. Extrapolated interferometer surface temperature $=680.6 \mathrm{~K}$. 


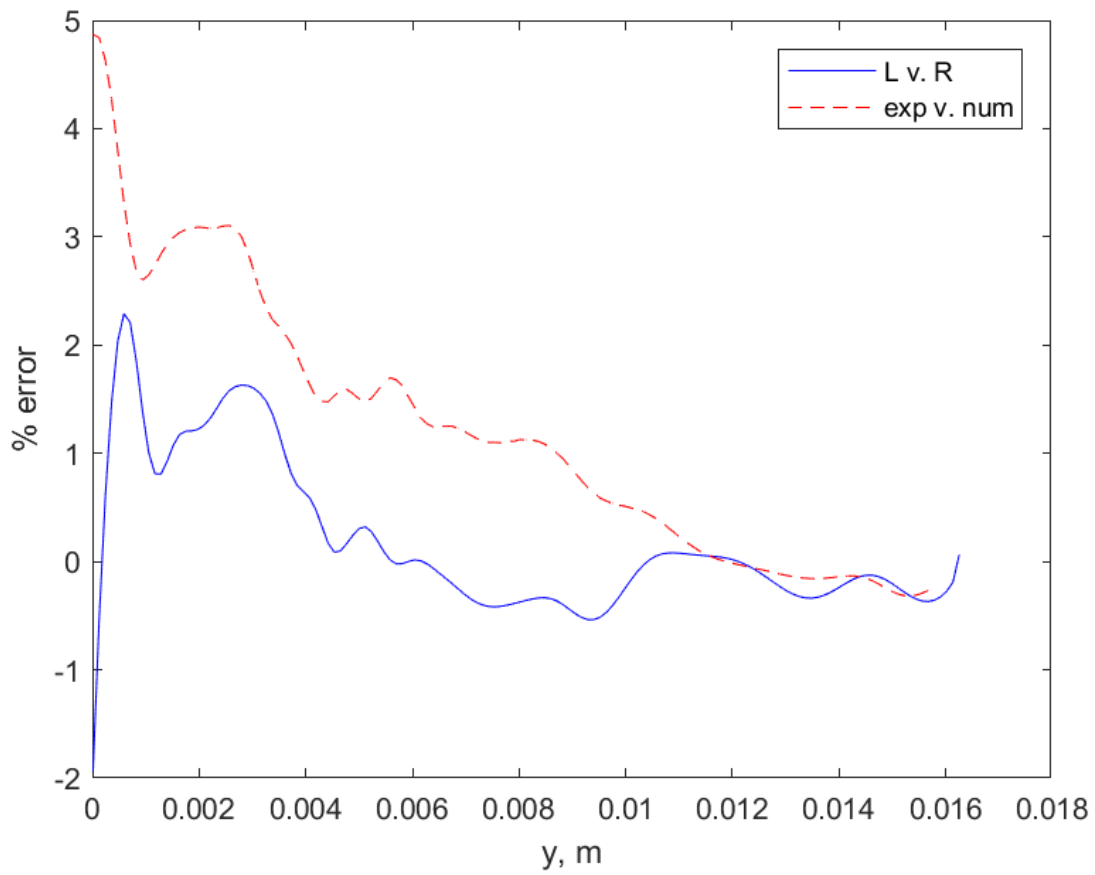

Figure B.33: Shot 44. Percent error in thermal boundary layer between both sides of experimental boundary layer profiles (red line) and percent error between predicted boundary layer profile from similarity solution and averaged experimental boundary layer profile (blue dashed line). 
Shot 45:
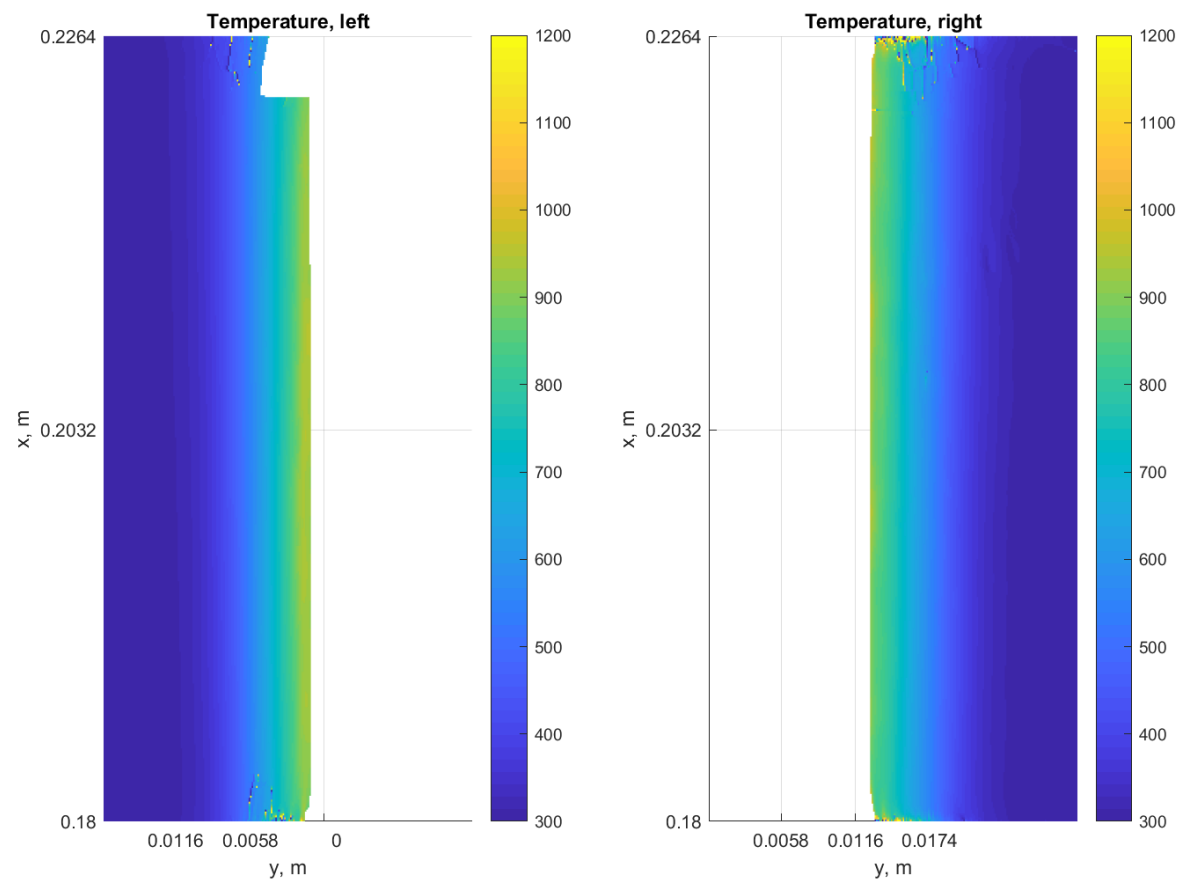

Figure B.34: Shot 45. Temperature fields from interferometry for nitrogen test. Surface temperature measured by pyrometer $=1000 \mathrm{~K}$. 


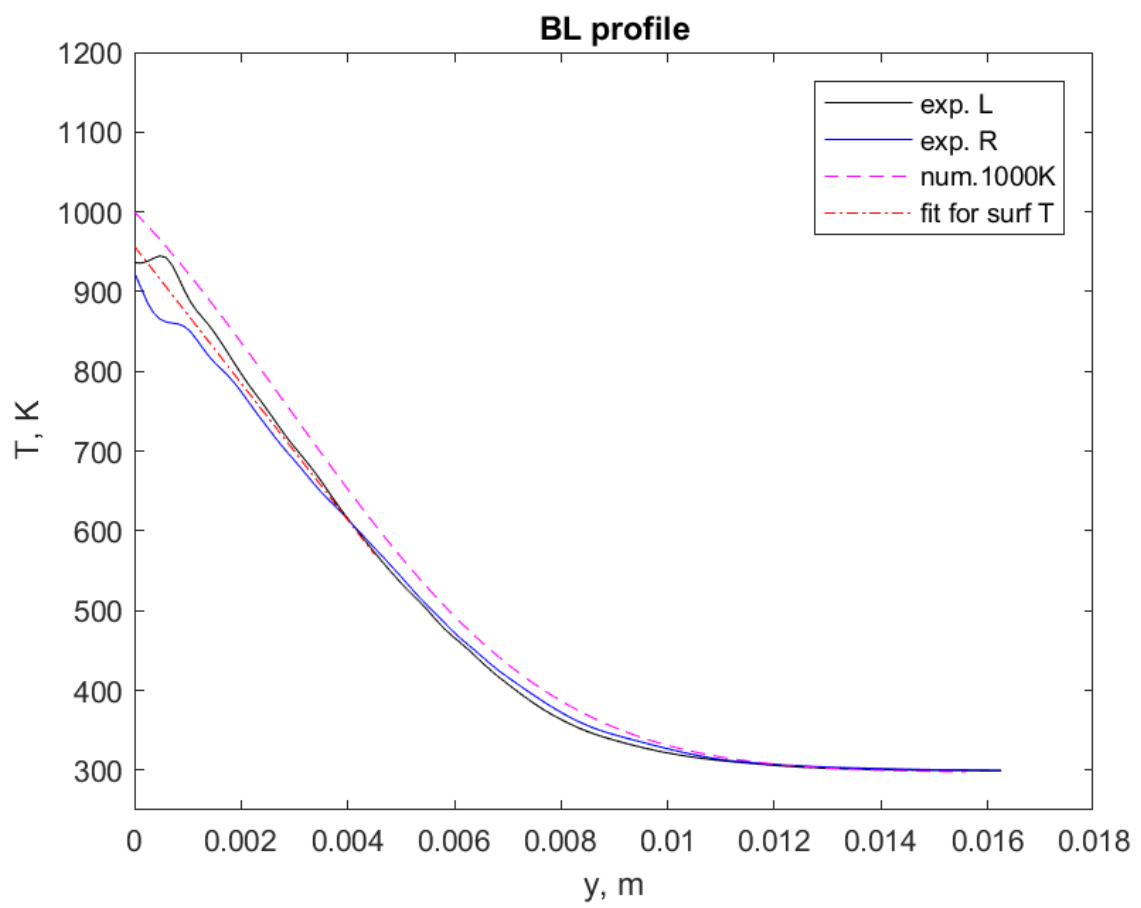

Figure B.35: Shot 45. Thermal boundary layer profiles for left (black line) and right (blue line) sides of experimental results compared with prediction of boundary layer profile (dashed magenta line) based on similarity solution for pyrometer surface temperature. Linear fit to averaged experimental profile (red dot-dashed line) used to extrapolate surface temperature from interferometer post processing. Extrapolated interferometer surface temperature $=956.9 \mathrm{~K}$. 


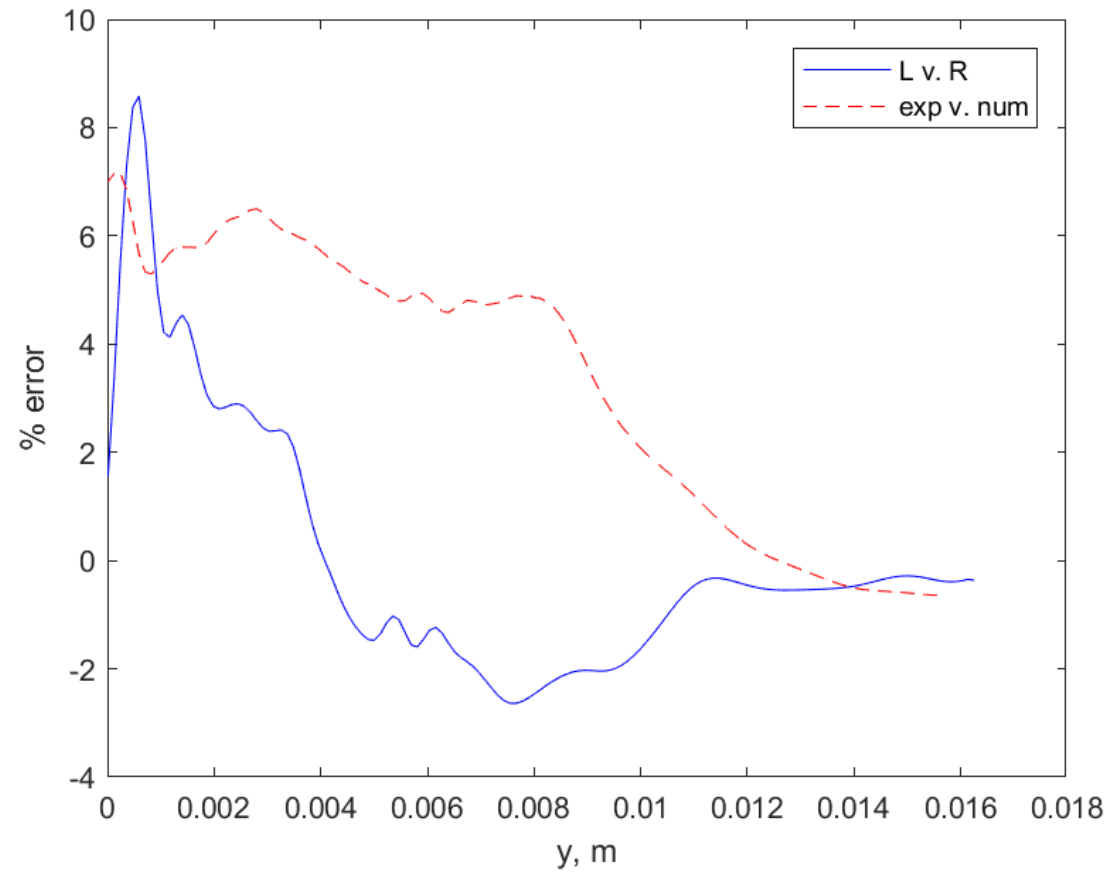

Figure B.36: Shot 45. Percent error in thermal boundary layer between both sides of experimental boundary layer profiles (red line) and percent error between predicted boundary layer profile from similarity solution and averaged experimental boundary layer profile (blue dashed line). 
Appendix $C$

INTERFEROMETER POST-PROCESSED RESULTS, HEXANE

\begin{tabular}{|c||ccccccc|}
\hline Cyl Tag & shot & pyr T $(\mathrm{K})$ & intf T $(\mathrm{K})$ & $\Delta \mathrm{T}(\mathrm{K})$ & \% diff. T & length $(\mathrm{s})$ & symmetry \\
\hline 50A & 62 & 1087 & 1088.7 & -1.7 & -0.2 & 30 & mostly symm. \\
50A & 63 & 1060 & 993.6 & 66.4 & 6.3 & 30 & very symm. \\
50A & 64 & 1070 & 1036.1 & 33.9 & 3.2 & 30 & very symm. \\
50C & 41 & 1106 & 996.6 & 109.4 & 9.9 & 30 & mostly symm. \\
50C & 52 & 1107 & 1012.7 & 94.3 & 8.5 & 30 & mostly symm. \\
50C & 53 & 1100 & 1003.4 & 96.6 & 8.8 & 30 & asymm. \\
75B & 16 & 1074 & 1059.3 & 14.3 & 1.3 & 30 & mostly symm. \\
75B & 31 & 1104 & 1006.5 & 97.7 & 8.8 & 30 & mostly symm. \\
75B & 32 & 1091 & 987.4 & 103.6 & 9.5 & 30 & asymm. \\
100A & 91 & 1038 & 951.2 & 86.8 & 8.4 & 30 & very symm. \\
100A & 93 & 1045 & 998.1 & 46.9 & 4.5 & 30 & very symm. \\
100A & 95 & 1004 & 973.9 & 30.1 & 3.0 & 30 & very symm. \\
100A & 101 & 1060 & 967.7 & 92.3 & 8.7 & 100 & very symm. \\
100A & 103 & 1011 & 952.9 & 58.1 & 5.7 & 100 & mostly symm. \\
100A & 106 & 1049 & 1012.6 & 36.4 & 3.5 & 100 & mostly symm. \\
100C & 84 & 1001 & 913.3 & 87.7 & 8.8 & 30 & mostly symm. \\
100C & 157 & 1107 & 790.7 & 316.3 & 28.6 & 100 & very symm. \\
100C & 164 & 1080 & 940.2 & 139.8 & 12.9 & 30 & asymm. \\
100C & 168 & 1070 & 919.8 & 150.2 & 14.0 & 30 & mostly symm. \\
100C & 169 & 1029 & 905.5 & 123.5 & 12.0 & 100 & mostly symm. \\
100C & 170 & 1062 & 909.4 & 152.6 & 14.4 & 100 & mostly symm. \\
200A & 123 & 1002 & 917.8 & 184.2 & 18.4 & 100 & mostly symm. \\
200A & 124 & 1002 & 833.9 & 168.1 & 16.8 & 100 & mostly symm. \\
200A & 127 & 1001 & 812.1 & 188.9 & 18.9 & 100 & mostly symm. \\
200A & 142 & 1028 & 843.6 & 184.4 & 17.9 & 100 & very symm. \\
200A & 143 & 1018 & 831.2 & 186.8 & 18.3 & 100 & very symm. \\
\hline
\end{tabular}

Table C.1: All processed interferometry results in stoichiometric hexane-air mixture.

\section{A}

No processable hexane shots for $25 \mathrm{~A}$, cylinder too narrow to find center accurately.

50A

Shot 62: 

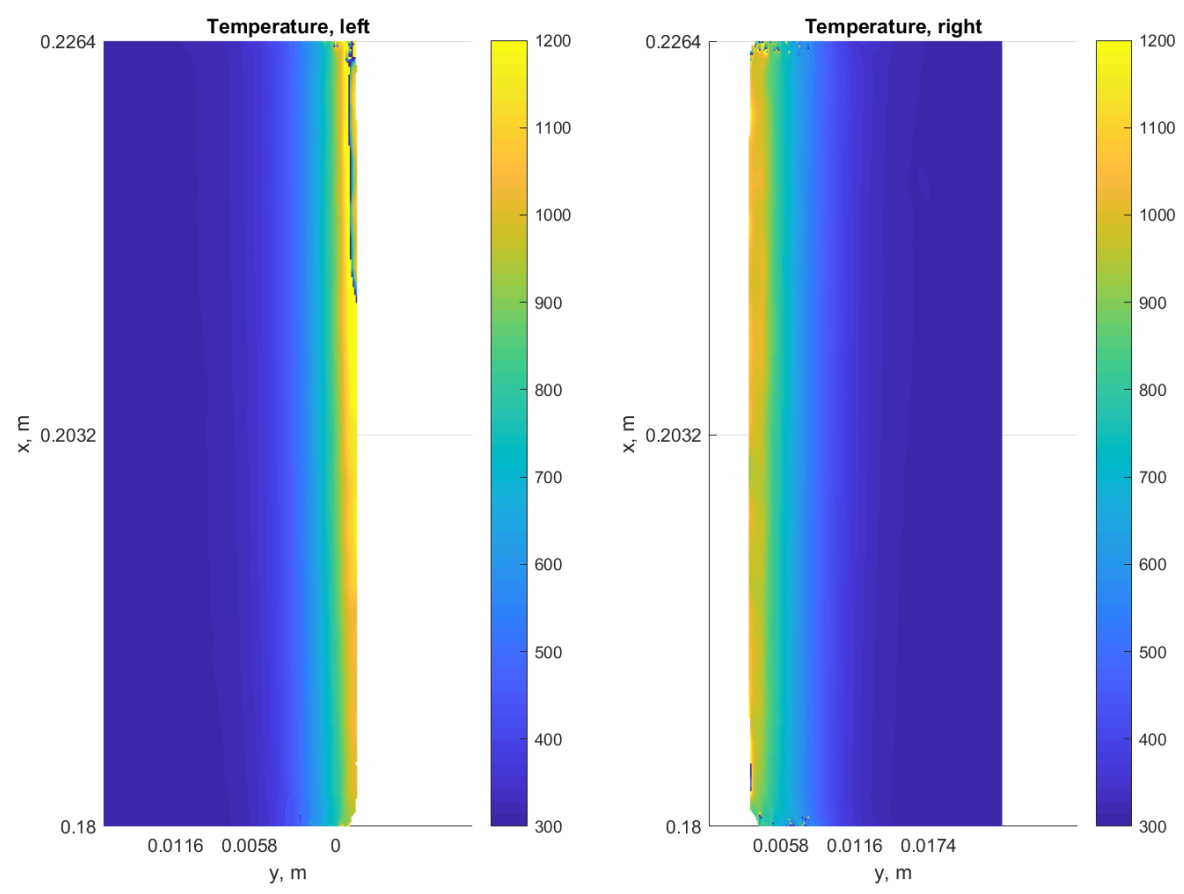

Figure C.1: Shot 62. Temperature fields from interferometry for hexane test. Surface temperature measured by pyrometer $=1087 \mathrm{~K}$. 


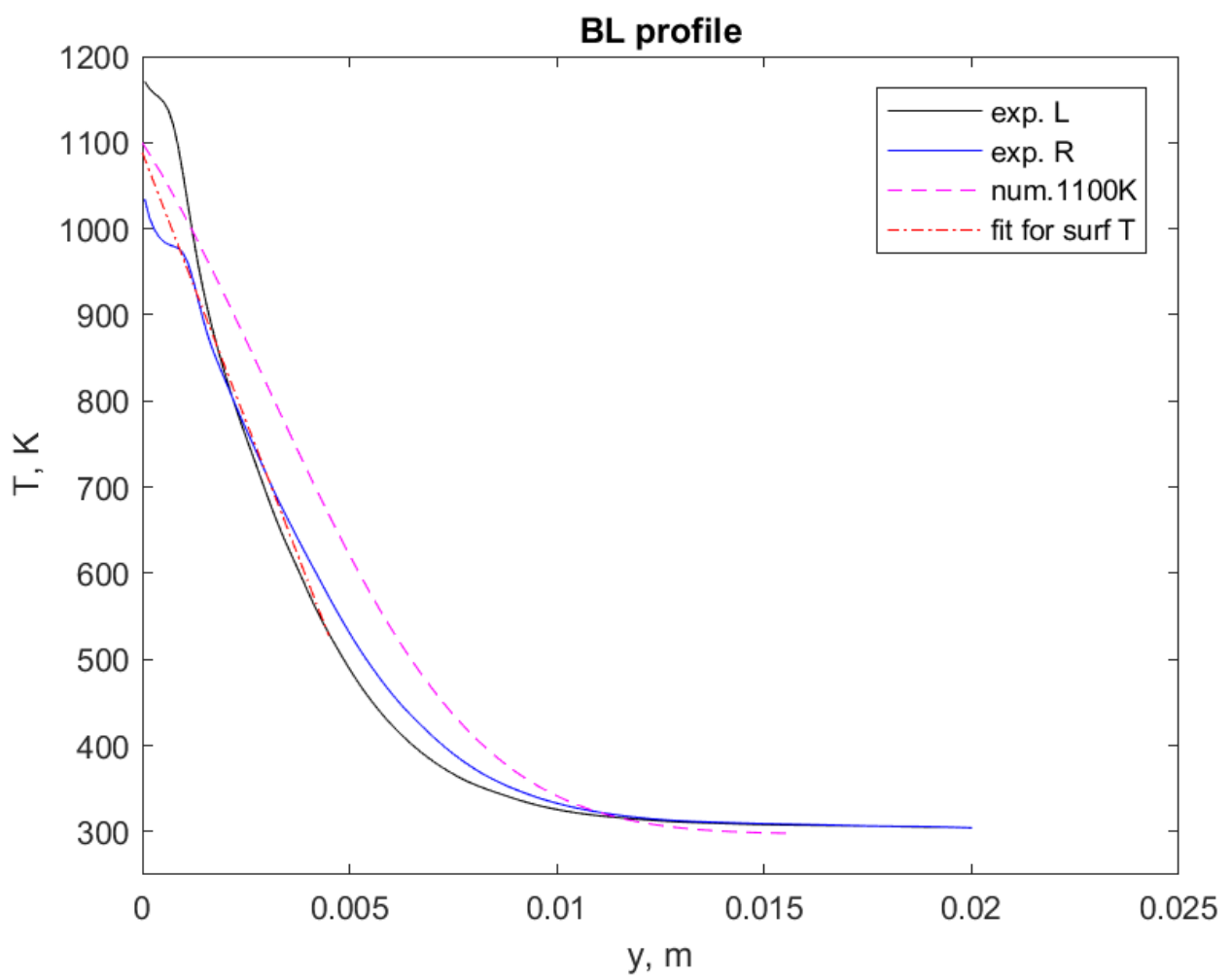

Figure C.2: Shot 62. Thermal boundary layer profiles for left (black line) and right (blue line) sides of experimental results compared with prediction of boundary layer profile (dashed magenta line) based on similarity solution for pyrometer surface temperature. Linear fit to averaged experimental profile (red dot-dashed line) used to extrapolate surface temperature from interferometer post processing. Extrapolated interferometer surface temperature $=1088.7 \mathrm{~K}$. 


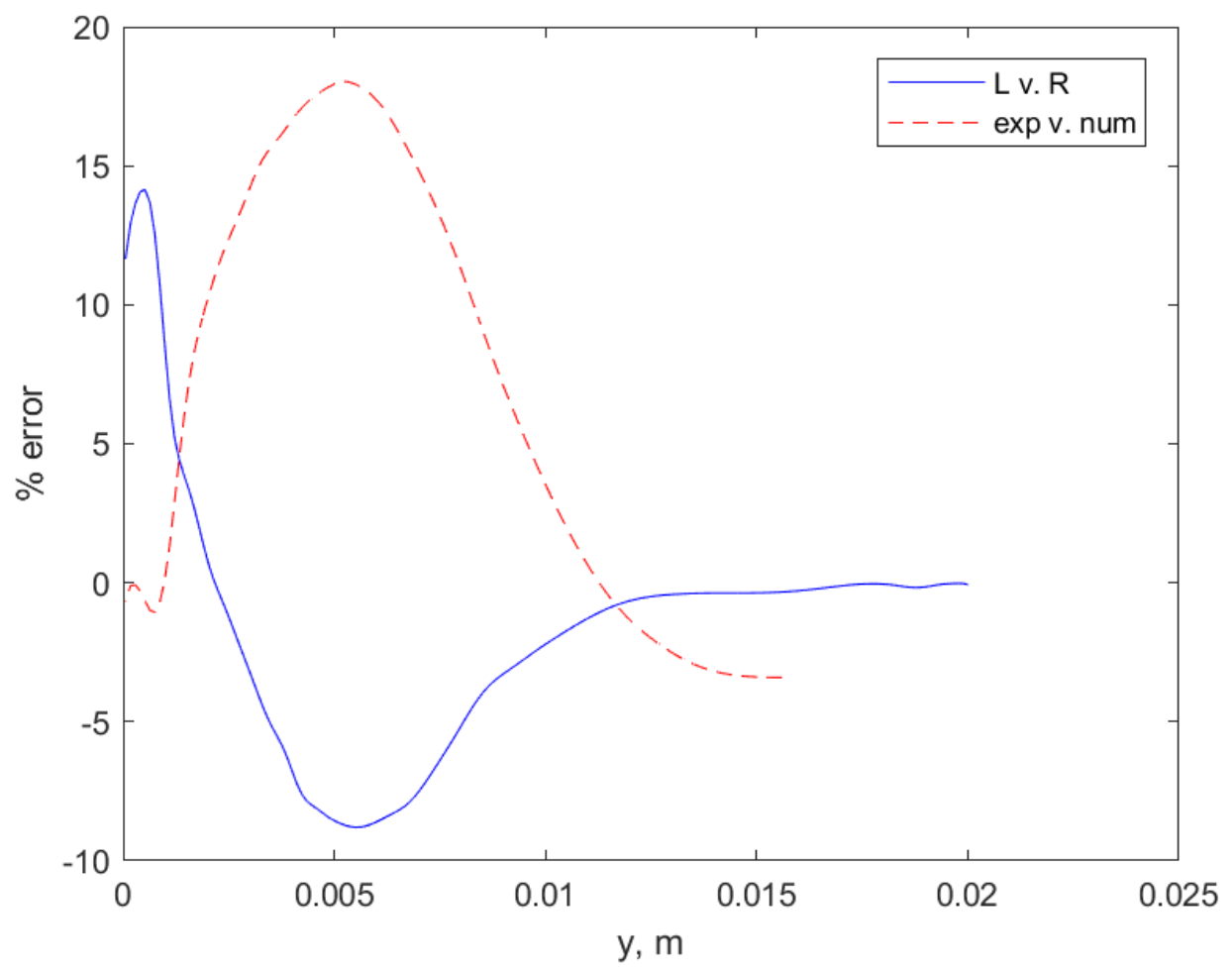

Figure C.3: Shot 62. Percent error in thermal boundary layer between both sides of experimental boundary layer profiles (red line) and percent error between predicted boundary layer profile from similarity solution and averaged experimental boundary layer profile (blue dashed line). 
Shot 63:
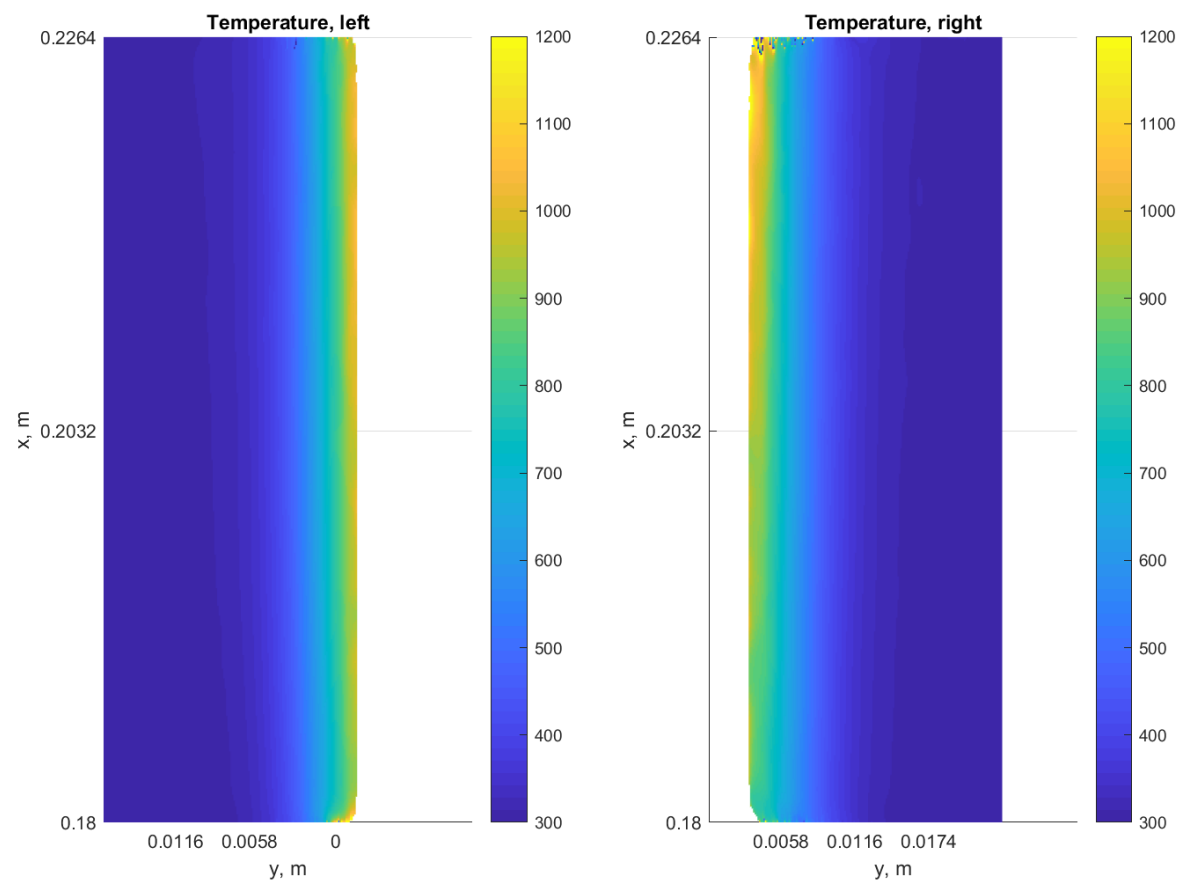

Figure C.4: Shot 63. Temperature fields from interferometry for hexane test. Surface temperature measured by pyrometer $=1060 \mathrm{~K}$. 


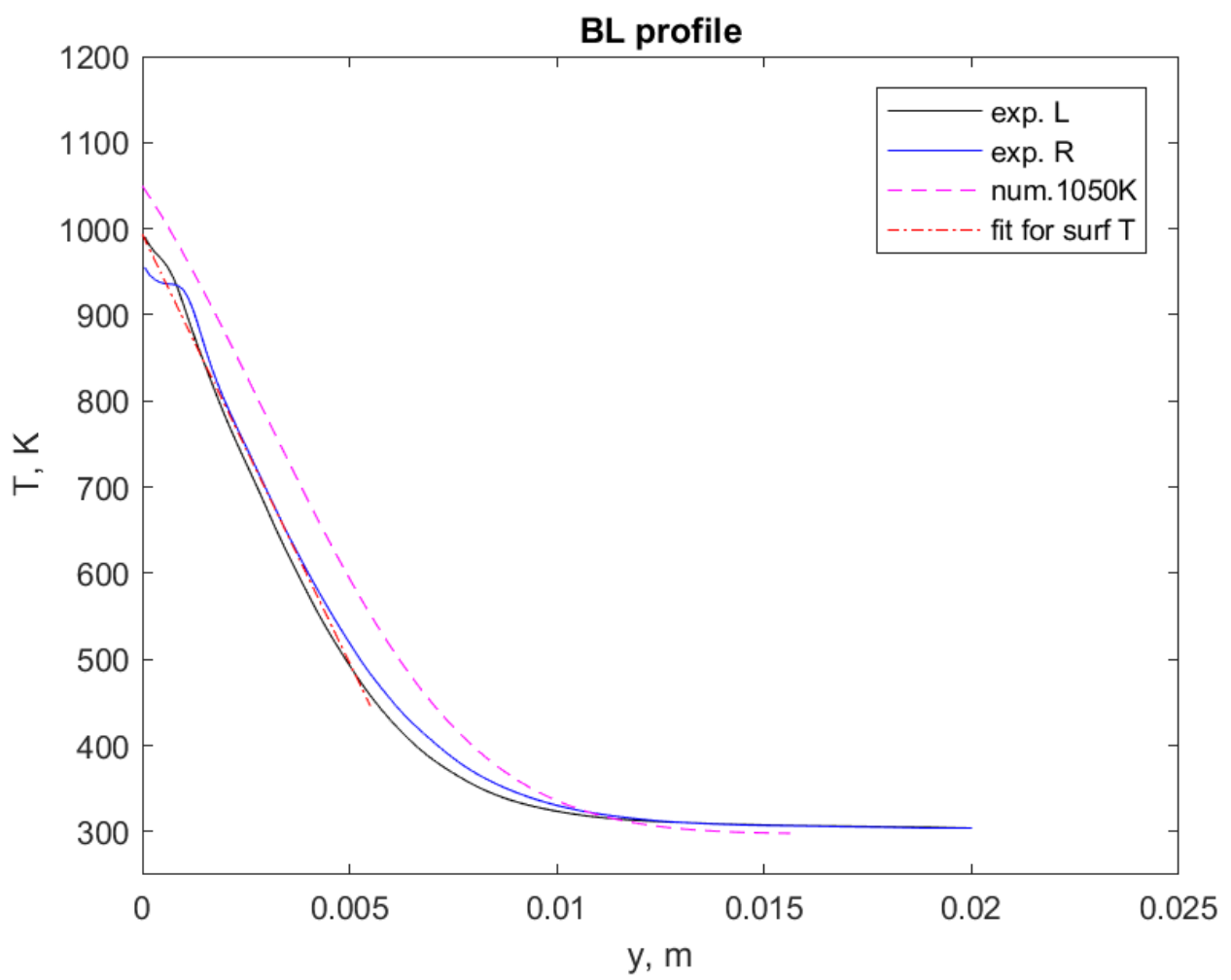

Figure C.5: Shot 63. Thermal boundary layer profiles for left (black line) and right (blue line) sides of experimental results compared with prediction of boundary layer profile (dashed magenta line) based on similarity solution for pyrometer surface temperature. Linear fit to averaged experimental profile (red dot-dashed line) used to extrapolate surface temperature from interferometer post processing. Extrapolated interferometer surface temperature $=993.6 \mathrm{~K}$. 


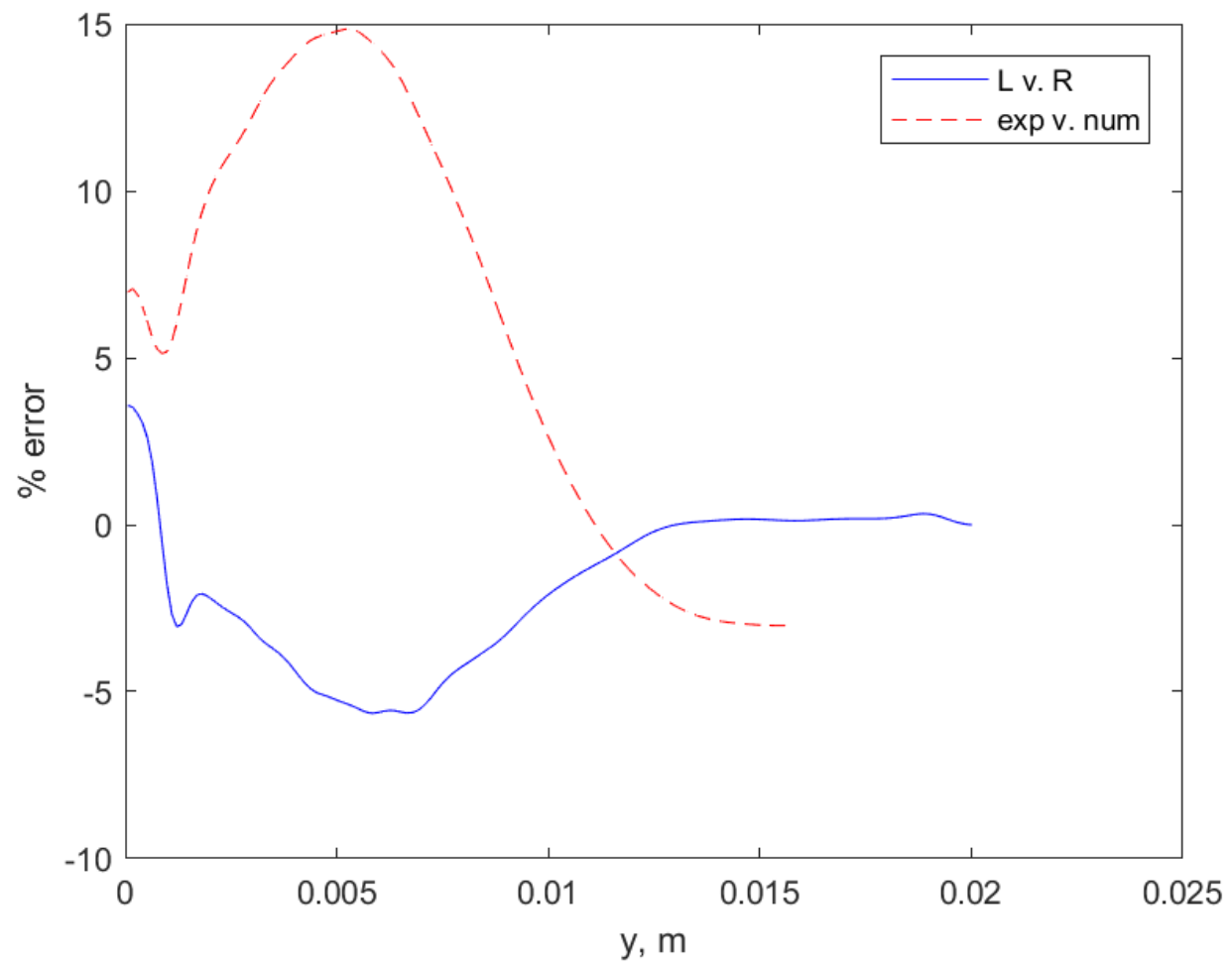

Figure C.6: Shot 63. Percent error in thermal boundary layer between both sides of experimental boundary layer profiles (red line) and percent error between predicted boundary layer profile from similarity solution and averaged experimental boundary layer profile (blue dashed line). 
Shot 64:
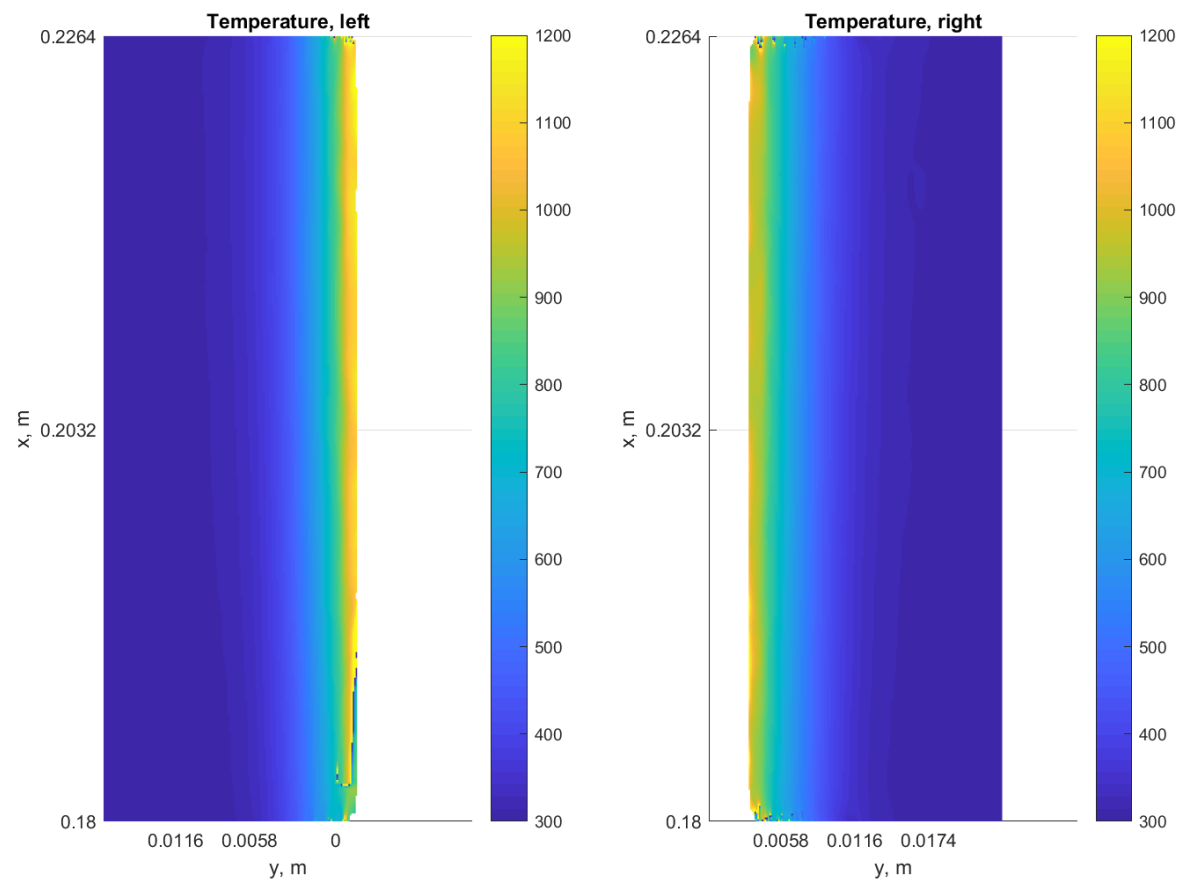

Figure C.7: Shot 64. Temperature fields from interferometry for hexane test. Surface temperature measured by pyrometer $=1070 \mathrm{~K}$. 


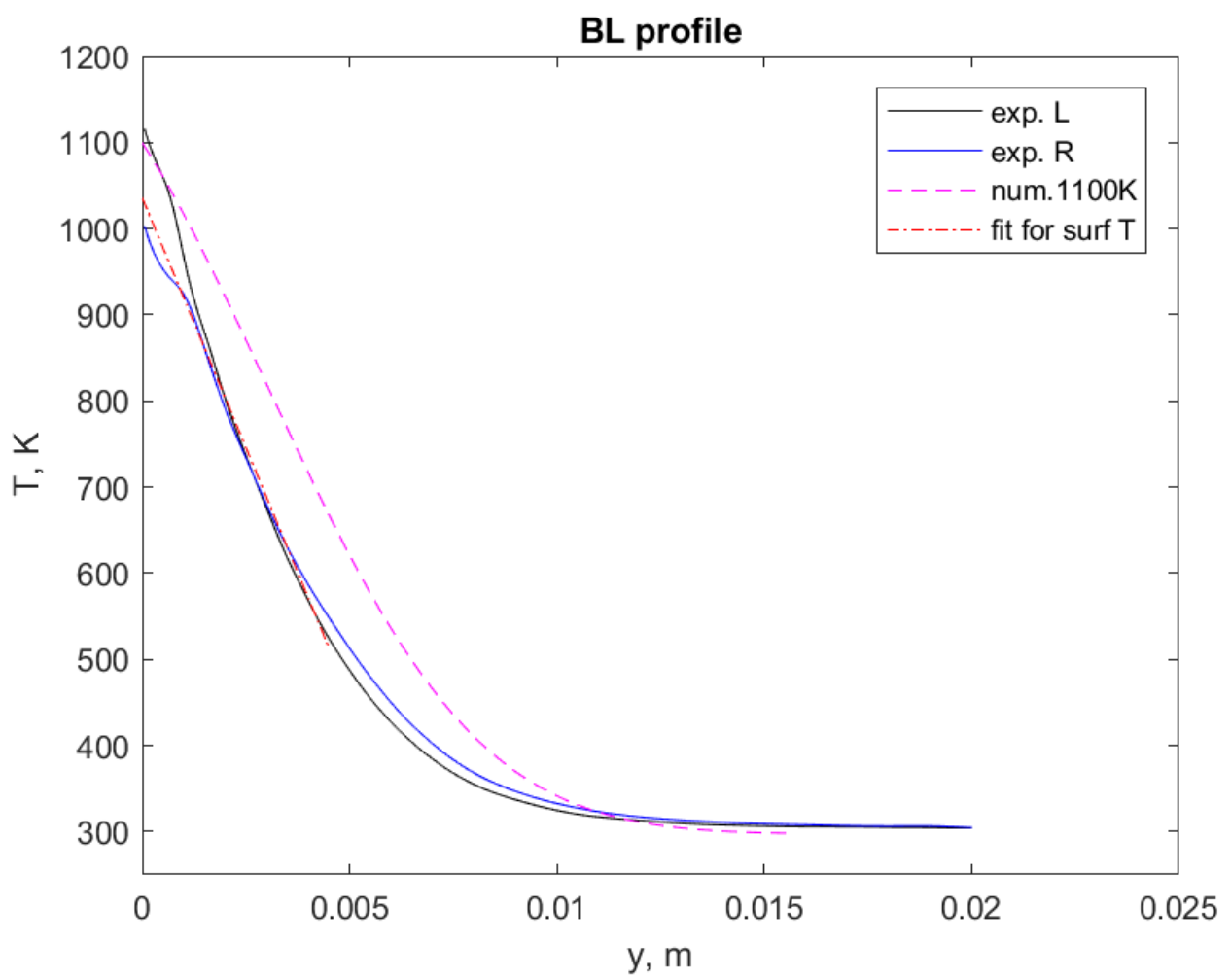

Figure C.8: Shot 64. Thermal boundary layer profiles for left (black line) and right (blue line) sides of experimental results compared with prediction of boundary layer profile (dashed magenta line) based on similarity solution for pyrometer surface temperature. Linear fit to averaged experimental profile (red dot-dashed line) used to extrapolate surface temperature from interferometer post processing. Extrapolated interferometer surface temperature $=1036.1 \mathrm{~K}$. 


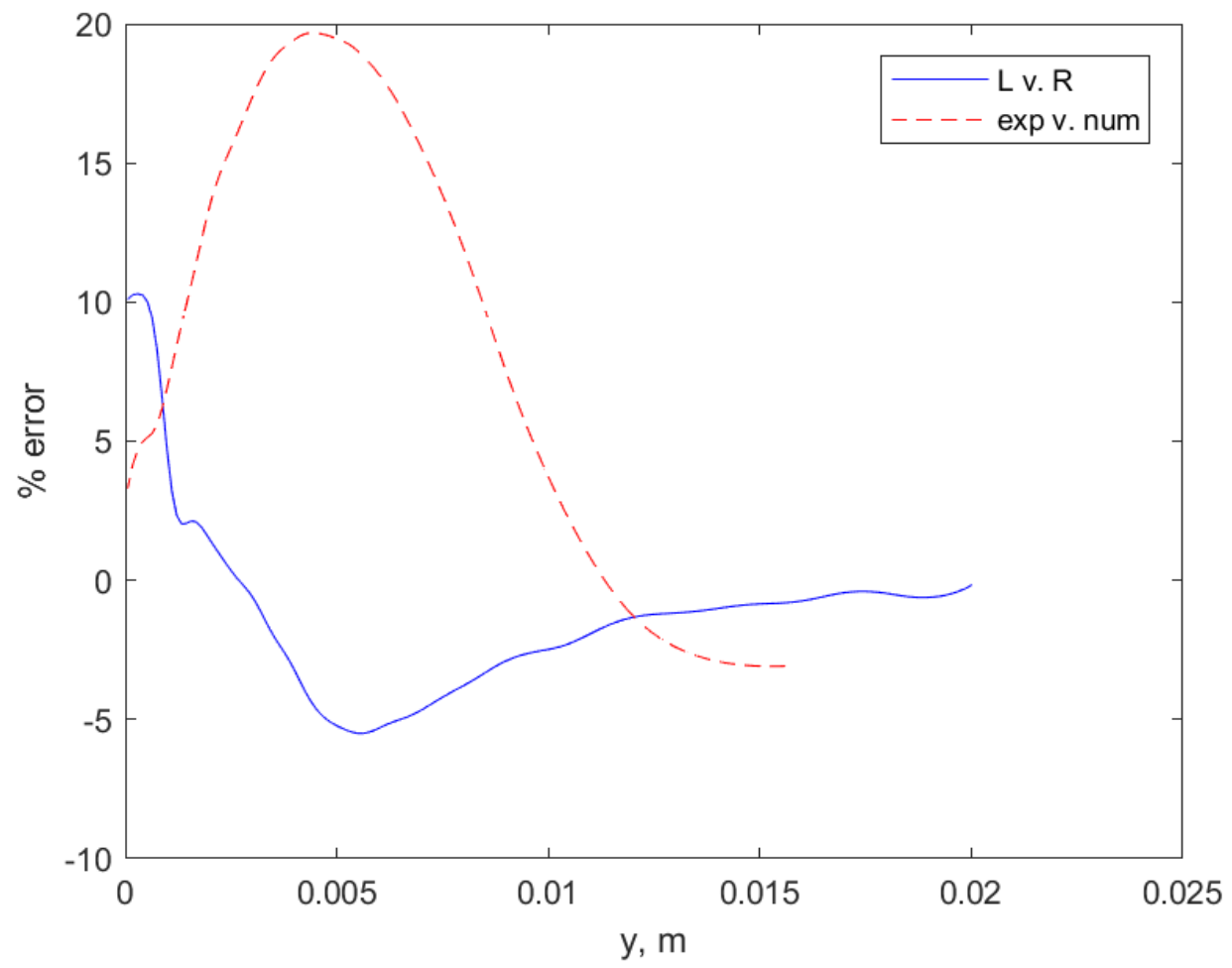

Figure C.9: Shot 64. Percent error in thermal boundary layer between both sides of experimental boundary layer profiles (red line) and percent error between predicted boundary layer profile from similarity solution and averaged experimental boundary layer profile (blue dashed line). 
50C

Shot 41:
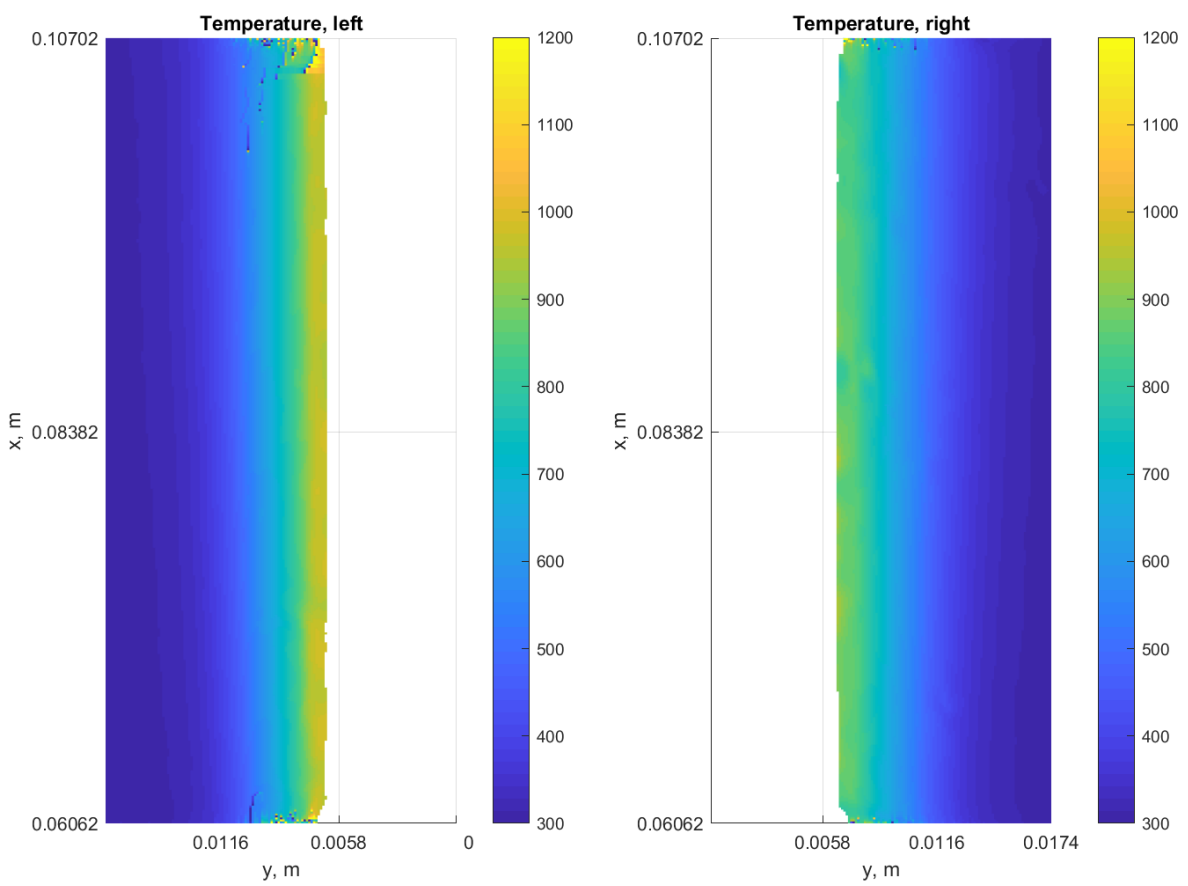

Figure C.10: Shot 41. Temperature fields from interferometry for hexane test. Surface temperature measured by pyrometer $=1106 \mathrm{~K}$. 


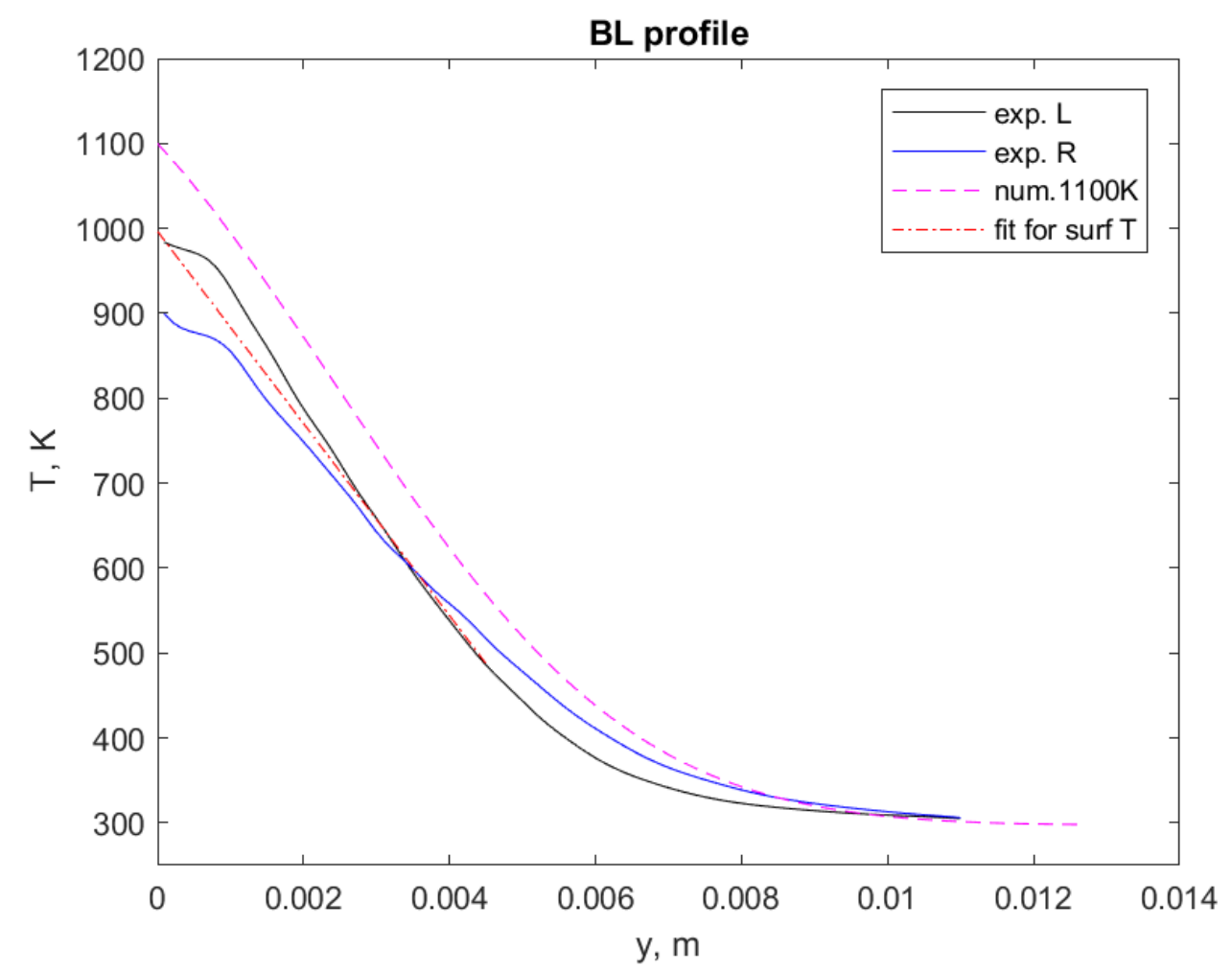

Figure C.11: Shot 41. Thermal boundary layer profiles for left (black line) and right (blue line) sides of experimental results compared with prediction of boundary layer profile (dashed magenta line) based on similarity solution for pyrometer surface temperature. Linear fit to averaged experimental profile (red dot-dashed line) used to extrapolate surface temperature from interferometer post processing. Extrapolated interferometer surface temperature $=996.6 \mathrm{~K}$. 


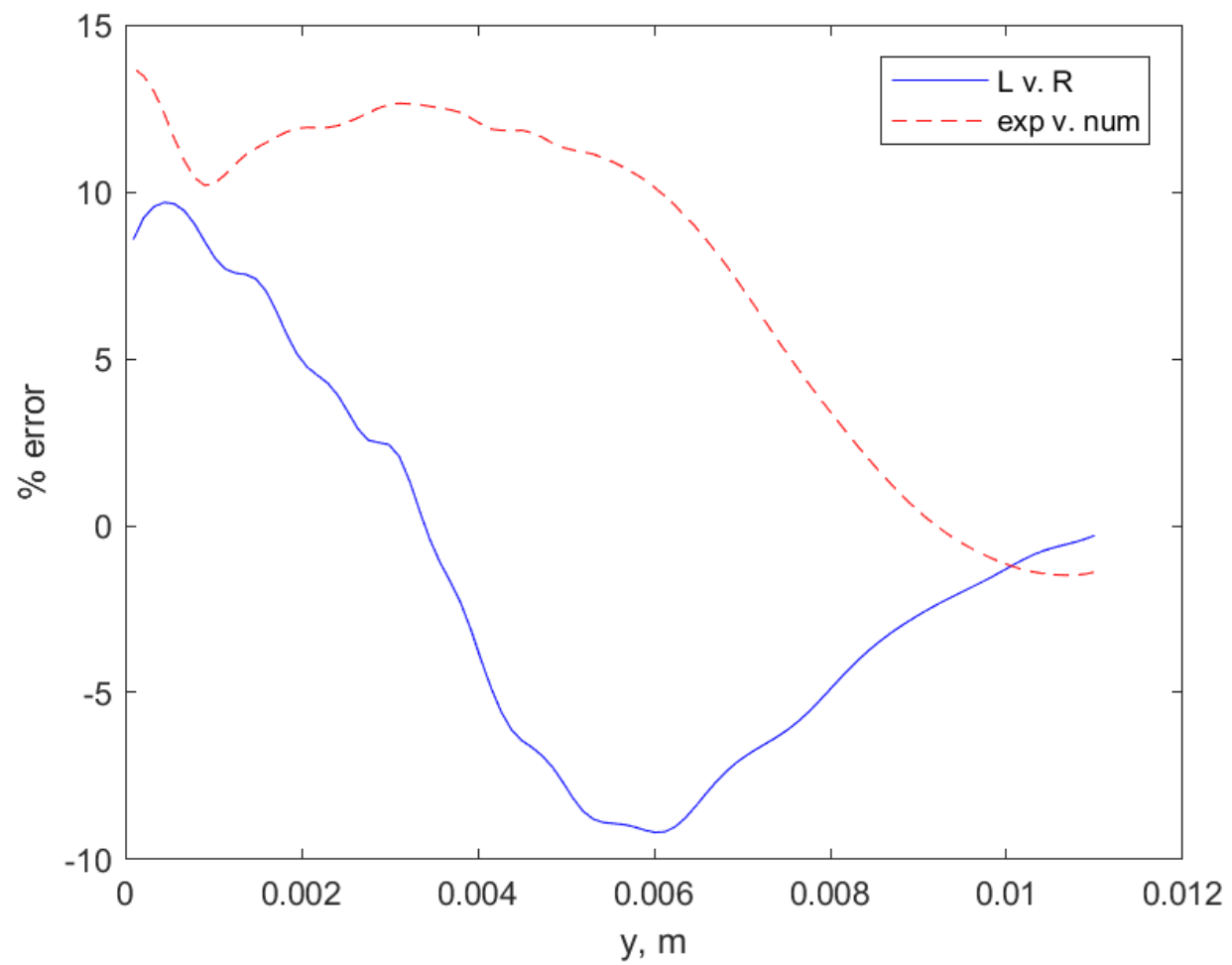

Figure C.12: Shot 41. Percent error in thermal boundary layer between both sides of experimental boundary layer profiles (red line) and percent error between predicted boundary layer profile from similarity solution and averaged experimental boundary layer profile (blue dashed line). 
Shot 52:
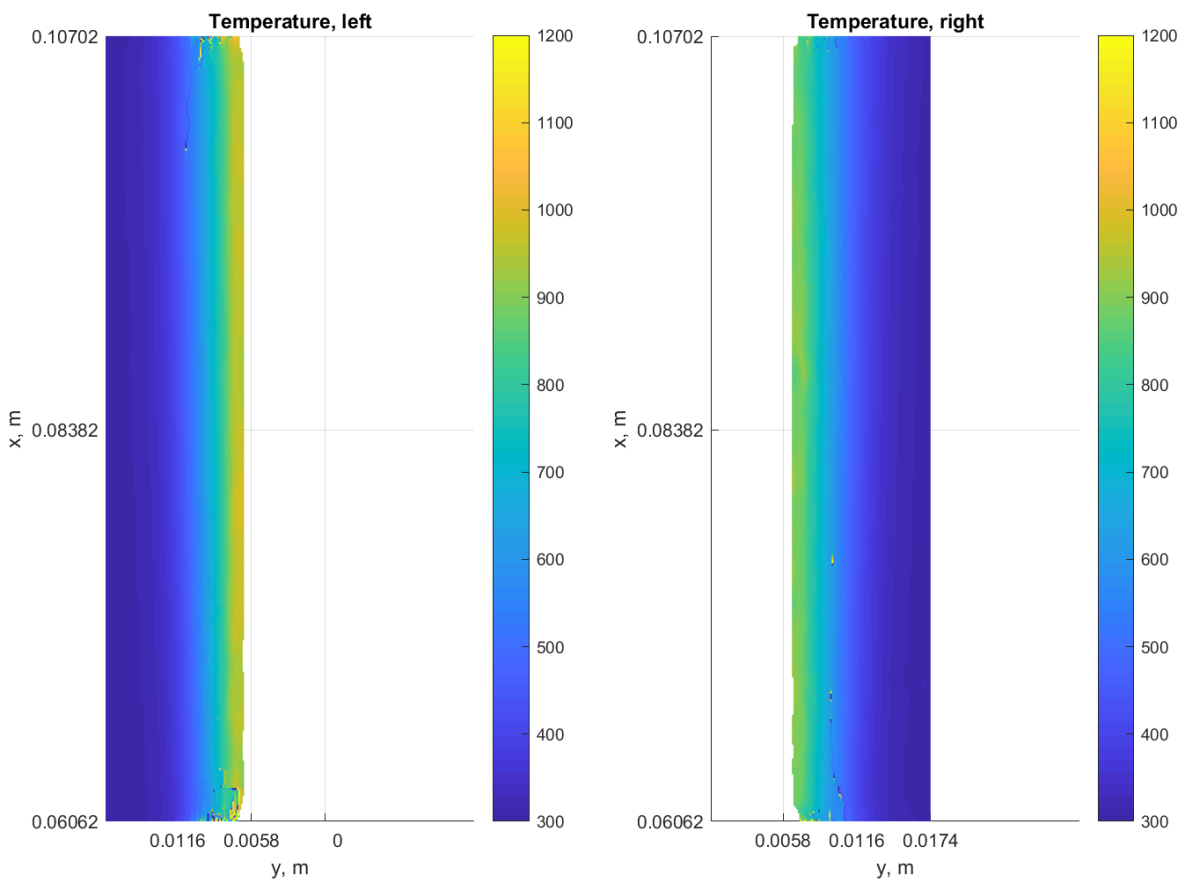

Figure C.13: Shot 52. Temperature fields from interferometry for hexane test. Surface temperature measured by pyrometer $=1107 \mathrm{~K}$. 


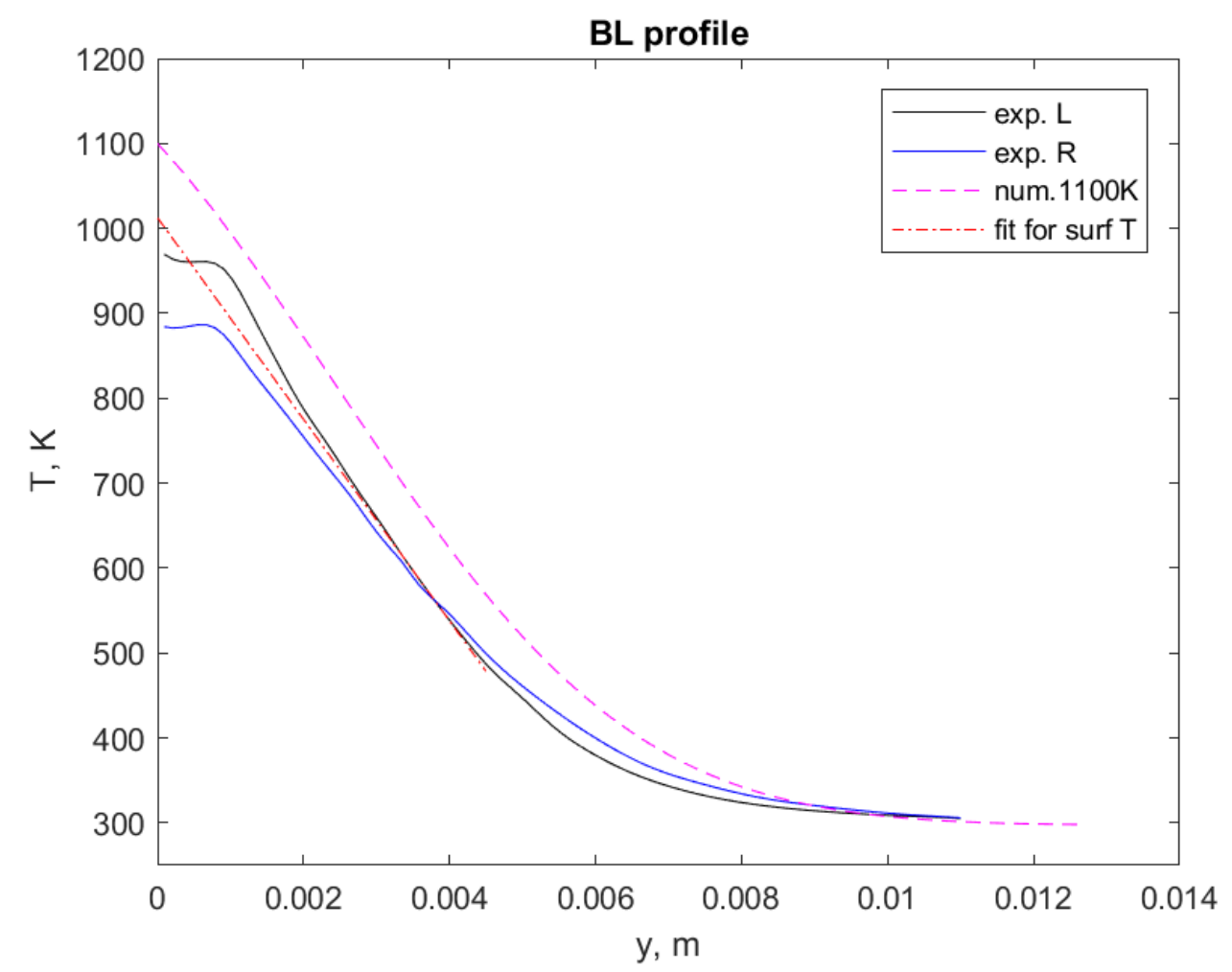

Figure C.14: Shot 52. Thermal boundary layer profiles for left (black line) and right (blue line) sides of experimental results compared with prediction of boundary layer profile (dashed magenta line) based on similarity solution for pyrometer surface temperature. Linear fit to averaged experimental profile (red dot-dashed line) used to extrapolate surface temperature from interferometer post processing. Extrapolated interferometer surface temperature $=1012.7 \mathrm{~K}$. 


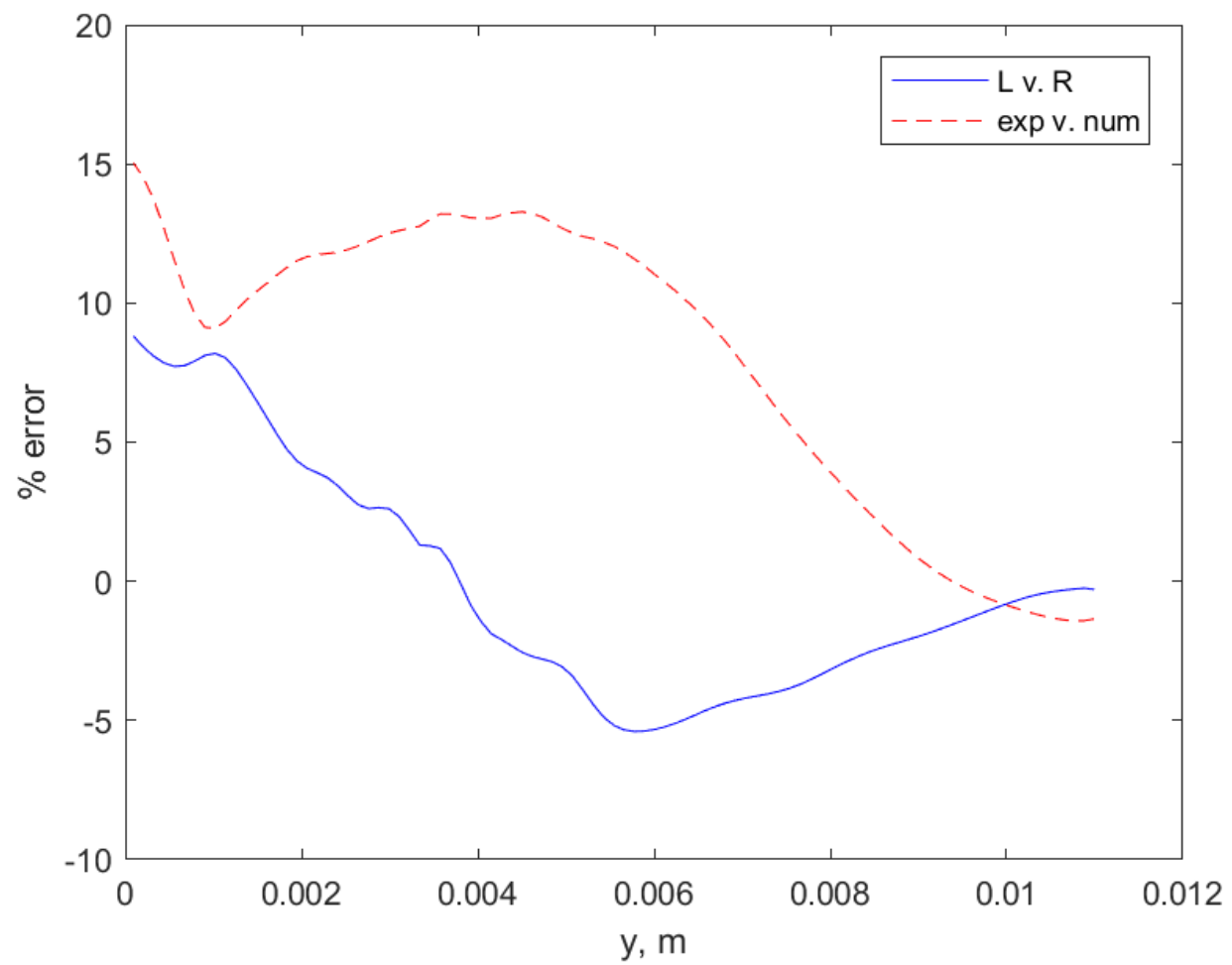

Figure C.15: Shot 52. Percent error in thermal boundary layer between both sides of experimental boundary layer profiles (red line) and percent error between predicted boundary layer profile from similarity solution and averaged experimental boundary layer profile (blue dashed line). 
Shot 53:
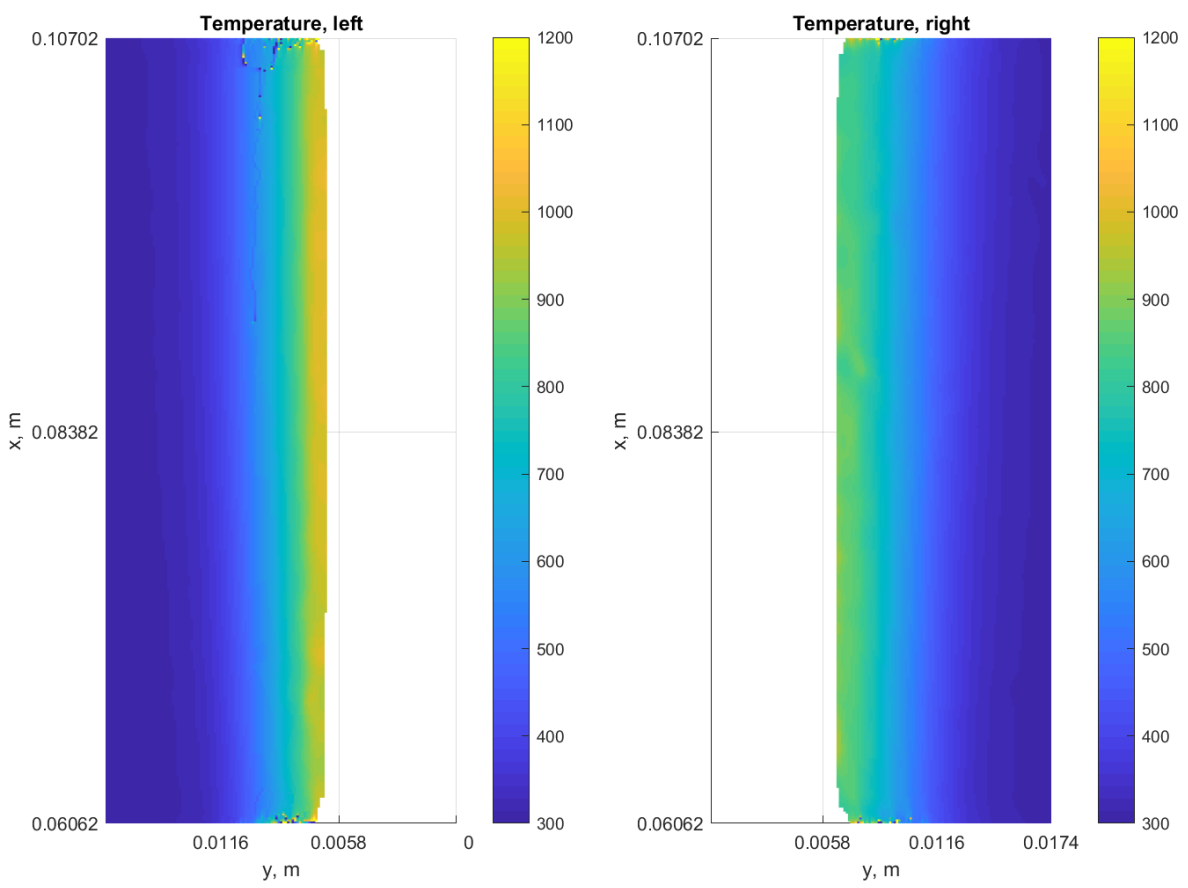

Figure C.16: Shot 53. Temperature fields from interferometry for hexane test. Surface temperature measured by pyrometer $=1100 \mathrm{~K}$. 


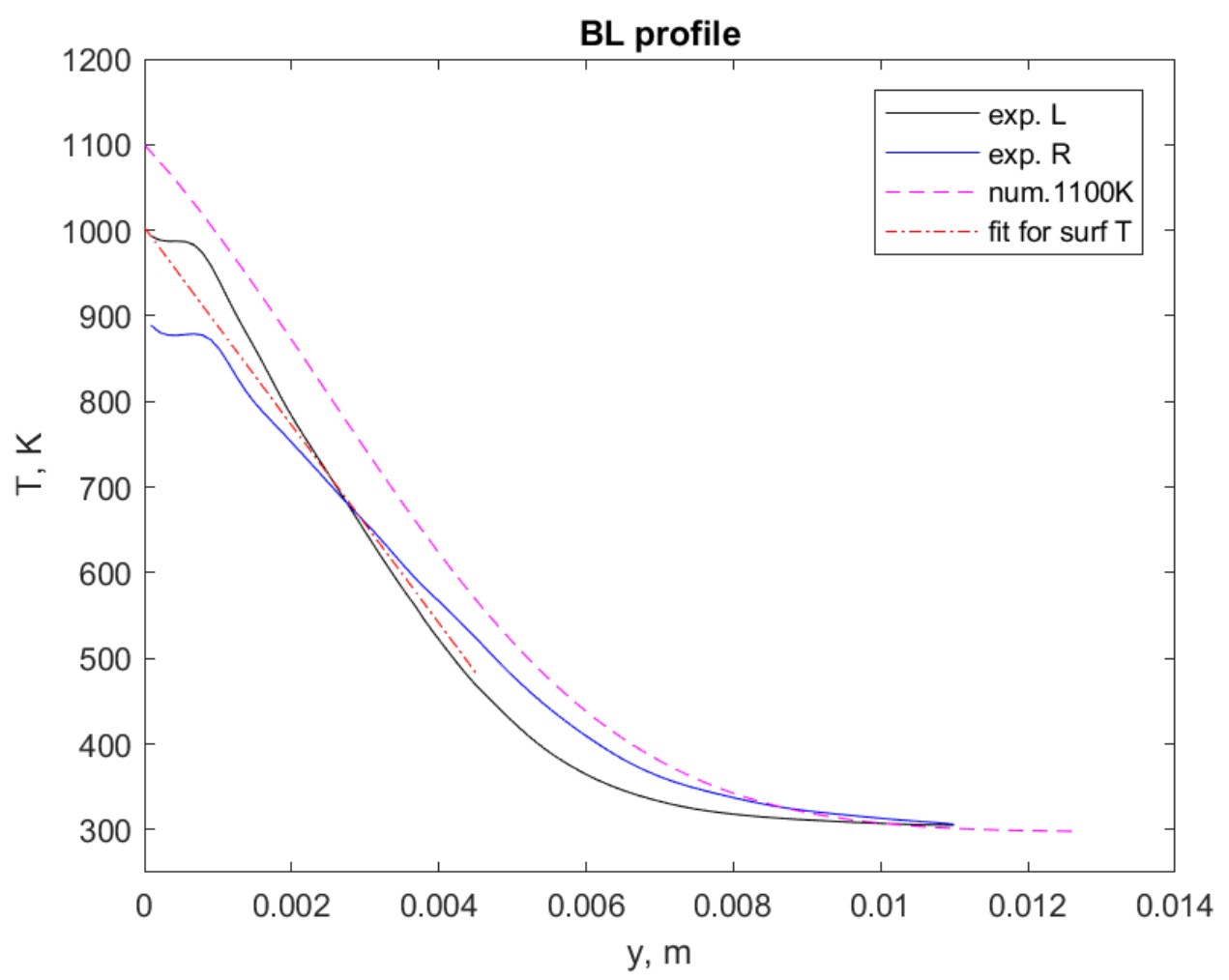

Figure C.17: Shot 53. Thermal boundary layer profiles for left (black line) and right (blue line) sides of experimental results compared with prediction of boundary layer profile (dashed magenta line) based on similarity solution for pyrometer surface temperature. Linear fit to averaged experimental profile (red dot-dashed line) used to extrapolate surface temperature from interferometer post processing. Extrapolated interferometer surface temperature $=1003.4 \mathrm{~K}$. 


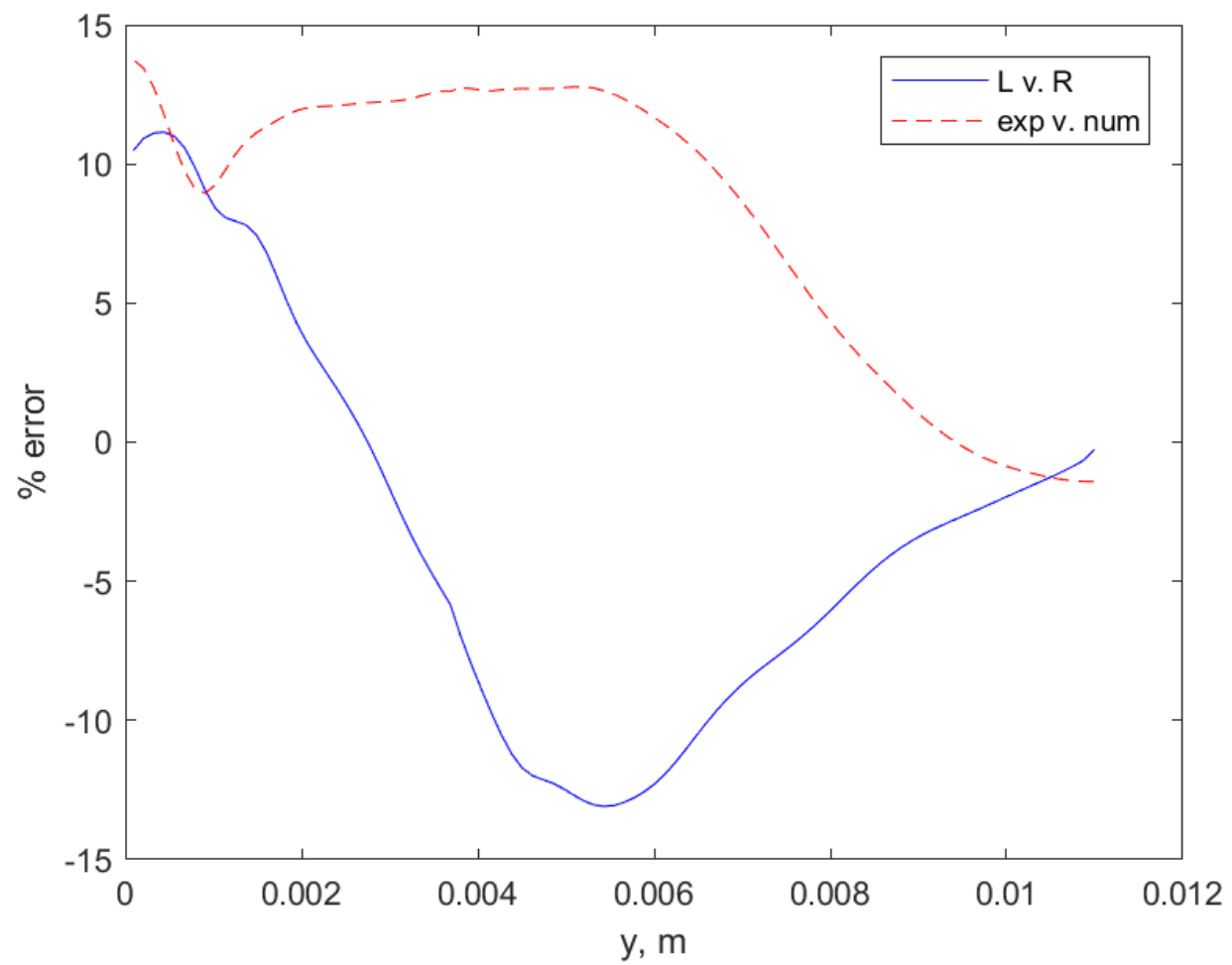

Figure C.18: Shot 53. Percent error in thermal boundary layer between both sides of experimental boundary layer profiles (red line) and percent error between predicted boundary layer profile from similarity solution and averaged experimental boundary layer profile (blue dashed line). 
75B

Shot 16:
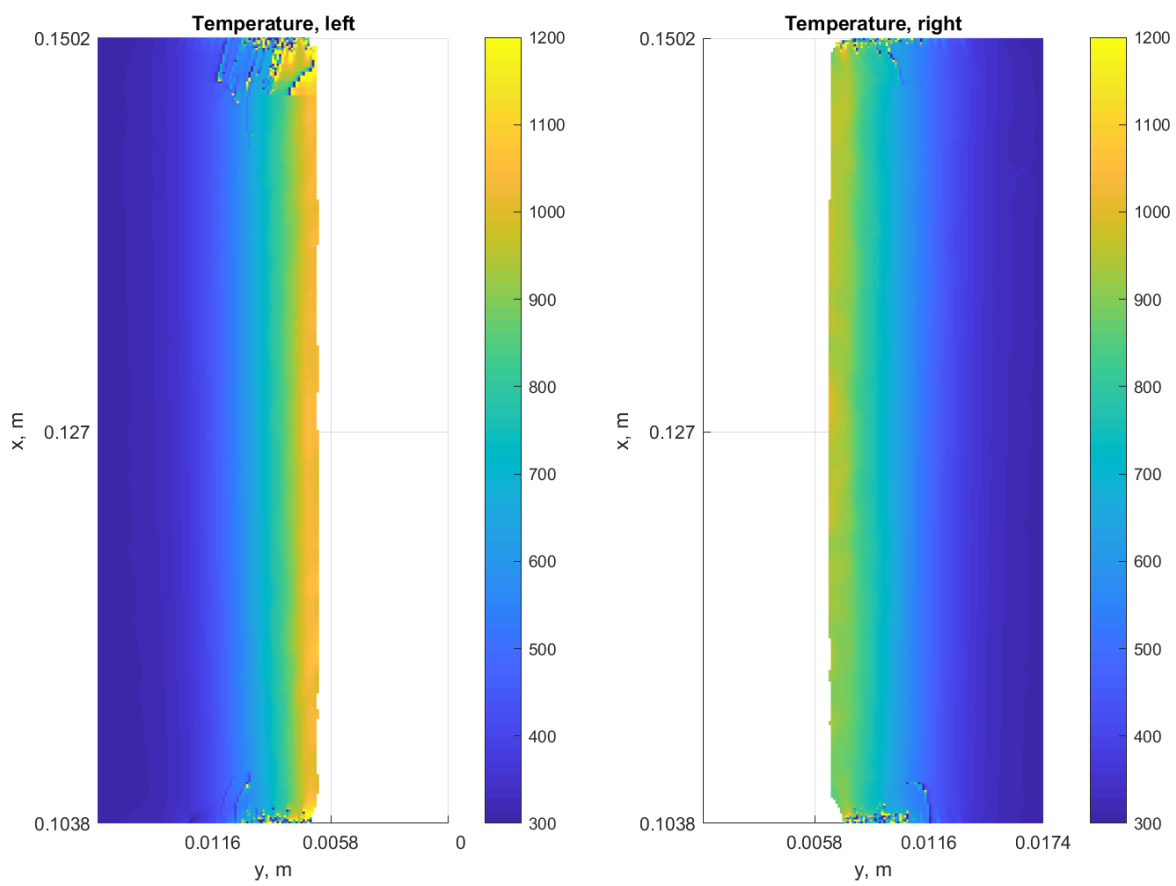

Figure C.19: Shot 16. Temperature fields from interferometry for hexane test. Surface temperature measured by pyrometer $=1074 \mathrm{~K}$. 


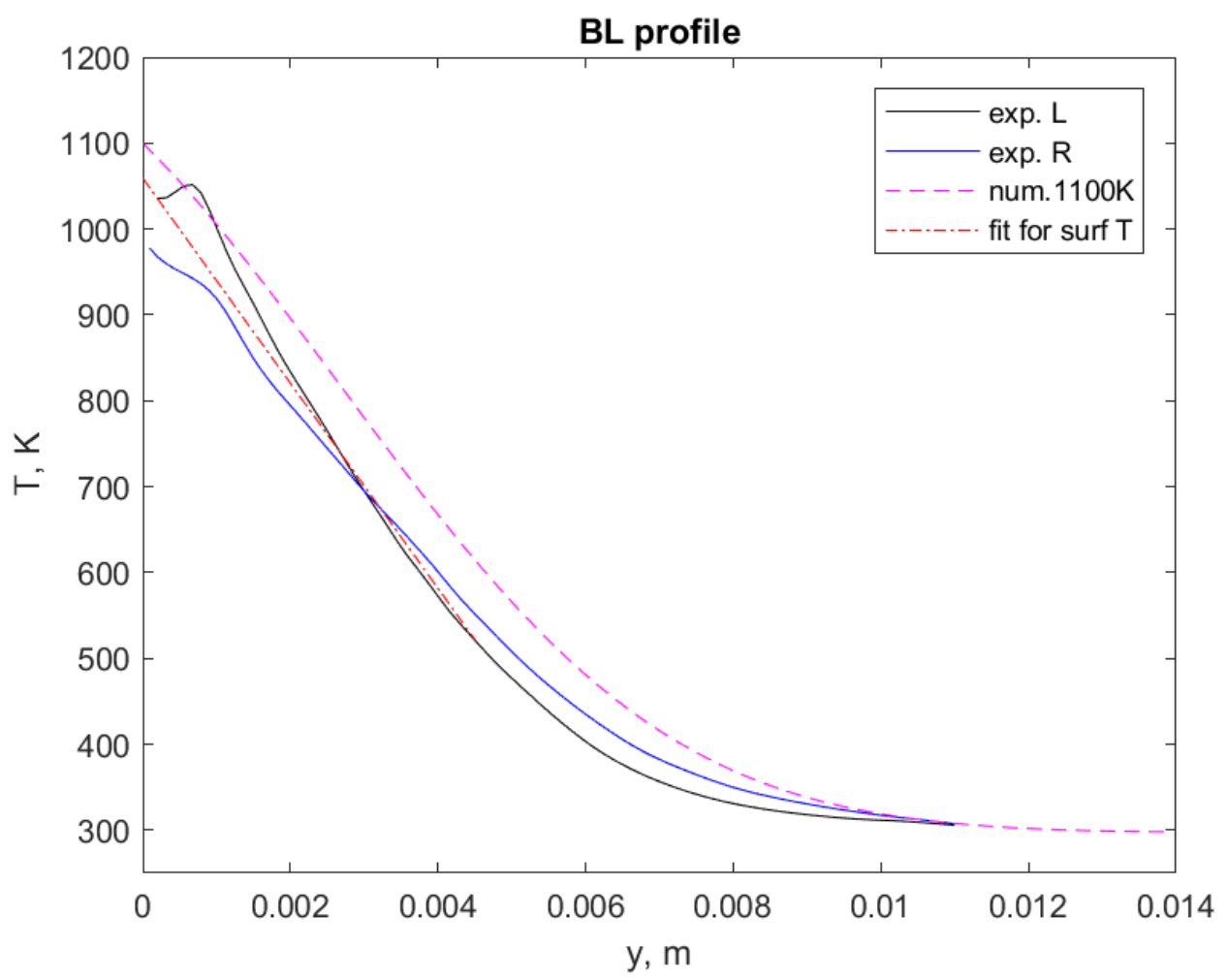

Figure C.20: Shot 16. Thermal boundary layer profiles for left (black line) and right (blue line) sides of experimental results compared with prediction of boundary layer profile (dashed magenta line) based on similarity solution for pyrometer surface temperature. Linear fit to averaged experimental profile (red dot-dashed line) used to extrapolate surface temperature from interferometer post processing. Extrapolated interferometer surface temperature $=1059.3 \mathrm{~K}$. 


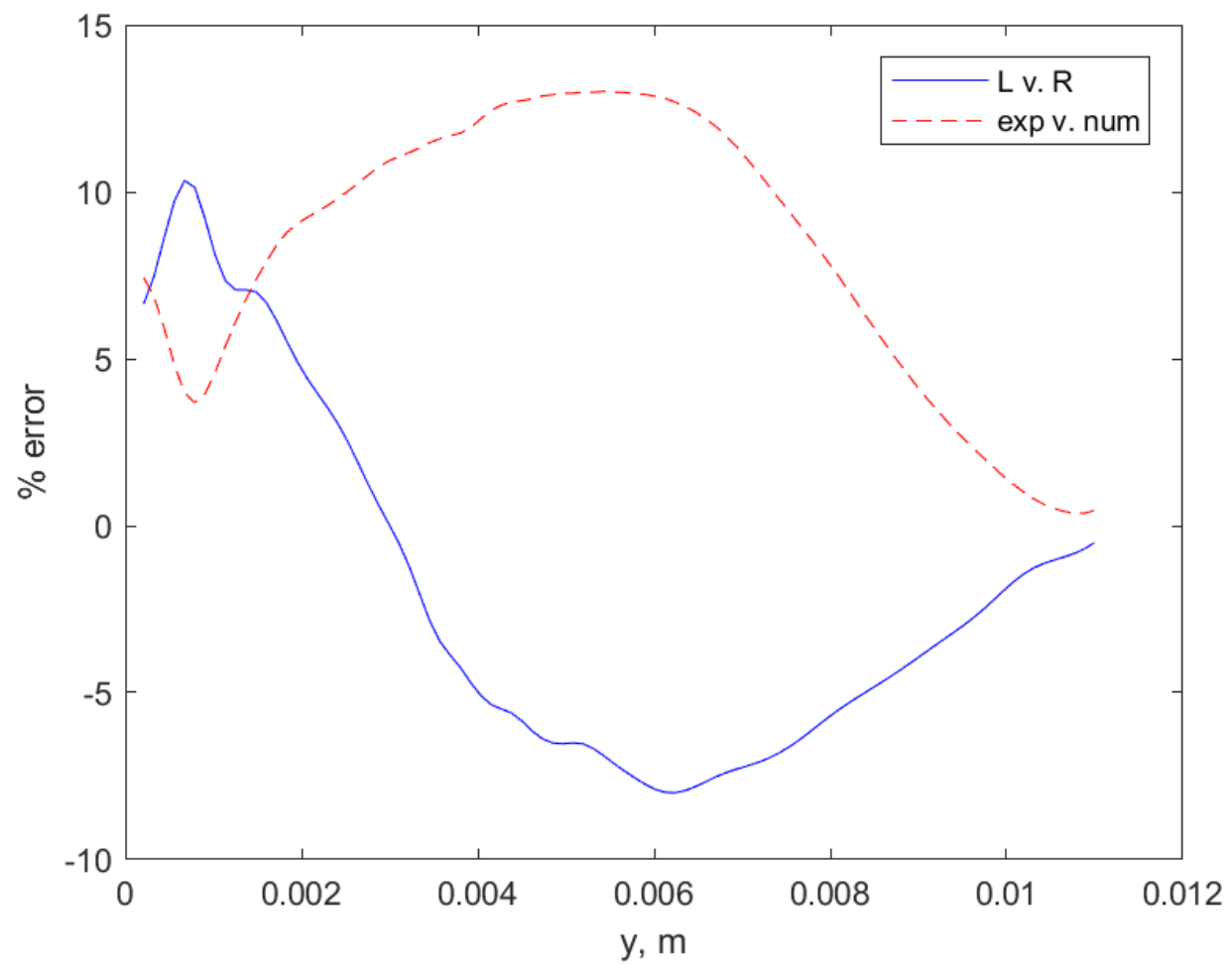

Figure C.21: Shot 16. Percent error in thermal boundary layer between both sides of experimental boundary layer profiles (red line) and percent error between predicted boundary layer profile from similarity solution and averaged experimental boundary layer profile (blue dashed line). 
Shot 31:
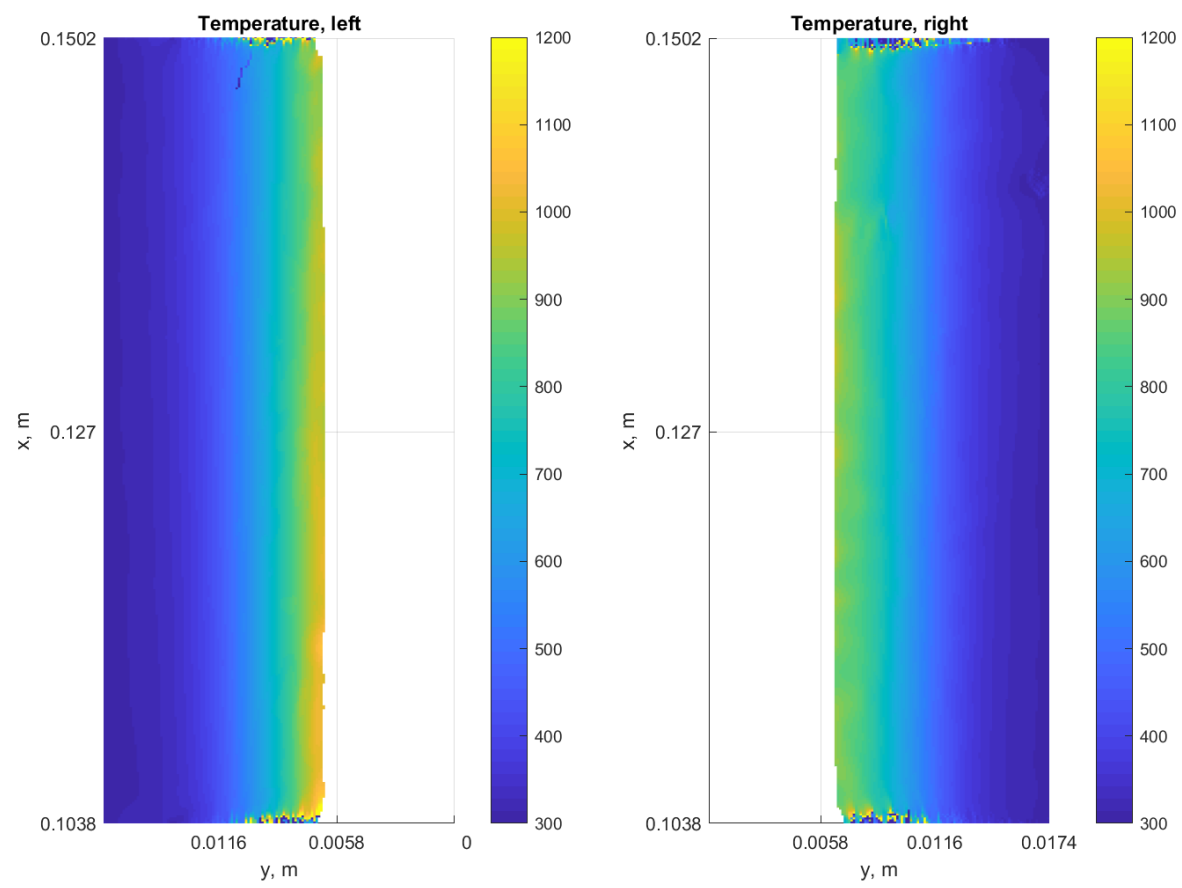

Figure C.22: Shot 31. Temperature fields from interferometry for hexane test. Surface temperature measured by pyrometer $=1104 \mathrm{~K}$. 


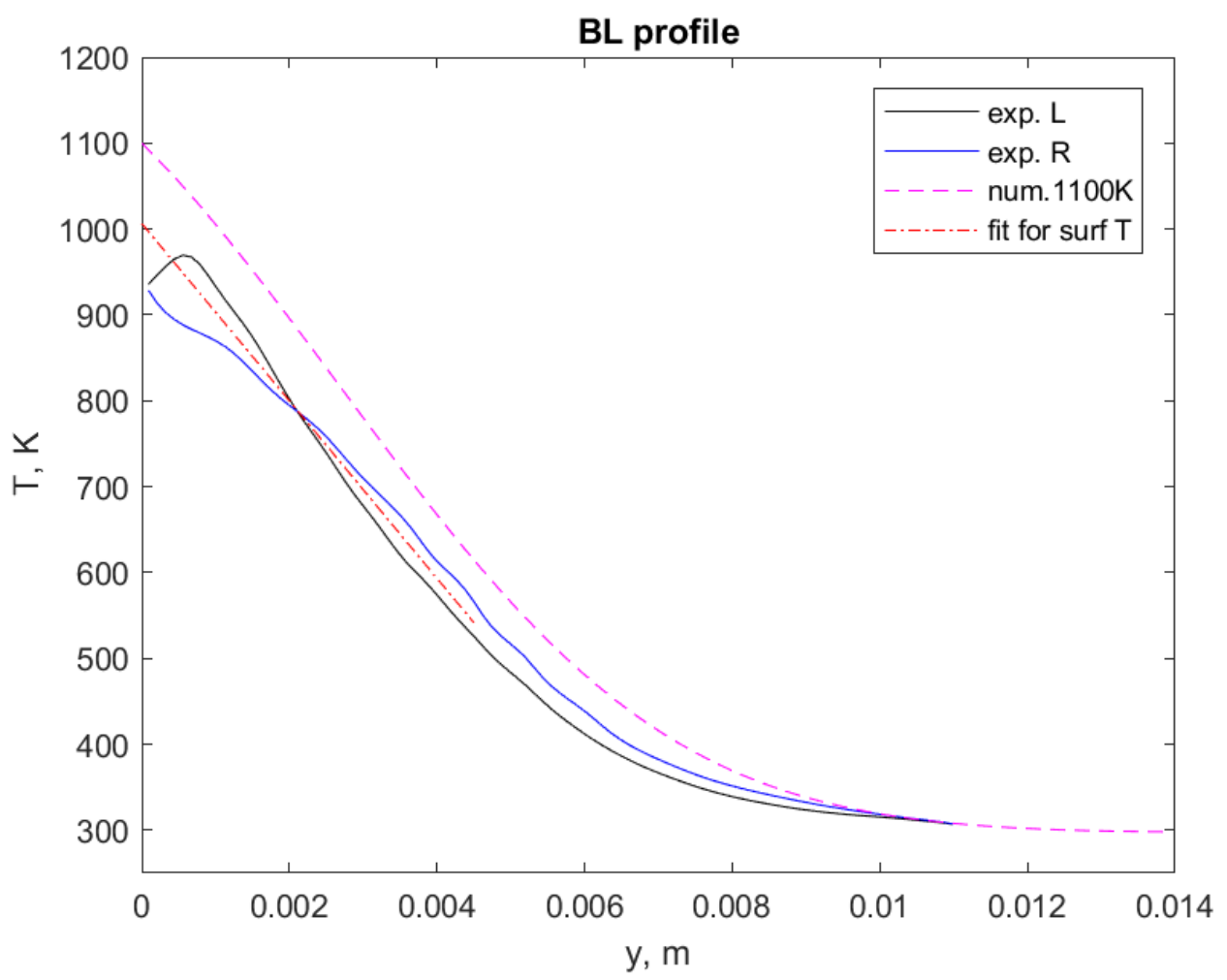

Figure C.23: Shot 31. Thermal boundary layer profiles for left (black line) and right (blue line) sides of experimental results compared with prediction of boundary layer profile (dashed magenta line) based on similarity solution for pyrometer surface temperature. Linear fit to averaged experimental profile (red dot-dashed line) used to extrapolate surface temperature from interferometer post processing. Extrapolated interferometer surface temperature $=1006.5 \mathrm{~K}$. 


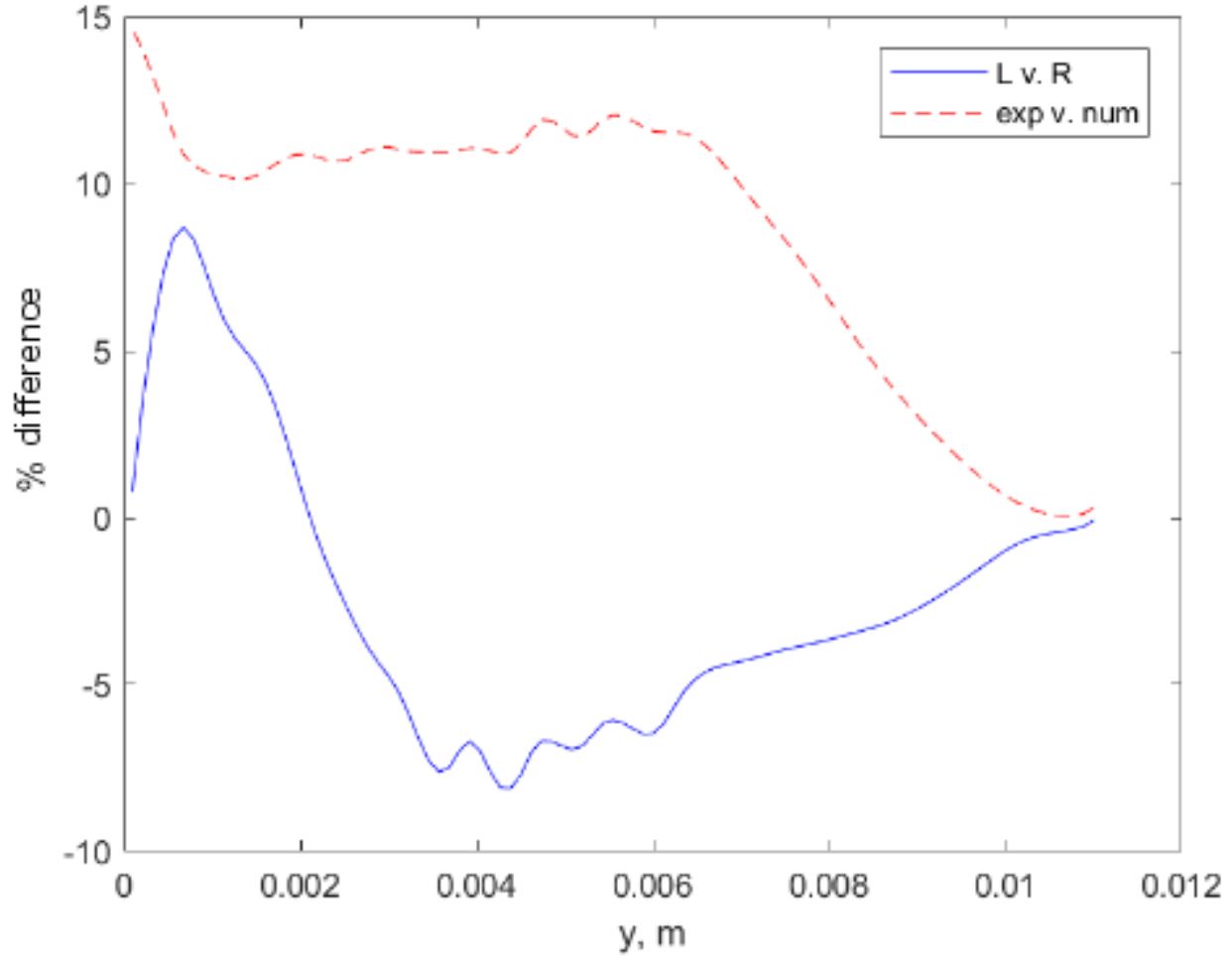

Figure C.24: Shot 31. Percent error in thermal boundary layer between both sides experimental boundary layer profiles (red line) and percent error between predicted boundary layer profile from similarity solution and averaged experimental boundary layer profile (blue dashed line). 
Shot 32:
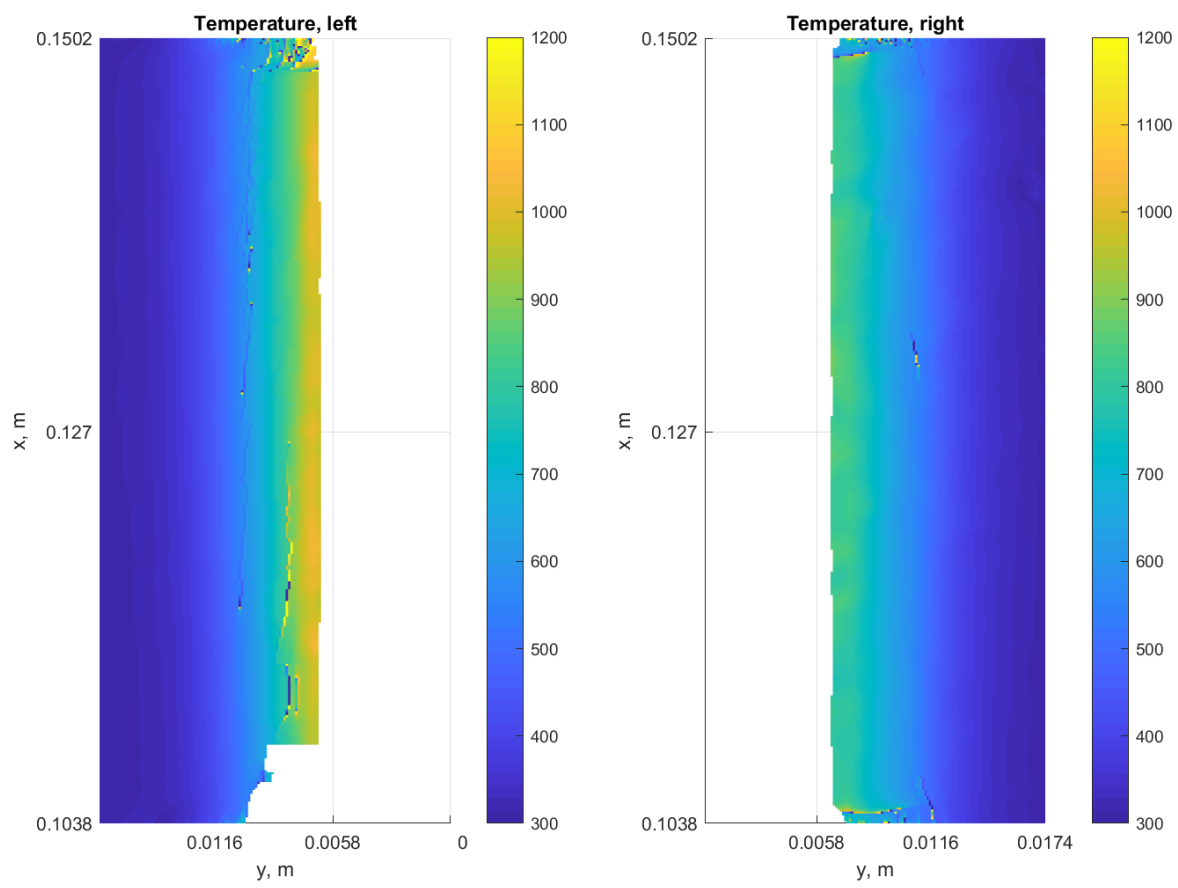

Figure C.25: Shot 32. Temperature fields from interferometry for hexane test. Surface temperature measured by pyrometer $=1091 \mathrm{~K}$. 


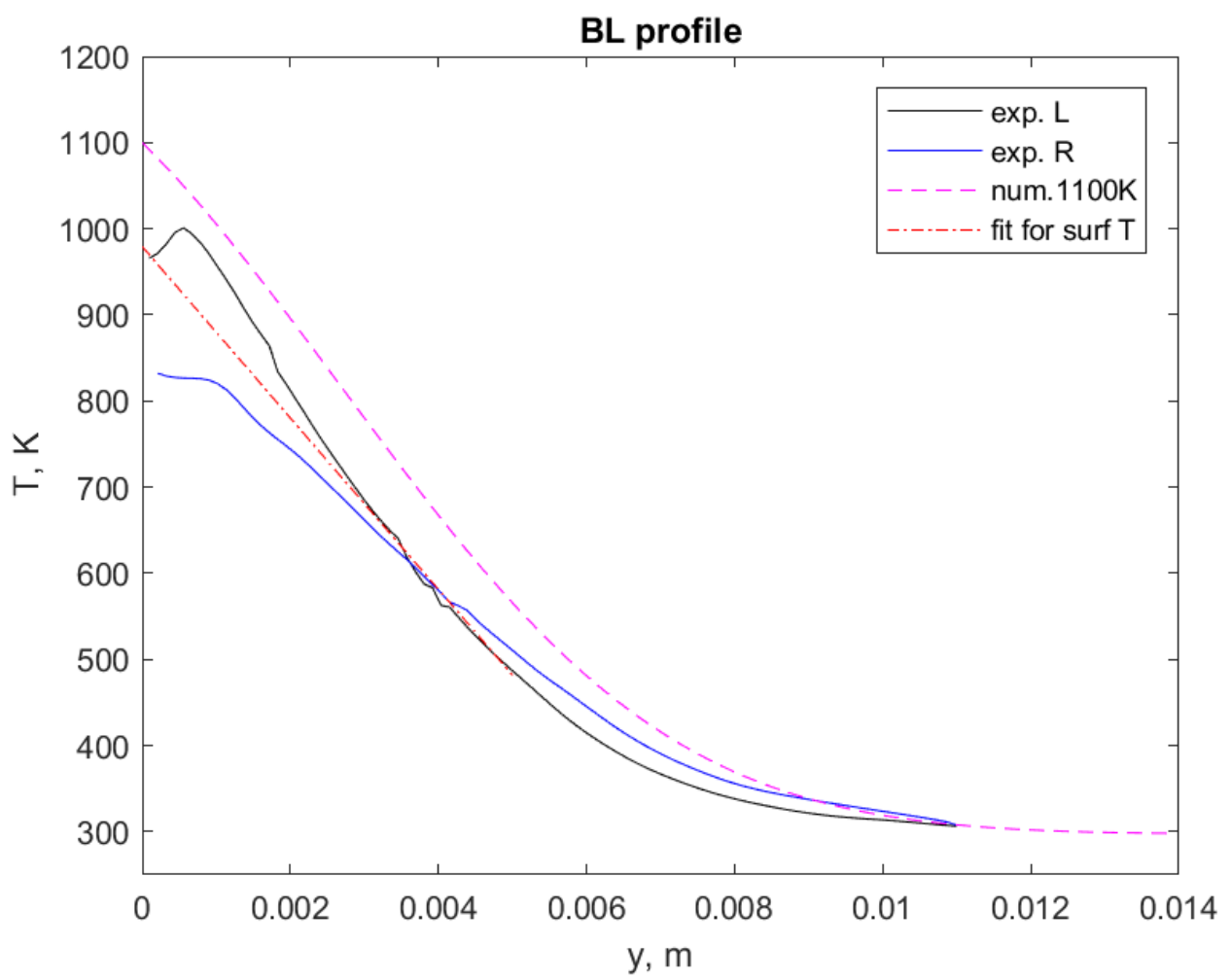

Figure C.26: Shot 32. Thermal boundary layer profiles for left (black line) and right (blue line) sides of experimental results compared with prediction of boundary layer profile (dashed magenta line) based on similarity solution for pyrometer surface temperature. Linear fit to averaged experimental profile (red dot-dashed line) used to extrapolate surface temperature from interferometer post processing. Extrapolated interferometer surface temperature $=987.4 \mathrm{~K}$. 


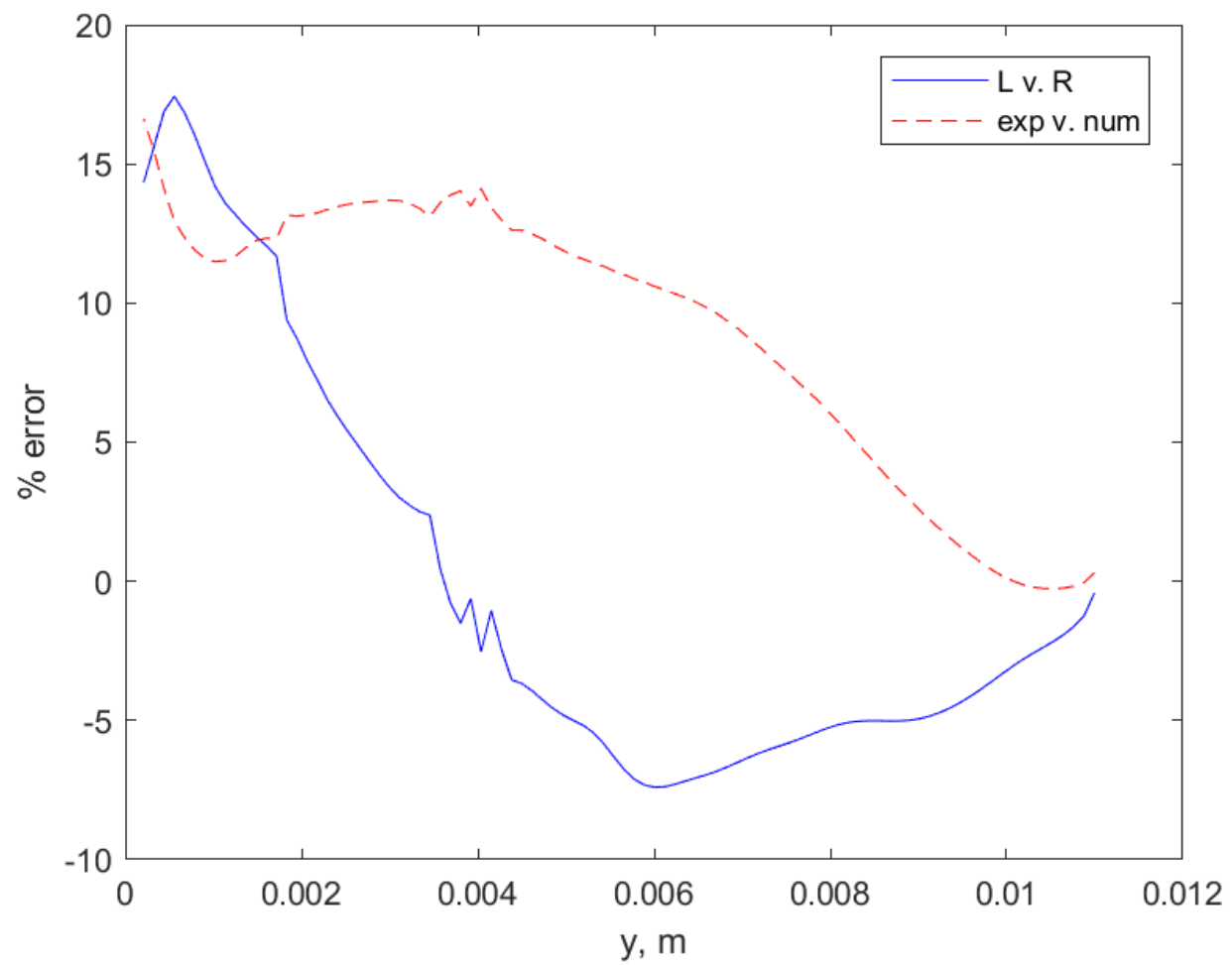

Figure C.27: Shot 32. Percent error in thermal boundary layer between both sides experimental boundary layer profiles (red line) and percent error between predicted boundary layer profile from similarity solution and averaged experimental boundary layer profile (blue dashed line). 
100A

Shot 91:

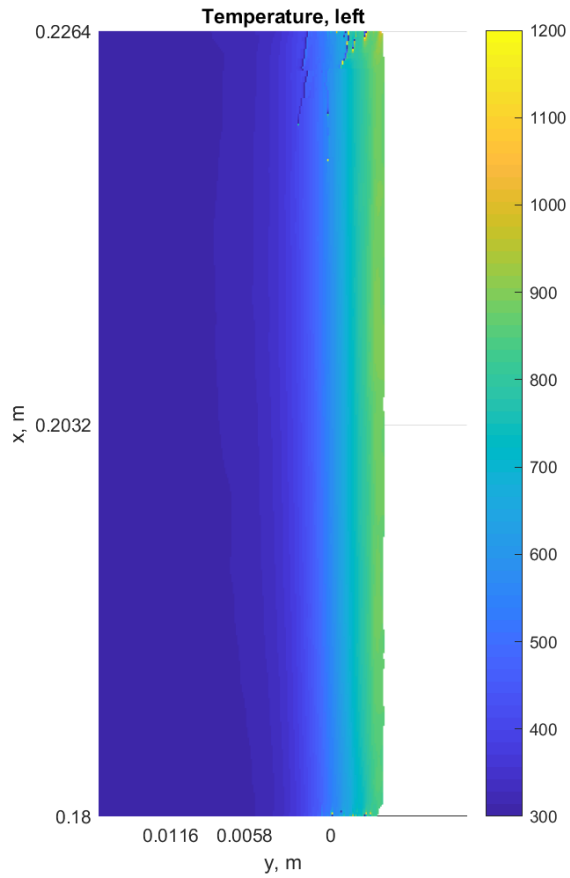

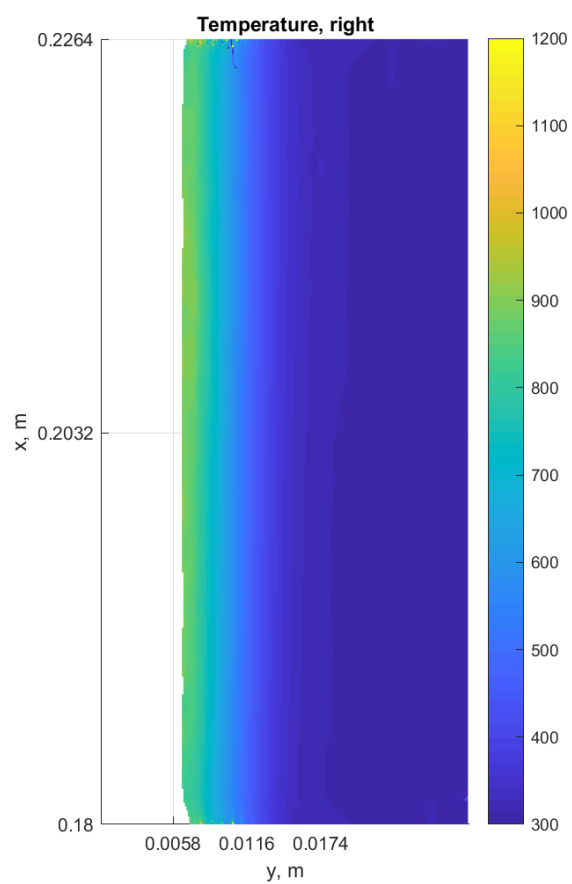

Figure C.28: Shot 91. Temperature fields from interferometry for hexane test. Surface temperature measured by pyrometer $=1028 \mathrm{~K}$. 


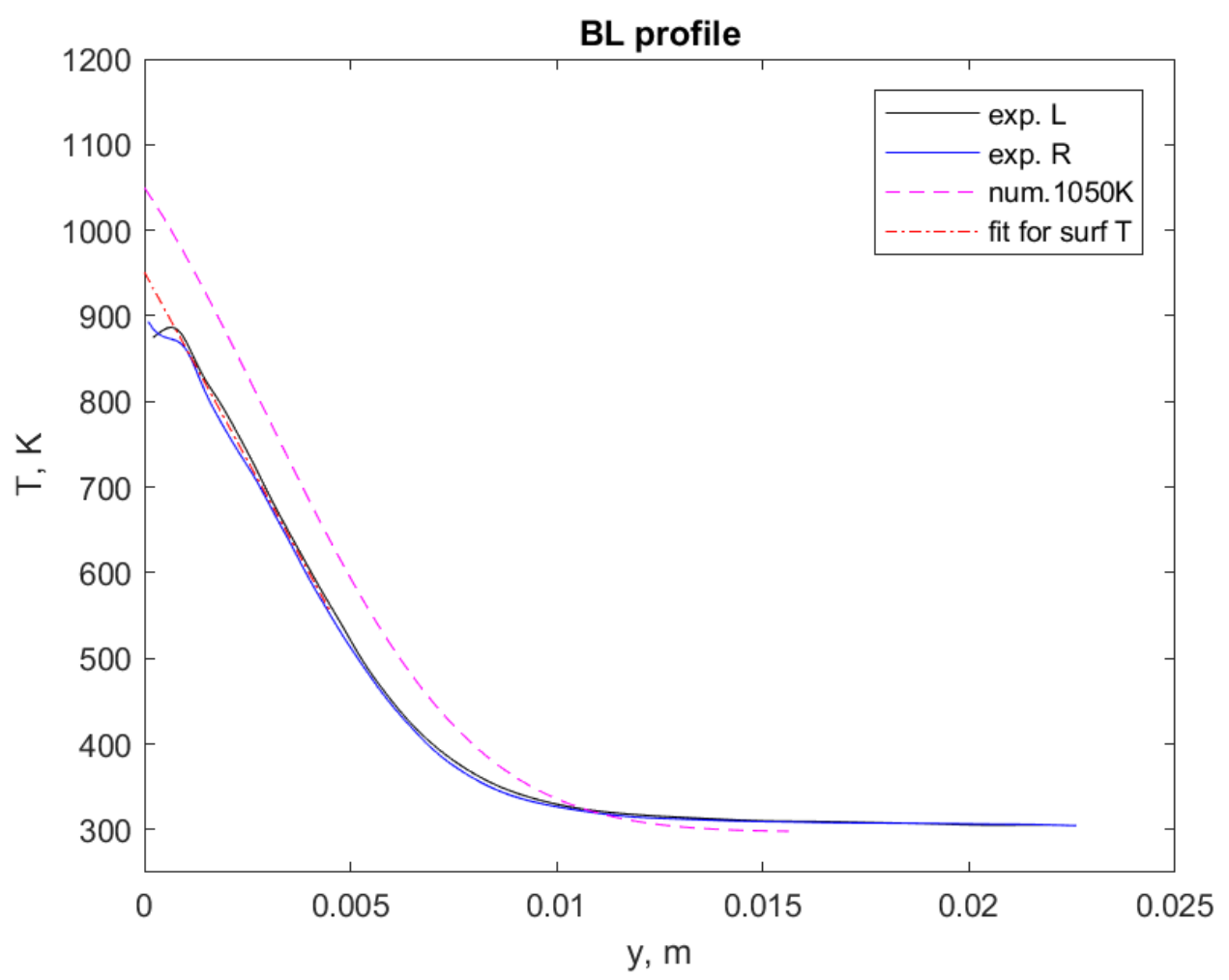

Figure C.29: Shot 91. Thermal boundary layer profiles for left (black line) and right (blue line) sides of experimental results compared with prediction of boundary layer profile (dashed magenta line) based on similarity solution for pyrometer surface temperature. Linear fit to averaged experimental profile (red dot-dashed line) used to extrapolate surface temperature from interferometer post processing. Extrapolated interferometer surface temperature $=951.2 \mathrm{~K}$. 


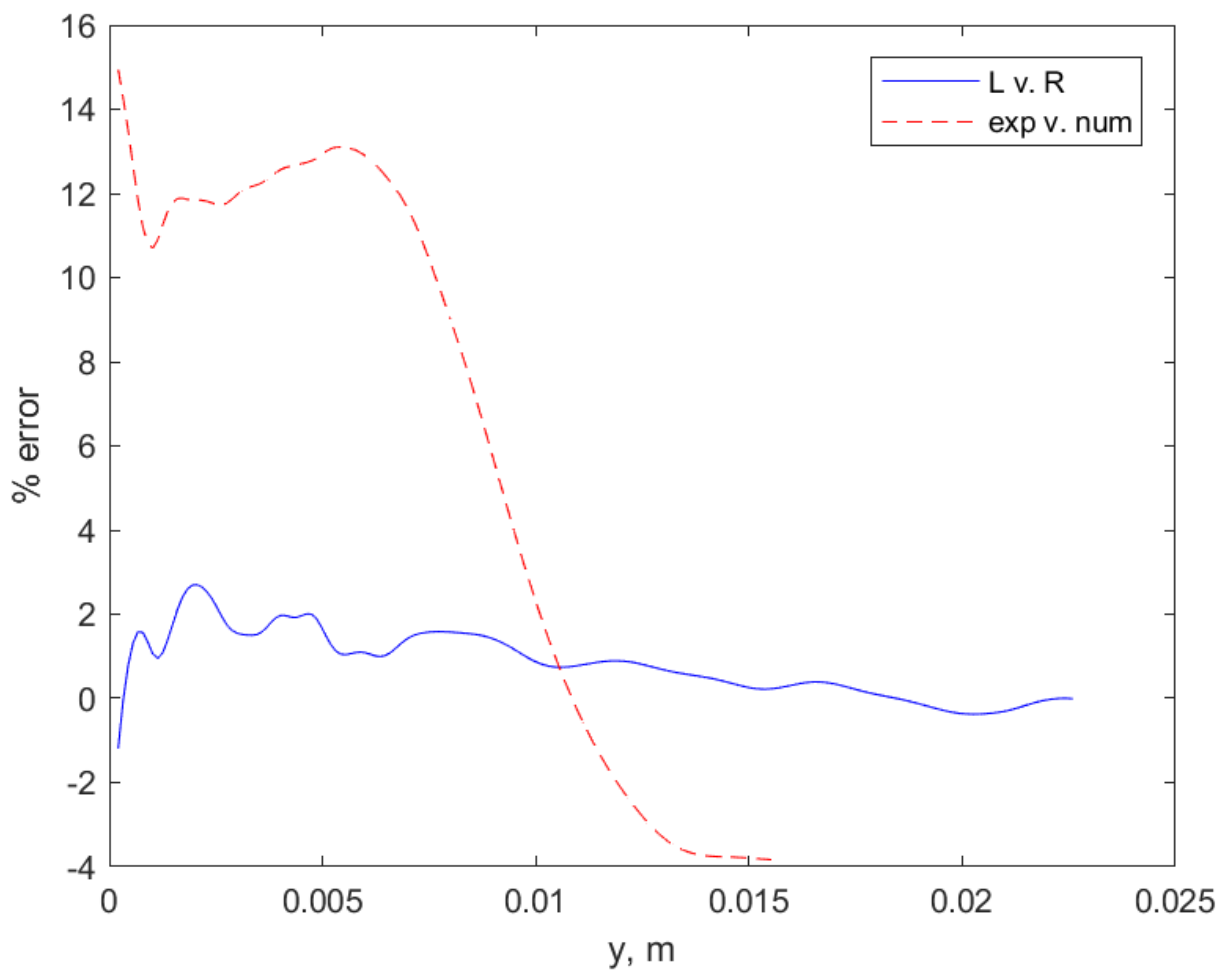

Figure C.30: Shot 91. Percent error in thermal boundary layer between both sides experimental boundary layer profiles (red line) and percent error between predicted boundary layer profile from similarity solution and averaged experimental boundary layer profile (blue dashed line). 
Shot 93:
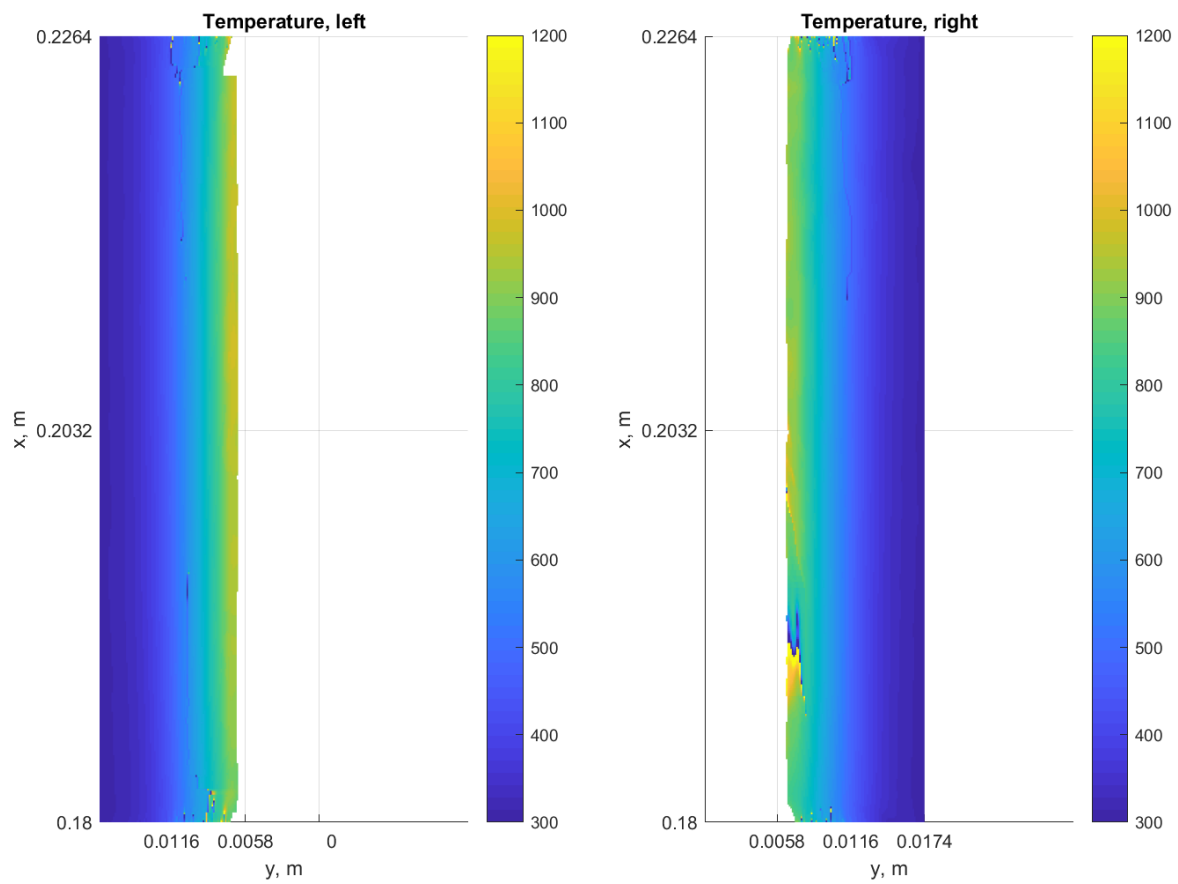

Figure C.31: Shot 93. Temperature fields from interferometry for nitrogen test. Surface temperature measured by pyrometer $=1045 \mathrm{~K}$. 


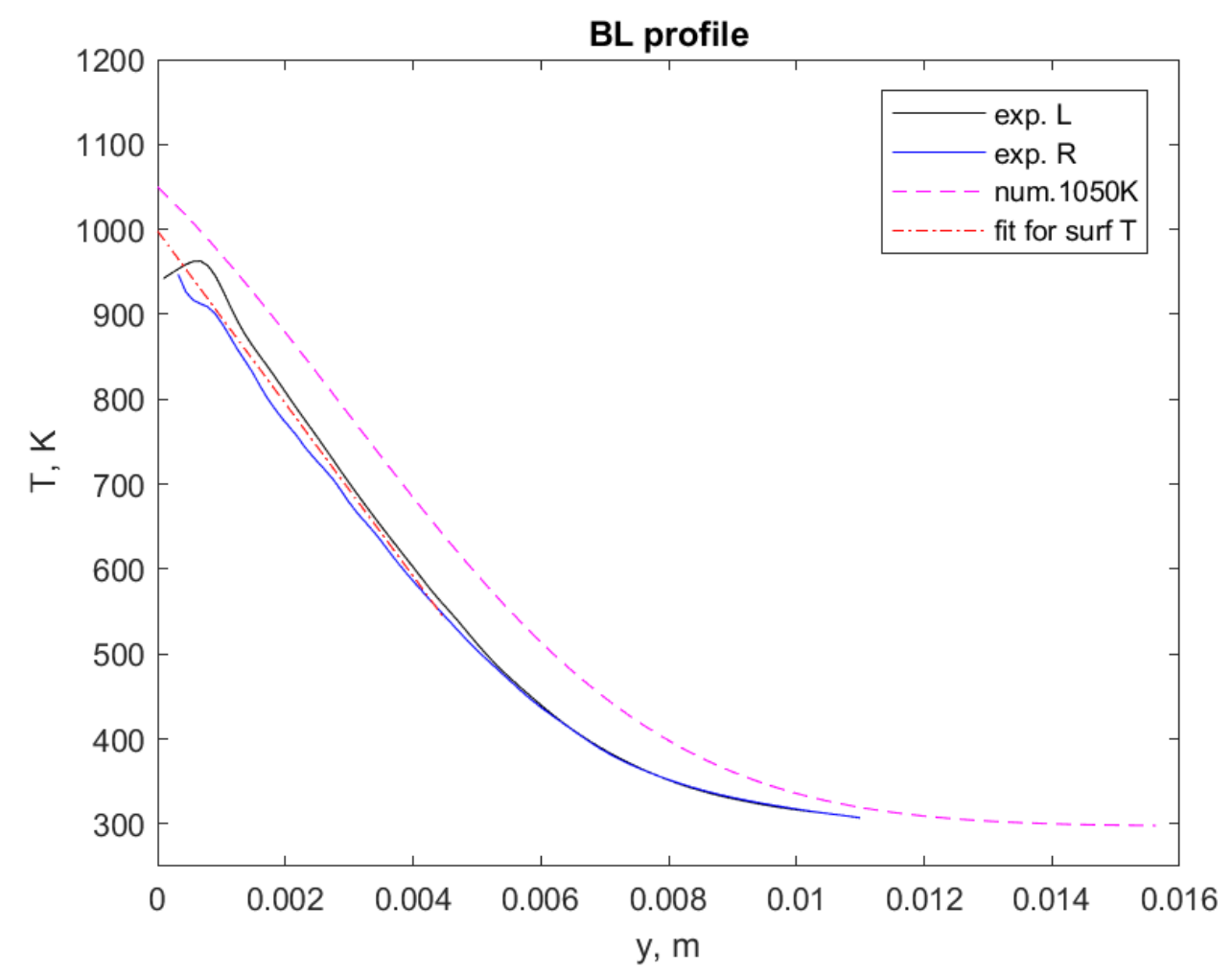

Figure C.32: Shot 93. Thermal boundary layer profiles for left (black line) and right (blue line) sides of experimental results compared with prediction of boundary layer profile (dashed magenta line) based on similarity solution for pyrometer surface temperature. Linear fit to averaged experimental profile (red dot-dashed line) used to extrapolate surface temperature from interferometer post processing. Extrapolated interferometer surface temperature $=998.1 \mathrm{~K}$. 


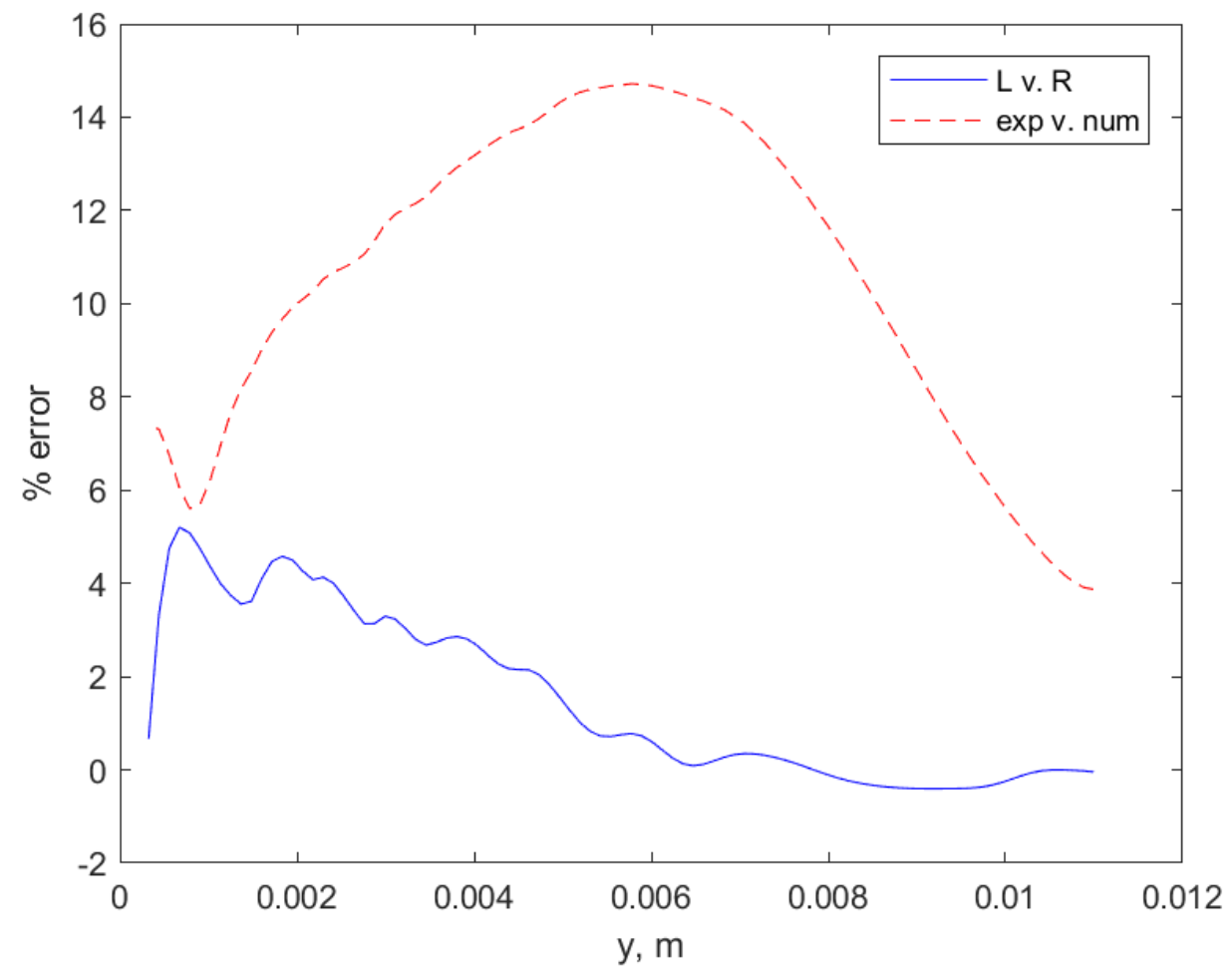

Figure C.33: Shot 93. Percent error in thermal boundary layer between both sides experimental boundary layer profiles (red line) and percent error between predicted boundary layer profile from similarity solution and averaged experimental boundary layer profile (blue dashed line). 
Shot 95:
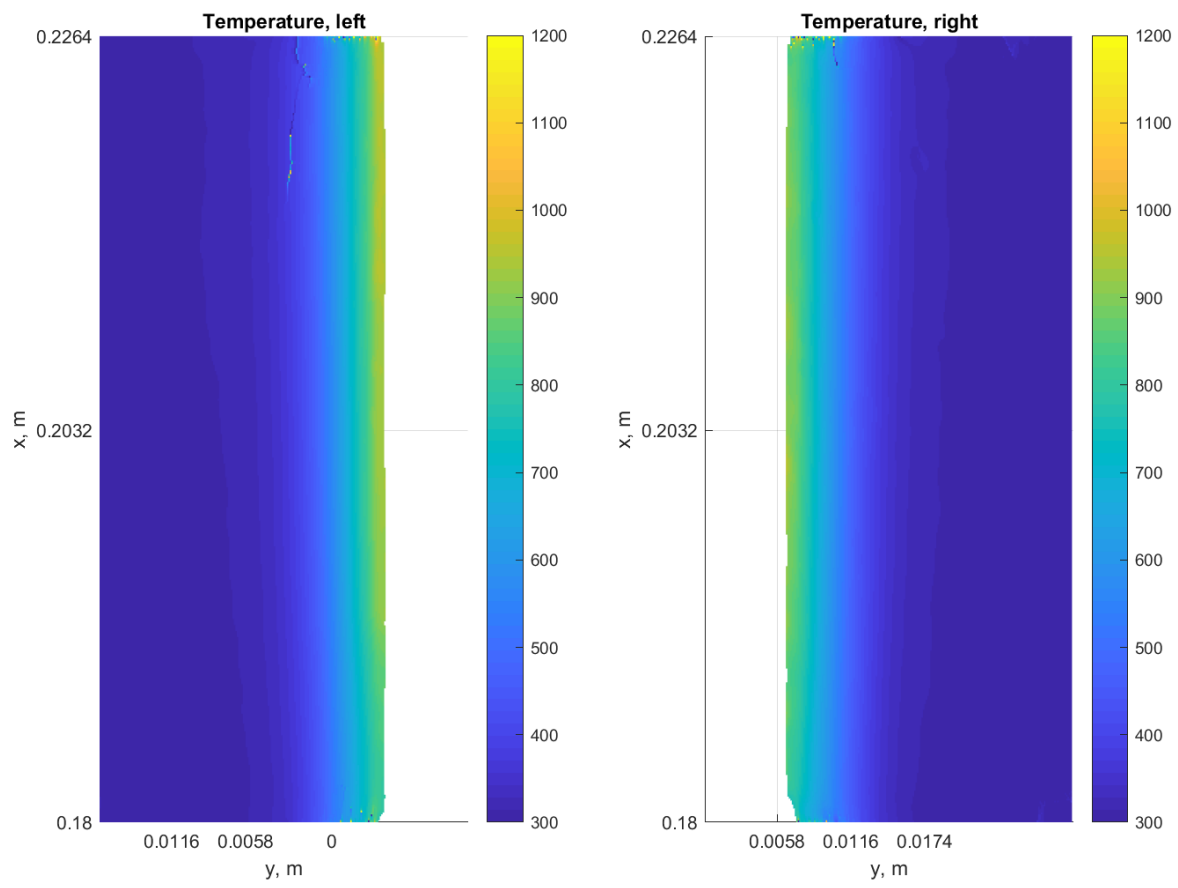

Figure C.34: Shot 95. Temperature fields from interferometry for nitrogen test. Surface temperature measured by pyrometer $=1004 \mathrm{~K}$. 


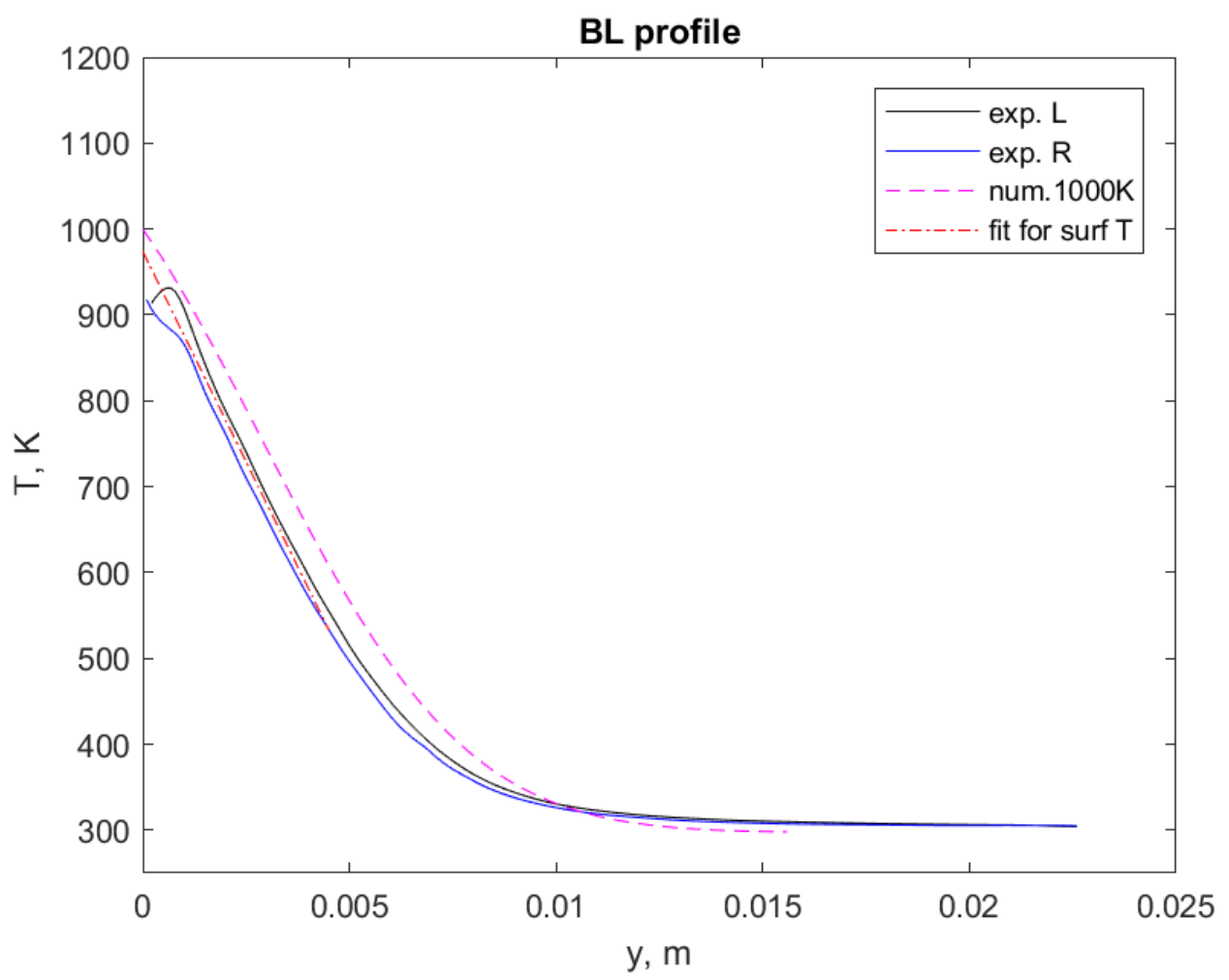

Figure C.35: Shot 95. Thermal boundary layer profiles for left (black line) and right (blue line) sides of experimental results compared with prediction of boundary layer profile (dashed magenta line) based on similarity solution for pyrometer surface temperature. Linear fit to averaged experimental profile (red dot-dashed line) used to extrapolate surface temperature from interferometer post processing. Extrapolated interferometer surface temperature $=973.9 \mathrm{~K}$. 


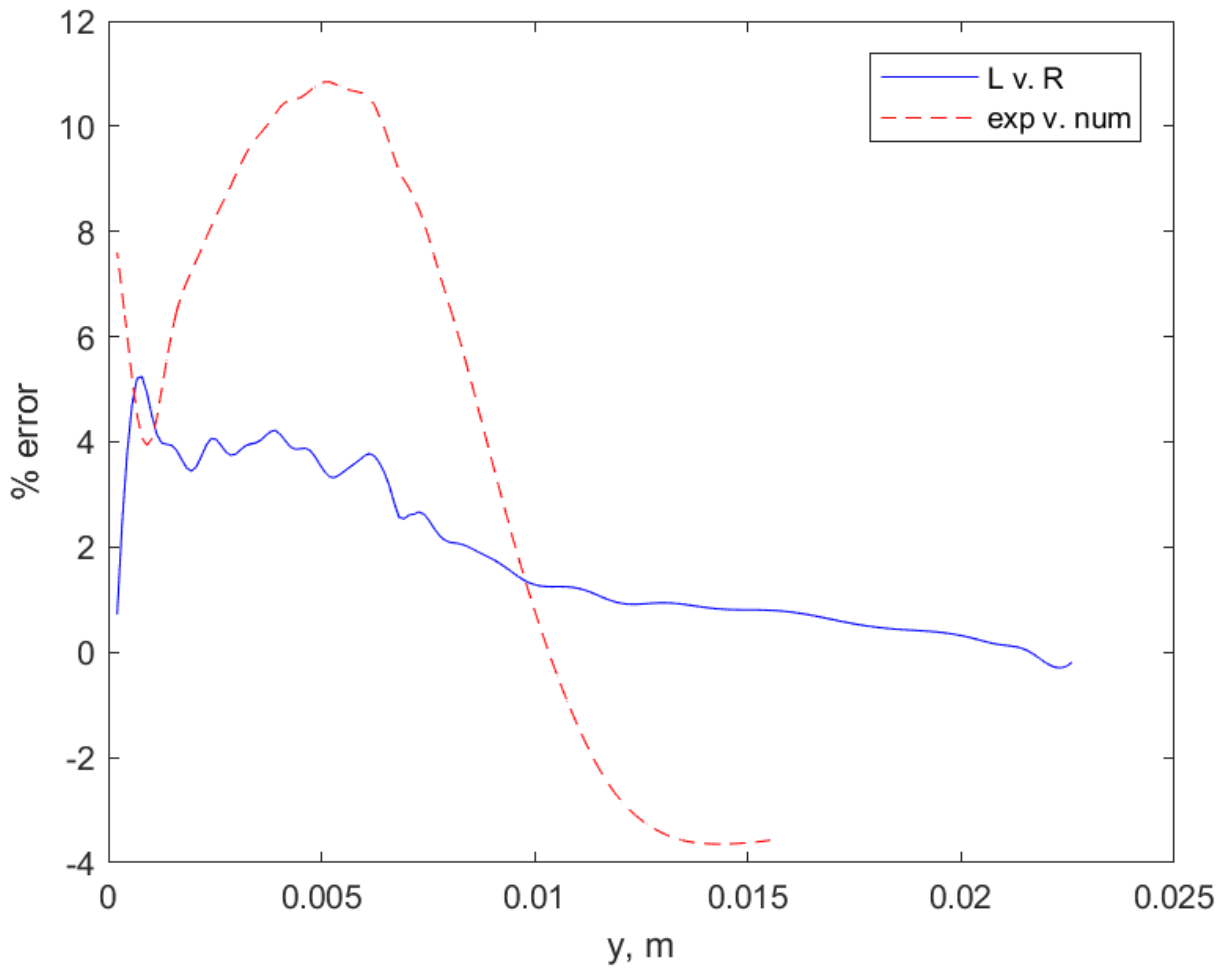

Figure C.36: Shot 95. Percent error in thermal boundary layer between both sides experimental boundary layer profiles (red line) and percent error between predicted boundary layer profile from similarity solution and averaged experimental boundary layer profile (blue dashed line). 
Shot 101:
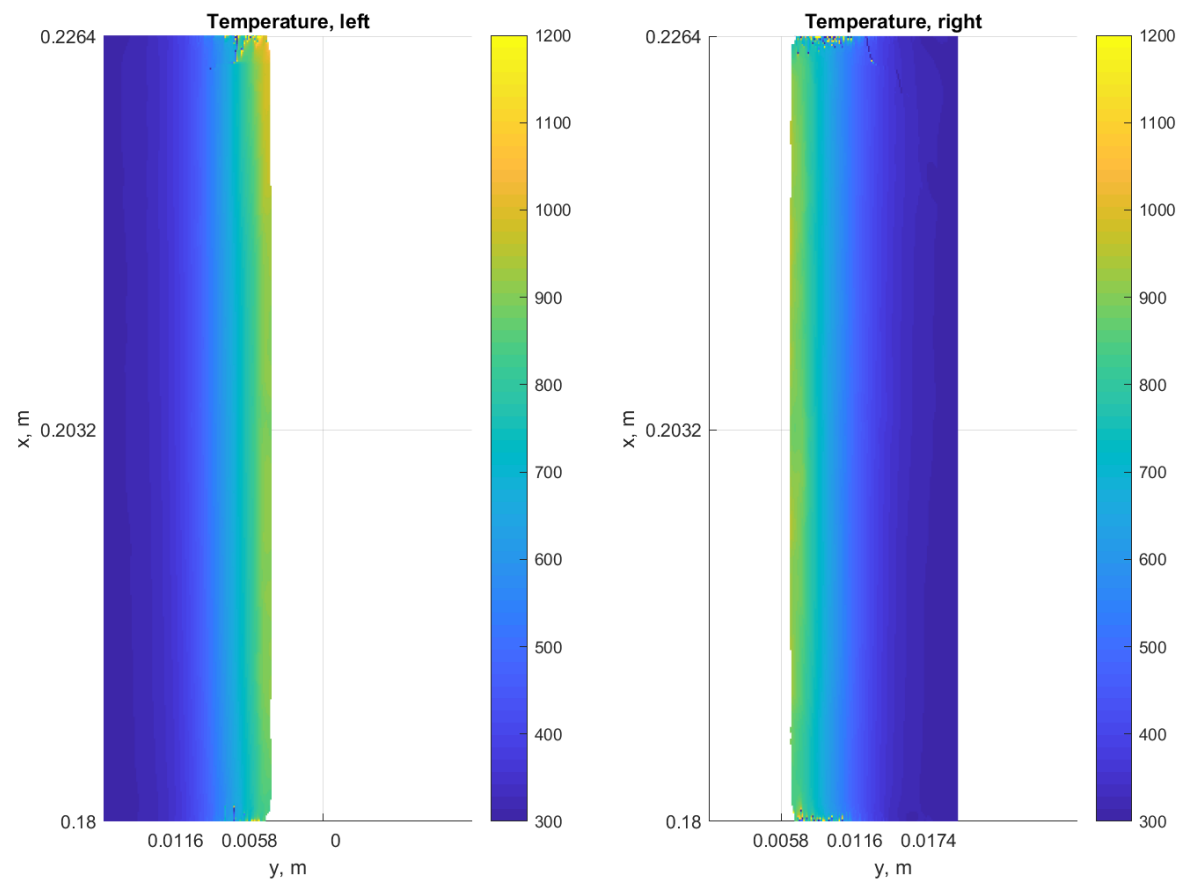

Figure C.37: Shot 101. Temperature fields from interferometry for nitrogen test. Surface temperature measured by pyrometer $=1060 \mathrm{~K}$. 


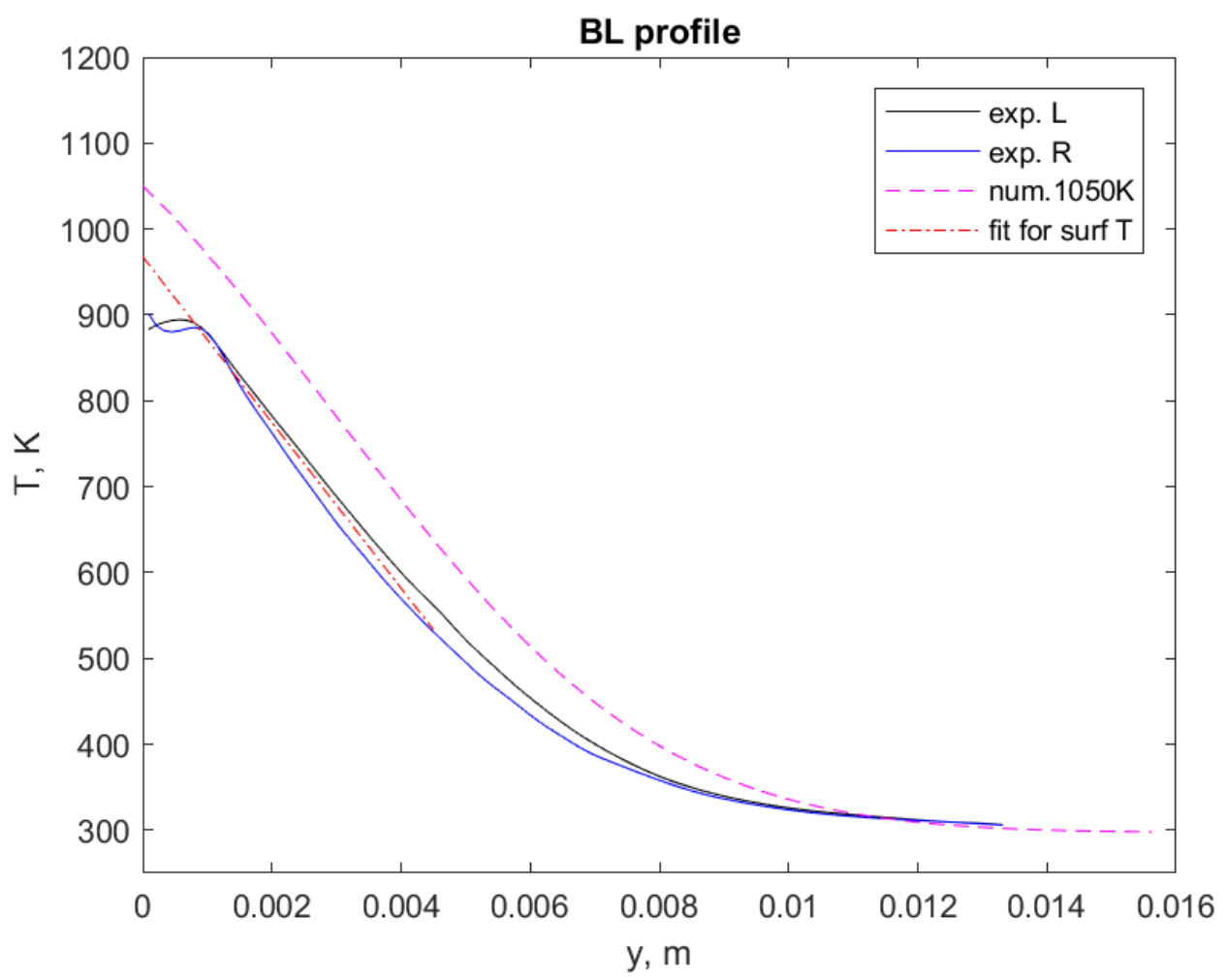

Figure C.38: Shot 101. Thermal boundary layer profiles for left (black line) and right (blue line) sides of experimental results compared with prediction of boundary layer profile (dashed magenta line) based on similarity solution for pyrometer surface temperature. Linear fit to averaged experimental profile (red dot-dashed line) used to extrapolate surface temperature from interferometer post processing. Extrapolated interferometer surface temperature $=967.7 \mathrm{~K}$. 


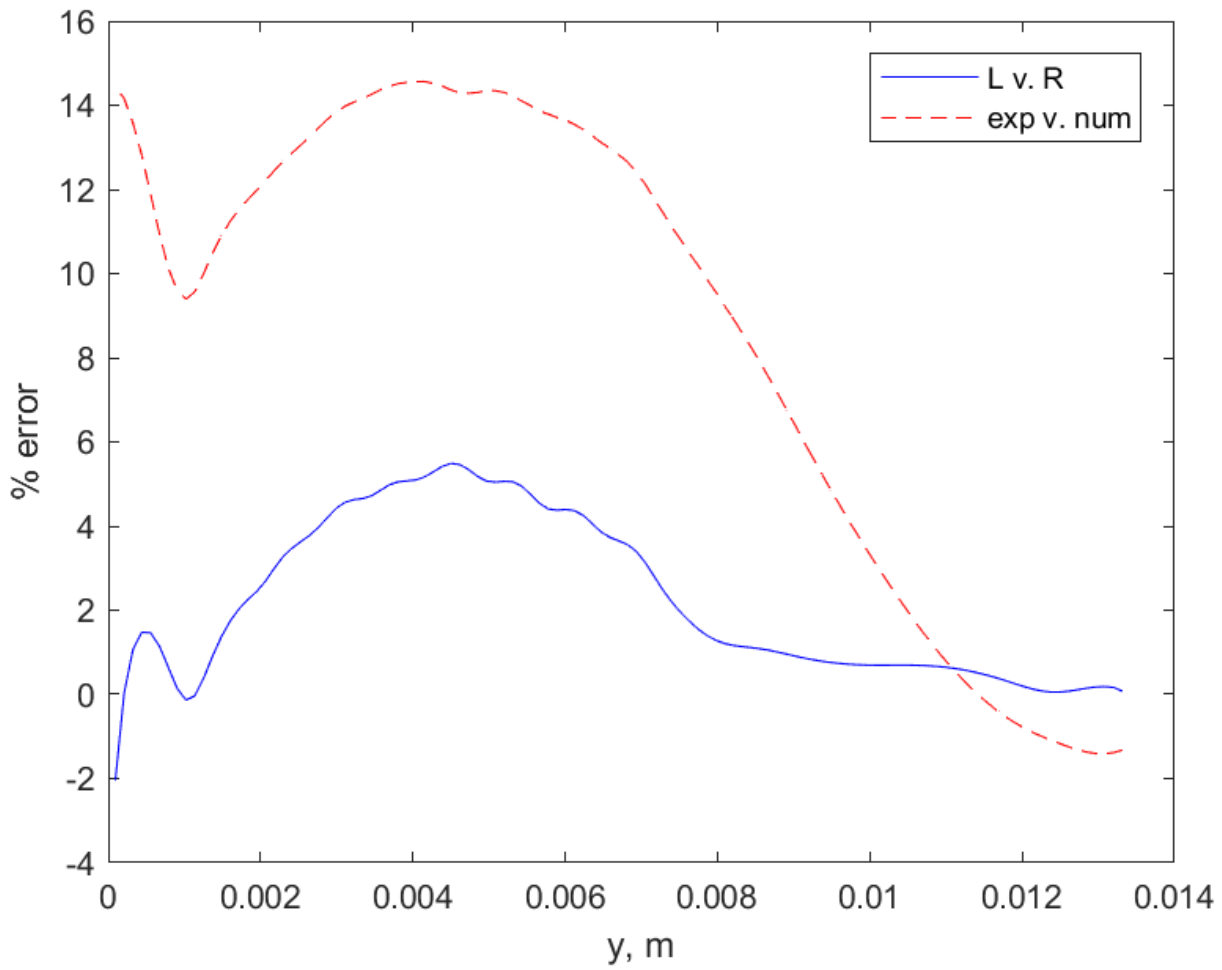

Figure C.39: Shot 101. Percent error in thermal boundary layer between both sides experimental boundary layer profiles (red line) and percent error between predicted boundary layer profile from similarity solution and averaged experimental boundary layer profile (blue dashed line). 
Shot 103:
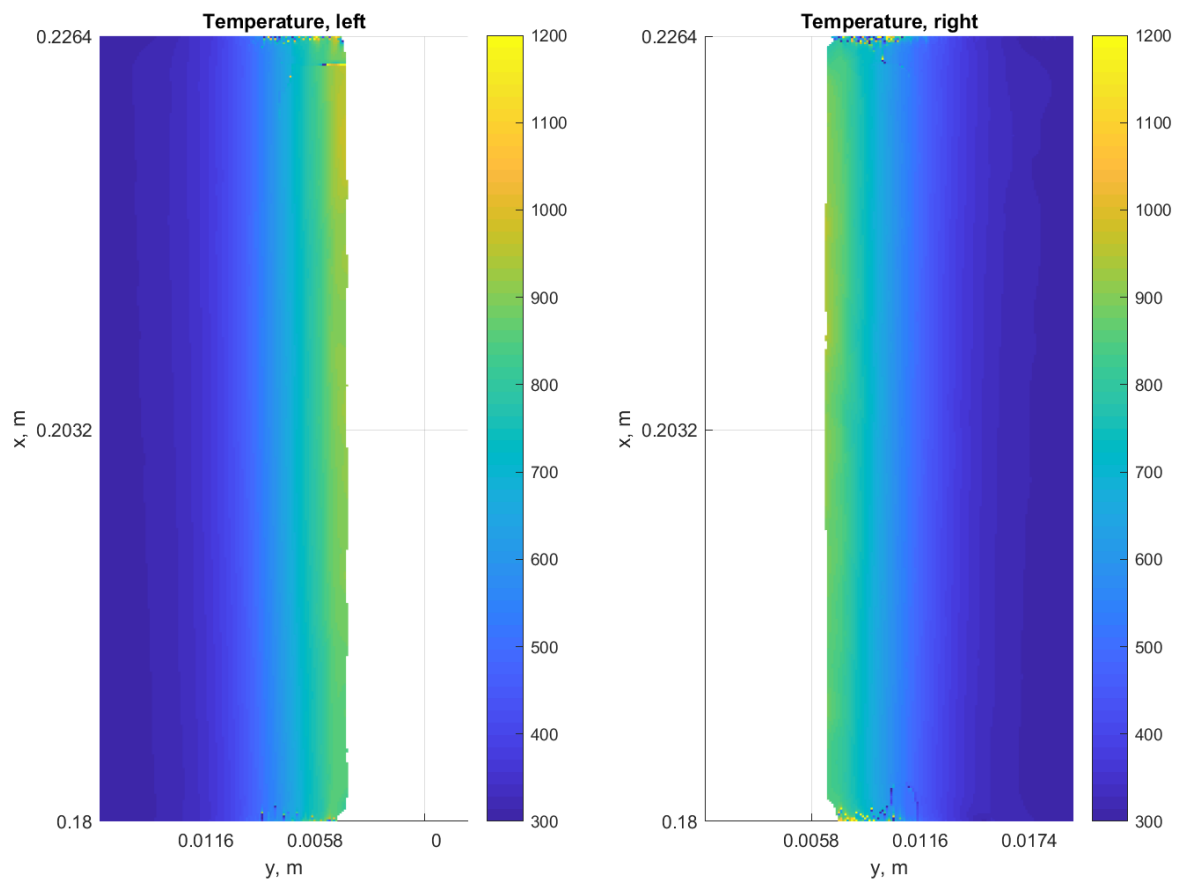

Figure C.40: Shot 103. Temperature fields from interferometry for nitrogen test. Surface temperature measured by pyrometer $=1011 \mathrm{~K}$. 


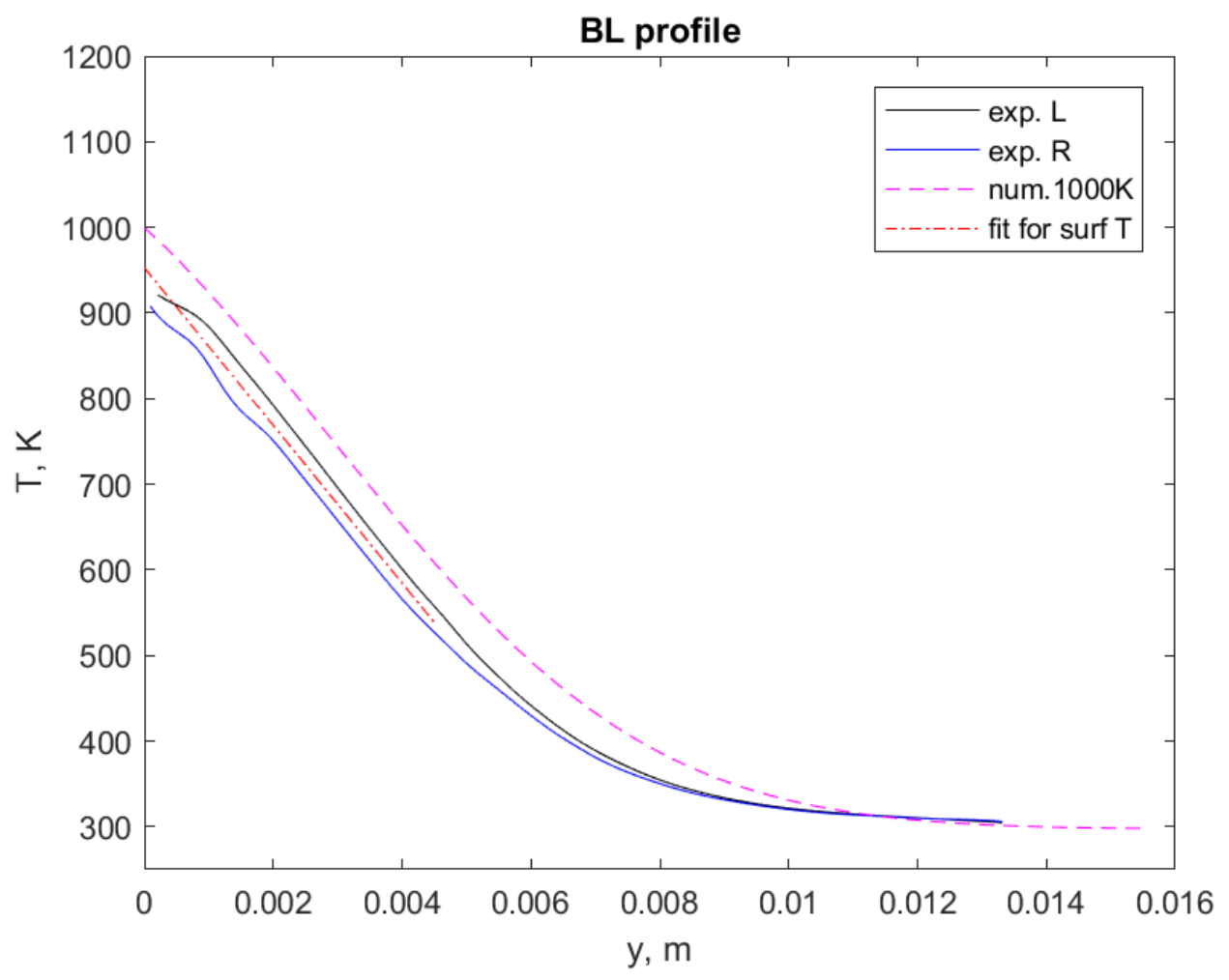

Figure C.41: Shot 103. Thermal boundary layer profiles for left (black line) and right (blue line) sides of experimental results compared with prediction of boundary layer profile (dashed magenta line) based on similarity solution for pyrometer surface temperature. Linear fit to averaged experimental profile (red dot-dashed line) used to extrapolate surface temperature from interferometer post processing. Extrapolated interferometer surface temperature $=952.9 \mathrm{~K}$. 


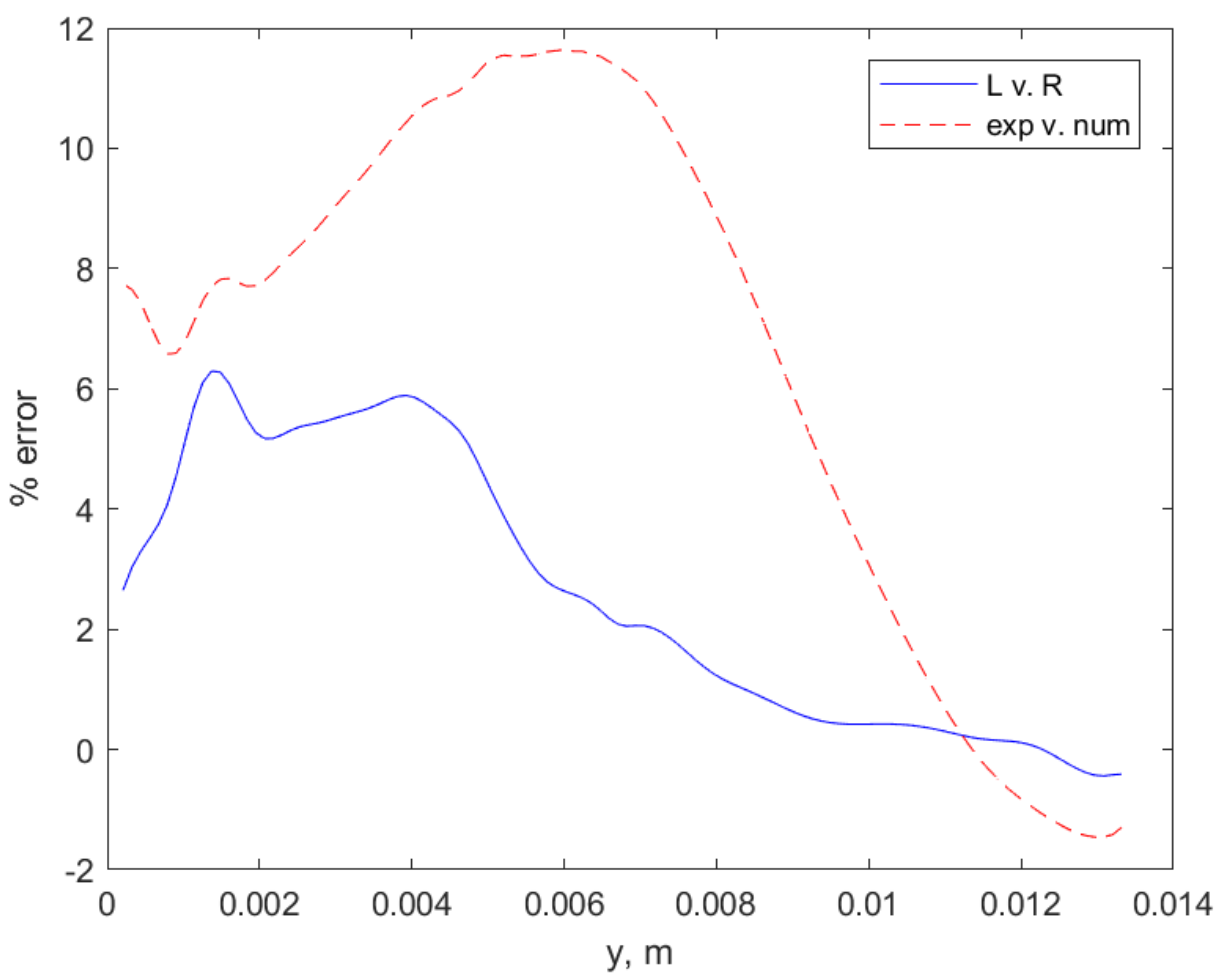

Figure C.42: Shot 103. Percent error in thermal boundary layer between both sides of experimental boundary layer profiles (red line) and percent error between predicted boundary layer profile from similarity solution and averaged experimental boundary layer profile (blue dashed line). 
Shot 106:
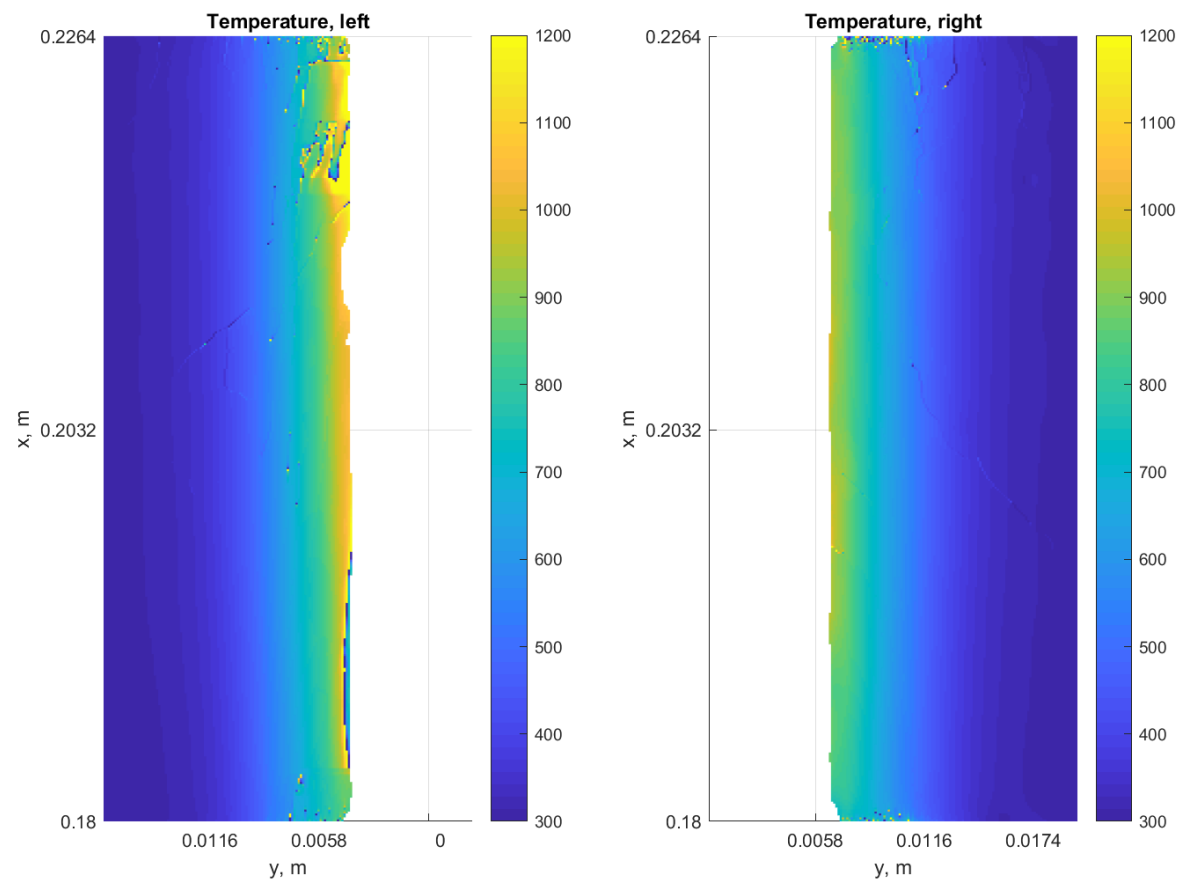

Figure C.43: Shot 106. Temperature fields from interferometry for nitrogen test. Surface temperature measured by pyrometer $=1049 \mathrm{~K}$. 


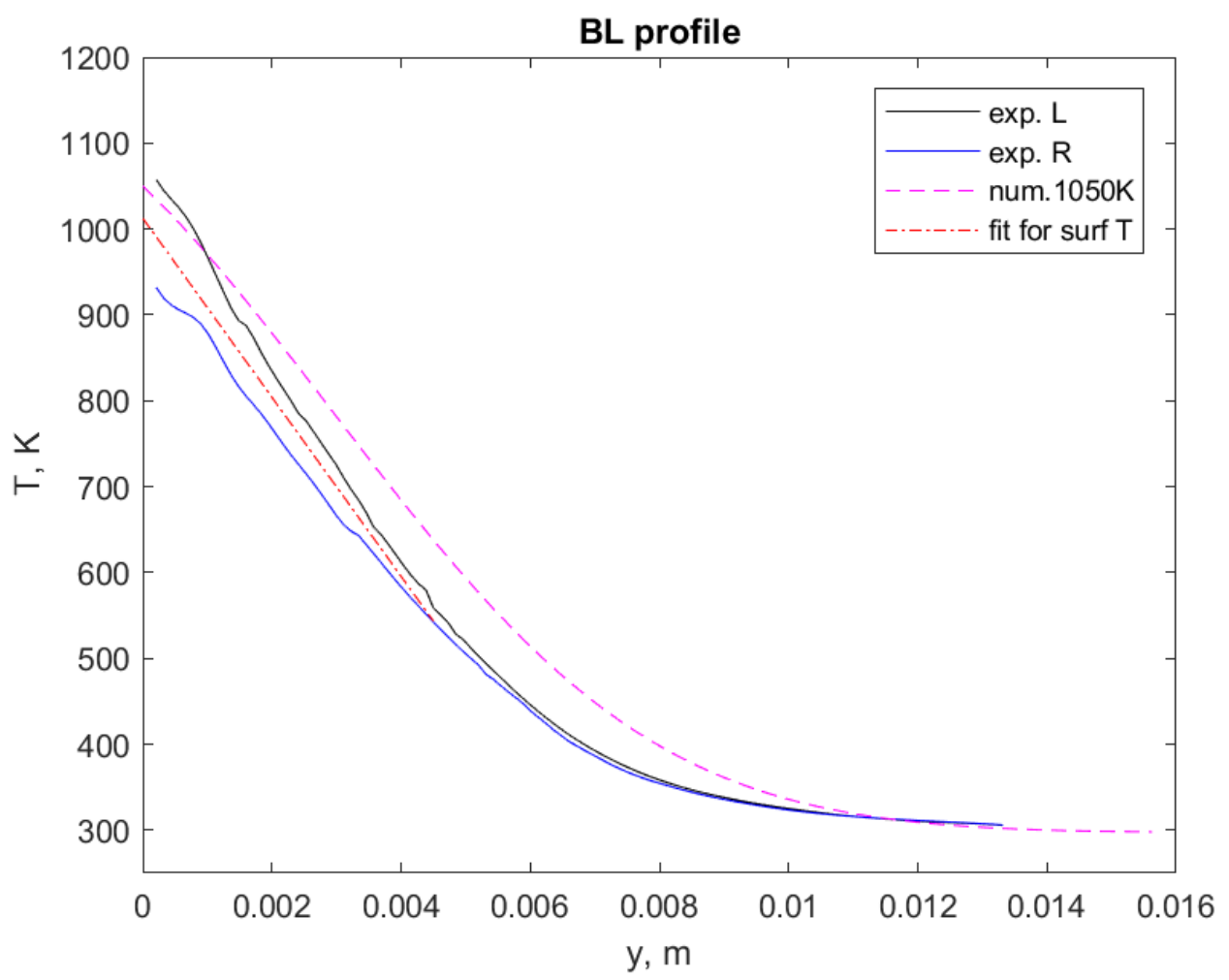

Figure C.44: Shot 106. Thermal boundary layer profiles for left (black line) and right (blue line) sides of experimental results compared with prediction of boundary layer profile (dashed magenta line) based on similarity solution for pyrometer surface temperature. Linear fit to averaged experimental profile (red dot-dashed line) used to extrapolate surface temperature from interferometer post processing. Extrapolated interferometer surface temperature $=1012.6 \mathrm{~K}$. 


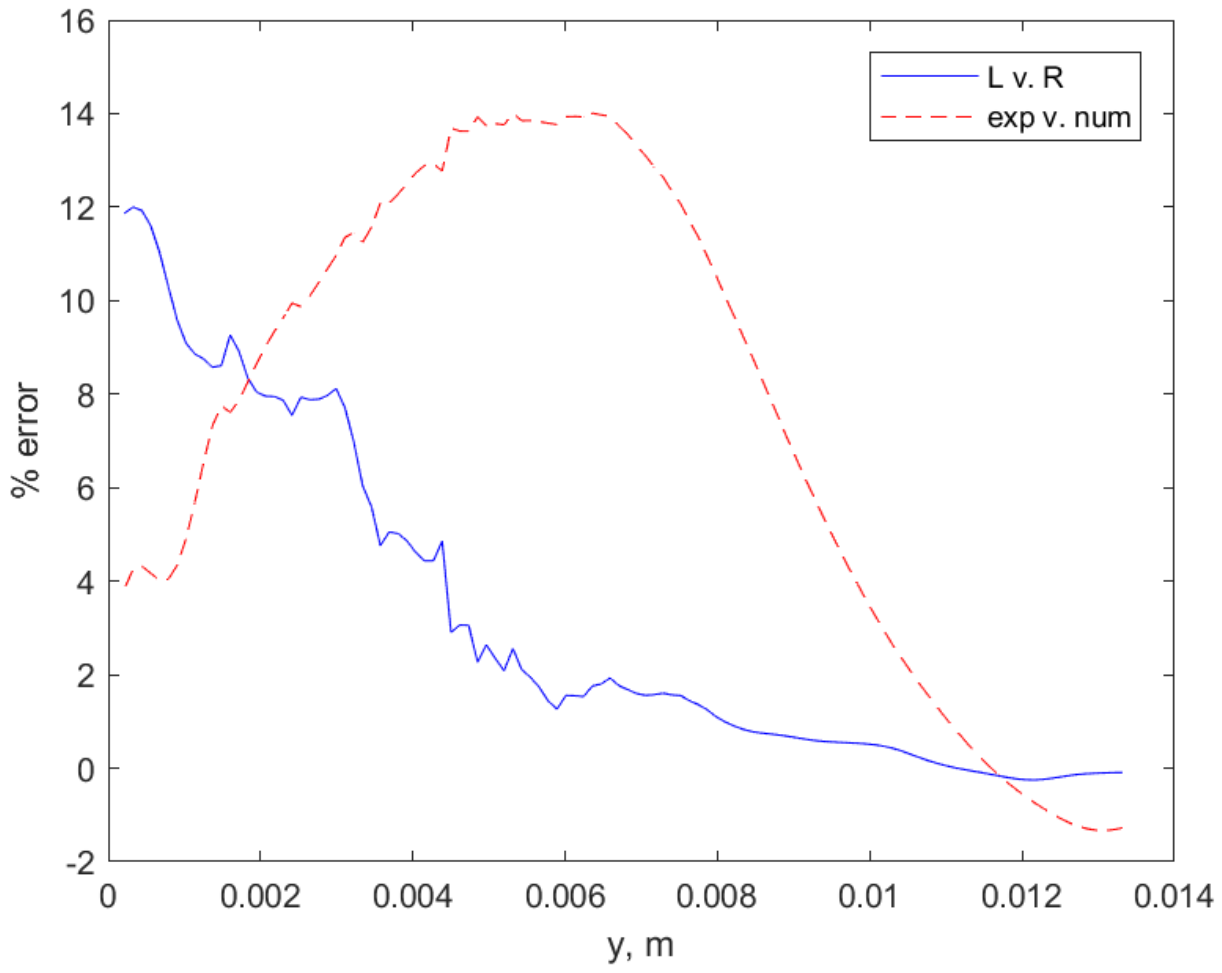

Figure C.45: Shot 106. Percent error in thermal boundary layer between both sides of experimental boundary layer profiles (red line) and percent error between predicted boundary layer profile from similarity solution and averaged experimental boundary layer profile (blue dashed line). 
100C

Shot84:

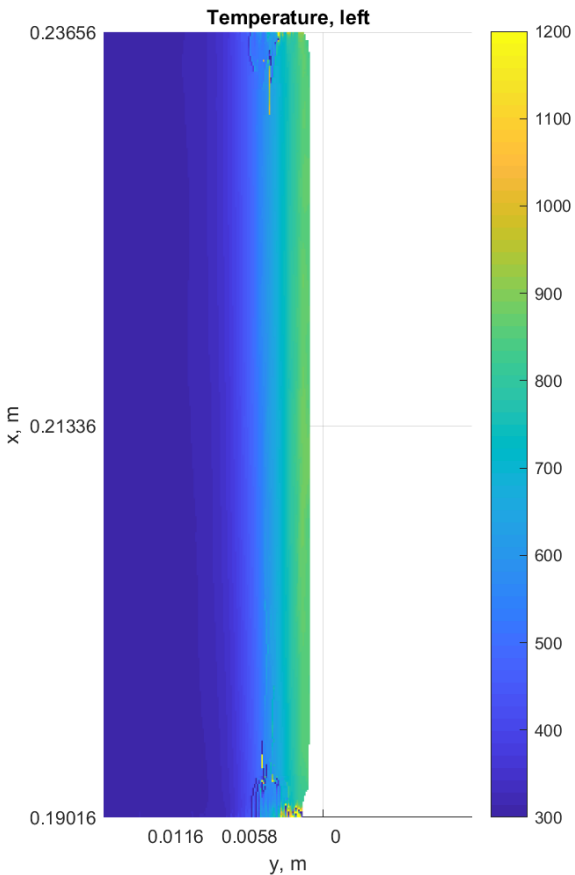

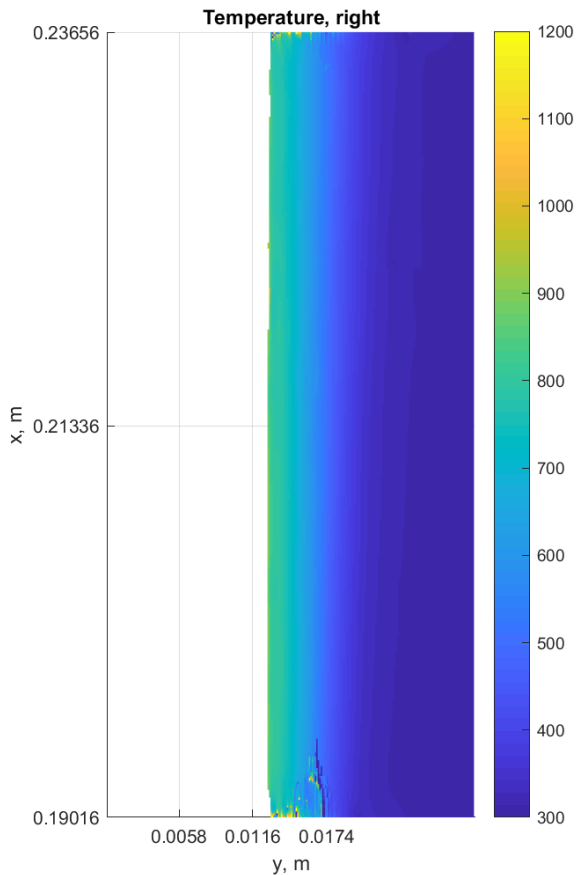

Figure C.46: Shot 84. Temperature fields from interferometry for nitrogen test. Surface temperature measured by pyrometer $=1001 \mathrm{~K}$. 


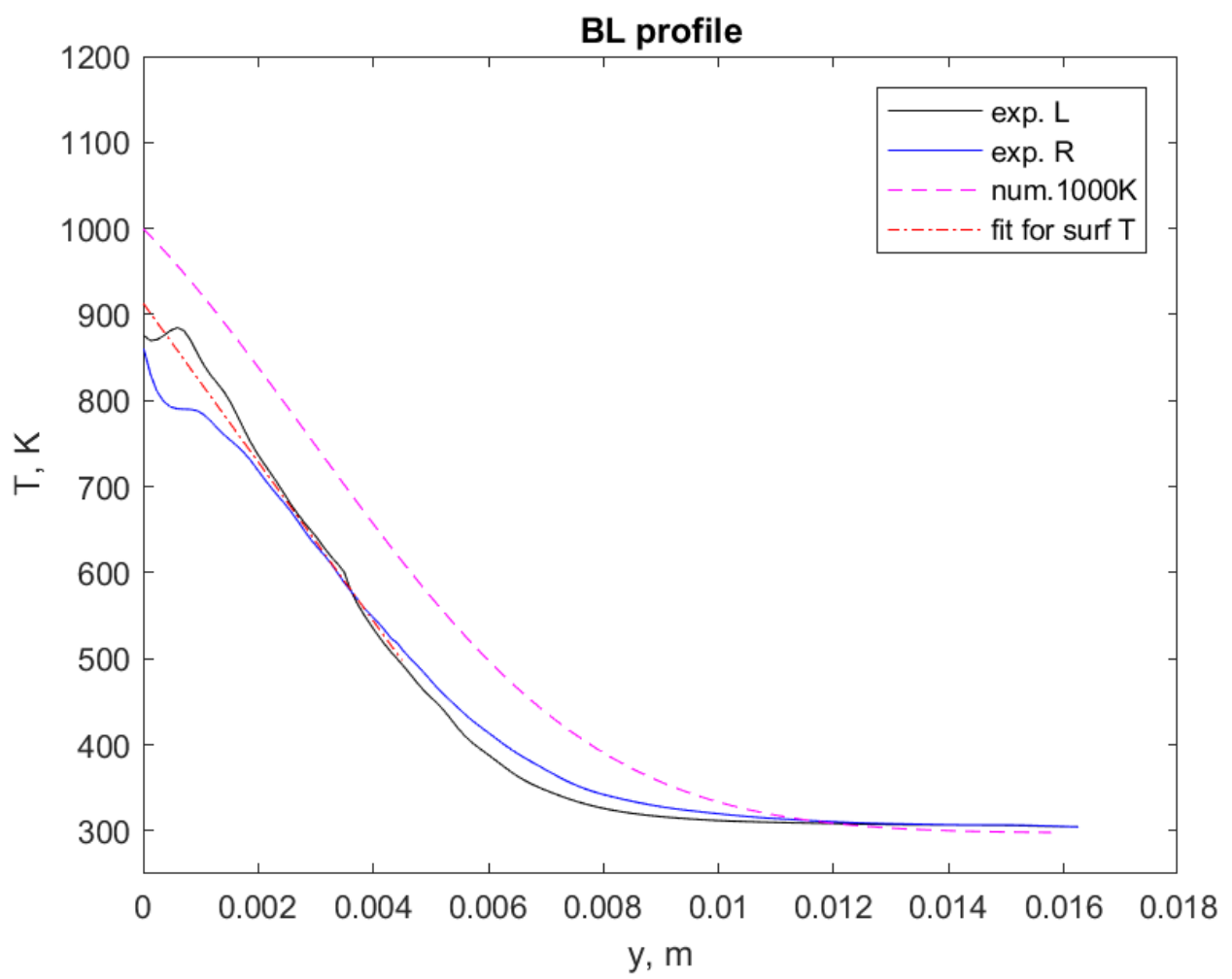

Figure C.47: Shot 84. Thermal boundary layer profiles for left (black line) and right (blue line) sides of experimental results compared with prediction of boundary layer profile (dashed magenta line) based on similarity solution for pyrometer surface temperature. Linear fit to averaged experimental profile (red dot-dashed line) used to extrapolate surface temperature from interferometer post processing. Extrapolated interferometer surface temperature $=913.3 \mathrm{~K}$. 


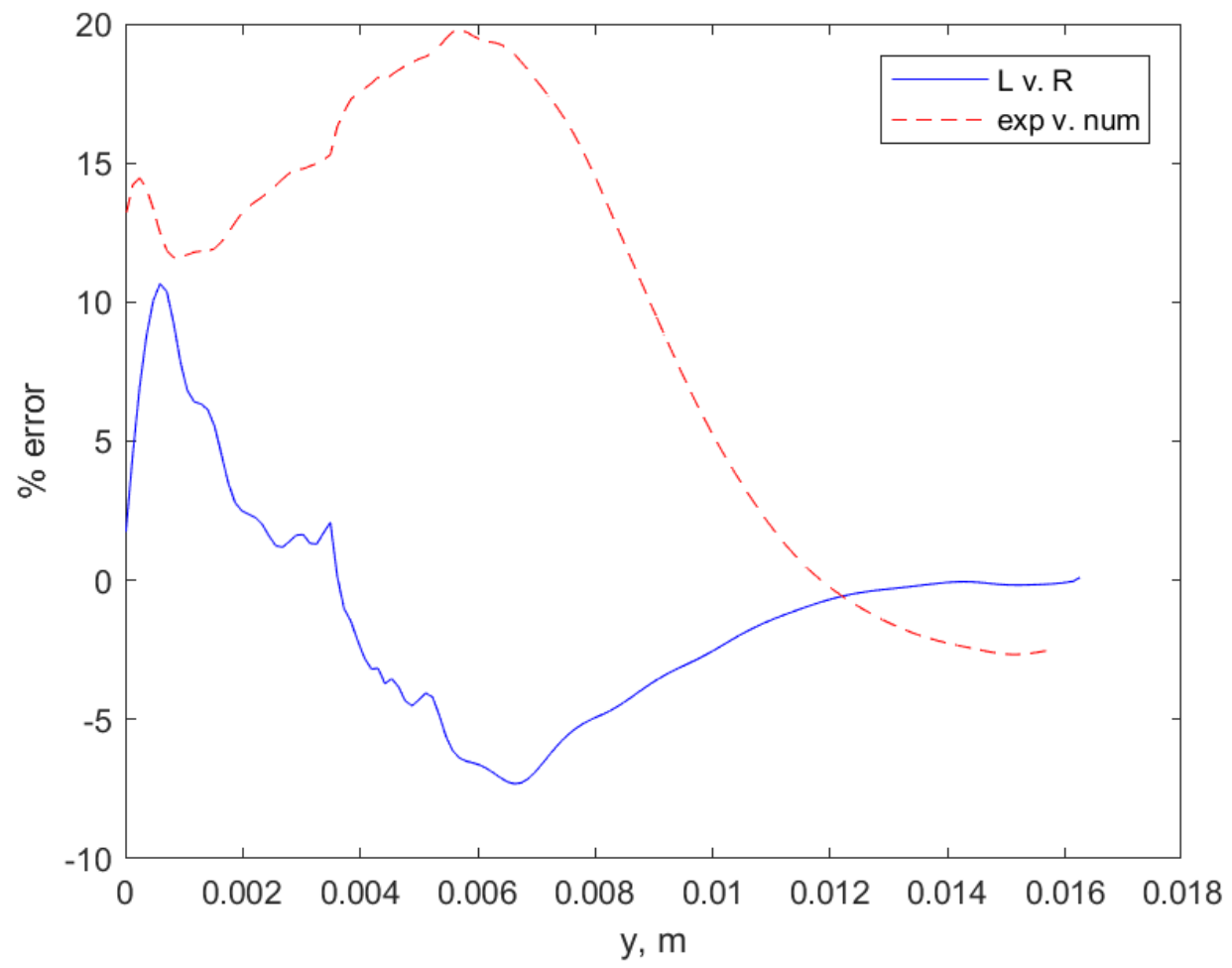

Figure C.48: Shot 84. Percent error in thermal boundary layer between both sides experimental boundary layer profiles (red line) and percent error between predicted boundary layer profile from similarity solution and averaged experimental boundary layer profile (blue dashed line). 
Shot 157:
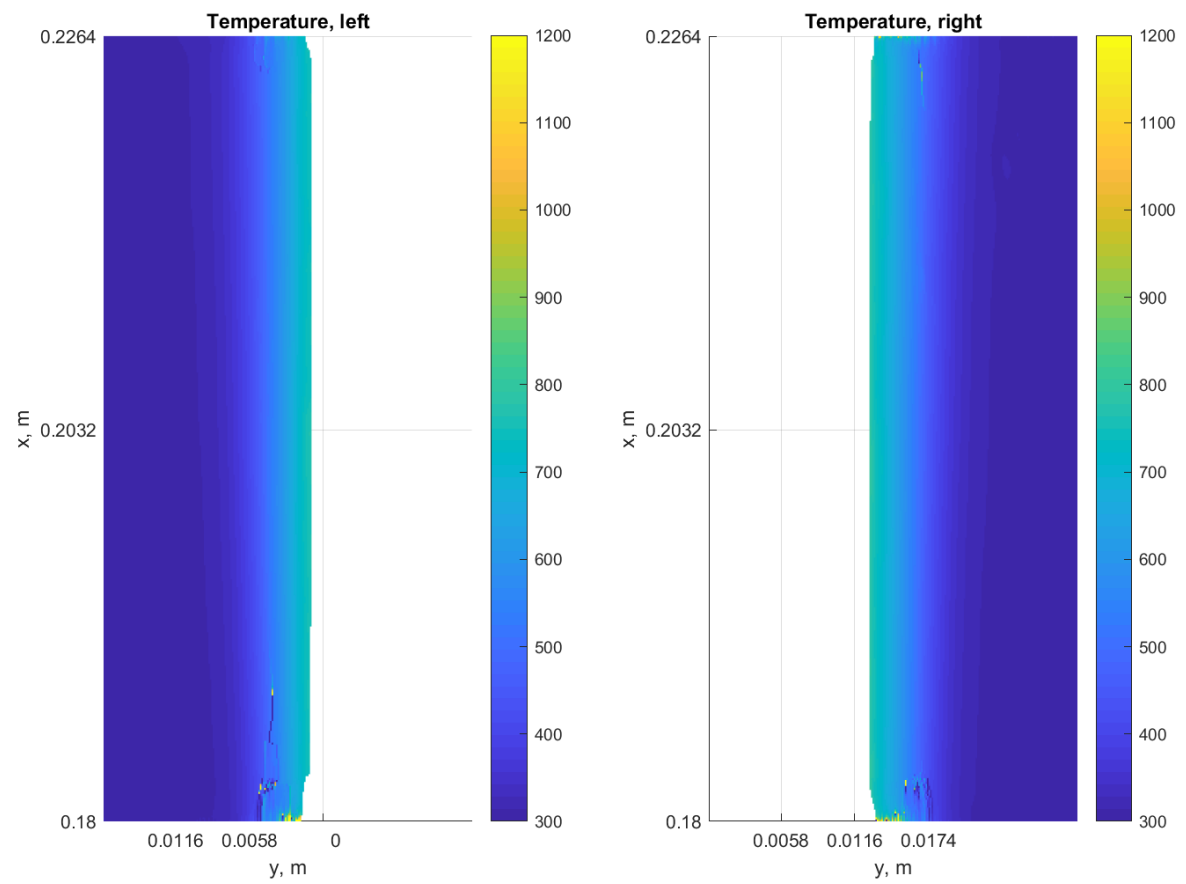

Figure C.49: Shot 157. Temperature fields from interferometry for nitrogen test. Surface temperature measured by pyrometer $=1107 \mathrm{~K}$. 


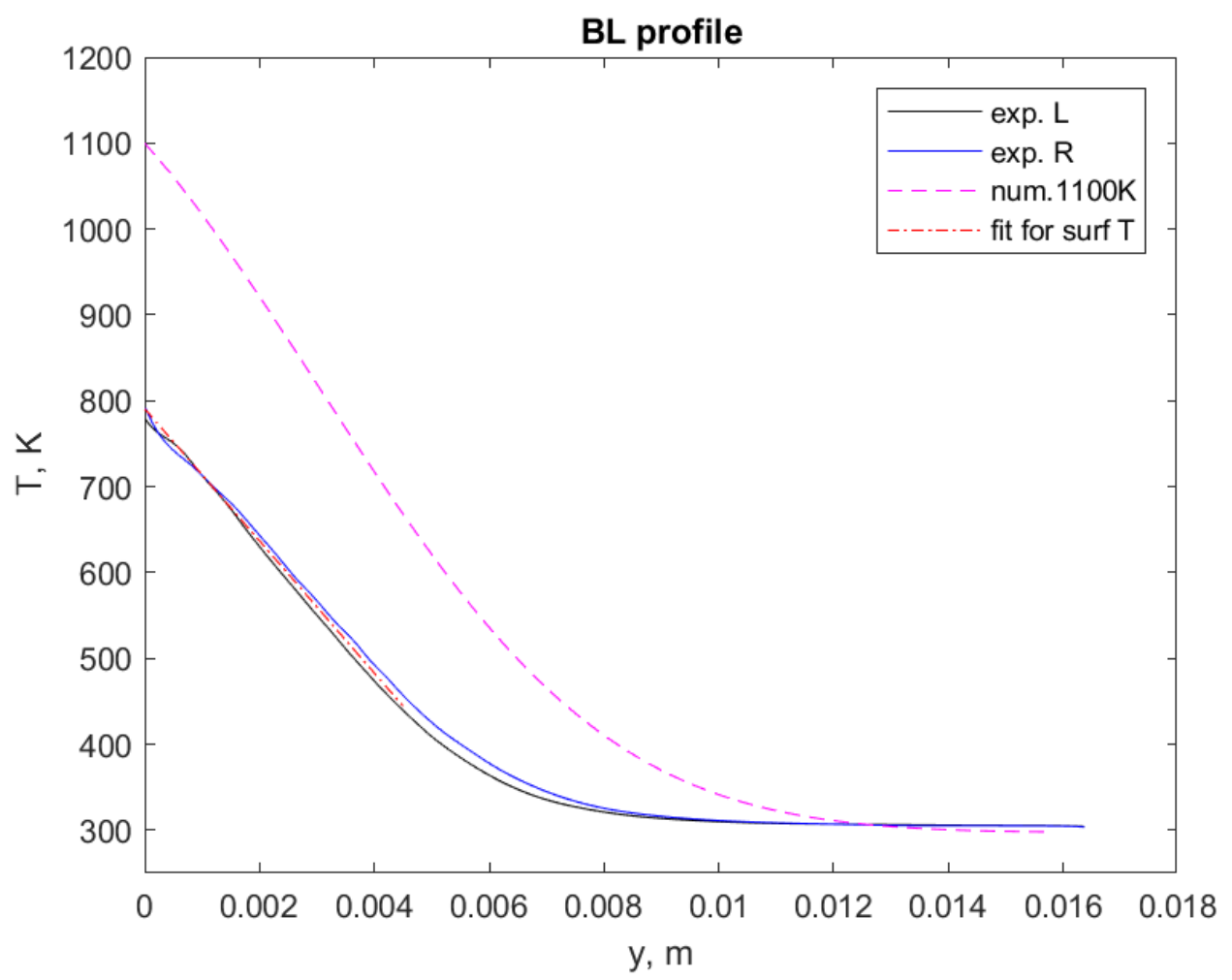

Figure C.50: Shot 157. Thermal boundary layer profiles for left (black line) and right (blue line) sides of experimental results compared with prediction of boundary layer profile (dashed magenta line) based on similarity solution for pyrometer surface temperature. Linear fit to averaged experimental profile (red dot-dashed line) used to extrapolate surface temperature from interferometer post processing. Extrapolated interferometer surface temperature $=790.7 \mathrm{~K}$. 


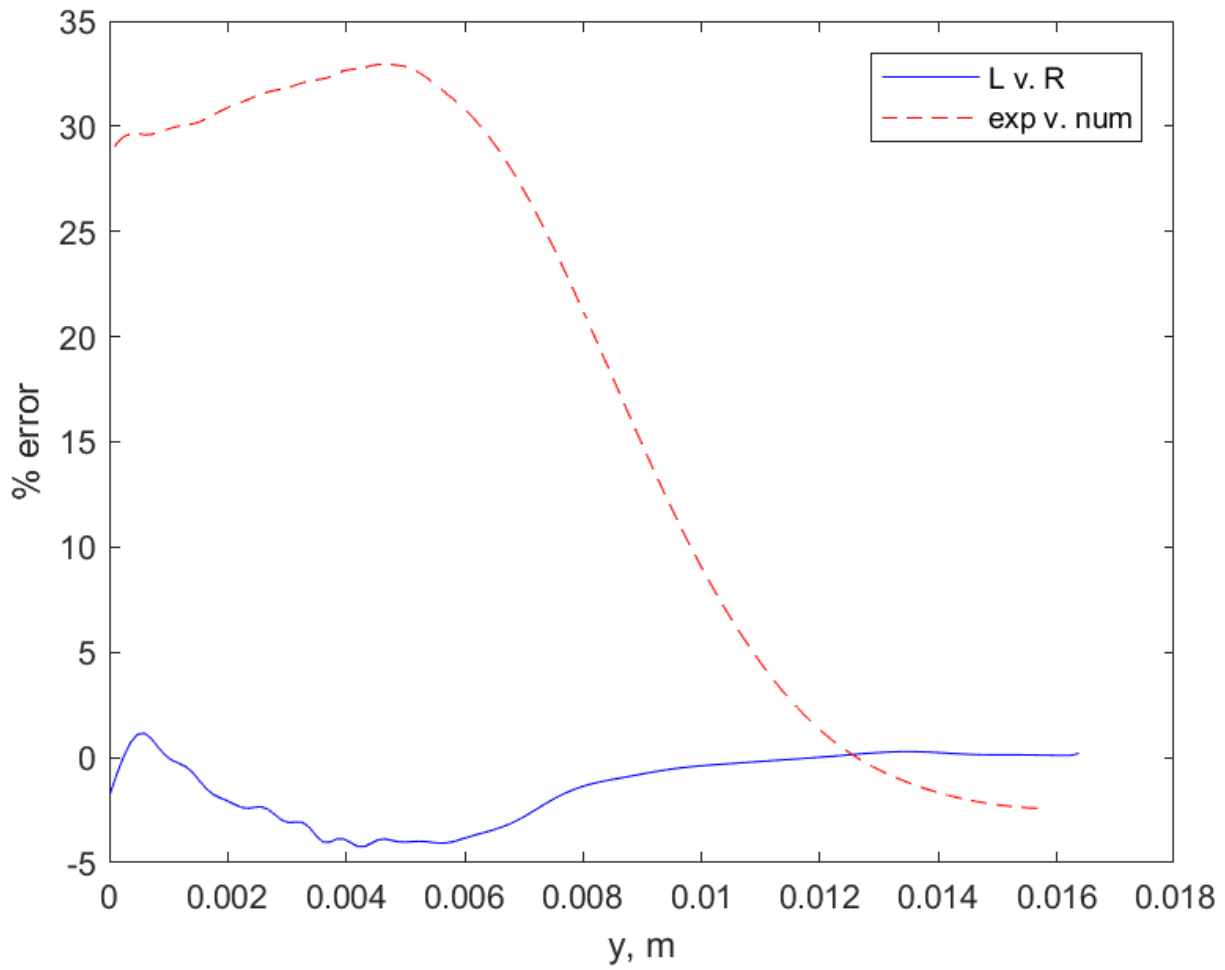

Figure C.51: Shot 157. Percent error in thermal boundary layer between both sides experimental boundary layer profiles (red line) and percent error between predicted boundary layer profile from similarity solution and averaged experimental boundary layer profile (blue dashed line). 
Shot 164:
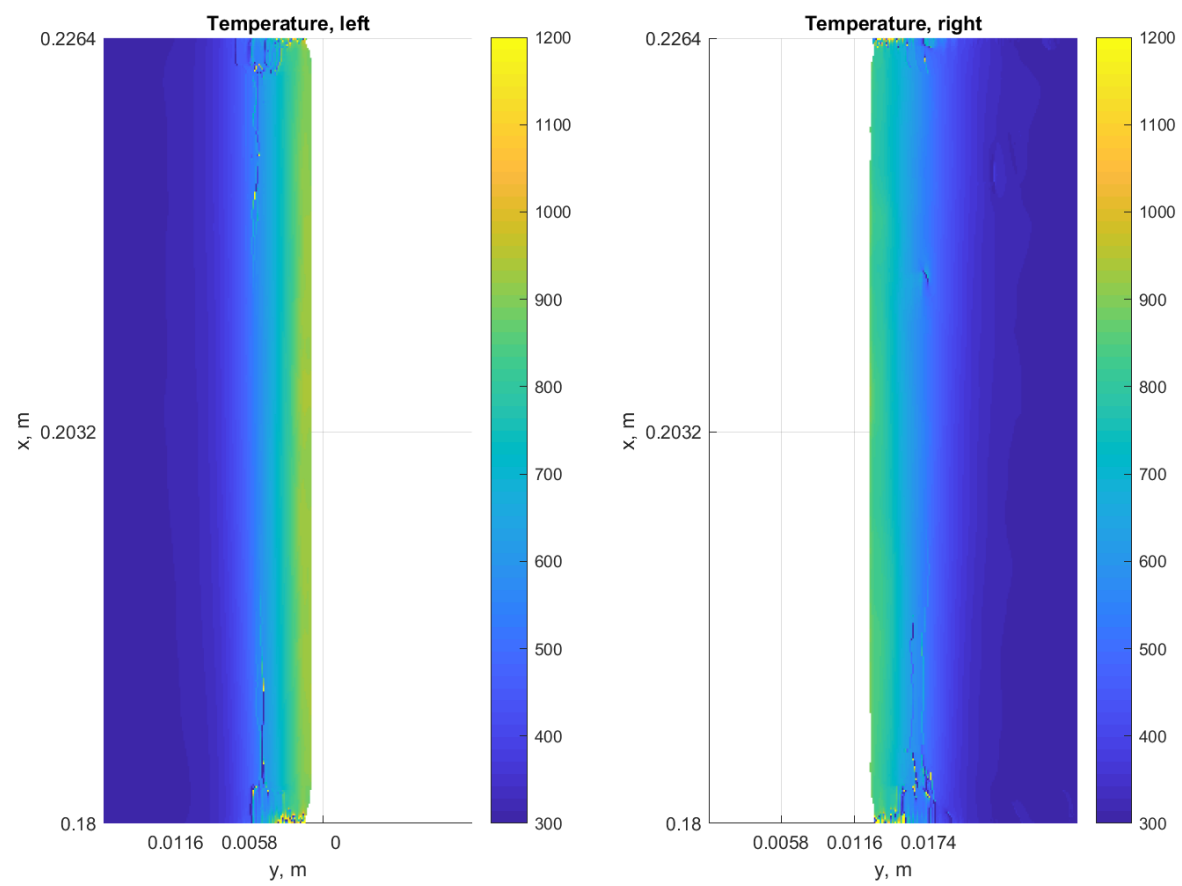

Figure C.52: Shot 164. Temperature fields from interferometry for nitrogen test. Surface temperature measured by pyrometer $=1080 \mathrm{~K}$. 


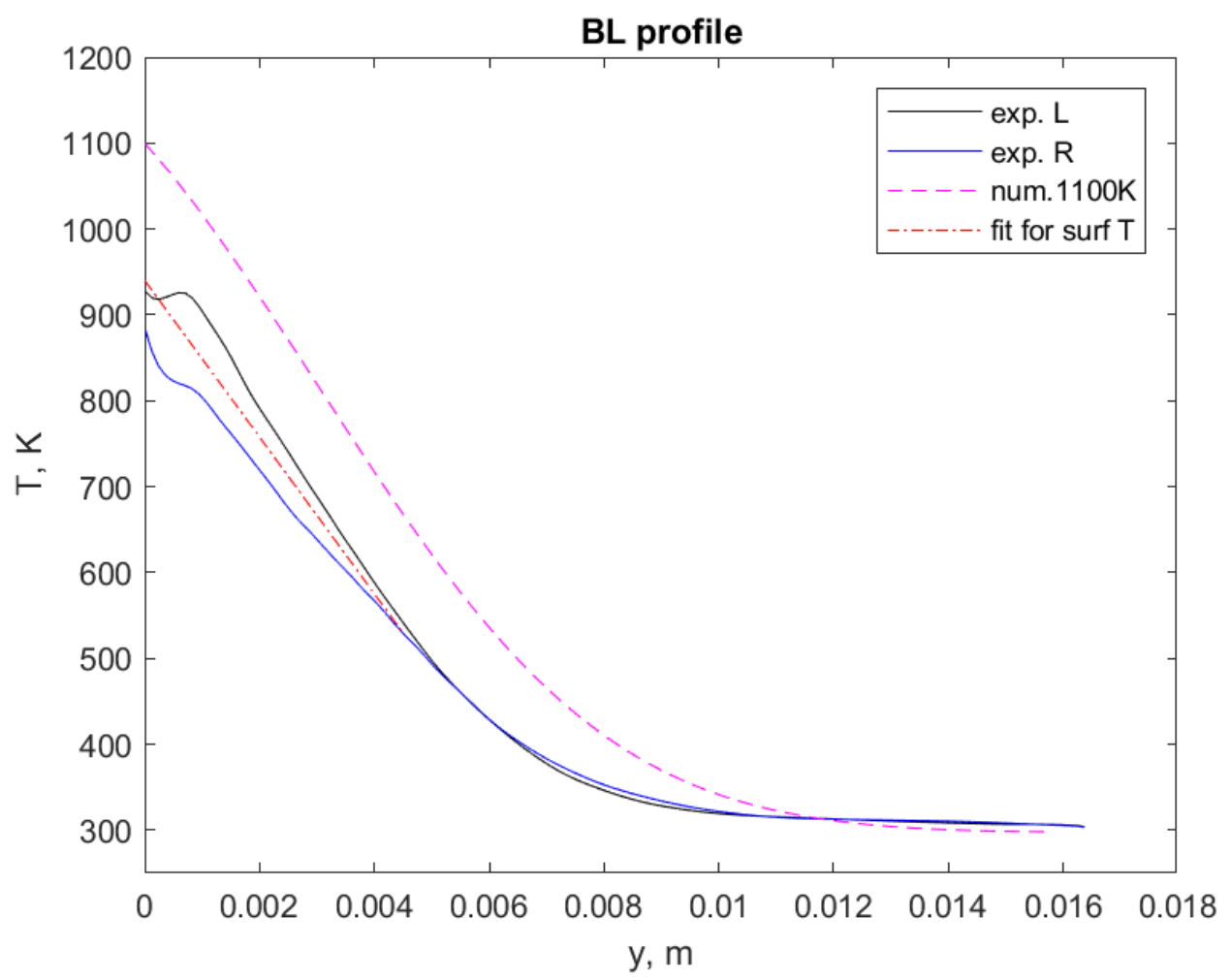

Figure C.53: Shot 164. Thermal boundary layer profiles for left (black line) and right (blue line) sides of experimental results compared with prediction of boundary layer profile (dashed magenta line) based on similarity solution for pyrometer surface temperature. Linear fit to averaged experimental profile (red dot-dashed line) used to extrapolate surface temperature from interferometer post processing. Extrapolated interferometer surface temperature $=940.2 \mathrm{~K}$. 


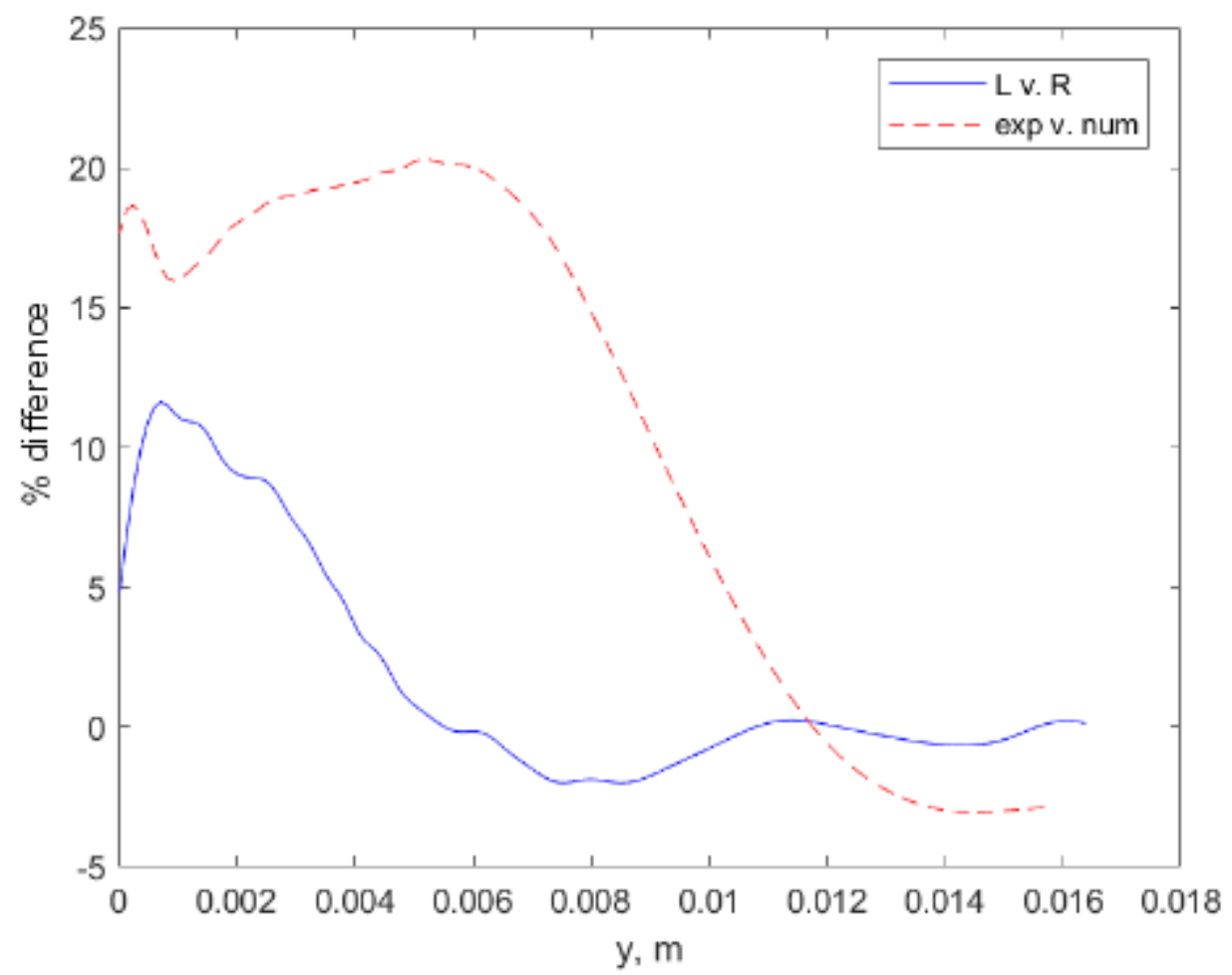

Figure C.54: Shot 164. Percent error in thermal boundary layer between both sides of experimental boundary layer profiles (red line) and percent error between predicted boundary layer profile from similarity solution and averaged experimental boundary layer profile (blue dashed line). 
Shot 168:
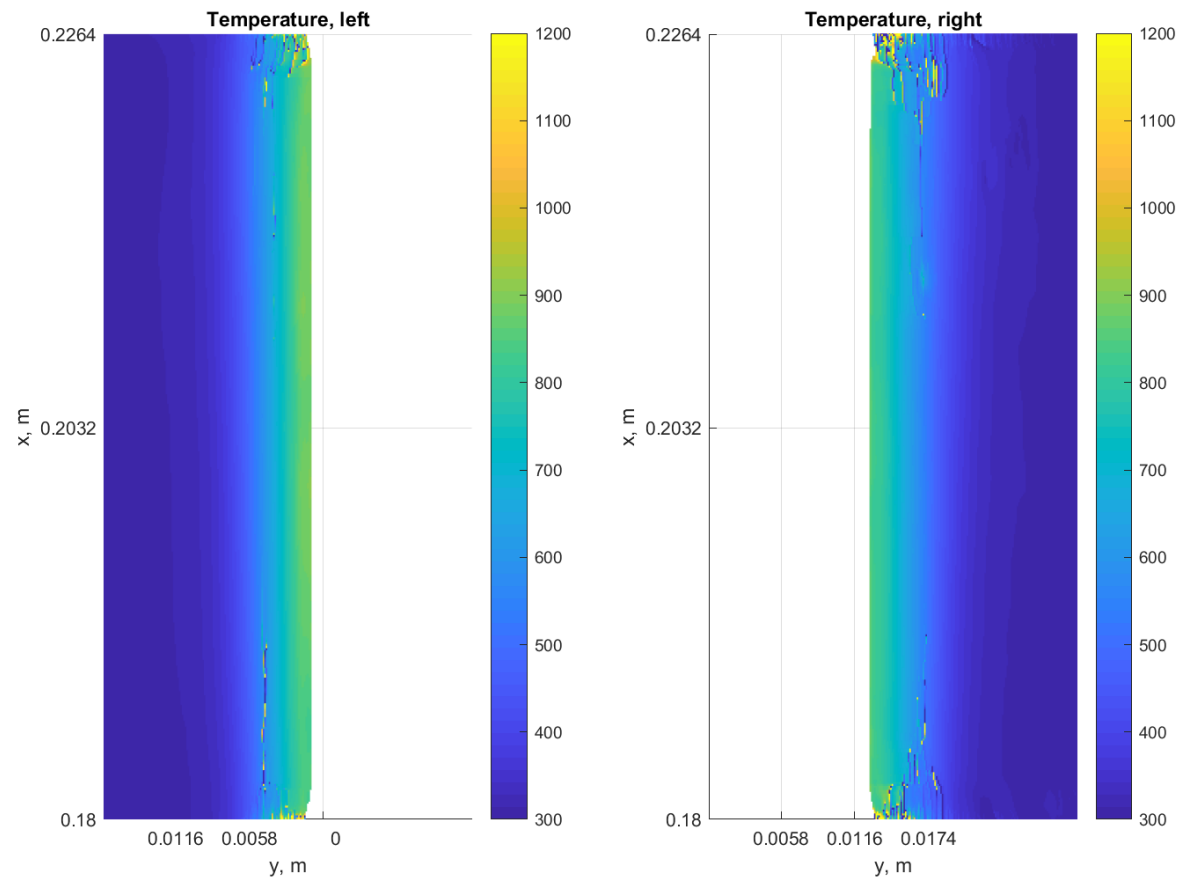

Figure C.55: Shot 168. Temperature fields from interferometry for nitrogen test. Surface temperature measured by pyrometer $=1070 \mathrm{~K}$.

Shot 169: 


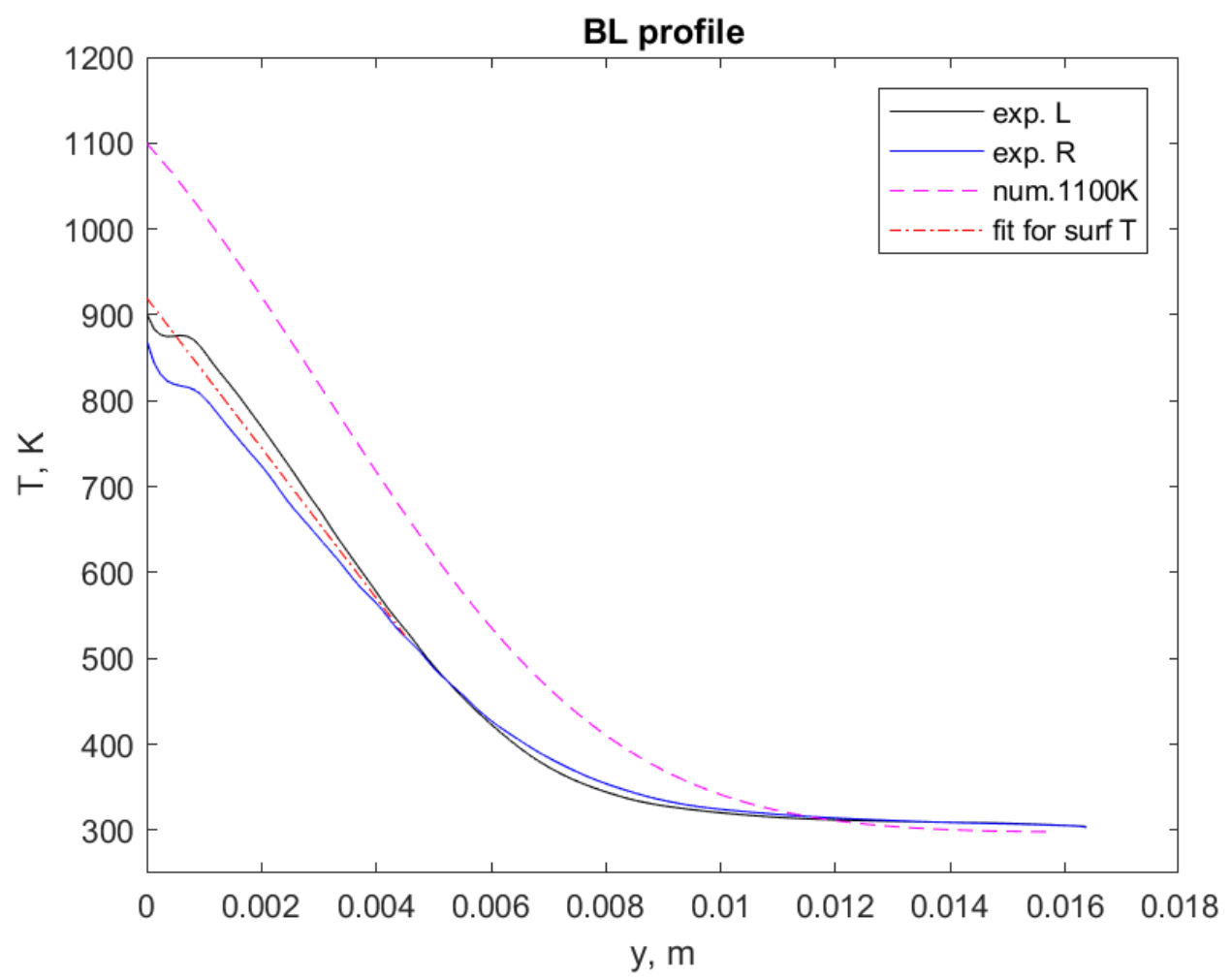

Figure C.56: Shot 168. Thermal boundary layer profiles for left (black line) and right (blue line) sides of experimental results compared with prediction of boundary layer profile (dashed magenta line) based on similarity solution for pyrometer surface temperature. Linear fit to averaged experimental profile (red dot-dashed line) used to extrapolate surface temperature from interferometer post processing. Extrapolated interferometer surface temperature $=919.8 \mathrm{~K}$. 


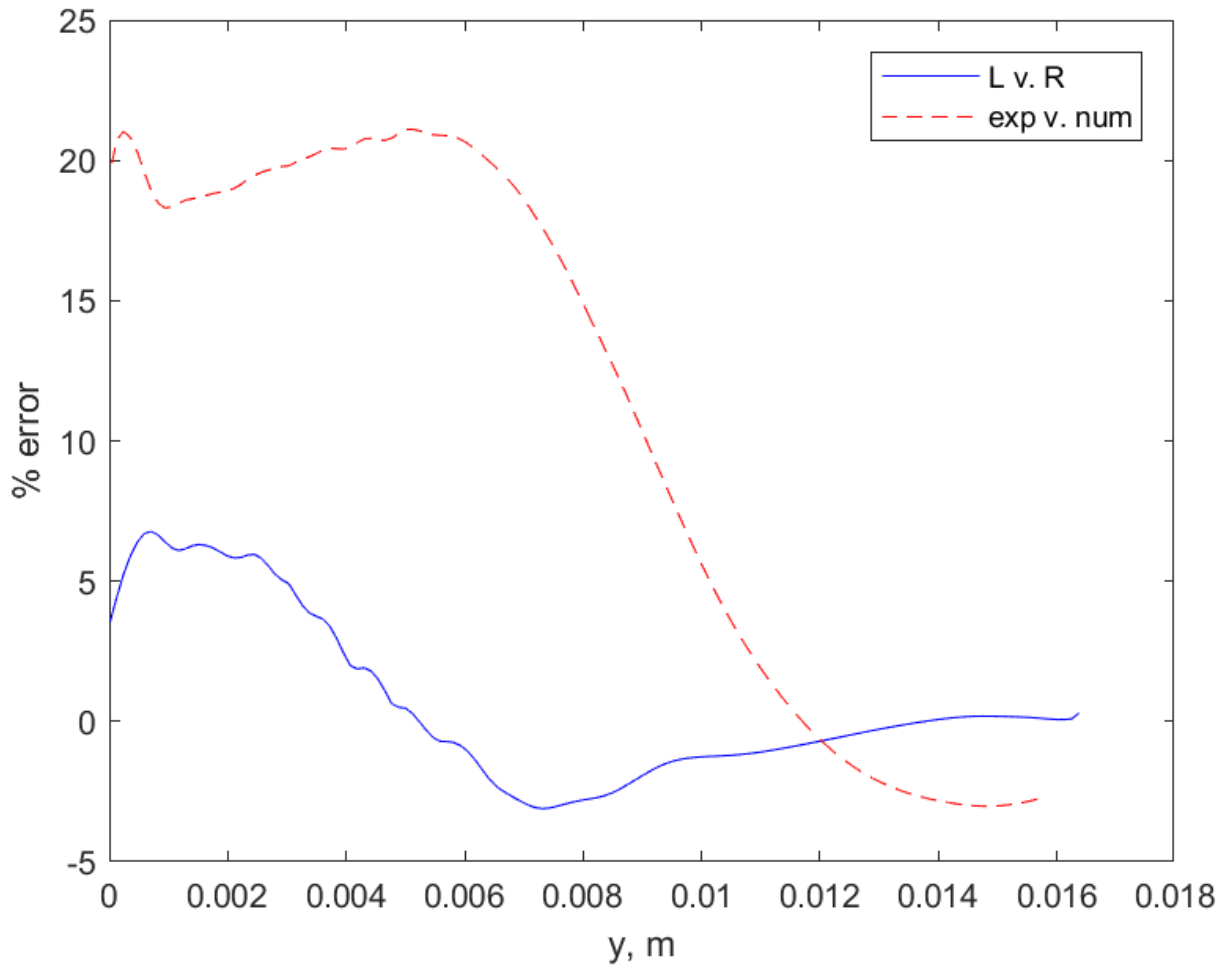

Figure C.57: Shot 168. Percent error in thermal boundary layer between both sides of experimental boundary layer profiles (red line) and percent error between predicted boundary layer profile from similarity solution and averaged experimental boundary layer profile (blue dashed line). 

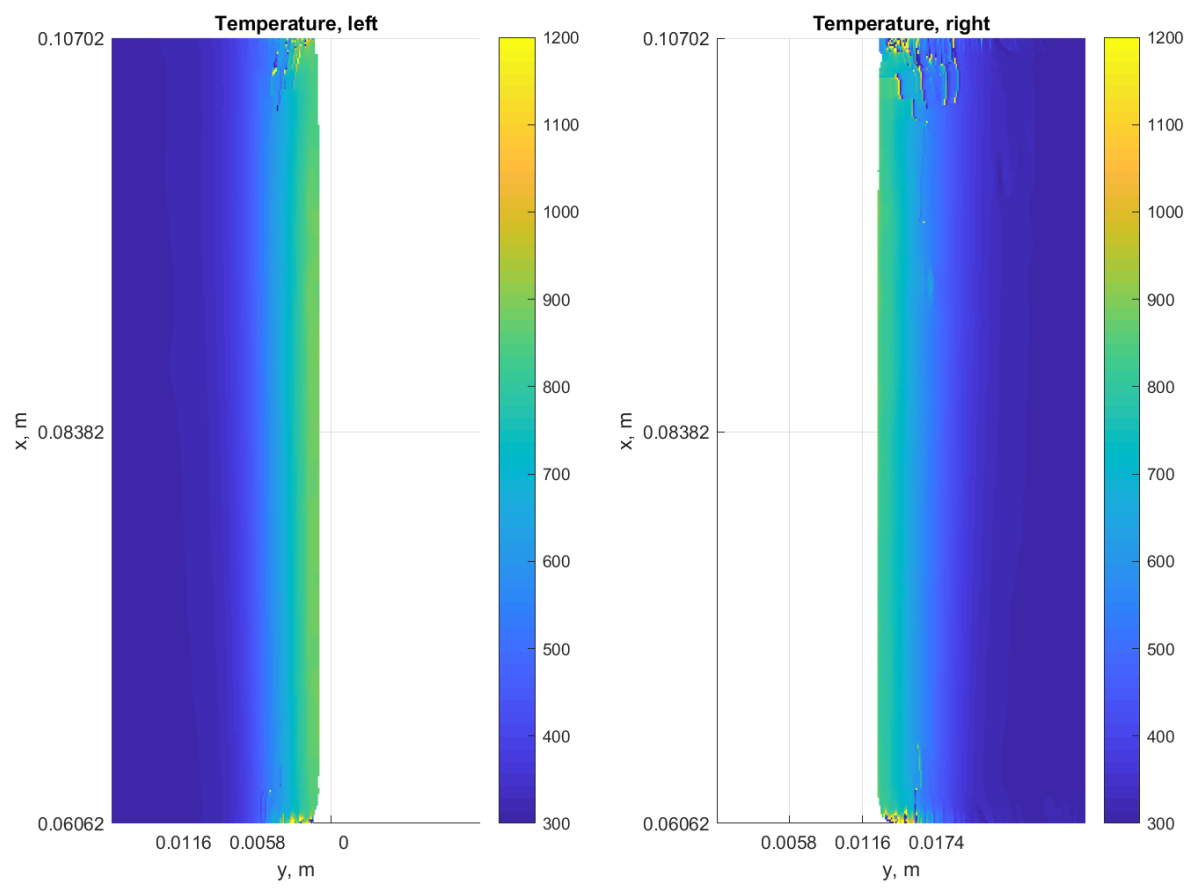

Figure C.58: Shot 169. Temperature fields from interferometry for nitrogen test. Surface temperature measured by pyrometer $=1029 \mathrm{~K}$. 


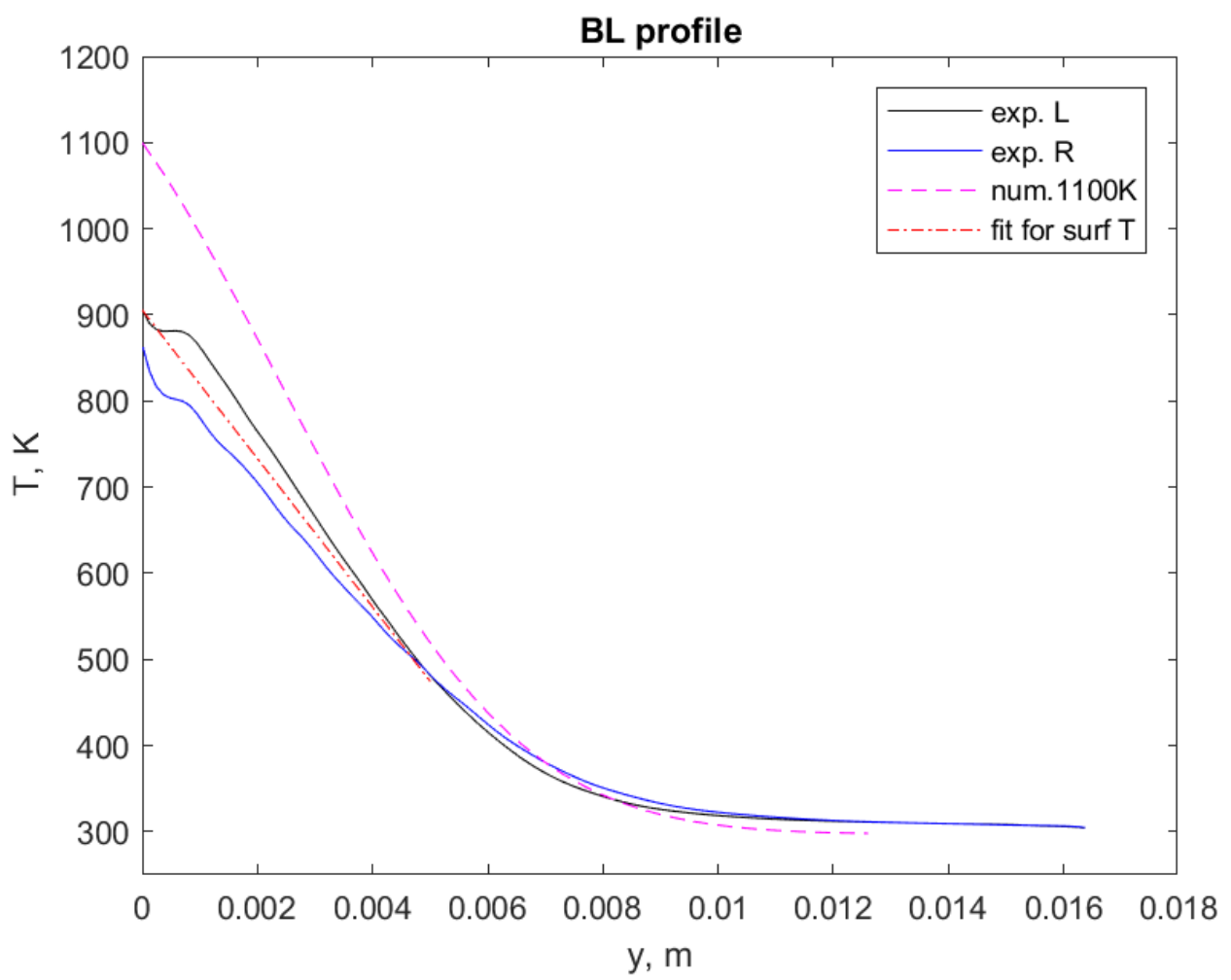

Figure C.59: Shot 169. Thermal boundary layer profiles for left (black line) and right (blue line) sides of experimental results compared with prediction of boundary layer profile (dashed magenta line) based on similarity solution for pyrometer surface temperature. Linear fit to averaged experimental profile (red dot-dashed line) used to extrapolate surface temperature from interferometer post processing. Extrapolated interferometer surface temperature $=905.5 \mathrm{~K}$. 


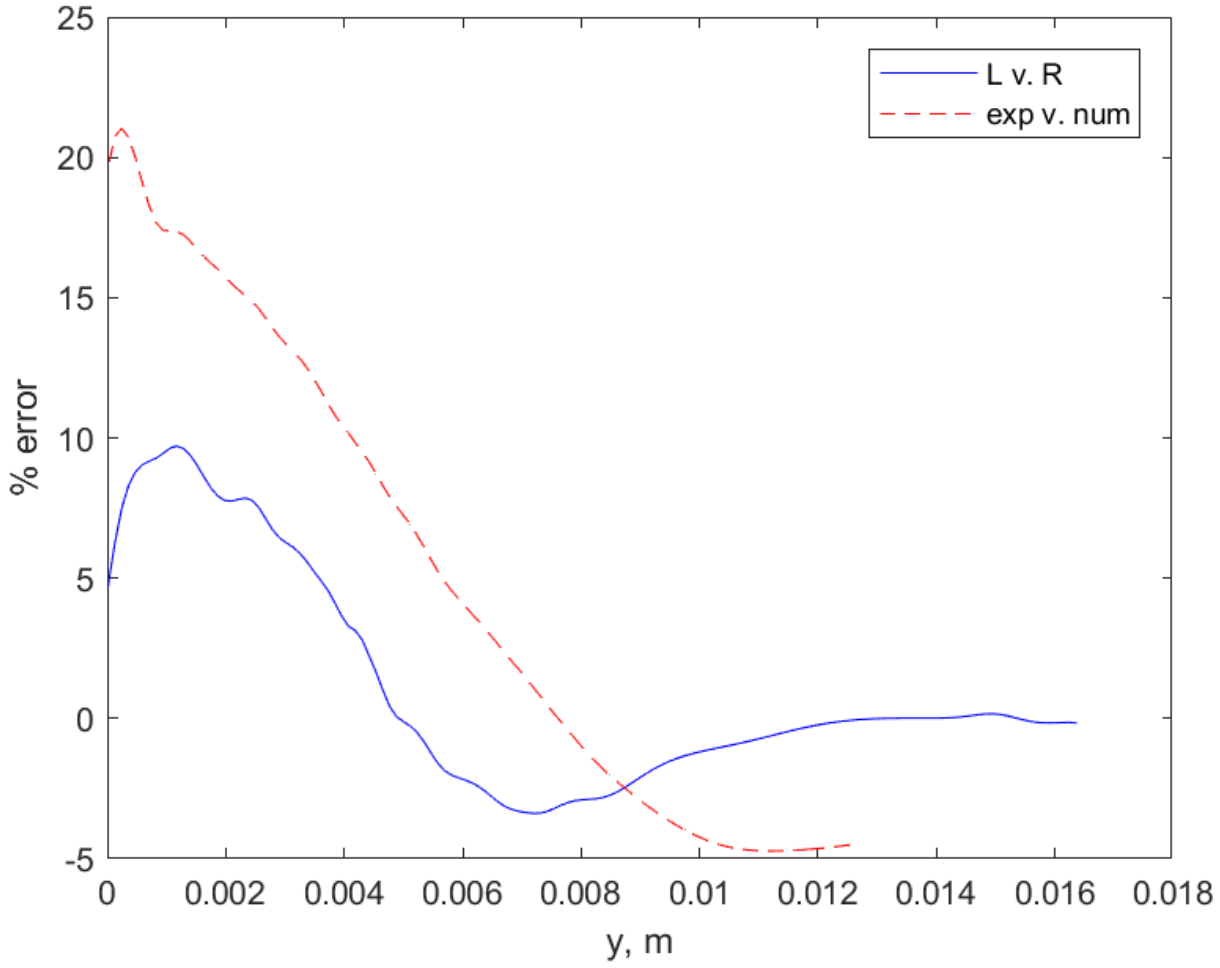

Figure C.60: Shot 169. Percent error in thermal boundary layer between both sides of experimental boundary layer profiles (red line) and percent error between predicted boundary layer profile from similarity solution and averaged experimental boundary layer profile (blue dashed line). 
Shot 170:
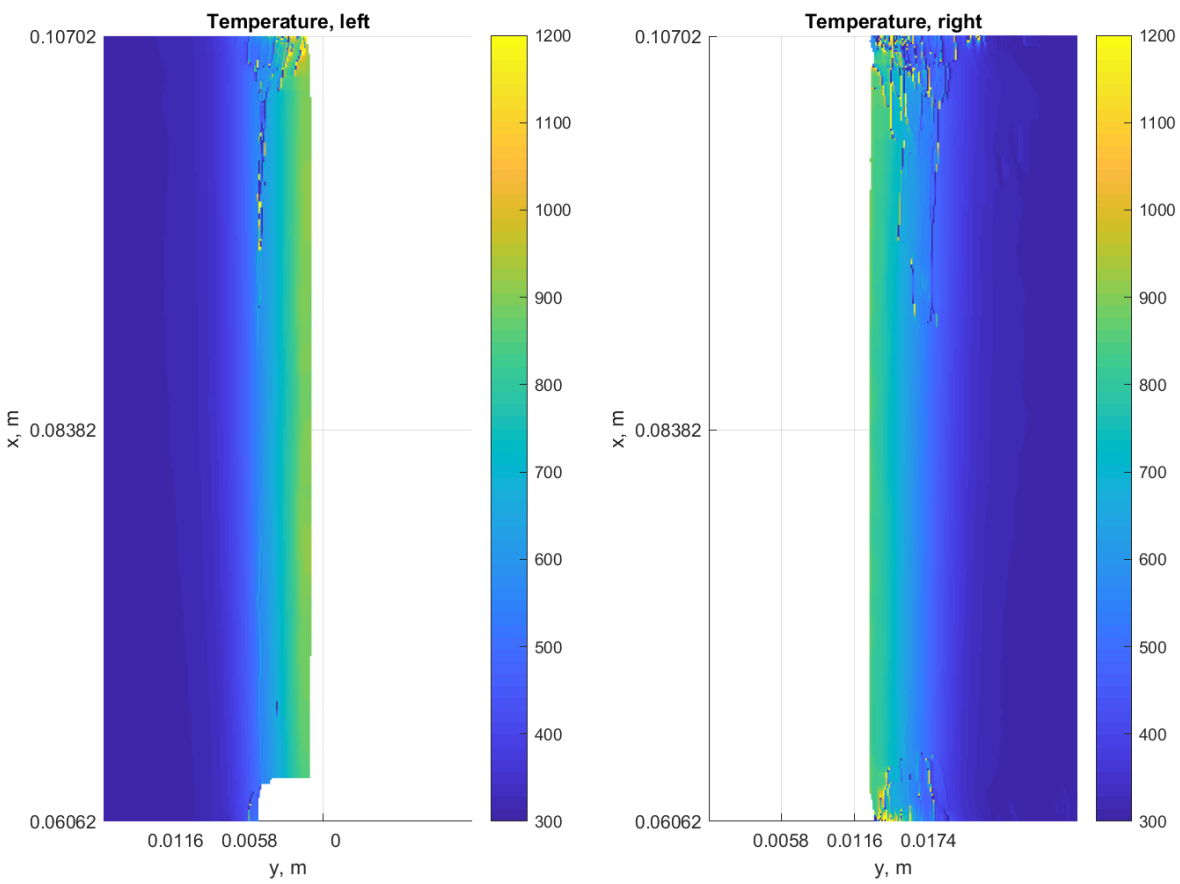

Figure C.61: Shot 170. Temperature fields from interferometry for nitrogen test. Surface temperature measured by pyrometer $=1062 \mathrm{~K}$. 


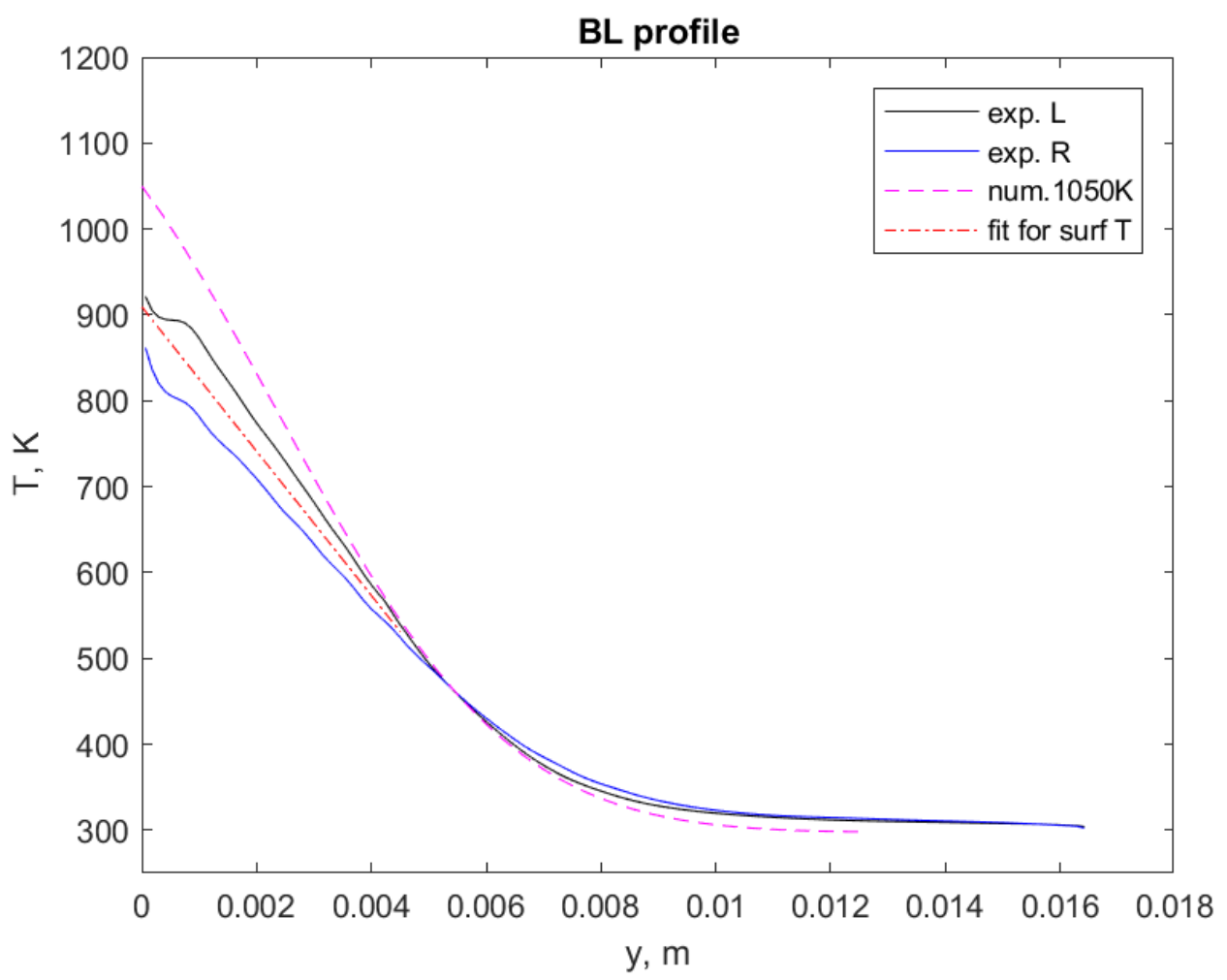

Figure C.62: Shot 170. Thermal boundary layer profiles for left (black line) and right (blue line) sides of experimental results compared with prediction of boundary layer profile (dashed magenta line) based on similarity solution for pyrometer surface temperature. Linear fit to averaged experimental profile (red dot-dashed line) used to extrapolate surface temperature from interferometer post processing. Extrapolated interferometer surface temperature $=909.4 \mathrm{~K}$. 


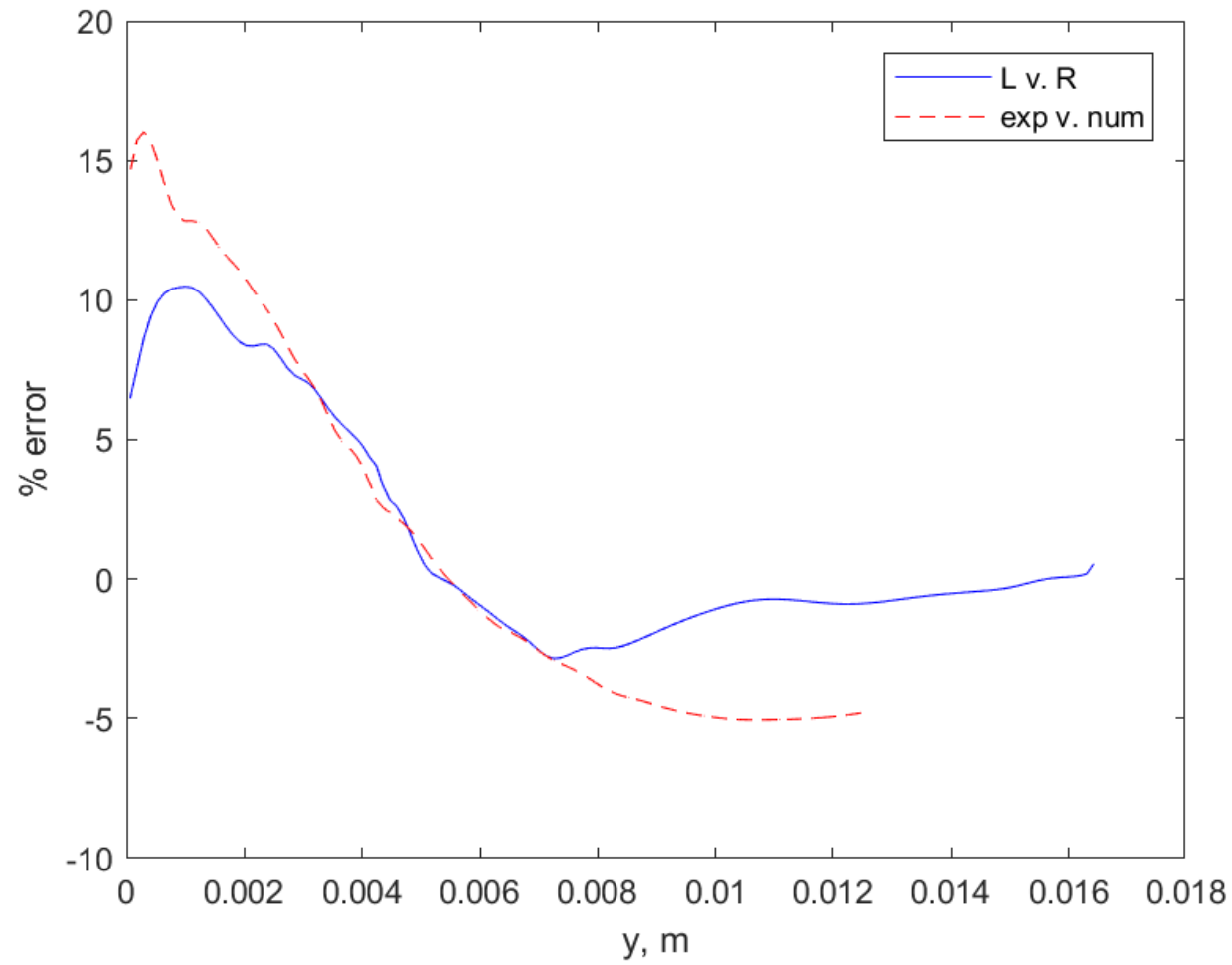

Figure C.63: Shot 170. Percent error in thermal boundary layer between both sides of experimental boundary layer profiles (red line) and percent error between predicted boundary layer profile from similarity solution and averaged experimental boundary layer profile (blue dashed line). 
200A

Shot 123:

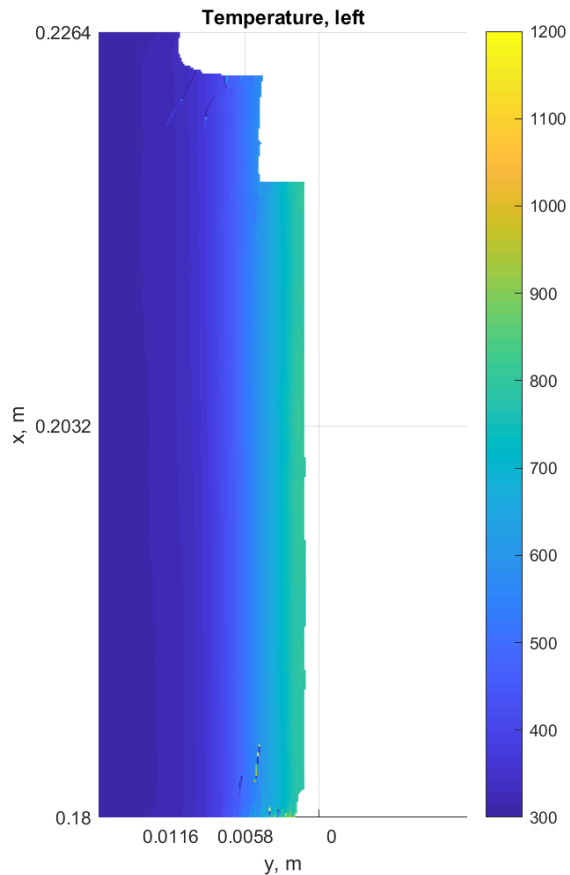

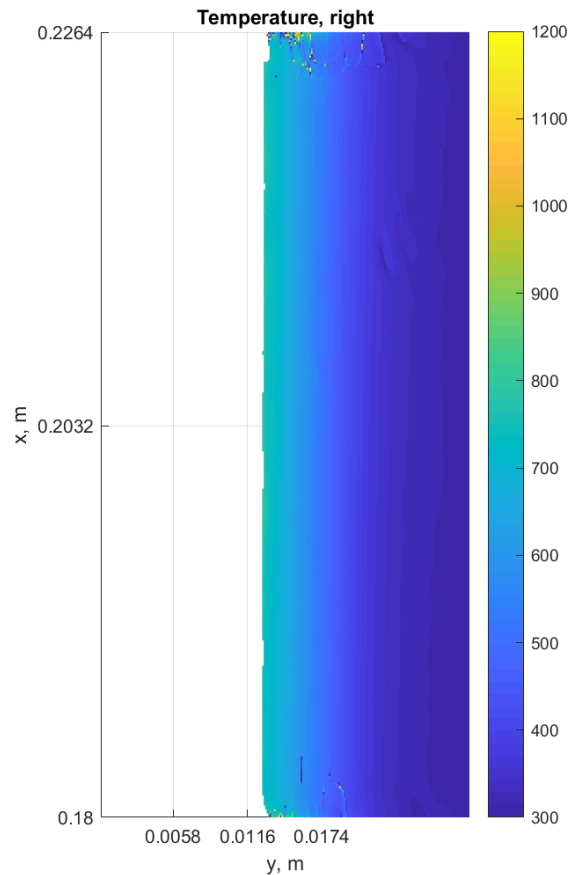

Figure C.64: Shot 123. Temperature fields from interferometry for nitrogen test. Surface temperature measured by pyrometer $=1002.0 \mathrm{~K}$. 


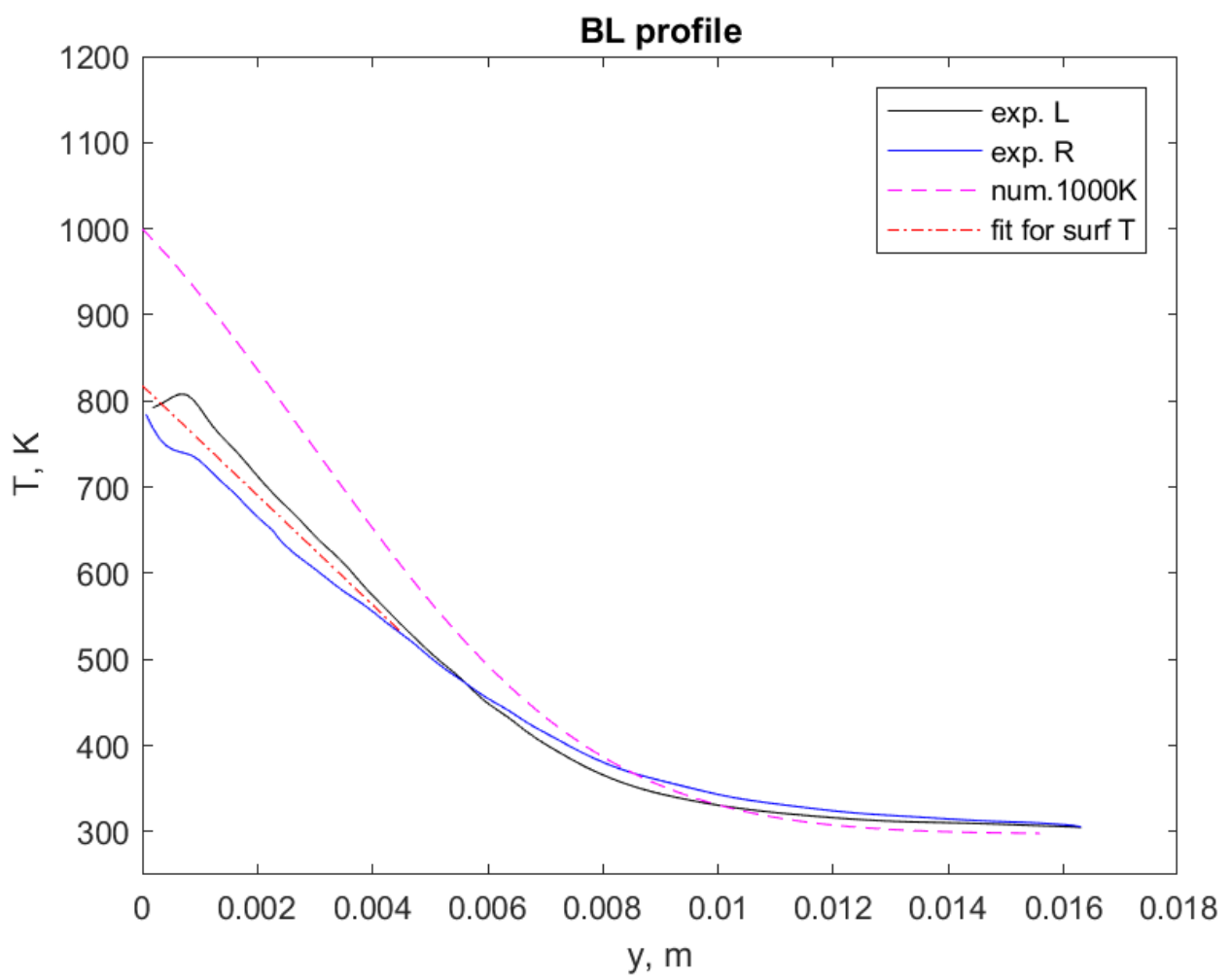

Figure C.65: Shot 123. Thermal boundary layer profiles for left (black line) and right (blue line) sides of experimental results compared with prediction of boundary layer profile (dashed magenta line) based on similarity solution for pyrometer surface temperature. Linear fit to averaged experimental profile (red dot-dashed line) used to extrapolate surface temperature from interferometer post processing. Extrapolated interferometer surface temperature $=817.8 \mathrm{~K}$. 


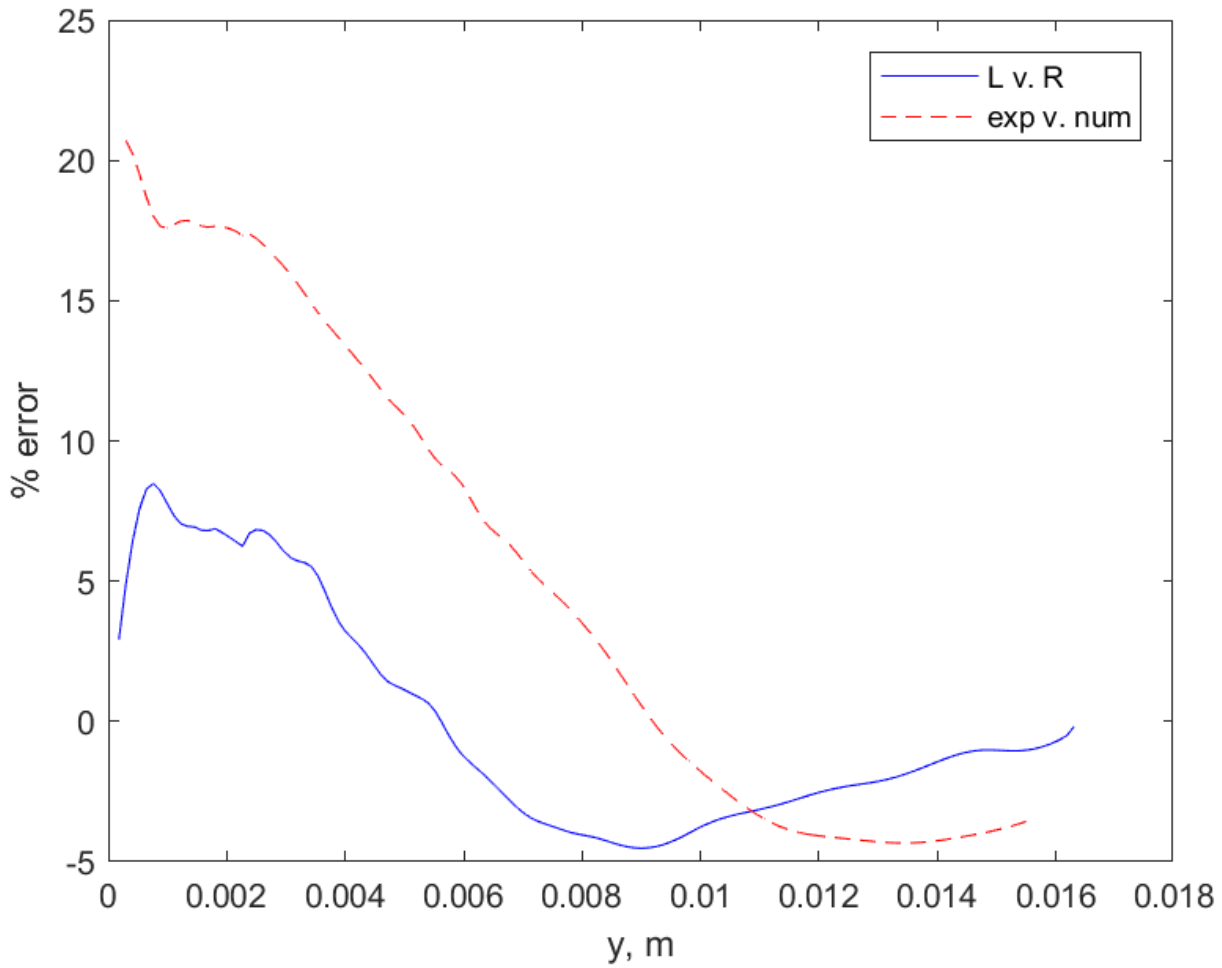

Figure C.66: Shot 123. Percent error in thermal boundary layer between both sides of experimental boundary layer profiles (red line) and percent error between predicted boundary layer profile from similarity solution and averaged experimental boundary layer profile (blue dashed line). 
Shot 124:
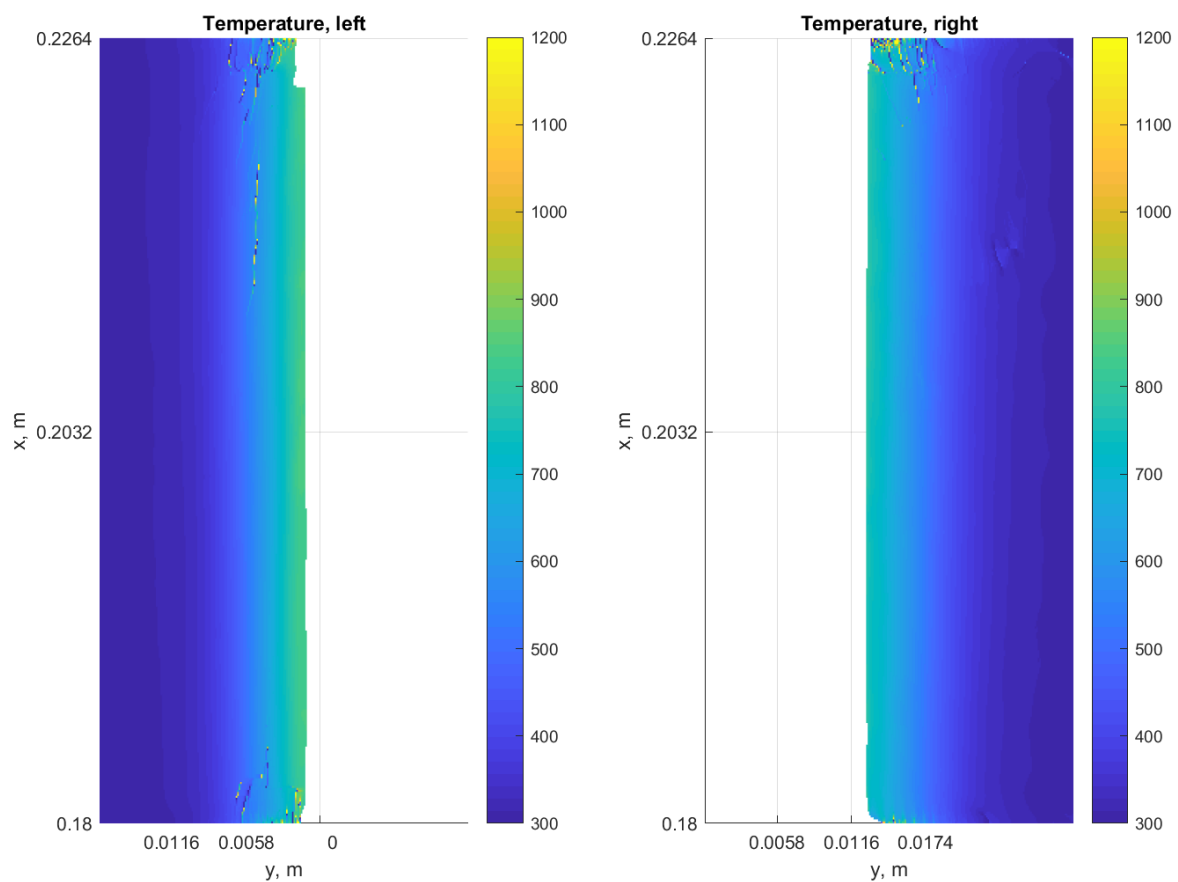

Figure C.67: Shot 124. Temperature fields from interferometry for nitrogen test. Surface temperature measured by pyrometer $=1002.0 \mathrm{~K}$. 


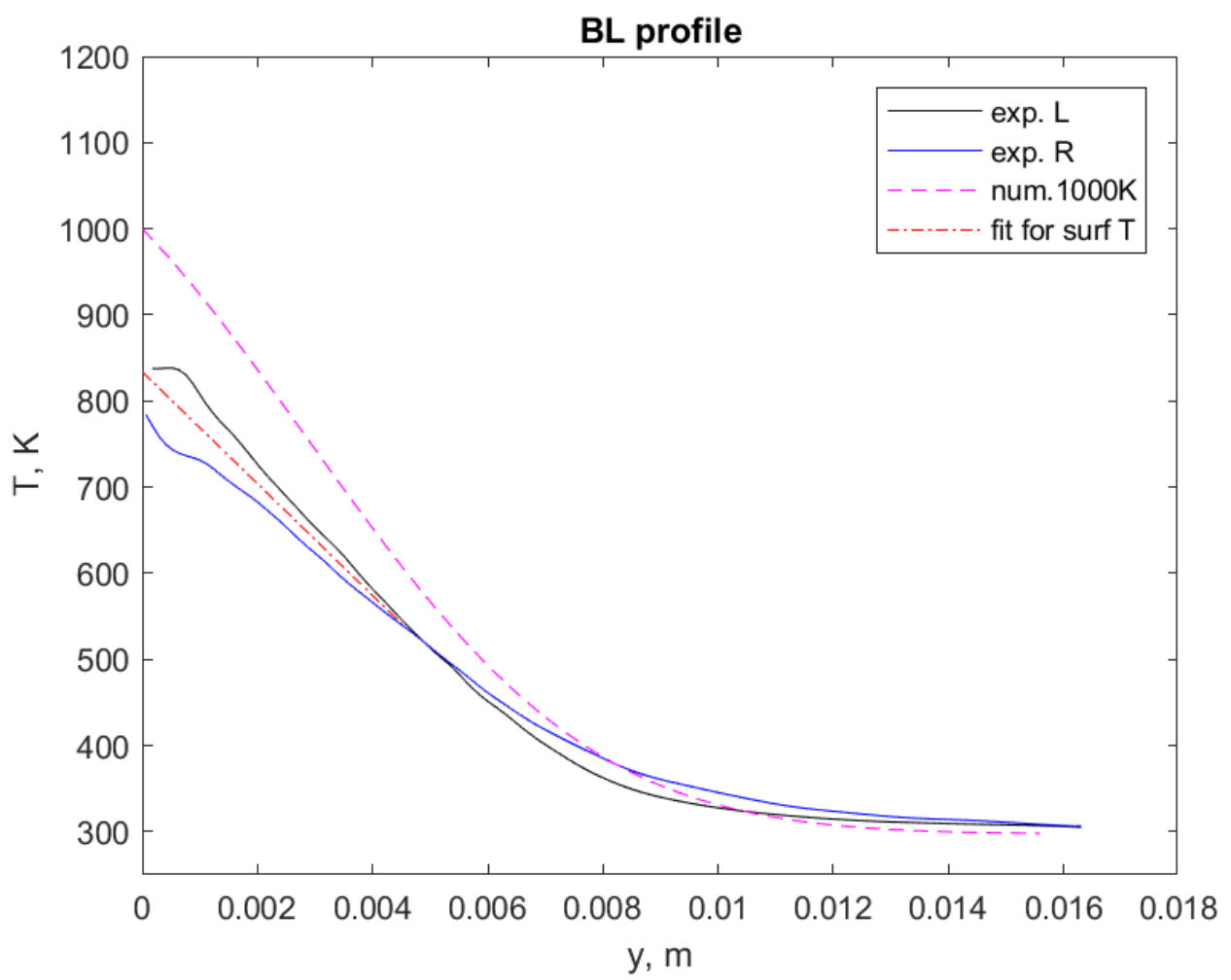

Figure C.68: Shot 124. Thermal boundary layer profiles for left (black line) and right (blue line) sides of experimental results compared with prediction of boundary layer profile (dashed magenta line) based on similarity solution for pyrometer surface temperature. Linear fit to averaged experimental profile (red dot-dashed line) used to extrapolate surface temperature from interferometer post processing. Extrapolated interferometer surface temperature $=833.9 \mathrm{~K}$. 


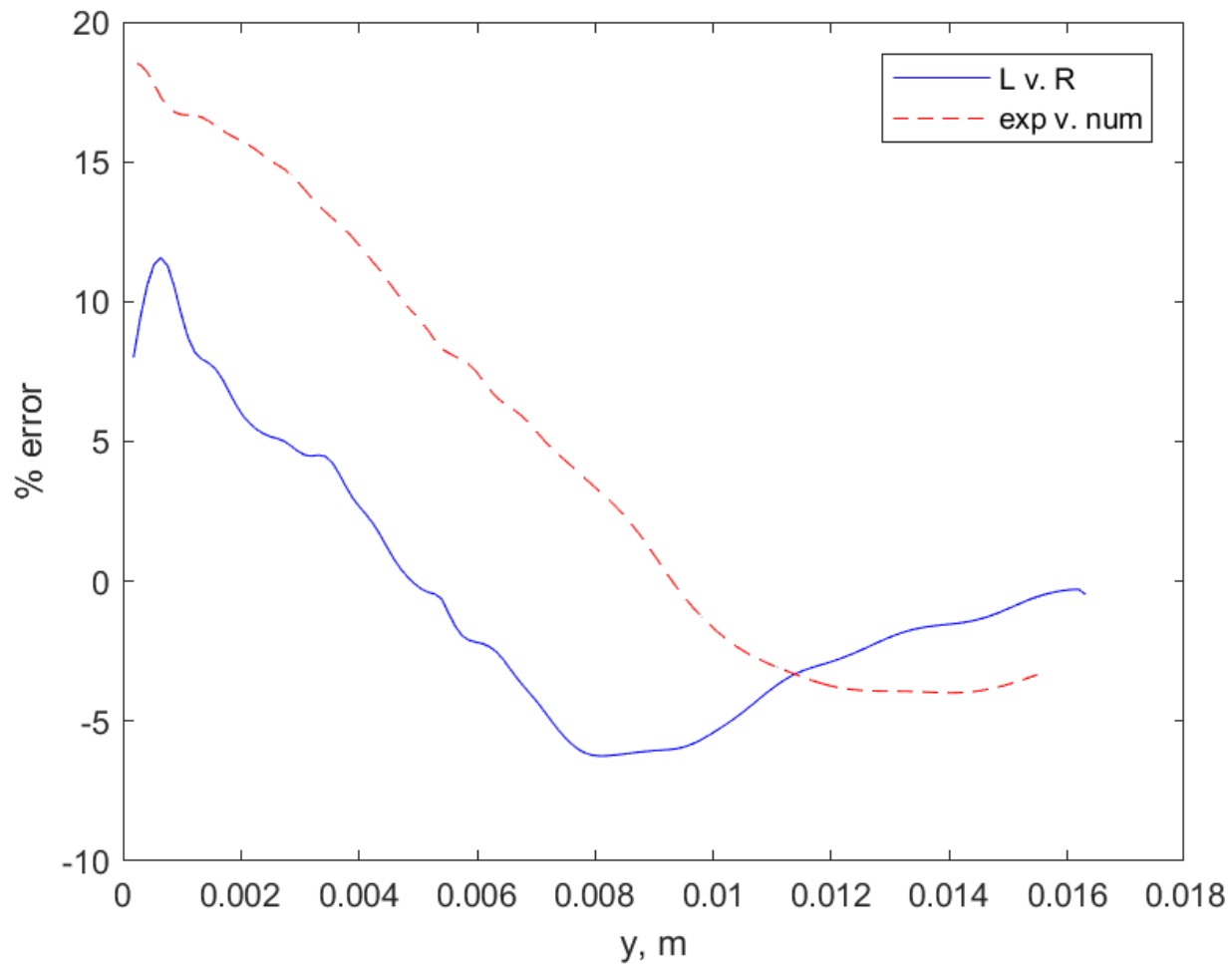

Figure C.69: Shot 124. Percent error in thermal boundary layer between both sides of experimental boundary layer profiles (red line) and percent error between predicted boundary layer profile from similarity solution and averaged experimental boundary layer profile (blue dashed line). 
Shot 127:
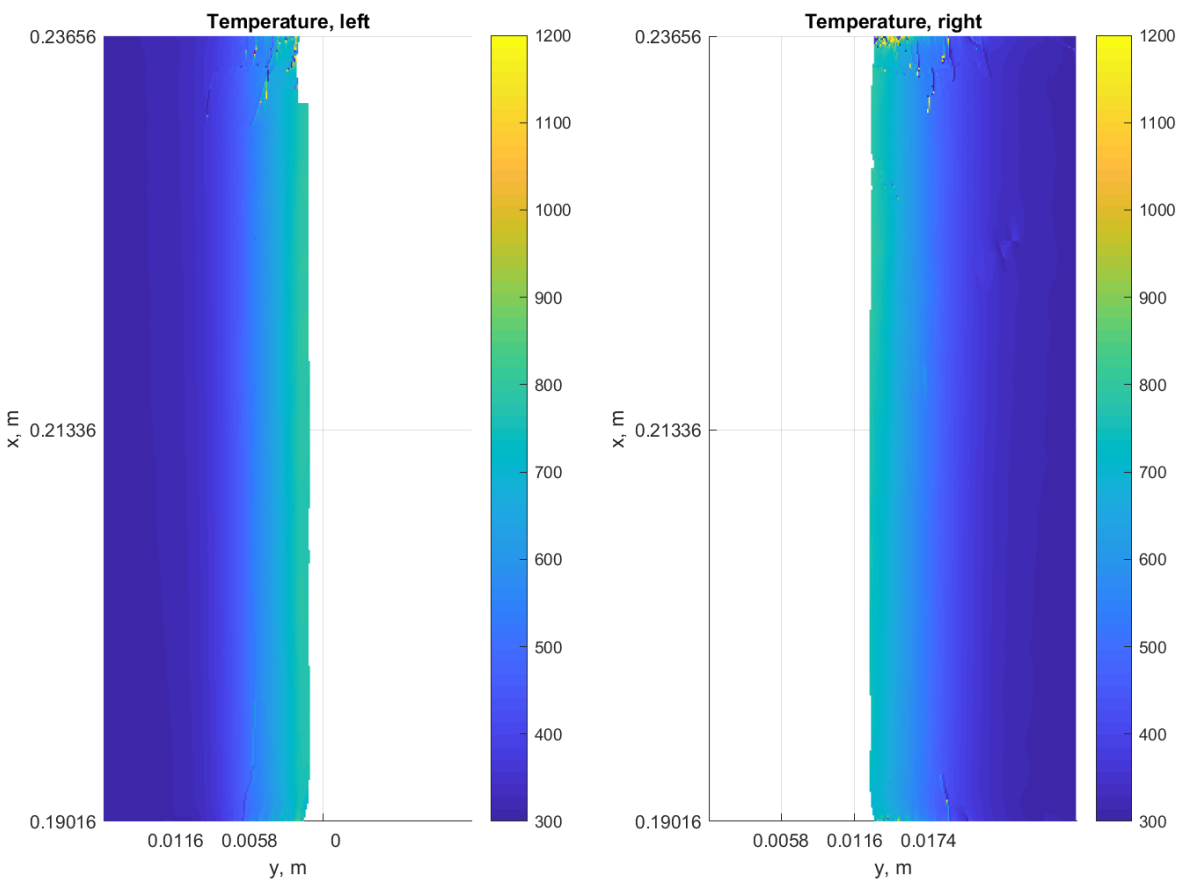

Figure C.70: Shot 127. Temperature fields from interferometry for nitrogen test. Surface temperature measured by pyrometer $=1001.0 \mathrm{~K}$. 


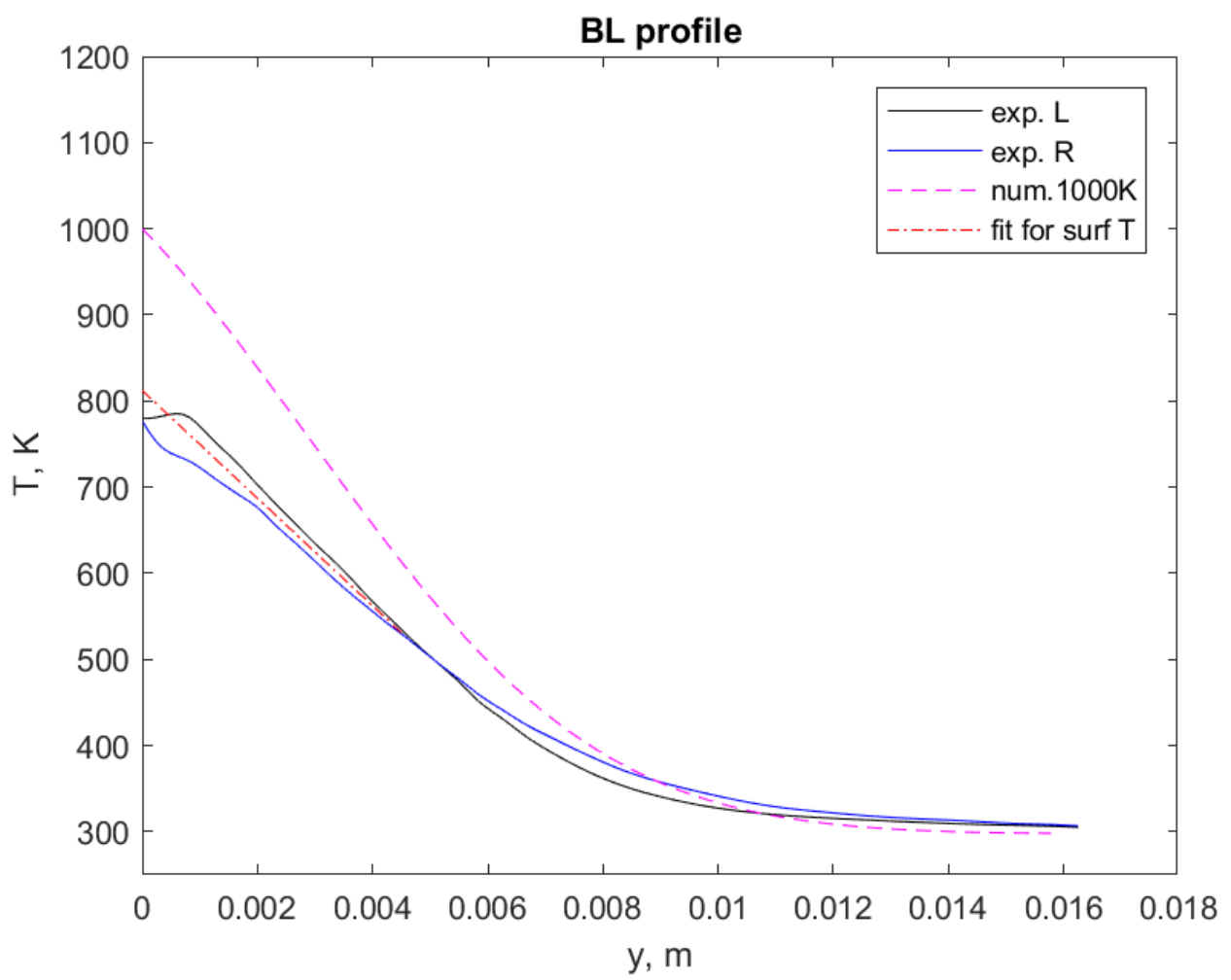

Figure C.71: Shot 127. Thermal boundary layer profiles for left (black line) and right (blue line) sides of experimental results compared with prediction of boundary layer profile (dashed magenta line) based on similarity solution for pyrometer surface temperature. Linear fit to averaged experimental profile (red dot-dashed line) used to extrapolate surface temperature from interferometer post processing. Extrapolated interferometer surface temperature $=812.1 \mathrm{~K}$. 


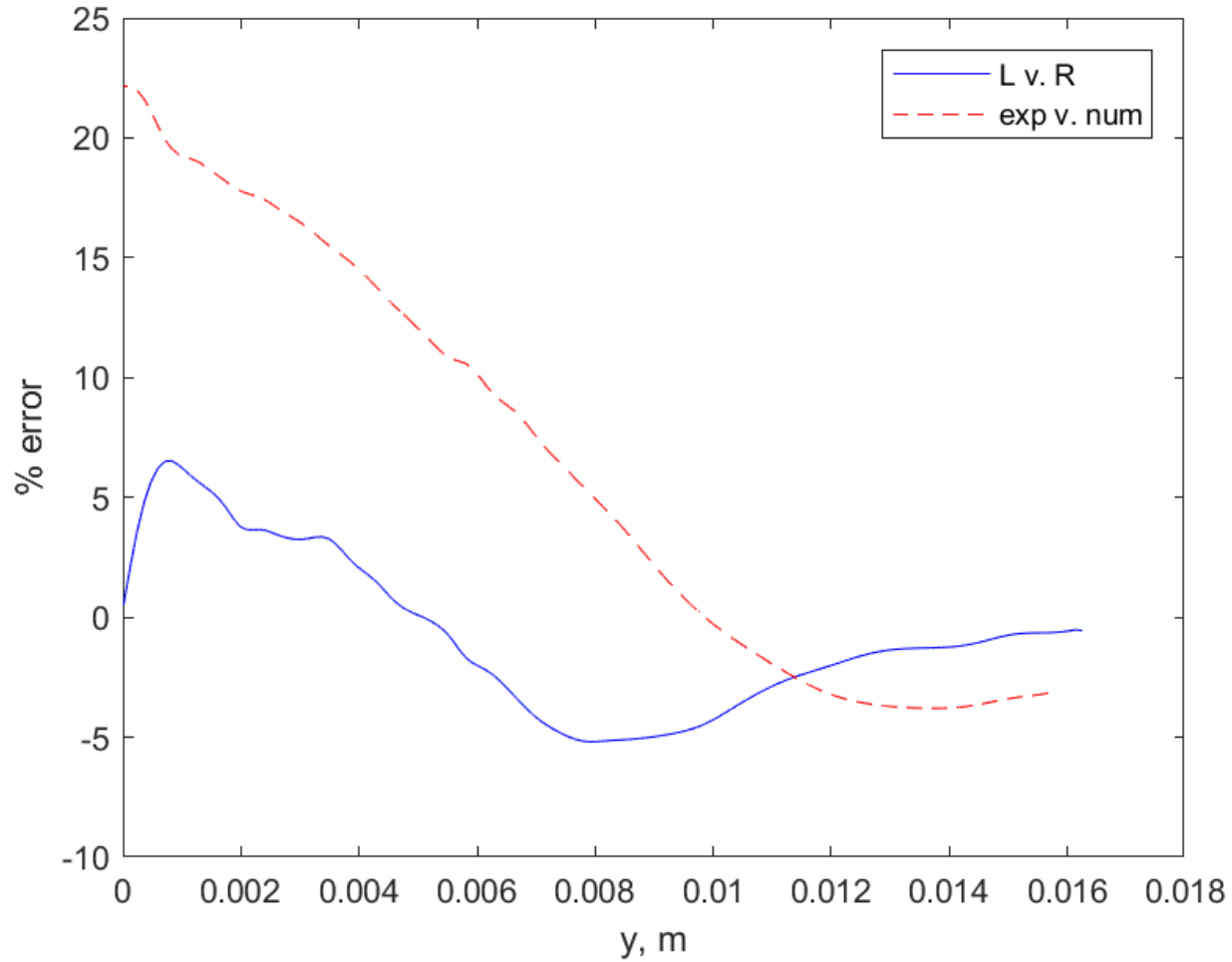

Figure C.72: Shot 127. Percent error in thermal boundary layer between both sides of experimental boundary layer profiles (red line) and percent error between predicted boundary layer profile from similarity solution and averaged experimental boundary layer profile (blue dashed line). 
Shot 142:
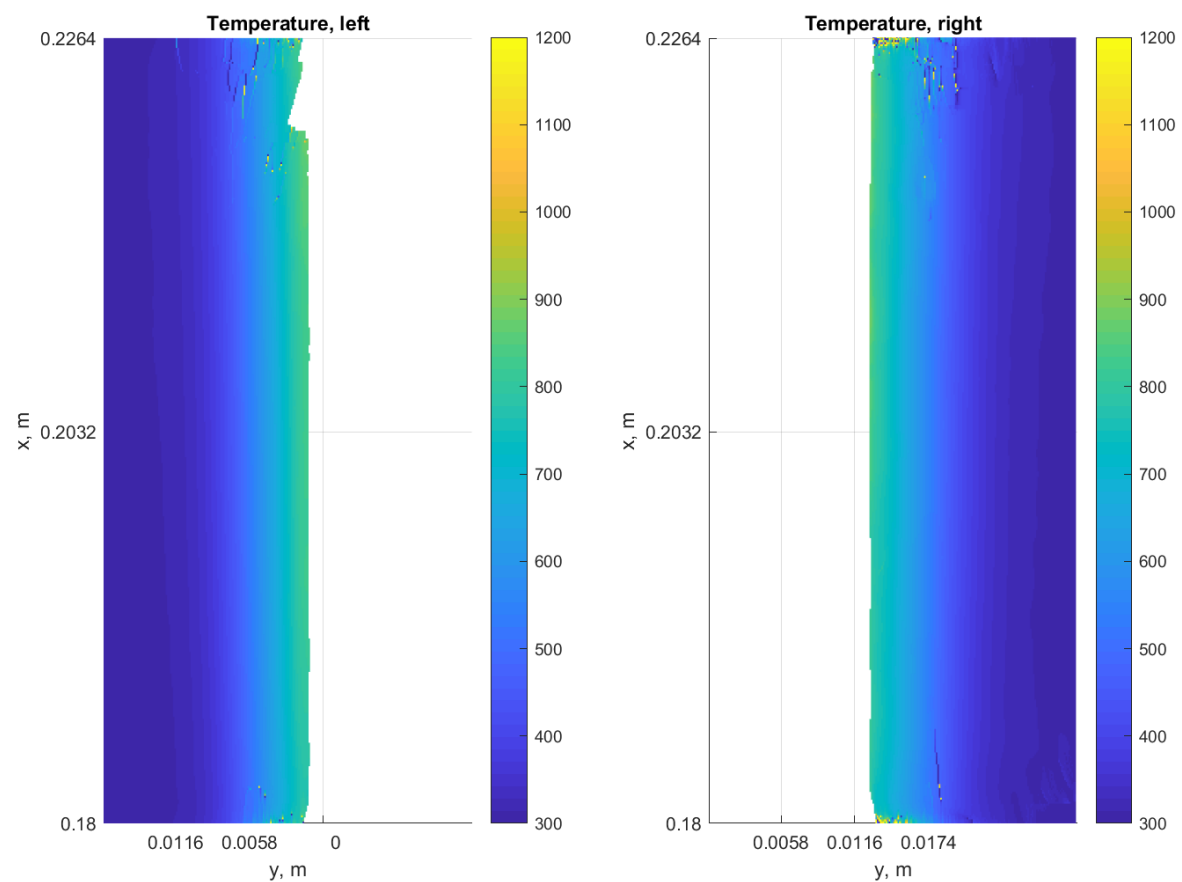

Figure C.73: Shot 142. Temperature fields from interferometry for nitrogen test. Surface temperature measured by pyrometer $=1028 \mathrm{~K}$. 


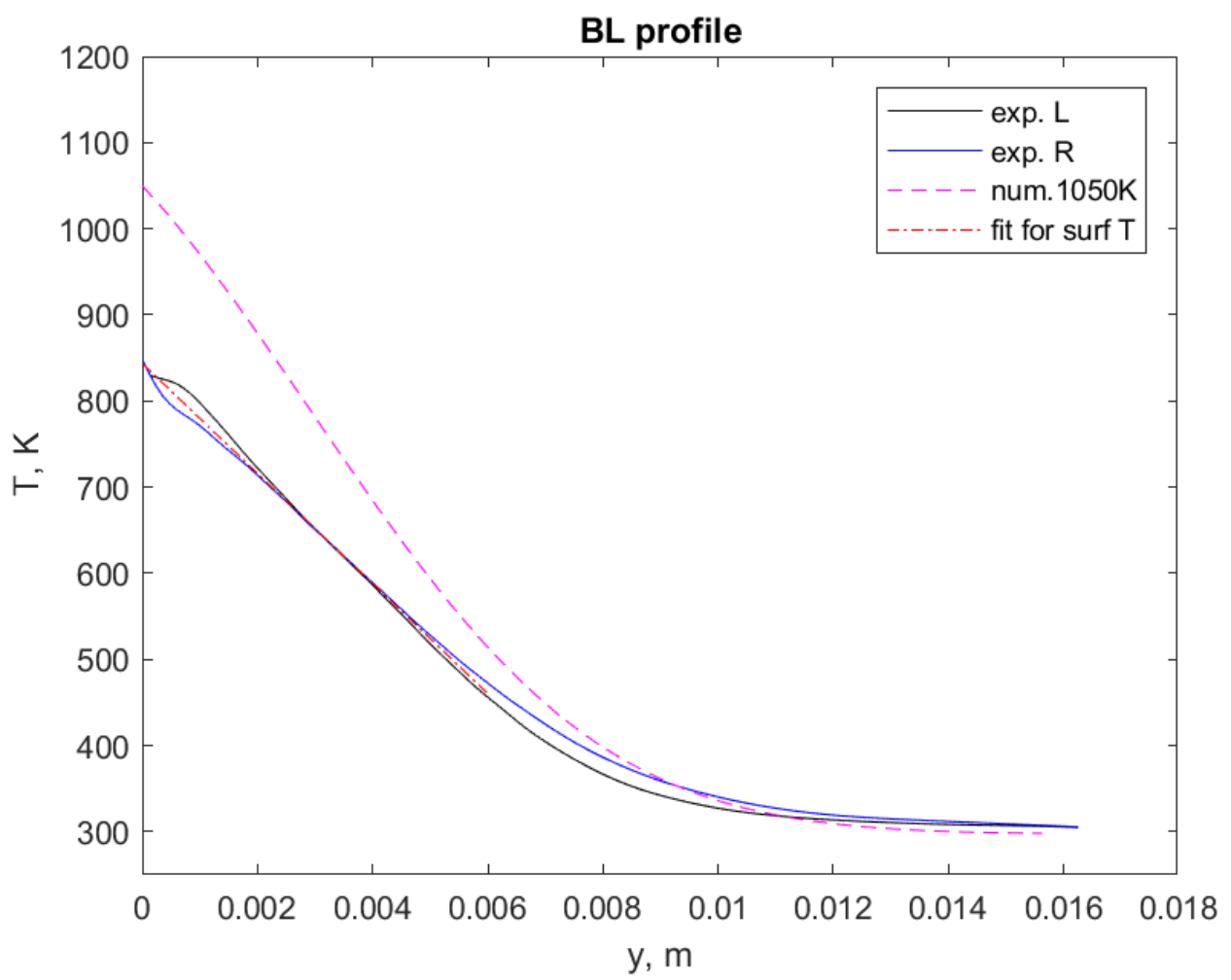

Figure C.74: Shot 142. Thermal boundary layer profiles for left (black line) and right (blue line) sides of experimental results compared with prediction of boundary layer profile (dashed magenta line) based on similarity solution for pyrometer surface temperature. Linear fit to averaged experimental profile (red dot-dashed line) used to extrapolate surface temperature from interferometer post processing. Extrapolated interferometer surface temperature $=843.6 \mathrm{~K}$. 


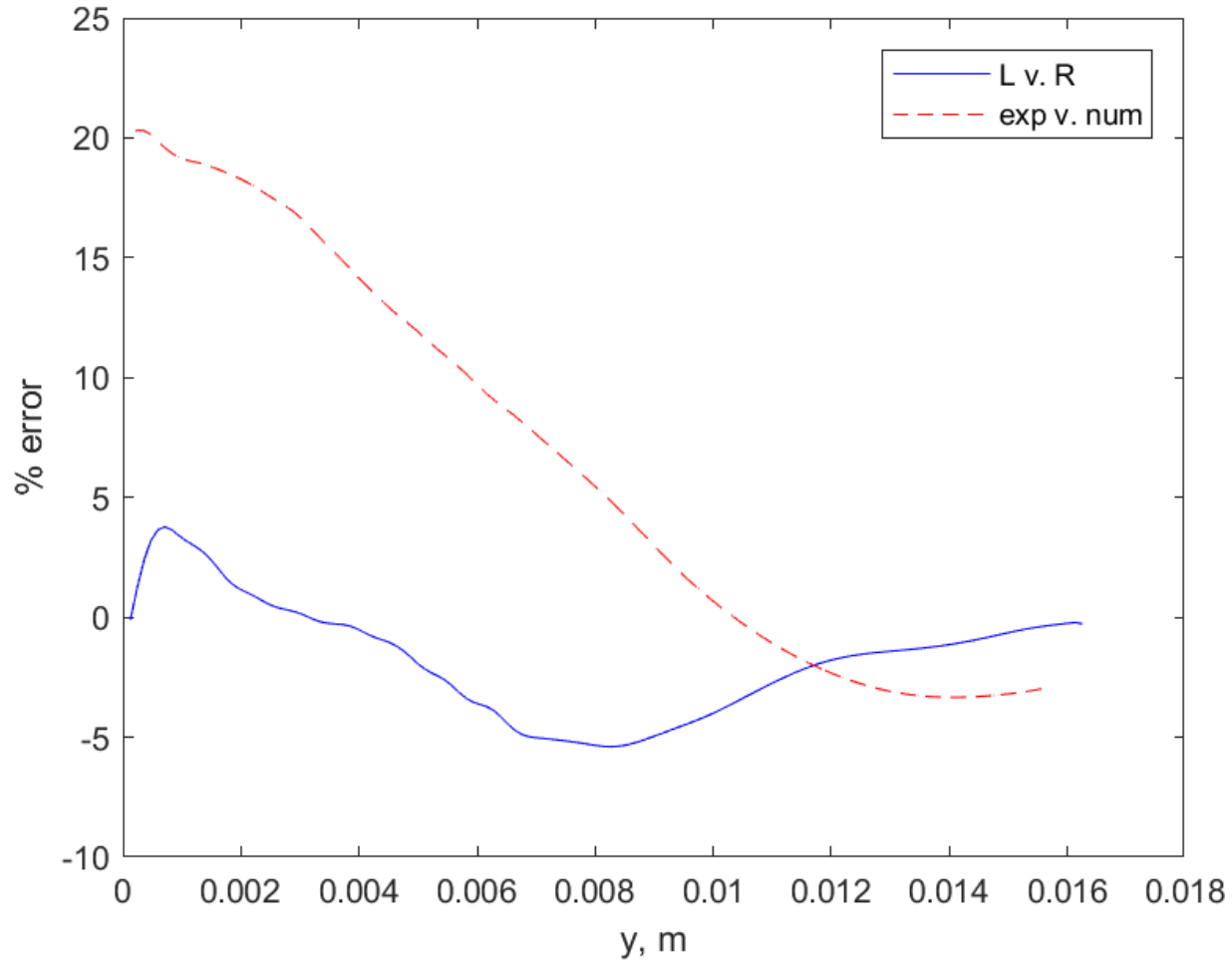

Figure C.75: Shot 142. Percent error in thermal boundary layer between both sides of experimental boundary layer profiles (red line) and percent error between predicted boundary layer profile from similarity solution and averaged experimental boundary layer profile (blue dashed line). 
Shot 143:
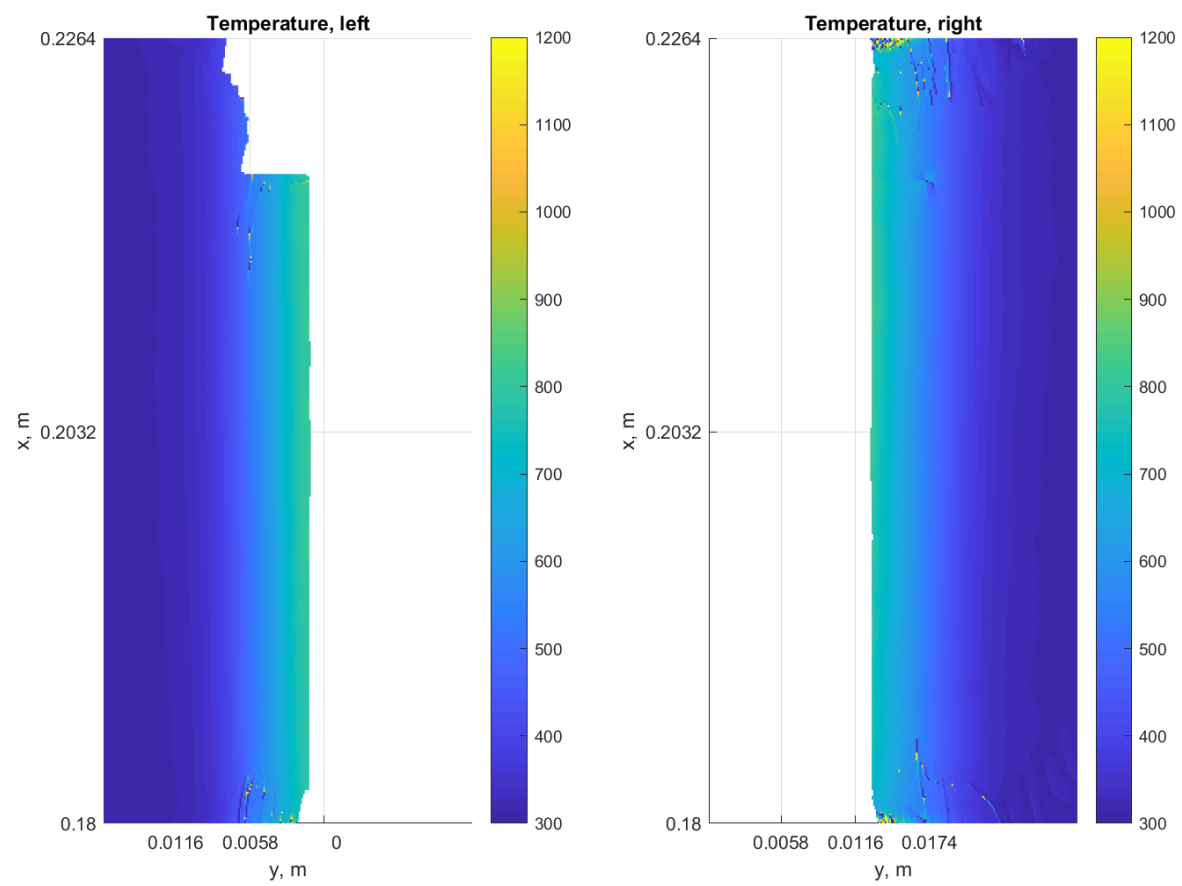

Figure C.76: Shot 143. Temperature fields from interferometry for nitrogen test. Surface temperature measured by pyrometer $=1018 \mathrm{~K}$. 


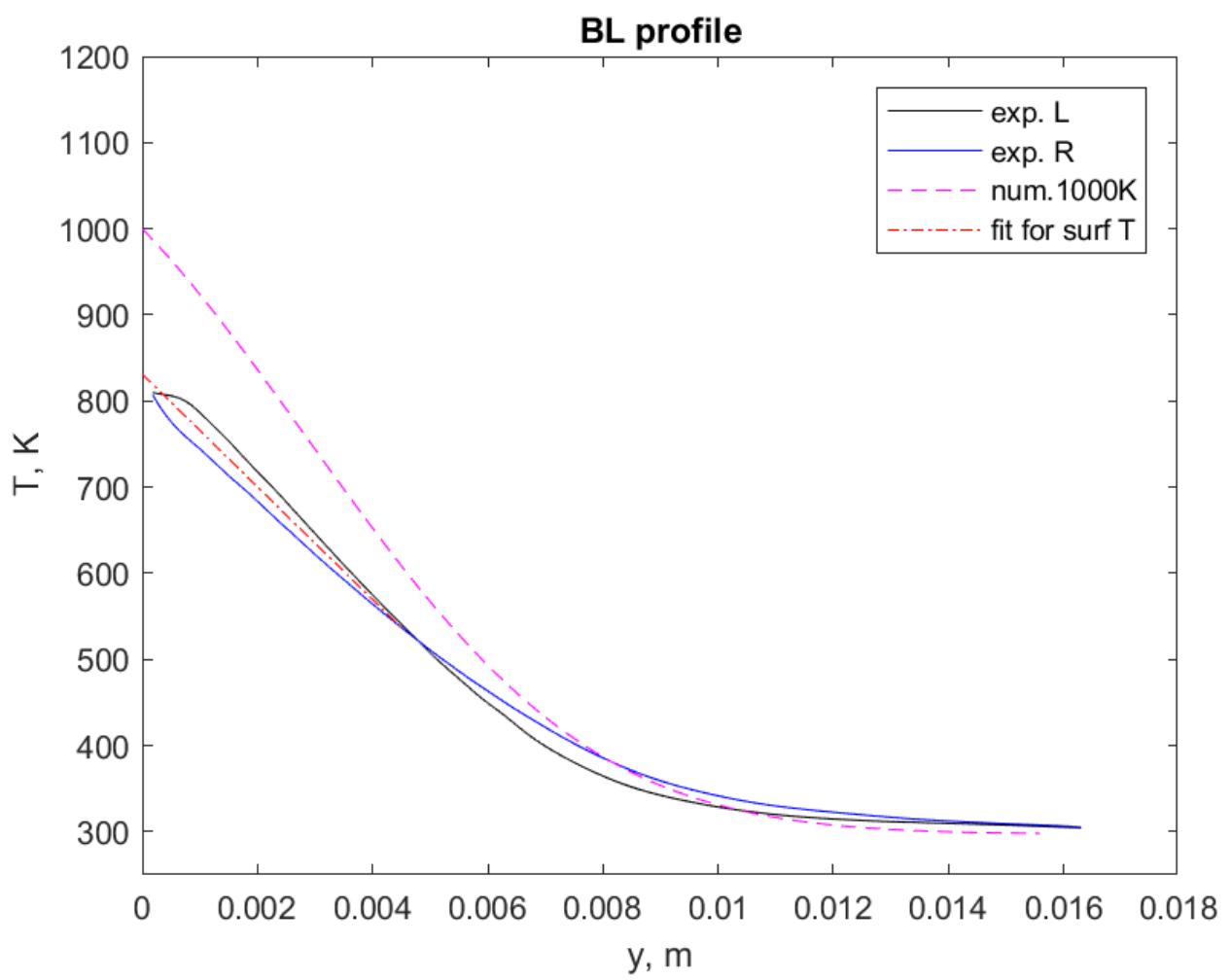

Figure C.77: Shot 143. Thermal boundary layer profiles for left (black line) and right (blue line) sides of experimental results compared with prediction of boundary layer profile (dashed magenta line) based on similarity solution for pyrometer surface temperature. Linear fit to averaged experimental profile (red dot-dashed line) used to extrapolate surface temperature from interferometer post processing. Extrapolated interferometer surface temperature $=831.2 \mathrm{~K}$. 


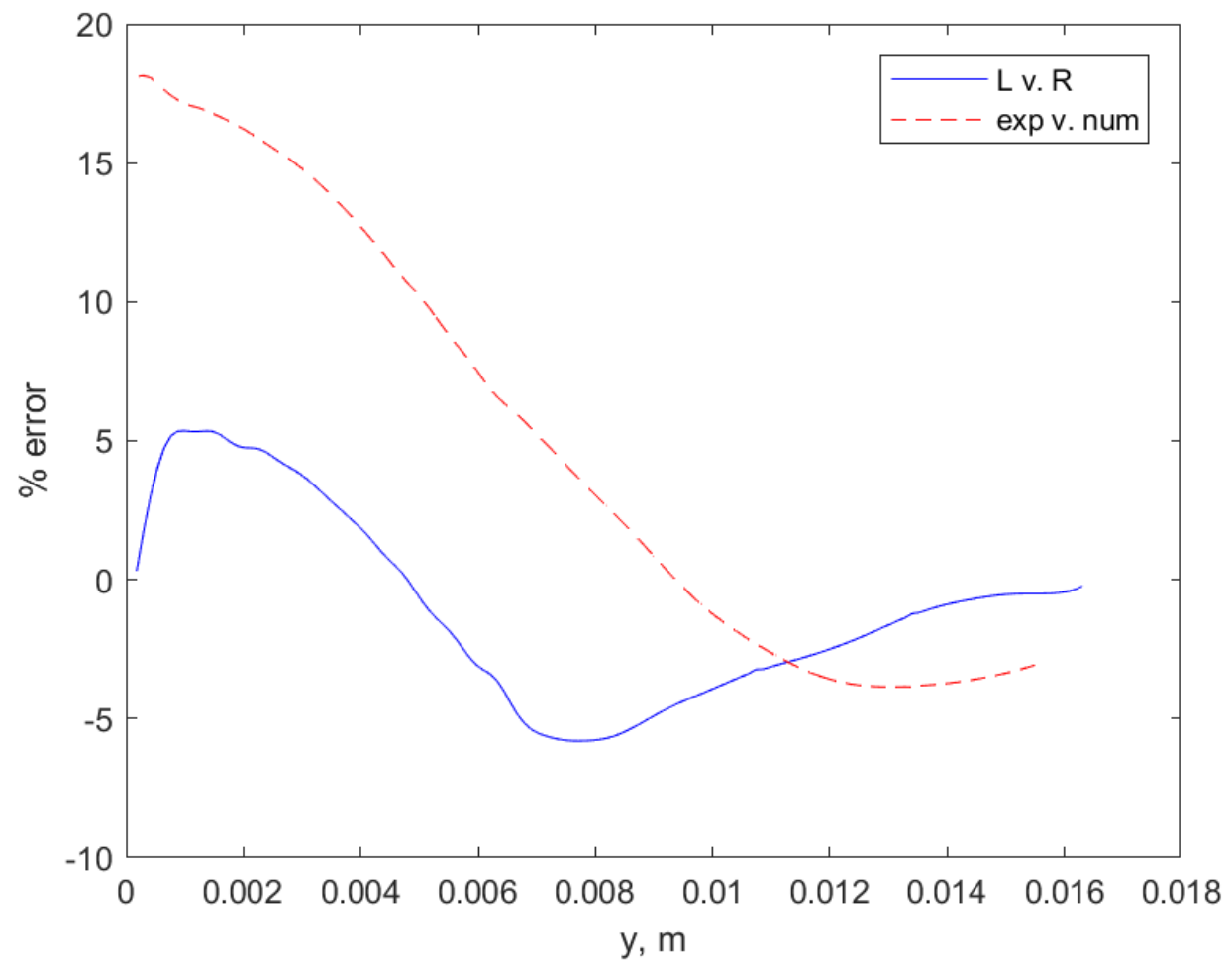

Figure C.78: Shot 143. Percent error in thermal boundary layer between both sides of experimental boundary layer profiles (red line) and percent error between predicted boundary layer profile from similarity solution and averaged experimental boundary layer profile (blue dashed line). 
Appendix D

\section{INTERFEROMETER POST-PROCESSED RESULTS, HYDROGEN}

\begin{tabular}{|c||ccccccc|}
\hline Cyl Tag & shot & pyr T $(\mathrm{K})$ & intf T $(\mathrm{K})$ & $\Delta \mathrm{T}(\mathrm{K})$ & $\%$ diff. T & length $(\mathrm{s})$ & symmetry \\
\hline 100C & 4 & 963 & 976.2 & -13.2 & -1.4 & 10 & mostly symm. \\
100C & 5 & 962 & 1039.9 & -77.9 & -18.1 & 1 & very symm. \\
\hline
\end{tabular}

Table D.1: All processed interferometry results in diluted hydrogen-air mixtures.

$100 \mathrm{C}$

Shot 4:
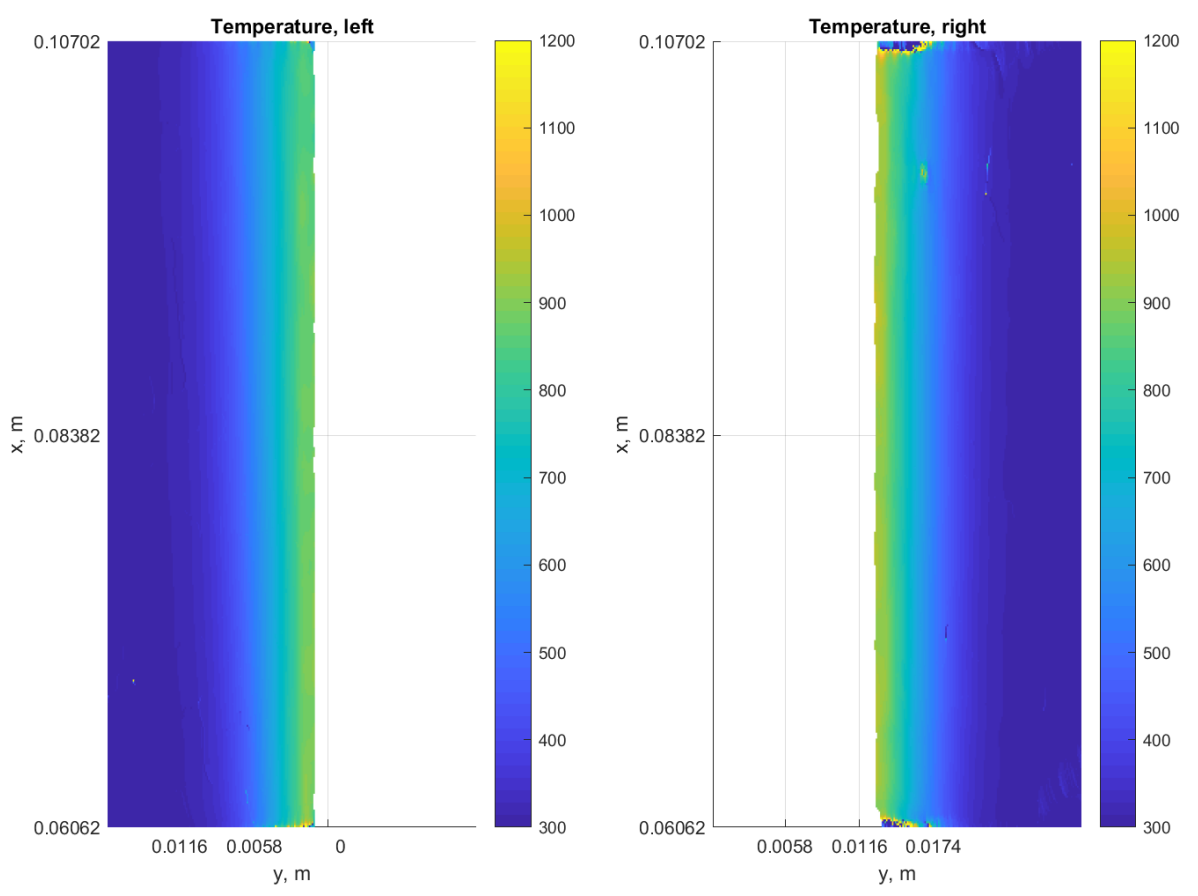

Figure D.1: Shot 4. Temperature fields from interferometry for hydrogen-air test. Surface temperature measured by pyrometer $=963 \mathrm{~K}$. 


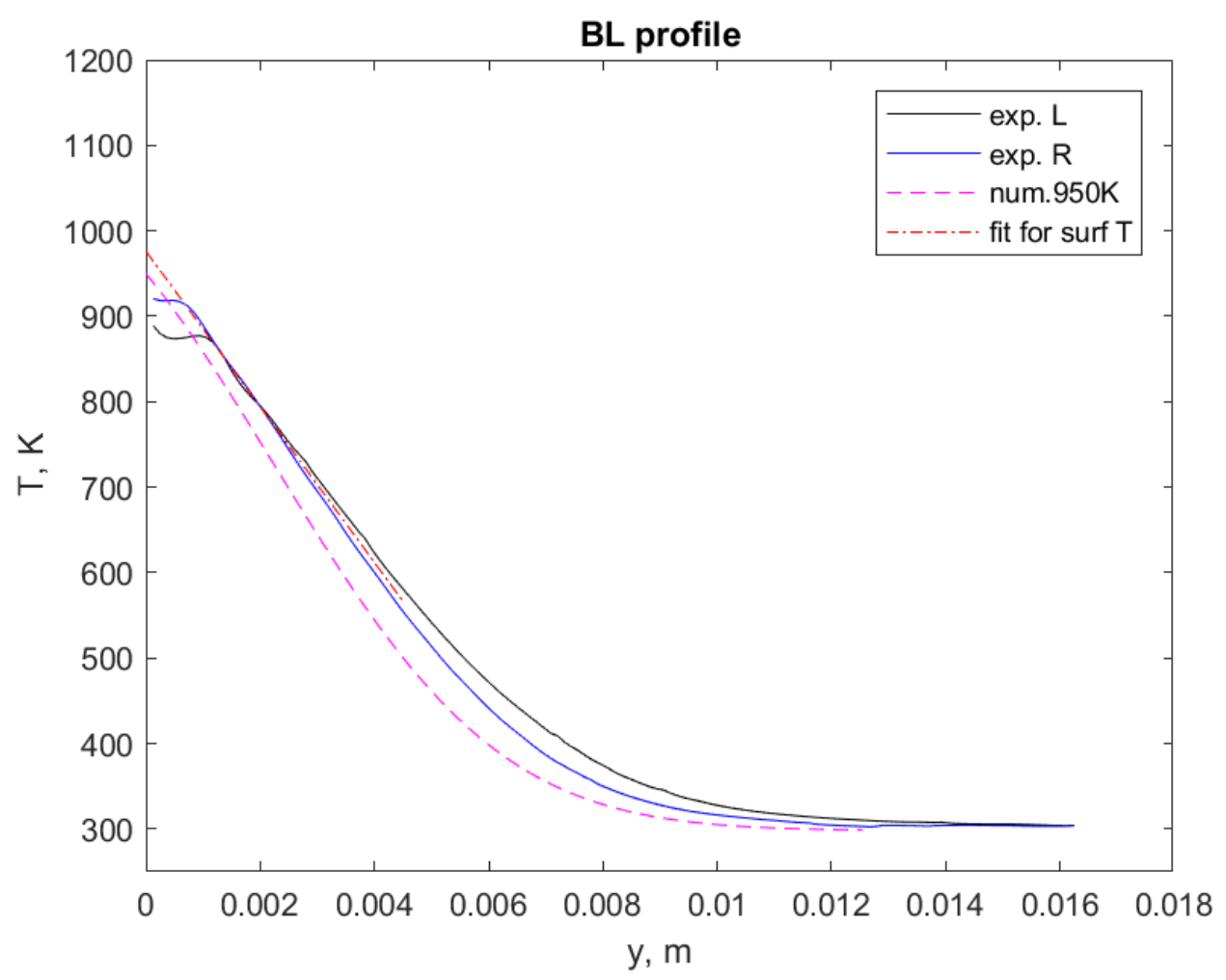

Figure D.2: Shot 4. Thermal boundary layer profiles for left (black line) and right (blue line) sides of experimental results compared with prediction of boundary layer profile (dashed magenta line) based on similarity solution for pyrometer surface temperature. Linear fit to averaged experimental profile (red dot-dashed line) used to extrapolate surface temperature from interferometer post processing. Extrapolated interferometer surface temperature $=976.2 \mathrm{~K}$. 


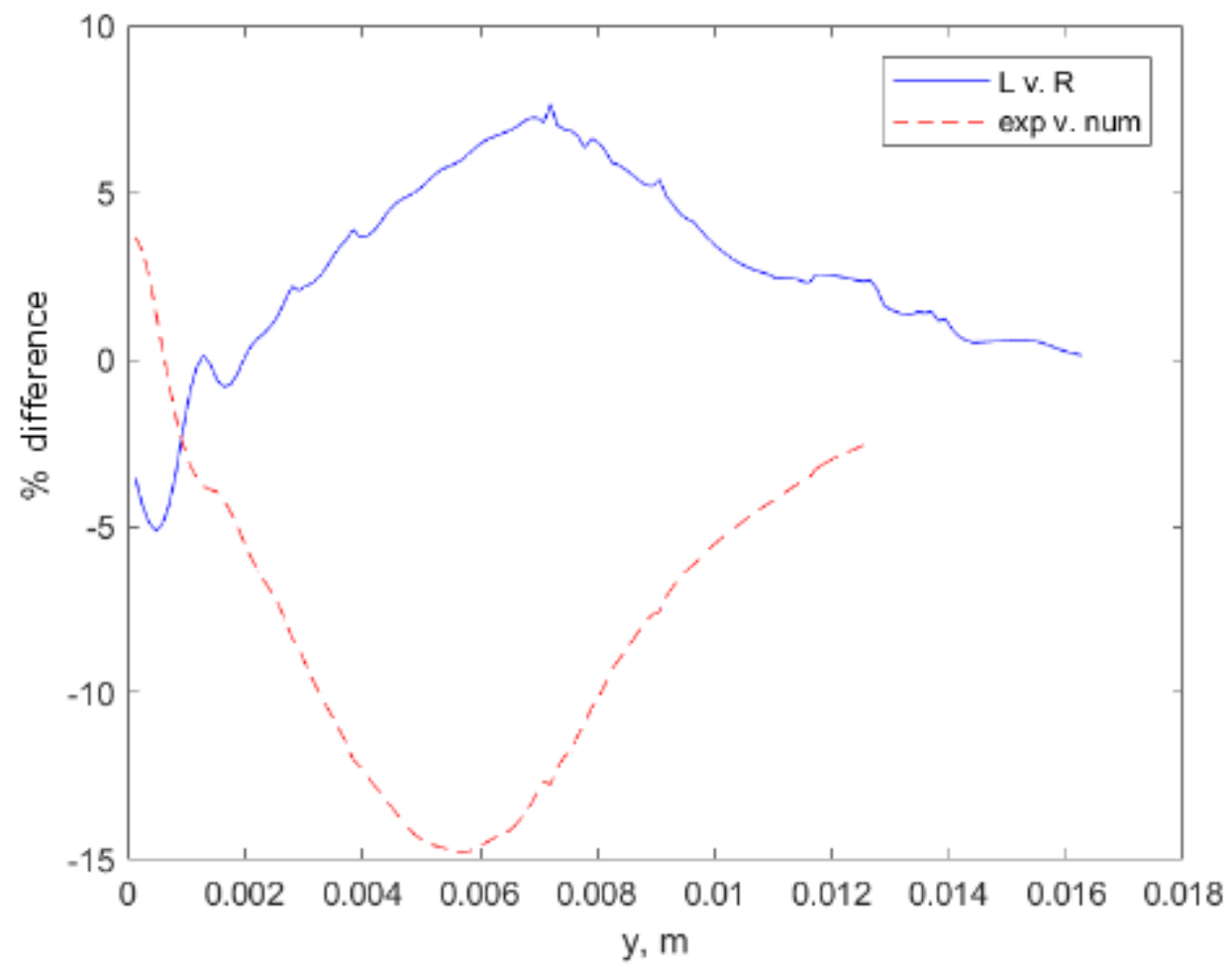

Figure D.3: Shot 4. Percent error in thermal boundary layer between both sides experimental boundary layer profiles (red line) and percent error between predicted boundary layer profile from similarity solution and averaged experimental boundary layer profile (blue dashed line). 
Shot 5:
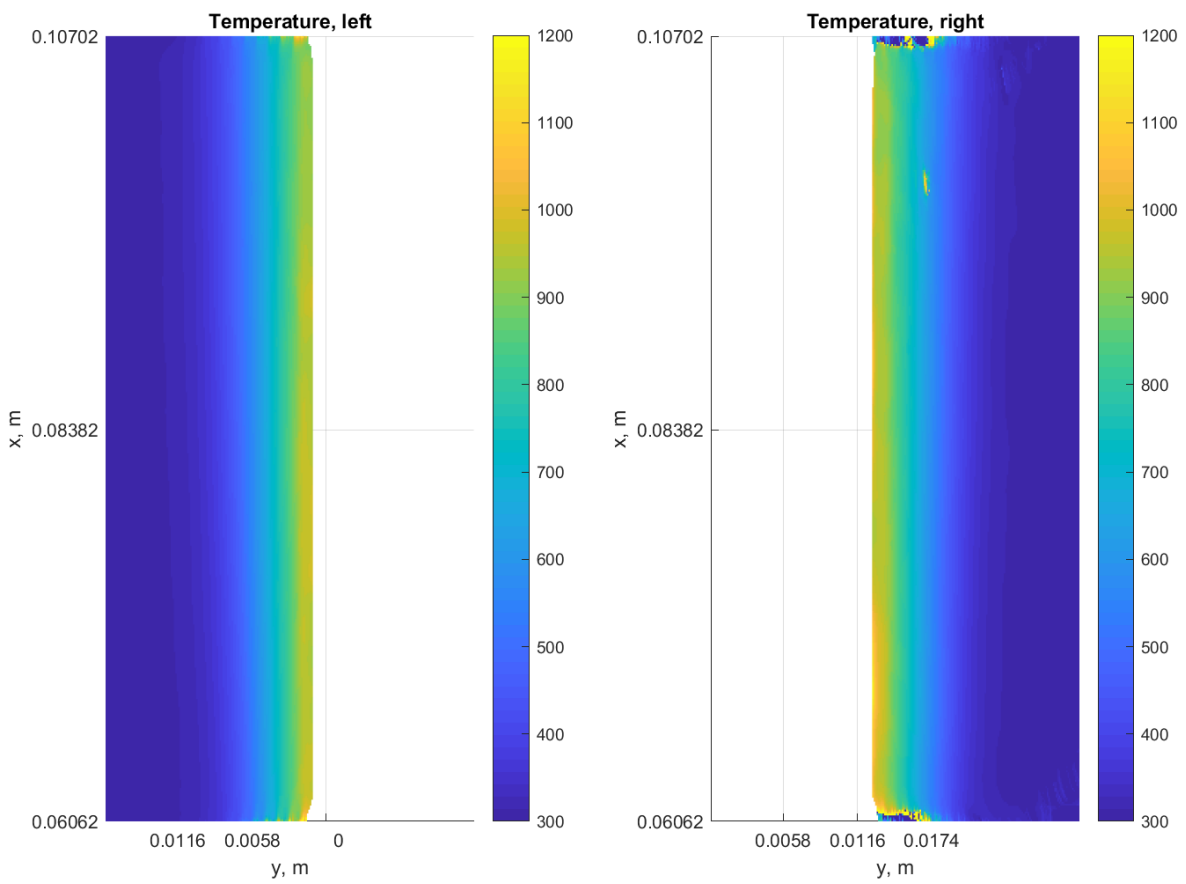

Figure D.4: Shot 5. Temperature fields from interferometry for hydrogen-air test. Surface temperature measured by pyrometer $=962 \mathrm{~K}$. 


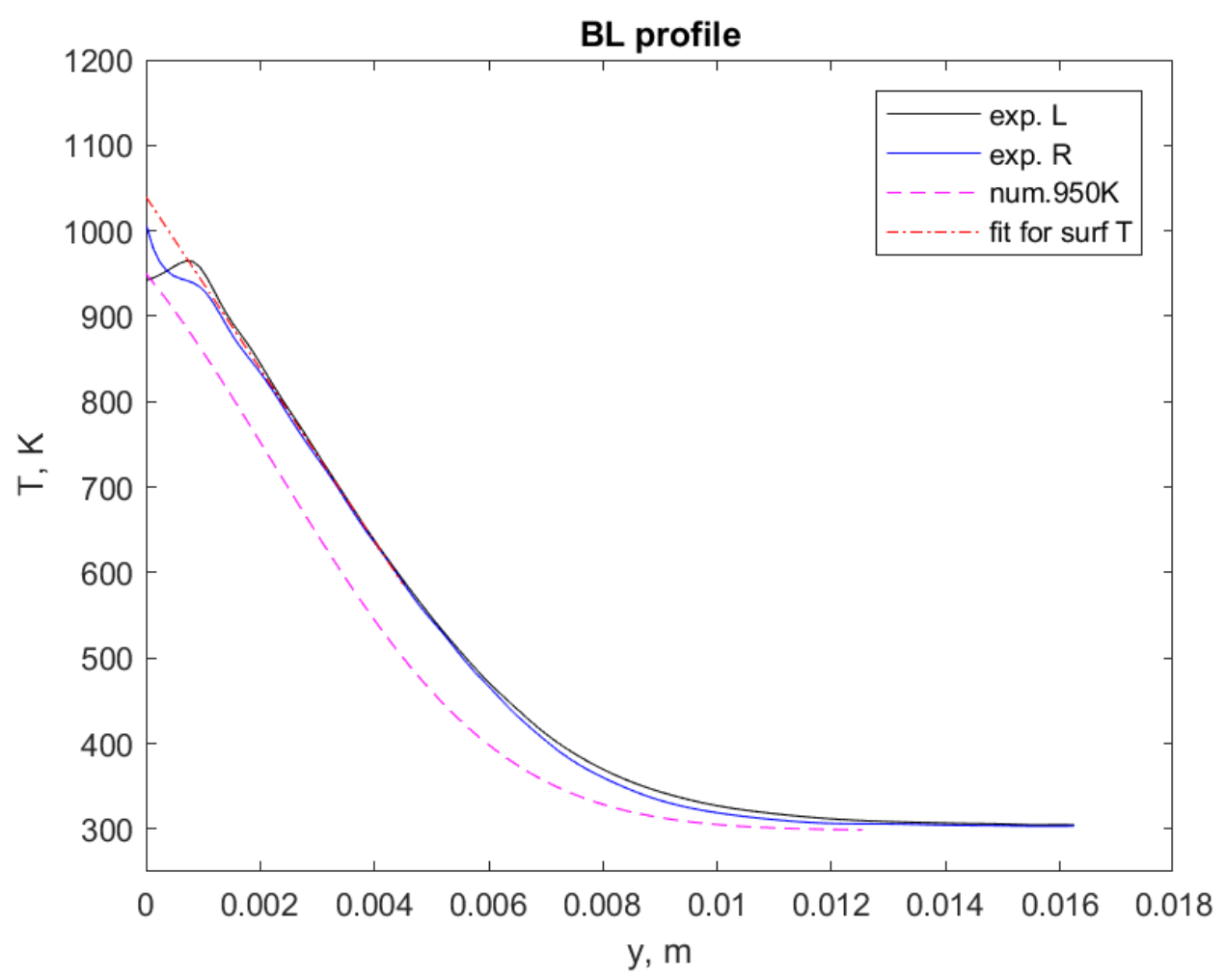

Figure D.5: Shot 5. Thermal boundary layer profiles for left (black line) and right (blue line) sides of experimental results compared with prediction of boundary layer profile (dashed magenta line) based on similarity solution for pyrometer surface temperature. Linear fit to averaged experimental profile (red dot-dashed line) used to extrapolate surface temperature from interferometer post processing. Extrapolated interferometer surface temperature $=1039.9 \mathrm{~K}$. 


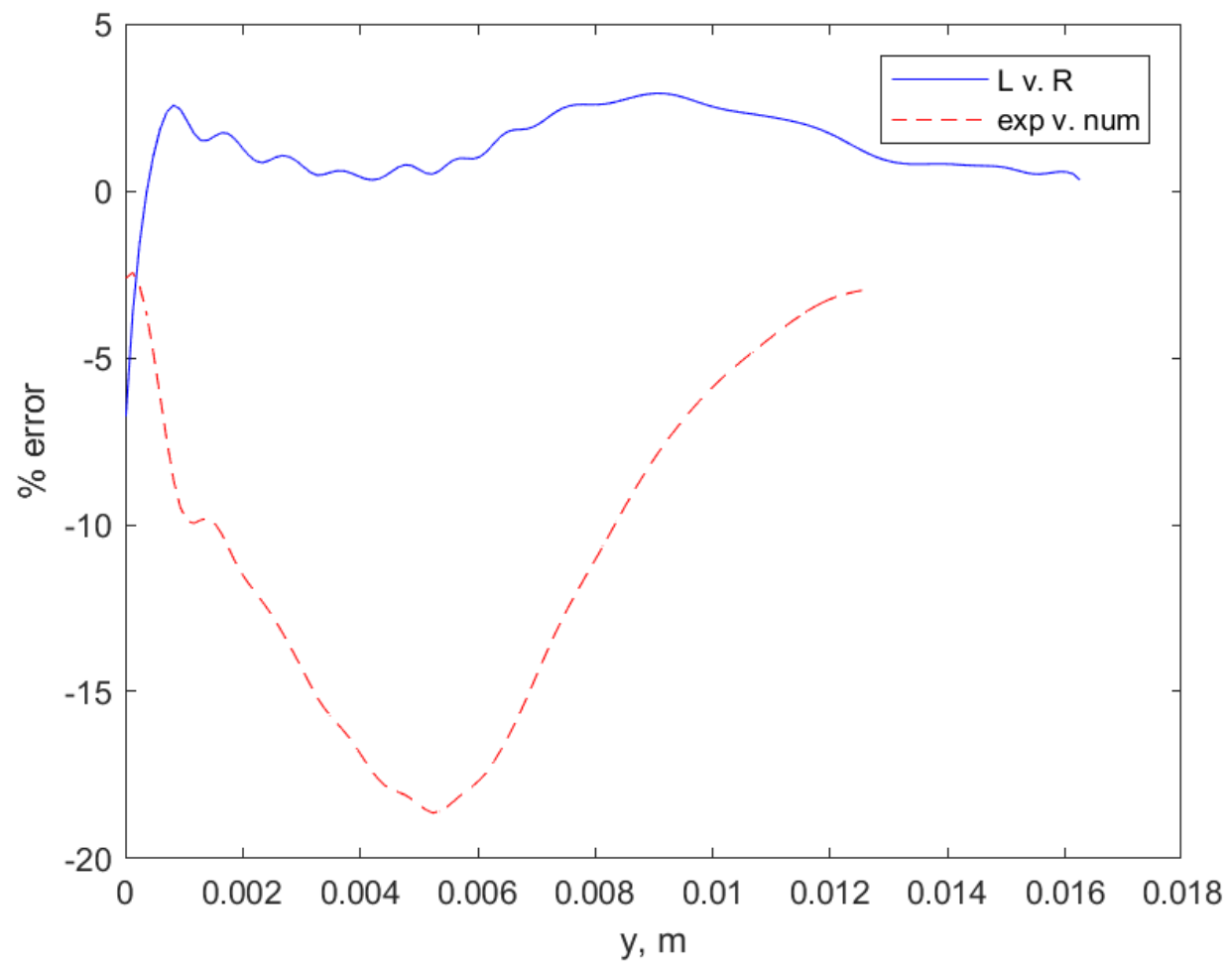

Figure D.6: Shot 5. Percent error in thermal boundary layer between both sides experimental boundary layer profiles (red line) and percent error between predicted boundary layer profile from similarity solution and averaged experimental boundary layer profile (blue dashed line). 
Appendix E

\section{ONE-DIMENSIONAL SIMULATION RESULTS}

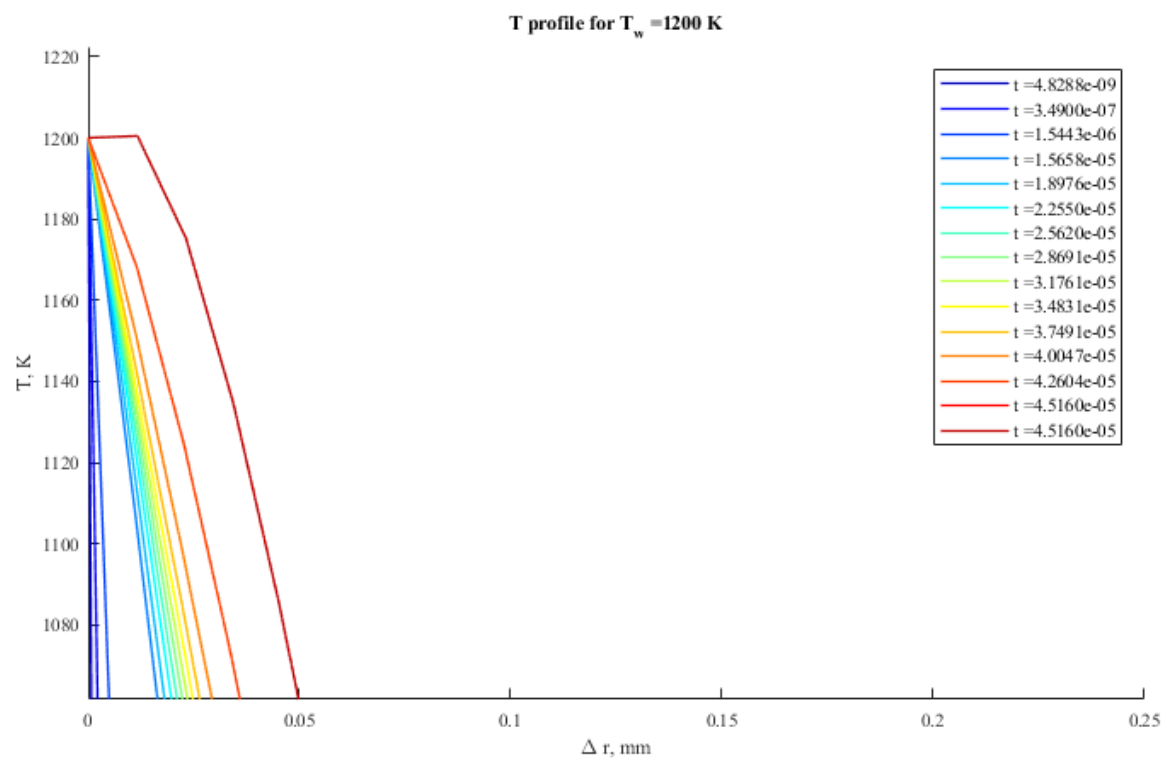

Figure E.1: Temperature profiles of supercritical case, up to $\mathrm{t}=\tau_{i g n, j_{q}}$. 


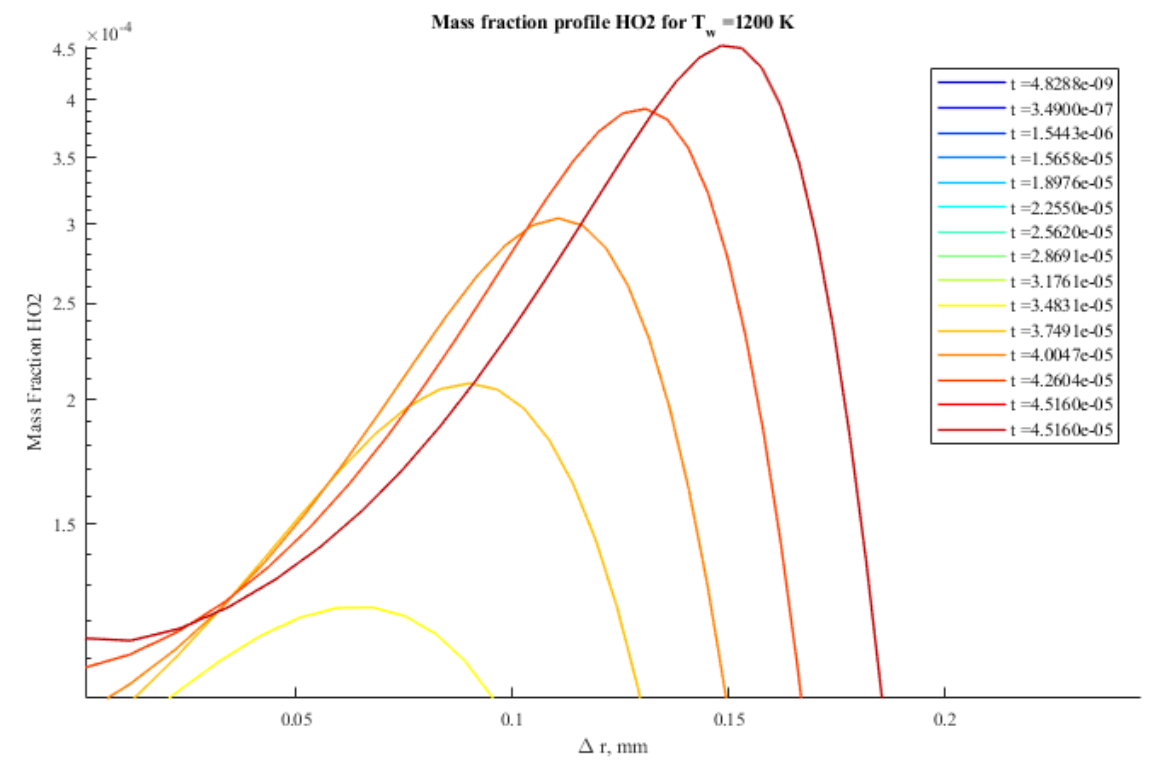

Figure E.2: $\mathrm{YHO}_{2}$ profiles of supercritical case, up to $\mathrm{t}=\tau_{i g n, j_{q}}$. 
Appendix F

\section{TEST SPREADSHEET}

F.1 Hexane 


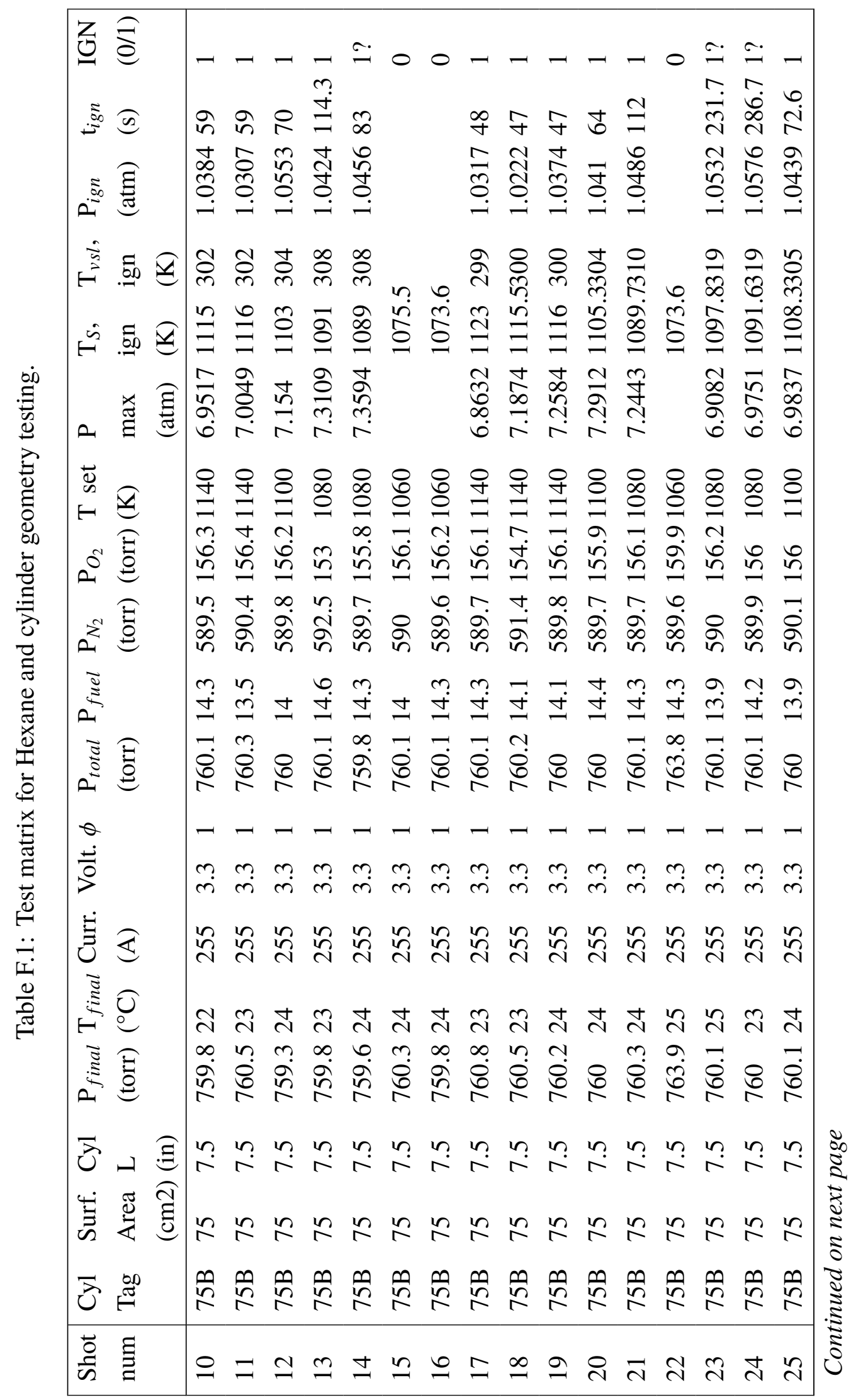




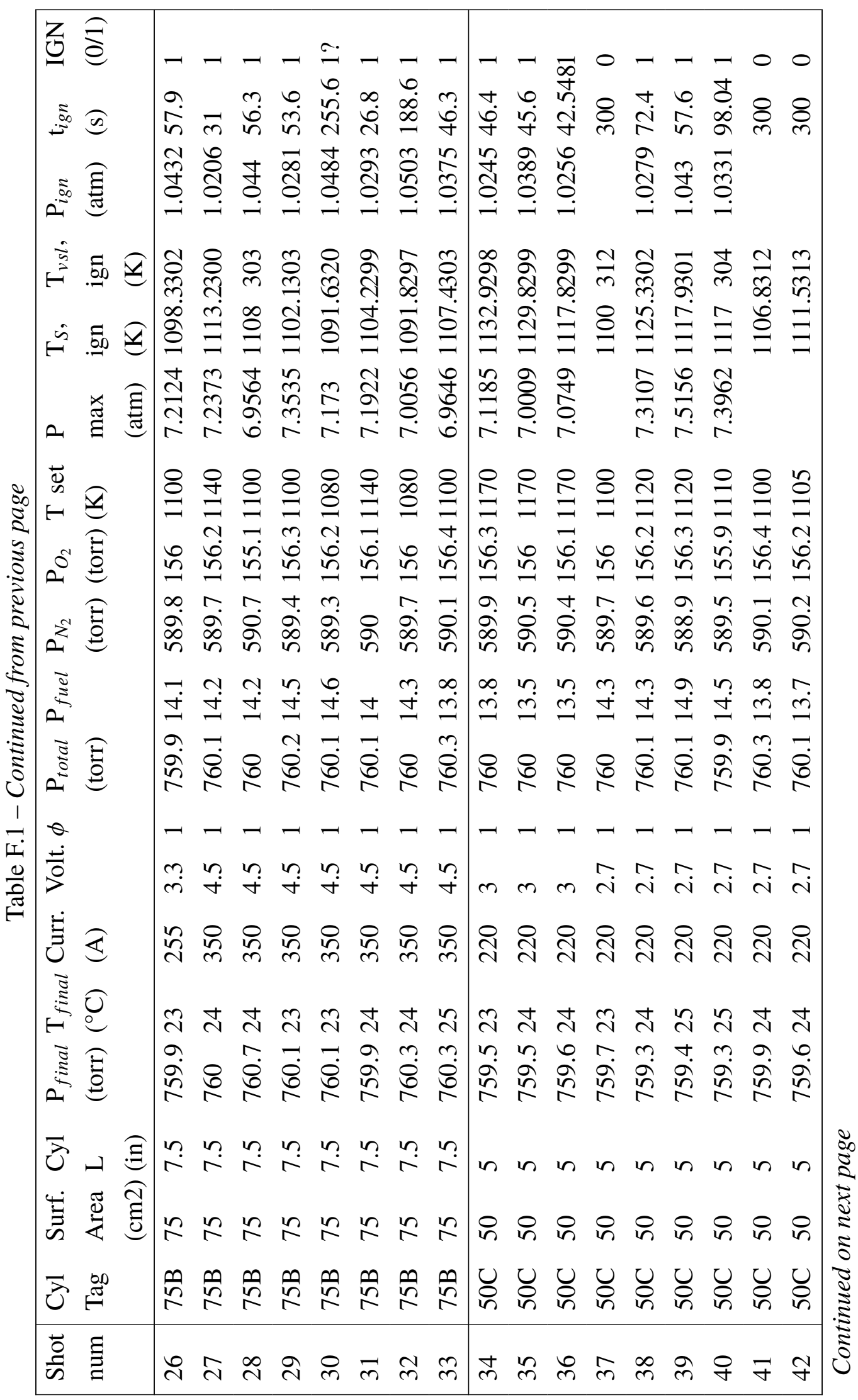




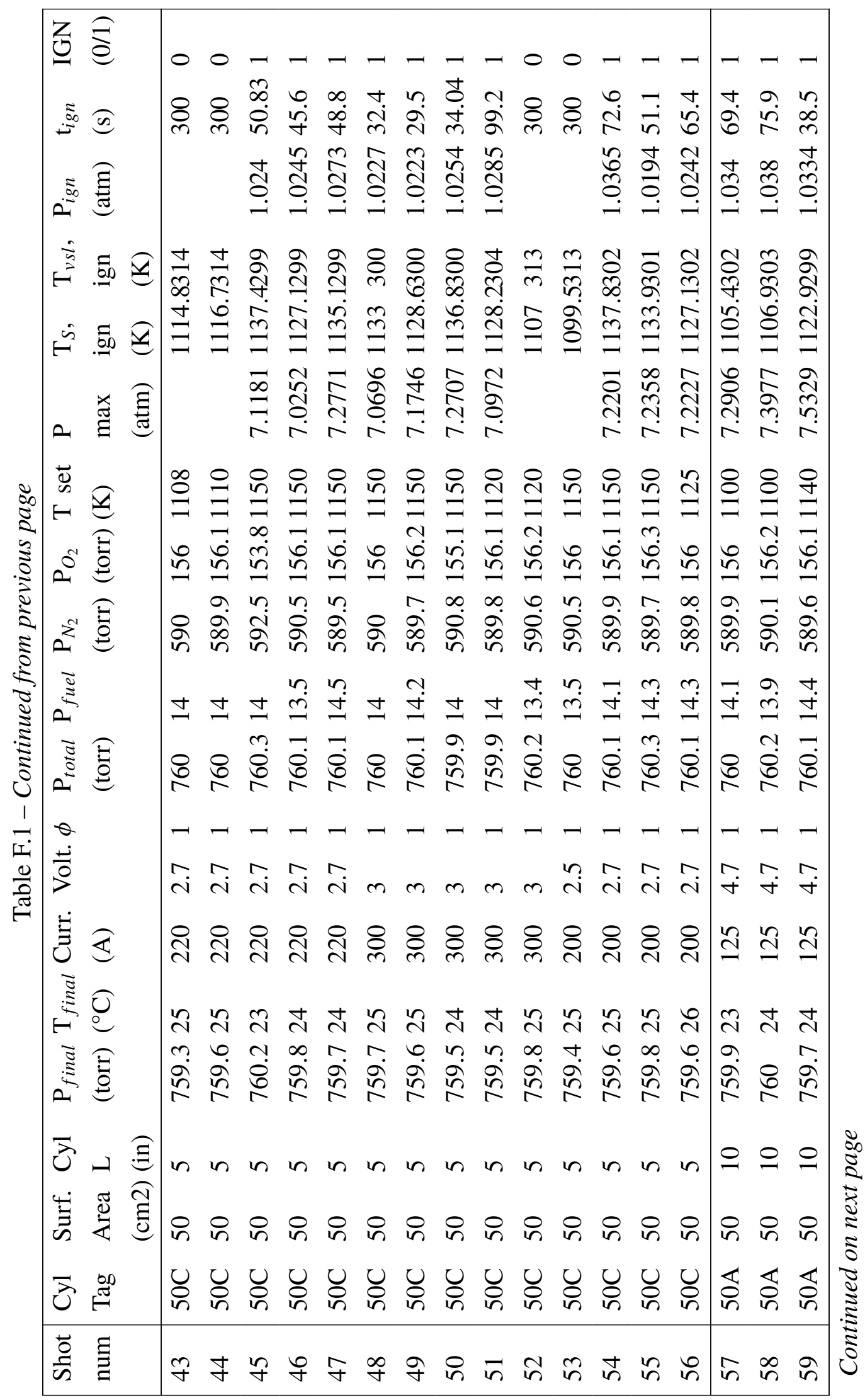




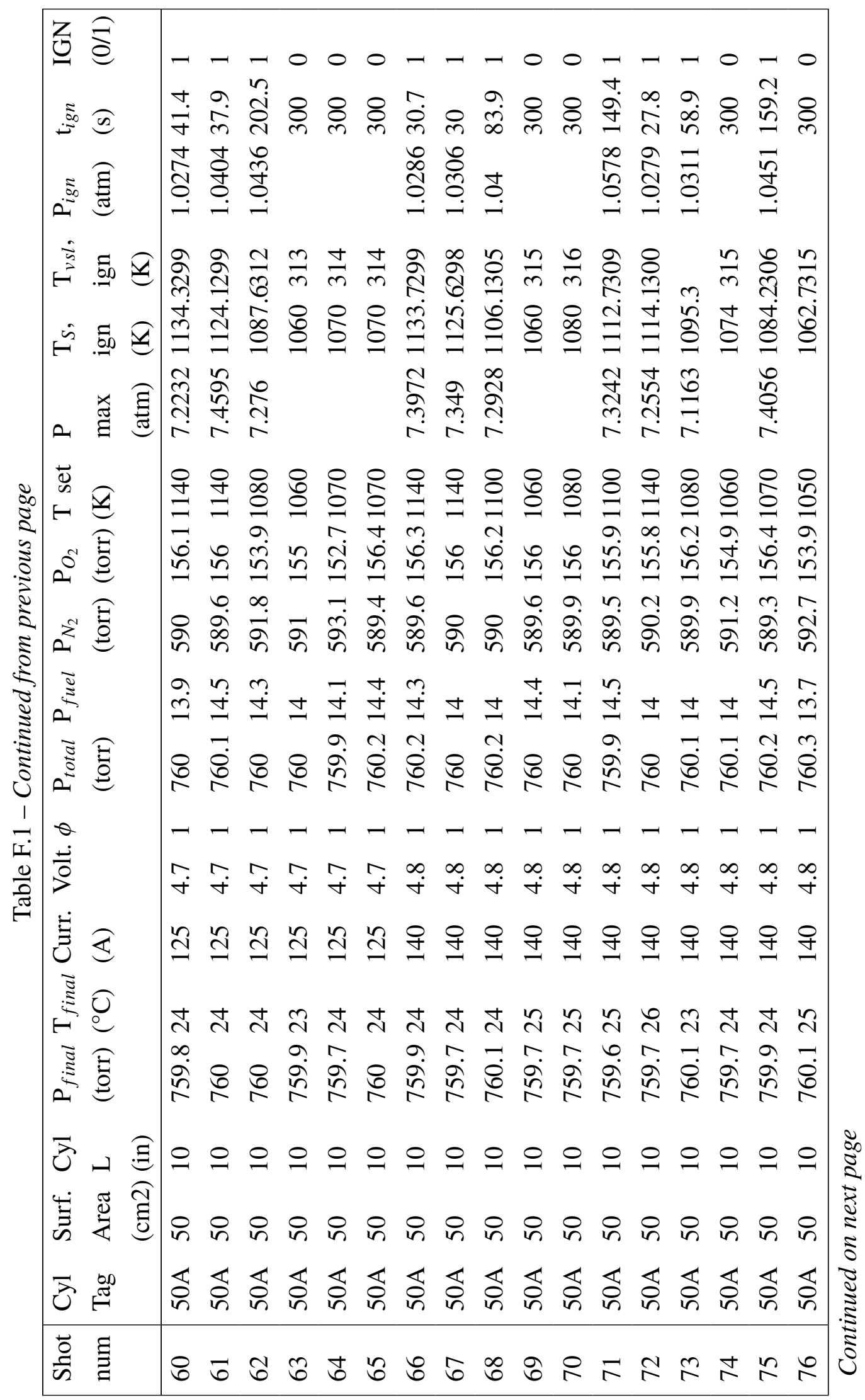




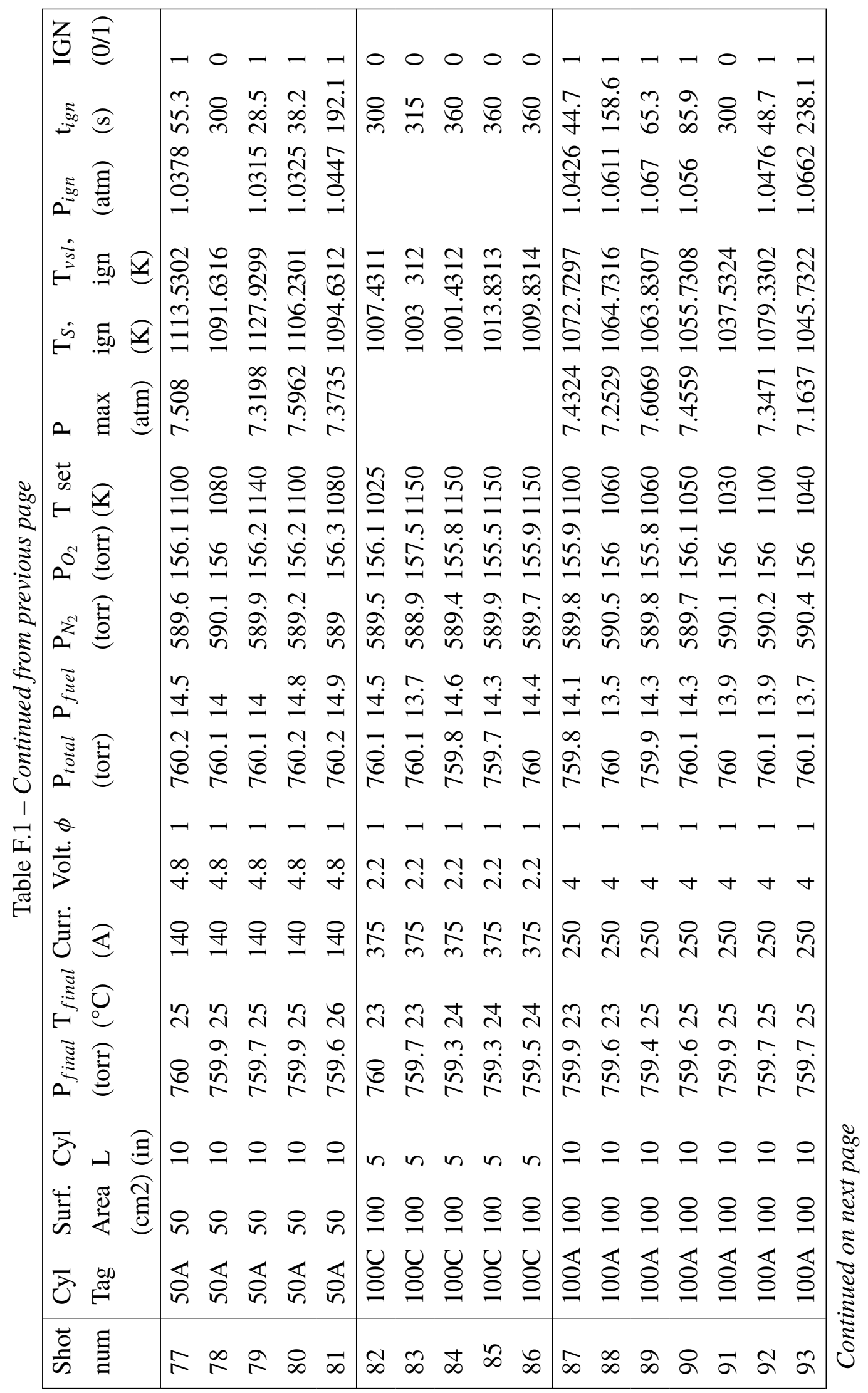




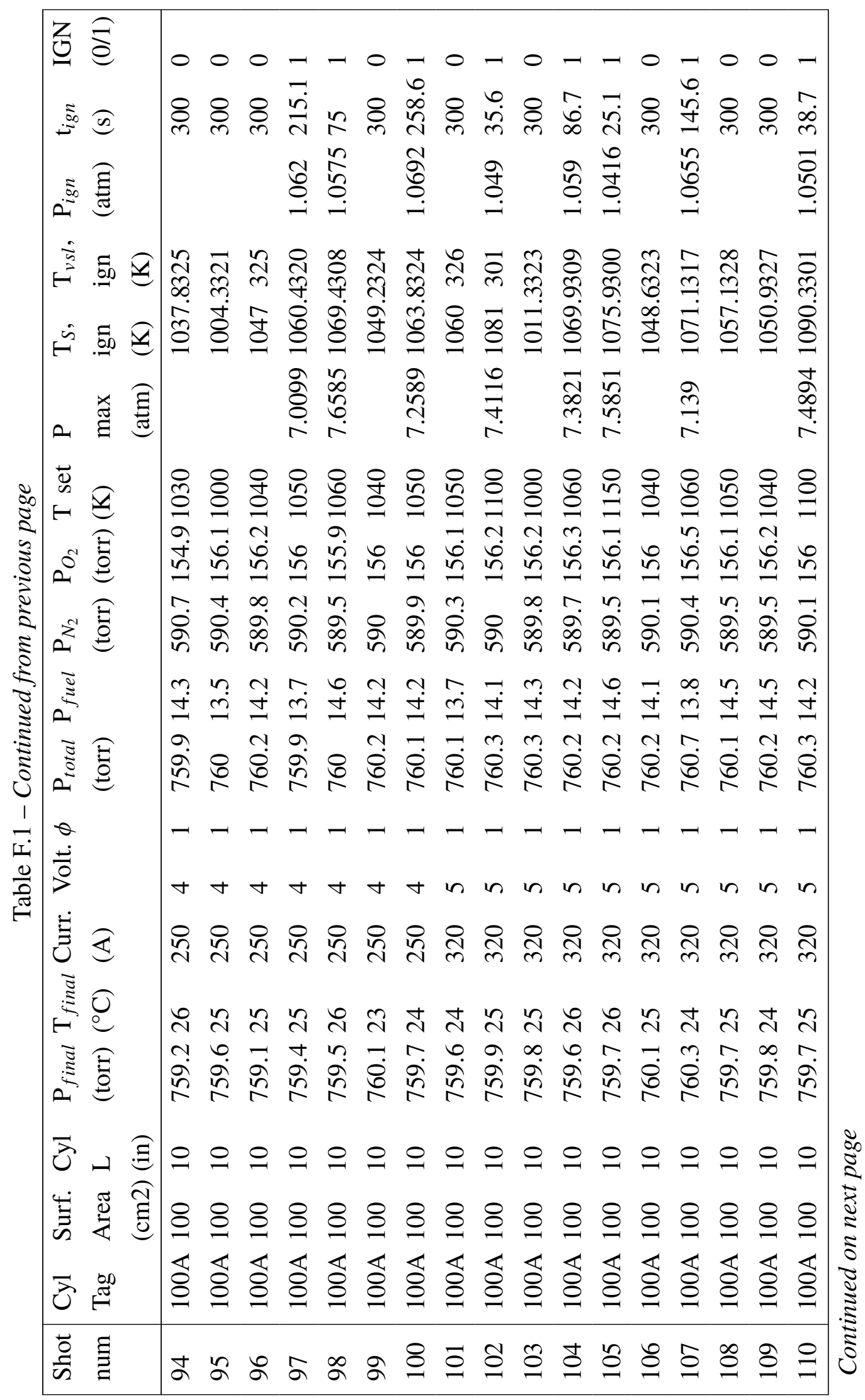




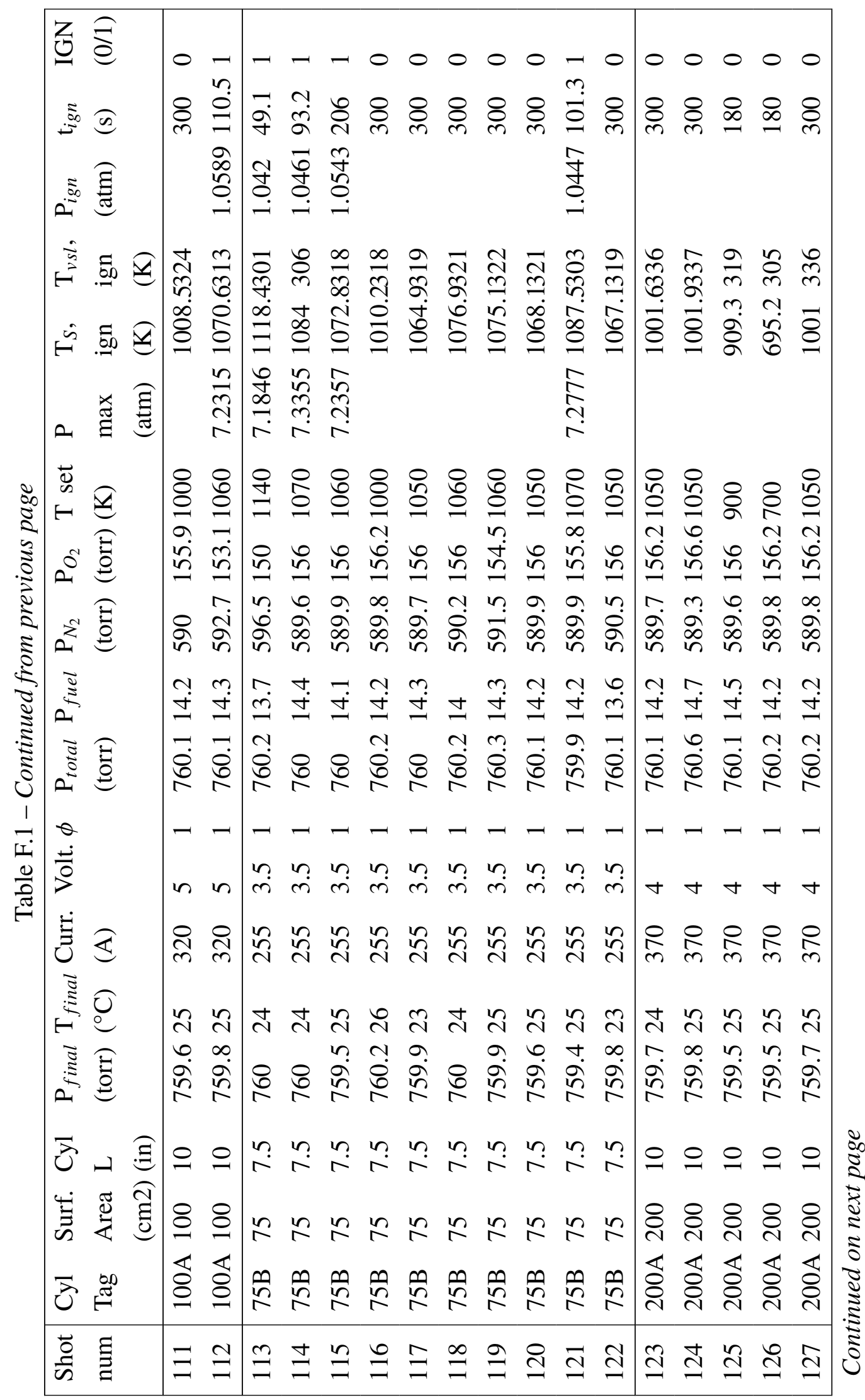




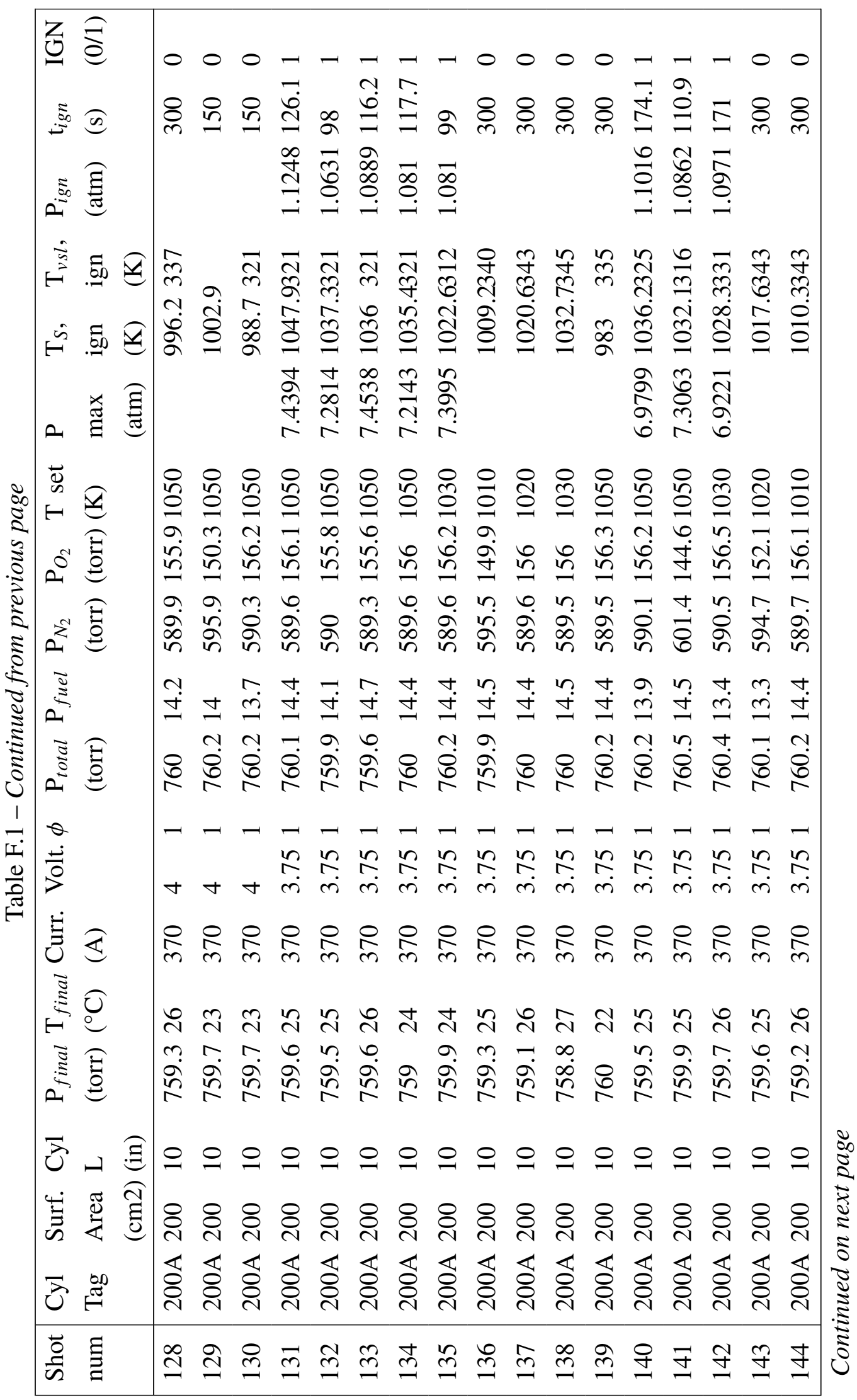




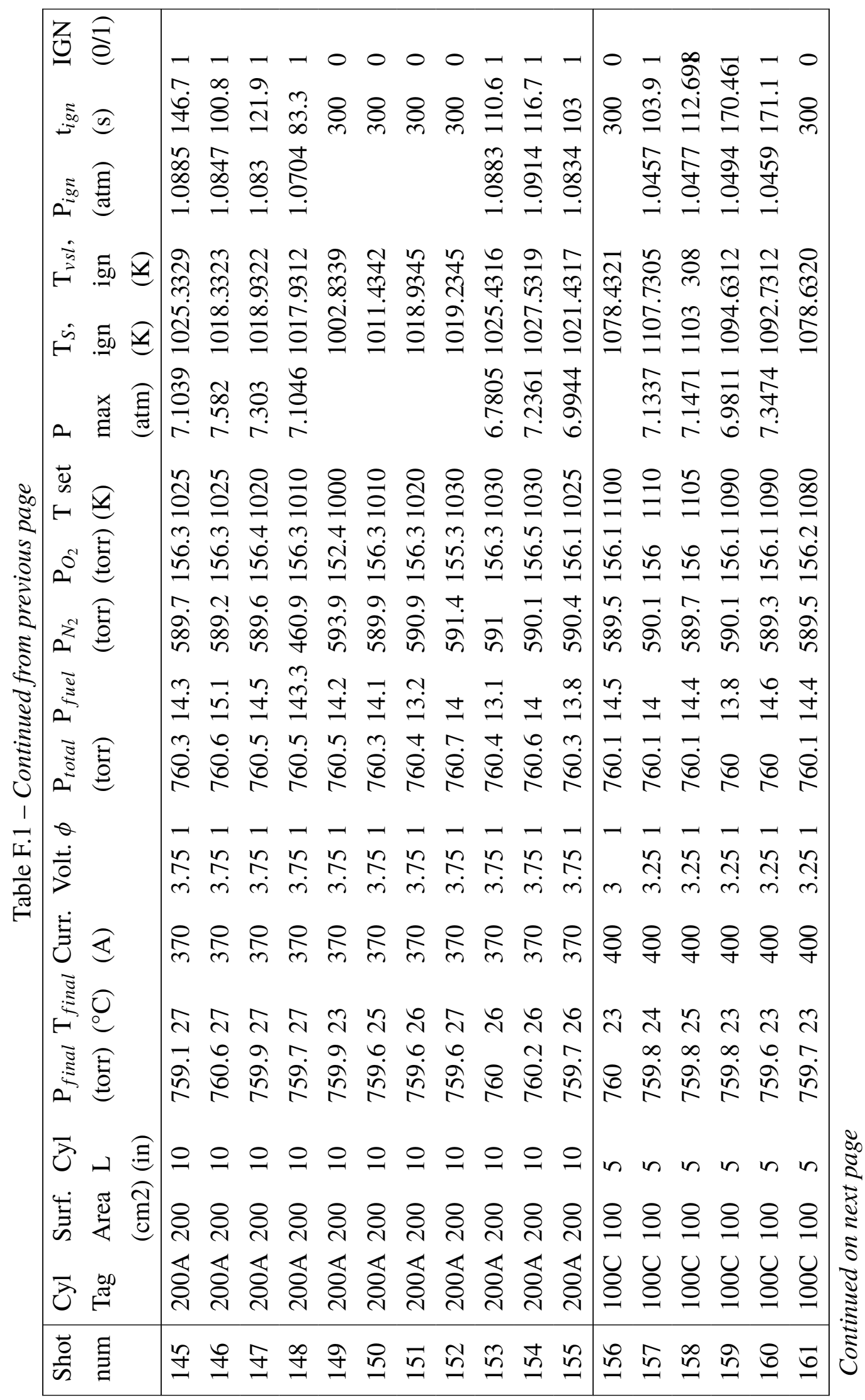




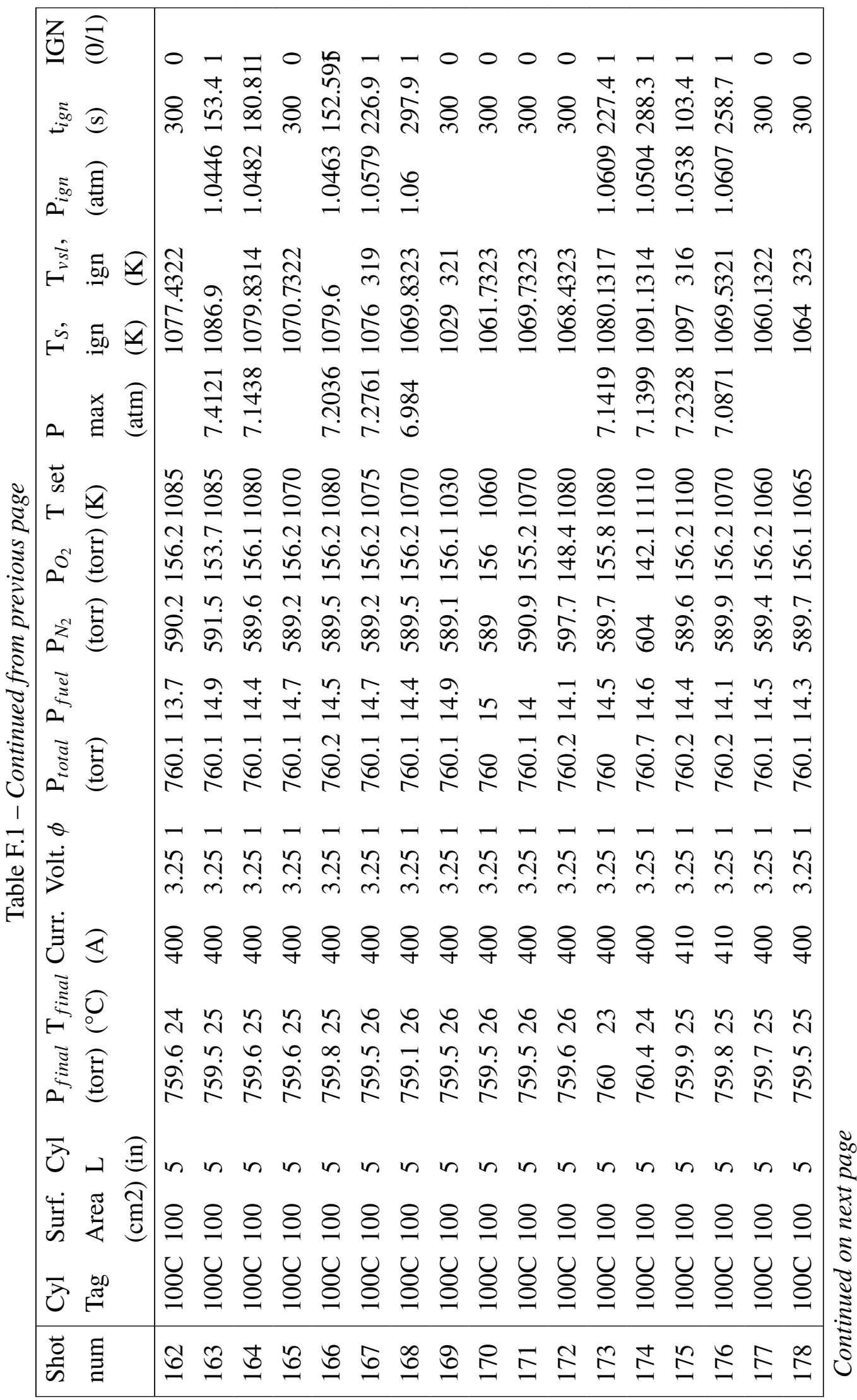




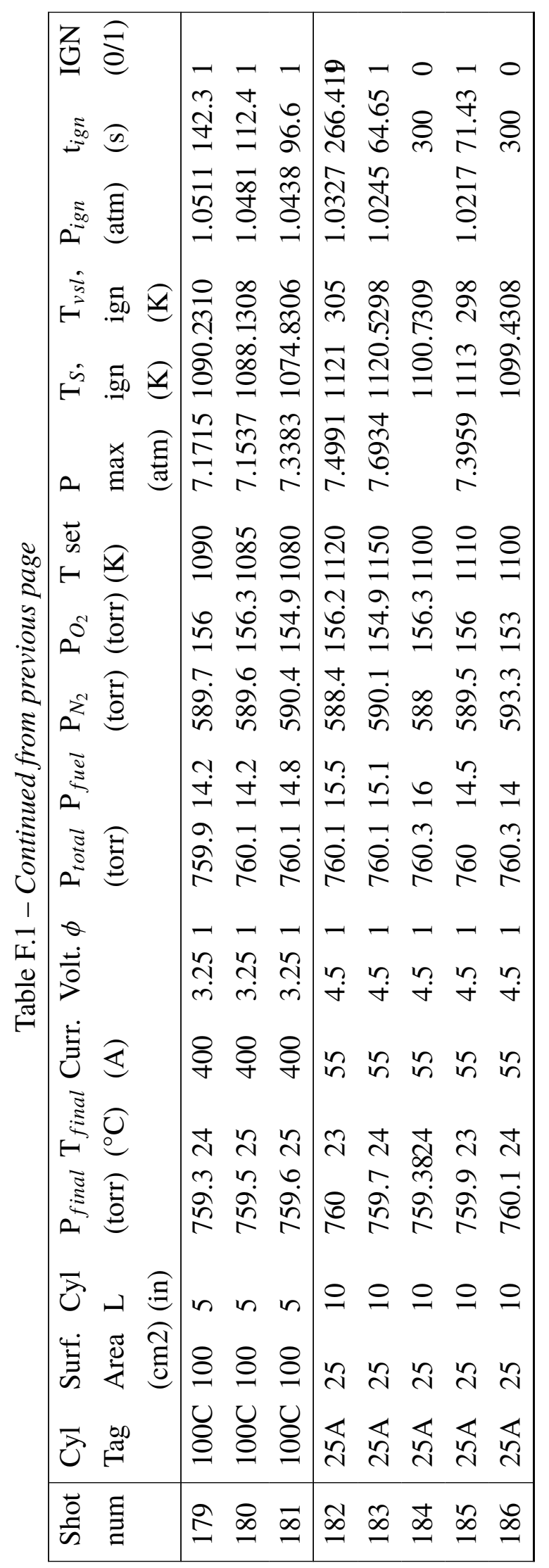


F.2 High-Temperature Hexane 


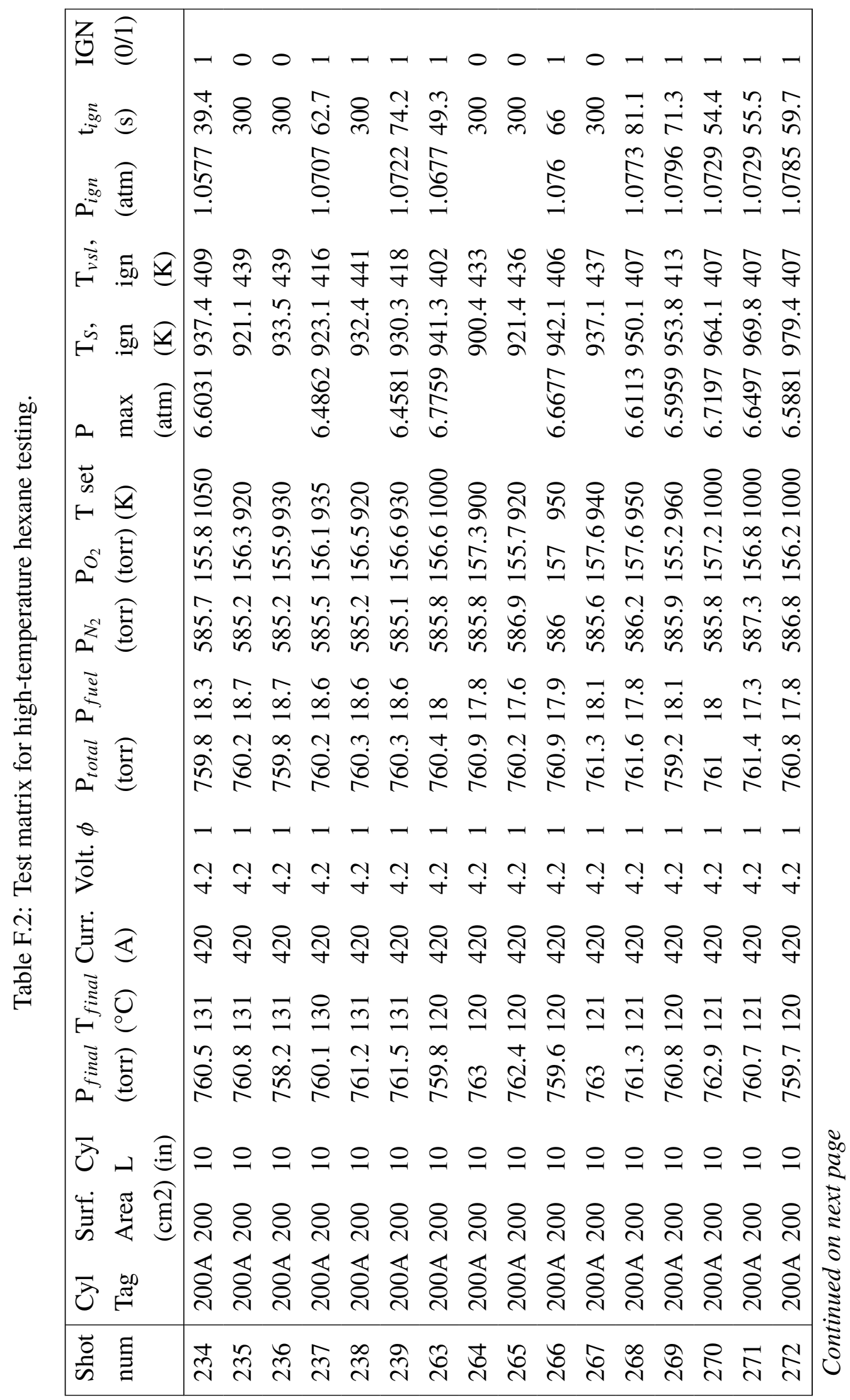




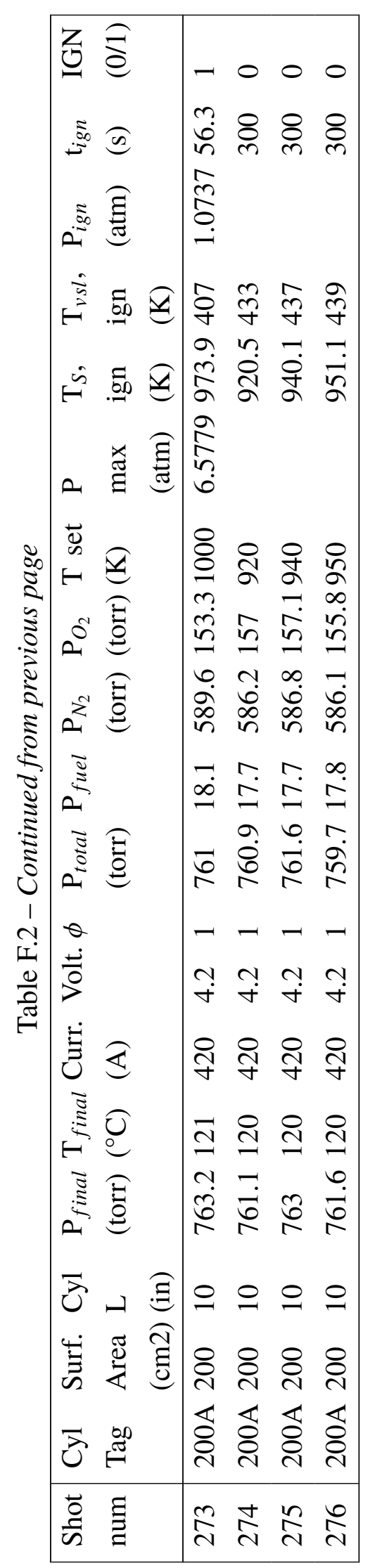




\section{F.3 Aachen}




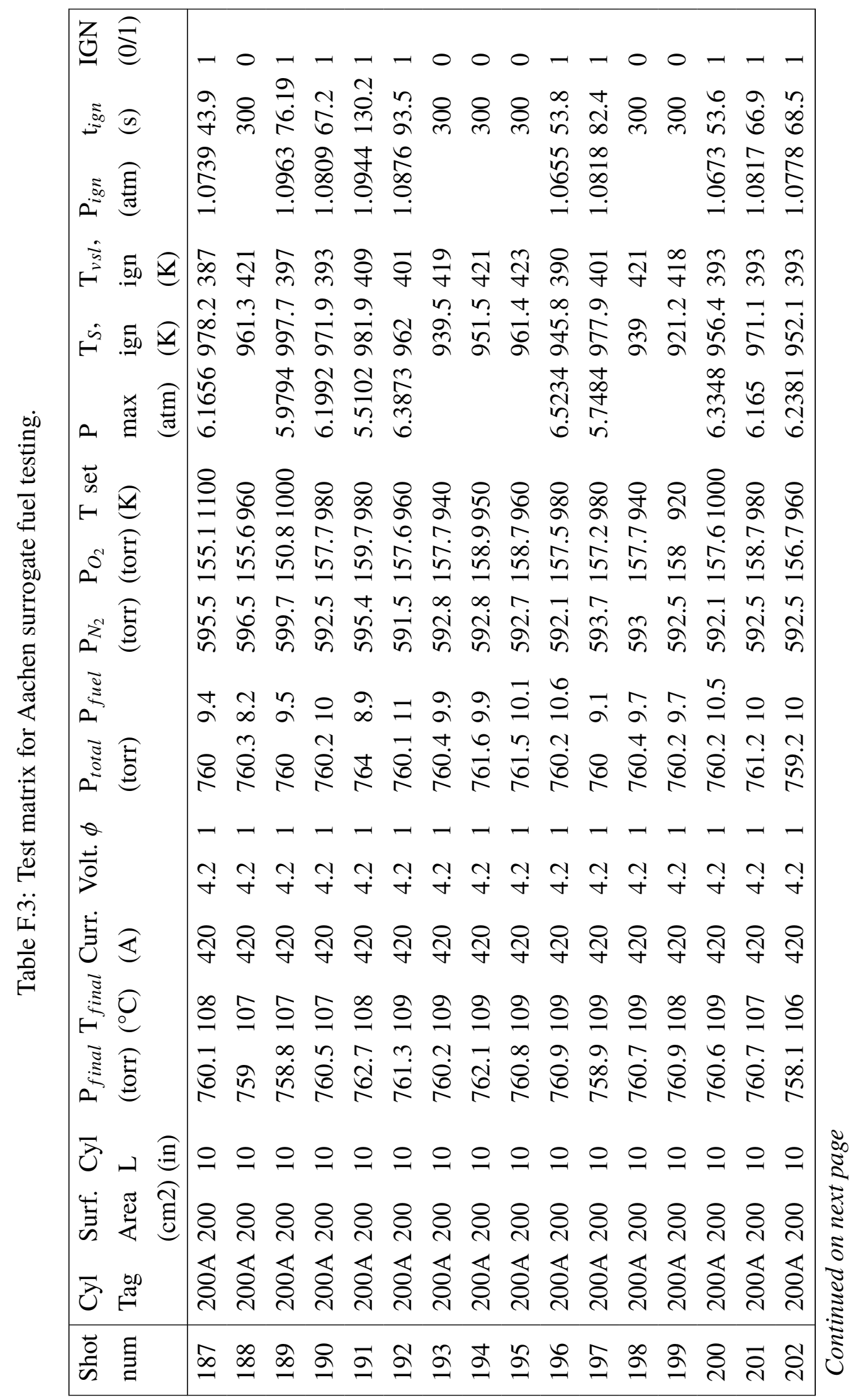




\begin{tabular}{|c|c|}
\hline 5 & \\
\hline O & $00-0 \rightarrow-1-0-$ \\
\hline 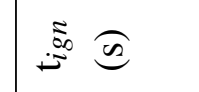 & 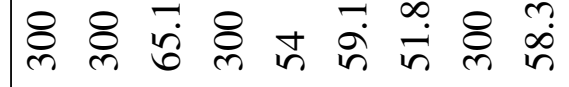 \\
\hline & 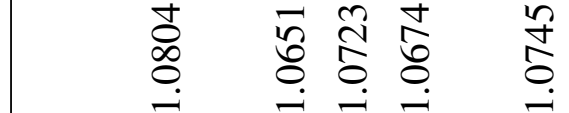 \\
\hline & f f \\
\hline & 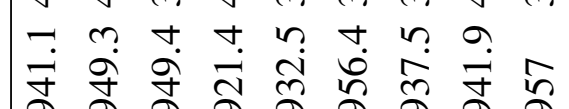 \\
\hline$\approx$ & 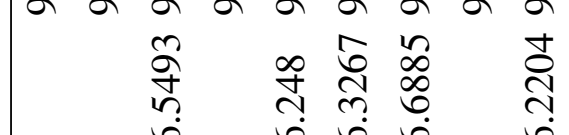 \\
\hline & \\
\hline & 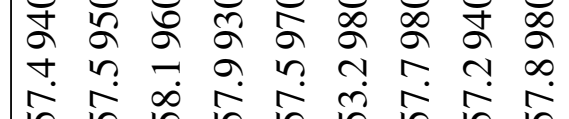 \\
\hline & 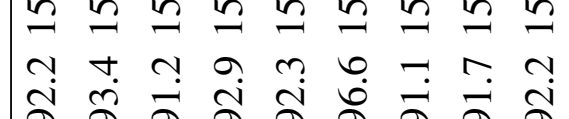 \\
\hline & \\
\hline & \\
\hline & ì \\
\hline & $-\gamma---\tau-\tau$ \\
\hline & 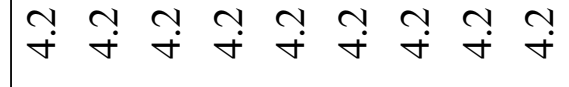 \\
\hline & 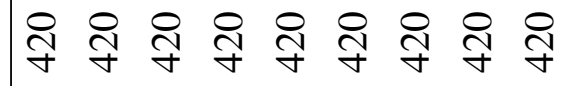 \\
\hline & $\underline{\sigma} \subseteq \stackrel{0}{\circ} \stackrel{0}{=}$ \\
\hline & 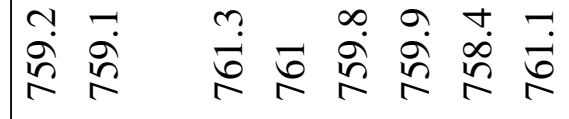 \\
\hline & 2 \\
\hline & \& \\
\hline & 这 \\
\hline & 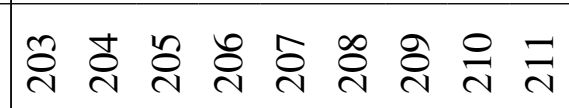 \\
\hline
\end{tabular}


F.4 JI 


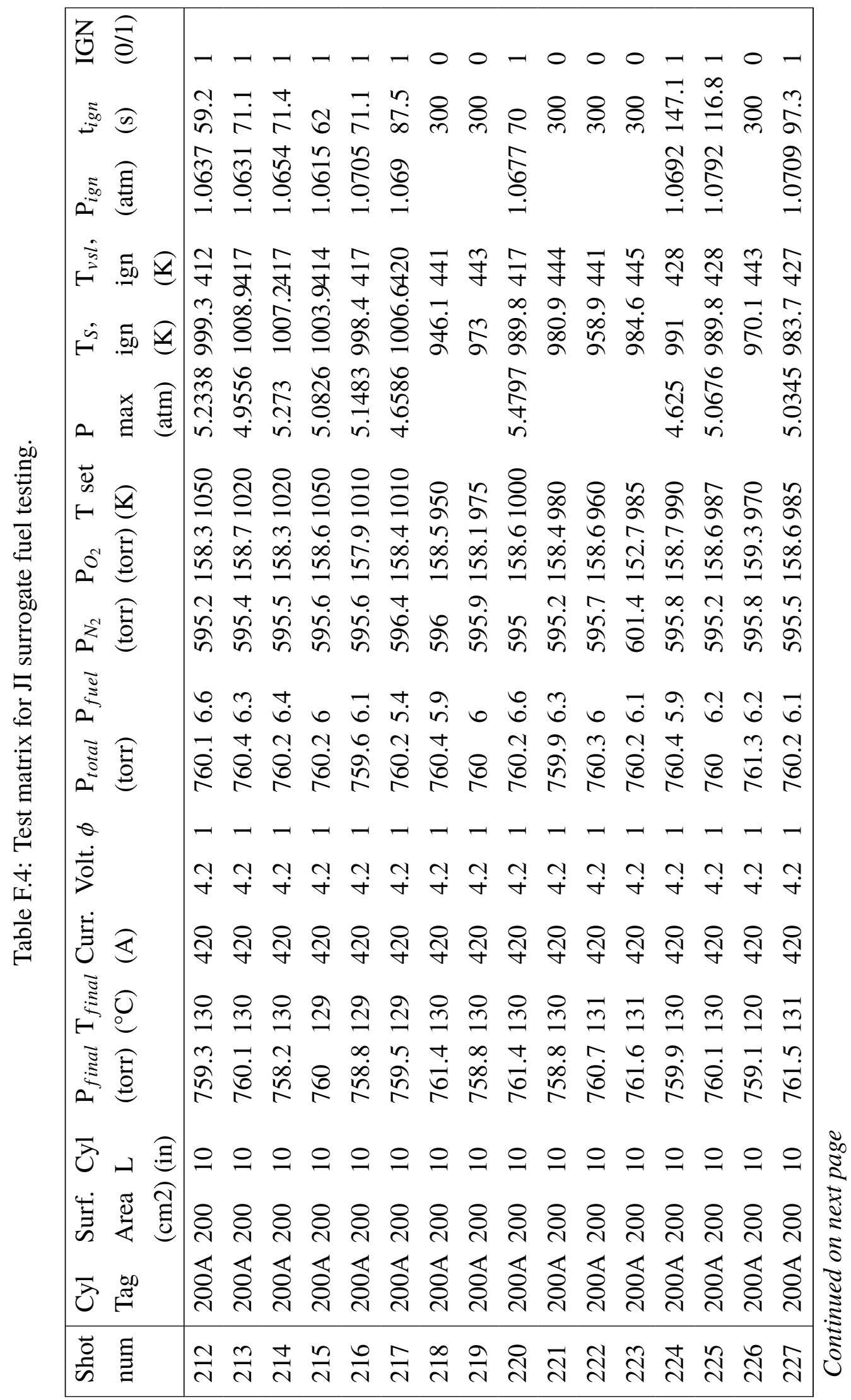




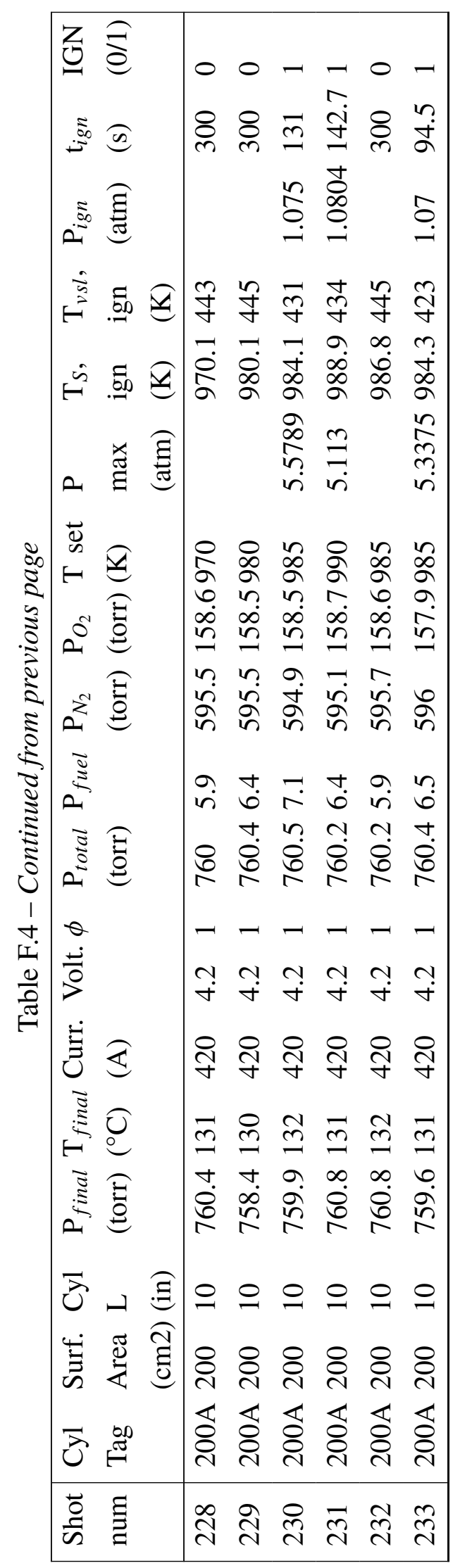


F.5 Jet A 


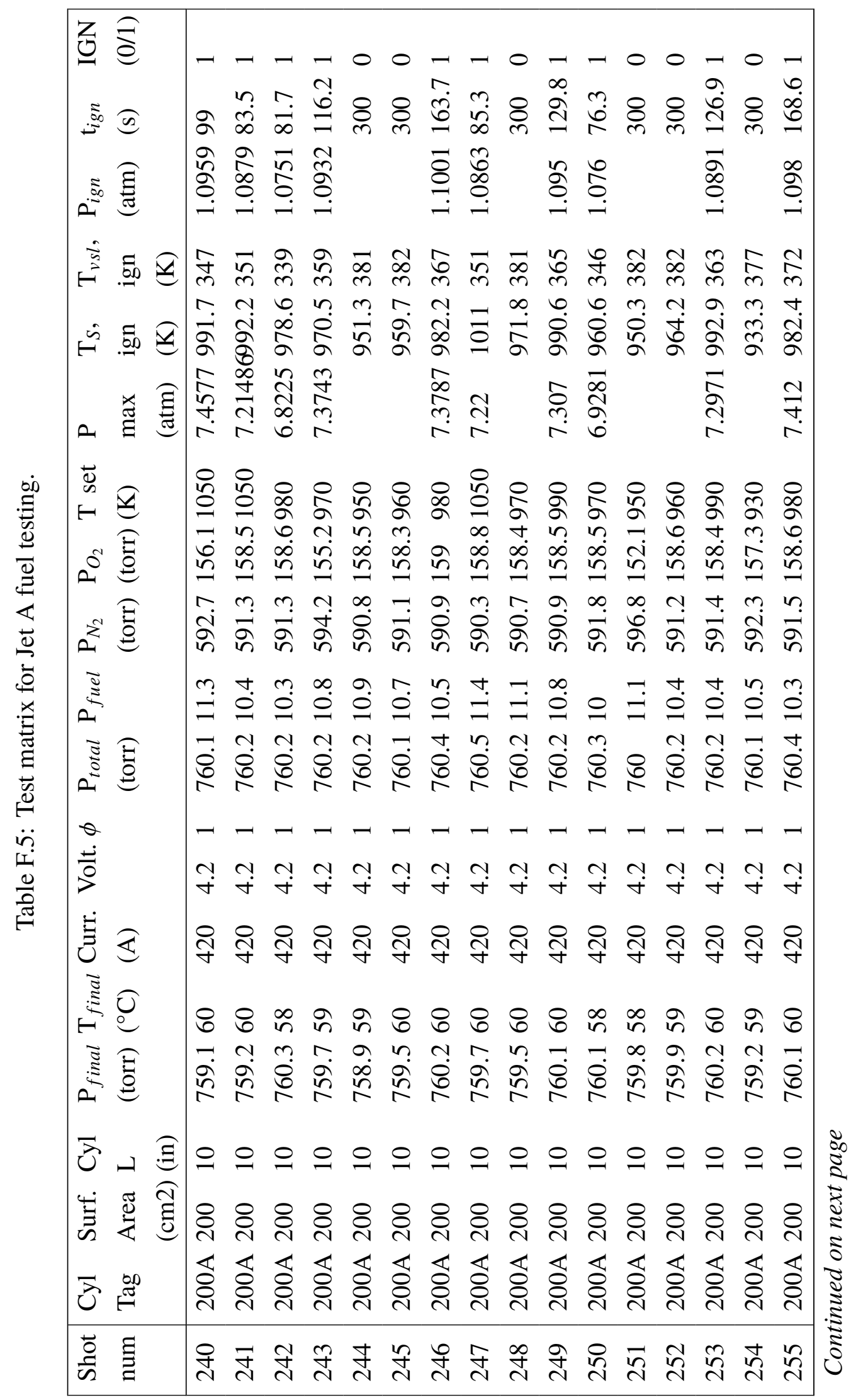




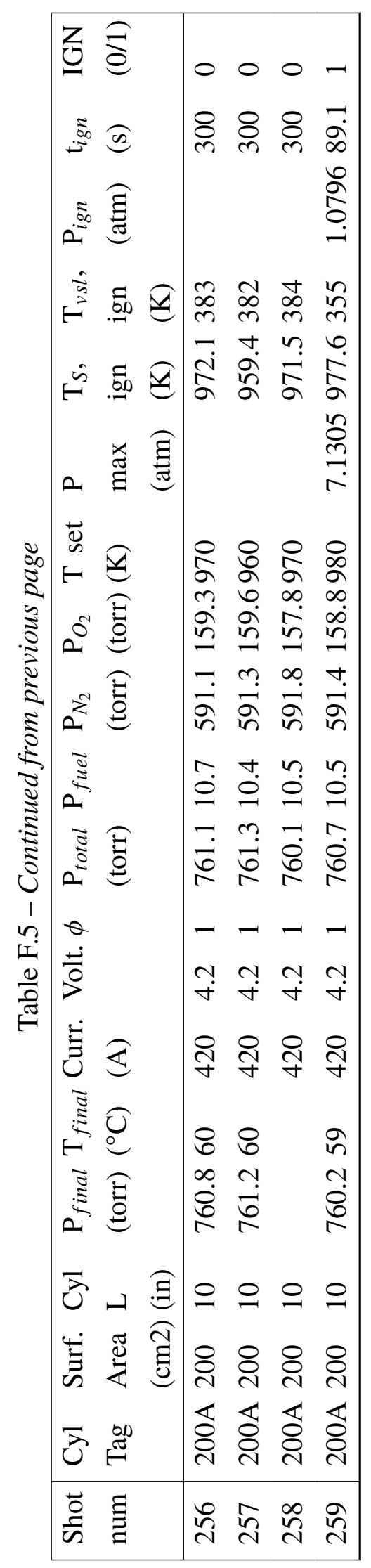


Appendix $G$

\section{ENGINEERING DRAWINGS}

\section{G.1 Support Structure}




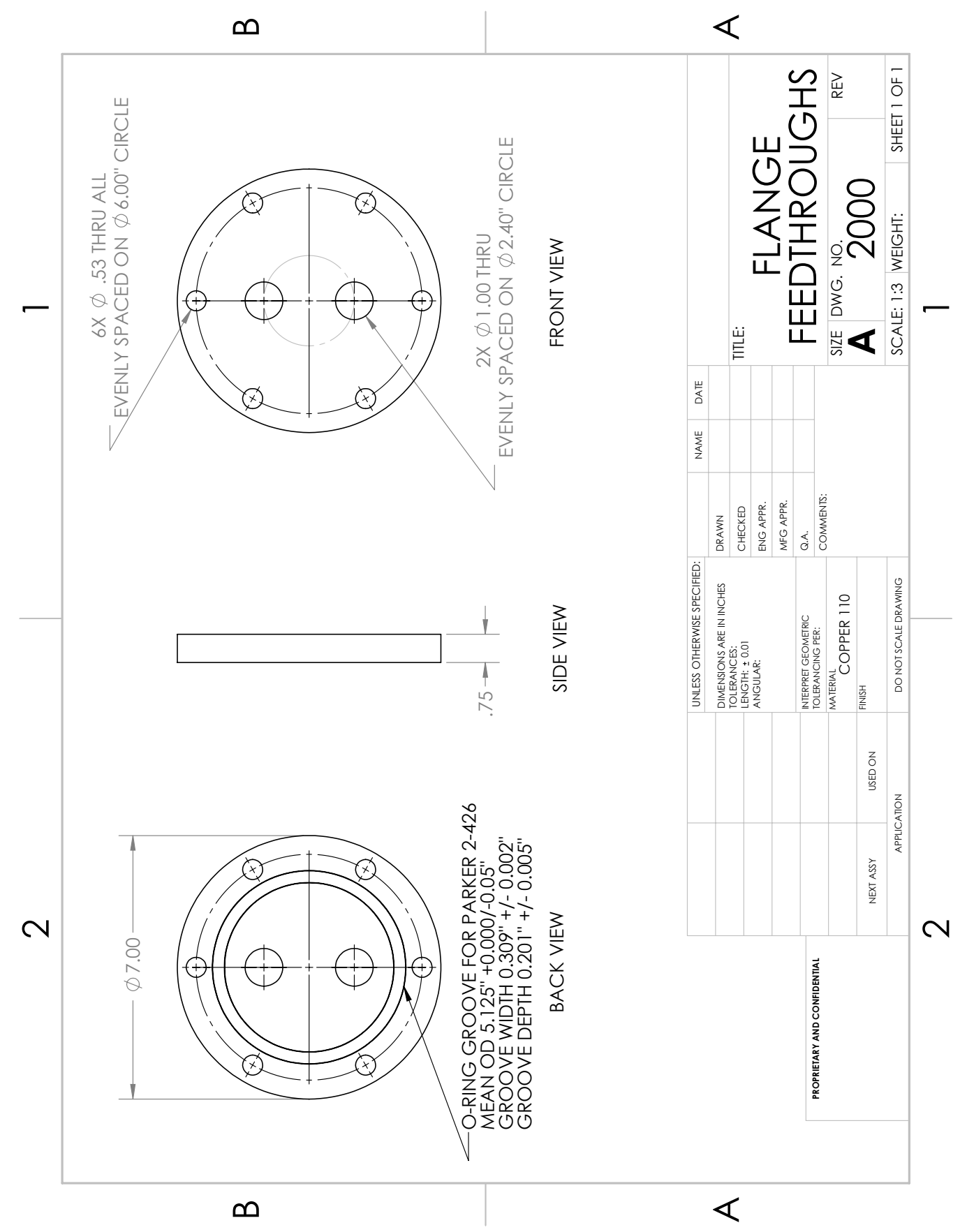




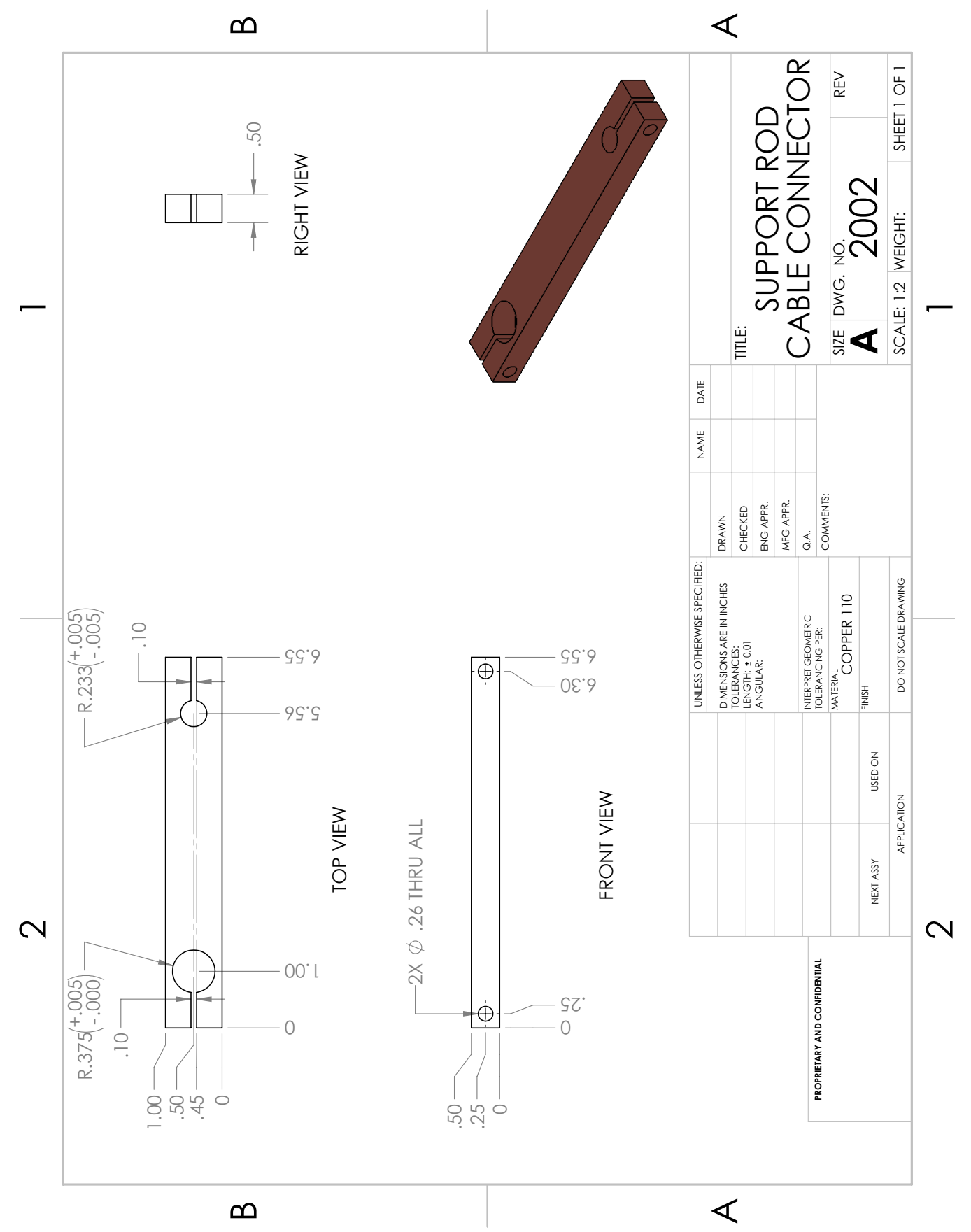




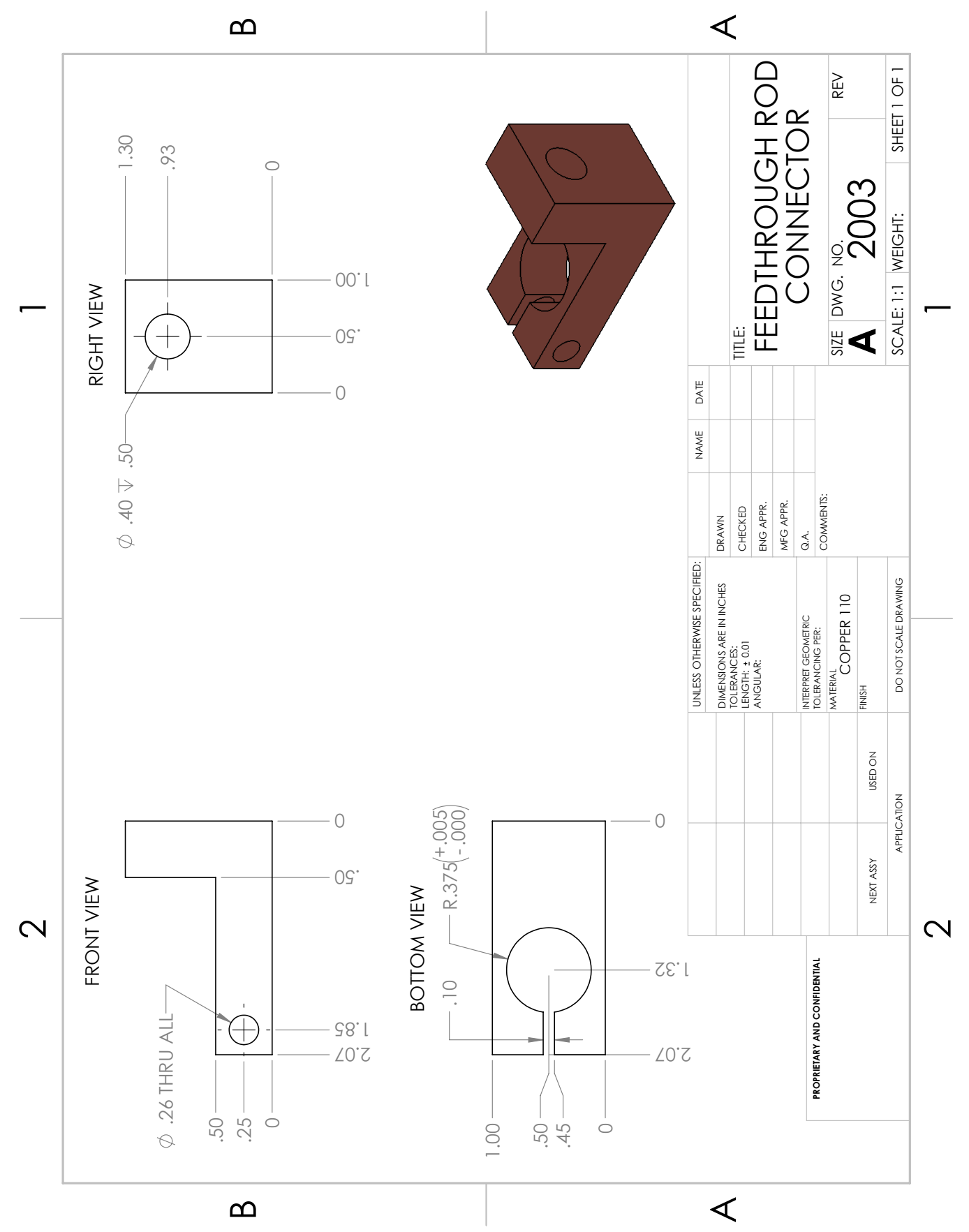




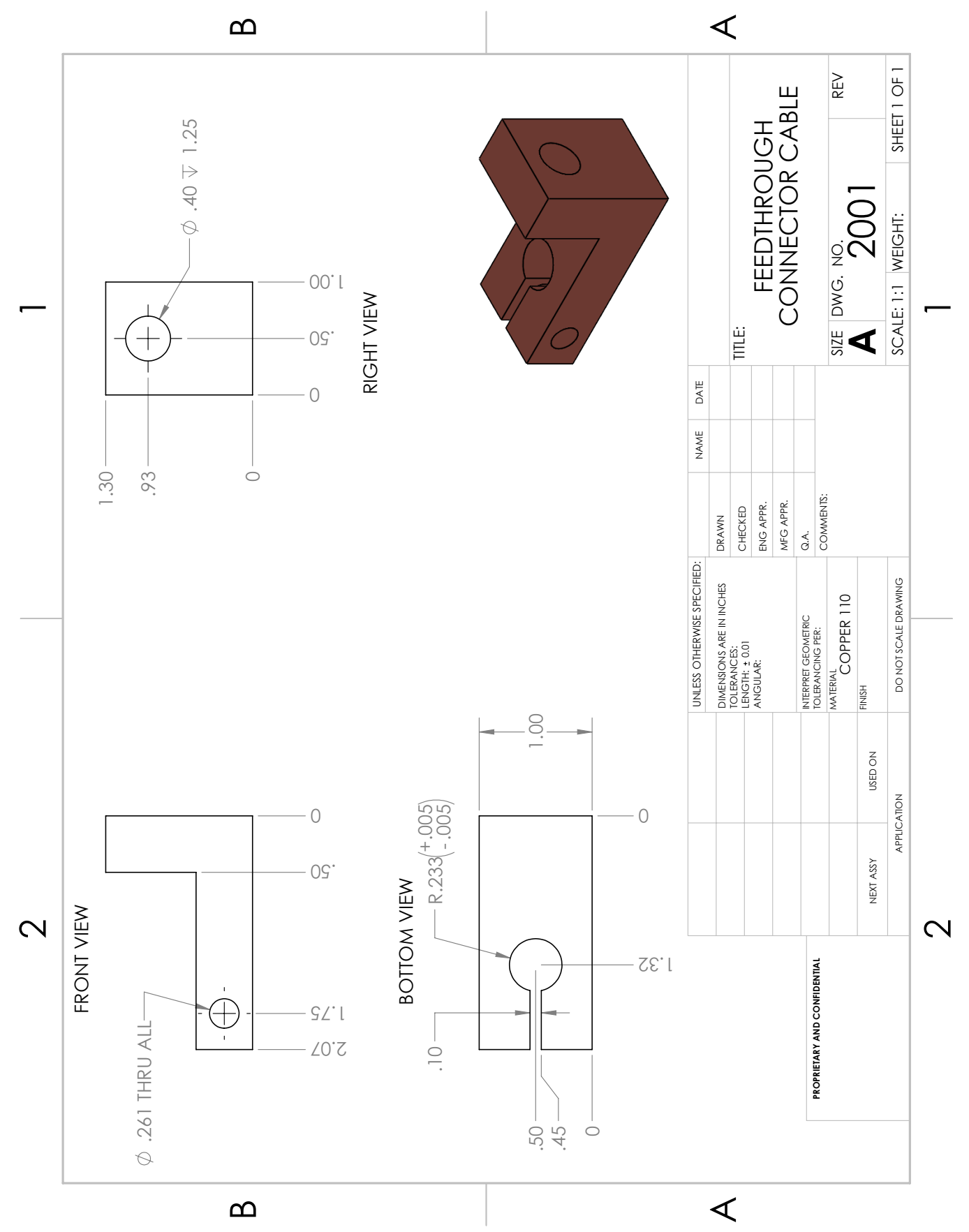




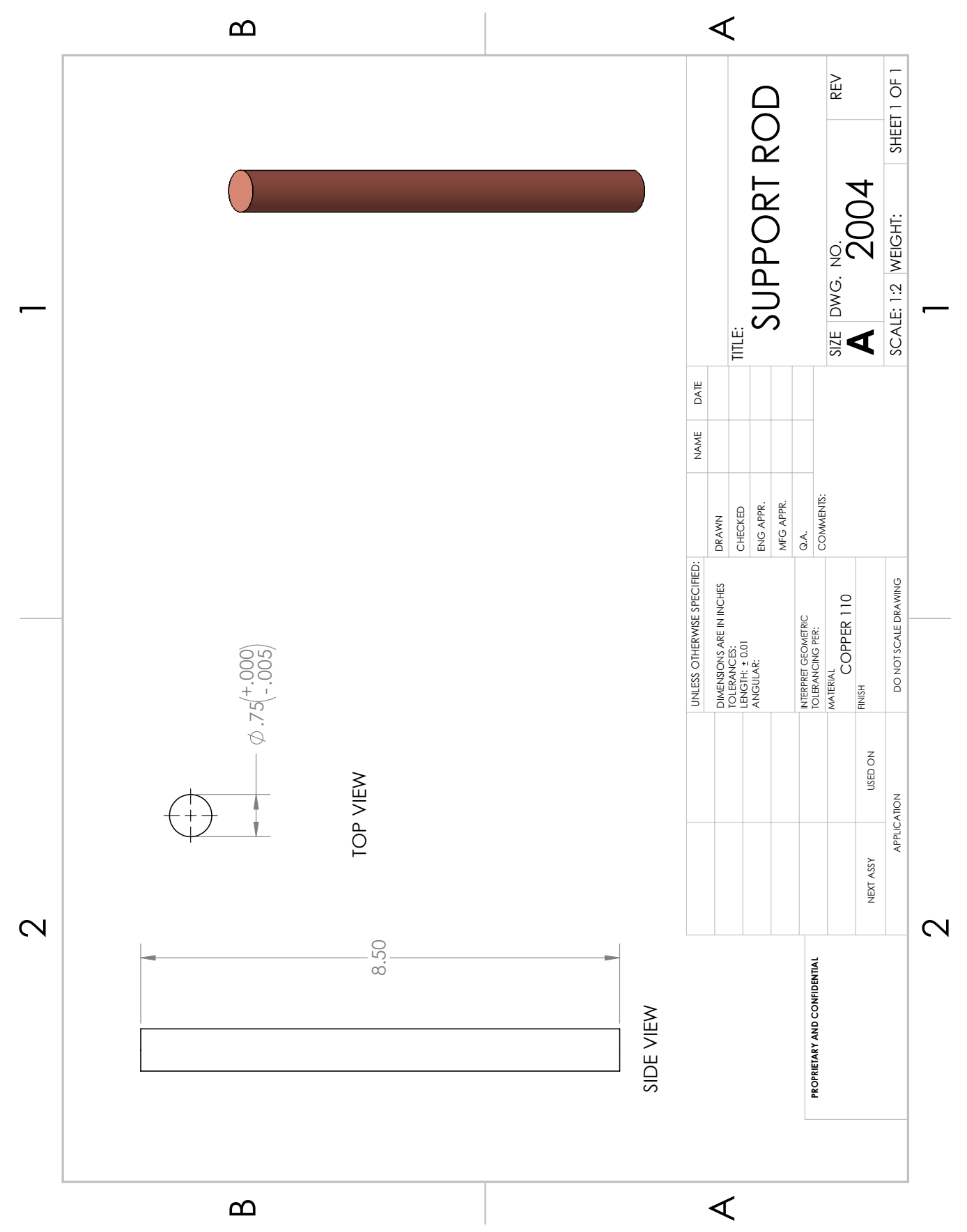




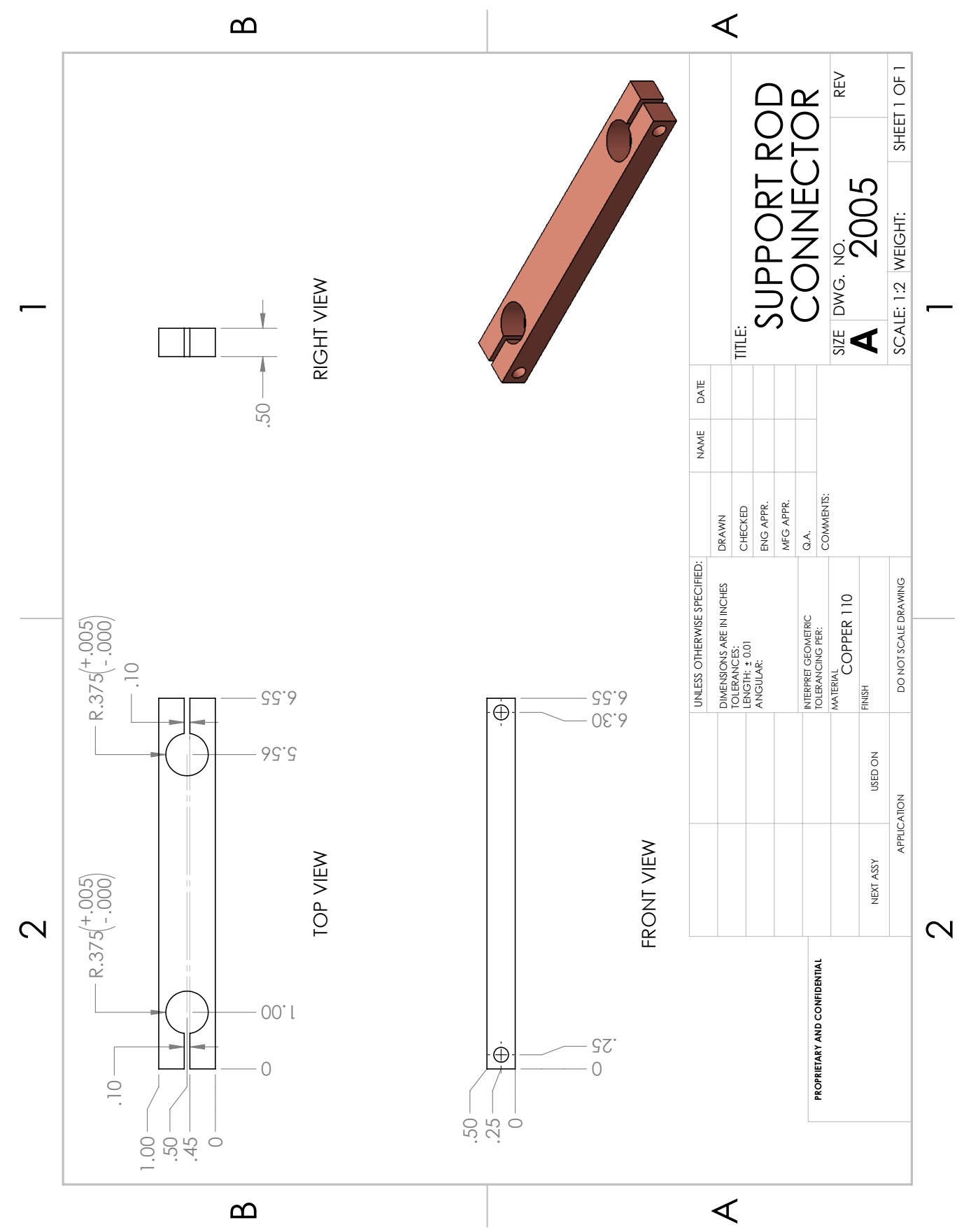


G.2 Test Cylinders 


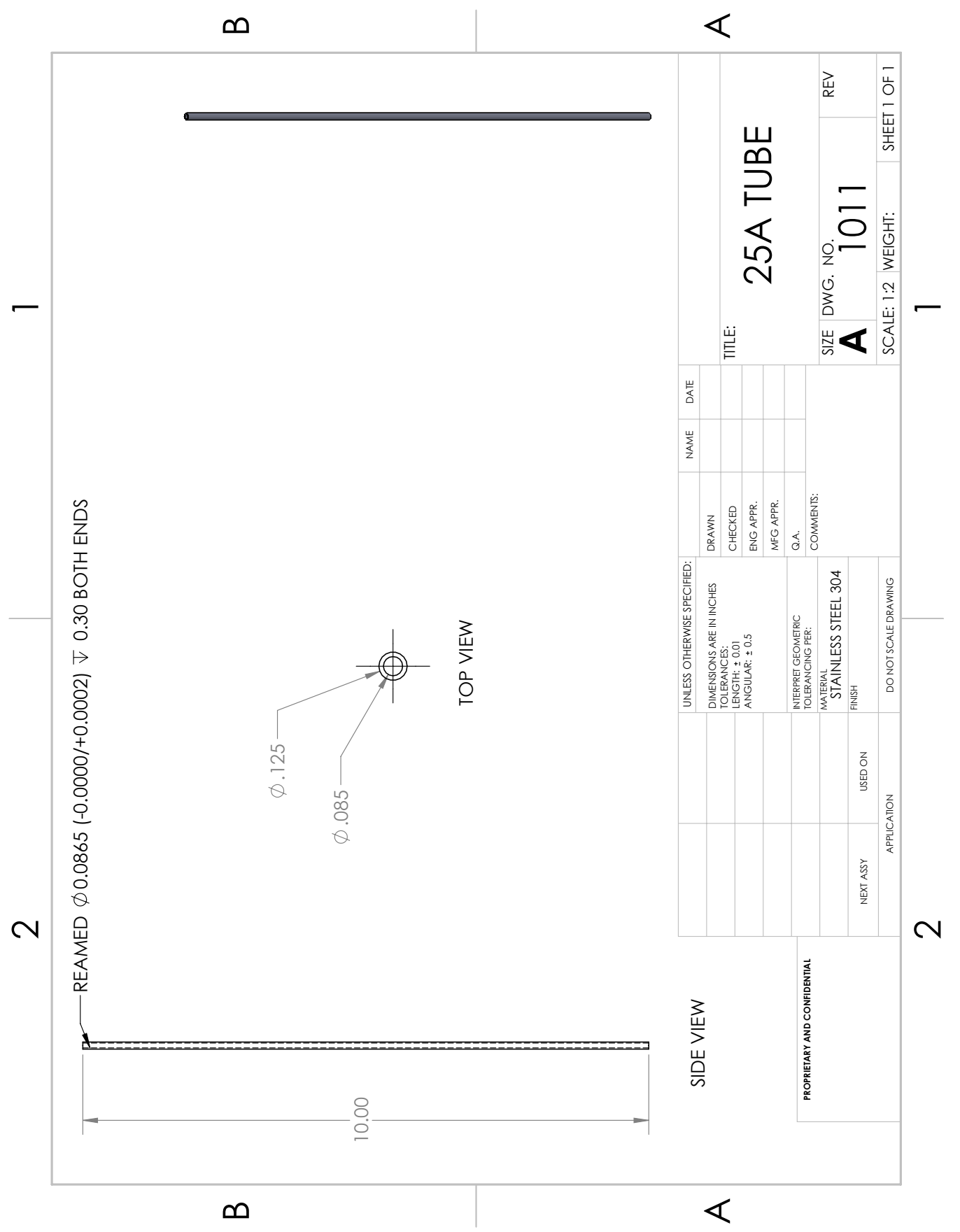




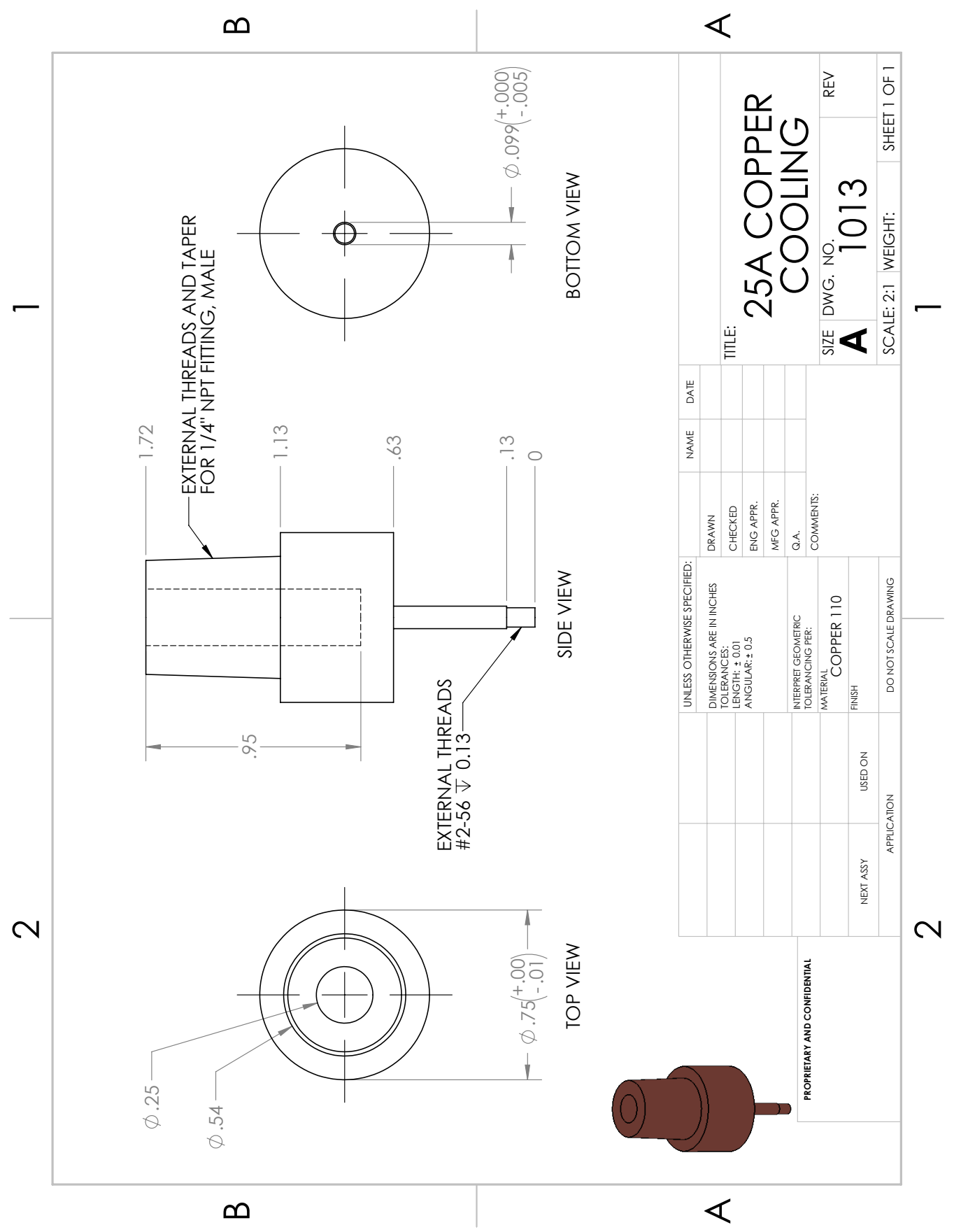




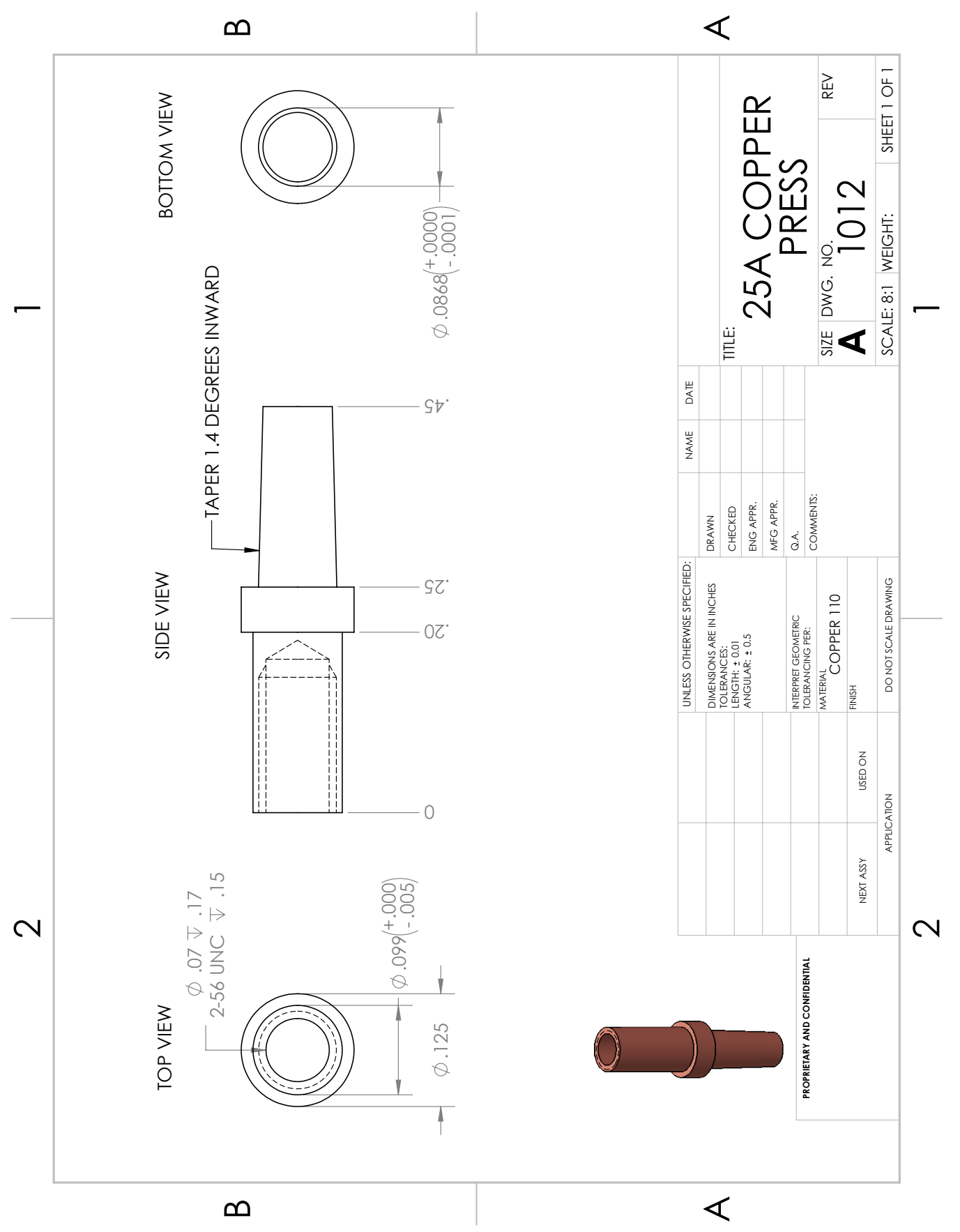




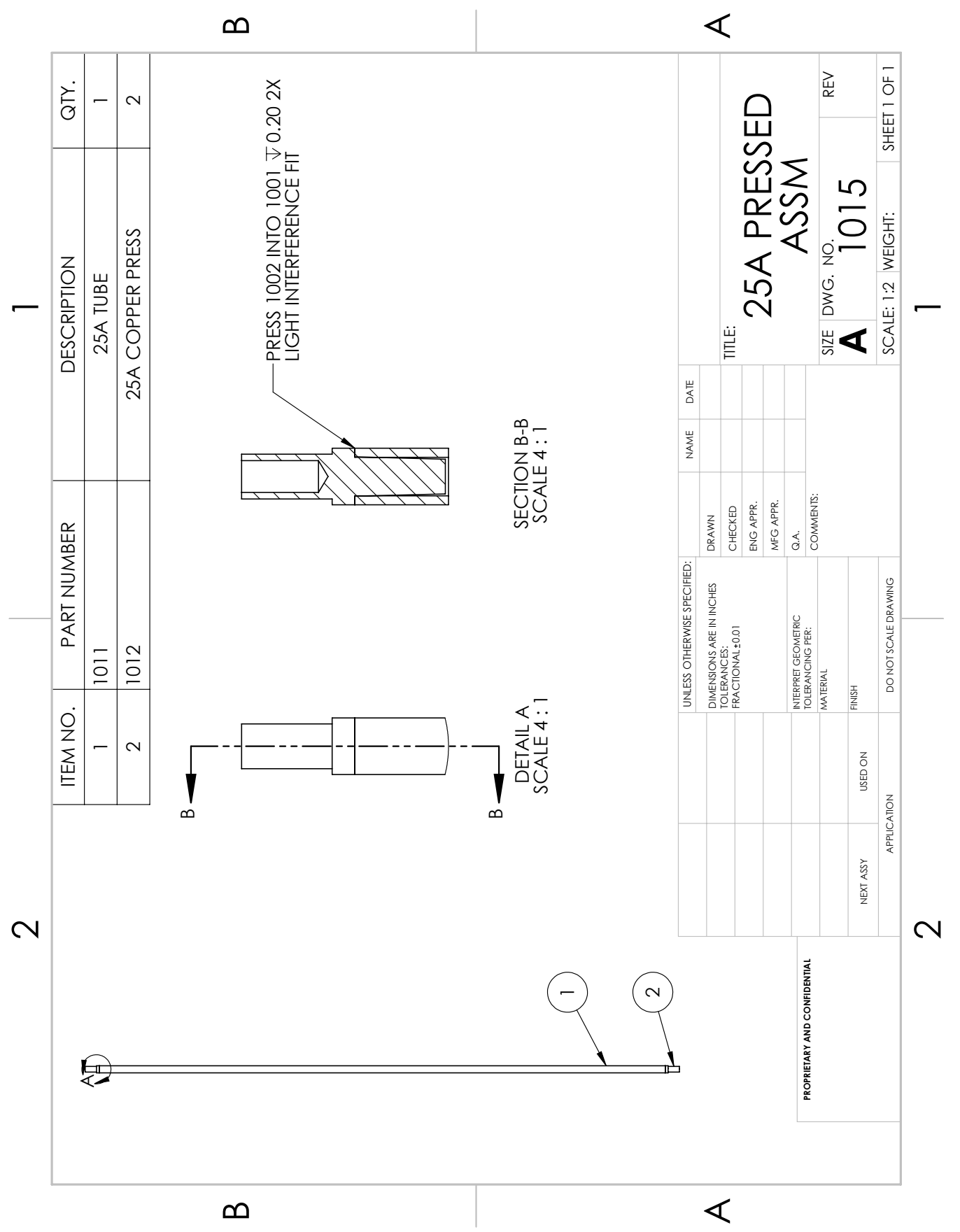




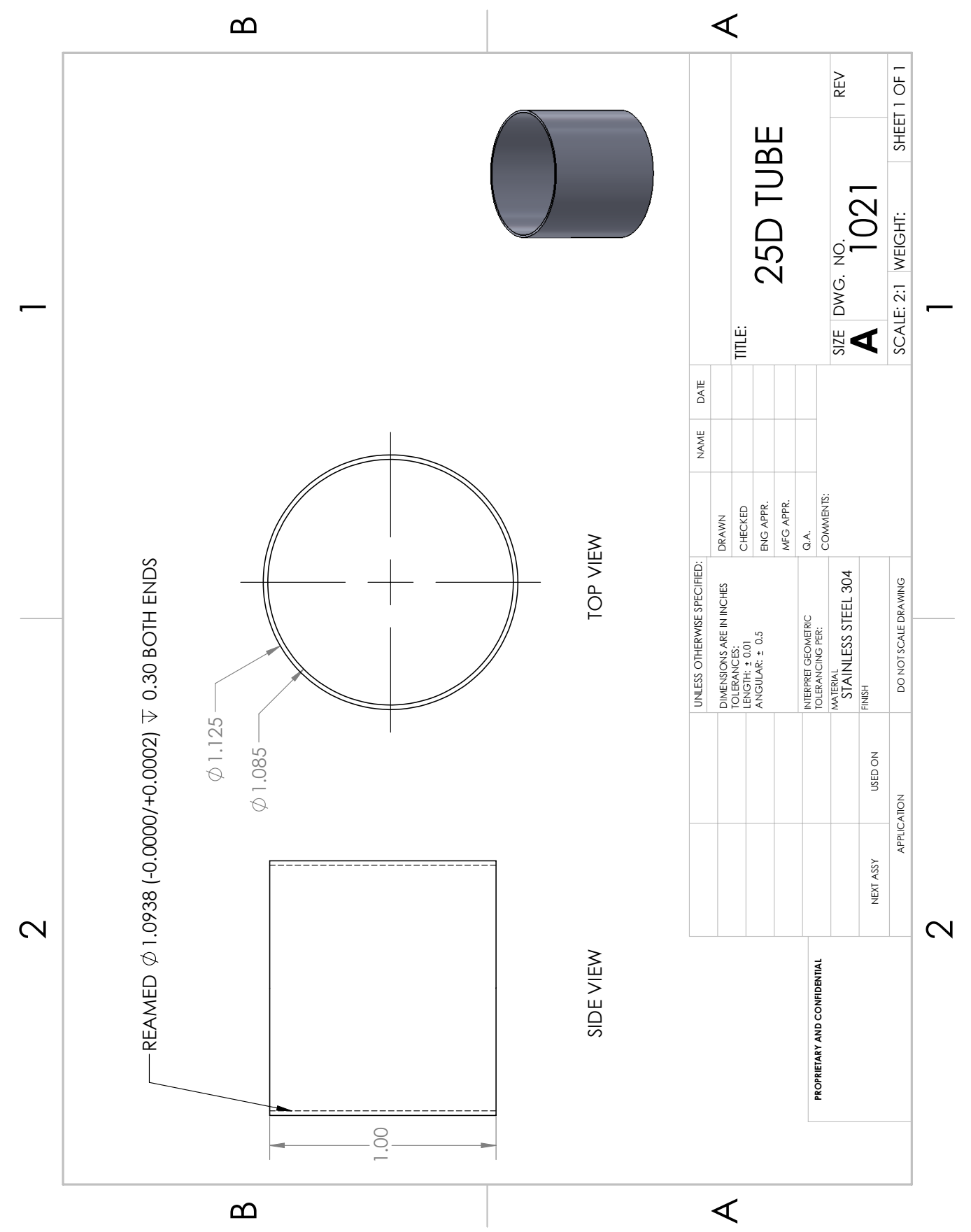




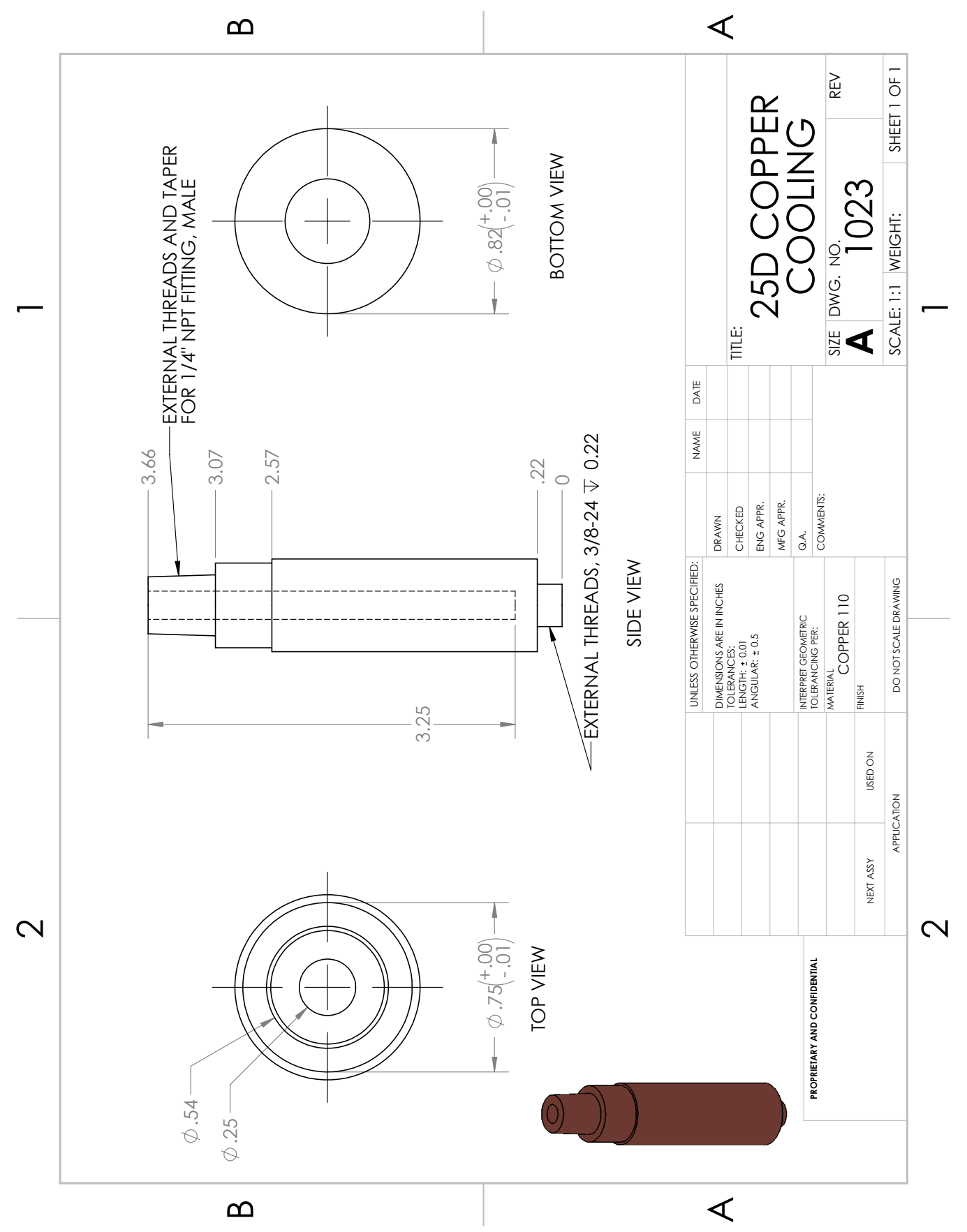




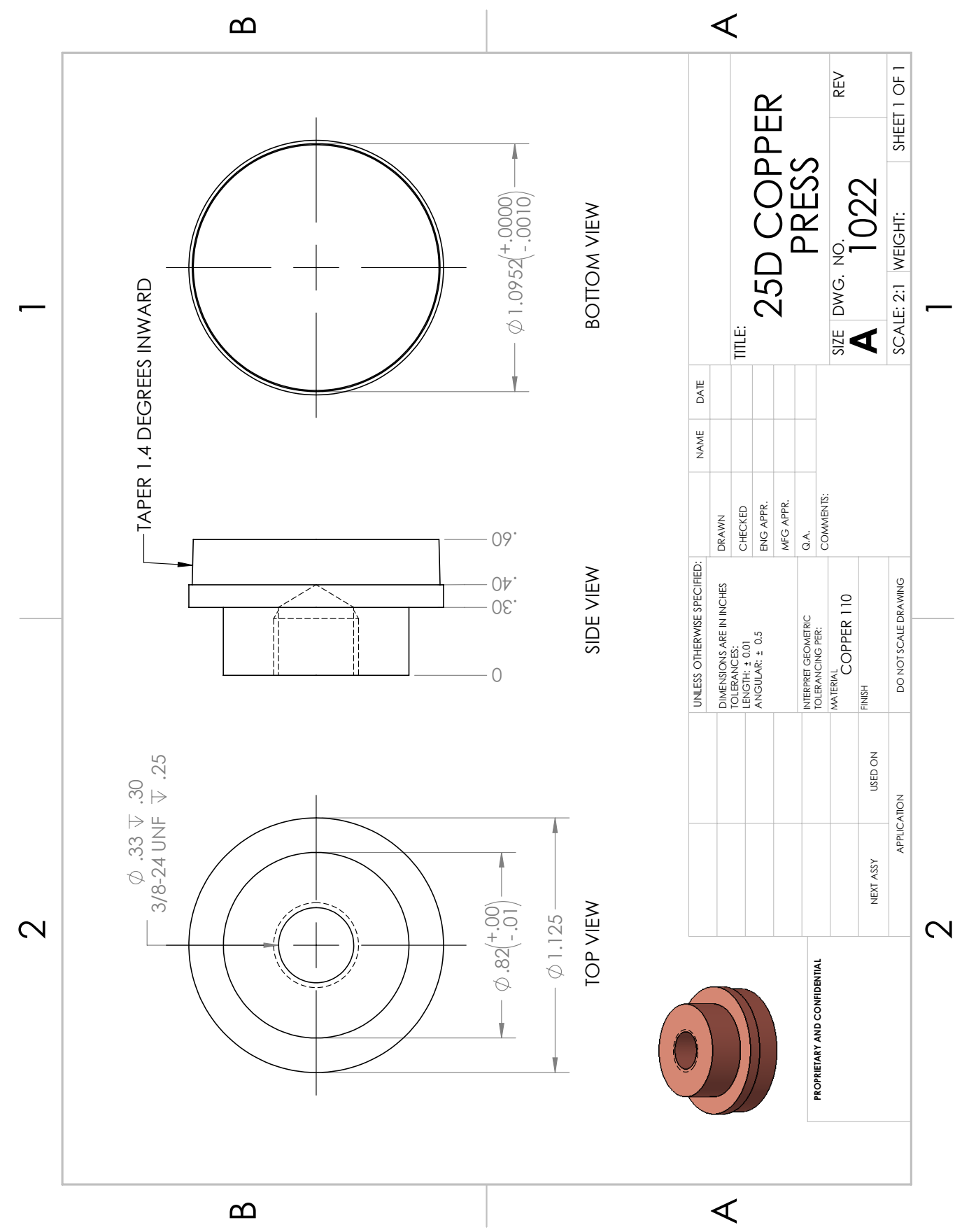




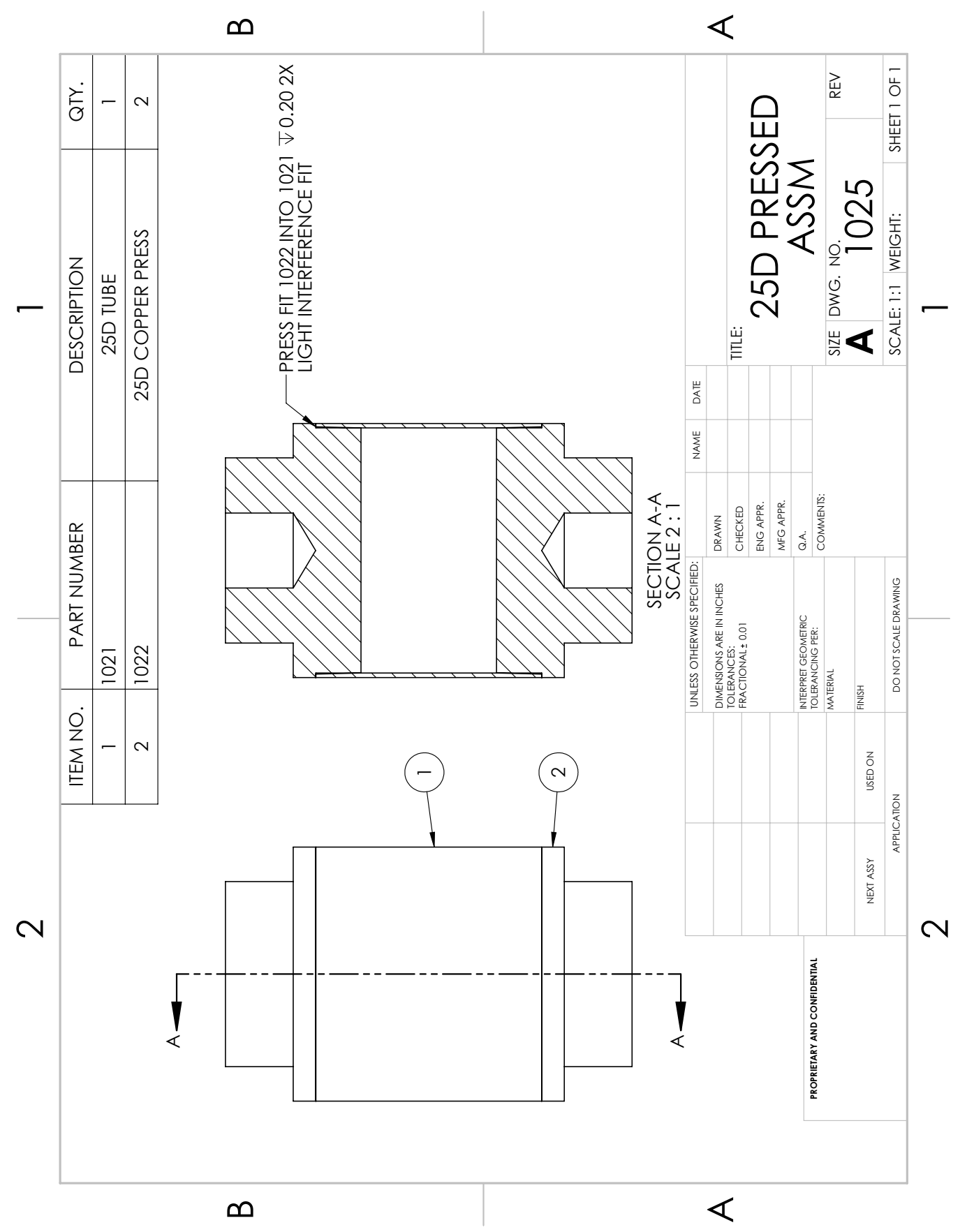




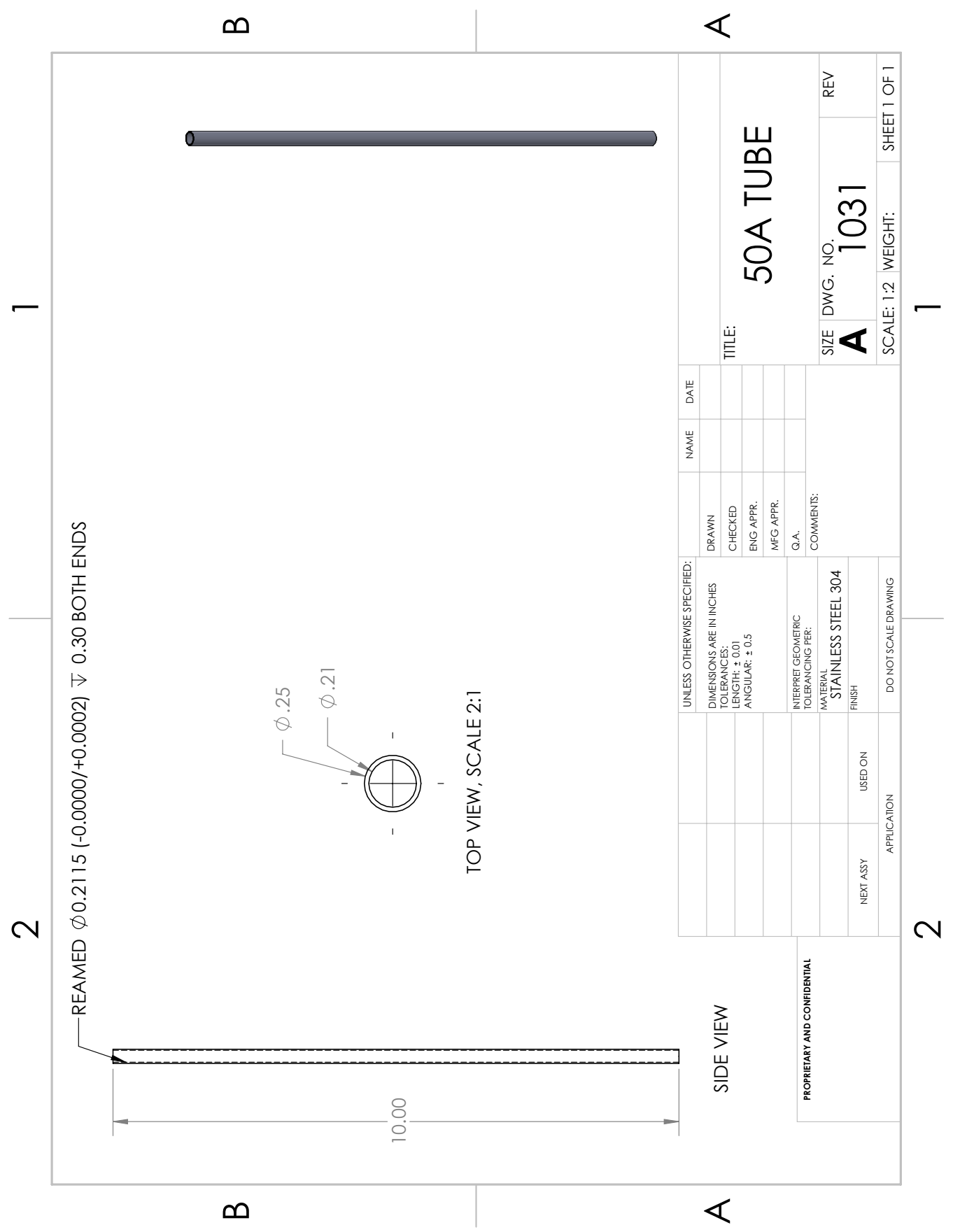




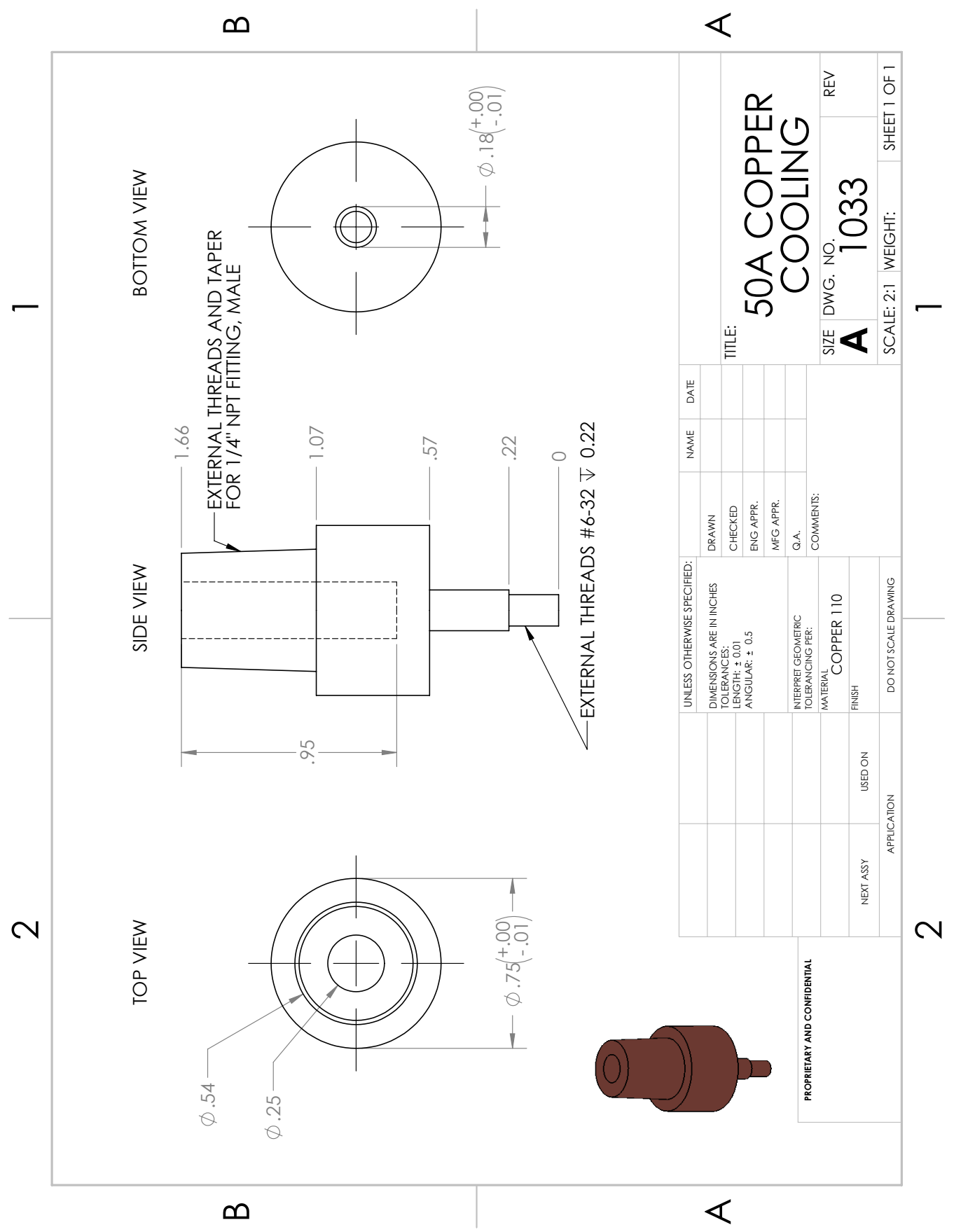




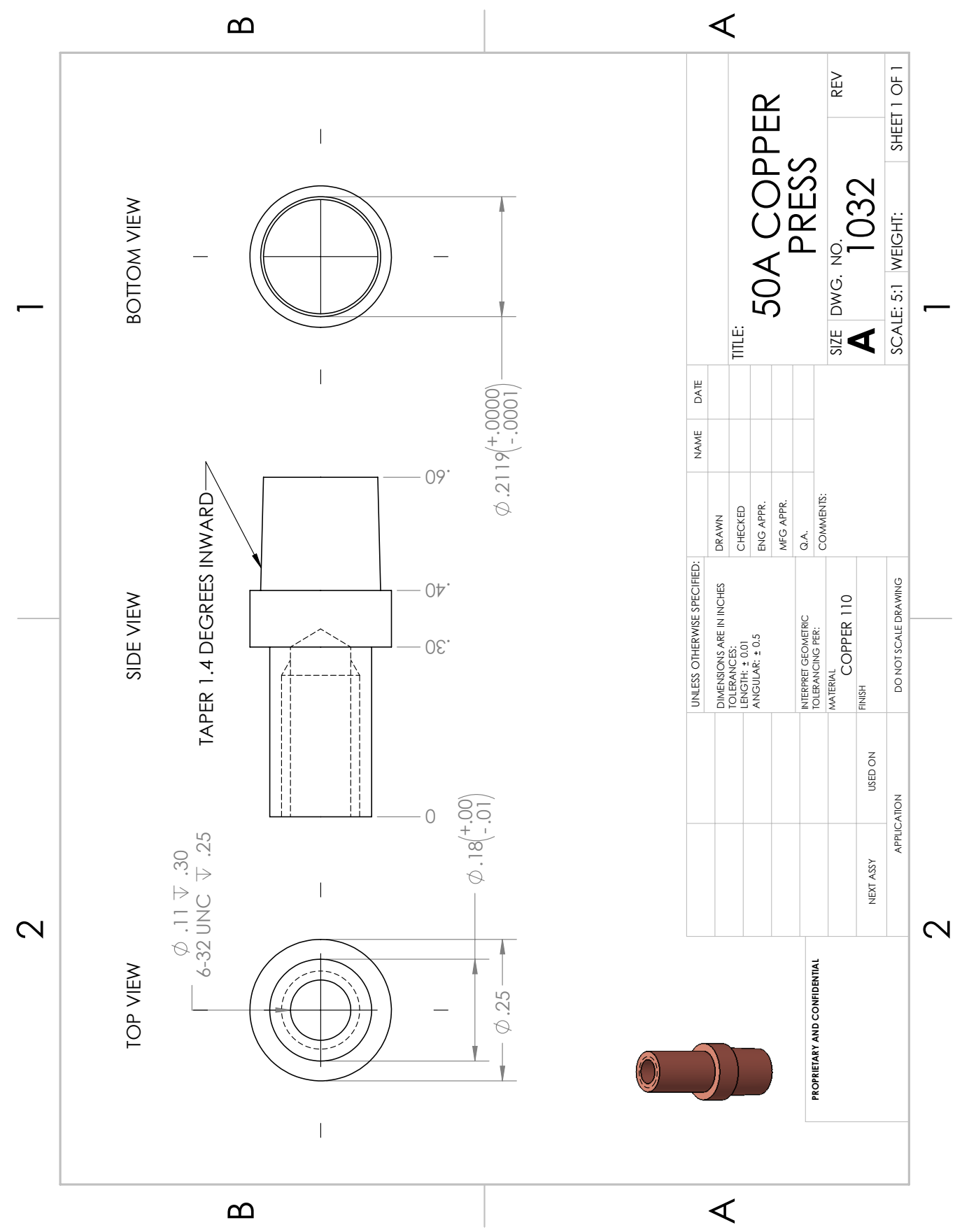




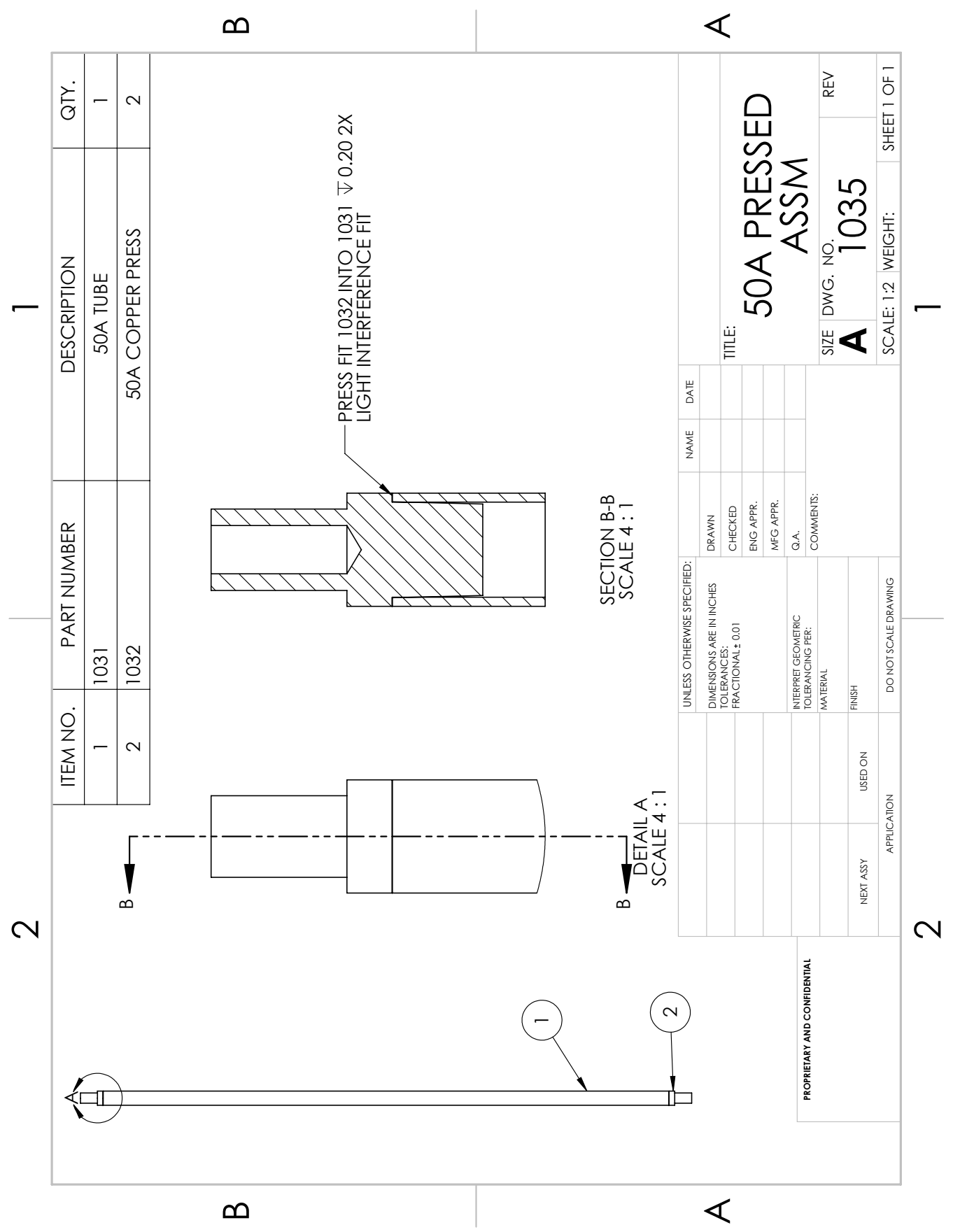




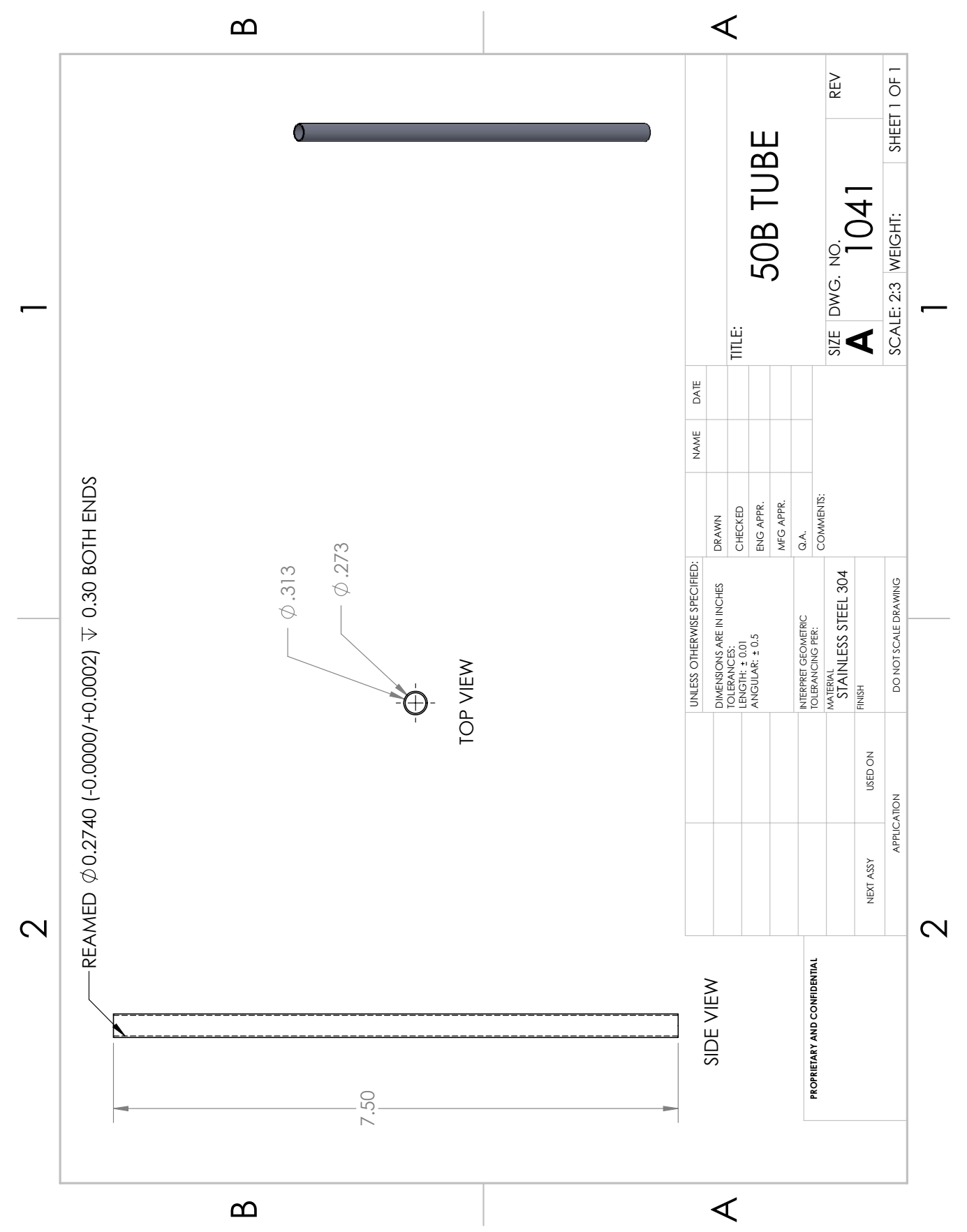




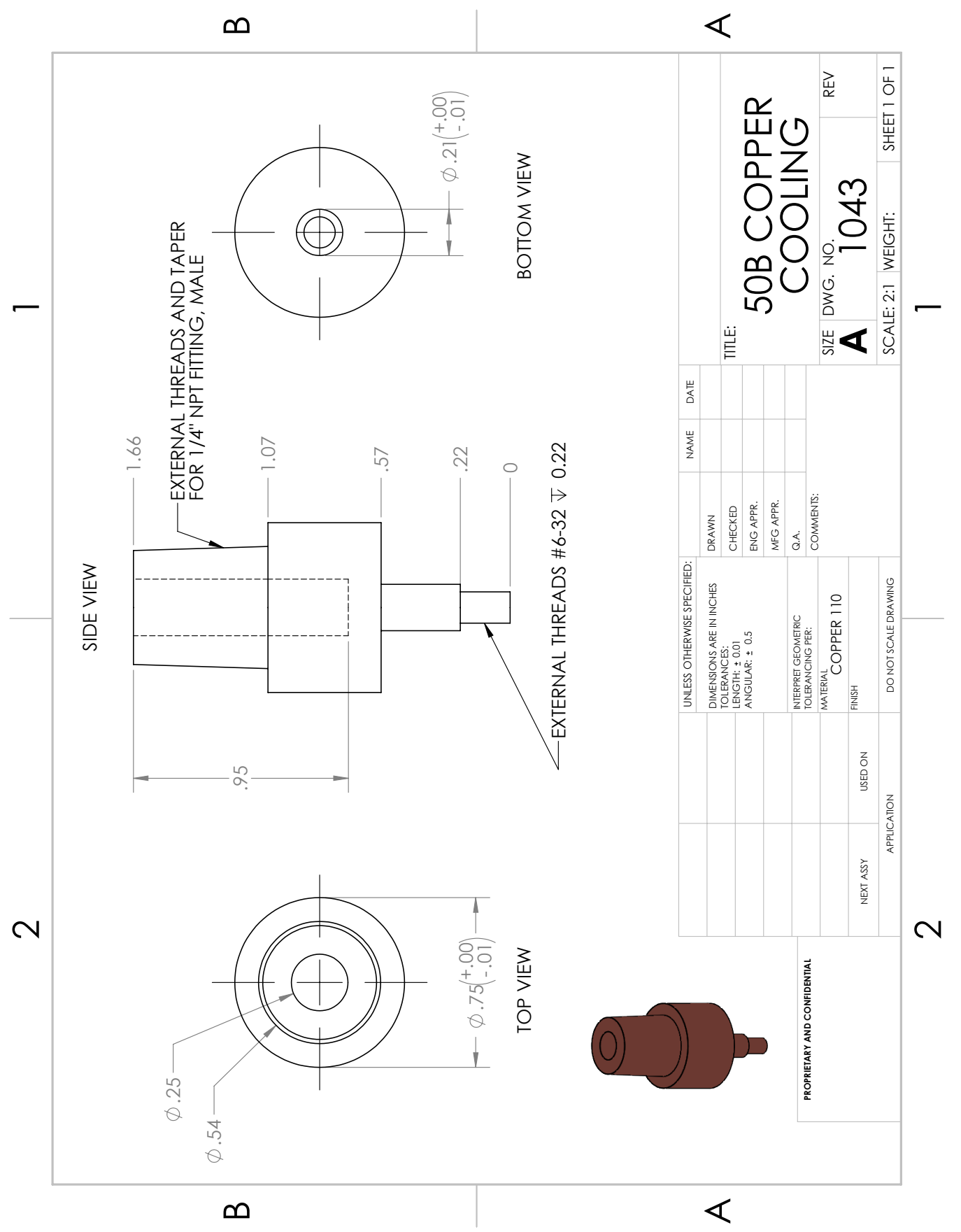




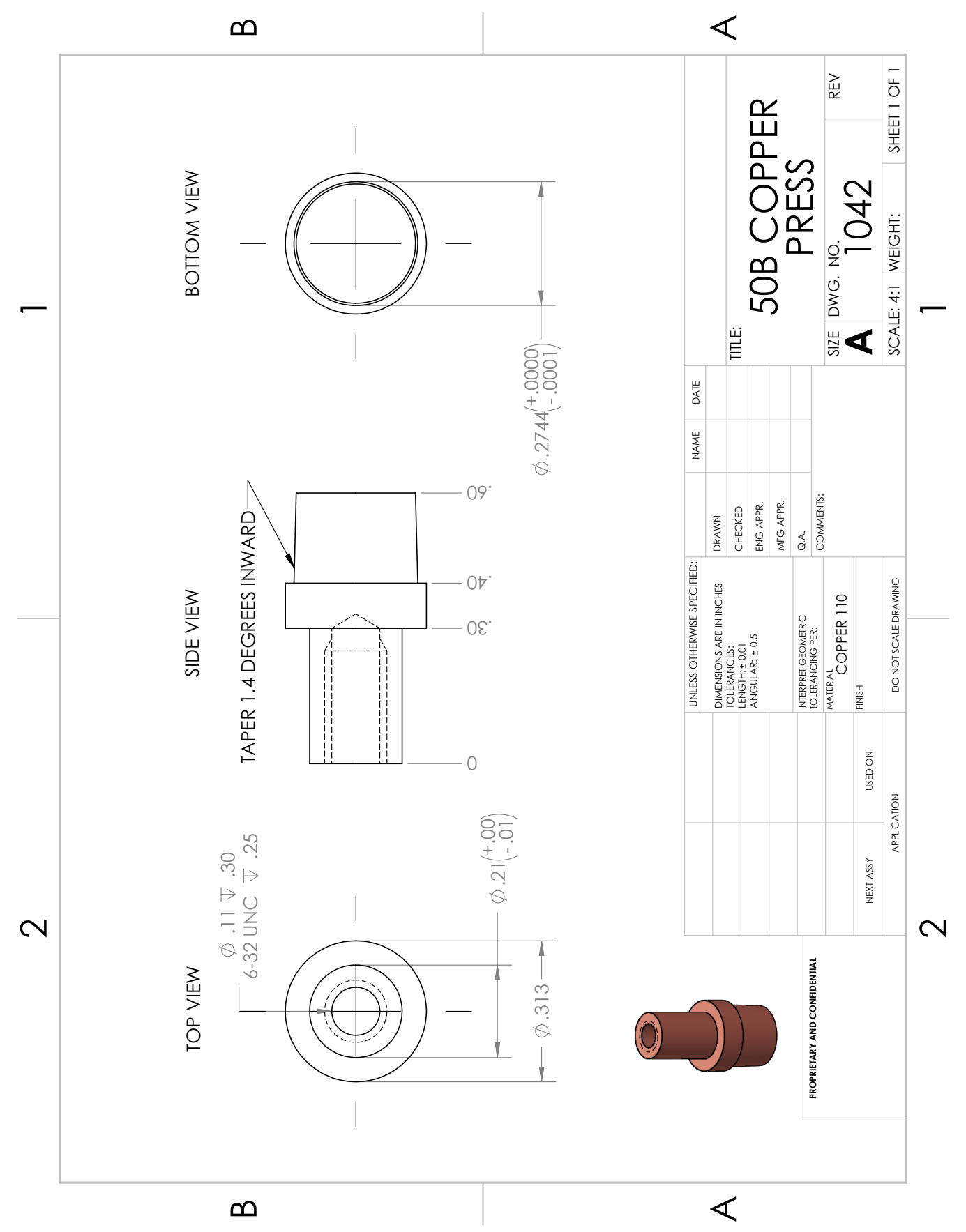




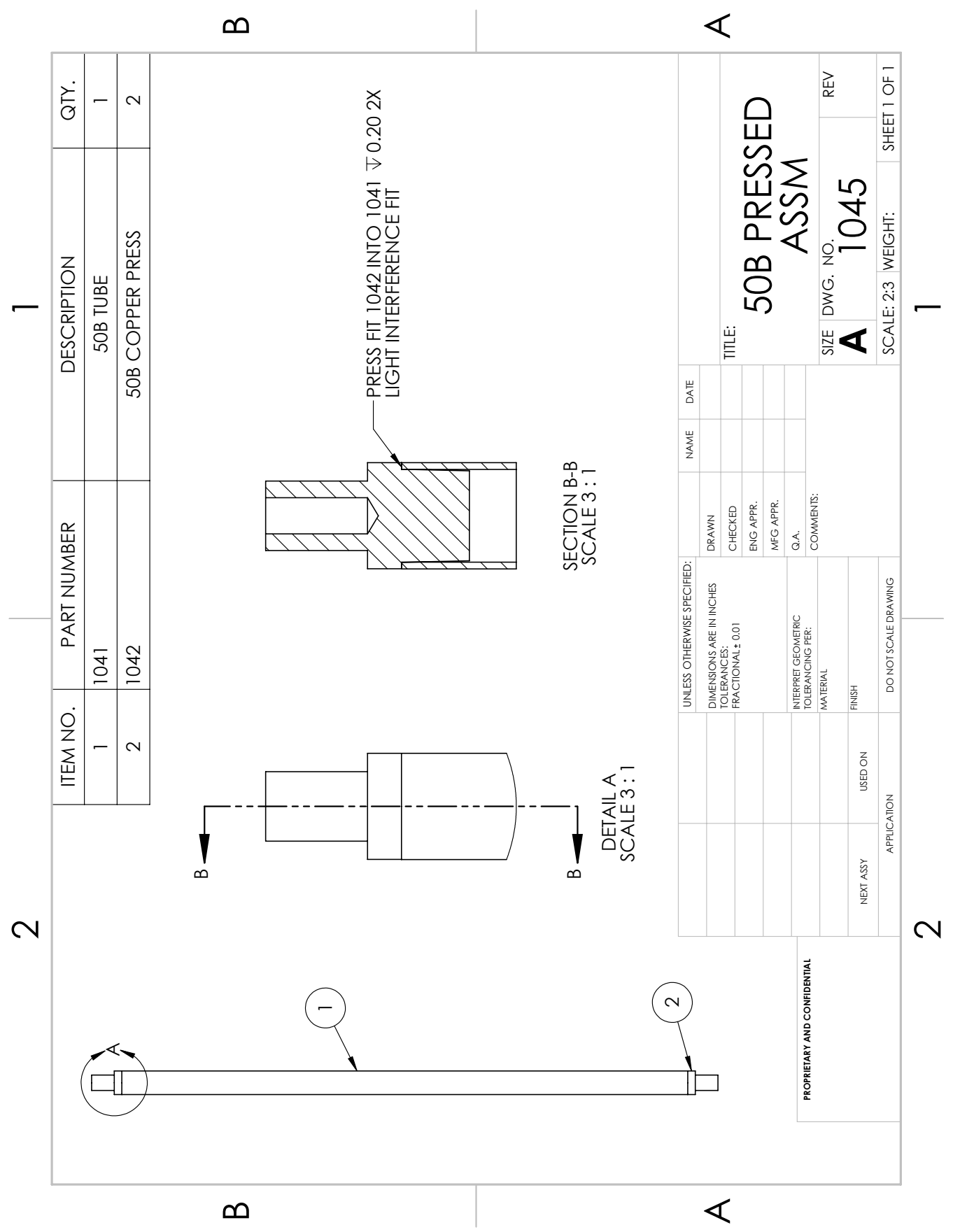




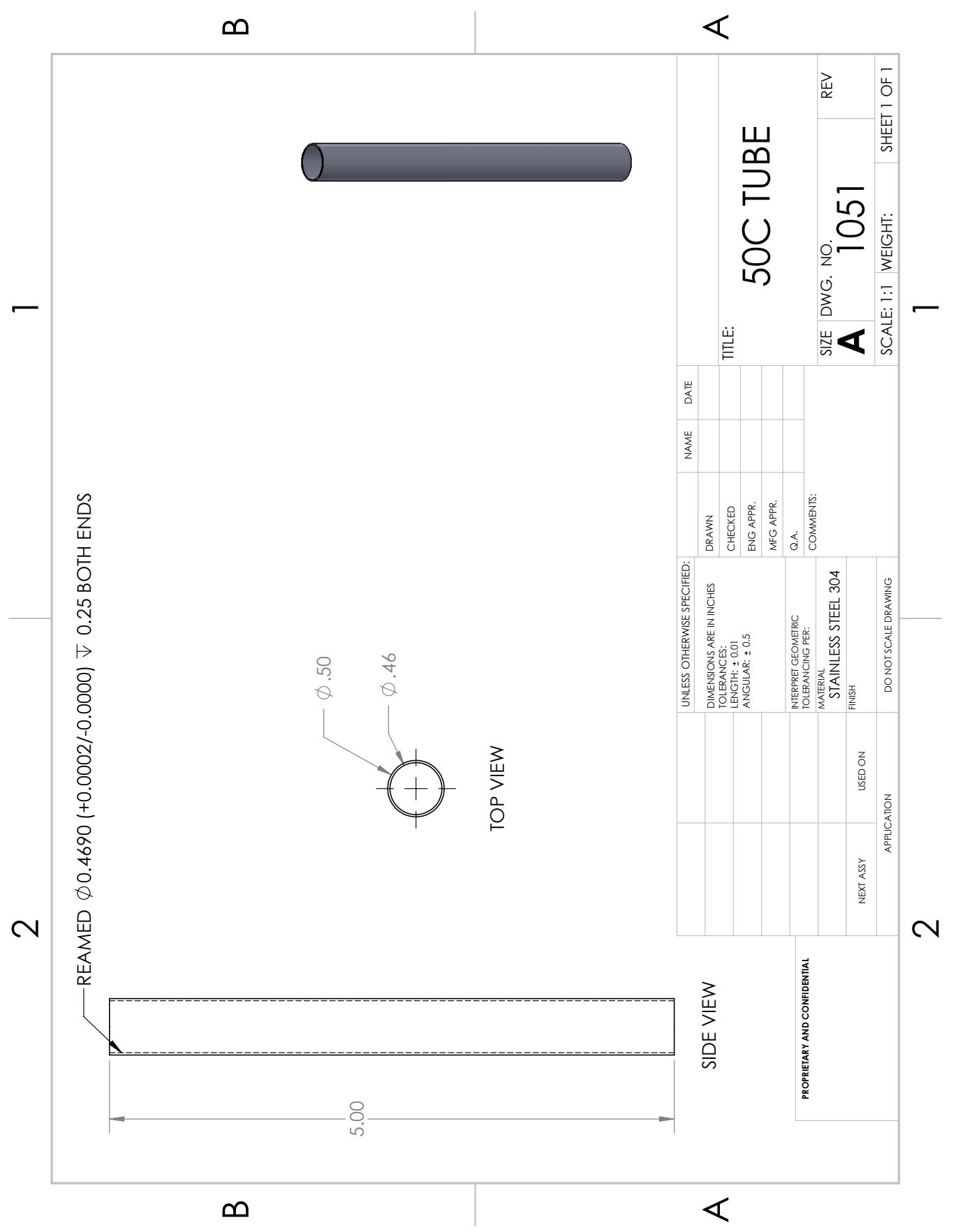




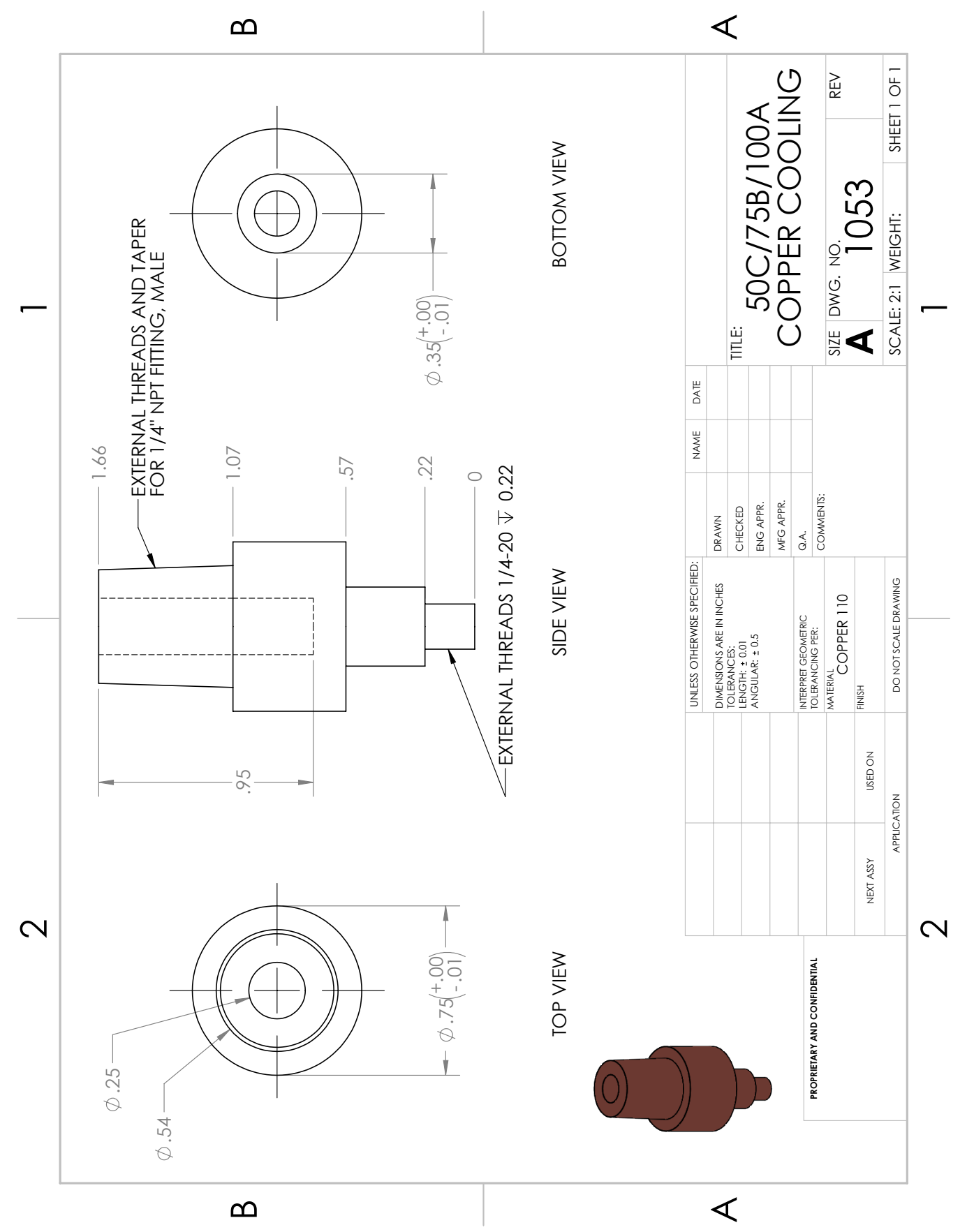




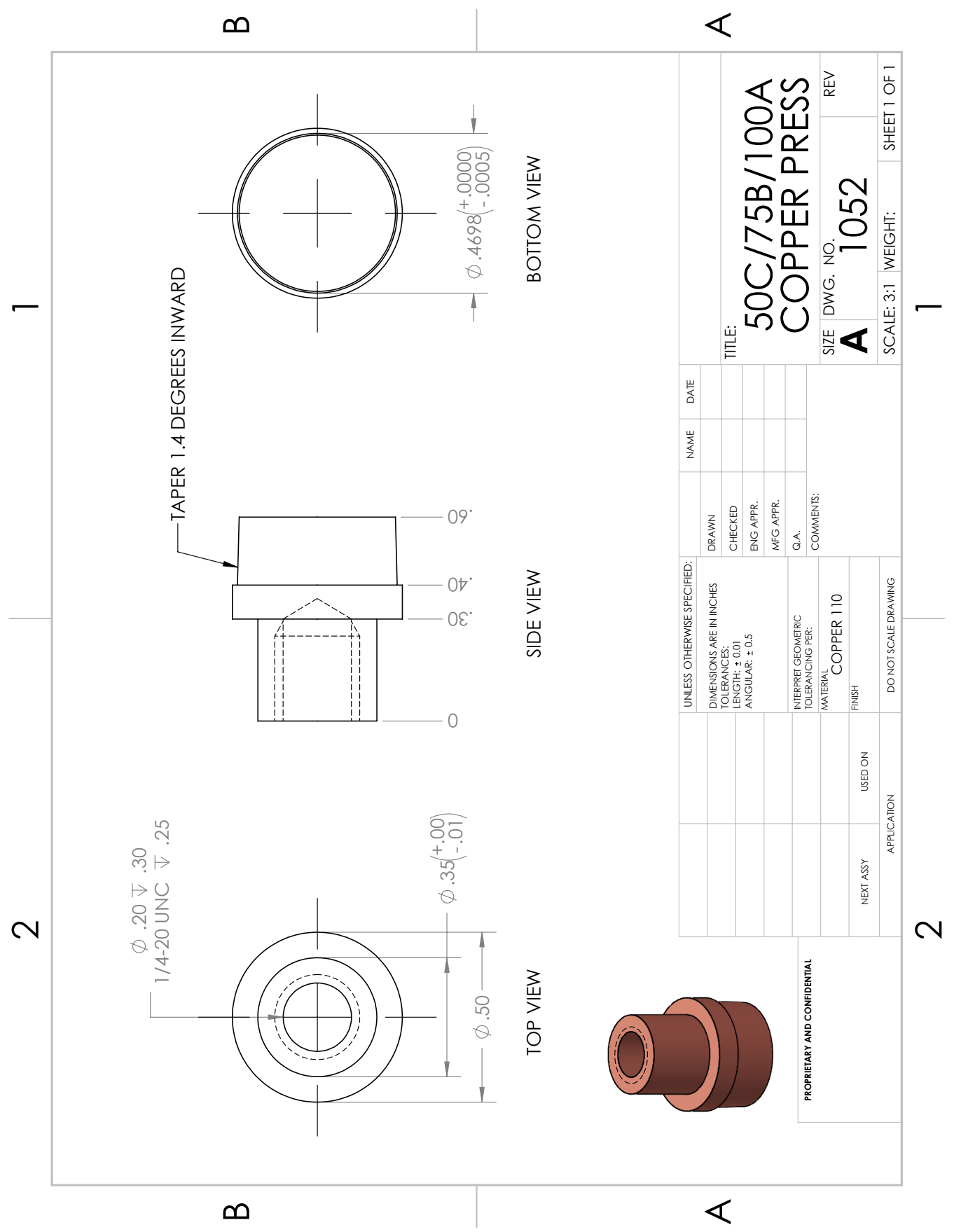




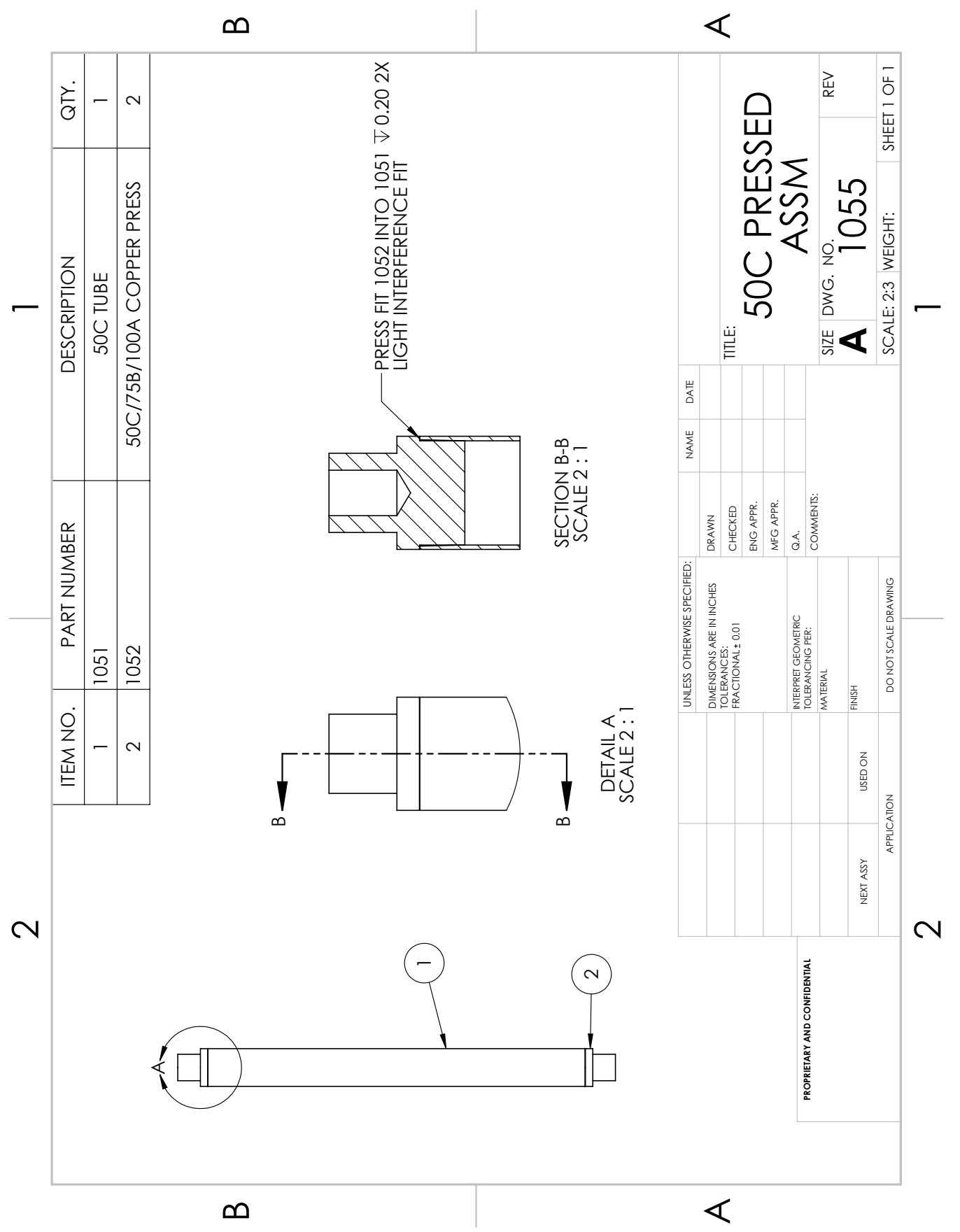




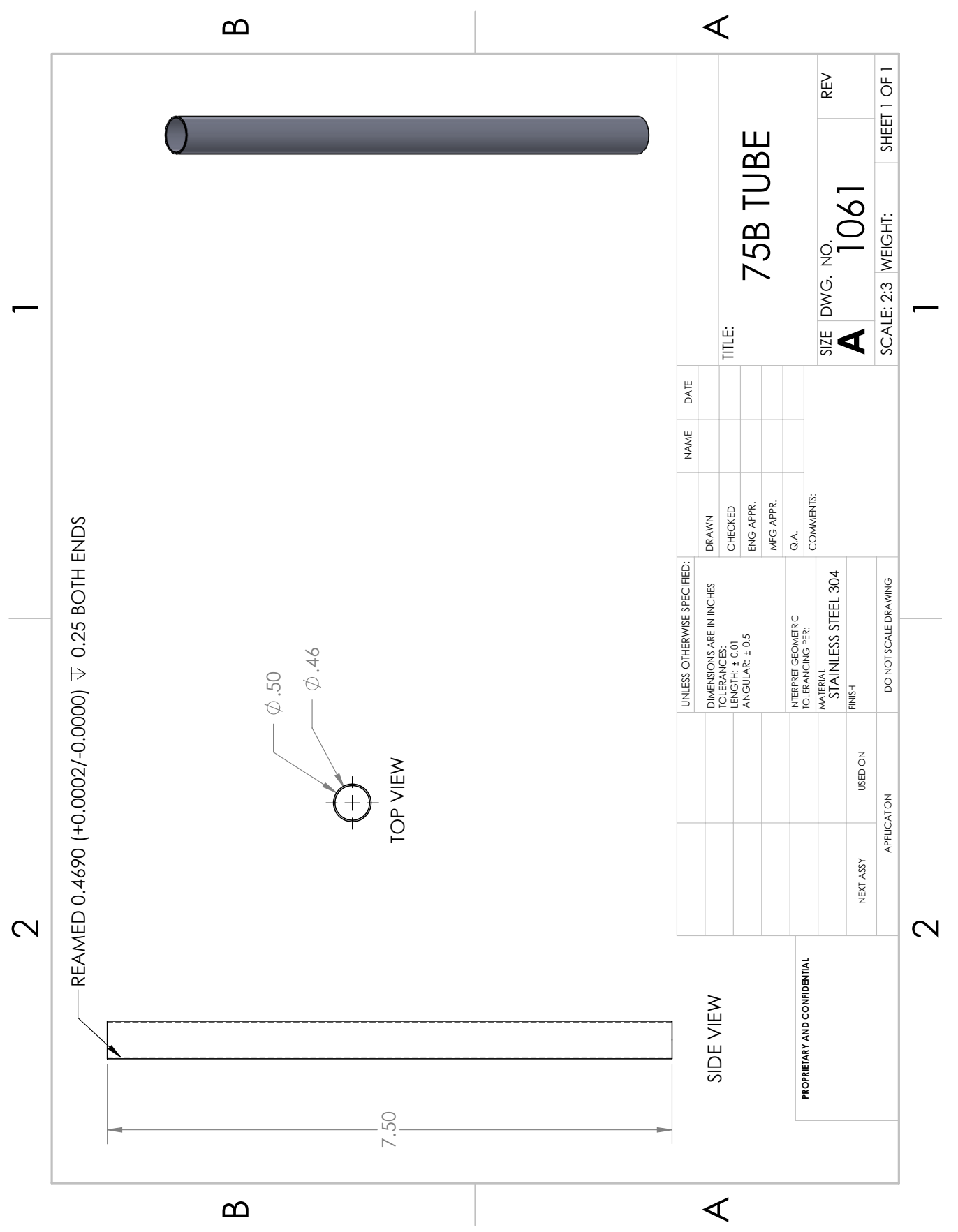




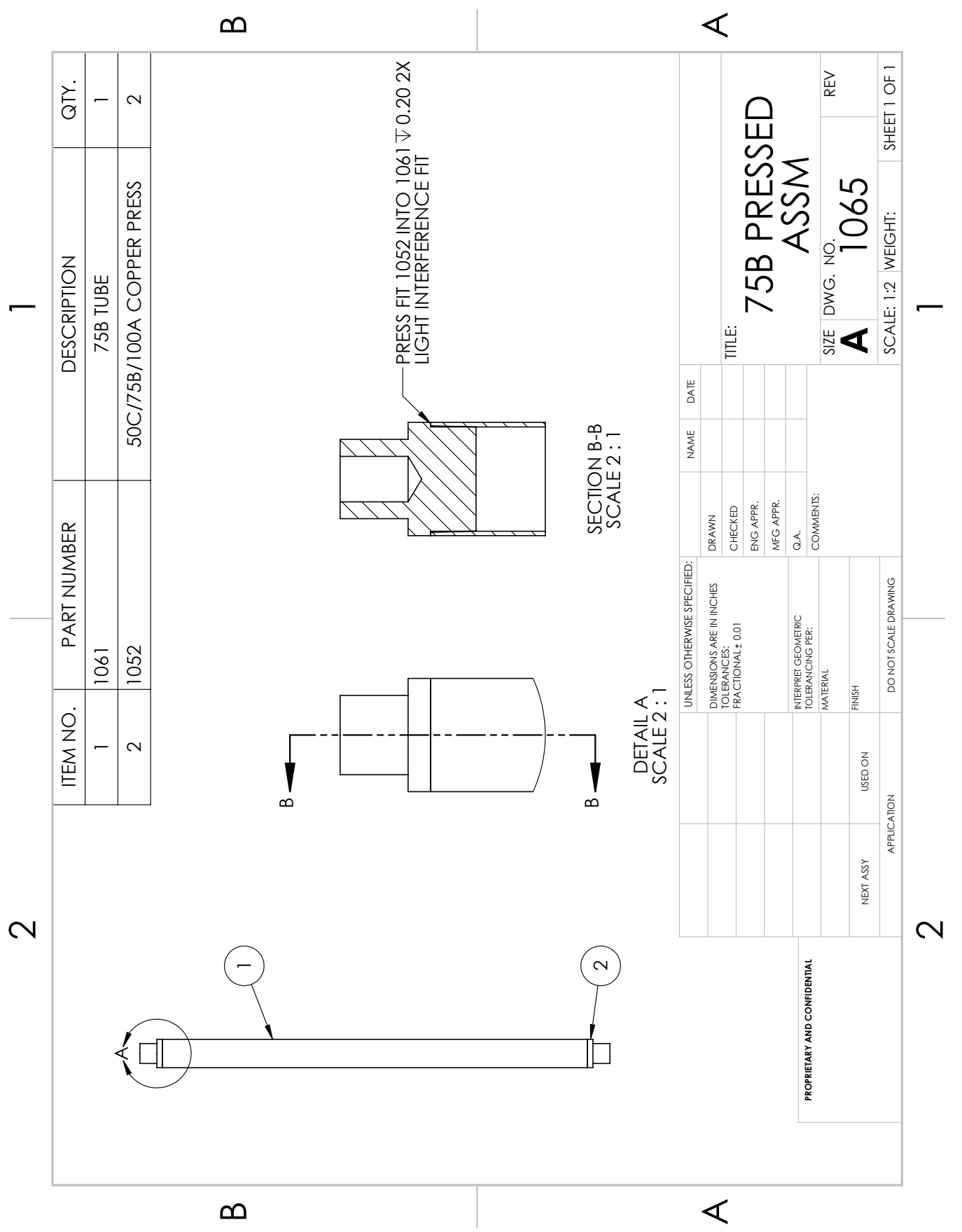




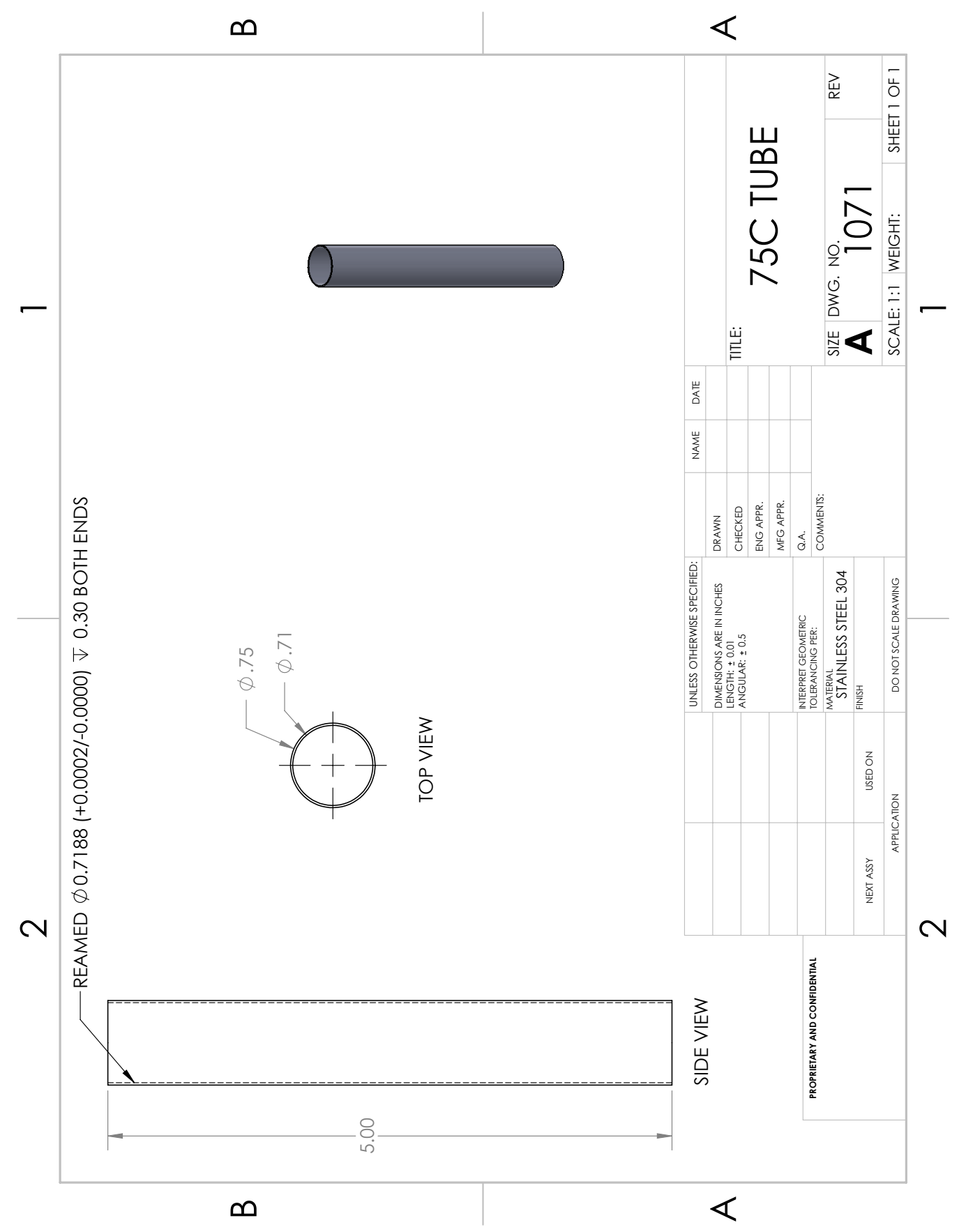




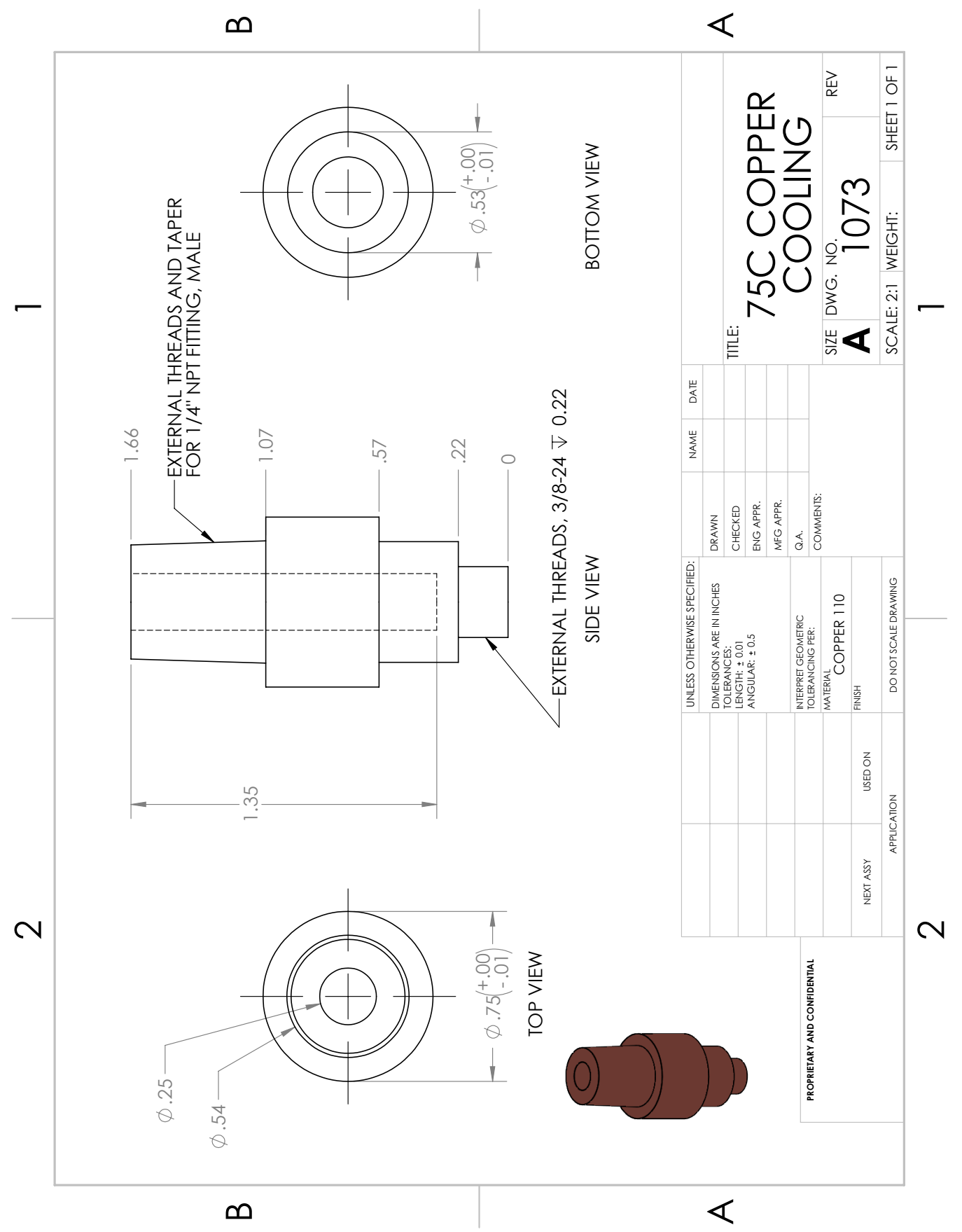




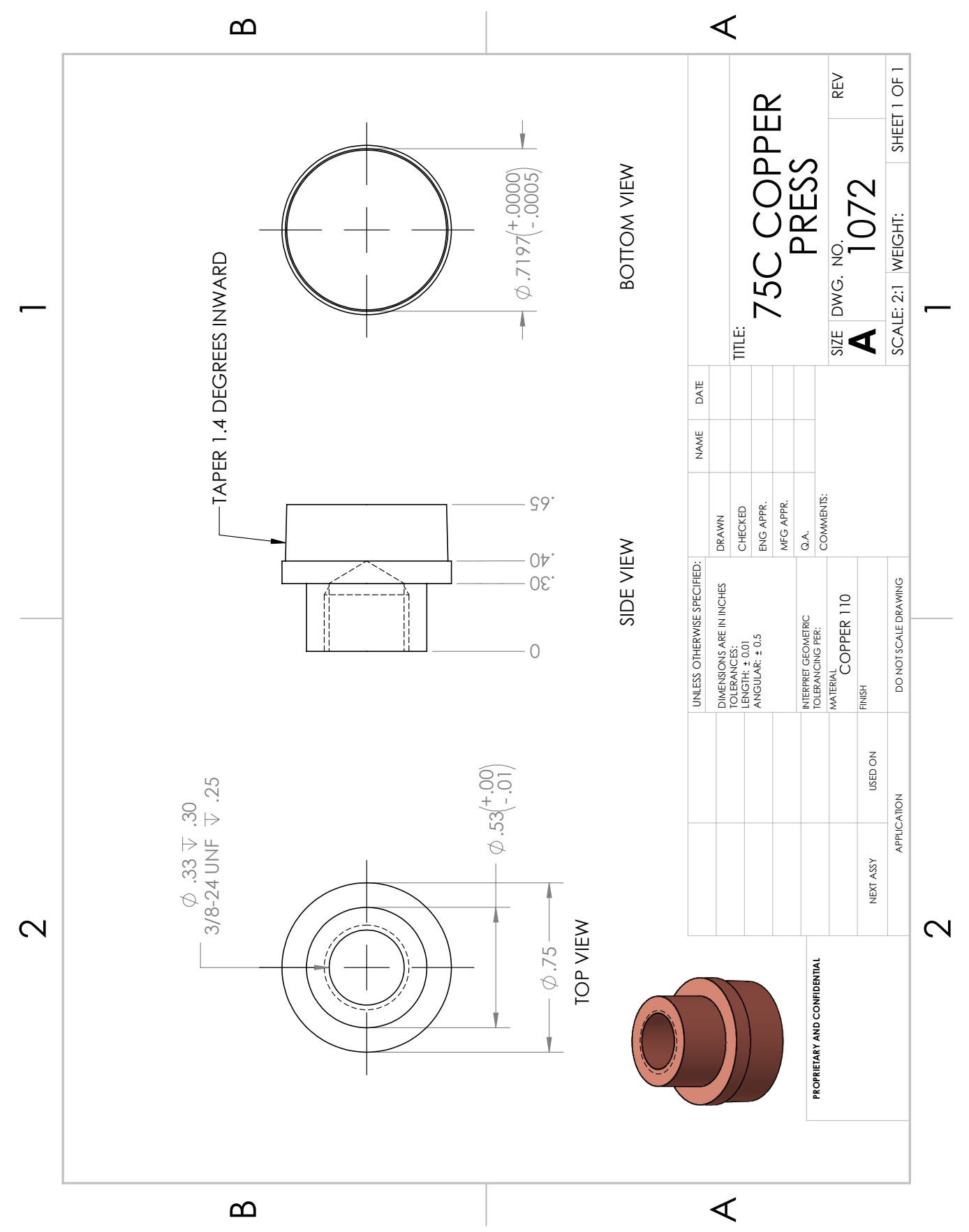




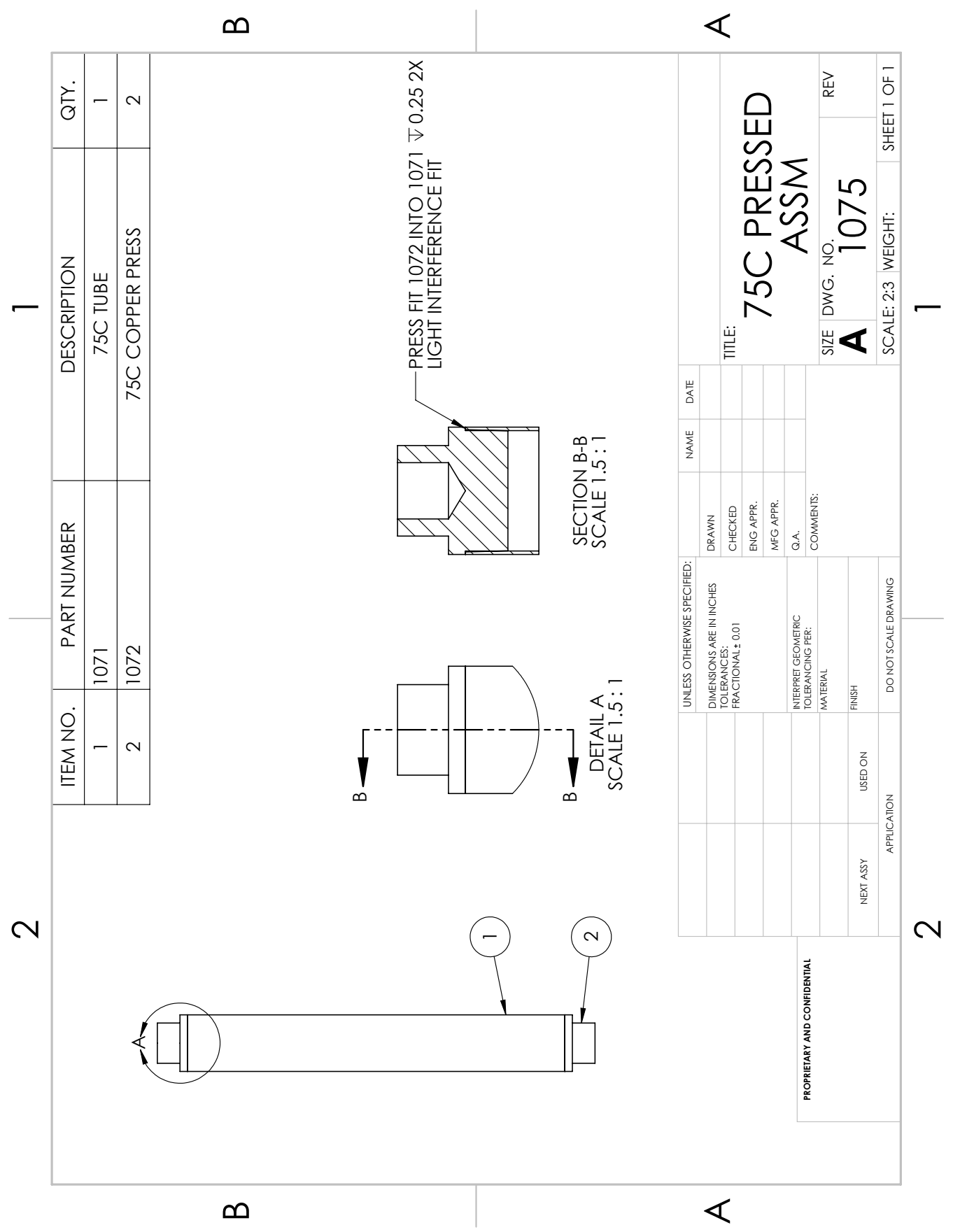




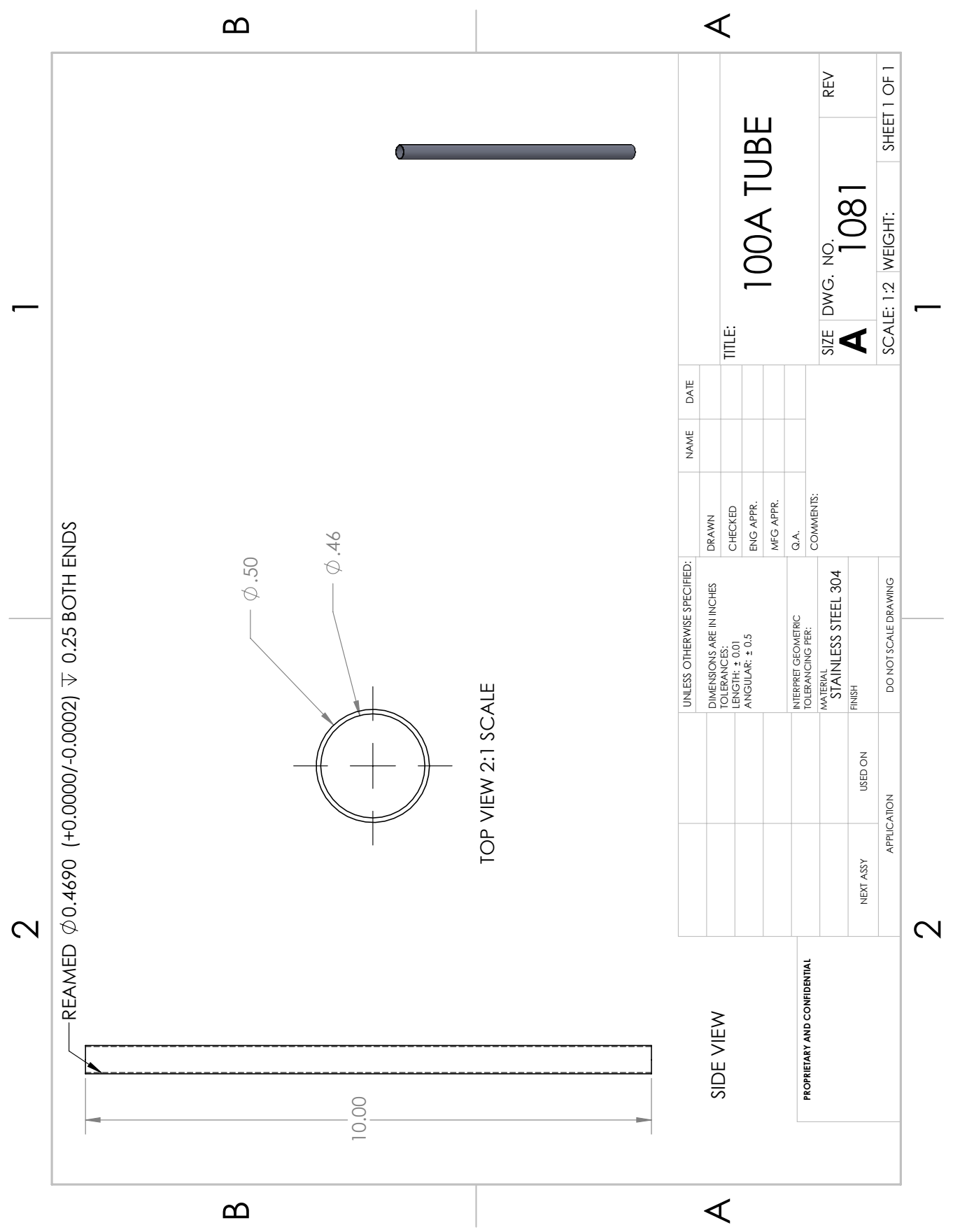




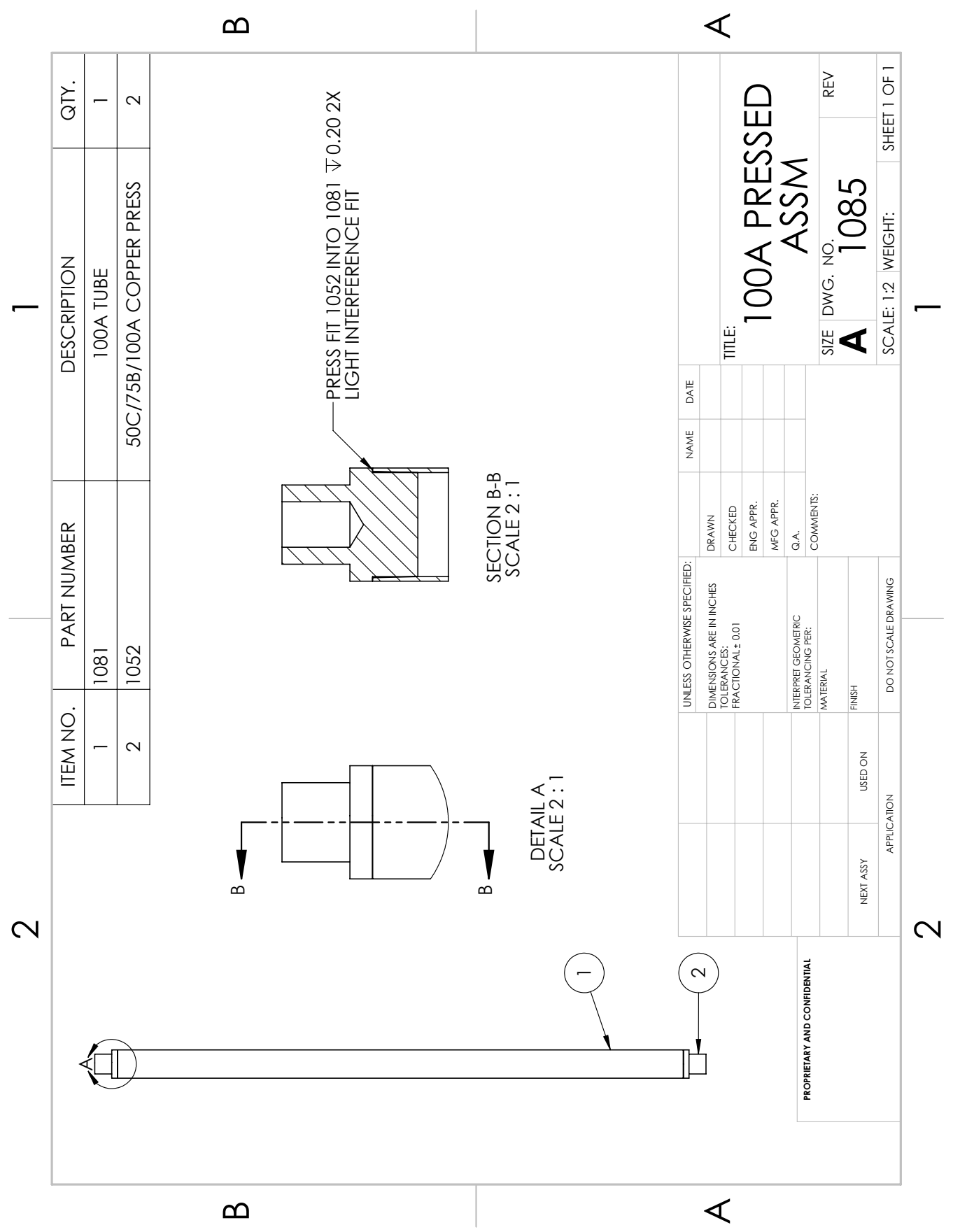




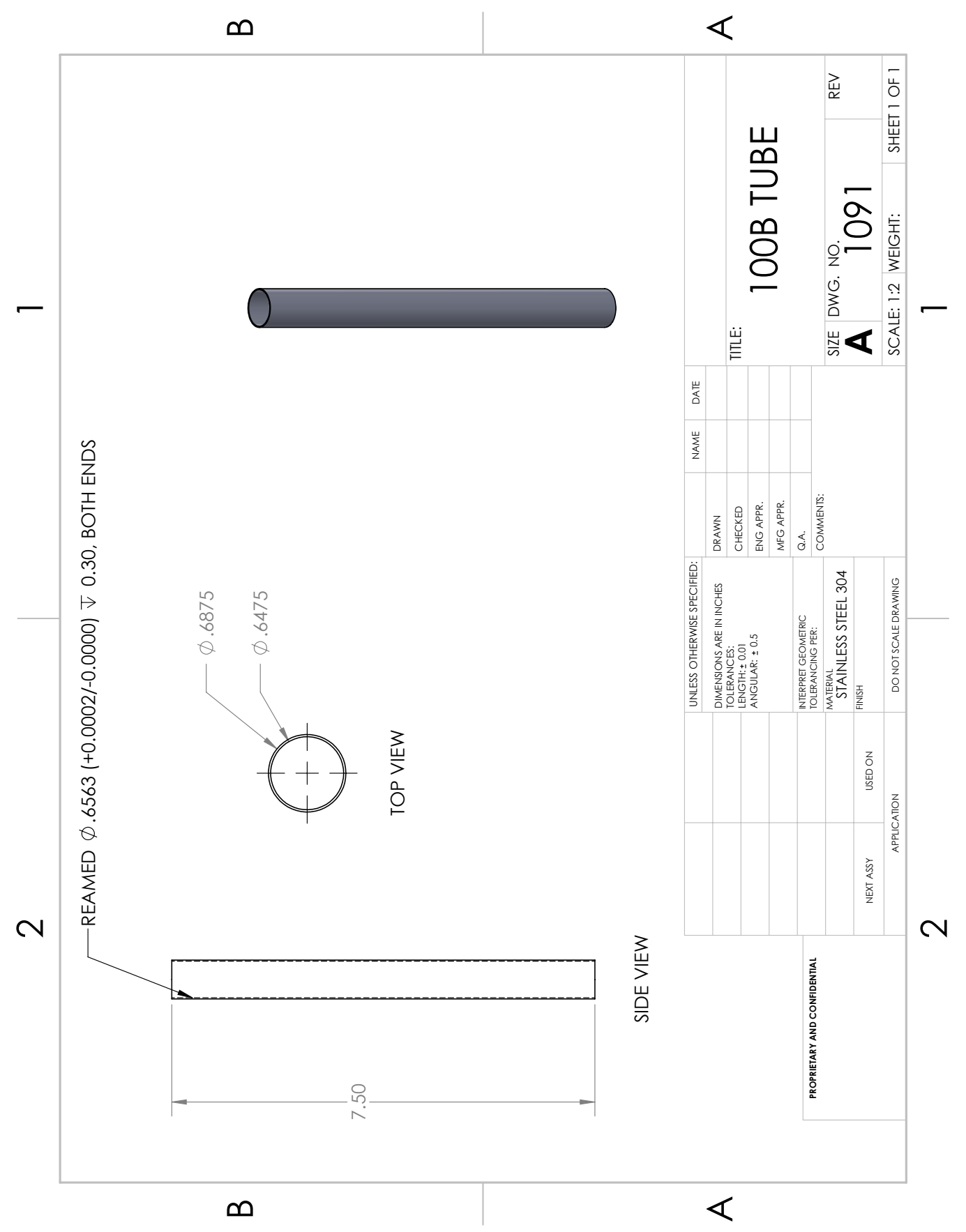




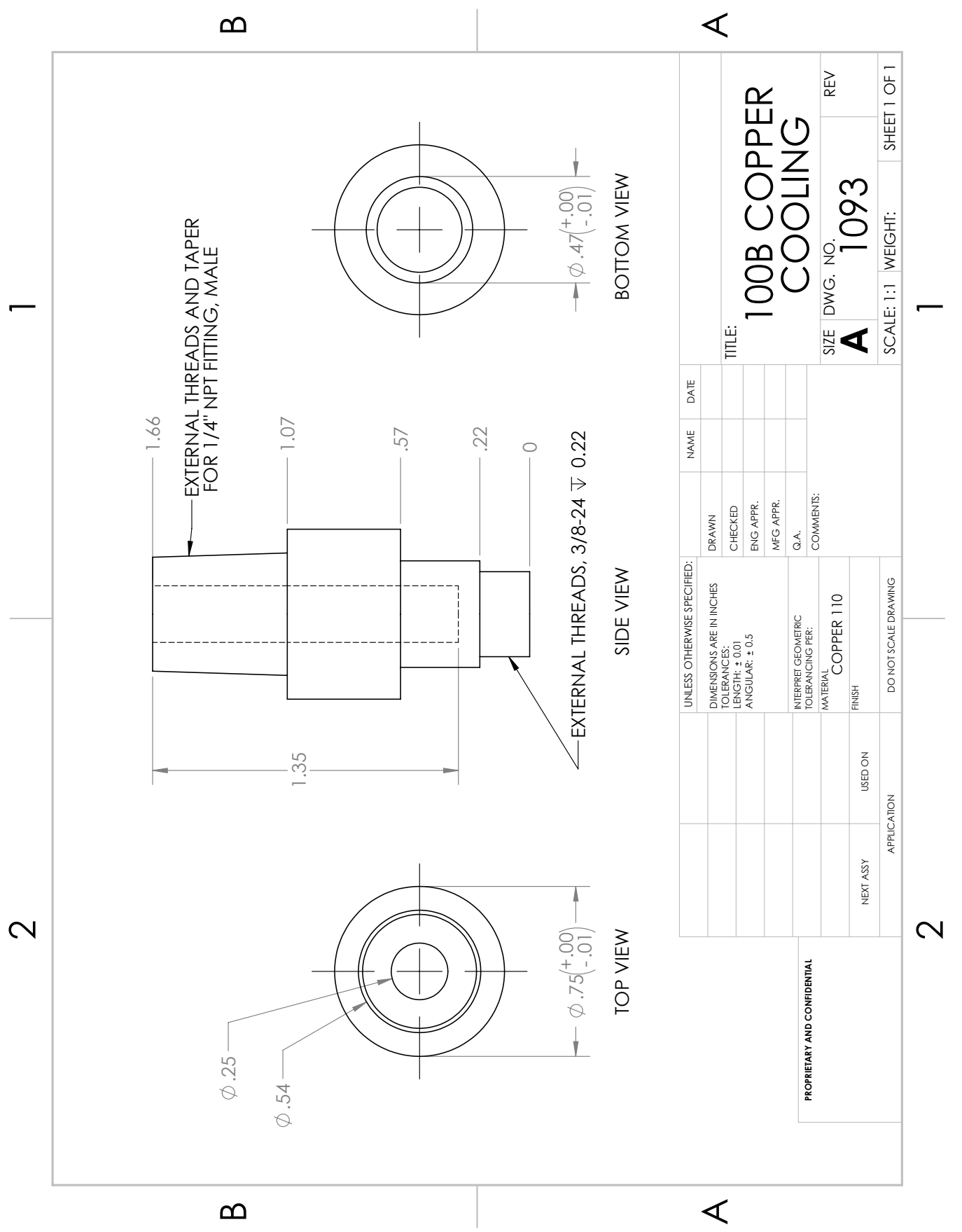




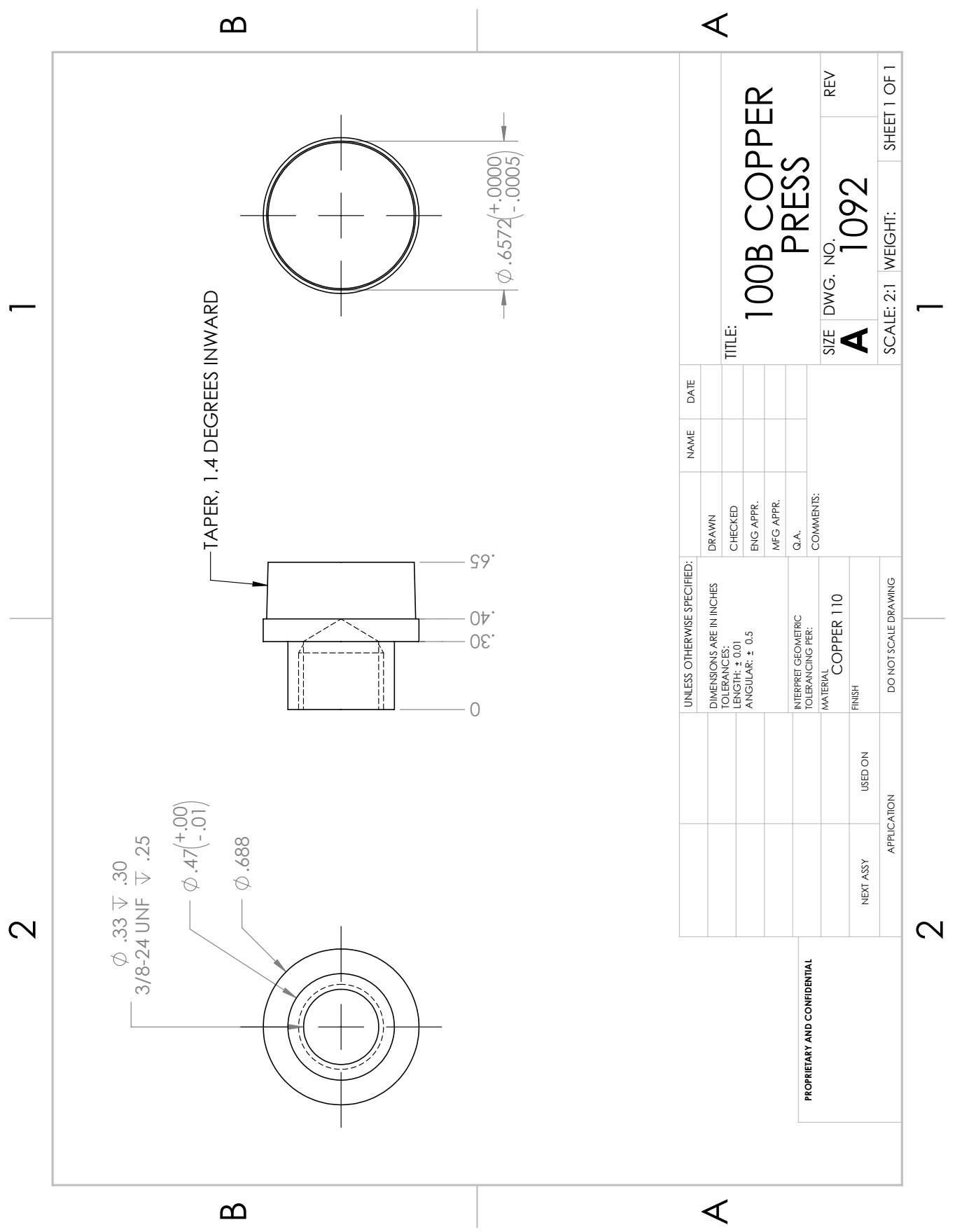




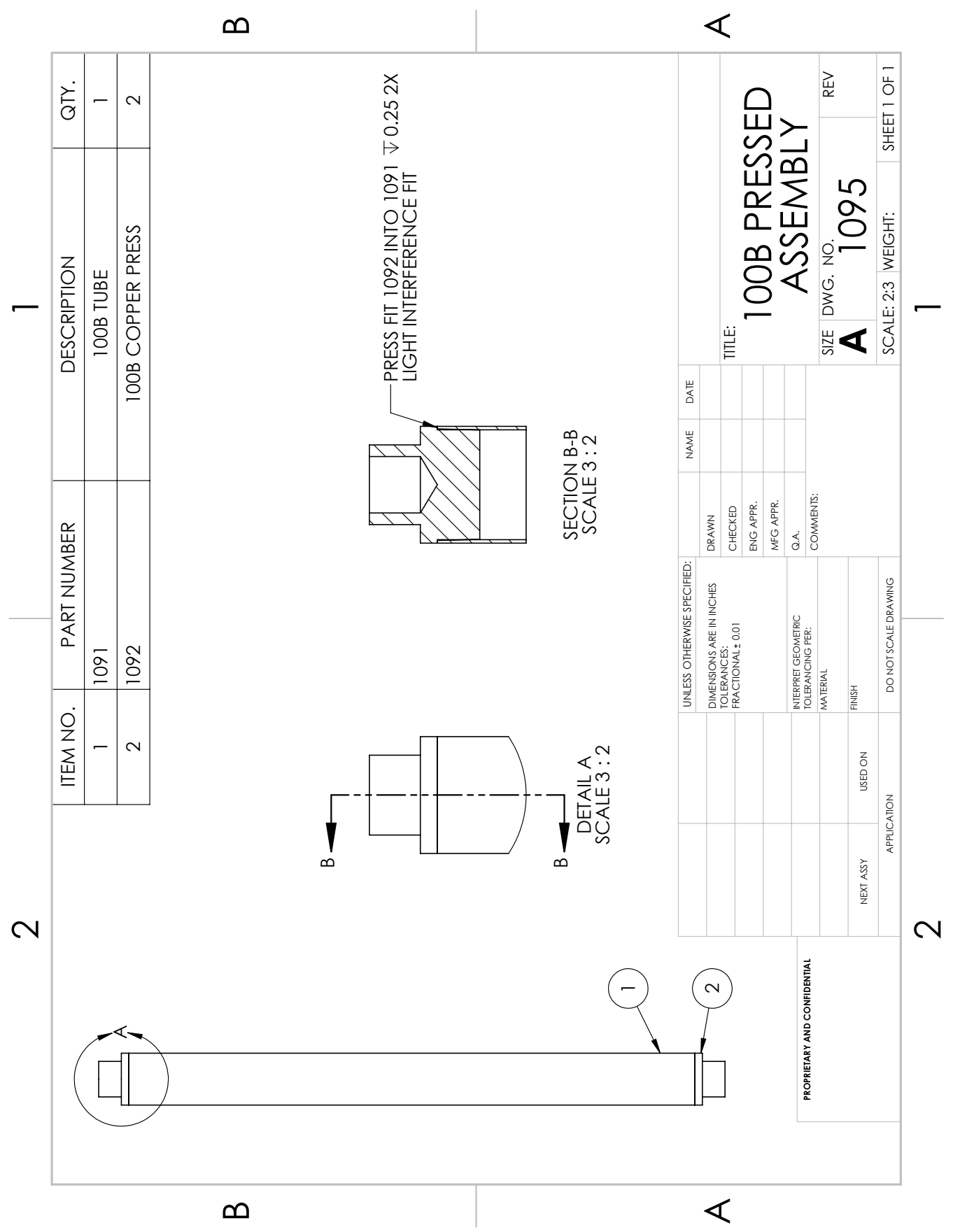




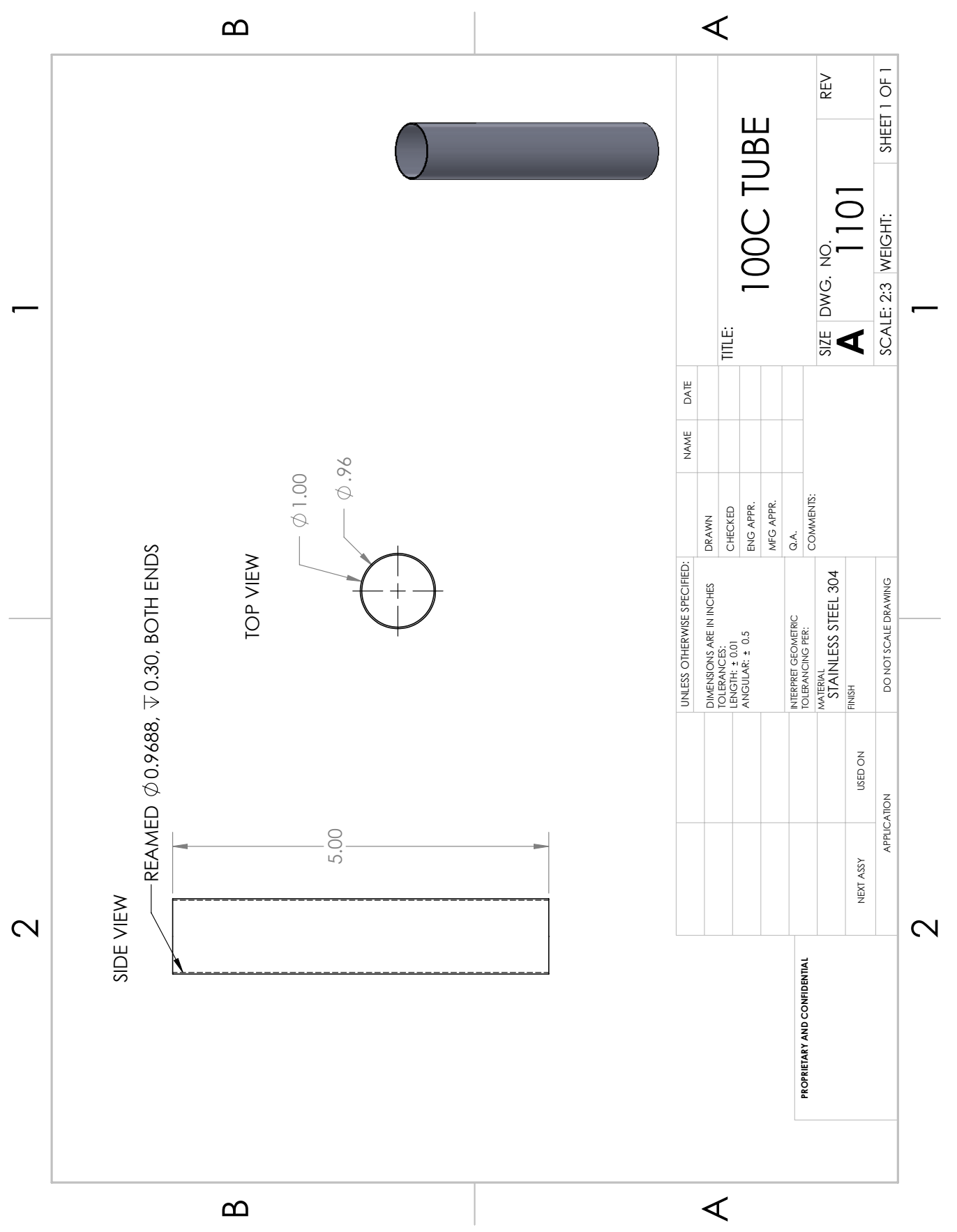




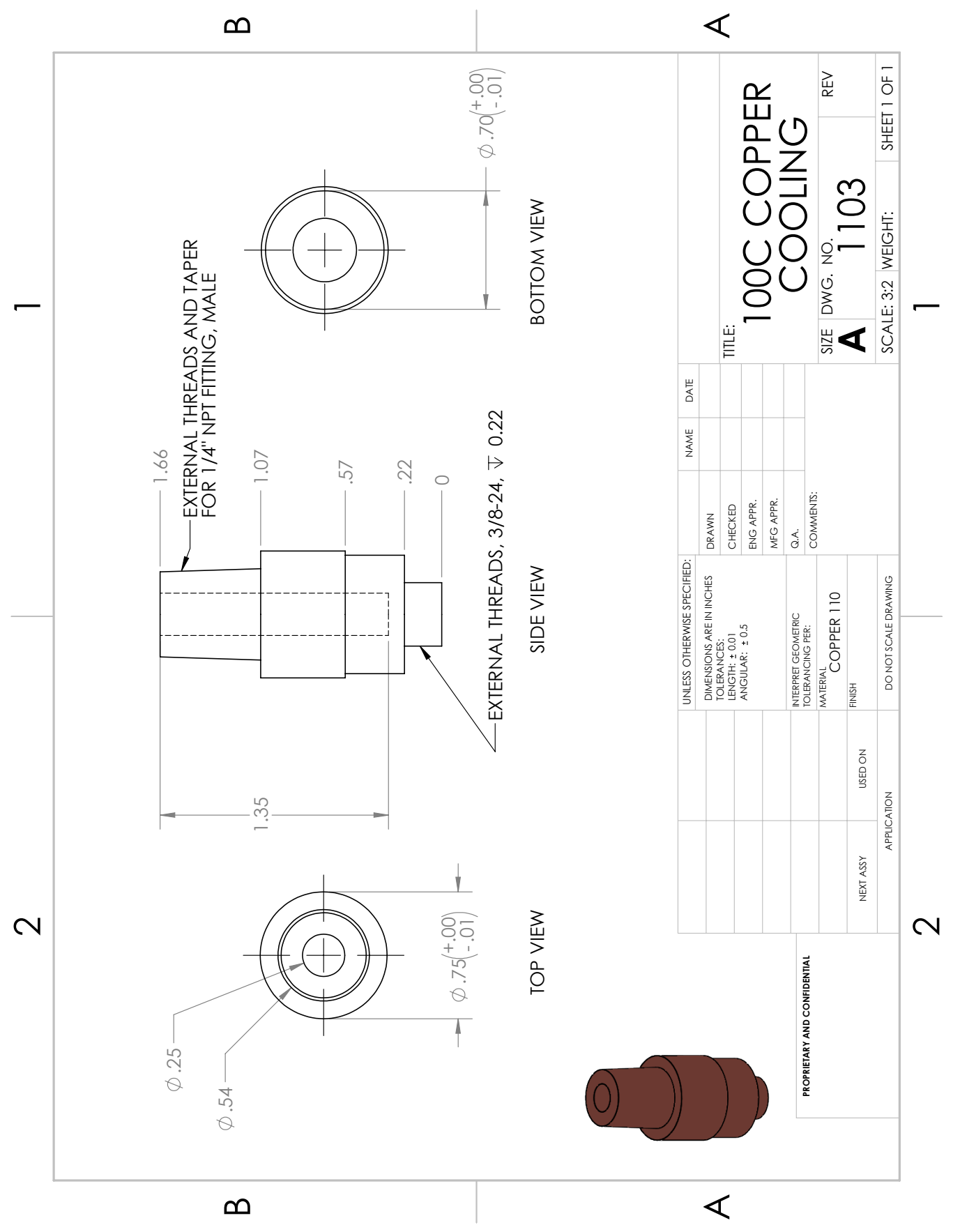




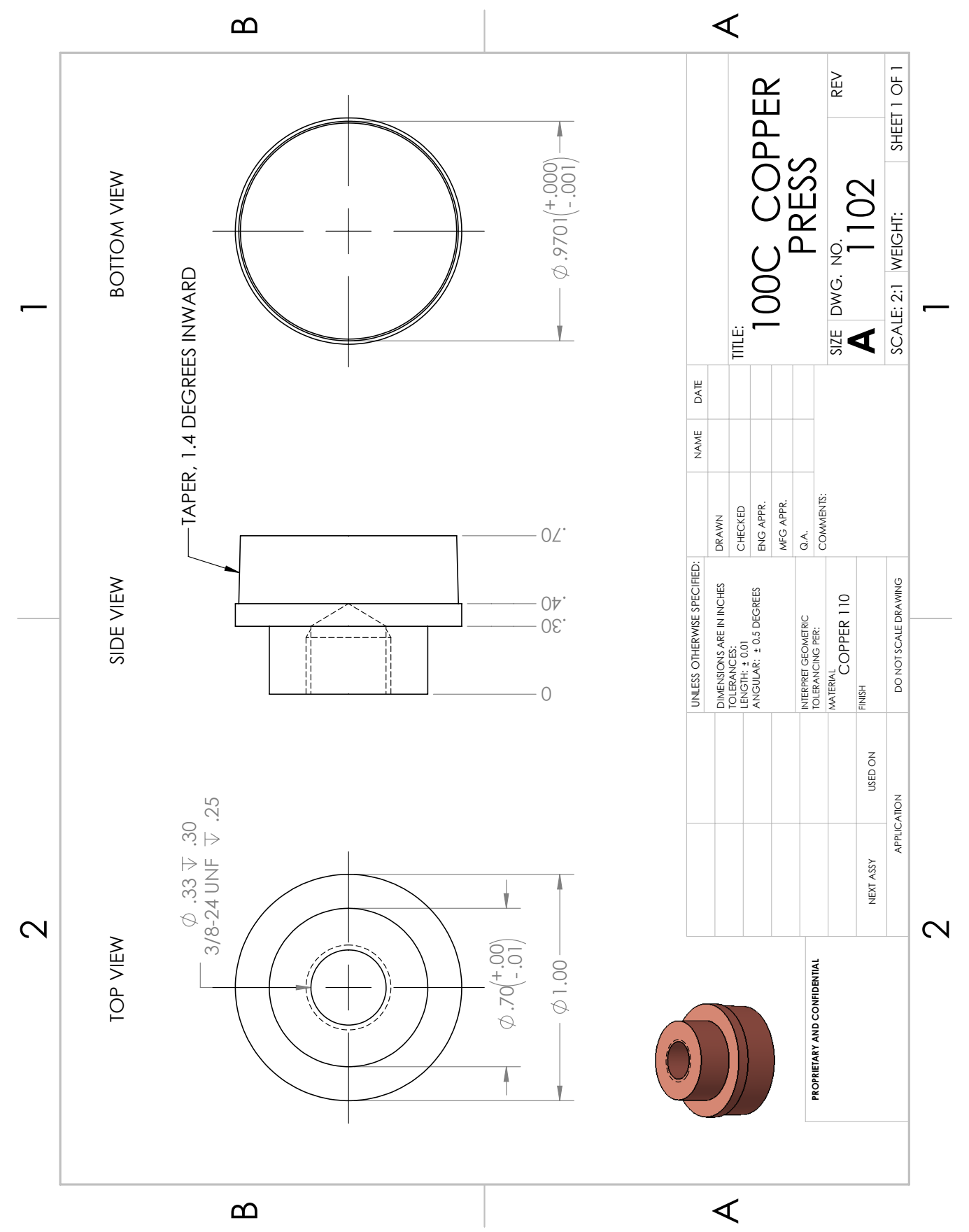




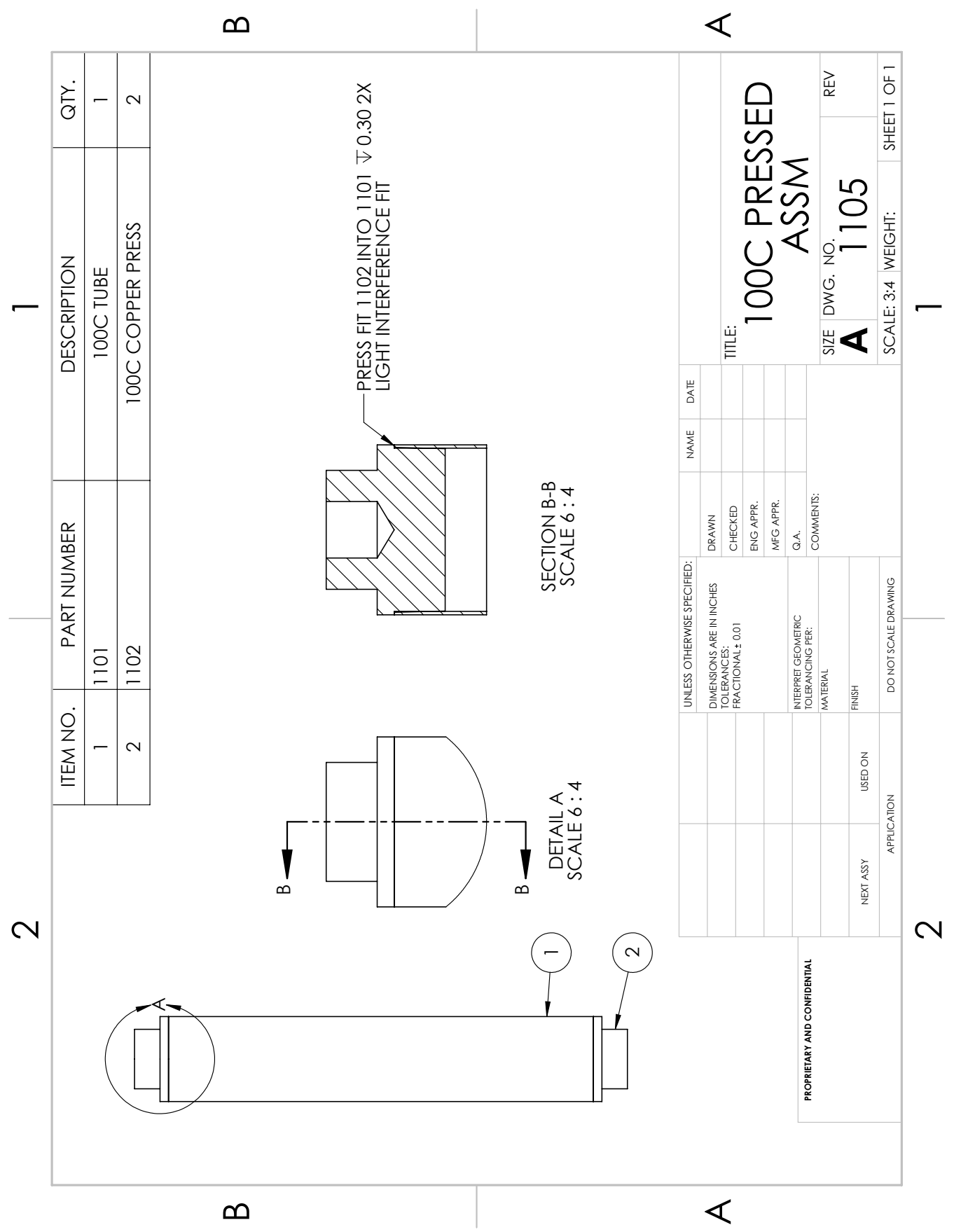




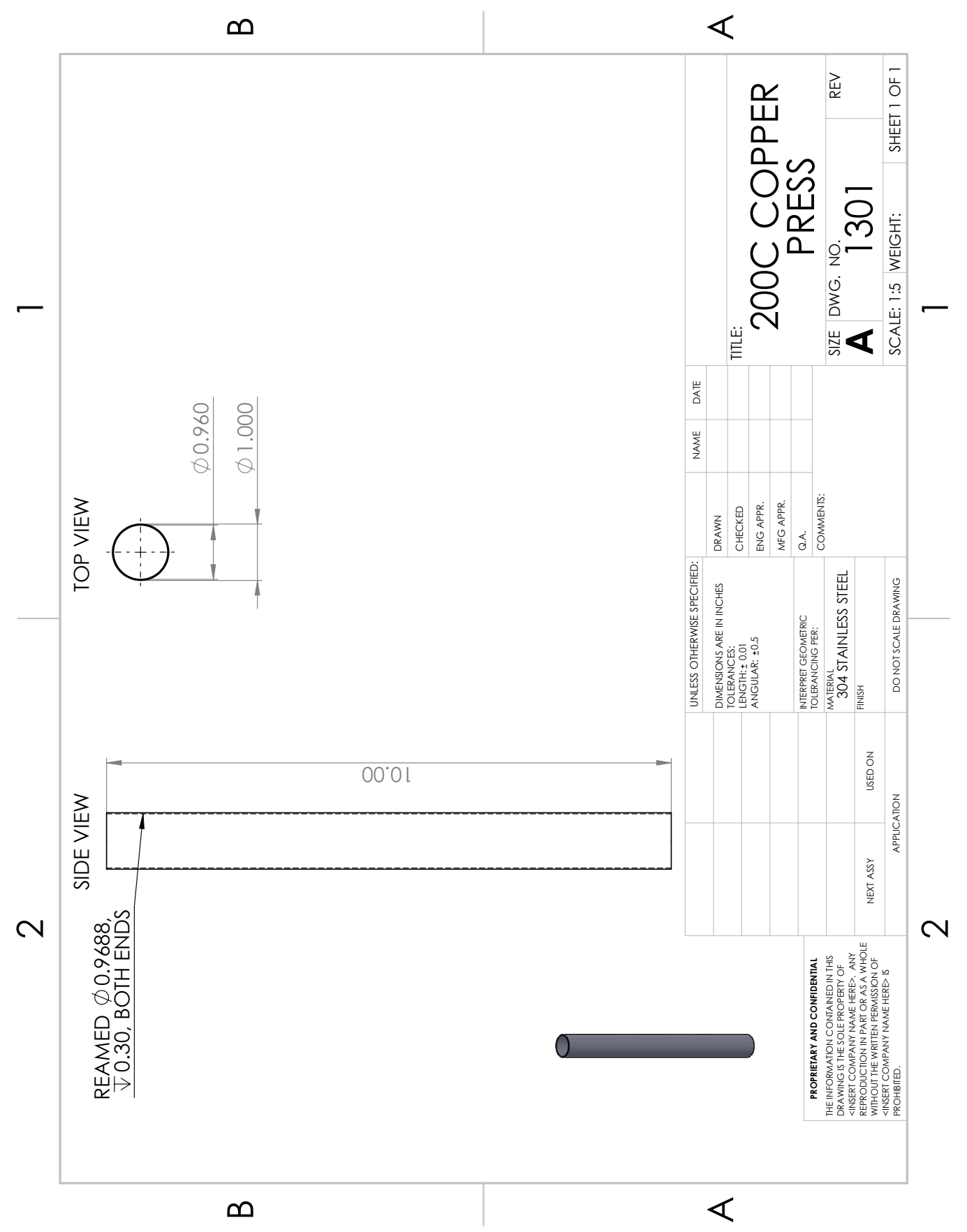


Appendix $H$

\section{EXPERIMENTAL CHECKLISTS}




\section{Heated Particle Ignition Test Check List $-\mathbf{n}-\mathrm{C}_{6} \mathrm{H}_{14}-\mathrm{O}_{2}-\mathrm{N}_{2}$}

Rev. November 15, 2019

Series:

Shot \#:

Date:-

RESULT:

Gas:

Mixture:

Equivalence ratio:_
$\mathrm{P}_{\text {preshot }}=$ Torr

$\mathrm{P}_{\text {ign }}$ :_ Torr

$\mathrm{P}_{\text {post-shot }}$ :

Torr

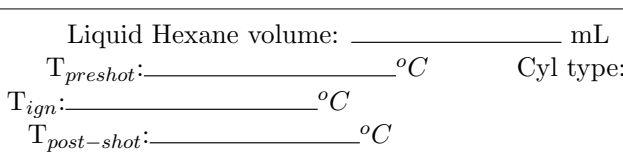

\section{Experiment Preparation:}

Turn on:

_ vacuum pump _ gas bottles _ hand valves _ Pressure amplifiers _ Magna PS

— heat exchanger on (MUST BE DONE 1ST) _ heat exchanger hand valves (MUST BE DONE 2ND)

_ Phantom camera _ Pyrometer _ C Coherent controller and laser

$\square$ Load software for the Phantom and Labview

$\square$ Ensure that lens cap has been removed from Phantom camera

\section{Experiment:}

$\square$ Open V2,V3,V4, and shutter and evacuate to $<100$ mtorr, then zero the Baratron gauge and amplifier

$\square$ Note voltage on pressure gauge at vacuum: $\mathrm{V}$, save trace as:

$\square$ Close V2 and V4 and turn on warning lights

$\square$ Open septum hand valve

$\square$ Fill hexane through septum using glass syringe to desired pressure

$\square$ Close septum hand valve

$\square$ Close door

$\square$ Fill with appropriate gases (fill order: liquid hexane, diluent, oxidizer), closing V2 and evacuating the line between gases by pushing VACUUM and FAST FILL together on the gas panel until vacuum gauge reads $<100$ mtorr

\begin{tabular}{|c|r|r|r|r|}
\hline Gas & Target Fraction & Target Partial Pressure & Target Final Pressure & Final Pressure \\
\hline Starting Pressure & - & - & - & Torr \\
\hline $\mathrm{n}^{-} \mathrm{C}_{6} \mathrm{H}_{14}$ & & Torr & Torr & Torr \\
\hline $\mathrm{N}_{2}$ & & Torr & Torr & Torr \\
\hline $\mathrm{O}_{2}$ & & Torr & Torr & Torr \\
\hline
\end{tabular}

$\square$ Close V2,V3 and evacuate the line and turn on mixer for 3 minutes 
Turn off mixer

$\square$ Note voltage on pressure gauge at final pressure: $\mathrm{V}$, save trace as:

$\square$ Take reference video with camera, save as:

$\square$ Open V3 and record the pressure and temperature

$\square$ Close V3

$\checkmark$ Set surface temperature on "cylinder-ramptopercT-pyromcontrol.vi" (must be less than 1050K) then press run. Tset:

$\square$ Set camera and oscilloscope to trigger

$\square$ Camera and oscilloscope should trigger off of pressure signal when ignition causes a pressure spike (camera triggers from oscilloscope trig out)

$\square$ Press spot control, stop scanning, STOP ALL on front panel of "cylinder-ramptopercT-pyromcontrol.vi" to end Labview script

$\square$ Increment shot counter on front panel of "cylinder-ramptopercT-pyromcontrol.vi"

$\square$ Save the interferometer video as:

Save oscilloscope trace as:

After gas has cooled (below 30C), record post-shot pressure and temperature

Note maximum pressure from pressure trace

$\square$ To evacuate vessel:

- If ignition: open V4 to evacuate

- if no ignition: backfill gas line with $\mathrm{N} 2$, open V2 and fill with N2 until dynamic pressure voltage has doubled $(0.9 \mathrm{~V})$ (dilute with $\mathrm{N}_{2}$ to a total pressure of 1500 torr $/ 2 \mathrm{~atm}$ - use dynamic gauge) and then open V4 to evacuate

\section{Experiment Shut-Down:}

$\square$ Check that all information has been entered in the test matrix, save

$\square$ Turn off:

— vacuum pump _ gas bottles _ hand valves _ Pressure amplifiers _ Magna PS

— heat exchanger hand valves off (MUST BE DONE 1st) _ heat exchanger off (MUST BE DONE 2nd)

_ Phantom camera _ Pyrometer _ Coherent controller and laser

$\square$ Put lens cap on Phantom camera and cover all lenses and mirrors

\section{NOTES:}




\section{Heated Cylinder Ignition Test Check List - Jet A-O $\mathrm{O}_{2}-\mathrm{N}_{2}$}

Rev. October 21, 2020

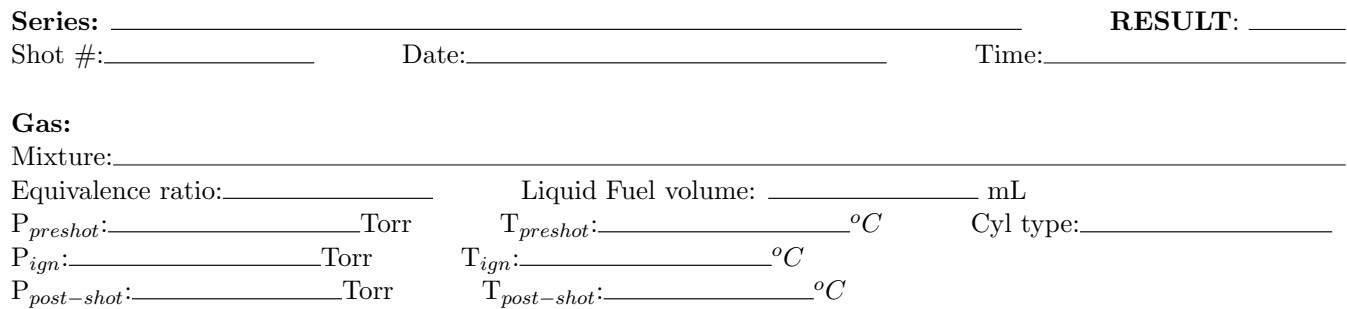

\section{Experiment Preparation:}

- Leak check vessel before experiment $(<0.1$ Torr/min)

- Turn on:

_ vacuum pump _ gas bottles _ hand valves _ Pressure amplifiers _ Magna PS

— heat exchanger on (MUST BE DONE 1ST) _ heat exchanger hand valves (MUST BE DONE 2ND)

_ Phantom camera _ Pyrometer _ C Coherent controller and laser

- Load software for the Phantom and Labview

- Ensure that lens cap has been removed from Phantom camera

- Attach open sample bottle to V6

\section{Experiment:}

1. Open V2,V3, and V4 and evacuate to $<100$ mtorr, then zero the Baratron gauge and amplifier

2. Note voltage on pressure gauge at vacuum:___ V, save trace as:

3. Close V2 and V4, open V5 and turn on warning lights

4. Open septum hand valve

5. Fill Jet A $\left(150 \mathrm{~mL} / 3 \mathrm{~kg} / \mathrm{m}^{3}\right)$ through septum using large capacity syringe

6. Close septum hand valve

7. Close door

8. Let vaporize for 10 mins, then measure partial pressure

9. Fill with appropriate gases (fill order: liquid Jet A, diluent, oxidizer), closing V2 and evacuating the line between gases by pushing VACUUM and FAST FILL together on the gas panel until vacuum gauge reads $<100$ mtorr 


\begin{tabular}{|c|r|r|r|r|}
\hline Gas & Target Fraction & Target Partial Pressure & Target Final Pressure & Final Pressure \\
\hline Starting Pressure & - & - & - & Torr \\
\hline Jet A & & Torr & Torr & Torr \\
\hline $\mathrm{N}_{2}$ & & Torr & Torr & Torr \\
\hline $\mathrm{O}_{2}$ & & Torr & Torr & Torr \\
\hline
\end{tabular}

1. Close V2,V3 and evacuate the line and turn on mixer for 3 minutes

2. Turn off mixer

3. Note voltage on pressure gauge at final pressure: $\mathrm{V}$, save trace as:

4. Take reference video with camera, save as:

5. Open V3 and record the pressure and temperature

6. Close V3

7. Set surface temperature on "cylinder-ramptopercT-pyromcontrol.vi" (must be less than 1050K) then press run. Tset:

8. Set camera and oscilloscope to trigger

9. Camera and oscilloscope should trigger off of pressure signal when ignition causes a pressure spike (camera triggers from oscilloscope trig out)

10. Press spot control, stop scanning, STOP ALL on front panel of "cylinder-ramptopercT-pyromcontrol.vi" to end Labview script

11. Increment shot counter on front panel of "cylinder-ramptopercT-pyromcontrol.vi"

12. Save the interferometer video as:

13. Save oscilloscope trace as:

14. After gas has cooled (below 30C), record post-shot pressure and temperature

15. Note maximum pressure from pressure trace

16. To evacuate vessel:

17. Push any remaining fuel out of vessel by:

(a) Loading vessel with $\mathrm{N} 2$ to $1.5 \mathrm{~atm} /-.675 \mathrm{~V}$

(b) Open V6 ('Fuel Out') to push remaining jet fuel into sample bottle

(c) Push fuel out by continuing to load with N2 (as needed)

(d) Close V6

18. Evacuate vessel to $<100$ mTorr to eliminate any trapped fuel

19. Close sample bottle

20. Remove sample bottle, dump excess jet fuel to waste bottle in bottle farm

\section{Experiment Shut-Down:}


- Check that all information has been entered in the test matrix, save

- Turn off:

_ vacuum pump _ gas bottles _ hand valves _ Pressure amplifiers _ Magna PS

_ heat exchanger hand valves off (MUST BE DONE 1st) _ _ heat exchanger off (MUST BE DONE 2nd)

_ Phantom camera _ Pyrometer _ Coherent controller and laser

- Put lens cap on Phantom camera and cover all lenses and mirrors

NOTES: 


\section{INDEX}

F

figures, $17,18,24,27,30,31,35,36,39,41,42,44,46,50,53,57,59,60,66,68$, 70, 78, 80, 84, 87, 89, 95, 97, 99, 101, 103, 108, 112, 113, 132, 137, 139, $142,144,150,151,164,165,192,193,195,197,198,202,203,206,209$. 221, 222, 224, 235, 237, 244, 246, 253, 255, 257, 259, 263, 265, 266, 407, 408

$\mathrm{T}$

tables, 27, 66, 92, 98, 122, 130, 137, 145, 156, 159, 166, 170, 171, 176, 180, 183, 260, 264, 283, 285, 322, 401 